Portland State University

PDXScholar

Dissertations and Theses

Dissertations and Theses

Spring 6-19-2017

\title{
Rhyolite Petrogenesis at Tower Mountain Caldera, OR
}

\author{
Elizabeth Ann Brown \\ Portland State University
}

Follow this and additional works at: https://pdxscholar.library.pdx.edu/open_access_etds

Part of the Geology Commons

Let us know how access to this document benefits you.

\section{Recommended Citation}

Brown, Elizabeth Ann, "Rhyolite Petrogenesis at Tower Mountain Caldera, OR" (2017). Dissertations and Theses. Paper 3997.

https://doi.org/10.15760/etd.5881

This Thesis is brought to you for free and open access. It has been accepted for inclusion in Dissertations and Theses by an authorized administrator of PDXScholar. Please contact us if we can make this document more accessible: pdxscholar@pdx.edu. 
Rhyolite Petrogenesis at Tower Mountain Caldera, OR

\section{by}

Elizabeth Ann Brown

A thesis submitted in partial fulfillment of the requirements for the degree of

Master of Science

in

Geology

Thesis Committee:

Martin J. Streck, Chair

John Schumacher

Richard Hugo

Portland State University

2017 


\begin{abstract}
Tower Mountain Caldera is the main feature of an Oligocene volcanic field located in the Umatilla National Forest, eastern Oregon. It is perfectly suited to investigate models of rhyolite petrogenesis as all of the important rock components for evaluating generation models are present in a single location and thus are presumably related; basalts, intermediate igneous rocks (which consist of older plutons and younger volcanic rocks, which are $~$ coeval with rhyolites), metamorphic basement rocks of significant grade, and rhyolites of varying composition. The formation of the caldera produced the Dale Tuff, which comprises the intra-caldera and outflow facies. ${ }^{40} \mathrm{Ar} /{ }^{39} \mathrm{Ar}$ dating places the age of the tuff at 32.66 $\pm 0.36 \mathrm{Ma}$. Post-caldera rhyolites erupted along apparent ring fractures and elsewhere. Radiometric U-Pb dating of zircons from three of these rhyolites yielded ages of $32.167 \pm 0.020 \mathrm{Ma}(\# \mathrm{CH} 07 \mathrm{a}), 31.798 \pm 0.012 \mathrm{Ma}(\# \mathrm{TM}$ ), and $31.426 \pm 0.016$ Ma (\#CH08a). All rhyolites at Tower Mountain range from low to high silica varieties. Some of the post-caldera rhyolites are chemically similar to the Dale Tuff, such as sample $\mathrm{CH} 07 \mathrm{a}$, and have compositions typical of rhyolites of calc-alkaline volcanic centers (I-type rhyolites), while others are similar to A-type rhyolites (CH08a and TM5). The ages indicate that the calc-alkaline rhyolites were followed by the A-type rhyolites. The petrogenetic relationships between the various rocks types were evaluated. Partial melt modeling based on experimental melts produced from crustal material indicates that batch partial melting of metamorphosed high silica crustal material modified by the addition of more primitive mafic material by assimilation/contamination is the most likely source for the Tower Mountain rhyolites.
\end{abstract}




\section{ACKNOWLEDGEMENTS}

Sincere thanks to my advisor, Dr. Martin Streck, for his patience and guidance. Thank you to Dr. John Schumacher and Dr. Rick Hugo for serving on my committee. A special extra thanks to Rick for training me in the use of the scanning electron microscope.

Thank you to Dr. Mark Schmitz at Boise State University for his help with U-Pb dating.

I have so much gratitude for the friendship, comradery, and help provided by my fellow students, particularly Karla Farley, Hillarie Sales, and Max Bordal. Team ANTS!

Thank you to my parents, who let me dig holes in the backyard as a kid, encouraged my rock collecting, and taught me the value of hard work.

Thank you to my husband, Justin Brown, for your support and patience, and for believing in me. 


\section{TABLE OF CONTENTS}

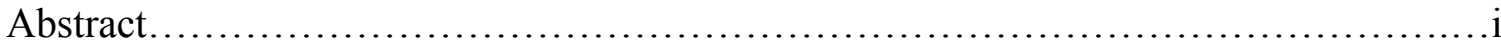

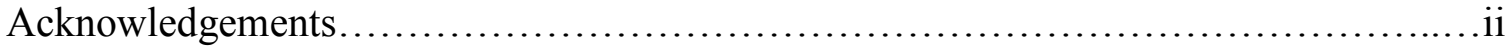

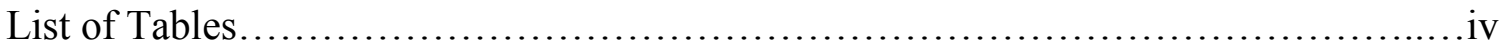

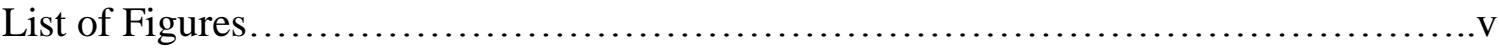

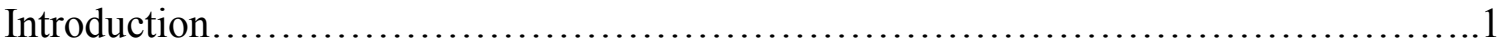

Background..............................................................

Methods....................................................................... 9

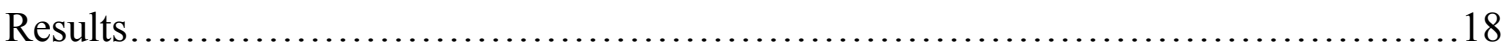

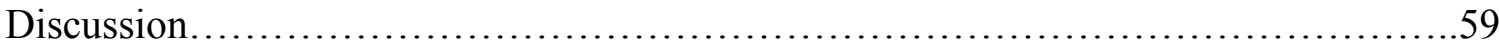

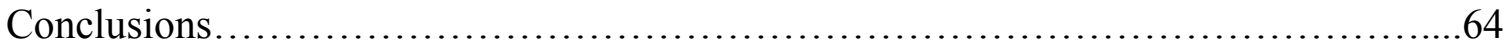

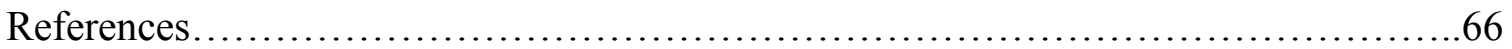

Appendices

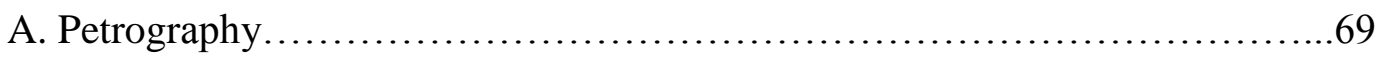

B. Geochemistry................................................... 79

C. Experimental Melts..................................................... 91

D. Partial Melt Modeling................................................167

E. Fractional Crystallization of Basalt.......................................268

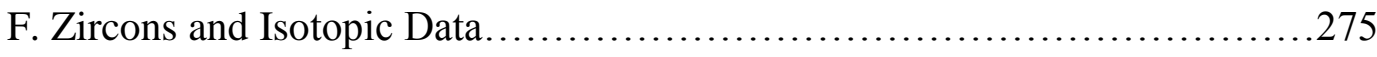




\section{LIST OF TABLES}

Table 1: Trace element partition coefficients for melting calculations ................16

Table 2: Trace element partition coefficients for basalt fractional crystallization

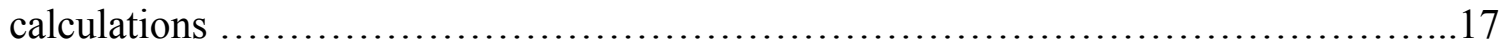

Table 3: Unnormalized trace element concentrations of basalt fractionation.............46

Table 4: Summary of partial melt modeling......................................55 


\section{LIST OF FIGURES}

Figure 1: Melt expulsion model for the generation of crystal poor rhyolites..............2

Figure 2: Simplified geologic map of the study area..................................

Figure 3: Stages of caldera formation................................................

Figure 4: Tower Mountain volcanic samples plotted onto a total alkali silica diagram....18

Figure 5: Plane light image of a thin section of sample CH08a......................20

Figure 6: Backscatter electron image of phenocrysts within sample CH08a............20

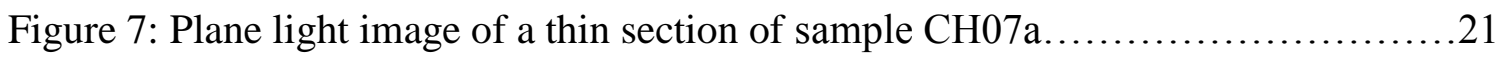

Figure 8: Backscatter electron image of phenocrysts within sample $\mathrm{CH} 07 \mathrm{a} \ldots \ldots \ldots \ldots . . .21$

Figure 9: Plane light image of a thin section of sample TM5 ........................22

Figure 10: Backscatter electron image of phenocrysts within sample TM5..............23

Figure 11: Plane light image of a thin section of sample $\mathrm{CH} 31 \ldots \ldots \ldots \ldots \ldots \ldots \ldots \ldots . \ldots 24$

Figure 12: Trace element spider diagram of TM rhyolites............................25

Figure 13: Trace element spider diagrams of rhyolite groups.........................27

Figure 14: Element/element scatterplots for representative rhyolite samples.............30

Figure 15: Plane light image of a thin section of sample G314-09-2 ..................31

Figure 16: Backscatter electron image of sample G314-09-2 .........................32

Figure 17: Trace element concentrations of basaltic and intermediate

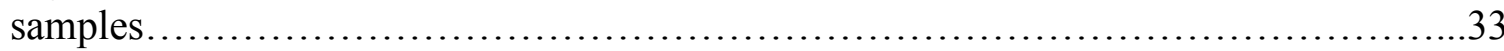

Figure 18: Ages in millions of years of rhyolite samples..............................34

Figure 19: ${ }^{40} \mathrm{Ar} /{ }^{39} \mathrm{Ar}$ weighted plateau age for the Dale Tuff...........................34

Figure 20: Plane light image of a thin section of sample MS12-47 .....................35

Figure 21: Backscatter electron image MS12-47...................................36 
Figure 22: Plane light image of a thin section of sample TM13-5................... 37

Figure 23: Plane light image of a thin section of sample TM13-7...................38

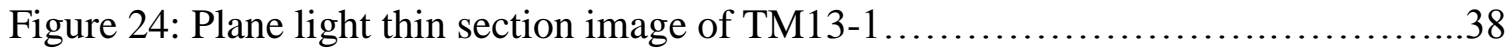

Figure 25: Spider diagram of the trace element concentrations of metamorphic

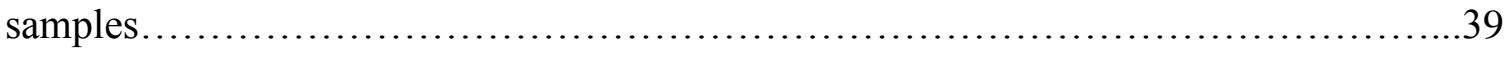

Figure 26: Plane light thin section image of sample G314-09-4....................41

Figure 27: Plane light thin section image of TM13-4...........................41

Figure 28: Plane light thin section image of sample TM13-3.....................42

Figure 29: Backscatter electron image of sample TM13-3........................43

Figure 30: Plane light image of a thin section of sample TM13-9.................44

Figure 31: Spider diagram of the trace element concentrations of the silicic and intermediate plutonic samples................................................. 45

Figure 32: Spider diagram of the results of fractional crystallization modeling of basalt

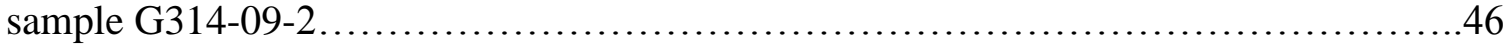

Figure 33: Energy dispersive X-ray spectrograph map of experimental melt MS1_.....49

Figure 34: Energy dispersive X-ray spectrograph map of experimental melt MS4 .......50

Figure 35: Energy dispersive X-ray spectrograph map of experimental melt MS5 ......51

Figure 36: Energy dispersive X-ray spectrograph map of experimental melt MS13.....52

Figure 37: Spider diagram for the partial melting of experimental melt MS4...........53

Figure 38: Spider diagram for the partial melting of experimental melt MS13.........54

Figure 39: Experimental melt glass compositions plotted onto a TAS diagram..........58

Figure 40: Modified experimental melt glass compositions plotted onto a TAS

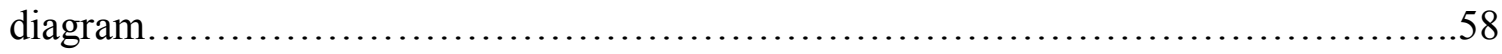




\section{INTRODUCTION}

There are two basic models for the petrogenesis of silicic (rhyolitic) magmas. The first model describes a process wherein the silicic melt is derived from basaltic parent magma, either by fractional crystallization alone, or by fractional crystallization combined with assimilation of crustal country rock. This model satisfactorily describes the production of small magma batches. In the other model, which can explain the production of larger magma bodies $\left(>10^{3} \mathrm{~km}^{3}\right)$, basaltic melts provide heat for the partial melting of crustal rocks (Riley et al., 2001; Bachmann \& Bergantz, 2004). Under certain conditions, silicic magmas can be generated via interactions between basaltic melts and metamorphic basement rocks (McCarthy \& Patiño Douce, 1997; Streck, 2002). This scenario also involves partial melting of metamorphic rocks, but in addition involves some material exchanges between the crustal protolith and mafic magmas. Either partial melting scenario may be followed by fractional crystallization. The melt expulsion model of Bachmann and Bergantz (2004), also called the "mush model" (Fig. 1), describes a scenario in which the production of voluminous crystal poor rhyolites is a result of the fractional crystallization or partial melting of compositionally intermediate plutonic bodies. As the intermediate magma body reaches approximately $50 \%$ crystallinity, the interstitial melt is of rhyolitic composition and the crystal mush becomes static. Being more buoyant, the melt escapes and rises to the top of the magma chamber forming an aphyric "rhyolite horizon". 
(a) Crystals kept in suspension by chaotic convection (low Reynolds \#) at crystallinities $<45 \%$.

(b) At $\sim 45-50 \%$ crystals, the interstitial melt is rhyolitic and convection comes to a halt, allowing melt extraction to begin by a combination of several processes (hindered settling, micro-settling, compaction) of the mush.

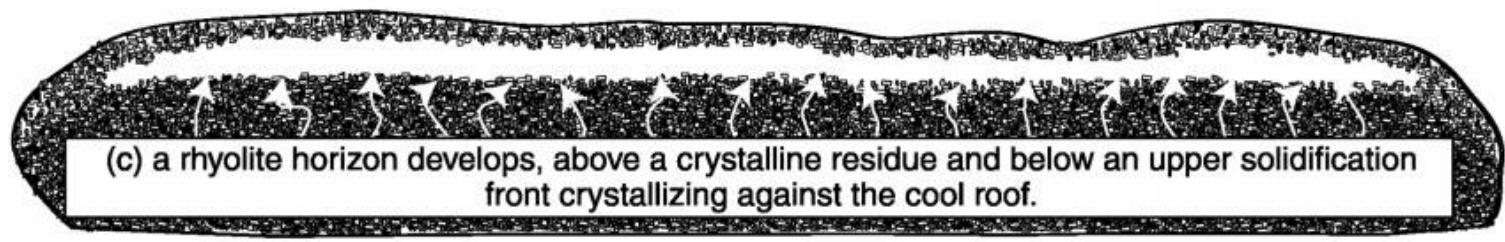

Fig. 1 The melt expulsion model, i.e. "mush model", for the generation of crystal poor rhyolites from Bachmann \& Bergantz (2004). This model describes a scenario in which the production of voluminous crystal poor rhyolites is a result of the fractional crystallization or partial melting of compositionally intermediate plutonic bodies.

The Tower Mountain Caldera in Eastern Oregon is perfectly suited to investigate models of rhyolite petrogenesis as all of the important rock components for evaluating generation models are present in a single location and thus are presumably related; basalts, intermediate igneous rocks (which consist of older plutons and younger volcanic rocks, which are approximately coeval with rhyolites), metamorphic basements rocks of significant grade, and rhyolites of varying composition. This study investigates the petrogenetic relationships between these components focusing on which rhyolite generation model applies. The complete record of components provides strong evidence for the origin of the rhyolites at Tower Mountain. 


\section{BACKGROUND}

\section{Geologic setting and formation of Tower Mountain Caldera}

The Tower Mountain (TM) Caldera (Fig. 2) is the main feature of a late Oligocene

bimodal volcanic field (Ferns, 2002) located in the Umatilla National Forest of eastern

Oregon. This area was mapped in 2001 by Mark Ferns of the Oregon Department of

Geology and Mineral Industries (DOGAMI).

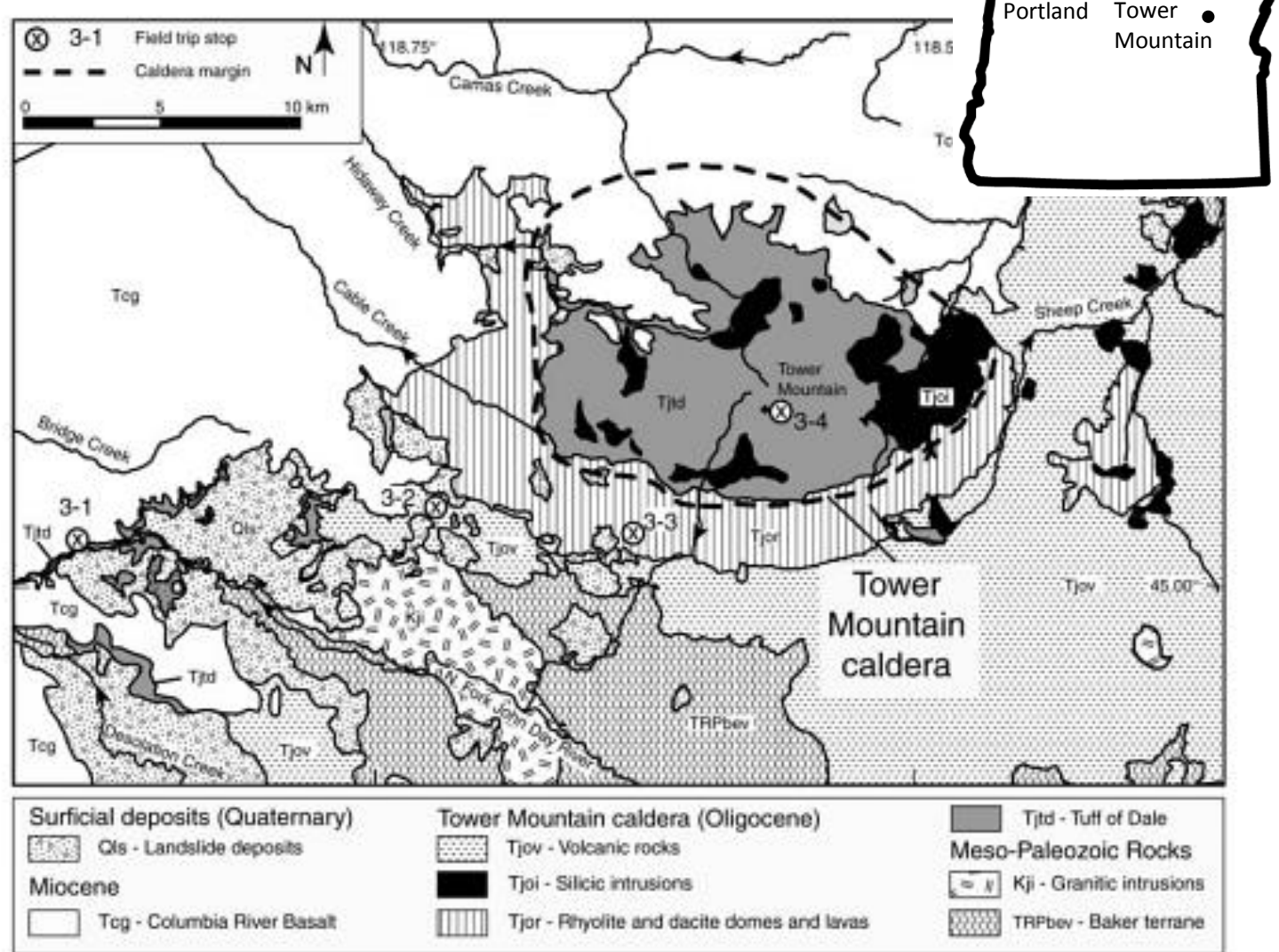

Fig. 2 Simplified geologic map of study area (McClaughry et al., 2009). The dashed line indicates the caldera margin. Note the onlapping of the Columbia River basalts to the north and west of the caldera. The intra-caldera facies consist primarily of silicic intrusions and the Dale Tuff. Facies to the south and east consist of other volcanics, metamorphosed accreted terranes, scattered silicic intrusions, and granitic intrusions, also called "stitching plutons". The map of Oregon (upper right) shows the location of the study area within the state. 
The TM volcanic field covers $500 \mathrm{~km}^{2}$ in the Blue Mountains Province. Based on previous age information and before our new age dating, the volcanic field was thought to have begun forming around 29.8 Ma where tholeiitic and alkali olivine basalts erupted at the junction between the southern margin of the Mountain Home Metamorphic Complex and the northern margin of the Baker Terrane (McClaughry et al., 2009). The Mountain Home Metamorphic Complex (MHMC) is described as a sequence of medium grade metamorphic rocks of both sedimentary and igneous origin (Anderson, 2013), while the northern margin of the Baker terrane is described as a $>25 \mathrm{~km}$ wide zone of "fault-bounded, imbricated slabs and slices of meta-igneous and meta-sedimentary rocks faulted into chert-argillite mélange" (Schwartz et al., 2010). Following the initial eruptions was the emplacement of debris flows and the eruption of a series of thick porphyritic dacite and andesite lavas.

The caldera-forming eruptions produced the Dale Tuff, which comprises the intra-caldera and outflow tuff (see Fig. 3 for stages of caldera formation). The subsidence of the caldera produced arcuate ring fractures from which rhyolite domes emerged. Eruptions during this period were of dacitic and rhyolitic composition. On the west side of the caldera these magmas are porphyritic, while the east side is dominated by aphyric magmas and ash flow tuffs. The dacites and rhyolites intruded the intra-caldera tuff and formed the resurgent core (McClaughry et al., 2009). The modern summit of Tower Mountain (2082 m) is located at the center of the resurgent core. While the stratigraphic relationships between volcanic units have not changed, it appears that actual eruption 
dates are slightly older and are tighter based on new data presented here (see Results section).

The existence of the caldera was unknown prior to the geologic mapping of this area.

While conducting the necessary fieldwork for map production in 1996, Mark Ferns and William Taubeneck discovered that Tower Mountain was at the center of an approximately 14-kilometer caldera. The intense Tower Mountain Fire of 1995 cleared out the thick lodgepole pine forest that had previously concealed the caldera.

Additionally, aeromagnetic data revealed a low gravity anomaly underlying the core of the caldera (DOGAMI, 2008).
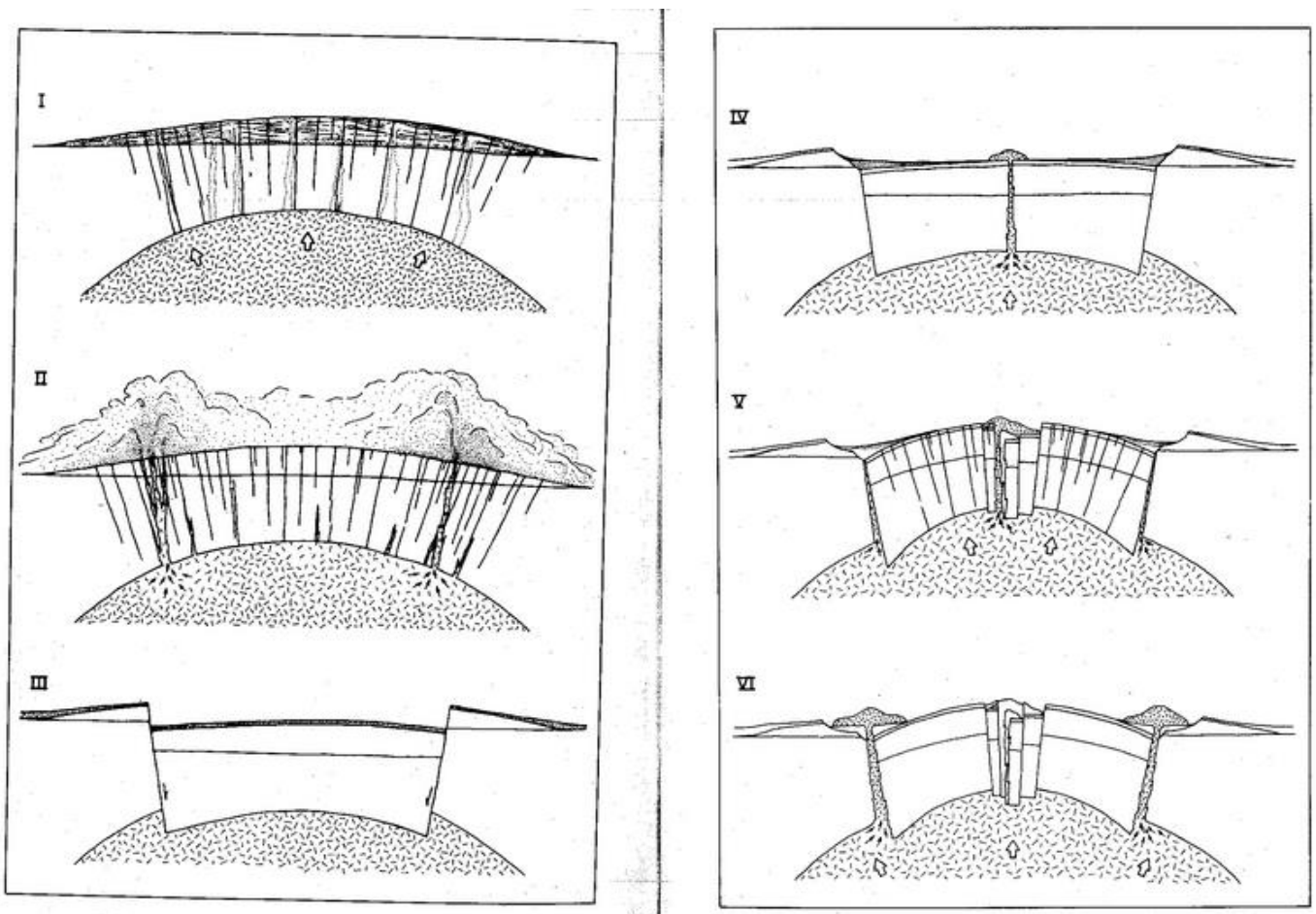

Fig. 3 Stages of caldera formation from Smith \& Bailey (1968). I: Regional tumescence generates ring fractures, which are visible at the surface. II: Explosive, caldera-forming eruptions. III: Removal of material causes caldera collapse. IV: Presurgence volcanism occurs, and along with sedimentation, begins to fill the crater. V: Resurgent doming occurs as magma accumulates below the surface. VI: Ring fracture volcanism. 


\section{$\underline{\text { Models and studies of rhyolite generation }}$}

McCarthy and Patiño Douce (1997) conducted melting experiments that modeled basaltic underplating of metapelite in order to study the role of basaltic melts in the production of high temperature felsic melts. The researchers found that the intrusion of tholeiitic basalt into metapelite country rock can produce "extensive" chemical interactions. The melts that result from these interactions range in composition from dacite to rhyolite at temperatures as high as $1150^{\circ} \mathrm{C}$ (McCarthy \& Patiño Douce, 1997). This is significant for a few reasons. First, because this temperature is well above the minimum melting temperature for water-saturated granite (i.e. "average" crustal material), secondly, because this violates the generally accepted idea that rhyolites are "cool" melts (750$900^{\circ} \mathrm{C}$ ), and finally, because this provides evidence of felsic melts forming from the interactions between basalt and metamorphic country rock.

Tamura and Tatsumi (2002) describe the bimodal (basaltic and rhyolitic) volcanism of the Izu-Bonin Arc. They suggest that in the case of island arc crust, saturated calcalkaline andesite forms a source region at depth and this source could then be reheated and remobilized by influxes of basalt. This is an excellent example of a scenario where basalt was not a material source for rhyolitic melt, but provided the necessary heat for partial melting of a compositional intermediate.

Thy, Beard and Lofgren (1990) conducted partial melting experiments on basalts and their metamorphic equivalents in order to determine a suitable protolith for Icelandic 
rhyolites, which constitute a rather high proportion of the crustal rock in that region. They found that dehydration melting of amphibolite generated a melt that was very similar to the rhyolites in question. Interestingly, they also state that the $\mathrm{K}_{2} \mathrm{O}$ content of the melts is a function of the $\mathrm{K}_{2} \mathrm{O}$ content of the starting material and did not find that this was the case for $\mathrm{FeO}$ and $\mathrm{Al}_{2} \mathrm{O}_{3}$.

The voluminous rhyolites of the Yellowstone Caldera have been studied by Girard and Stix (2009, 2010). In their investigation of the Upper Basin Member rhyolites (2009), they found that these rhyolites were not produced from a single source, but rather originated from six independent magma batches. In their study of the Central Plateau Member rhyolites (2010), they developed a model via trace element geochemical modeling that requires a large "differentiation reservoir" that is heated and replenished episodically by silicic magmas. This process forms smaller magma chambers, which then erupt within a period of a few hundred to a few thousand years. In both of these cases they suggest that the protoliths were themselves silicic, and explain the production of the intra-caldera rhyolites via the crystal mush model.

Szymanowski et al. (2015) investigated the bimodal volcanics of the Yellowstone-Snake River Plain volcanic province. They argue that the scarcity of intermediate volcanics in rhyolitic-basaltic bimodal systems has led to models that require "large degrees" of crustal melting. The authors argue that intermediate melt inclusions found within rhyolitic ignimbrites, as well as mineral phases which are in equilibrium with 
intermediate melts, suggest that crustal reservoirs of intermediate composition, which remain mostly unerupted, provide material to the rhyolitic melt via assimilation-fractional crystallization. The addition of less evolved material produces the composition observed in the erupted rhyolites.

Streck (2002) describes the relationship between basalts, metamorphic xenoliths, and the voluminous rhyolitic Rattlesnake Tuff of eastern Oregon. Metasedimentary or metavolcanic lithic fragments are found as inclusions in the tuff and are interpreted as samples of crustal material. This is of relevance to this study as the lithology of these xenoliths is similar to that of the Mountain Home Metamorphic Complex and Baker Terranes, and are likely related to the accreted terranes of the Blue Mountains. Streck suggests that these rhyolites were formed via partial melting of the crust followed by fractional crystallization.

\section{$\underline{\text { Study relevance and objectives }}$}

The Tower Mountain Caldera is petrologically poorly studied despite existing rock exposures providing an unusual combination of all important rock components for evaluating petrogenetic models of rhyolite formation. This study sought to fill this gap by investigating how rhyolites are generated at Tower Mountain. This was done in the context of previously proposed rhyolite petrogenesis models (described above), which were evaluated for their applicability to the TM suite. Given the variety of exposed crustal rocks around Tower Mountain, a second focus was to determine which of the 
crustal lithologies makes for a more suitable protolith by partial melting, with or without hybridization.

\section{METHODS}

\section{Sampling}

Hand samples were collected by M.J. Streck from the study area over a period of three years. Samples were taken from the main rhyolite units and from select units of basaltic to andesitic lava flows of the Tower Mountain volcanic series as established on published geologic maps. In addition to volcanic rocks, all major country rock units were also sampled. Samples were then selected for thin section preparation and whole rock geochemical analysis via XRF and ICP-MS. Samples were selected on the basis of providing wide areal coverage, lithological variation, and freshness of sample (i.e. avoiding altered samples).

\section{$\underline{\text { Petrographic analysis }}$}

Petrographic characteristics of the samples were determined using plane- and crossedpolarized transmitted light. Features from thin section analyses include: mineral occurrences, size, and proportions, as well as mineral and rock textures (see Appendix A).

$\underline{\text { Bulk rock analysis }}$ 
Major and trace element analyses were performed at the GeoAnalytical Lab at Washington State University using X-ray Fluorescence (XRF) and Inductively Coupled Plasma-Mass Spectrometry (ICP-MS). Detailed methods and data can be found in Appendix B.

\section{$\underline{\text { Experimental melts and analysis }}$}

During a sabbatical stay of M.J. Streck at the University of Hannover, Germany, a series of melting experiments were performed using a cold seal apparatus in the experimental petrology lab at Hannover. Pressure conditions for all experiments were $500 \mathrm{MPa}$ corresponding to a mid-crustal depth of $\sim 18 \mathrm{~km}$. Temperature ranged from 750 to above $1040^{\circ} \mathrm{C}$ and water conditions ranged from pure dehydration experiments (no external water added, i.e. dry) to $\sim 6$ weight percent. Redox conditions where kept at $\sim \mathrm{QFM}+2$ and experiments lasted on average seven days. Four TM crustal lithologies (biotite schist, biotite fels, amphibolite, and granodiorite) were used as protolith compositions. In addition, intrusions of basaltic to andesitic composition were approximated by mixing protoliths with glasses of differing compositions. This was done to evaluate material exchanges between crustal rocks and intruding magmas. Natural samples of high-alumina basalt (HAOT), Fe rich andesite, and an icelandite from eastern Oregon were chosen. Glasses of all three mafic rocks were produced in the lab at Hannover. The glass component of the experiments were analyzed by Streck at the University of Stuttgart using a CAMECA SX-50 microprobe. Analytical conditions were a $5 \mu \mathrm{m}$ beam, an 
accelerating voltage of $15 \mathrm{kV}$, a low beam current $(8 \mathrm{nA})$ counting $\mathrm{Na}$ and $\mathrm{K}$ first for 10 seconds, $\mathrm{Ba}$ and $\mathrm{P}$ for 30 seconds, and all other elements for 20 seconds.

Analysis of the experimental charges was completed via scanning electron microscope at the Center for Electron Microscopy and Nanofabrication at Portland State University. The instrument used was the Zeiss Sigma VP FEG SEM, a variable pressure field emission SEM equipped with Oxford EDS/WDS detectors and E-beam lithography system. Energy-dispersive X-ray spectroscopy (EDS) in conjunction with the Oxford AZtec software (2013) was used to determine mineral identification, phase proportions and to produce phase maps. The AZtec software groups materials into phases based on composition, but cannot provide mineral identities. The software does provide the weight percent and atomic percent of the elements in each phase. Atomic percentages were used in formula recalculations to determine phase identities. Backscatter electron (BSE) images were also obtained via the Zeiss Sigma. BSE images provide qualitative compositional data; the shade of grey is an indication of average atomic number (Z). Black areas indicate holes or cracks (no electron return) while white areas indicate a relatively high average Z. Results of these analyses can be found in Appendix A.

Isotopic dating

${ }^{40} \mathrm{Ar} /{ }^{39} \mathrm{Ar}$ incremental heating experiments were performed at Oregon State University. Crystallization ages on acid-rinsed, fresh feldspars were determined by the ${ }^{40} \mathrm{Ar} /{ }^{39} \mathrm{Ar}$ incremental heating method at the Noble Gas Mass Spectrometry lab at OSU. All 
samples were loaded into quartz vials containing small quantities of mineral monitor FCT-3 biotite and irradiated at the OSU TRIGA research reactor. Samples were heated in a double-vacuum, thermocouple-controlled resistance furnace. After each heating step, followed by gas cleanup, the isotopic composition of argon was analyzed using a MAP 215-50 mass spectrometer. ArArCALC software provided by Koppers (2002) was used to reduce the isotopic data and make age calculations. Further details of analytical procedures are described in Duncan and Keller (2004) and on the OSU laboratory website: http://www.coas.oregonstate.edu/research/mgg/chronology.html.

Uranium-lead isotopic dating of zircons was completed at the Isotope Geology Laboratory at Boise State University. This was done via laser ablation inductively coupled plasma mass spectrometry. LA-ICP MS also provides trace element concentration data, which can be found in Appendix F. Feldspar was handpicked from mineral separates in order to obtain a common lead correction factor via thermal ionization mass spectrometry (TIMS). Further details of analytical procedures can be found on the BSU laboratory website: https://earth.boisestate.edu/isotope/analyticalcapabilities/.

\section{$\underline{\text { Model calculations }}$}

Partition coefficients (also called distribution coefficients or D-values) needed for model calculations were selected for the identified mineral phases based on both published and unpublished data. Equation 1 is the general equation for determining a partition 
coefficient where $D$ is the partition coefficient, $i$ is a particular element, $l$ is the liquid phase (melt), $s$ is the solid phase (mineral), and $C$ is the concentration of the particular element, $i$, in either phase $l$ or $s$ :

$$
D_{i}^{l-s}=\frac{C_{i}^{l}}{C_{i}^{s}}
$$

Some partition coefficients for zircon were not found in the literature, and so were calculated using the trace element data obtained via LA-ICP MS collected as part of the $\mathrm{U}-\mathrm{Pb}$ zircon dating. This was done by determining the average concentration of each trace element in the zircons, then determining the average concentration of each trace element in the bulk rhyolite samples (melt), and dividing the first value by the second. This was done for each rhyolite sample (CH08a, TM5, and CH07a). The results of these calculations were then averaged, and the resulting D-values used in subsequent calculations. Table 1 contains the partition coefficients used in calculations concerning the experimental melts. Table 2 contains the partition coefficients used in calculations concerning basalt sample G314-09-2.

Using the normalized phase proportions (holes and melt phase removed, solid phase proportions recalculated to $100 \%$ ) provided by AZtec, bulk partition coefficients were calculated for each element using Equation 2, where $\bar{D}$ is the bulk partition coefficient, $i$ is a particular element, $s$ is a particular solid phase (mineral), $V$ is the volume percent 
concentration of the particular mineral, and $D$ is the partition coefficient calculated with Equation 1:

$$
\bar{D}_{i}=\sum V_{s} D_{i}^{S}
$$

The bulk partition coefficients were then used in calculating the trace element concentrations of the partial melt of the melting experiments. Equation 3 is the partial batch melting equation where $C$ is the concentration of a particular element, $i, C^{o}$ is the original concentration in the solid mineral phase (and the concentration in the whole system), $C^{l}$ is the concentration in the liquid phase (melt), $D$ is the bulk partition coefficient, and $F$ is the melt fraction:

$$
\frac{C_{i}^{l}}{C_{i}^{o}}=\frac{1}{D_{i}^{\frac{s}{l}}(1-F)+F}
$$

Equation 4 is the partial fractional melting equation where $C$ is the concentration of a particular element, $i, C^{o}$ is the original concentration in the solid mineral phase (and the concentration in the whole system), $C^{l}$ is the concentration in the liquid phase (melt), $C^{s}$ is the concentration remaining in the solid phase, $D$ is the bulk partition coefficient, and $F$ is the melt fraction:

$$
\frac{C_{i}^{l}}{C_{i}^{S}}=\frac{1}{D}(1-F)^{\frac{1}{(D-1)}}
$$


Trace element concentrations were calculated for $\sim \pm 20 \%$ the melt percentage contained in a particular experimental melt using both the batch and fractional melting equations. The results of these calculations can be found in Appendix D. The concentration values were then normalized using the primitive mantle normalizing values of Sun and McDonough (1989). The resulting spider diagrams were then compared to those of the parent material and to observed rhyolite compositions in order to determine the model of best fit. These can be found in Appendix D.

To test the viability of rhyolite production via fractional crystallization of basalt, this scenario was also modeled. Equation 5 is Rayleigh fractionation where $C$ is the concentration of a particular element, $i, C^{o}$ is the original concentration in the solid phase prior to melting (parent), $C^{l}$ is the concentration after melting (daughter), $D$ is the bulk partition coefficient, and $F$ is the melt fraction remaining:

$$
\frac{C_{i}^{l}}{C_{i}^{o}}=F^{D-1}
$$

Trace element concentrations were calculated for remaining melt values of $5 \%$ to $1 \%$ at intervals of $0.05 \%$ using the partition coefficients listed in Table 2. As with the experimental melts, these values were then normalized using the primitive mantle normalizing values of Sun and McDonough (1989). The resulting spider diagrams were then compared to observed rhyolites in order to determine the model of best fit. 
Table 1. Trace element partition coefficients for melting calculations

\begin{tabular}{|c|c|c|c|c|c|c|}
\hline & Cs & $\mathrm{Rb}$ & $\mathrm{Ba}$ & Th & $\mathrm{U}$ & $\mathrm{Nb}$ \\
\hline plagioclase & 0.07 & 0.02 & 0.5 & 0.01 & 0.01 & 0.01 \\
\hline alkali feldspar & 0.052 & 0.2 & 7 & 0.03 & 0.055 & 0.03275 \\
\hline quartz & 0.005 & 0.005 & 0.005 & 0.005 & 0.005 & 0.005 \\
\hline clinopyroxene & 0.009 & 0.01 & 0.01 & 0.01 & 0.01 & 0.009 \\
\hline orthopyroxene & 0.005 & 0.005 & 0.005 & 0.005 & 0.005 & 0.005 \\
\hline amphibole & 0.01 & 0.02 & 0.4 & 0.01 & 0.01 & 1 \\
\hline biotite & 0.7 & 1.5 & 8 & 0.005 & 0.005 & 1.7 \\
\hline FeTi oxides & 0.005 & 0.05 & 0.005 & 0.08 & 0.08 & 1.2 \\
\hline apatite & 0.7 & 1.5 & 0.2 & 1 & 1 & 1.7 \\
\hline zircon & 0.005 & 0.05 & 0.005 & 41.84 & 192.05 & 1.08 \\
\hline & $\mathrm{Ta}$ & $\mathrm{La}$ & $\mathrm{Ce}$ & $\mathrm{Pb}$ & $\operatorname{Pr}$ & $\mathrm{Sr}$ \\
\hline plagioclase & 0.01 & 0.3 & 0.2 & 0.4 & 0.18 & 6 \\
\hline alkali feldspar & 0.047 & 0.138 & 0.06 & 0.8 & 0.048 & 11.8 \\
\hline quartz & 0.005 & 0.005 & 0.005 & 0.005 & 0.005 & 0.005 \\
\hline clinopyroxene & 0.009 & 0.2 & 0.5 & 0.03 & 1 & 0.25 \\
\hline orthopyroxene & 0.005 & 0.01 & 0.01 & 0.009 & 0.015 & 0.008 \\
\hline amphibole & 1 & 0.5 & 0.9 & 0.08 & 1.5 & 2 \\
\hline biotite & 1.7 & 0.01 & 0.01 & 0.17 & 0.01 & 0.6 \\
\hline FeTi oxides & 1.4 & 0.22 & 0.2 & 0.1 & 0.17 & 0.01 \\
\hline apatite & 0.2 & 46.2 & 65.7 & 0.2 & 70 & 2 \\
\hline zircon & 2 & 0.24 & 0.54 & 0.1 & 0.5 & 0.01 \\
\hline & $\mathrm{Nd}$ & $\mathrm{Zr}$ & Hf & $\mathrm{Sm}$ & $\mathrm{Eu}$ & $\mathrm{Tb}$ \\
\hline plagioclase & 0.15 & 0.01 & 0.01 & 0.1 & 2.5 & 0.08 \\
\hline alkali feldspar & 0.035 & 0.03 & 0.036 & 0.02 & 4.75 & 0.024 \\
\hline quartz & 0.005 & 0.005 & 0.005 & 0.005 & 0.005 & 0.005 \\
\hline clinopyroxene & 2 & 0.2 & 0.2 & 4 & 4.8 & 5.5 \\
\hline orthopyroxene & 0.02 & 0.03 & 0.03 & 0.04 & 0.06 & 0.09 \\
\hline amphibole & 2 & 0.65 & 0.64 & 4 & 4.8 & 5 \\
\hline biotite & 0.01 & 0.05 & 0.05 & 0.01 & 0.15 & 0.015 \\
\hline FeTi oxides & 0.15 & 0.8 & 0.75 & 0.11 & 0.13 & 0.08 \\
\hline apatite & 101.5 & 0.6 & 0.7 & 130 & 155 & 110 \\
\hline zircon & 0.68 & 3224.17 & 1589.76 & 3.42 & 2.42 & 33.69 \\
\hline & Dy & $\mathrm{Y}$ & $\mathrm{Yb}$ & $\mathrm{Lu}$ & & \\
\hline plagioclase & 0.07 & 0.07 & 0.07 & 0.07 & & \\
\hline alkali feldspar & 0.024 & 0.05 & 0.02 & 0.016 & & \\
\hline quartz & 0.005 & 0.005 & 0.005 & 0.005 & & \\
\hline clinopyroxene & 5 & 4.8 & 3.8 & 3.6 & & \\
\hline orthopyroxene & 0.18 & 0.4 & 0.7 & 0.65 & & \\
\hline amphibole & 4.8 & 4 & 3.3 & 2.5 & & \\
\hline biotite & 0.02 & 0.03 & 0.04 & 0.05 & & \\
\hline FeTi oxides & 0.09 & 0.09 & 0.09 & 0.1 & & \\
\hline apatite & 76.2 & 162 & 40 & 20 & & \\
\hline zircon & 64.89 & 106.45 & 385.75 & 0.1 & & \\
\hline \multicolumn{7}{|c|}{ Bacon and Druitt, 1988} \\
\hline \multicolumn{7}{|l|}{ Bea et al., 1994} \\
\hline \multicolumn{7}{|l|}{ Broderick, 2008} \\
\hline \multicolumn{7}{|l|}{ Brown, 2013} \\
\hline \multicolumn{7}{|c|}{ Streck and Grunder, 1997} \\
\hline Streck, unpublis & & & & & & \\
\hline
\end{tabular}


Table 2. Trace element partition coefficients for basalt fractional crystallization calculations

\begin{tabular}{|c|c|c|c|c|c|c|}
\hline & Cs & $\mathrm{Rb}$ & $\mathrm{Ba}$ & Th & $\mathrm{U}$ & $\mathrm{Nb}$ \\
\hline plagioclase & 0.007 & 0.002 & 0.05 & 0.001 & 0.001 & 0.001 \\
\hline clinopyroxene & 0.0009 & 0.001 & 0.001 & 0.001 & 0.001 & 0.0009 \\
\hline \multirow{2}{*}{$\begin{array}{l}\text { FeTi oxides } \\
\text { olivine }\end{array}$} & 0.0005 & 0.005 & 0.0005 & 0.008 & 0.008 & 0.12 \\
\hline & 0.05 & 0.04 & 0.03 & 0.03 & 0.04 & 0.01 \\
\hline \multirow{5}{*}{$\begin{array}{l}\text { plagioclase } \\
\text { clinopyroxene } \\
\text { FeTi oxides } \\
\text { olivine }\end{array}$} & $\mathrm{Ta}$ & $\mathrm{La}$ & $\mathrm{Ce}$ & $\mathrm{Pb}$ & $\operatorname{Pr}$ & \multirow[b]{11}{*}{$\mathrm{Lu}$} \\
\hline & 0.001 & 0.03 & 0.02 & 0.04 & 0.018 & \\
\hline & 0.0009 & 0.02 & 0.05 & 0.003 & 0.1 & \\
\hline & 0.14 & 0.015 & 0.016 & 0.01 & 0.017 & \\
\hline & 0.03 & 0.03 & 0.002 & 0.0001 & 0.0002 & \\
\hline \multirow{5}{*}{$\begin{array}{l}\text { plagioclase } \\
\text { clinopyroxene } \\
\text { FeTi oxides } \\
\text { olivine }\end{array}$} & $\mathrm{Sr}$ & $\mathrm{Nd}$ & $\mathrm{Zr}$ & $\mathrm{Hf}$ & $\mathrm{Sm}$ & \\
\hline & 0.6 & 0.015 & 0.001 & 0.001 & 0.01 & \\
\hline & 0.025 & 0.2 & 0.02 & 0.02 & 0.4 & \\
\hline & 0.001 & 0.026 & 0.08 & 0.075 & 0.024 & \\
\hline & 0.02 & 0.0023 & 0.06 & 0.04 & 0.0108 & \\
\hline \multirow{5}{*}{$\begin{array}{l}\text { plagioclase } \\
\text { clinopyroxene } \\
\text { FeTi oxides } \\
\text { olivine }\end{array}$} & $\mathrm{Eu}$ & $\mathrm{Tb}$ & Dy & $\mathrm{Y}$ & $\mathrm{Yb}$ & \\
\hline & 0.25 & 0.008 & 0.007 & 0.007 & 0.007 & 0.007 \\
\hline & 0.48 & 0.55 & 0.5 & 0.48 & 0.38 & 0.36 \\
\hline & 0.025 & 0.019 & 0.009 & 0.009 & 0.018 & 0.01 \\
\hline & 0.03 & 0.03 & 0.0263 & 0.0036 & 0.0313 & 0.0383 \\
\hline \multicolumn{2}{|c|}{$\begin{array}{l}\text { *Bacon and Druitt, } \\
1988\end{array}$} & & & \multicolumn{2}{|c|}{$\begin{array}{l}\text { *Streck and } \\
\text { Grunder,1997 }\end{array}$} & \\
\hline \multicolumn{3}{|c|}{ Fujimaki et al., 1984} & & \multicolumn{3}{|c|}{ *Streck, unpublished data } \\
\hline \multicolumn{3}{|c|}{ Lemarchand et al., 1987} & & \multicolumn{3}{|c|}{ Villemant et al., 1981} \\
\hline \multicolumn{3}{|c|}{ McKenzie and O'Nions, 1987} & & \multirow{2}{*}{\multicolumn{3}{|c|}{$\begin{array}{l}\text { Estimated or interpolated } \\
\text { values } \\
\text { *Original reference values } \\
\text { divided by } 10\end{array}$}} \\
\hline \multicolumn{3}{|c|}{ Nielsen et al., 1992} & & & & \\
\hline
\end{tabular}




\section{RESULTS}

\section{VOLCANIC ROCKS}

Based on a total alkali-silica classification diagram (LeBas et al., 1986), volcanic samples from the Tower Mountain Caldera range from rhyolite to basalt (Figure 4).

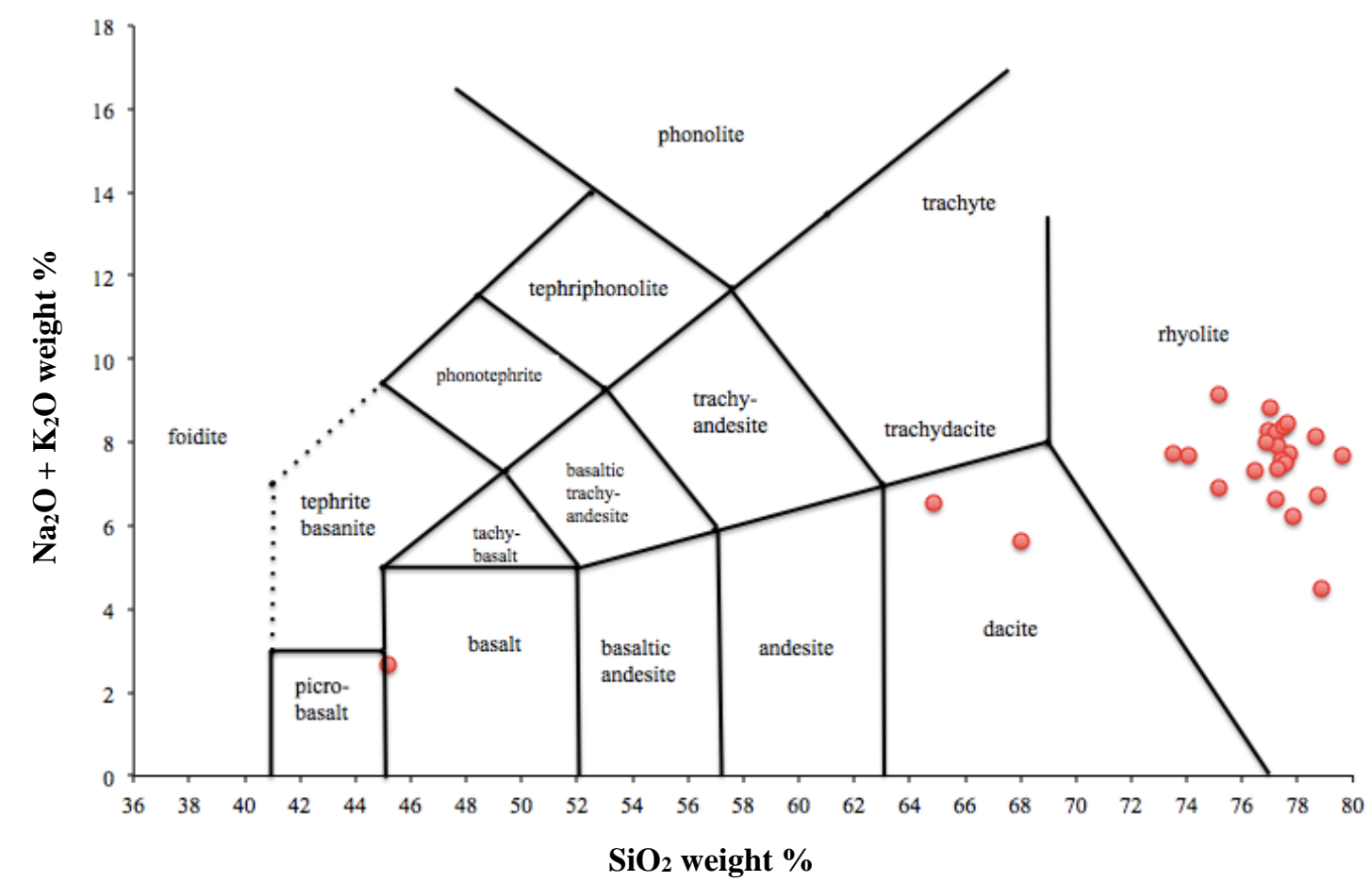

Fig. 4 Tower Mountain volcanic samples plotted onto a total alkali silica (TAS) diagram. The single basalt sample plots on the border between picrobasalt and basalt, indicating low silica content. The rhyolitic samples form a loose cluster between $\sim 6$ to 8 weight percent total alkali and $\sim 74$ to 80 weight percent silica.

\section{Rhyolite Petrography}

Overall, the Tower Mountain rhyolites are typical in terms of mineral assemblage; quartz, alkali feldspar and plagioclase feldspar are the main phases with biotite, amphibole, pyroxene, FeTi oxides, and zircon as accessory phases. In terms of texture, the rhyolites tend to be glassy with sparse phenocrysts. Samples CH08a, CH07, TM5, and CH31 (Dale 
Tuff) are described in detail below to illustrate the similarities and differences among the samples.

The groundmass of $\mathrm{CH} 08 \mathrm{a}$ is glassy and highly fractured with perlitic texture. There is obvious flow banding. Phenocrysts account for approximately 5-7\% of the sample area. Alkali feldspars in the form of sanidine are the most noticeable and largest minerals in the sample, accounting for $\sim 50 \%$ of total phenocrysts. These are mostly anhedral, although some have a euhedral shape. These crystals are fractured and have inclusions of FeTi oxides and zircon. Biotite accounts for $\sim 25 \%$ of the total phenocrysts. These contain zircon and FeTi oxides inclusions, and are extremely anhedral. Quartz accounts for $\sim 6 \%$ of the phenocrysts. It is highly anhedral and highly fractured. Figure 5 is a plane light image of a thin section of $\mathrm{CHO8a}$, and Figure 6 is a BSE image of phenocrysts within this sample.

Sample CH07a is composed of a glassy and homogenous groundmass which is light in color, and comprises $\sim 90 \%$ of the sample by area, while phenocrysts make up $\sim 10 \%$ of the total sample area. The most abundant phenocryst is plagioclase feldspar (andesine) at $\sim 55 \%$ modal abundance. Plagioclase phenocrysts are euhedral to subhedral. Iron oxides account for $\sim 30 \%$ of phenocrysts, and are found both scattered throughout the sample and as inclusions in biotite. Approximately $15 \%$ of phenocrysts are biotite, which are highly fractured. Zircon was observed as an accessory phase and is primarily found as inclusions. Flow structures are visible in the groundmass attesting that it is rhyolite lava 
flow. Figure 7 is a plane light image of a thin section of $\mathrm{CHO7a}$, and Figure 8 is a BSE image of phenocrysts within this sample.

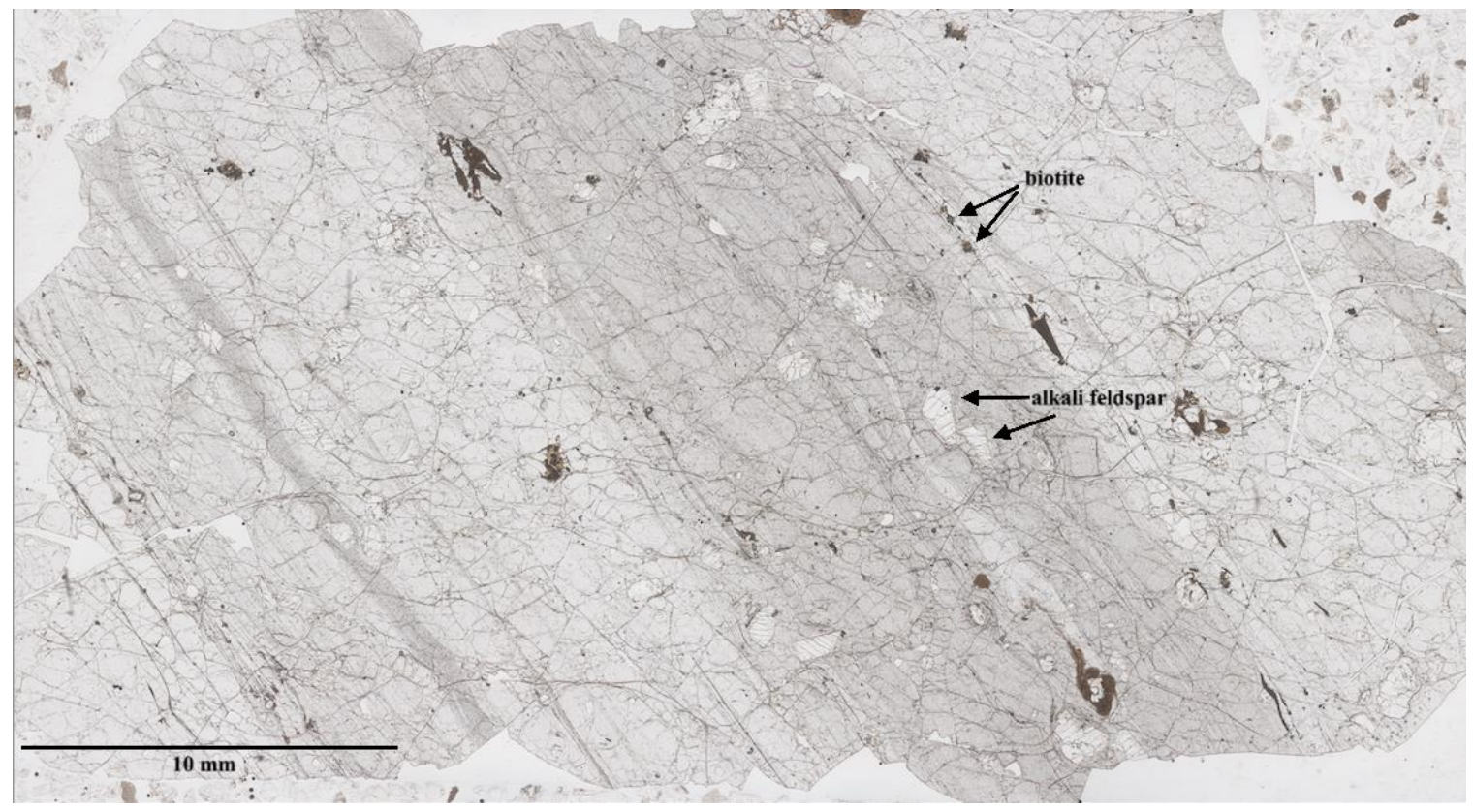

Fig. 5 Plane light image of a thin section of sample $\mathrm{CH} 08$ a; rhyolite lava flow. Note the flow banding, perlitic texture, visible phenocrysts, and overall light color. Scale bar is 10

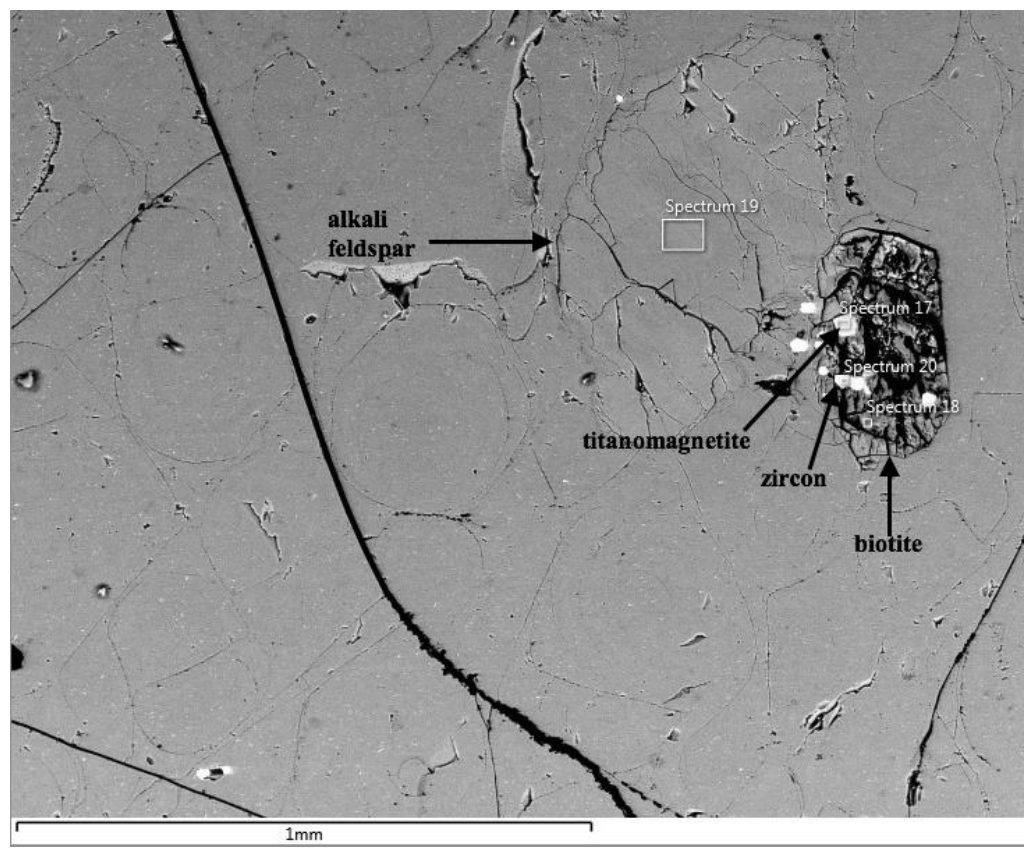
$\mathrm{mm}$. See text for detailed description.

Fig. 6 Backscattered electron (BSE) image of phenocrysts within sample CH08a. The perlitic texture of the groundmass is visible, as are large cracks, indicating a glassy texture. Visible in this image are phenocrysts of titanoFeTi oxides, biotite, alkali feldspar, and zircon. The alkali feldspar and groundmass are of similar shades of grey, indicating similar compositions. Scale bar is $1 \mathrm{~mm}$. 


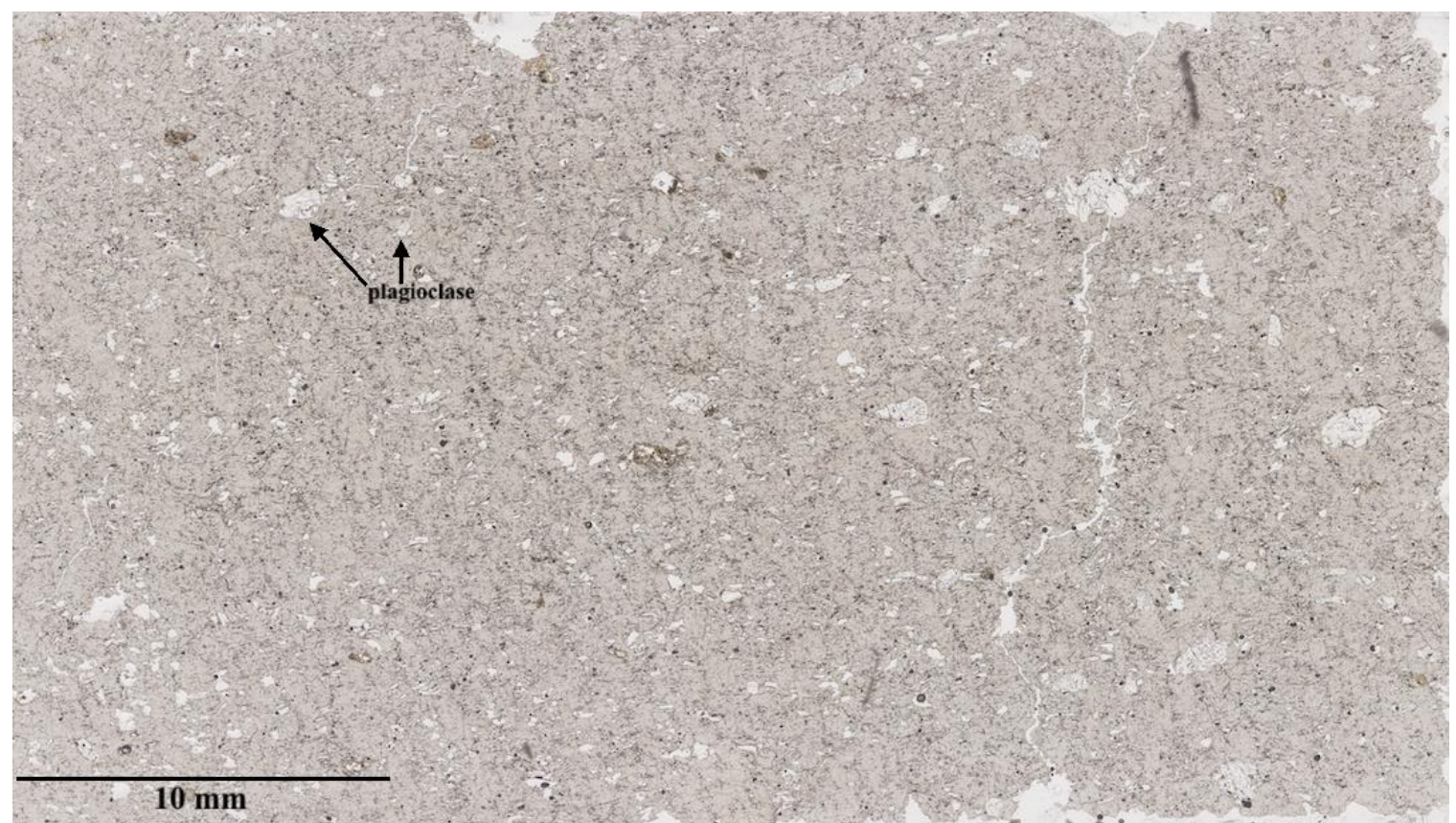

Fig. 7 Plane light image of a thin section of sample $\mathrm{CH} 07$ a; rhyolite lava flow. Note the homogeneous texture of the groundmass, visible feldspar phenocrysts (discernable by the roughly rectangular shape and faint grey fracture lines), and overall light color. Biotite phenocrysts are too small to be visible at this scale. See text for detailed description.

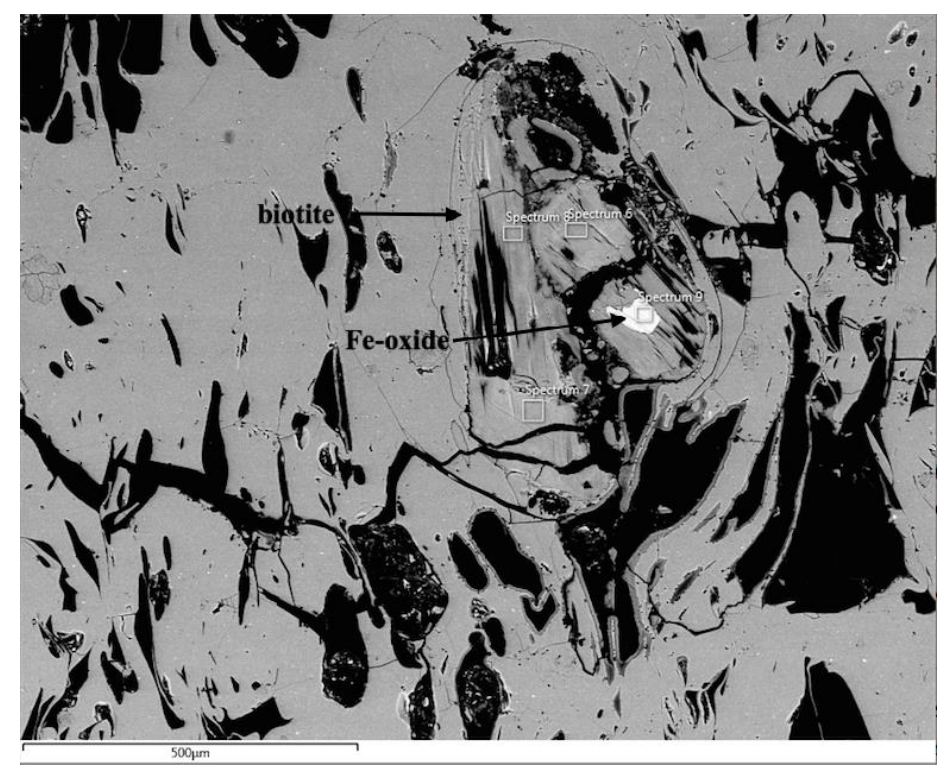

Fig 8. Backscatter electron (BSE) image of phenocrysts within sample CH07a. Visible in the image is a twinned phenocryst of biotite with an iron oxide inclusion. The groundmass displays flow structures and irregularly shaped holes and cracks, indicating a glassy texture. Scale bar is $500 \mu \mathrm{m}$.

The groundmass of sample TM5 is glassy, predominantly transparent, highly fractured, and shows distinctive flow banding. It is darker in color than either $\mathrm{CH} 08$ a or $\mathrm{CH} 07 \mathrm{a}$.

Phenocrysts are sparse at $\sim 10 \%$ of the sample area. The largest and most abundant of the 
phenocrysts, $\sim 70 \%$, are alkali feldspar (anorthoclase), which are euhedral to anhedral, tend to be highly fractured, and are found with inclusions of titanoFeTi oxides and zircon. Many show compositional zoning. Clinopyroxene phenocrysts are less abundant, 20\%, typically highly euhedral, and fractured. TitanoFeTi oxides comprises $\sim 6 \%$ of phenocrysts, and zircon, $4 \%$. Figure 9 is a plane light image of a thin section of TM5. Figure 10 is a BSE image of phenocrysts within this sample.

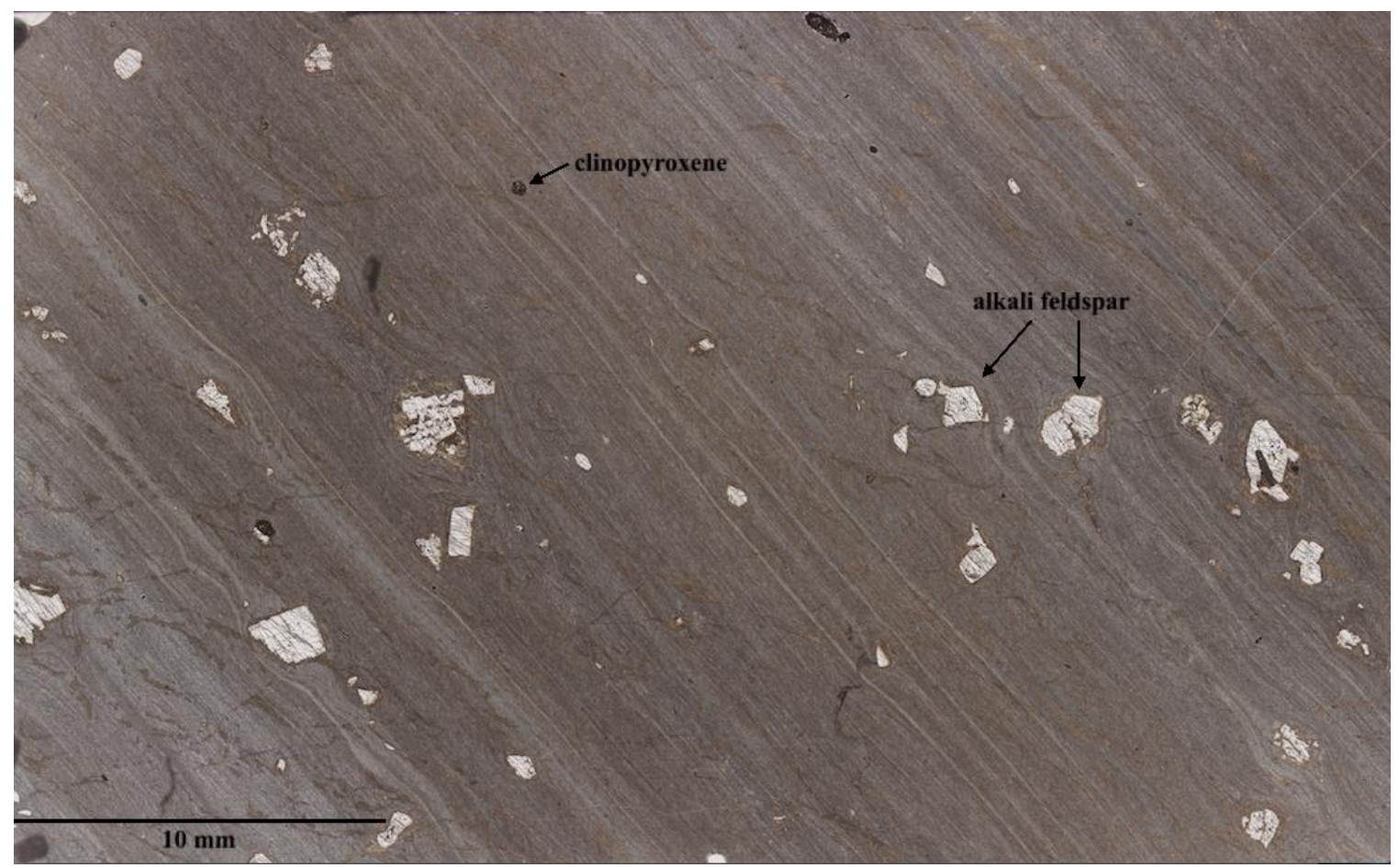

Fig. 9 Plane light image of a thin section of sample TM5; rhyolite lava flow. Note the flow banding and fine-grained texture of the groundmass, and the sparse, but visible, phenocrysts. Overall, the sample is darker in color than either CH08a or CH07a. See text for detailed description. 


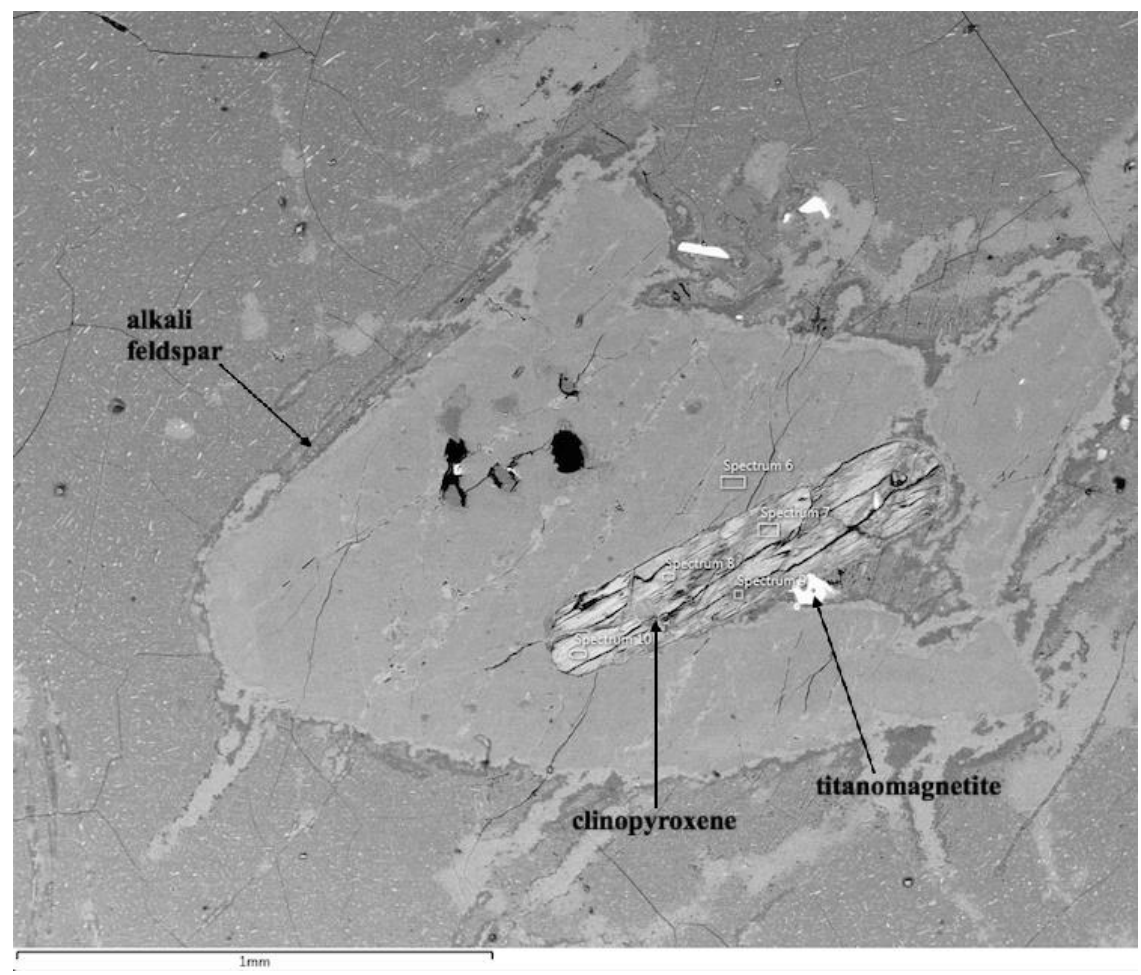

Fig. 10

Backscattered electron (BSE) image of phenocrysts within sample TM5. The fine-grained texture of the groundmass is visible as are intersecting cracks, indicating a glassy texture. Visible in this image are phenocrysts of alkali feldspar, clinopyroxene, and titanoFeTi oxides. The scale bar is 1 $\mathrm{mm}$.

The Dale Tuff (CH31) is the outflow tuff of the Tower Mountain Caldera. It has the typical glass shard texture with sparse phenocrysts. A mere $4 \%$ of the area is occupied by phenocrysts. Of these, plagioclase feldspar is the most abundant. The normalized mineral assemblage is as follows: $\sim 90 \%$ plagioclase feldspar, $\sim 10 \%$ oxides, $<1 \%$ clinopyroxene. The feldspar phenocrysts are euhedral to subhedral and are quite small at just a few millimeters in length. There are abundant small, rounded oxides throughout the sample. Two subhedral pyroxene phenocrysts were observed, which were comparable in size to the feldspar phenocrysts. Very sparse lithic fragments of $\sim 3 \mathrm{~mm}$ contain plagioclase laths, euhedral amphibole, and rounded oxides, suggesting that the fragments are basaltic in origin. Figure 11 is a plane light image of a thin section of $\mathrm{CH} 31$. 


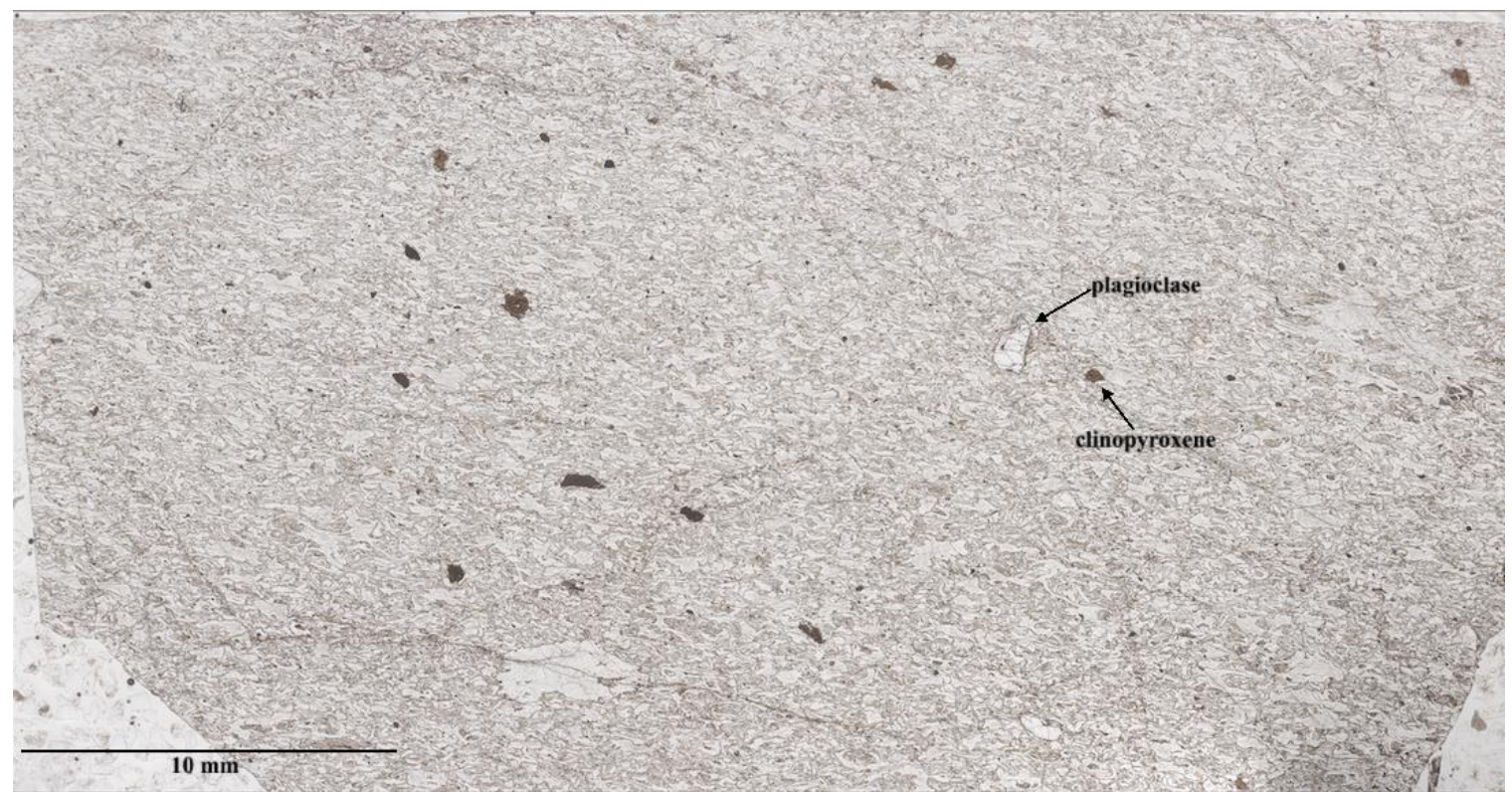

Fig. 11 Plane light image of a thin section of sample CH31, Dale Tuff. The overall texture is glassy with sparse phenocrysts and lithic fragments. See text for detailed description.

\section{$\underline{\text { Rhyolite Geochemistry }}$}

Detailed results of geochemical analysis can be found in Table B1 in Appendix B. The rhyolites have been divided into five groups based on geochemistry and lithology; 1) low silica, 2) A-like, 3) Dale Tuff-like, 4) Dale Tuff, and 5) high silica. It should be noted that there are rhyolites that have silica values similar to those observed in the high and low silica groups, but have not been categorized as such due to some other outstanding characteristic(s). $\mathrm{SiO}_{2}$ content among all rhyolites ranges from $\sim 73.5 \%$ to $\sim 78.9 \%$. $\mathrm{FeO}^{*}$ is consistently low and ranges from $\sim 0.4 \%$ to $\sim 1.9 \%$. $\mathrm{CaO}$ is also consistently low at $\sim 0.2 \%$ to $\sim 1.7 \%$. $\mathrm{Na}_{2} \mathrm{O}$ ranges from $\sim 1.4 \%$ to $4.1 \%$, and $\mathrm{K}_{2} \mathrm{O}$ from $\sim 3.1 \%$ to $\sim 6.0 \%$. These ranges are consistent with what is expected for rhyolite. 
Figure 12 shows a primitive mantle normalized (Sun and McDonough, 1989) trace element spider diagram for all Tower Mountain rhyolite samples for which ICP MS data was obtained. Overall, the rhyolites show a similar trace element concentration pattern; an overall decrease from $\mathrm{Rb}$ to $\mathrm{Lu}$, peaks at $\mathrm{U}$ and $\mathrm{Pb}$, and troughs at $\mathrm{Ba}, \mathrm{Nb}, \mathrm{Sr}$, and $\mathrm{Eu}$.

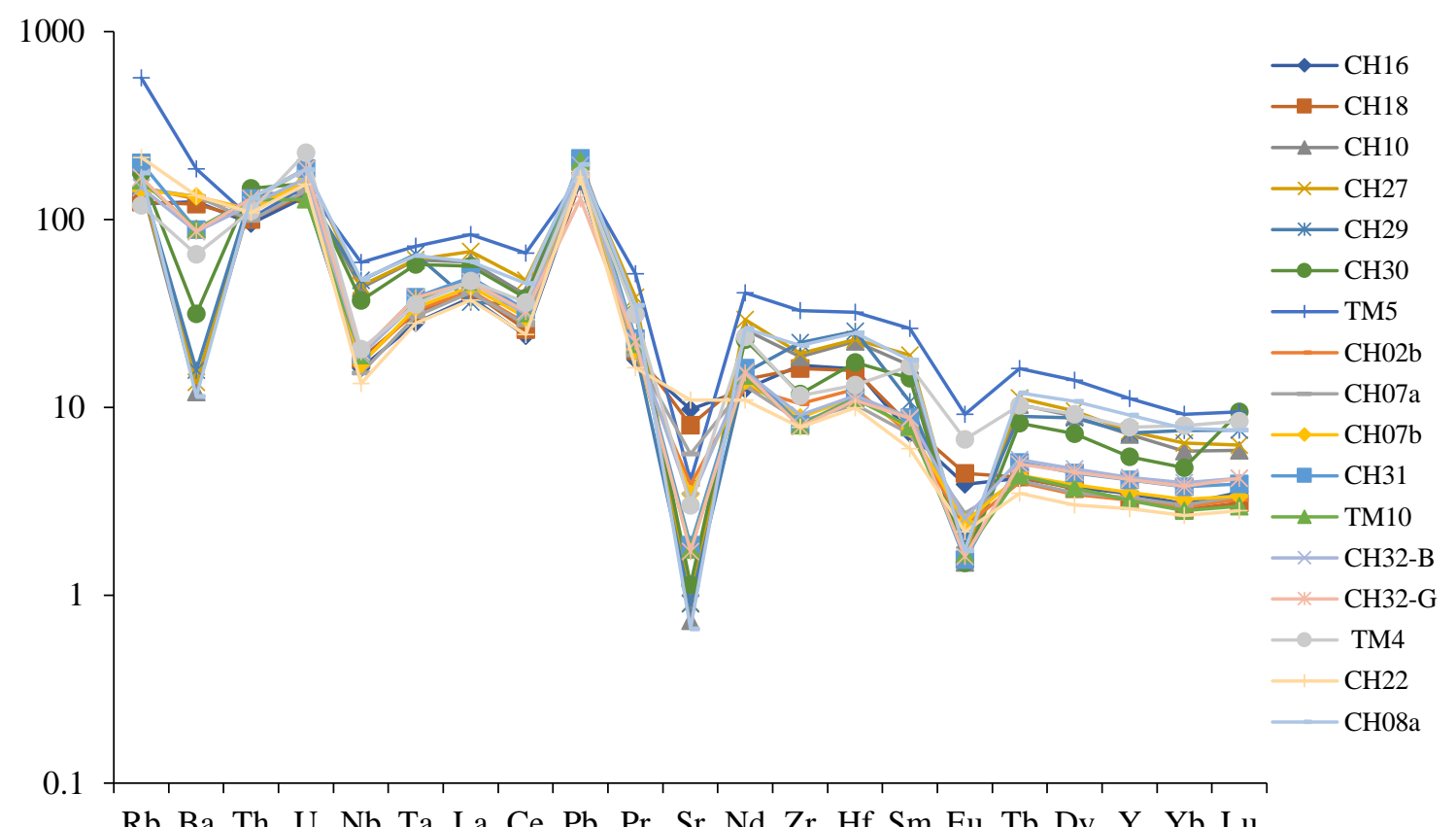

Fig. 12 Primitive mantle normalized (Sun and McDonough, 1989) trace element spider diagram for all Tower Mountain rhyolite samples for which ICP MS data was obtained. These values are listed in Table 1B.

Figure 13 shows the normalized trace element concentrations on individual spider diagrams for each of the distinct rhyolite groups; low-silica, A-like, Dale Tuff, Dale Tufflike, and high-silica. Note that the scale is retained from Figure 12 to Figure 13. Although all of the rhyolites show similar patterns of enrichement and depletion, there are subtle but distinct differences among groups. Compared to the primitive mantle, all are 100 times more enriched in the more incompatible elements. This enrichment decreases to just above that observed in the primitive mantle as the elements become less 
incompatible. There are exceptions to this trend, most notably at uranium, lead, niobium, strontium, and europium. The uranium peak is relatively small; 50 times thorium enrichment, except in the case of the low silica group where uranium concentration is $\sim 35$ times that of thorium. In contrast, the peaks observed at lead jump back up to $\sim 100$ times those observed in the primitive mantle. In the case of the low silica and Dale Tufflike groups, the rhyolites are more enriched in lead than rubidium. This is also the case for sample TM4 of the high silica rhyolites. Niobium depletion is relatively uniform across the groups showing concentrations around 15 times that observed in the primitive mantle. The exception to this is seen in the A-like rhyolites, where enrichment is $\sim 40$ times. The negative anomaly observed at strontium is the greatest for the A-like rhyolites. The Dale Tuff group shows an anomaly at strontium that is similarly strong. For the Dale Tuff-like group and sample TM4 of the high silica group, the negative anomaly at strontium is $\sim 3$ times. Sample $\mathrm{CH} 22$ of the high silica group does not show a trough at strontium. Strontium concentrations are $\sim 8$ times that of the primitive mantle for the low silica group, the highest concentration among the rhyolites. The negative anomalies at europium follow the trends of strontium, being the least pronounced in the low silica group and the most pronounced in the A-like group. A negative anomaly at barium is observed for the A-like rhyolites, the Dale Tuff, and for sample TM4 of the high silica rhyolites. The anomaly is strongest for the A-like rhyolites, varying from 15 to 32 times that observed in the primitive mantle (excluding sample TM5, which can be seen to vary significantly from the rest of the samples in this group in terms of concentrations for nearly all trace elements). 

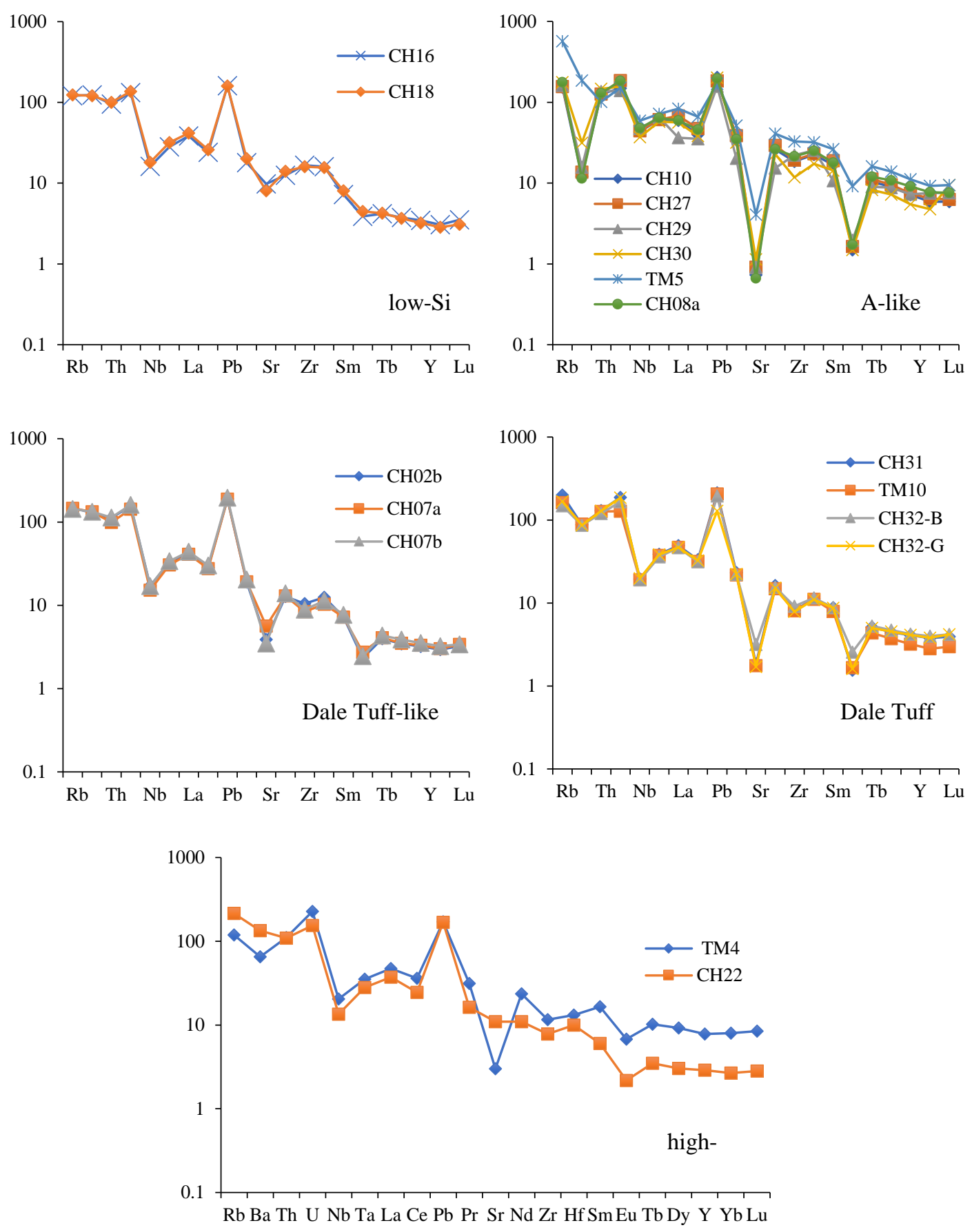

Fig. 13 Primitive mantle normalized (Sun and McDonough, 1989) spider diagrams of the trace element concentrations of rhyolite samples separated into their respective groups. 
It is difficult to discuss the high silica rhyolites as a cohesive group. A cursory examination of Figure 13 reveals that these samples are quite different in terms of trace element concentrations, some of which as been discussed above. In addition to these differences, it should be noted that sample TM4 is more enriched in the less incompatible elements than is sample $\mathrm{CH} 22$ by $\sim 3$ times.

A-type rhyolites are usually defined as being alkali rich, that is, rhyolites of this type tend to follow the rule $\mathrm{Na}_{2} \mathrm{O}+\mathrm{K}_{2} \mathrm{O}>\mathrm{Al}_{2} \mathrm{O}_{3}$. However, this is not the only parameter for defining A-type rhyolites; trace element patterns must also be taken into consideration (Whalen et al., 1987). Although the rhyolites here described as "A-like" are not particularly alkali-rich, these samples do show similar trace element concentration patterns as would be expected for A-type rhyolites, specifically in regards to niobium, zirconium, cerium, and yttrium. Though this not particularly apparent in the above spider diagrams, patterns in trace element concentrations are further revealed by element/element scatterplots. These are presented for all rhyolite groups in Figure 14.

Scatterplots A and B in Figure 14 demonstrate how trace element concentrations might be used to delineate A-type and I-type rhyolites (Whalen et al., 1987). Scatterplot A shows how the concentration of niobium varies with the ratio of $1000 \mathrm{Ga} / \mathrm{Al}$. The A-like rhyolites (red squares), cluster loosely in the A-type region, with higher concentrations of $\mathrm{Nb}$ combined with higher ratios of $1000 \mathrm{Ga} / \mathrm{Al}$. The remaining rhyolites cluster tightly (with the exception of high silica sample $\mathrm{CH} 22$, blue asterisk) at relatively low 
concentrations of niobium with moderate ratios of $1000 \mathrm{Ga} / \mathrm{Al}$. Scatterplot B shows that the variation of the niobium with zirconium can also be used to distinguish A-type and Itype rhyolites. Here, the A-like rhyolites show a positive linear relationship between these element concentrations, with a clustering of points at moderately high concentrations of both elements. The I-type rhyolites show two distinct patterns; the Dale-tuff like, Dale Tuff and high silica rhyolites cluster tightly at relatively low concentrations of both niobium and zirconium. The low silica rhyolites (blue triangles) show similar niobium concentrations, but elevated zirconium concentrations.

Scatterplot $\mathrm{C}$ of Figure 14 shows the variation of yttrium with cerium. There is a positive linear correlation between these two elements ranging from $\sim 30 \mathrm{ppm}$ cerium and $\sim 13$ ppm yttrium to $\sim 118 \mathrm{ppm}$ cerium and $\sim 50 \mathrm{ppm}$ yttrium. The A-type rhyolites again show the most elevated concentrations of both elements. The high silica rhyolites show a marked difference in trace element concentrations with sample $\mathrm{CH} 22$ plotting among the Dale Tuff-like rhyolites and sample TM4 plotting with A-like rhyolites. Scatterplot D shows the variation of the sum of the concentrations of cerium, zirconium, niobium, and yttrium with lanthanum. Here, samples fall into a rough positive linear trend from $\sim 24$ ppm lanthanum and $\sim 154 \mathrm{ppm} \mathrm{Ce}+\mathrm{Zr}+\mathrm{Nb}+\mathrm{Y}$ to $\sim 57 \mathrm{ppm}$ lanthanum and $\sim 576 \mathrm{ppm}$ $\mathrm{Ce}+\mathrm{Zr}+\mathrm{Nb}+\mathrm{Y}$. The Dale Tuff and Dale Tuff-like rhyolites cluster closely around the lower end of the trend, while the low silica rhyolites show slightly elevated $\mathrm{Ce}+\mathrm{Zr}+\mathrm{Nb}$ $+\mathrm{Y}$ concentrations in comparison. As a group, the A-like rhyolites show the highest concentrations of both $\mathrm{Ce}+\mathrm{Zr}+\mathrm{Nb}+\mathrm{Y}$ and lanthanum as compared to the other rhyolite 
groups, the exception being sample $\mathrm{CH} 29$, which contains only $23.9 \mathrm{ppm} \mathrm{La}$, the lowest concentration among the samples plotted here. According to Whalen et al., A-type rhyolites contain $>350 \mathrm{ppm} \mathrm{Ce}+\mathrm{Zr}+\mathrm{Nb}+\mathrm{Y}$. A-type sample $\mathrm{CH} 30$ falls $\sim 50 \mathrm{ppm}$ short of the boundary, but all other A-type samples are at or above the established boundary.
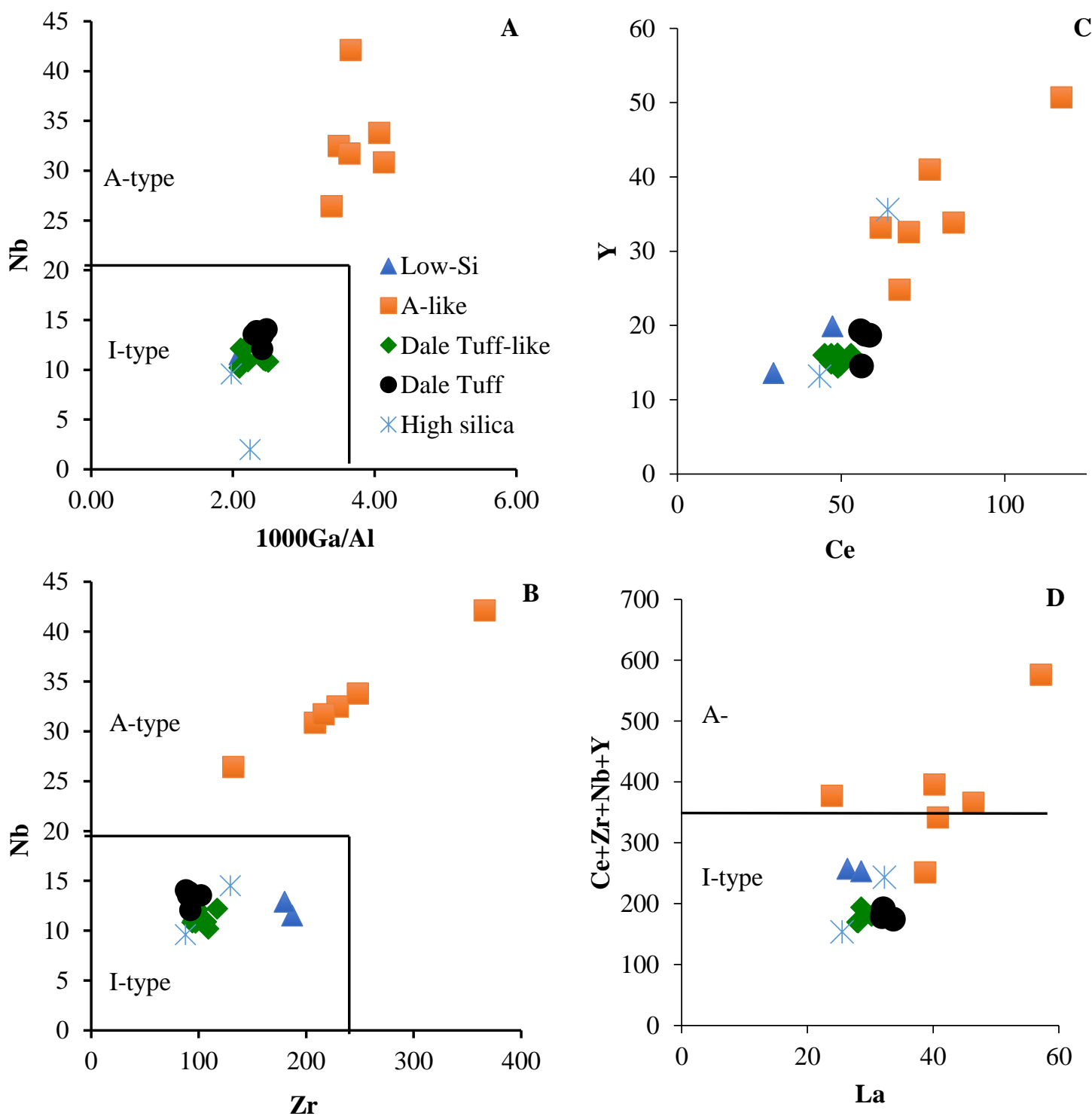

Fig. 14 Element/element variation diagrams for representative rhyolite samples (Table B1). Plots are after Whalen et al., 1987. A shows the variation of niobium with $10000 \mathrm{Ga} / \mathrm{Al}$. B shows the variation of zirconium with niobium $\mathrm{C}$ shows the variation of yttrium with cerium, and D, the variation of the sum of cerium, zirconium, niobium, and yttrium with lanthanum. 


\section{Basaltic Petrography}

Sample G314-09-2 was the only basalt sample analyzed from the study area (basalt lava flows are rare among TM volcanic rocks), which, by location and stratigraphy, may be considered as a parent magma for the rhyolites. Sample G314-09-2 is intergranular in texture; the angular interstices between plagioclase grains are occupied by grains of ferromagnesian minerals; olivine, pyroxene, and iron titanium oxides. Phenocryst modal abundance is as follows: $\sim 55 \%$ plagioclase, $\sim 40 \%$ pyroxene, $\sim 3 \%$ olivine, and $\sim 2 \% \mathrm{Fe}-\mathrm{Ti}$ oxides. Figure 15 is a plane light thin section image and Figure 16 is a BSE image of this sample.

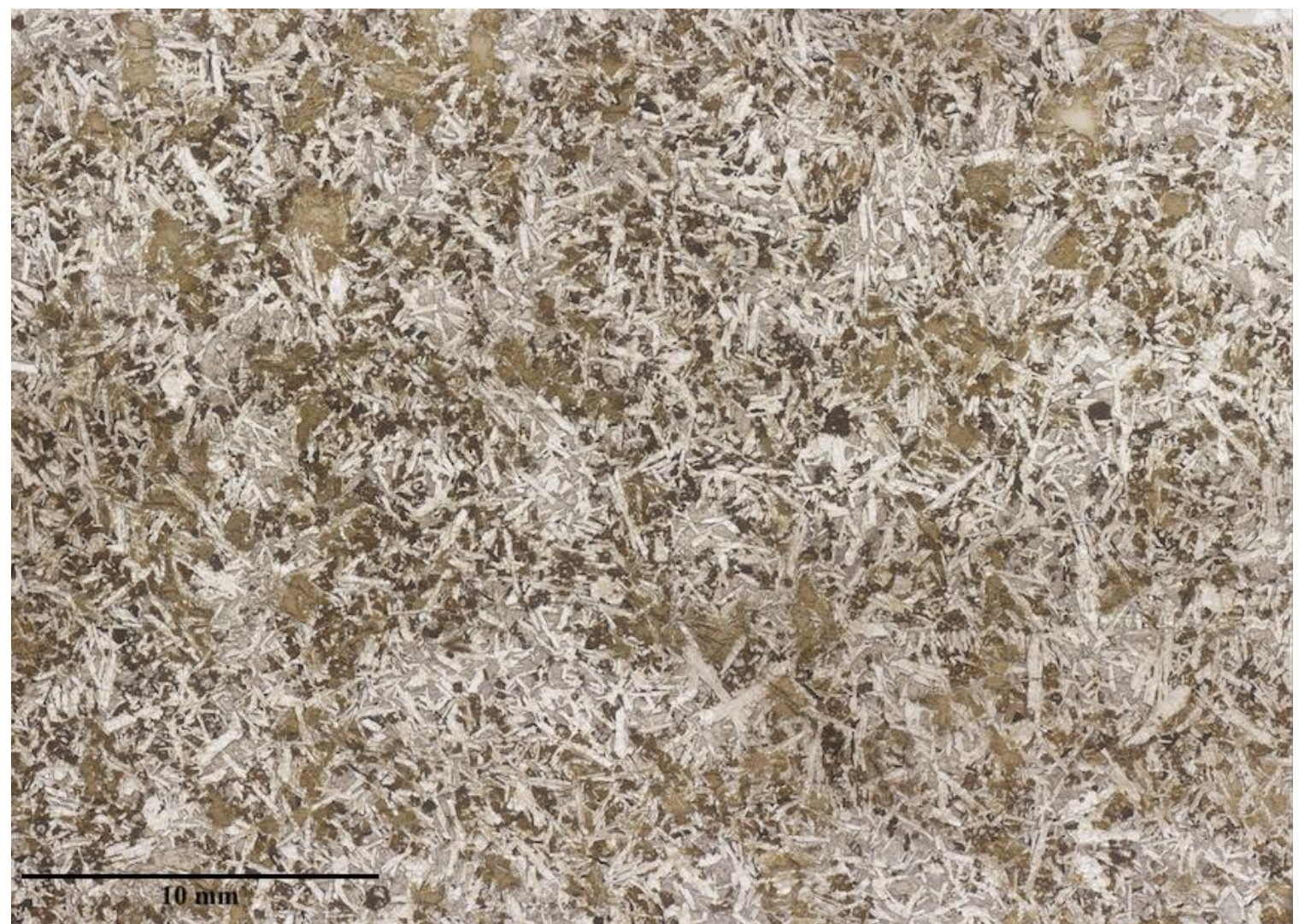

Fig. 15 Plane light image of a thin section of sample G314-09-2, basalt. The overall texture is intergranular; interlocking plagioclase feldspar laths (light color minerals) make up the majority of the groundmass with olivine, pyroxene and oxides in the interstitial spaces. See text for detailed description. 


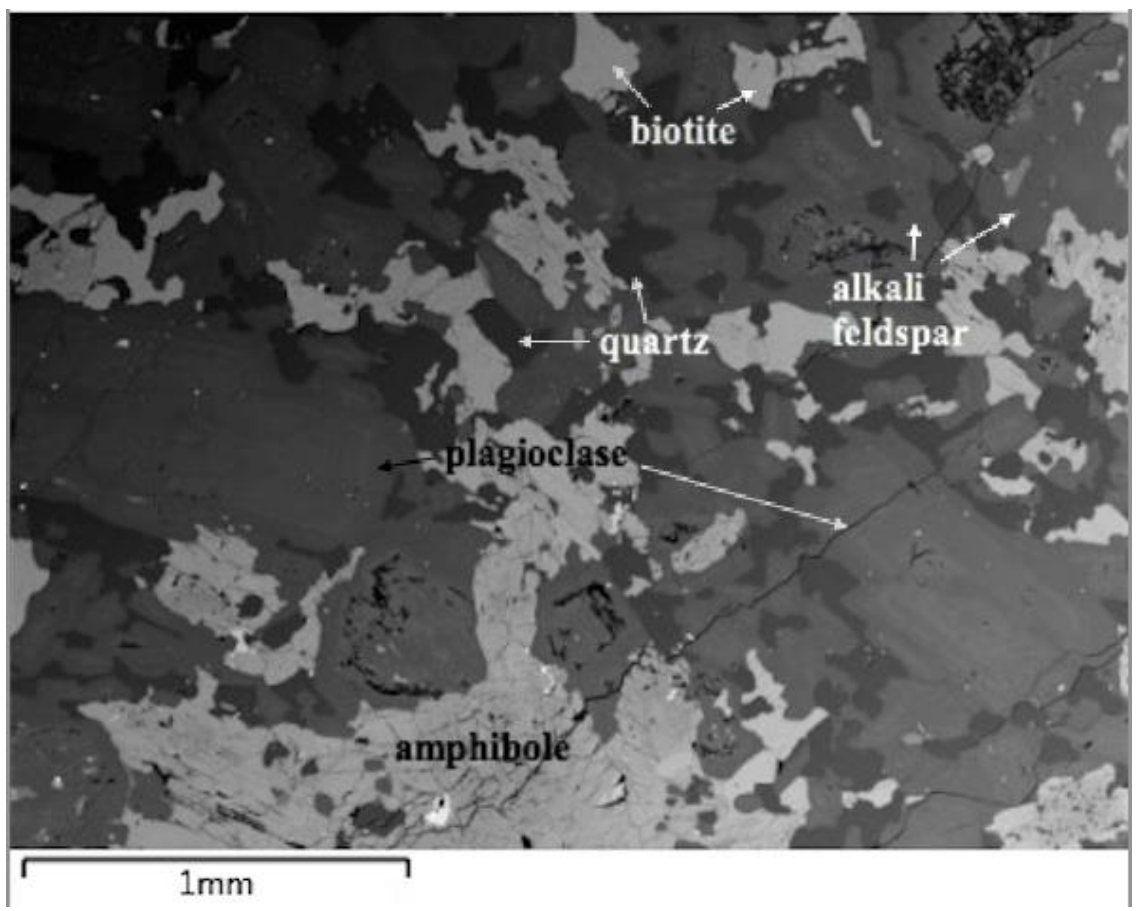

Fig. 16 Backscattered electron (BSE) image of basalt sample G314-092. The dark gray areas are plagioclase, while black areas represent holes. Note the intergranular texture. There is evidence of significant secondary mineralization and alteration. For example, the high electron return in the cracks, which is likely due to remobilization of calcium by water, and the interstitial dendritic textures.

\section{$\underline{\text { Geochemistry of basalt and intermediate rocks }}$}

Table B2 in Appendix B contains the geochemical data for basaltic and intermediate samples. Figure 17 below shows the normalized trace element concentrations of basalt sample G314-09-2 and intermediate (dacitic) samples CH01 and TM6. These are the only mafic and intermediate samples for which ICP MS trace element concentrations were obtained. 


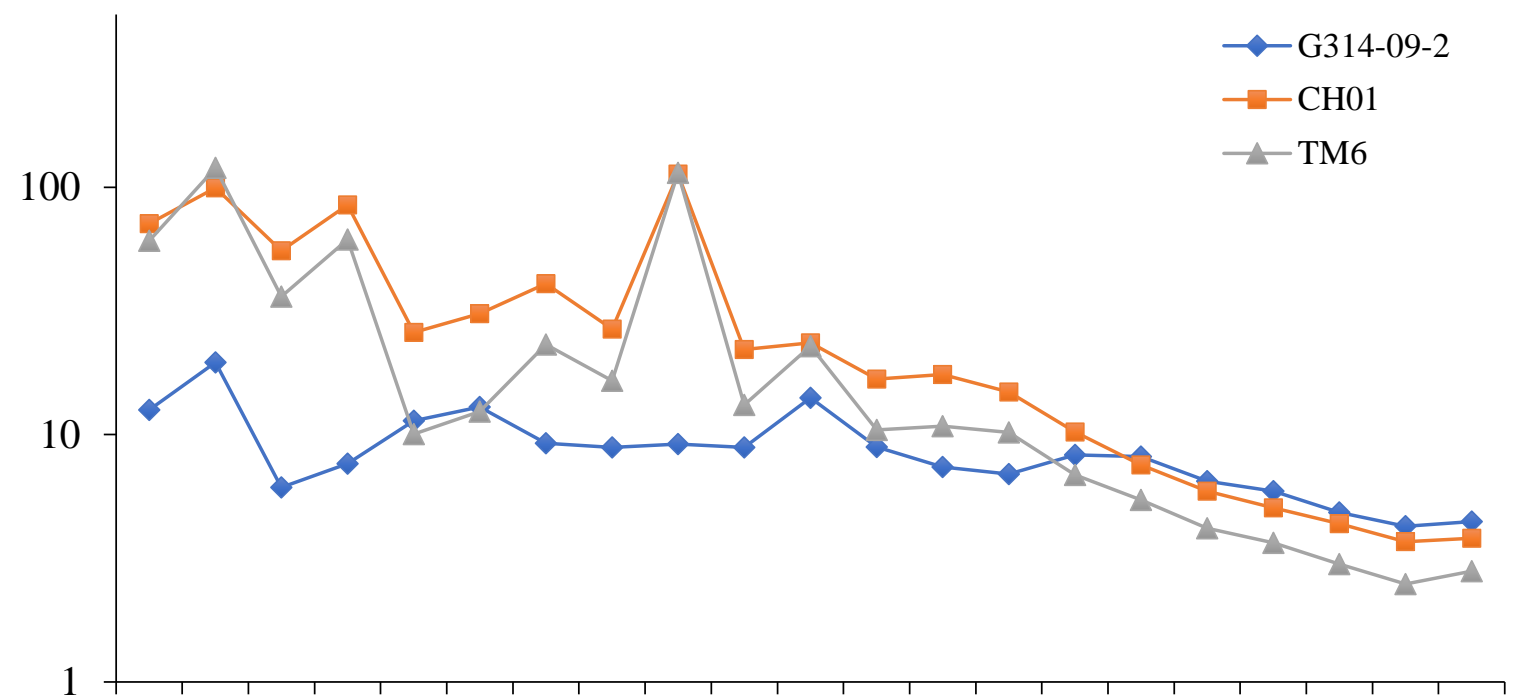

$\mathrm{Rb} \mathrm{Ba}$ Th $\mathrm{U}$ Nb Ta $\mathrm{La} \mathrm{Ce} \mathrm{Pb}$ Pr $\mathrm{Sr} \mathrm{Nd} \mathrm{Zr}$ Hf Sm Eu Tb Dy Y Yb Lu

Fig. 17 Primitive mantle normalized (Sun and McDonough, 1989) spider diagram of the trace element concentrations of basaltic (G314-09-2) and intermediate (CH01, TM6) samples.

\section{$\underline{\text { Isotopic Dating of Rhyolites }}$}

$\mathrm{U}-\mathrm{Pb}$ isotopic dating of zircon and ${ }^{40} \mathrm{Ar} /{ }^{39} \mathrm{Ar}$ isotopic dating of feldspar were utilized to determine the age of the Tower Mountain rhyolites. Images, compositional data, detailed isotopic data and temperature of formation data for the zircons can be found in Appendix F. Figure 18 contains a summary of likely zircon ages which are: $31.426 \pm 0.016 \mathrm{Ma}$ for CH08a (A-like), $31.798 \pm 0.012$ Ma for TM5 (A-like), $32.169 \pm 0.024$ Ma for CH07a (Dale Tuff-like, I-type), and 32.345 \pm 0.01 for MS12-49 (Dale Tuff, I-type). ${ }^{40} \mathrm{Ar} /{ }^{39} \mathrm{Ar}$ dating was performed on the Dale Tuff. The results are listed in Table F3 in Appendix F and shown in Figure 19. The weighted plateau age was determined to be $32.66 \pm 0.36$ Ma. The ages indicate that the calc-alkaline/I-type rhyolites (Dale Tuff and Dale Tufflike) were followed by the A-like rhyolites. 


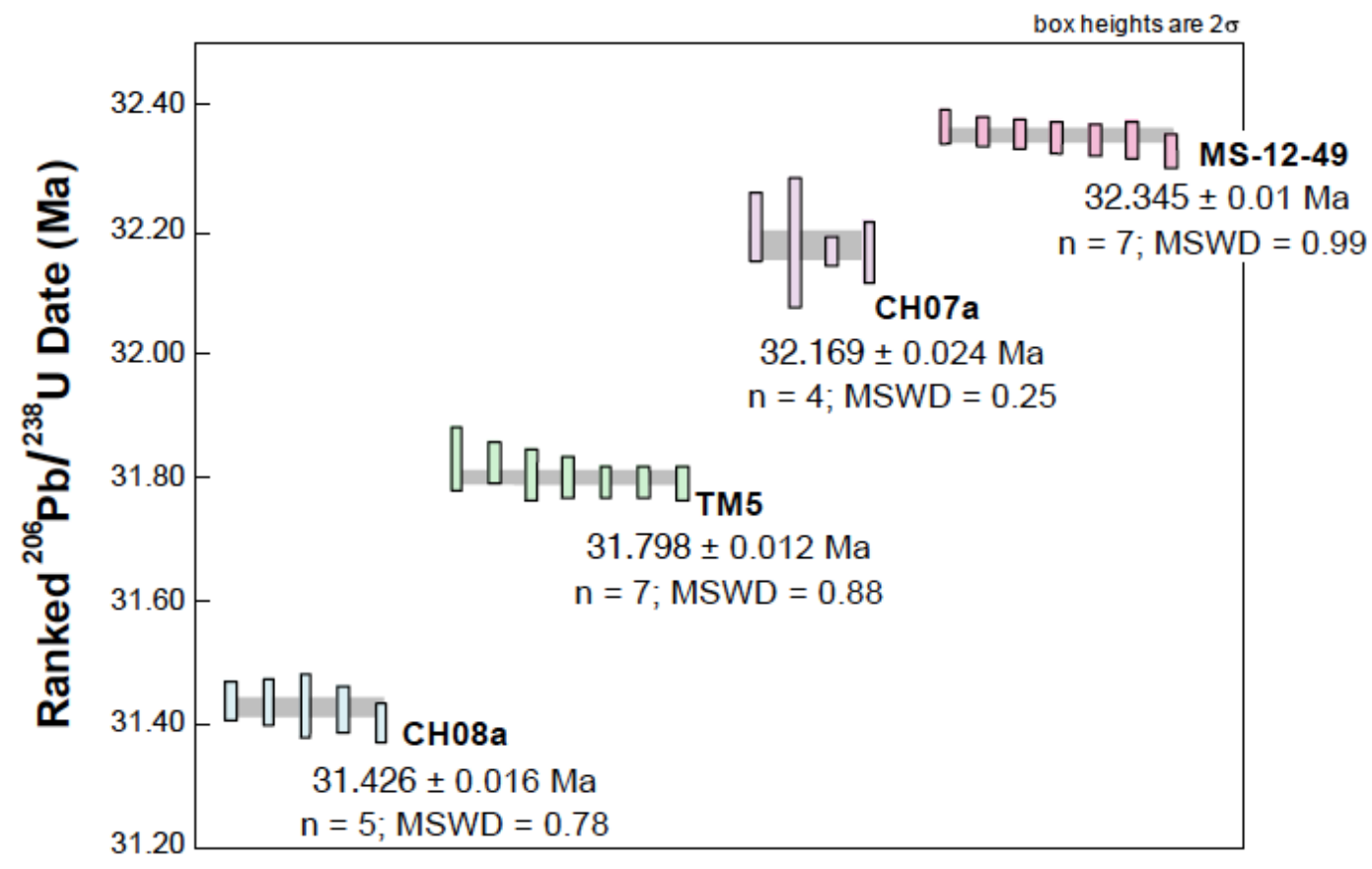

Fig. 18 Ages in millions of years of rhyolite samples CH08a (A-like), TM5 (A-like), CH07a (Dale Tuff-like, I-type), and MS12-49 (Dale Tuff, I-type) via chemical abrasionisotope dilution thermal ionization mass spectrometry (CA-ID TIMS). The colored bars indicate the probability age ranges. $\mathrm{n}=$ number of samples, i.e. number of zircon crystals. MSWD = mean square weighted deviation (Schmitz, 2016).

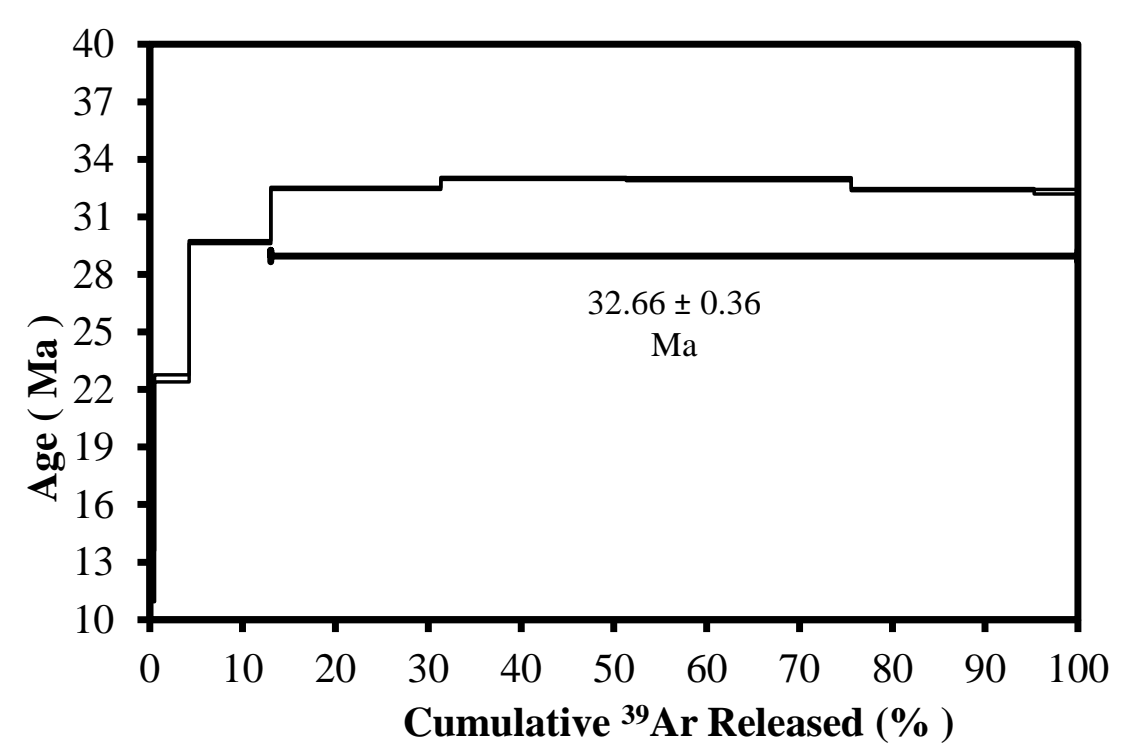

Fig. $19{ }^{40} \mathrm{Ar} /{ }^{39} \mathrm{Ar}$ weighted plateau age for the Dale Tuff. The weighted mean ages are calculated by weighting each age analysis by the inverse of the variance. The weighted mean error is calculated using the method of Taylor (1982). The plateau must comprise at least

two contiguous steps and indicates the most probable age, which in this case is $32.66 \pm$ $0.36 \mathrm{Ma}$. 


\section{CRUSTAL ROCKS}

\section{Metamorphic Samples}

The metamorphic samples range widely in composition and grade. Several of these samples will be discussed in detail in this section.

Sample MS12-47 is a biotite fels, which is a metamorphic rock found in abundance in the study area. It should be noted that the term "fels" is used here in reference to the texture as opposed to the hornfels metamorphic facies. The texture is partially lepidoblastic and partially granoblastic with a pure quartz groundmass. Mineral phase modal abundance is as follows: $\sim 70 \%$ quartz, $\sim 13 \%$ biotite, $\sim 7 \%$ plagioclase (albite), $\sim 8 \%$ alkali feldspar (orthoclase), and $\sim 2 \%$ muscovite. The mineral assemblage indicates that the protolith may be an immature sandstone. Feldspars show alteration to sericite. Figure 20 is a plane light image of a thin section of MS12-47; Figure 21 is a BSE image.

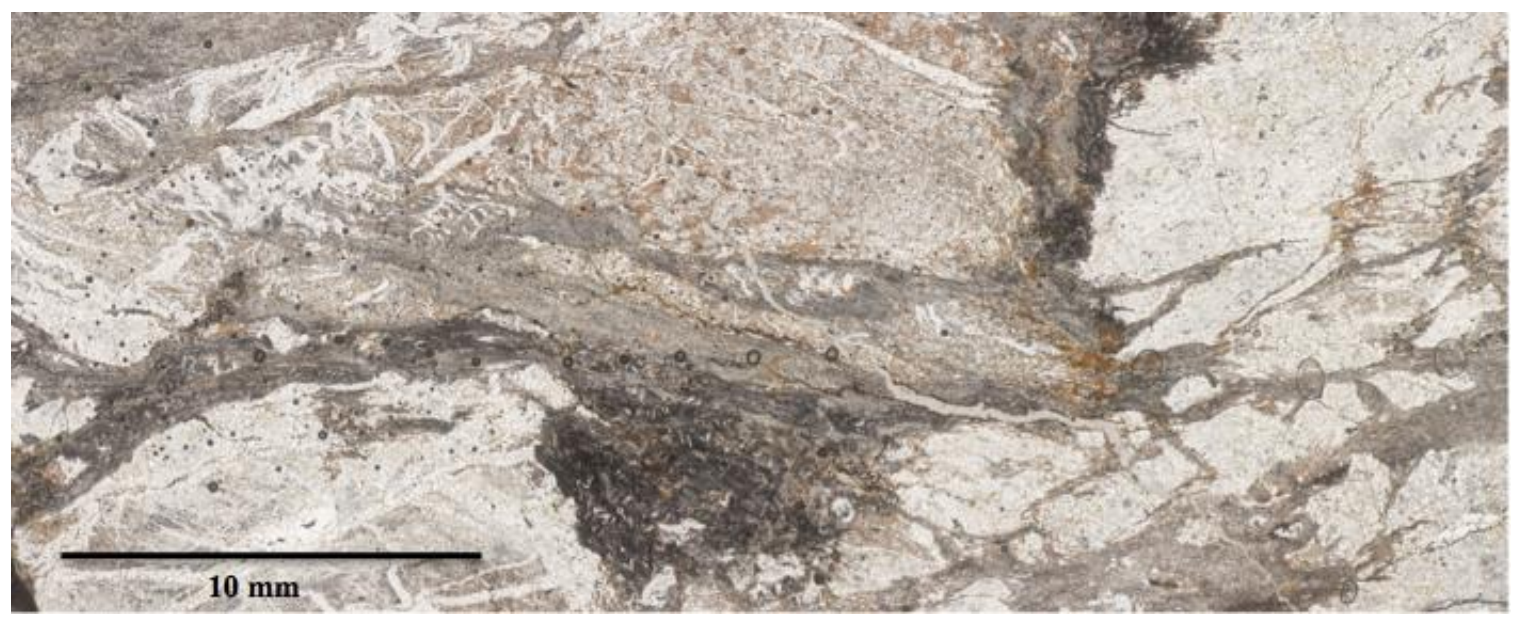

Fig. 20 Plane light image of a thin section of sample MS12-47, biotite fels. Quartz is the primary mineral phase, which is light grey in color in the above image. See text for detailed description. 


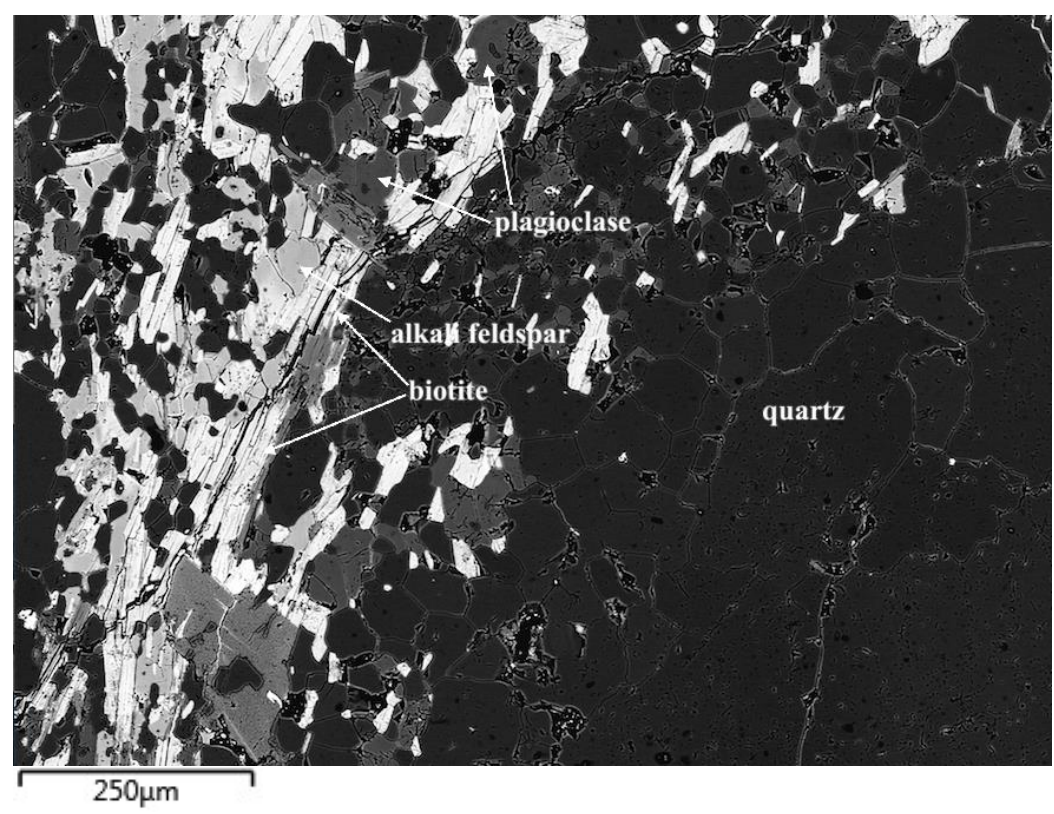

Fig. 21 Backscattered electron (BSE) image MS12-47, biotite fels. The texture is partially lepidoblastic with incipient schistosity (left side of image) with a pure quartz groundmass (right side of image). Porphyroblasts are of quartz, biotite, plagioclase, alkali feldspar, and muscovite.

Sample TM13-5 is amphibolite, which is formed when basalt undergoes medium-grade regional metamorphism. It is medium-grained in texture, shows weak to moderate foliation/incipient schistosity, and contains primarily amphibole and plagioclase feldspar with biotite and chlorite as accessory phases. Large hornblende porphyroblasts and actinolite laths make up over $50 \%$ of the sample by area. Plagioclase accounts for $\sim 40 \%$; oxides, $\sim 10 \%$, and biotite and chlorite, $<1 \%$. Figure 22 is a plane light image of a thin section of TM13-5.

TM13-7 is mica schist. It is medium-grained with strong foliation and contains mainly quartz and biotite, and to a lesser extent, muscovite and chlorite. There are zones that contain only quartz and here the grains are interlocking and show strong undulatory extinction. Chevron-like folds can be seen overprinting the original schistose orientation of the grains. Figure 23 is a plane light thin section image of TM13-7. 


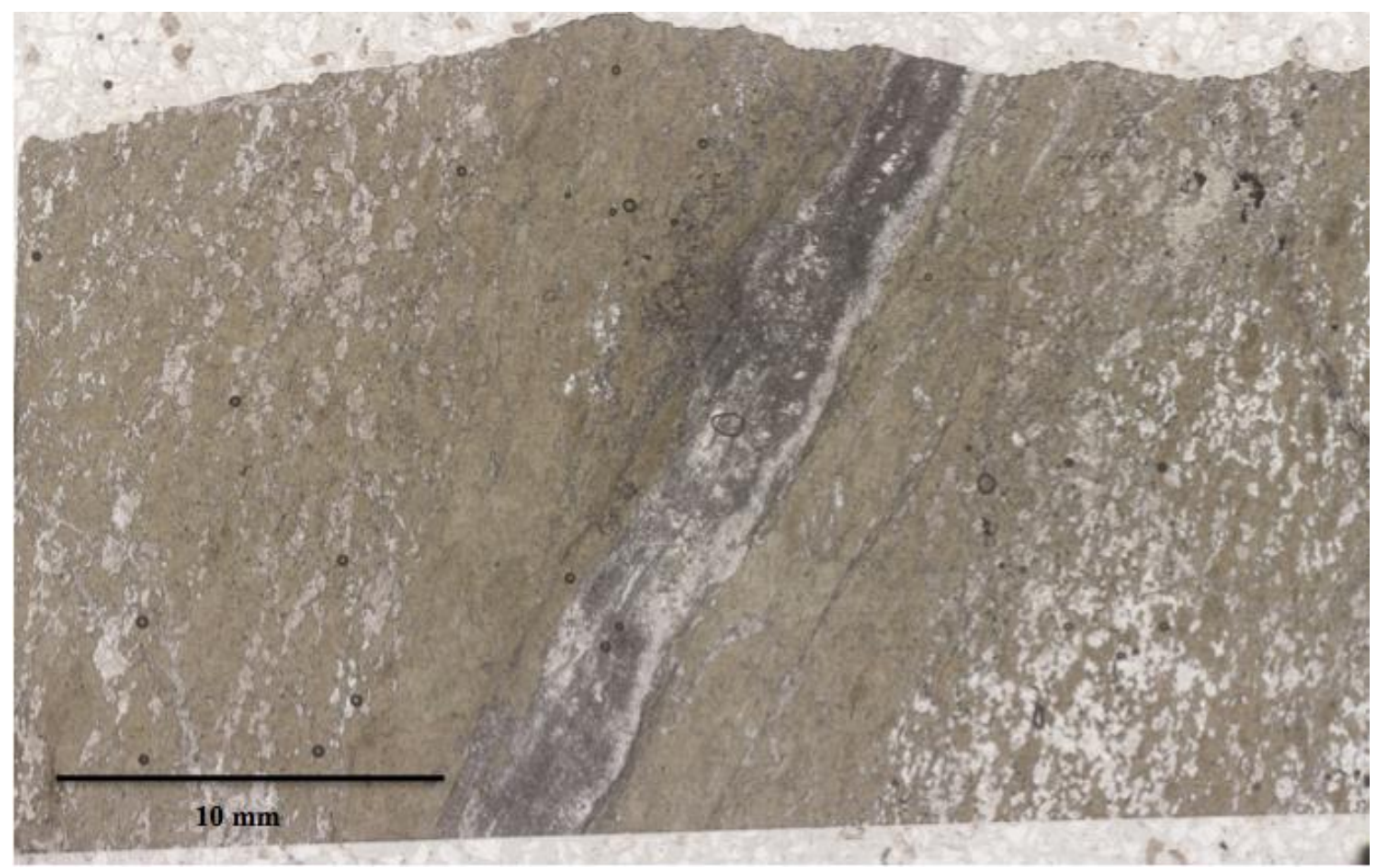

Fig. 22 Plane light image of a thin section of sample TM13-5, amphibolite. Weak foliation of mineral grains is visible. The primary mineral phase is amphibole, which lends the sample its overall green hue. The light grey areas are plagioclase. See text for detailed description.

TM13-1 is a biotite schist. It is fine-grained with well-developed foliation. Quartz shows sutured boundaries, and some grains show undulating extinction. Biotite displays moderate parallelism with amphibole porphyroblasts found among biotite laths.

Segregation of minerals into mica-rich and quartz-rich domains parallel to the schistosity is observed. There are few $(\sim 1-2 \%)$ oxides. Figure 24 is a plane light thin section image of sample TM13-1.

\section{Geochemistry of metamorphic samples}

Figure 25 shows the normalized trace element concentrations of metamorphic samples for which ICP MS data were obtained. From only brief observation of this figure, it can 


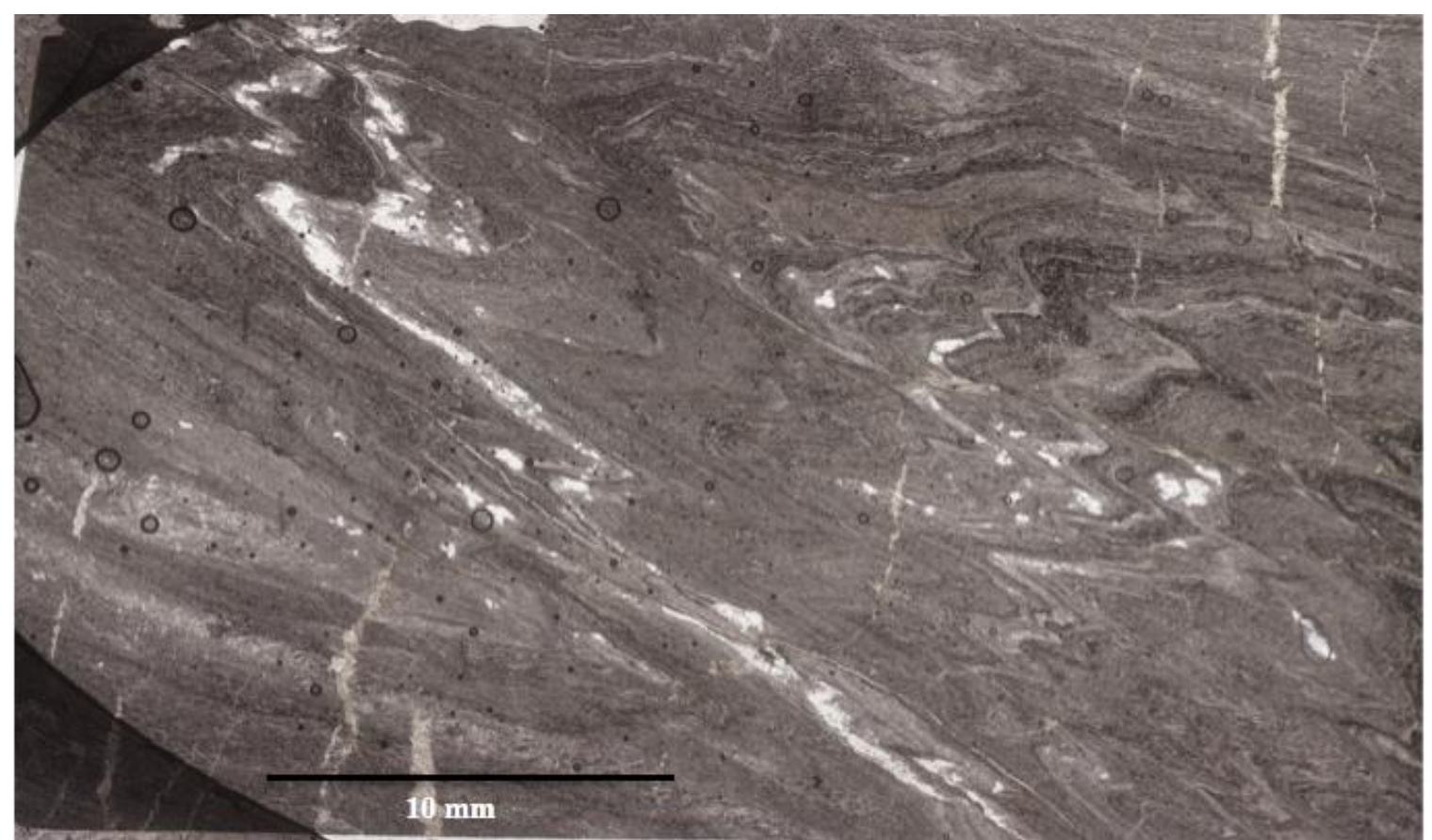

Fig. 23 Plane light image of a thin section of sample TM13-7, biotite schist. Strong foliation and chevron-like folding are visible. The light areas are the "quartz only" zones. See text for detailed description.

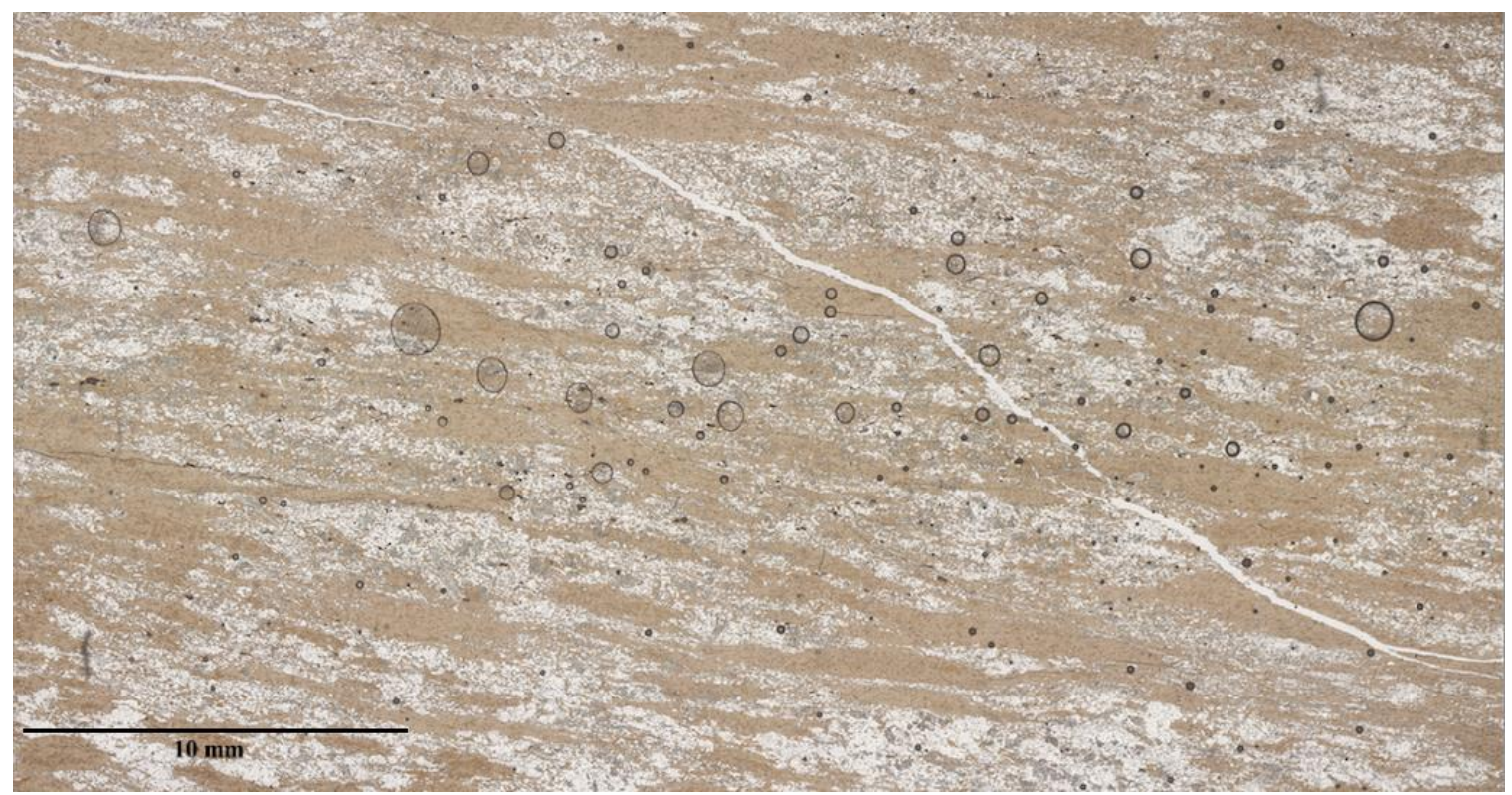

Fig. 24 Plane light thin section image of TM13-1, biotite schist. The light areas are zones of quartz while the darker areas are biotite. Careful observation also reveals the greenish hue of amphibole. Weak to moderate foliation can be seen. See text for further discussion. 
be seen that the metamorphic samples are compositionally diverse. Rubidium enrichment ranges from $\sim 350$ times that observed in the primitive mantle (TM13-1, biotite schist) to $\sim 2$ times that of the primitive mantle (TM13-5, amphibolite). This diversity of enrichment decreases as incompatibility decreases. That is, there is a narrower range of enrichment values observed for lutetium; all samples fall between $\sim 3$ and 10 times the concentrations observed in the primitive mantle. All samples show a peak at barium and uranium and a trough at thorium. Samples MS12-47, TM13-1, TM13-6, TM13-7b peak at lead, while sample TM13-7 (mica schist) displays a negative anomaly and sample TM135 (amphibolite) shows neither. Samples TM13-5 and TM13-1 peak at strontium, while samples MS12-47, TM13-7 and TM13-7b show a trough here. TM13-6 (micaceous quartzite) displays neither. Samples TM13-7b (mica schist) and TM13-5 (amphibolite) display mirrored patterns for zirconium and hafnium; a peak and a trough, respectively.

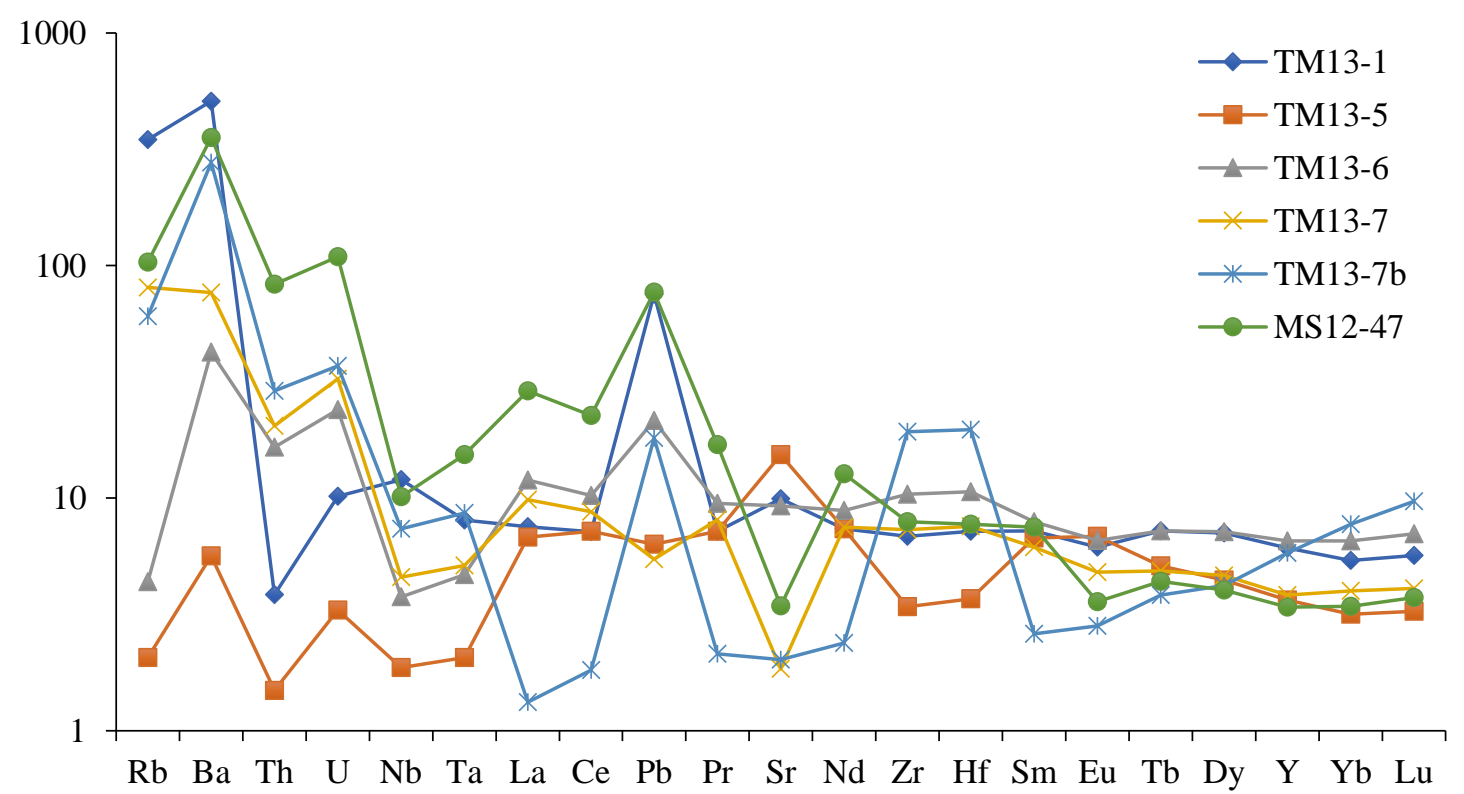

Fig. 25 Primitive mantle normalized (Sun and McDonough, 1989) spider diagram of the trace element concentrations of metamorphic samples. TM13-1 is biotite schist, TM13-5 is amphibolite, TM13-6 is micaceous quartzite, TM13-7 and 13-7b are mica schist, and MS12-47 is biotite fels. 


\section{$\underline{\text { Petrography of plutonic samples }}$}

Tower Mountain plutonic rocks are of two types, silicic and intermediate. Examples of the petrography of each type are discussed below.

Sample G314-09-4 is granite with 75.97 weight percent $\mathrm{SiO}_{2}$ and mineral modal abundance as follows: $\sim 80 \%$ quartz, $\sim 15 \%$ alkali feldspar, $\sim 3 \%$ plagioclase feldspar, $\sim 1 \%$ garnet, $\sim 1 \%$ biotite, $<1 \%$ chlorite. The granite displays interlocking grains of quartz with both alkali feldspar and sparse plagioclase feldspar. Some quartz display undulatory extinction. The feldspars are partially seritized. Graphic texture, the intergrowth of quartz and alkali feldspar, is observed. Sparse, yet large ( $\sim 2 \mathrm{~mm})$, and highly fractured garnet phenocrysts are observed. The undulatory extinction of quartz and presence of chlorite point to low-grade metamorphism. Figure 26 is a plane light image of sample G314-09-4.

Sample TM13-4 is granite with 72.91 weight percent $\mathrm{SiO}_{2}$. It displays large, interlocking phenocrysts of quartz, alkali feldspar, plagioclase, and biotite. Feldspars show strong compositional zoning. There are excellent examples of graphic texture and feldspar exsolution lamellae throughout. Accessory chlorite and muscovite are subhedral. The modal abundance of minerals present is as follows: $\sim 60 \%$ alkali-feldspar, $~ 25 \%$ quartz, $\sim 10 \%$ biotite, $<5 \%$ plagioclase feldspar, $\sim 1 \%$ chlorite, and $<1 \%$ muscovite. Figure 27 is a plane light thin section image of TM13-4. 


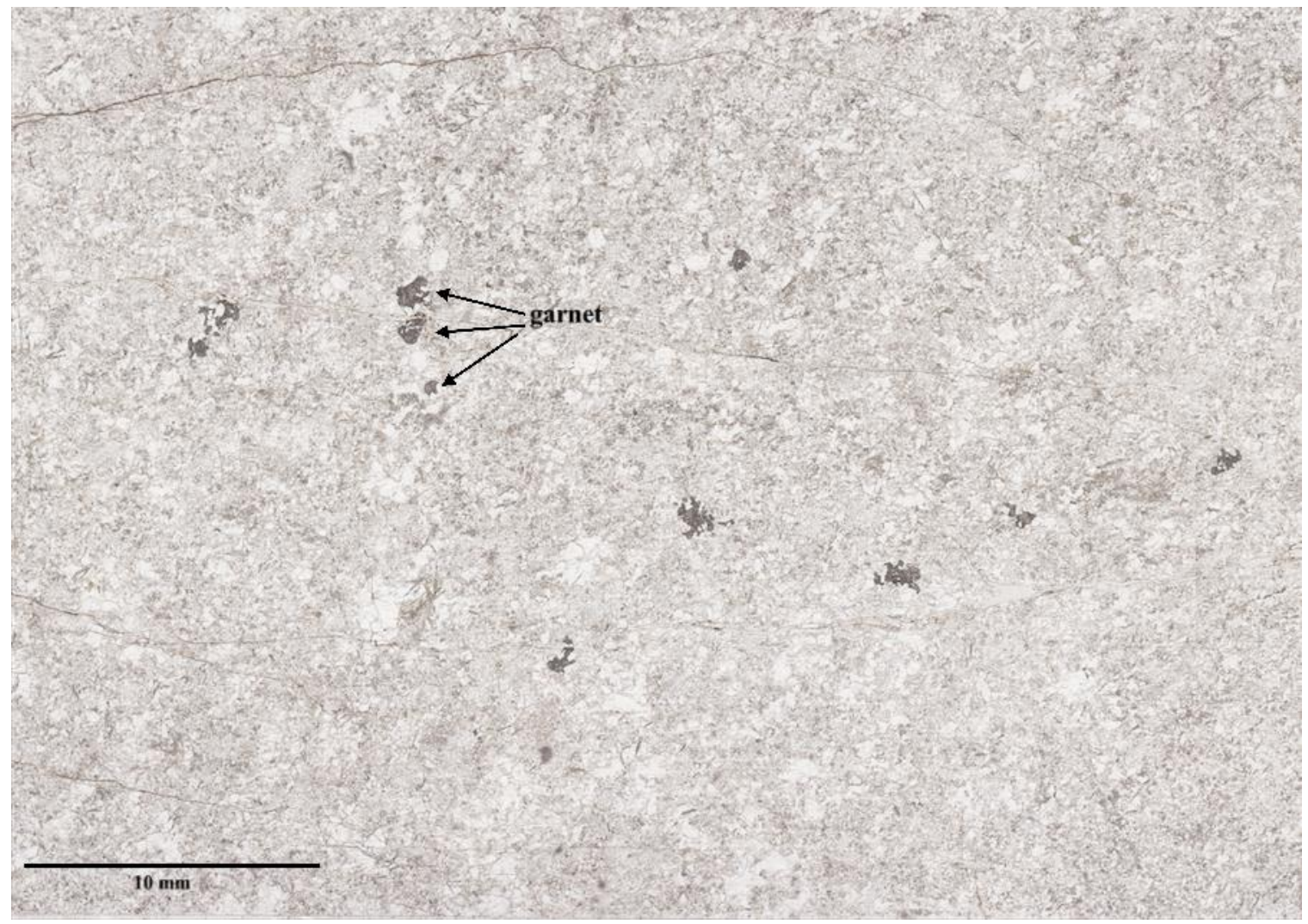

Fig. 26 Plane light thin section image of sample G314-09-4, granite. Note the overall light color imparted by felsic minerals and the interlocking texture of phenocrysts. The relatively large and dark "blotchy" areas are fractured garnet phenocrysts. A faint greenish hue is imparted by chlorite and biotite. See text for further discussion.

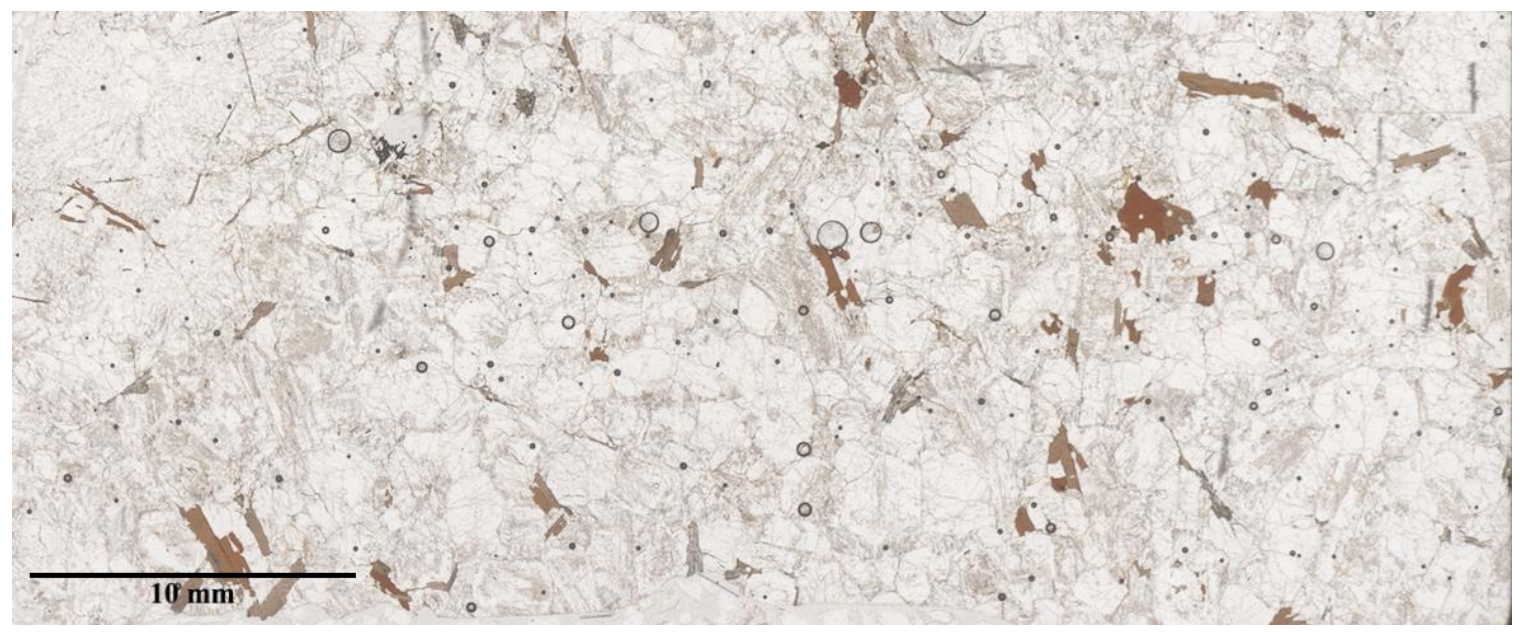

Fig. 27 Plane light thin section image of TM13-4, granite. Compare to Figure 25. The overall light color is an indication of the abundance of silicic minerals; quartz, alkalifeldspar, and plagioclase feldspar. The greenish brown laths and blades are biotite. See text for further discussion. 
Sample TM13-3 is granodiorite with 62.52 weight percent $\mathrm{SiO}_{2}$. It is medium to largegrained and the texture is phaneritic. Phenocrysts are of plagioclase feldspar, alkali feldspar, amphibole, biotite, and quartz. Feldspars are both euhedral and subhedral with strong compositional zoning and abundant twinning. This zoning can be seen in Figure 29 , which is a BSE image of this sample. Subhedral to anhedral amphibole are located in interstitial spaces. The modal abundance of minerals is as follows: 25\% quartz, 20\% plagioclase feldspar, 20\% alkali feldspar, $20 \%$ amphibole, $~ 5 \%$ biotite, $<1 \%$ chlorite, $<1 \%$ apatite. Figure 28 is a plane light thin section image of TM13-3; Figure 29 is a BSE image of the same.

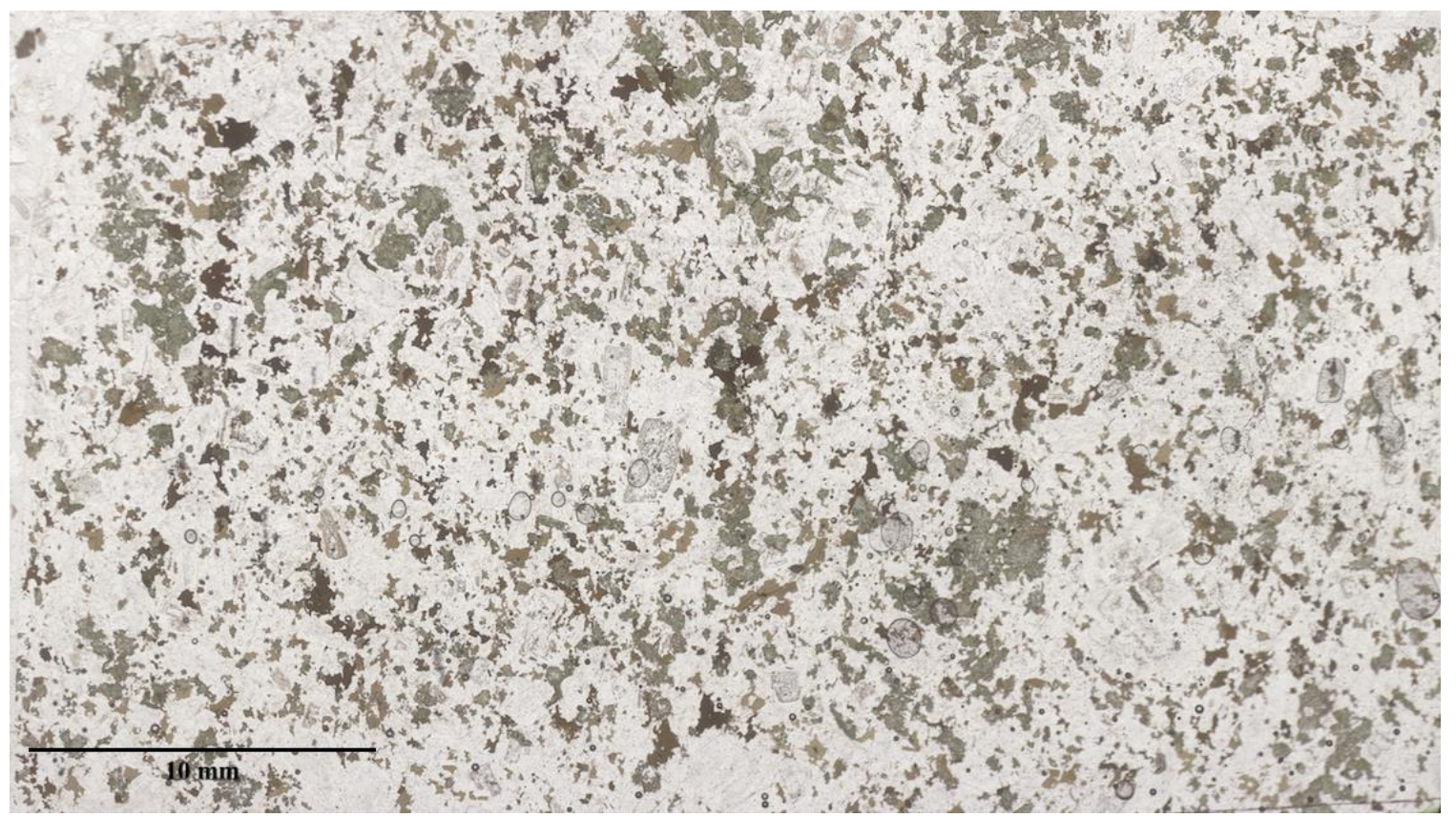

Fig. 28 Plane light thin section image of sample TM13-3, granodiorite. Note the interlocking texture of the phenocrysts and the mix of mafic and felsic minerals, which is characteristic of an intermediate igneous rock. See text for further discussion. 


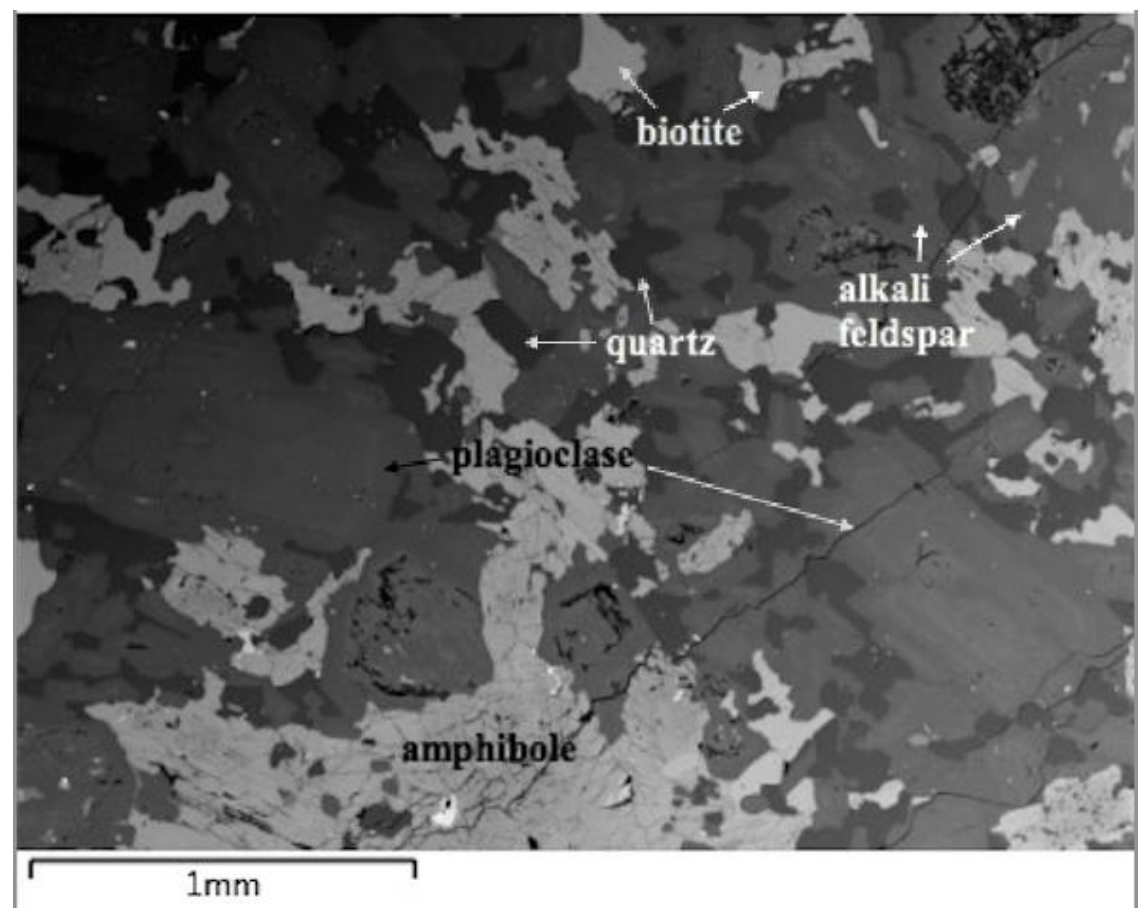

Fig. 29

Backscattered electron (BSE) image of sample TM13-3, granodiorite. Note the interlocking texture of the phenocrysts. The compositional zoning of plagioclase feldspar can be seen.

Sample TM13-9 is diorite with 62.00 weight percent $\mathrm{SiO}_{2}$. Figure 30 is a plane light thin section image of this sample. The mineral modal abundance is as follows: $50 \%$ plagioclase feldspar, $\sim 20 \%$ biotite, $\sim 10 \%$ quartz, $~ 10 \%$ amphibole, $\sim 5 \%$ alkali feldspar, $\sim 5 \%$ oxides. The texture is phaneritic with some plagioclase phenocrysts greater than 5 $\mathrm{mm}$. Plagioclase is euhedral and shows strong compositional zoning. All other phenocrysts are euhedral to subhedral with oxides being relatively large and equant.

\section{Geochemistry of plutonic samples}

All geochemical data can be found in Table B3 in Appendix B. Figure 31 is a primitive mantle normalized (Sun and McDonough 1989) spider diagram of the trace element concentrations of the intermediate and silicic plutonic rocks for which ICP MS data was obtained. All samples are enriched in all trace elements as compared to the primitive mantle. This enrichment varies from $\sim 100$ times for $\mathrm{Rb}$ and $\mathrm{Pb}$, to just above primitive 


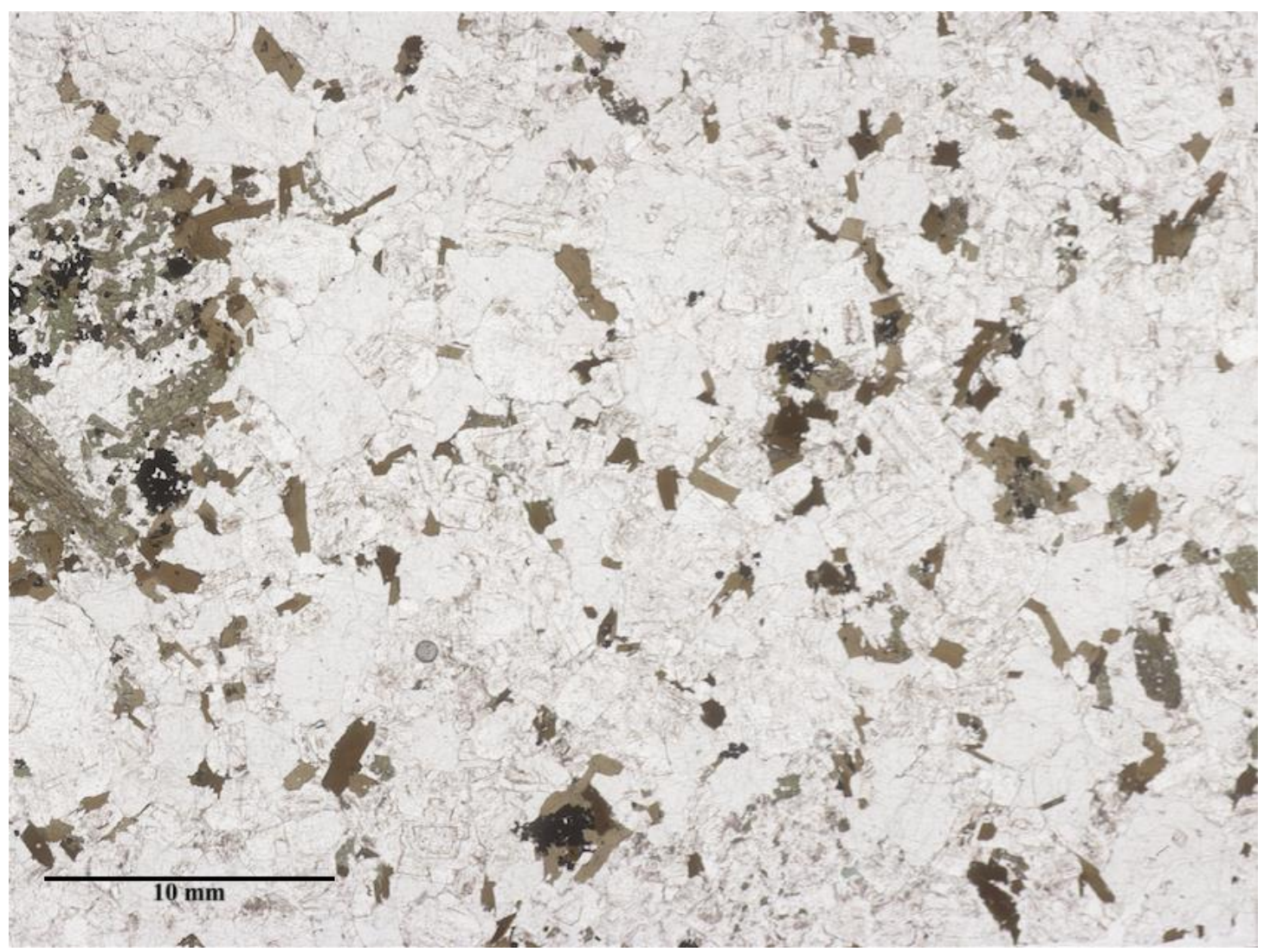

Fig. 30 Plane light image of a thin section of sample TM13-9, diorite. This sample contains interlocking plagioclase feldspar, quartz, biotite, and amphibole with accessory oxides. Note the relatively large and highly euhedral plagioclase feldspar, which constitutes the bulk of this sample. See text for further discussion.

mantle concentrations for HREE. All samples except G314-09-4 (granite) show a peak at barium, where this sample shows a negative anomaly. A similar pattern is seen at strontium, but here TM13-2 (granite dike) shows neither a peak nor trough, while all other samples except G314-09-4 show a peak. Samples G314-09-4 and TM13-2 show slight troughs at zirconium. G314-09-4 has a negative europium anomaly; TM13-2 shows a slight anomaly here, as well. It is worth noting that sample TM13-4, which is granite, is geochemically more similar to samples TM13-3 (granodiorite) and TM13-9 (diorite) than it is to the other granitic samples. 


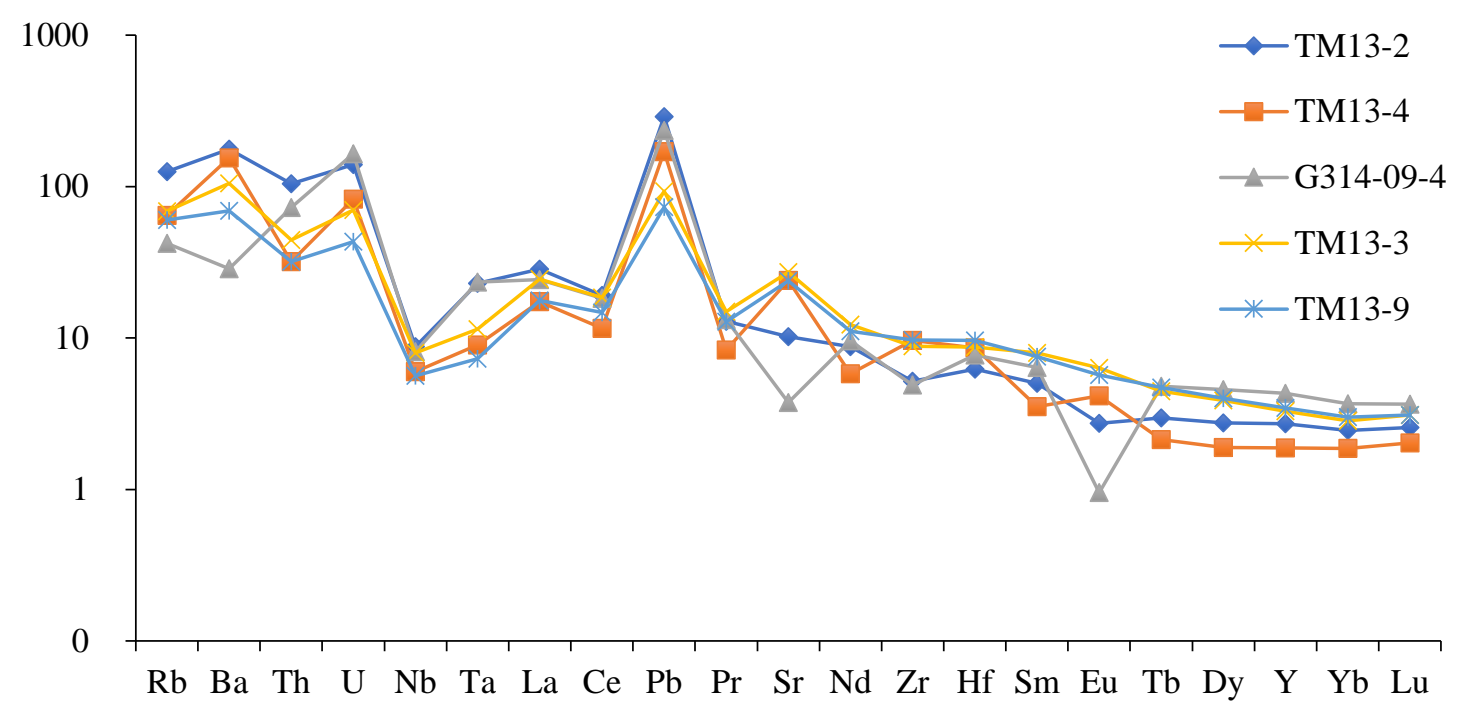

Fig. 31 Primitive mantle normalized (Sun and McDonough, 1989) spider diagram of the trace element concentrations of the silicic and intermediate plutonic samples. TM13-2 is a granite dike, TM13-4 is pegmatitic granite, G314-09-4 is granite, TM13-3 is granodiorite, and TM13-9 is diorite.

\section{MODELING RHYOLITE PETROGENESIS}

\section{Fractional crystallization}

Basalt sample G314-09-2 was used to investigate the possibility of the formation of rhyolites via fractional crystallization. When a basalt undergoes fractional crystallization it takes $>90 \%$ crystallization for the remaining liquid to reach rhyolitic compositions (Grove and Gerlach, 1982). Therefore, modeling was done only for remaining liquids of $5 \%$ or less. The unnormalized results of these calculations are listed in Table 3 . Figure 32 shows a primitive mantle normalized spider diagram comparing the results of modeled fractional crystallization of the basalt with the observed trace element concentrations of representative rhyolites. The model produces a flattened pattern where it is compared to that of the rhyolites. For the basalt, a mild peak is seen at barium, which is not shared by the rhyolites. Additionally, the strong lead peak that is shared among all rhyolites is 
absent. Troughs occur at thorium, strontium, and europium. Troughs also occur at strontium and europium among the rhyolites. Overall, the fit between the basalt and the rhyolites is unsatisfactory.

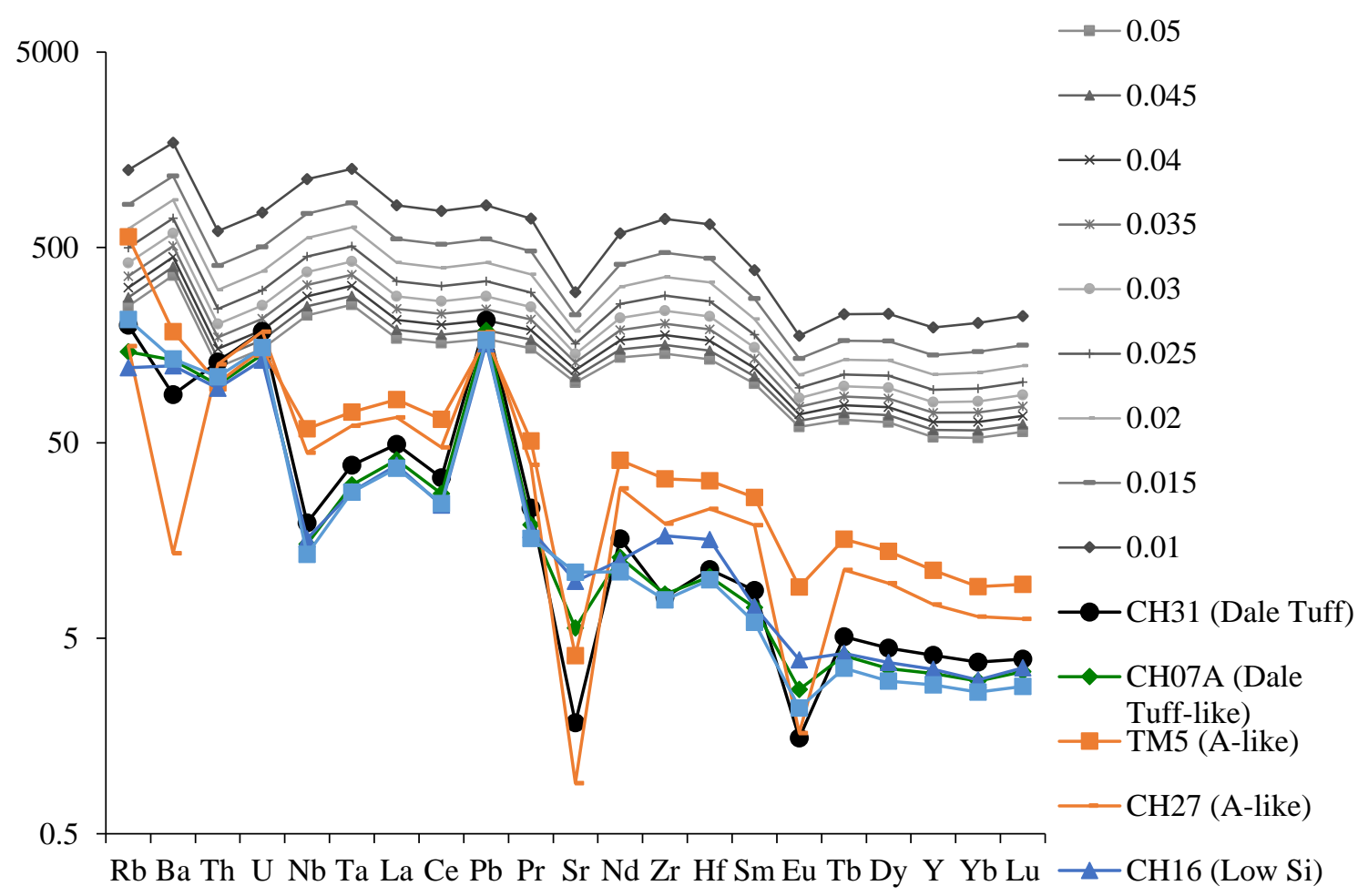

Fig. 32 Primitive mantle normalized (Sun and McDonough, 1989) spider diagram of the results of fractional crystallization modeling of basalt sample G314-09-2. The trace element concentrations at differing remaining melt fractions (5-1\%) are represented by the greyscale lines with the remaining melt fractions listed in the key.

Table 3. Unnormalized trace element concentrations of basalt fractionation

\begin{tabular}{crrrrrrrrr}
\hline \hline \multicolumn{8}{c}{ melt fraction remaining } \\
\hline $\mathrm{ppm}$ & \multicolumn{1}{c}{0.05} & \multicolumn{1}{c}{0.045} & \multicolumn{1}{c}{0.04} & \multicolumn{1}{c}{0.035} & 0.03 & \multicolumn{1}{c}{0.025} & 0.02 & 0.015 & \multicolumn{1}{c}{0.01} \\
\hline $\mathrm{Rb}$ & 158.7 & 176.3 & 198.3 & 226.6 & 264.2 & 316.9 & 395.9 & 527.4 & 790.3 \\
$\mathrm{Ba}$ & 2515.2 & 2786.2 & 3124.0 & 3556.7 & 4131.2 & 4931.7 & 6125.4 & 8100.3 & 12010.5 \\
$\mathrm{Th}$ & 10.3 & 11.5 & 12.9 & 14.8 & 17.2 & 20.7 & 25.8 & 34.4 & 51.5 \\
$\mathrm{U}$ & 3.2 & 3.5 & 4.0 & 4.5 & 5.3 & 6.4 & 7.9 & 10.6 & 15.8 \\
$\mathrm{Nb}$ & 160.5 & 178.2 & 200.4 & 228.9 & 266.9 & 320.1 & 399.8 & 532.4 & 797.3 \\
$\mathrm{Ta}$ & 10.4 & 11.6 & 13.0 & 14.9 & 17.4 & 20.8 & 26.0 & 34.6 & 51.8 \\
$\mathrm{La}$ & 117.6 & 130.3 & 146.2 & 166.5 & 193.5 & 231.1 & 287.2 & 380.2 & 564.3 \\
$\mathrm{Ce}$ & 287.3 & 318.2 & 356.7 & 405.9 & 471.2 & 562.2 & 697.9 & 922.1 & 1365.6 \\
\hline
\end{tabular}




\begin{tabular}{crrrrrrrrr}
\hline $\mathrm{Pb}$ & 12.1 & 13.4 & 15.1 & 17.2 & 20.0 & 23.9 & 29.7 & 39.3 & 58.4 \\
$\mathrm{Pr}$ & 42.1 & 46.5 & 52.1 & 59.1 & 68.4 & 81.3 & 100.5 & 132.1 & 194.1 \\
$\mathrm{Sr}$ & 2149.6 & 2304.5 & 2491.0 & 2720.8 & 3012.5 & 3398.1 & 3937.9 & 4762.3 & 6225.3 \\
$\mathrm{Nd}$ & 184.9 & 203.6 & 226.6 & 255.9 & 294.5 & 347.6 & 425.9 & 553.5 & 800.6 \\
$\mathrm{Zr}$ & 1601.3 & 1777.0 & 1996.3 & 2277.8 & 2652.5 & 3176.0 & 3959.4 & 5260.9 & 7853.0 \\
$\mathrm{Hf}$ & 41.4 & 45.9 & 51.6 & 58.9 & 68.5 & 82.1 & 102.3 & 136.0 & 203.1 \\
$\mathrm{Sm}$ & 44.6 & 48.6 & 53.7 & 60.0 & 68.2 & 79.3 & 95.5 & 121.4 & 170.1 \\
$\mathrm{Eu}$ & 10.1 & 10.9 & 11.8 & 12.9 & 14.3 & 16.1 & 18.7 & 22.7 & 29.7 \\
$\mathrm{~Tb}$ & 7.1 & 7.7 & 8.4 & 9.3 & 10.5 & 12.1 & 14.4 & 18.0 & 24.6 \\
$\mathrm{Dy}$ & 47.0 & 51.1 & 56.1 & 62.4 & 70.5 & 81.5 & 97.3 & 122.2 & 168.6 \\
$\mathrm{Y}$ & 244.0 & 265.5 & 291.8 & 324.8 & 367.6 & 425.5 & 508.9 & 641.0 & 887.5 \\
$\mathrm{Yb}$ & 26.1 & 28.6 & 31.6 & 35.3 & 40.2 & 46.9 & 56.5 & 72.0 & 101.3 \\
$\mathrm{Lu}$ & 4.2 & 4.6 & 5.1 & 5.7 & 6.5 & 7.6 & 9.2 & 11.7 & 16.5 \\
\hline
\end{tabular}

\section{PARTIAL MELTING}

\section{Experimental melts}

The typical rhyolitic mineral assemblage of quartz, alkali feldspar and plagioclase feldspar as main phases, with biotite, amphibole, pyroxene, FeTi oxides, and zircon as accessory phases, is not observed in the majority of experimental melts (see Appendix C). Plagioclase is the dominant feldspar observed, with alkali feldspar being found in only two of the experimental melts. Additionally, quartz is not observed as a distinct mineral phase in the majority of experimental melts. Mineral assemblages that are consistent with dacite (plagioclase feldspar, amphibole, biotite, and pyroxene) are frequently observed. Typical mafic mineral assemblages, such as plagioclase with clinopyroxene, are infrequently observed.

The average major-element concentrations of the glass (i.e. "melt" phase) were determined via electron microprobe. The $\mathrm{SiO}_{2}$ weight percent and alkali $\left(\mathrm{Na}_{2} \mathrm{O}+\mathrm{K}_{2} \mathrm{O}\right)$ 
weight percent were used to classify the experimental melts via the TAS system (LeBas et al., 1986). The summary of these results is found in Table 4. The majority are classified as rhyolite, however, nearly as many melts are classified as dacite. The minority are classified as andesite. Table 4 also contains a summary of the results of partial melt modeling, which is based on the observation and interpretation of the spider diagrams. Common exceptions to "best fit" are HREE, Sr, Eu, Ba, and Th. Exceptions at $\mathrm{Zr}, \mathrm{Hf}, \mathrm{Nd}$ and $\mathrm{Pb}$ were also observed. The parent materials for which the batch melt model most closely fits the observed data of the rhyolites are TM13-3 (granodiorite), MS12-47 (biotite fels), and mixtures that contain MS12-47.

Capsule preparation data, backscattered electron images, phase proportions, compositional analysis, etc. for the experimental melts can be found in Appendix C. Four examples of the results of these experiments are presented below.

The glass of experimental melt MS1 contains an average of $73.89 \% \mathrm{SiO}_{2}$ and $8.08 \%$ total alkali classifying it as rhyolitic. MS1 produced a mineral assemblage of quartz, orthopyroxene, and pyrite from sample MS12-47 (biotite fels). Figure 33 is a phase map of MS1. The melt occupies $\sim 75 \%$ of the total area; phenocrysts are $\sim 25 \%$ of the area. The modal abundance of mineral phases is as follows: $\sim 79 \%$ quartz, $\sim 21 \%$ orthopyroxene, and $\sim 0.2 \%$ pyrite. Quartz is euhedral to subhedral and fairly equant, while orthopyroxene presents two morphologies; tabs and laths. Pyrite appears as rounded inclusions in pyroxene. 


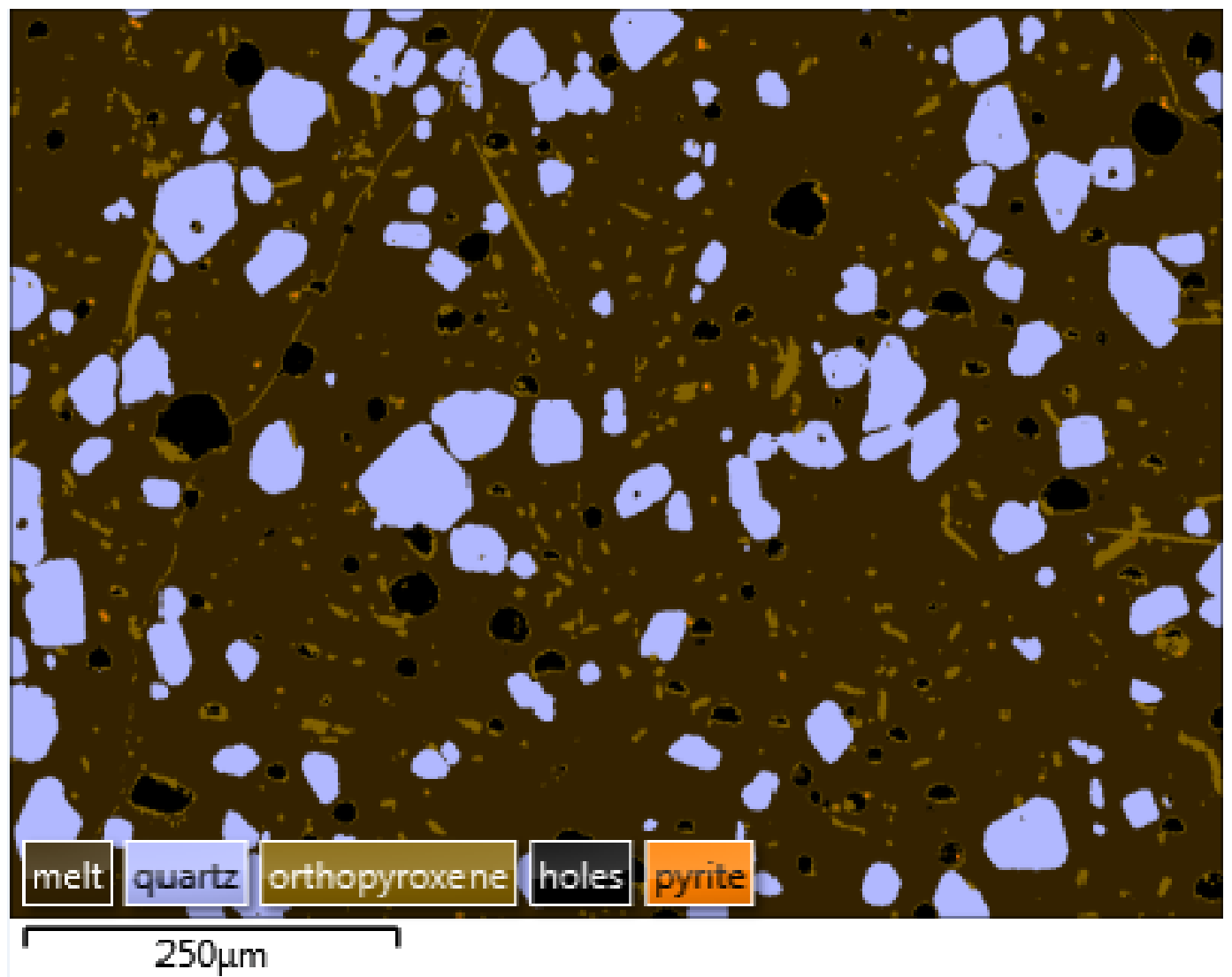

Fig. 33 Energy dispersive X-ray spectrograph (EDS) map of experimental melt MS1 created using the Oxford AZtec software. The order of phases in the key indicates the relative abundance of each phase; melt, quartz, orthopyroxene, pyrite.

The glass of experimental melt MS4 contains an average of $78.1 \% \mathrm{SiO}_{2}$ and $4.29 \%$ total alkali classifying it as rhyolitic. MS4 produced a mineral assemblage of quartz, plagioclase, orthopyroxene, and pyrite from sample MS12-47 (biotite fels). Figure 34 is a phase map of MS4. The melt occupies $\sim 41 \%$ of the total area; phenocrysts, $\sim 56 \%$. The modal abundance of mineral phases is as follows: $~ 75 \%$ quartz, $\sim 14 \%$ plagioclase, $\sim 11 \%$ orthopyroxene, and $\sim 0.02 \%$ pyrite. There are two populations of quartz; the first is larger, subhedral, equant, and rounded, while the second is smaller and ranges from rounded and fairly equant to nearly tabular. Orthopyroxene also presents two 
morphologies; rounded blocks and laths, with some phenocrysts appearing as intermediates between these. Plagioclase is subhedral and blocky. Pyrite appears as inclusions in orthopyroxene.

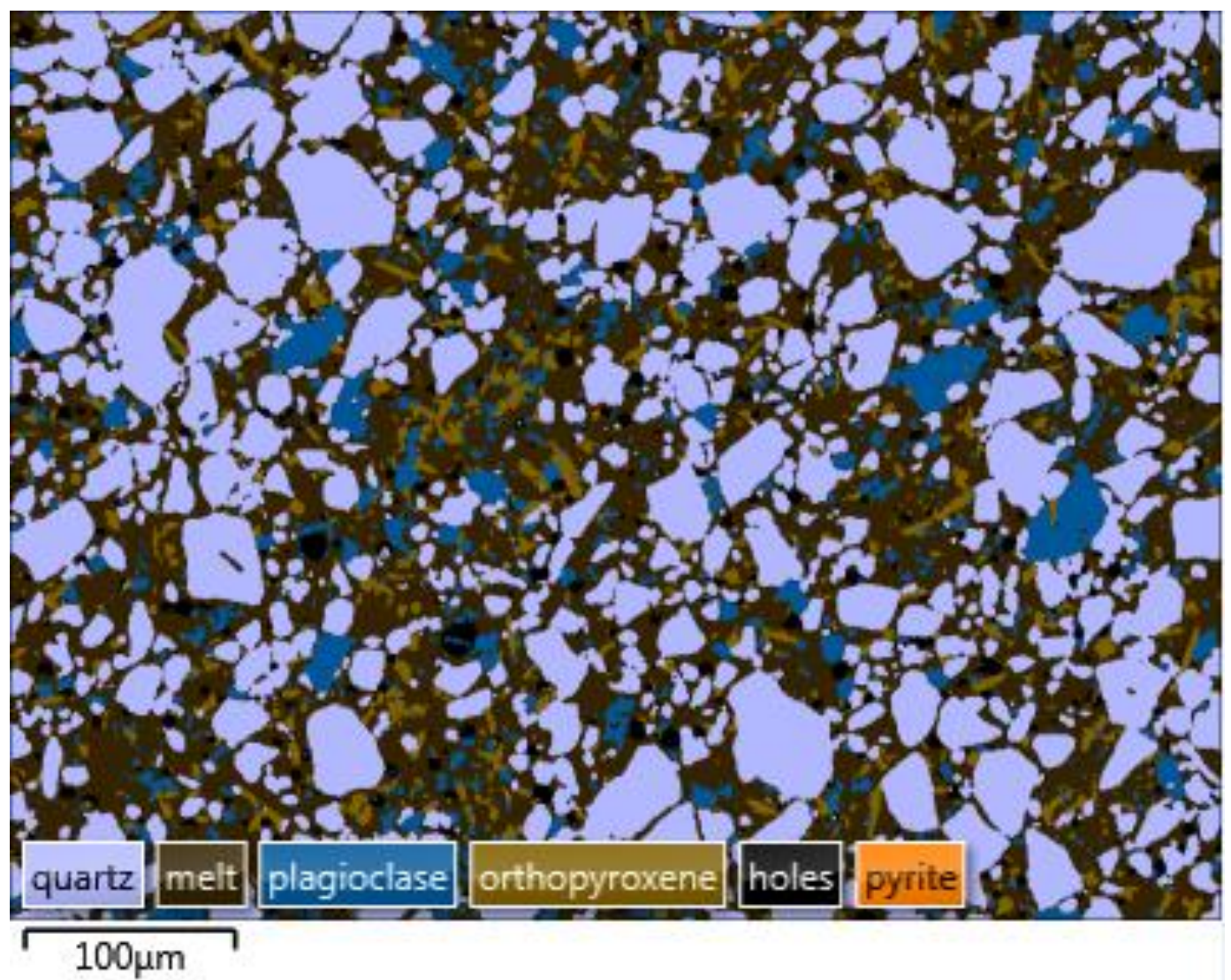

Fig. 34 Energy dispersive X-ray spectrograph (EDS) map of experimental melt MS4 created using the Oxford AZtec software. The order of phases in the key indicates the relative abundance of each phase; quartz, melt, plagioclase, orthopyroxene, and pyrite.

The glass of experimental melt MS5 contains an average of $68.62 \% \mathrm{SiO}_{2}$ and $5.70 \%$ total alkali, classifying it as dacitic. MS5 produced a mineral assemblage of plagioclase feldspar, orthopyroxene, clinopyroxene, FeTi oxides, and apatite from sample TM13-3 (granodiorite). Figure 35 is a phase map of MS5. The melt occupies $\sim 70 \%$ of the sample area; phenocrysts occupy $\sim 27 \%$. The modal abundance of mineral phases is as follows: $\sim 60 \%$ plagioclase feldspar, $\sim 26 \%$ orthopyroxene, $\sim 5 \%$ clinopyroxene, $\sim 2 \%$ FeTi oxides, 
and $\sim 0.3 \%$ apatite. Plagioclase is subhedral to anhedral as is clinopyroxene.

Orthopyroxene presents two morphologies; rounded blocks and laths. FeTi oxides is rather large and rounded in appearance. Apatite is subhedral and rounded; it is seen both as a distinct accessory phase and as inclusions in pyroxene and feldspar. FeTi oxides is rather large and rounded in appearance.

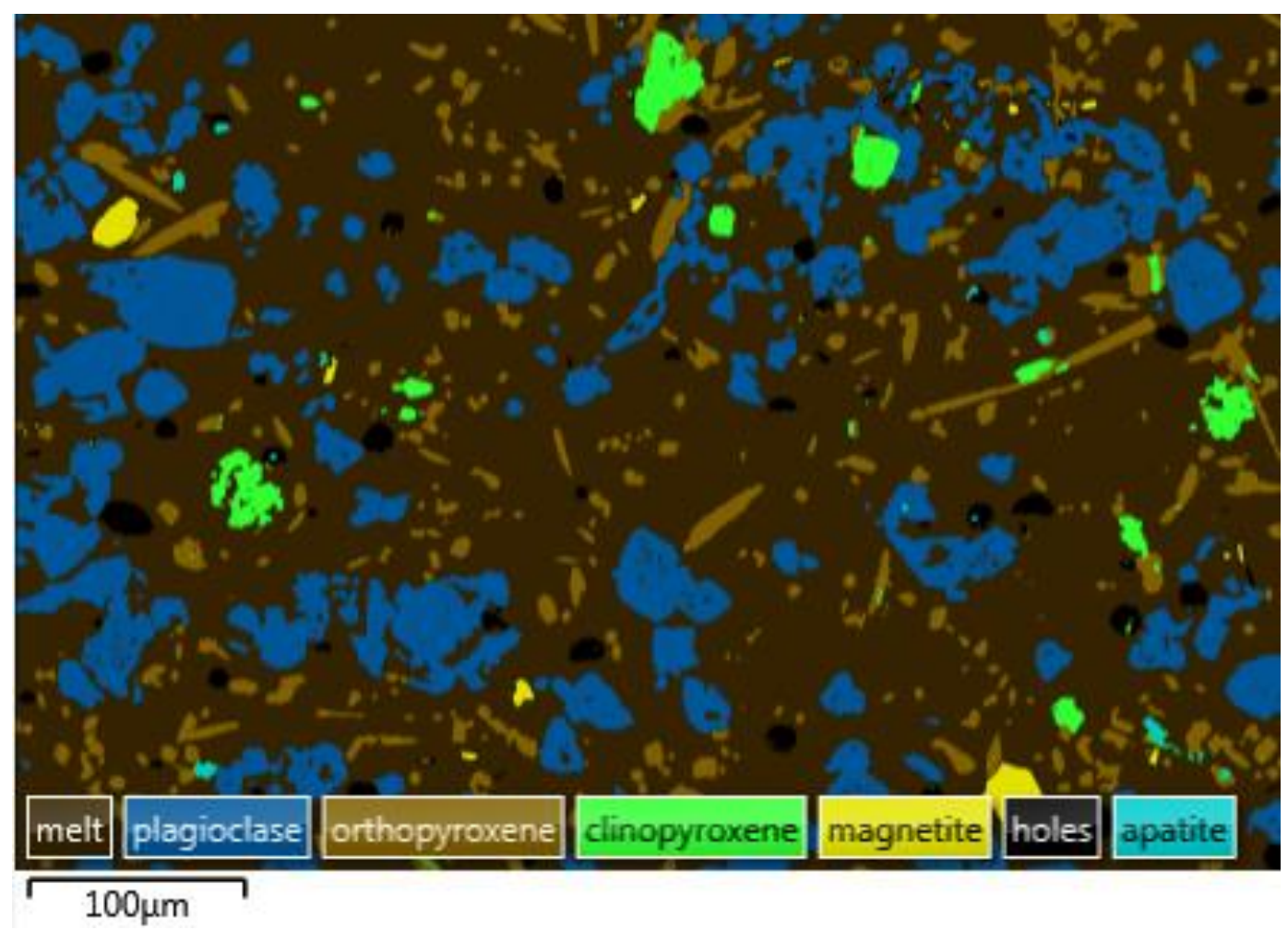

Fig. 35 Energy dispersive X-ray spectrograph (EDS) map of experimental melt MS5 created using the Oxford AZtec software. The order of phases in the key indicates the relative abundance of each phase; melt, plagioclase, orthopyroxene, clinopyroxene, FeTi oxides, apatite.

The glass of MS13 contains an average of $67.26 \% \mathrm{SiO}_{2}$ and $6.16 \%$ total alkali, classifying it as dacitic. MS13 produced a mineral assemblage of plagioclase, orthopyroxene, clinopyroxene, and amphibole from TM13-3 (granodiorite). Figure 36 is a phase map of MS13. The melt occupies $\sim 73 \%$ of the total area, phenocrysts, $\sim 26 \%$. The 
modal abundance of mineral phases is as follows: $\sim 59 \%$ plagioclase, $\sim 37 \%$

orthopyroxene, $\sim 4 \%$ clinopyroxene, $\sim 0.8 \%$ amphibole. Plagioclase is subhedral to

anhedral, as is clinopyroxene. Orthopyroxene presents two morphologies; rounded blocks

and laths. Amphibole is found either as inclusions within plagioclase or is anhedral and rounded in appearance.

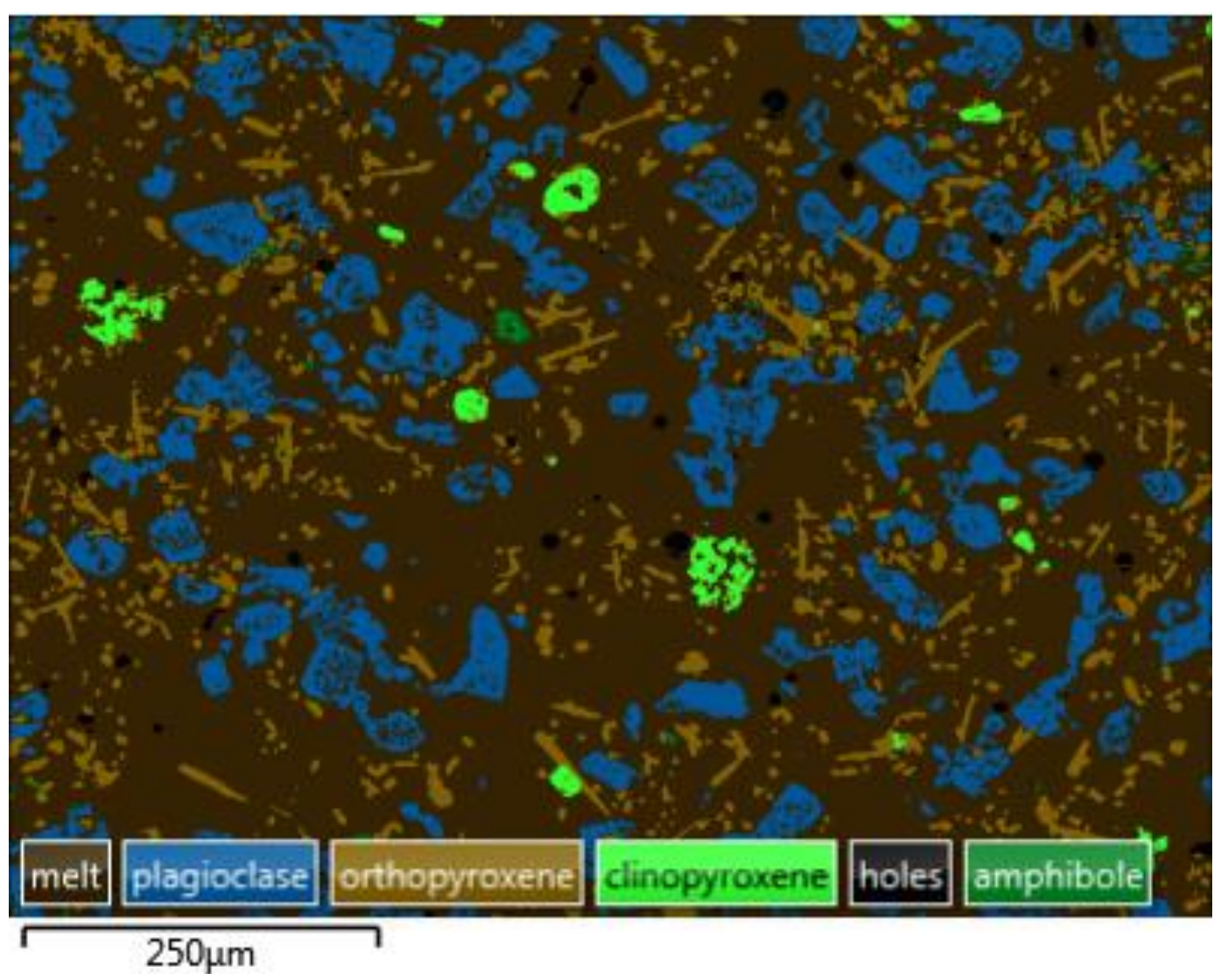

Fig. 36 Energy dispersive X-ray spectrograph (EDS) map of experimental melt MS13 created using the Oxford AZtec software. The order of phases in the key indicates the relative abundance of each phase; melt, plagioclase, orthopyroxene, clinopyroxene, and amphibole.

\section{$\underline{\text { Partial melt models }}$}

Detailed results of the batch and fractional partial melt models can be found in Appendix 

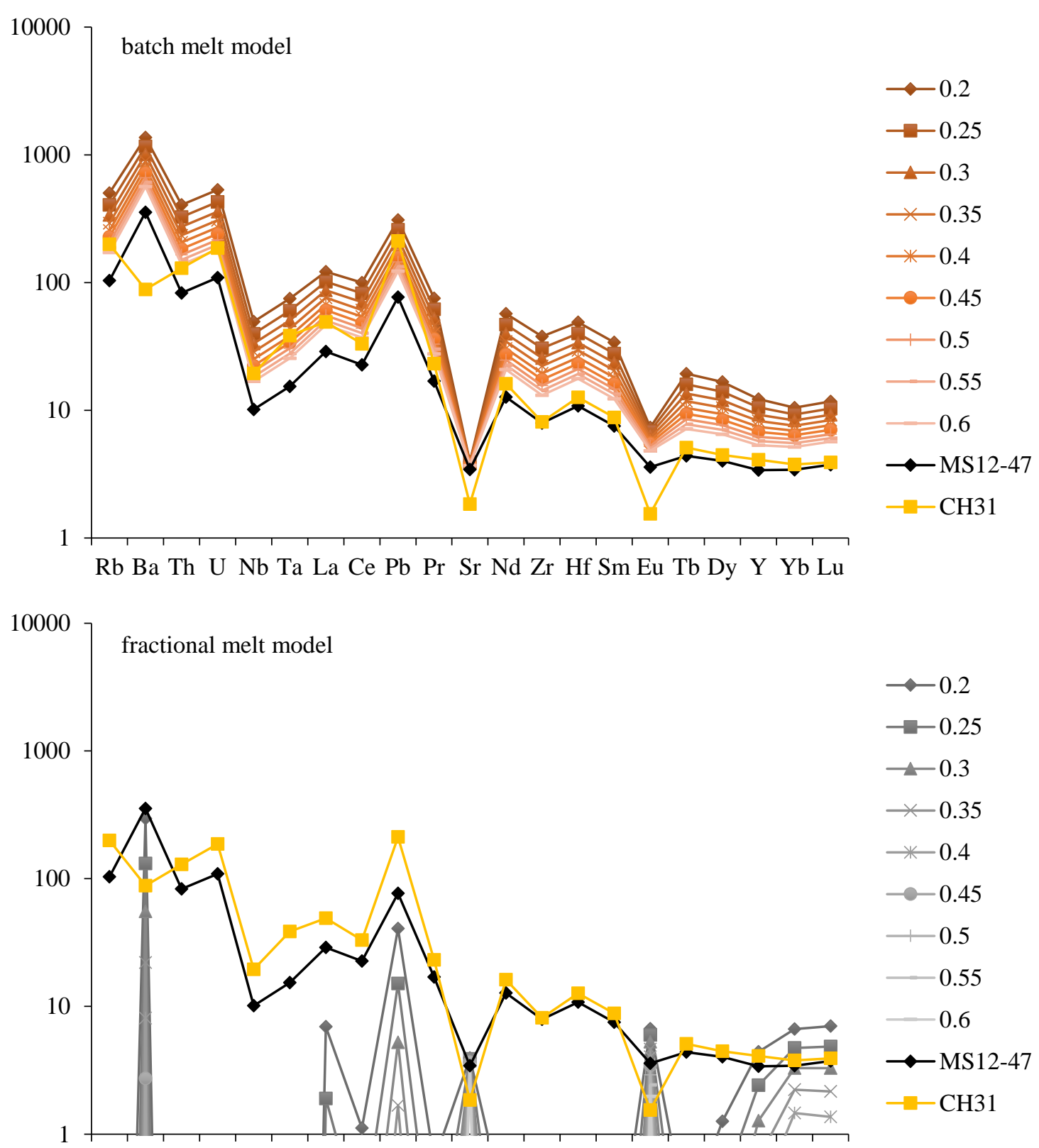

$\mathrm{Rb}$ Ba Th U Nb Ta La Ce Pb Pr Sr Nd Zr Hf Sm Eu Tb Dy Y Yb Lu

Fig. 37 Primitive mantle normalized (Sun and McDonough, 1989) spider diagram for the partial melting of experimental melt MS4. The top graph shows the results of the batch melt model; the bottom shows the results of the fractional melt model. The melt percentages are approximately $\pm 20 \%$ that observed in the experimental melt (MS4) as determined by the AZtec software. The trace element concentrations of the parent material, sample MS12-47 (biotite fels), are indicated by the black diamonds. The trace element concentrations of the Dale Tuff, sample $\mathrm{CH} 31$, which is included for comparison, are indicated by the yellow squares. 


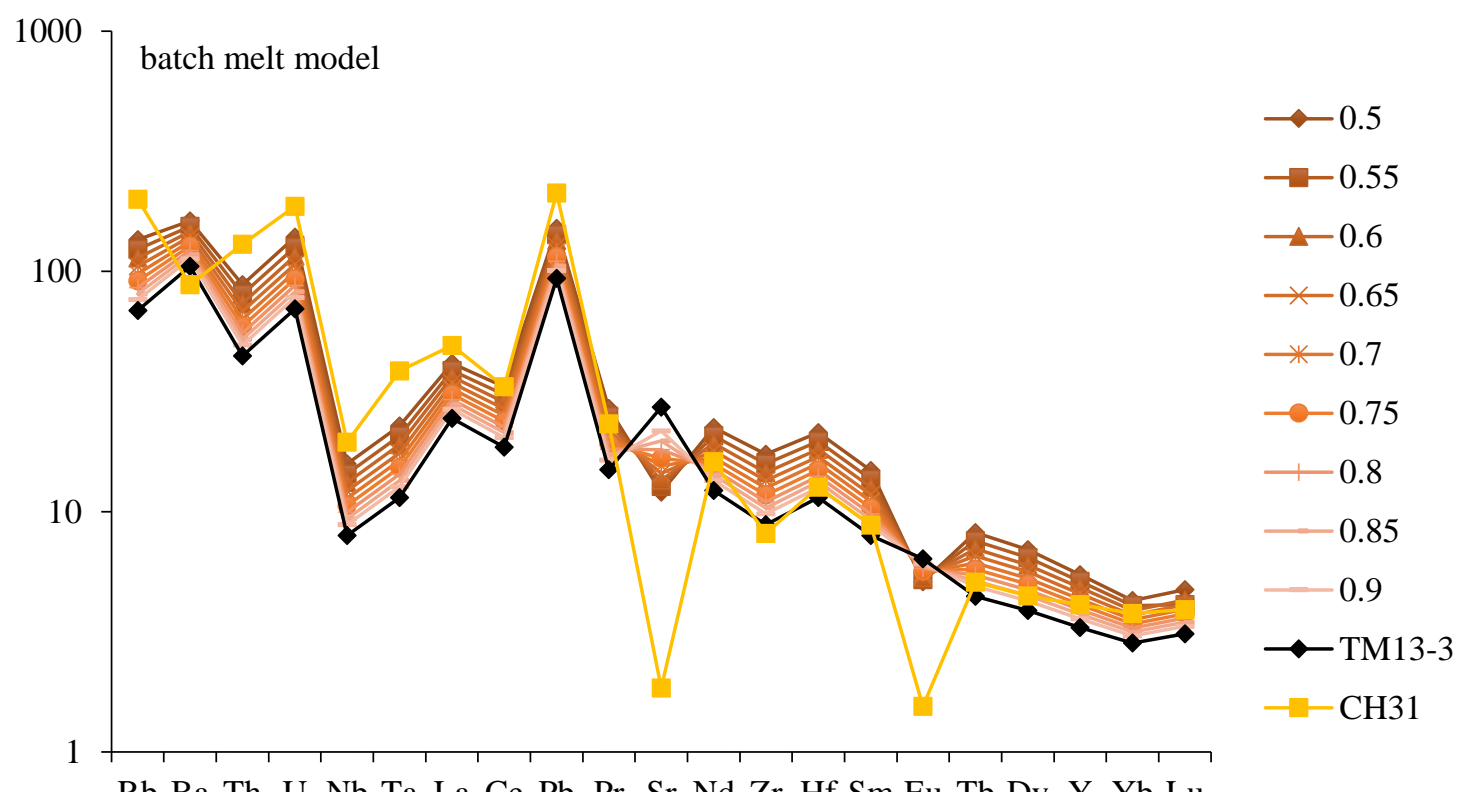

$\mathrm{Rb} \mathrm{Ba}$ Th U Nb Ta La Ce Pb Pr Sr Nd Zr Hf Sm Eu Tb Dy Y Yb Lu

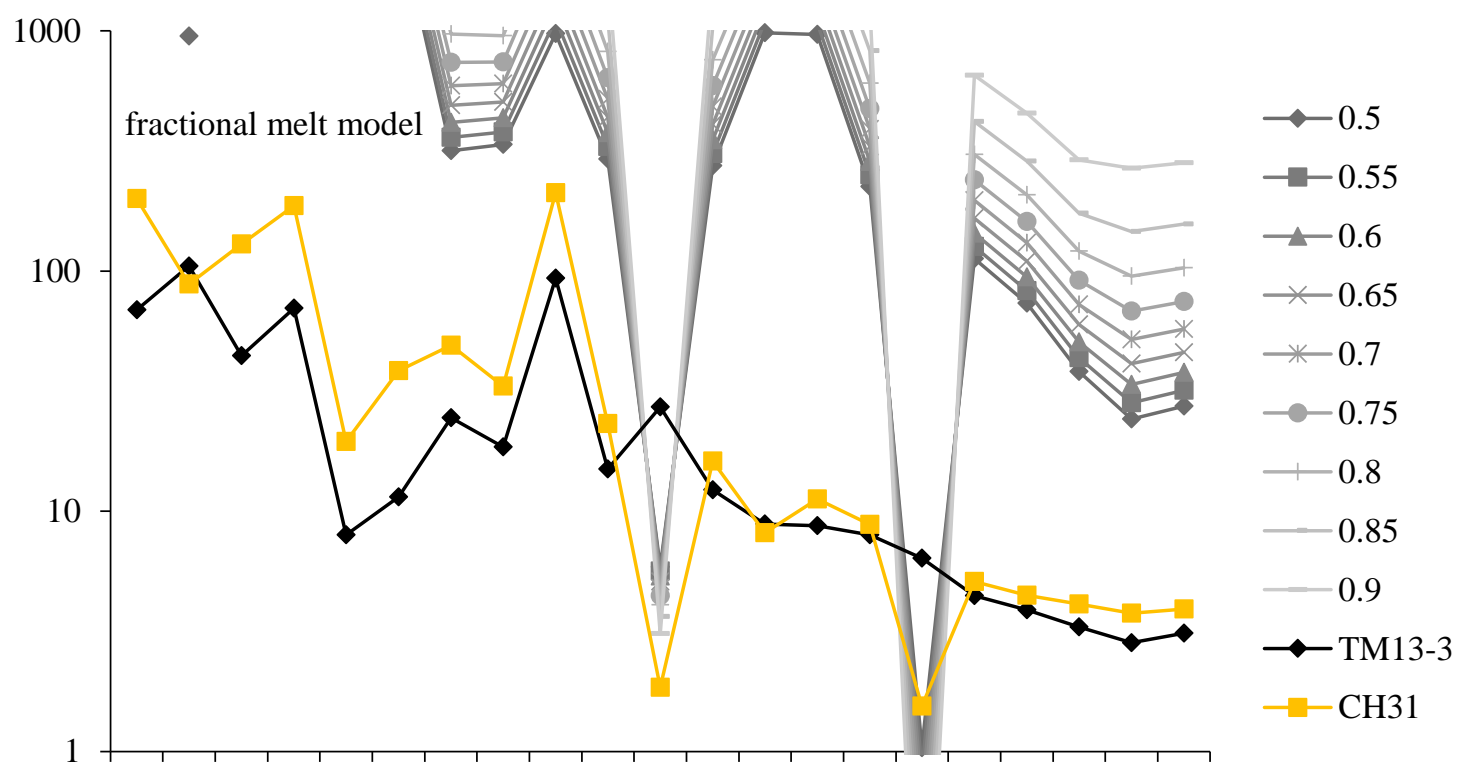

$\mathrm{Rb} \mathrm{Ba}$ Th U Nb Ta La Ce Pb Pr Sr Nd Zr Hf Sm Eu Tb Dy Y Yb Lu

Fig. 38 Primitive mantle normalized (Sun and McDonough, 1989) spider diagram for the partial melting of experimental melt MS13. The top graph shows the results of the batch melt model; the bottom shows the results of the fractional melt model. The melt percentages are approximately $\pm 20 \%$ that observed in the experimental melt (MS13) as determined by the AZtec software. The trace element concentrations of the parent material, sample TM13-3 (granodiorite), are indicated by the black diamonds. The trace element concentrations of the Dale Tuff, sample $\mathrm{CH} 31$, which is included for comparison, are indicated by the yellow squares. 
D. Trace element concentrations were calculated for melt percentages that were $\sim \pm 20 \%$ that observed in a given experimental melt. Figures 37 and 38 show examples of the results of modeling presented as spider diagrams. From these, the fit of the model can be determined visually. (Spider diagrams for all experimental melts are in Appendix D.) In general, the batch melt model was a better fit than the fractional melt model, although this fit is not perfect. In Figure 37, it can be seen that the Dale Tuff has a trough at barium, which is not captured by the model. Additionally, the europium anomaly is more pronounced in the Dale Tuff than in the model. This is also true, but to a lesser extent, for the trough at strontium. In Figure 38, it can be seen that the model data fails to capture the trace element pattern of the Dale Tuff from rubidium to barium, and again at strontium and europium.

Table 4. Summary of partial melt modeling

\begin{tabular}{|c|c|c|c|c|c|c|}
\hline $\begin{array}{l}\text { experimental } \\
\text { melt }\end{array}$ & parent & $\begin{array}{c}\mathrm{SiO}_{2} \\
\text { weight } \\
\text { percent }\end{array}$ & $\begin{array}{c}\text { Total } \\
\text { alkali } \\
\text { weight } \\
\text { percent }\end{array}$ & $\begin{array}{c}\text { TAS } \\
\text { classification }\end{array}$ & $\begin{array}{l}\text { best fit } \\
\text { model }\end{array}$ & $\begin{array}{l}\text { notable } \\
\text { exceptions } \\
\text { to fit }\end{array}$ \\
\hline MS1 & MS12-47 & 73.89 & 8.08 & rhyolite & batch & \\
\hline MS3 & TM13-5 & 69.38 & 1.89 & dacite & neither & \\
\hline MS4 & MS12-47 & 78.08 & 4.29 & rhyolite & batch & \\
\hline MS5 & TM13-3 & 68.62 & 5.70 & dacite & batch & \\
\hline MS6 & TM13-5 & 61.77 & 1.97 & andesite & batch & $\mathrm{Sr}, \mathrm{Eu}$ \\
\hline MS8 & TM13-3 & 73.08 & 6.29 & rhyolite & batch & \\
\hline MS9 & TM13-5 & 58.51 & 2.78 & andesite & batch & \\
\hline MS13 & TM13-3 & 67.26 & 6.16 & dacite & batch & \\
\hline MS15 & MS12-47 & 73.20 & 6.16 & rhyolite & batch & \\
\hline MS16 & TM13-3 & 76.55 & 6.04 & rhyolite & batch & \\
\hline MS17 & TM13-5 & 70.42 & 7.06 & rhyolite & batch & $\mathrm{Sr}, \mathrm{Eu}$ \\
\hline MS18 & MS12-47 & - & - & - & batch & $\mathrm{Eu}$ \\
\hline MS19 & TM13-5 & 67.83 & 1.23 & dacite & batch & $\mathrm{Sr}, \mathrm{Eu}$ \\
\hline MS22 & TM13-5 & 53.25 & 2.47 & basaltic andesite & batch & HREE \\
\hline MS23 & TM13-1 & - & - & - & batch & $\begin{array}{l}\mathrm{Ba}, \mathrm{Sr}, \mathrm{Zr} \text {, } \\
\mathrm{Eu}\end{array}$ \\
\hline
\end{tabular}




\begin{tabular}{|c|c|c|c|c|c|c|}
\hline MS26 & $50 \mathrm{M} 2 \mathrm{C}$ & 71.11 & 7.01 & rhyolite & batch & \\
\hline MS27 & $50 \mathrm{M} 3 \mathrm{~A}$ & - & - & - & batch & \\
\hline MS28 & $50 \mathrm{M} 3 \mathrm{~B}$ & 62.96 & 9.93 & dacite & batch & \\
\hline MS29 & $50 \mathrm{M} 3 \mathrm{C}$ & 69.83 & 6.77 & dacite & batch & \\
\hline MS30 & $50 \mathrm{M} 1 \mathrm{~A}$ & 68.96 & 5.40 & dacite & batch & \\
\hline MS33 & $50 \mathrm{M} 1 \mathrm{D}$ & - & - & - & batch & HREE \\
\hline MS34 & $50 \mathrm{M} 2 \mathrm{D}$ & 55.34 & 5.32 & basaltic andesite & batch & HREE \\
\hline MS35 & $50 \mathrm{M} 3 \mathrm{D}$ & 55.63 & 5.51 & basaltic andesite & batch & \\
\hline MS37 & TM13-1 & 65.76 & 7.55 & dacite & batch & Ba, HREE \\
\hline MS38 & TM13-3 & 71.86 & 6.98 & rhyolite & batch & Sr. Eu \\
\hline MS39 & TM13-3 & 65.30 & 5.60 & dacite & batch & \\
\hline MS40 & TM13-5 & 64.17 & 2.87 & dacite & batch & $\mathrm{Pb}, \mathrm{HREE}$ \\
\hline MS41 & TM13-5 & 64.93 & 3.21 & dacite & batch & $\begin{array}{l}\mathrm{Pb}, \mathrm{Zr}, \mathrm{Hf}, \\
\text { HREE }\end{array}$ \\
\hline MS43 & $50 \mathrm{M} 2 \mathrm{~B}$ & 72.59 & 7.44 & rhyolite & batch & \\
\hline MS44 & $50 \mathrm{M} 2 \mathrm{C}$ & 73.51 & 7.00 & rhyolite & batch & Th \\
\hline MS45 & $50 \mathrm{M} 3 \mathrm{~A}$ & 73.14 & 8.63 & rhyolite & batch & \\
\hline MS46 & 50M3B & 67.39 & 10.01 & dacite & batch & $\mathrm{Ba}$ \\
\hline MS47 & $50 \mathrm{M} 3 \mathrm{C}$ & 73.93 & 7.56 & rhyolite & batch & Nd, HREE \\
\hline MS48 & $50 \mathrm{M} 3 \mathrm{~A}$ & 71.12 & 7.25 & rhyolite & batch & \\
\hline MS49 & $50 \mathrm{M} 3 \mathrm{~A}$ & 70.76 & 5.61 & dacite & batch & \\
\hline MS50 & $50 \mathrm{M} 2 \mathrm{~A}$ & 72.23 & 7.33 & rhyolite & batch & \\
\hline MS51 & $50 \mathrm{M} 2 \mathrm{~A}$ & 70.26 & 4.92 & dacite & batch & \\
\hline MS52 & $50 \mathrm{M} 1 \mathrm{~A}$ & 68.62 & 4.27 & dacite & batch & \\
\hline MS53 & $50 \mathrm{M} 1 \mathrm{~A}$ & 63.18 & 6.75 & dacite & batch & \\
\hline MS55 & $50 \mathrm{M} 1 \mathrm{~B}$ & 57.75 & 10.55 & trachy andesite & batch & HREE \\
\hline MS57 & 50M1D & 52.80 & 4.64 & basaltic andesite & batch & HREE \\
\hline MS58 & $50 \mathrm{M} 1 \mathrm{~A}$ & 63.98 & 3.73 & dacite & batch & \\
\hline MS59 & $50 \mathrm{M} 2 \mathrm{C}$ & 60.15 & 5.65 & andesite & batch & Th \\
\hline MS60 & MS12-47 & 72.79 & 10.78 & rhyolite & batch & \\
\hline MS62 & $50 \mathrm{M} 2 \mathrm{~A}$ & - & - & - & batch & $\mathrm{Ba}$ \\
\hline MS63 & $50 \mathrm{M} 2 \mathrm{~A}$ & 72.88 & 6.89 & rhyolite & batch & \\
\hline MS65 & $50 \mathrm{MAB}$ & - & - & - & batch & \\
\hline
\end{tabular}

The glass of the experimental melts was analyzed via EMP. The results of this analysis were used to plot the melts onto a TAS diagram thereby providing the rock type classification. Figure 39 shows the results of the unmodified experimental melts, that is, those to which mafic glass was not added. Sample TM13-5, amphibolite, produced melts 
that were of basaltic to dacitic composition. Silica content ranges from 53.25 weight percent to $70.42 \%$. Alkali content ranges from $1.22 \%$ to $7.06 \%$. Sample TM13-3, granodiorite, produced melts that were of dacitic to rhyolitic composition. Silica content ranges from $65.30 \%$ to $76.55 \%$. Alkali content ranges from $5.60 \%$ to $7.00 \%$. Sample MS12-47 (biotite fels) produced melts that were of dacitic to rhyolitic composition. Silica content ranges from $63.98 \%$ to $78.08 \%$. Alkali content ranges from $3.73 \%$ to $10.78 \%$. Sample TM13-1 (biotite schist) produced a melt that was of trachydacitic composition. Silica content is $65.76 \%$ and alkali content is $7.55 \%$. In general, the unmodified experimental melts show a greater variation in silica content than total alkali content.

Figure 40 shows the results of the modified experimental melts, that is, those to which mafic glass was added. Sample TM13-5, amphibolite, produced melts that were of basaltic composition. Silica content ranges from 52.8 weight percent to $55.34 \%$. Alkali content ranges from $4.64 \%$ to $5.51 \%$. Sample TM13-3, granodiorite, produced melts that were of andesitic to rhyolitic composition. Silica content ranges from $60.15 \%$ to $73.93 \%$. Alkali content ranges from 5.65\% to 7.01\%. Sample MS12-47 (bitote fels) produced melts that were of dacitic to rhyolitic composition. Silica content ranges from $63.18 \%$ to 73.14\%. Alkali content ranges from 3.73\% to 8.63\%. Sample TM13-1 (biotite schist) produced melts that were of trachyandesitic to trachydacitic composition. Silica content ranges from $57.75 \%$ to $67.39 \%$. Alkali content ranges from $7.44 \%$ to $10.55 \%$. In general, the modified experiment melts also show a greater variation in silica content than total alkali content. 


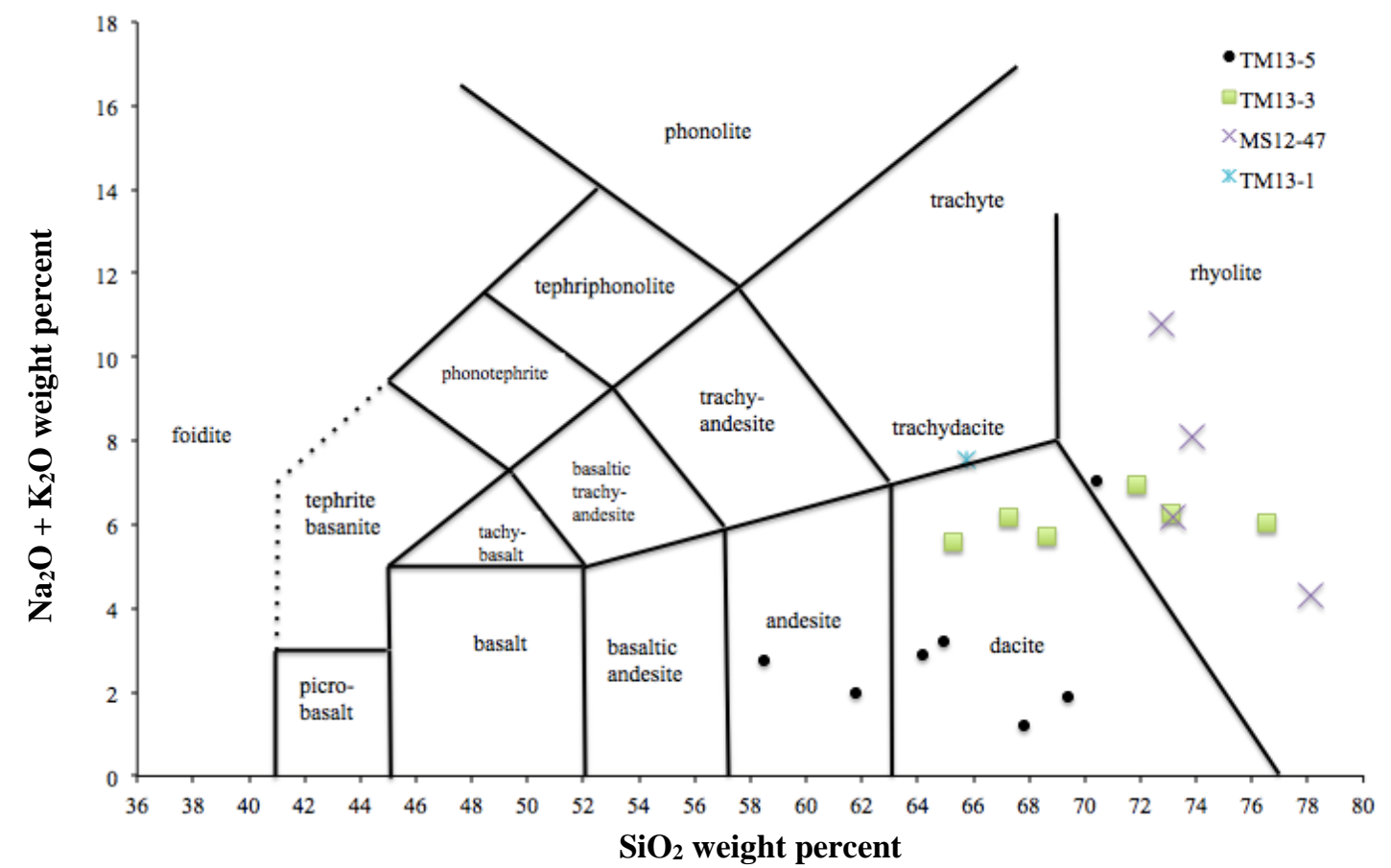

Fig. 39 Unmodified experimental melt glass compositions plotted onto a TAS diagram. The protolith samples are indicated in the key; TM13-5 (amphibolite), TM13-3 (granodiorite), MS12-47 (biotite fels), and TM13-1 (biotite schist).

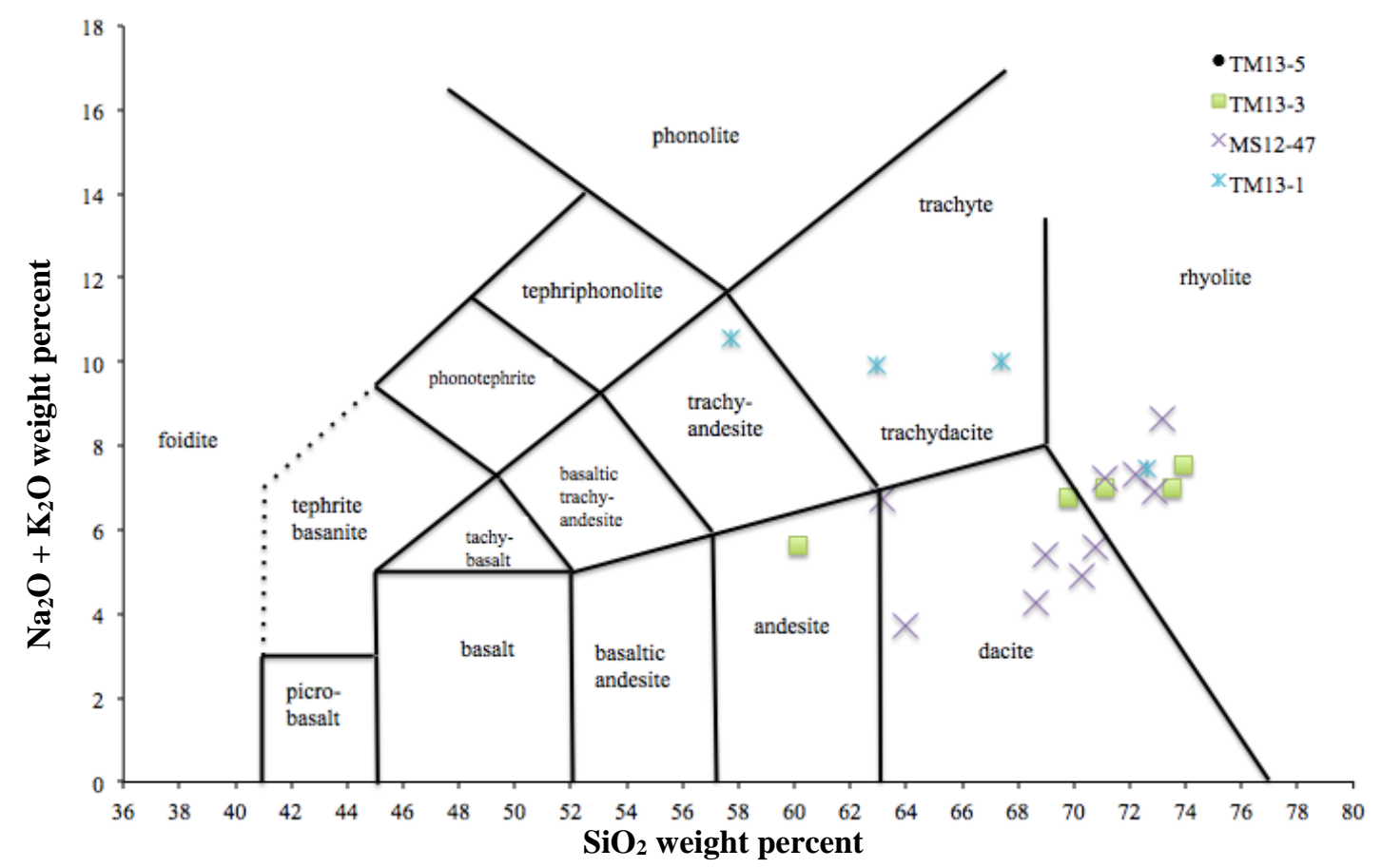

Fig. 40 Modified experimental melt glass compositions plotted onto a TAS diagram. The protolith samples are indicated in the key; TM13-5 (amphibolite), TM13-3 (granodiorite), MS12-47 (biotite fels), and TM13-1 (biotite schist). 


\section{DISCUSSION}

\section{TRANSITION FROM CALC-ALKALINE TO A-TYPE RHYOLITES}

Calc-alkaline (I-type) rhyolites are low in iron and magnesium while being high in calcium, sodium, and aluminum. They are produced from hydrous, oxidized melts, and are frequently associated with oceanic/continental subduction zones. A-type rhyolites, in contrast, are sub-alkaline to peralkaline, contain relatively high iron/magnesium ratios, as well as high silica, sodium, and potassium. These rhyolites are low in calcium and strontium, while being enriched in the high field strength elements and the rare earth elements, with the notable exception of europium. A-type rhyolites tend to be relatively anhydrous, and are often produced after a previous episode of crustal melting (Elliot, 2003; Whalen, et al., 1987).

Numerous studies (Dorsey and LaMaskin, 2008; Schwartz, et al., 2010; etc.) indicate that the Blue Mountains Province began with the protracted accretion of oceanic terranes onto the existing North American continental crust. Oceanic crustal material is quite hydrous due to interactions with seawater (Elliot, 2003). Assuming that partial melting of this crustal material is the source of the TM rhyolites, it follows that initial rhyolitic eruptions would also be hydrous, and therefore quite explosive, and carry geochemical signatures consistent with subduction. Additionally, mineralogical evidence, i.e. the presence of water-bearing phases such as biotite, indicates that these rhyolites are indeed hydrous. 
It is convenient to think of partial melting as a distillation or evolutionary processes, meaning that subsequent eruptions from the same source material will be relatively enriched in the more incompatible elements and depleted in volatiles, such as water, as well as the more compatible elements. As discussed above, the results of isotopic dating of TM rhyolites indicate that the eruption of calc-alkaline (I-type) rhyolites occurred first followed by eruption of A-like rhyolites. This suggests that these two groups of rhyolites are derived from the same source material.

\section{TIMING OF RHYOLITE ERUPTIONS}

The results of the ${ }^{40} \mathrm{Ar} /{ }^{39} \mathrm{Ar}$ and $\mathrm{U}-\mathrm{Pb}$ isotopic dating provide a clear picture of the timing of eruptions for the rhyolite groups. The caldera-forming eruption produced the Dale Tuff (I-type rhyolite), which comprises the intra-caldera and outflow tuff. Isotopic dating places the age of the tuff at $32.66 \pm 0.36 \mathrm{Ma}\left({ }^{40} \mathrm{Ar} /{ }^{39} \mathrm{Ar}\right)$ and $32.345 \pm 0.01(\mathrm{U}-\mathrm{Pb})$. Postcaldera rhyolites then erupted along apparent ring fractures and elsewhere (see Figure 2). $\mathrm{U}-\mathrm{Pb}$ dating of zircons from three of these rhyolites yielded ages of $32.169 \pm 0.024 \mathrm{Ma}$ for CH07a (Dale Tuff-like/I-type), $31.798 \pm 0.012$ Ma for TM5 (A-like), and 31.426 \pm 0.016 Ma for CH08a (A-like). The ages indicate that the calc-alkaline rhyolites (I-type, Dale Tuff and Dale Tuff-like) were followed by the A-type rhyolites and that effusive rhyolite eruptions restarted without much hiatus after caldera formation.

\section{PARTIAL MELTING MODELS FOR RHYOLITES}

Metamorphic rocks as source for rhyolites 
The region of the Blue Mountains Province in which the Tower Mountain Caldera formed consists of the Baker Terrane and the Mountain Home Metamorphic Complex. Crustal material in this region is primarily composed of metamorphic rocks along with igneous intrusions (stitching plutons). As has been discussed, partial melting of crustal material is a strong candidate for rhyolite generation. Further evidence of a crustal source is provided by Seligman, et al. (2014) in which the authors investigated stable oxygen isotope signatures of eastern Oregon calderas, including Tower Mountain. They found

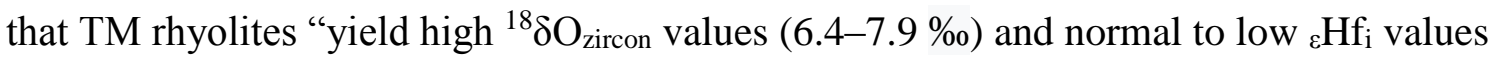
(5.2-12.6), indicating crustal melting of high- ${ }^{18} \delta \mathrm{O}$ supracrustal rocks.”

Crustal material that may have previously been an unsuitable candidate for rhyolite generation via partial melting may be transformed by metamorphic processes into a more suitable protolith. Three metamorphic samples were used in the production of experimental melts; biotite fels (MS12-47), amphibolite (TM13-5), and biotite schist (TM13-1). Of these three, experimental melts produced from the biotite fels tended toward rhyolitic (Fig. 39). Melts produced from amphibolite were typically dacitic, though andesite, basaltic andesite, and a single rhyolite were also produced. Melts produced from the biotite schist were dacitic. The results of partial melt modeling (Appendix D) support the partial batch melting of biotite fels (MS12-47) as the most likely source of a rhyolitic melt. This is also consistent with the results of calculating incompatible trace element concentrations of the partial melts produced from melting MS12-47 based on observed residual phase proportions (see multiple Figures in 
Appendix D). However, the $\mathrm{SiO}_{2}$ weight percent of this sample is quite high, $80.28 \%$, which is higher than any found in the TM rhyolites, but the $\mathrm{SiO}_{2}$ of partial melts are lower than the bulk rock composition of MS12-47. The addition of material with a more primitive composition (lower silica, lower concentration of incompatible elements) would help in lowering the silica content of the partial melts to match TM rhyolites, and for inconsistencies observed in the trace element concentration patterns, i.e. the trough observed at barium and the strong europium anomaly (Figs. 37 and 38).

\section{$\underline{\text { Intermediate plutonic rocks as source for rhyolites }}$}

The melt expulsion model of Bachmann and Bergantz (2004), also called the "mush model" (see Figure 1), describes a scenario in which the production of voluminous crystal poor rhyolites, which is an apt description for the Tower Mountain rhyolites, is a result of the fractional crystallization or partial melting of compositionally intermediate plutonic bodies (Bachmann \& Bergantz, 2004). As the intermediate magma body reaches approximately $50 \%$ crystallinity, the interstitial melt is of rhyolitic composition and the crystal mush becomes static. Being more buoyant, the melt escapes and rises to the top of the magma chamber forming an aphyric "rhyolite horizon", which then erupts at the surface.

Szymanowski, et al. (2015) found that intermediate melts, which the authors classified as those containing 57-67 weight percent $\mathrm{SiO}_{2}$, can be found in association with main-stage rhyolitic volcanism. They propose a "fractionation-dominated evolution including some 
assimilation". This type of AFC (assimilation-fractional crystallization) model requires that "significant amounts" of intermediate cumulates remain in the crust. This allows the partial melt to then move towards a rhyolitic composition (Szymanowski et al., 2015).

As can be seen from the results of the experimental melts (Appendix D), partial melting of plutonic intermediate sample TM13-3 (granodiorite) does produce an exceptionally good fit, although only half of the melts produced from this protolith can be classified as rhyolites, with the other half being classified as dacites (see Table 4, Figs. 39 and 40). Figure 30, which shows the normalized trace element concentrations of intermediate samples (TM13-3, TM13-9), confirms a positive anomaly at both uranium and lead. However, negative anomalies at barium and europium are absent. The low silica rhyolites (samples $\mathrm{CH} 16$ and $\mathrm{CH} 18$, which are also classified as I-type) lack a barium trough and show only a mild europium anomaly. It is possible that these rhyolites are the product of partial melting of an intermediate plutonic body, either by melt expulsion or AFC.

\section{FRACTIONAL CRYSTALIZATION MODEL FOR RHYOLITES}

A silicic melt may be derived from a basaltic parent magma, either by fractional crystallization alone, or by fractional crystallization combined with assimilation of crustal country rock. This model satisfactorily describes the production of only small magma batches, and so is not favored for caldera-forming eruptions (Riley et al., 2001; Bachmann \& Bergantz, 2004; Szymanowski, et al., 2015). However, the possibility of rhyolite production as the result of the fractional crystallization of basalt was explored via 
sample G314-09-2. It can be seen from Figure 32 that the fractional crystallization of basalt produces a poor fit for the rhyolites. Overall, the trace element concentration pattern derived from the fractional crystallization of basalt is quite flat, that is, it lacks the "peaks" and "troughs" seen in the rhyolite spider diagrams. For example, the positive lead anomaly, which is shared among all rhyolite samples, is conspicuously absent in the fractional crystallization model. Fractional crystallization of basalt is thus unlikely the process by which rhyolites were generated. However, underplating of crustal rocks (metamorphic and intermediate plutonic) by basalt is very likely the ultimate cause for rhyolite volcanism, providing the heat necessary to induce partial melting.

\section{CONCLUSIONS}

Tower Mountain Caldera is the main feature of an Oligocene volcanic field located in the Umatilla National Forest, eastern Oregon. The TM rhyolites have been divided into five groups based mostly on geochemistry and lithology; 1) low silica (I-type), 2) A-like, 3) Dale Tuff-like (I-type), 4) Dale Tuff (I-type), and 5) high silica (I-type). The formation of the caldera produced the Dale Tuff, which comprises the intra-caldera and outflow tuff. Isotopic dating places the age of the tuff at $32.66 \pm 0.36 \mathrm{Ma}\left({ }^{40} \mathrm{Ar} /{ }^{39} \mathrm{Ar}\right)$ and $32.345 \pm$ $0.01(\mathrm{U}-\mathrm{Pb})$. Post-caldera rhyolites then erupted along apparent ring fractures and elsewhere. U-Pb dating of zircons from three of these rhyolites yielded ages of $32.167 \pm$ $0.020 \mathrm{Ma}$ (CH07a), $31.798 \pm 0.012 \mathrm{Ma}$ (TM5), and $31.426 \pm 0.016 \mathrm{Ma}$ (CH08a). The ages indicate that calc-alkaline rhyolites (I-type) were followed by the A-type rhyolites and that effusive rhyolite eruptions restarted without much hiatus after caldera formation. 
Temporal and geochemical data indicate that the I-type and A-type rhyolites are likely derived from the same source material. Partial melt modeling based on experimental melts produced from crustal material indicates that batch partial melting of metamorphosed high silica crustal material modified by the addition of more primitive mafic material by assimilation/contamination is the most likely source. This modeling also suggests that a likely source for the low silica rhyolites is partial melting of Cretaceous plutons of dioritic to granodioritic composition followed by some fractionation to reduce $\mathrm{Ba}, \mathrm{Sr}$ and Eu. Fractional crystallization of basalt is unlikely the process by which TM rhyolites were generated. However, underplating of crustal rocks (metamorphic and intermediate plutonic rocks) by basalt is very likely the ultimate cause for rhyolite volcanism, providing the heat necessary to induce partial melting. 


\section{REFERENCES}

Anderson, B.S., 2013, Magmatism, metamorphism, and deformation in the Mountain Home Metamorphic Complex, Blue Mountains, Province, Oregon, and its role in late Jurassic deformation in the western North American Cordillera: Masters Thesis, The University of Alabama.

Bachmann, O., and Bergantz, G.W., 2004, On the origin of crystal-poor rhyolites: Extracted from batholithic crystal mushes: Journal of Petrology, v. 45, no. 8, p. $1565-1582$. Bindeman, I.N., and Valley, J.W., 2000, Formation of low- $\delta 180$ rhyolites after caldera collapse at Yellowstone, Wyoming, USA: Geology, v. 28, p. 719-722.

Bacon, C.R., and Druitt, T.H., 1988, Compositional evolution of the zoned calcalkaline magma chamber of Mount Mazama, Crater Lake, Oregon: Contributions to Mineralogy and Petrology, v. 98, no. 2, p. 224-256.

Bea, F., Pereira, M.D., and Stroh, A., 1994, Mineral/leucosome trace-element partitioning in a peraluminous migmatite (a laser ablation ICP-MS study): Chemical Geology, v. 117 , p. 291-312.

Blundy, J., and Dalton, J., 2000, Experimental comparison of trace element partitioning between clinopyroxene and melt in carbonate and silicate systems, and implications for mantle metasomatism: Contributions to Mineralogy and Petrology, v. 139, p. 356-371

Broderick, C.A., 2008, The origin of sulfur-rich apatites in silicic magmas: Masters Thesis, Portland State University.

Dorsey, R.J., and LaMaskin, T.A., 2008, Mesozoic collision and accretion of oceanic terranes in the Blue Mountains province of northeastern Oregon: Insights from the stratigraphic record: Arizona Geological Society Digest, v. 22, p. 325-332

Duncan, R.A., and Keller, R.A., 2004, Radiometric ages for basement rocks from the Emperor Seamounts, ODP Leg 197: Geochemistry Geophysics Geosystems, v. 5, p. $1525-2017$.

Elliot, T., 2003, Tracers of the Slab: Inside the Subduction Factory: American Geophysical Union, Geophysical Monograph 138, p. 23-45.

Ewart, A., and Griffin, W.L., 1994, Application of proton-microprobe data to traceelement partitioning in volcanic-rocks: Chemical Geology, v. 117, p. 251-284.

Ferns, M.L., 2002, Tower Mountain: A northeast Oregon late Oligocene caldera: Geological Society of America Annual Meeting Abstract.

Girard, G., and Stix, J., 2009, Magma recharge and crystal mush rejuvenation associated with early post-collapse Upper Basin member rhyolites, Yellowstone Caldera, Wyoming: Journal of Petrology, v. 50, no. 11, p. 2095-2125.

Girard, G., and Stix, J., 2010, Rapid extraction of discrete magma batches from a large differentiating magma chamber: the Central Plateau member rhyolites, Yellowstone Caldera, Wyoming: Contrib Mineral Petrol, v. 160, p. 441-465.

Grove, T.L., and Gerlach, D.C., 1982, Origin of calc-alkali series lavas at Medicine Lake Volcano by fractionation, assimilation and mixing: Contributions to Mineralogy and Petrolog, v. 80, no. 2, p. 160-182. 
Knaack, C., Cornelius, S., and Hooper, P.,1994, Trace element analyses of rocks and minerals by ICP-MS: Washington State University, Open-File Report.

Koppers, A.A.P., 2002, ArArCALC Microsoft Excel ${ }^{\circledR}$ 97-2000-XP application: Computers and Geosciences, v. 28, no. 5, p. 605-619.

LeBas, M.J., Lemaitre, R.W., Streckeisen, A., and Zanettin, B., 1986, A chemical classification of volcanic-rocks based on the total alkali silica diagram: Journal of Petrology, v. 27, no. 3, p. 745-750.

McCarthy, T.C., and Patiño Douce, A.E., 1997, Experimental evidence for high temperature felsic melts formed during basaltic intrusion of the deep crust: Geology, v. 25, p. 463-466.

McClaughry, J.D., Ferns, M.L., Streck, M.J., Patridge, K.A., and Gordon, C.L., 2009, Paleogene caldera of central and eastern Oregon: Eruptive sources of widespread tuffs in the John Day and Clarno Formations: Geological Society of America Field Trip Guide 15, 874 p.

Nash, W.P., and Crecraft, H.R., 1985, Partition coefficients for trace elements in silicic magmas: Geochimica et Cosmochimica, v. 49, no. 2, p. 309-322.

Johnson, D.M., Hooper, P.R., and Conrey, R.M., 1999, XRF analysis of rocks and minerals for major and trace elements on a single low dilution Li-tetraborate fused bead: Advances in X-Ray Analysis, v. 41, p. 843-867.

Oregon Department of Geology and Mineral Industries (DOGAMI), 2008, Granite to Ukiah, Grant County, Oregon: Field Trip Guide, webpage: http://www.oregongeology.org/sub/fieldtrips/ukiah-granite/default.htm.

Oxford Instruments, 2013, AZtec EDS Software

Riley, T.R., Leat, P.T., Pankhurst, R.J., and Harris, C., 2000, Origins of large volume rhyolitic volcanism in the Antarctic Peninsula and Patagonia by crustal melting: Journal of Petrology, v. 42, no. 6, p. 1043-1065.

Schmitz, M.D., 2016, Report of investigation: Sample: CH-08a, TM5, CH-07a, MS-1249; Boise State University Isotope Geology Laboratory, 9 p.

Schwartz, J.J., Snoke, A.W., Frost, C.D., Barnes, C.G., Gromet, P.L., and Johnson, K., 2010, Analysis of the Wallowa-Baker terrane boundary: Implications for tectonic accretion in the Blue Mountains province, northeastern Oregon: Geological Society of America Bulletin, v. 122, no. 3-4, p. 517-536.

Seligman, A.N., Bindeman, I.N., McClaughry, J., Stern, R.A., and Fisher, C., 2014, The earliest low and high $\delta^{18} \mathrm{O}$ caldera-forming eruptions of the Yellowstone plume: implicatuons for the 30-40 Ma Oregon calderas and speculations on plumetriggered delaminations: Frontiers in Earth Science, v. 2, p. 1-9.

Sisson, T.W., 1991, Pyroxene-high silica rhyolite trace-element partition-coefficients measured by ion microbe: Geochimica et Cosmochimica Acta, v. 55. no. 6, p. 575-585.

Smith, R.L., and Bailey, R.A., 1968, Resurgent cauldrons: Geological Society of America Memoirs, v. 116, p. 613-662.

Streck, M.J., 2002, Partial melting to produce high-silica rhyolites of a young bimodal 
suite: Compositional constraints among rhyolites, basalts, and metamorphic xenoliths from the Harney Basin, Oregon: International Journal of Earth Science, v. 91, p. 583-593.

Streck, M.J., and Grunder, A.L., 1997, Compositional gradients and gaps in high-silica rhyolites of the Rattlesnake Tuff, Oregon: Journal of Petrology, v. 38, no. 1, p. 133-163.

Sun, S.S. and McDonough, W.F., 1989, Chemical and isotopic systematics of oceanic basalts: implications for mantle composition and processes: Geological Society, London, Special Publications, v. 42, p. 313-3445.

Szymanowski, D., Ellis, B.S., Bachmann, O., Guillong, M., and Phillips, W.M., 2015, Bridiging basalts and rhyolites in the Yellowstone-Snake River Plain volcanic province: The elusive intermediate step: Earth and Planetary Science Letters, v. 415, p. 80-89.

Tamura, Y., and Tatsumi, Y., 2002, Remelting of an andesitic crust as a possible origin for rhyolitic magma in oceanic arcs: an example from the Izu-Bonin Arc: Journal of Petrology, v. 43, no. 6, p. 1029-1047.

Taylor, J.R., 1982, An introduction to error analysis: The study of uncertainties in physical measurements: University Science Books, Mill Valley, California, $270 \mathrm{p}$.

Thy, P., Beard, J.S., and Lofgren, G.E., 1990, Experimental constrains on the origin of Iceland rhyolites: The Journal of Geology, v. 98, no. 3, p. 417-421.

Watson, E.B., and Green, T.H., 1981, Apatite/liquid partition coefficients for the rare earth elements and strontium: Earth and Planetary Science Letters, v. 56, p. 405421.

Whalen, J.B., Currie, K.L. and Chappell, B.W., 1987, A-Type Granites - Geochemical Characteristics, Discrimination and Petrogenesis. Contributions to Mineralogy and Petrology, v. 95, no. 4, p. 407-419.

Wilson, M., 2007, Igneous petrogenesis: A global tectonic approach: Springer, Dordrecht, The Netherlands, 480 p. 


\section{VOLCANIC ROCKS \\ $\underline{\text { Rhyolites }}$}

\section{APPENDIX A: PETROGRAPHY}

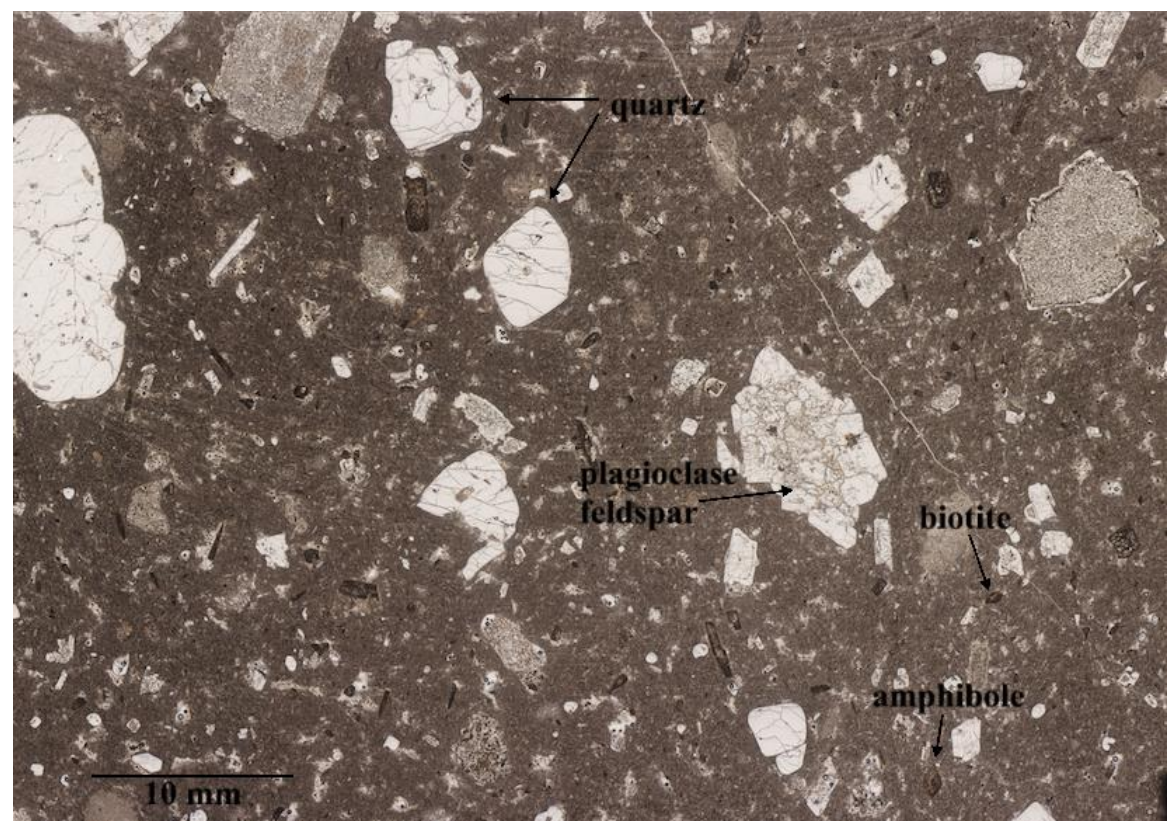

Fig. A1 Plane light thin section image of sample TM4. Phenocrysts account for $\sim 40 \%$ of the area of the sample, with remainder being fine-grained groundmass. The modal abundance of mineral phases is as follows: $\sim 40 \%$ quartz, $\sim 40 \%$ plagioclase feldspar, $~ 15 \%$ amphibole, and $\sim 5 \%$ biotite. Quartz is subhedral and rounded. Plagioclase is euhedral to subhedral and often intergrown. It frequently shows perfect twinning and exsolution lamellae. Amphibole is relatively small and remarkably euhedral. Biotite is relatively small and anhedral.

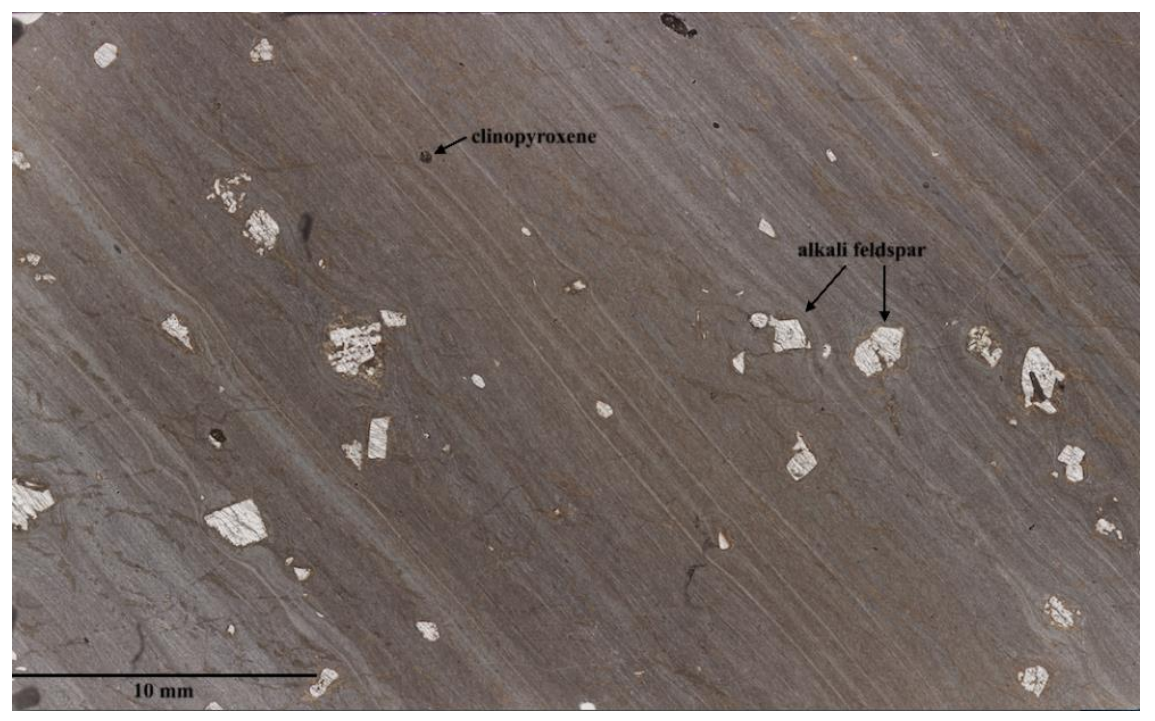

Fig. A2 Plane light thin section image of sample TM5. This sample is glassy, predominantly transparent, highly fractured, and shows distinctive flow banding. Phenocrysts are sparse at $\sim 10 \%$ of the sample area. The largest and most abundant of the phenocrysts, $\sim 70 \%$, are alkali feldspar (anorthoclase), which are euhedral to anhedral, tend to be highly fractured, and are found with inclusions of titanoFeTi oxides and zircon. Many show compositional zoning. Clinopyroxene phenocrysts are less abundant, $\sim 20 \%$, typically highly euhedral, and fractured. TitanoFeTi oxides comprises $\sim 6 \%$ of phenocrysts, and zircon, $\sim 4 \%$. 


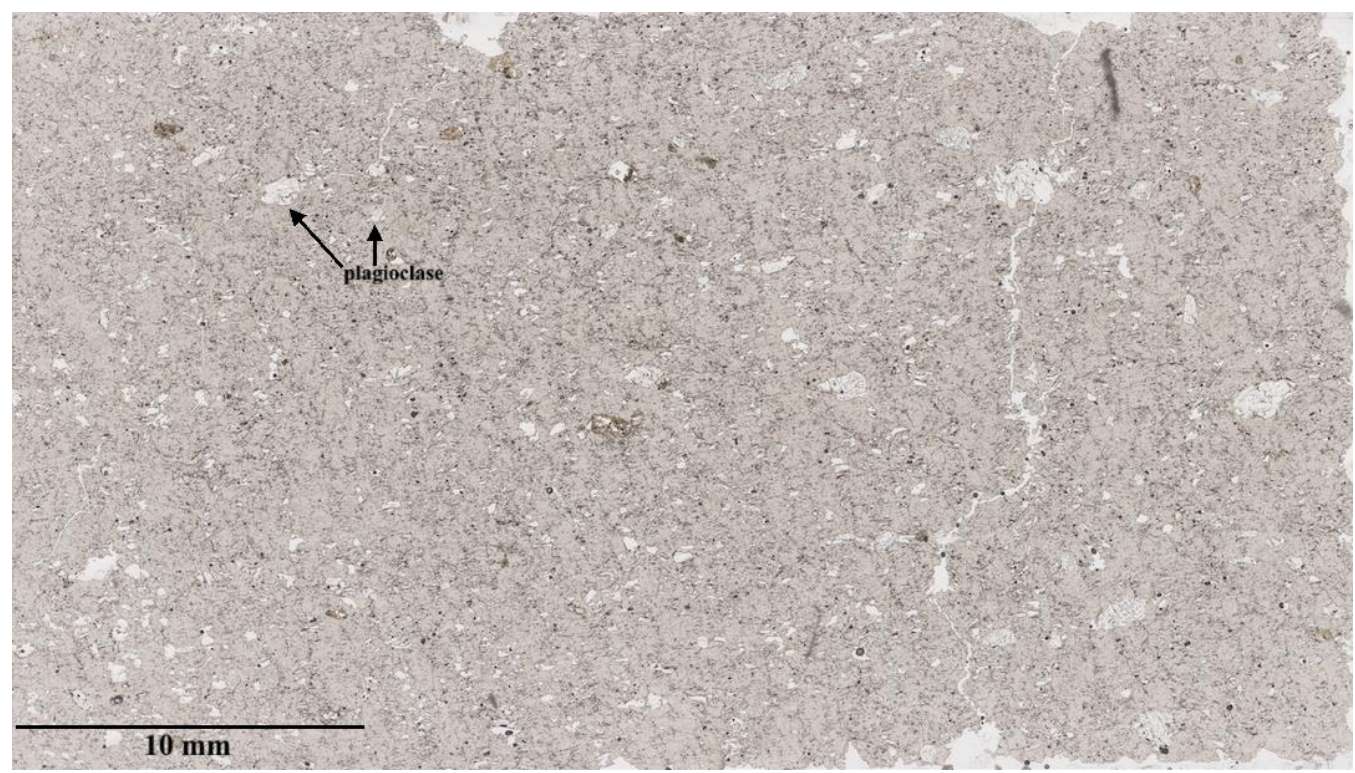

Fig. A3 Plane light thin section image of sample $\mathrm{CH} 07 \mathrm{a}$. It is composed of a glassy and homogenous groundmass which is light in color, and which comprises $~ 90 \%$ of the sample by area, while phenocrysts make up $\sim 10 \%$ of the total sample area. The most abundant phenocryst is plagioclase feldspar (andesine) at $\sim 55 \%$ modal abundance. Plagioclase phenocrysts are euhedral to subhedral. Iron oxides account for $\sim 30 \%$ of phenocrysts, and are found both scattered throughout the sample and as inclusions in biotite.

Approximately $15 \%$ of phenocrysts are biotite, which show heavy weathering. Zircon was observed as an accessory phase and is primarily found as inclusions. Flow structures are visible in the groundmass. These structures, as well as the low abundance and small size of the phenocrysts indicate that $\mathrm{CHO7a}$ represents a rhyolite flow.

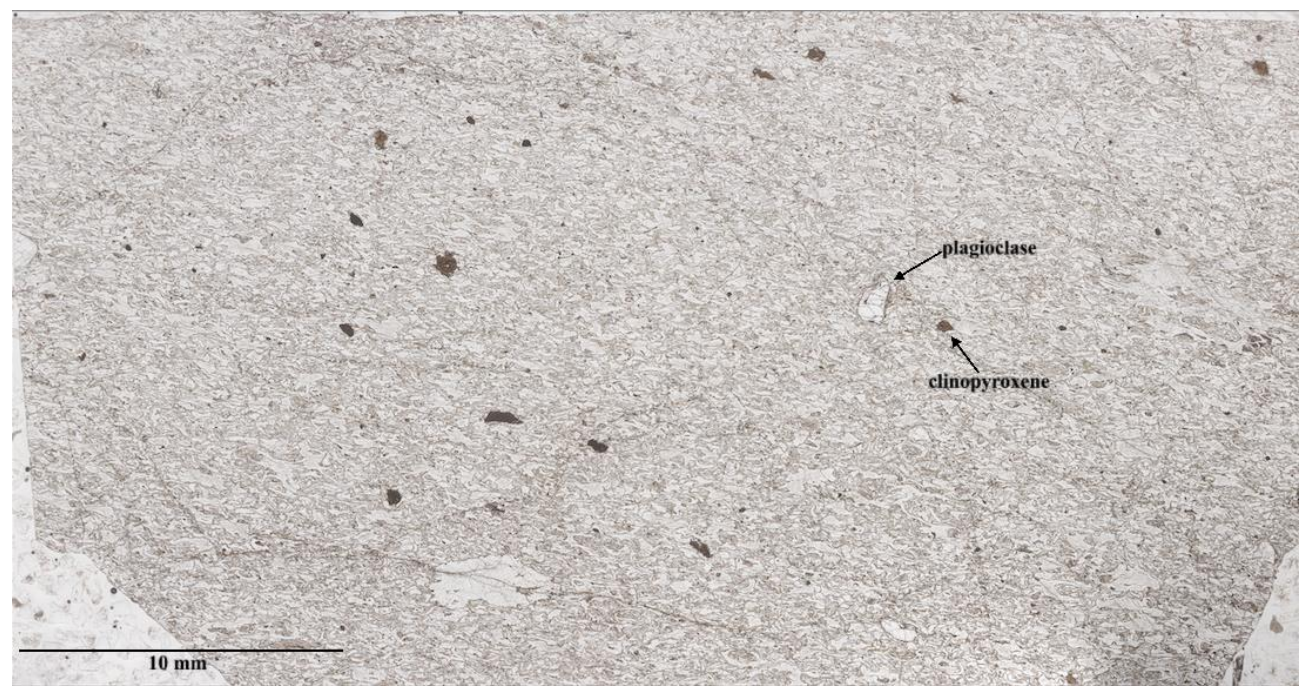

Fig. A4 Plane light thin section image of sample CH31, Dale Tuff. It has the typical glass shard texture with sparse phenocrysts. A mere $4 \%$ of the area is occupied by phenocrysts. Of these, plagioclase feldspar is the most abundant. The normalized mineral assemblage is as follows: $\sim 90 \%$ plagioclase feldspar, $\sim 10 \%$ oxides, $<1 \%$ clinopyroxene. The feldspar phenocrysts are euhedral to subhedral and are quite small at just a few millimeters in length. There are abundant small, rounded oxides throughout the sample. Two subhedral pyroxene phenocrysts were observed, which were comparable in size to the feldspar phenocrysts. Very sparse lithic fragments of $\sim 3 \mathrm{~mm}$ contain plagioclase laths, euhedral amphibole, and rounded oxides, suggesting that the fragments are basaltic in origin. 


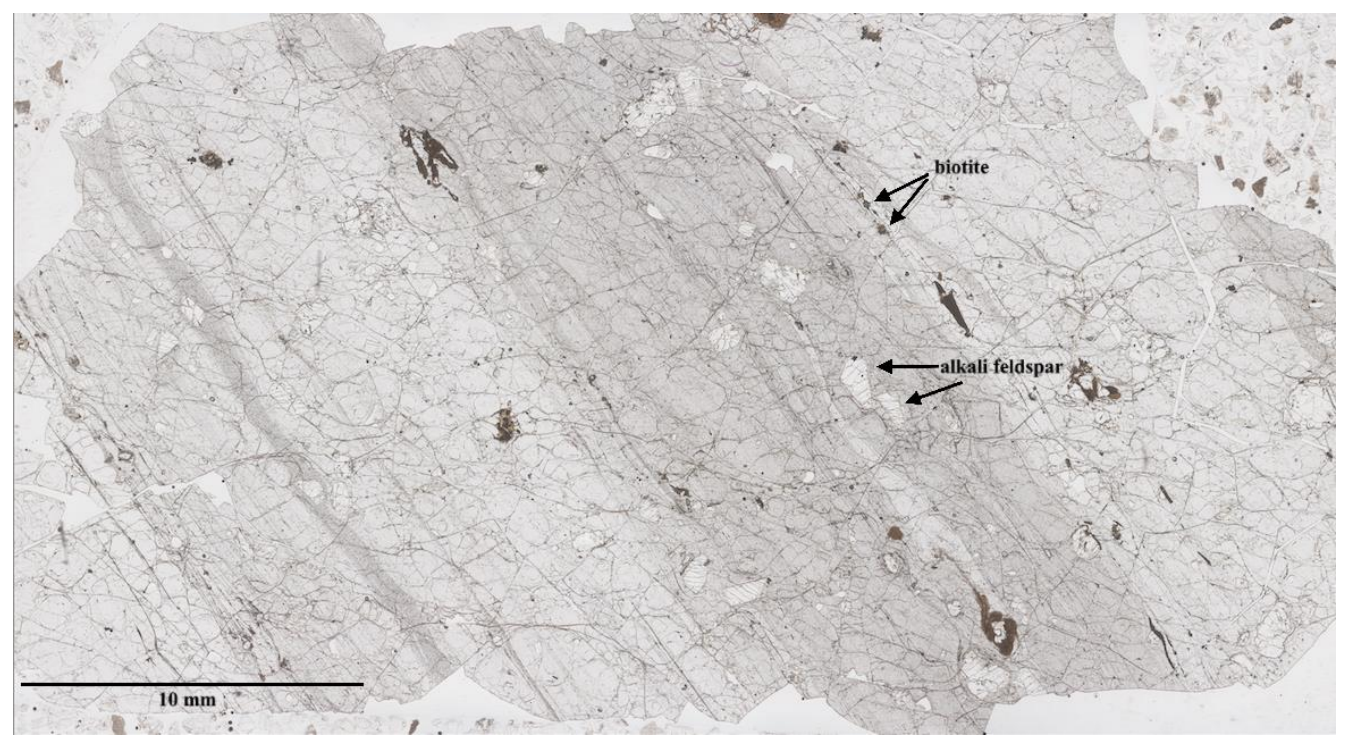

Fig. A5 Plane light thin section image of sample CH08a. It is glassy and highly fractured with perlitic texture. There is obvious flow banding. Phenocrysts account for approximately 5-7\% of the sample area. Alkali feldspars in the form of sanidine are the most noticeable and largest minerals in the sample, accounting for $\sim 50 \%$ of total phenocrysts. They are mostly anhedral, although some have a euhedral shape. These crystals are highly weathered, fractured and have inclusions of FeTi oxides and zircon. Biotite accounts for $\sim 25 \%$ of the total phenocrysts. These contain zircon and FeTi oxides inclusions, are extremely anhedral, and highly weathered. Quartz accounts for $\sim 6 \%$ of the phenocrysts; it is highly anhedral and fractured.

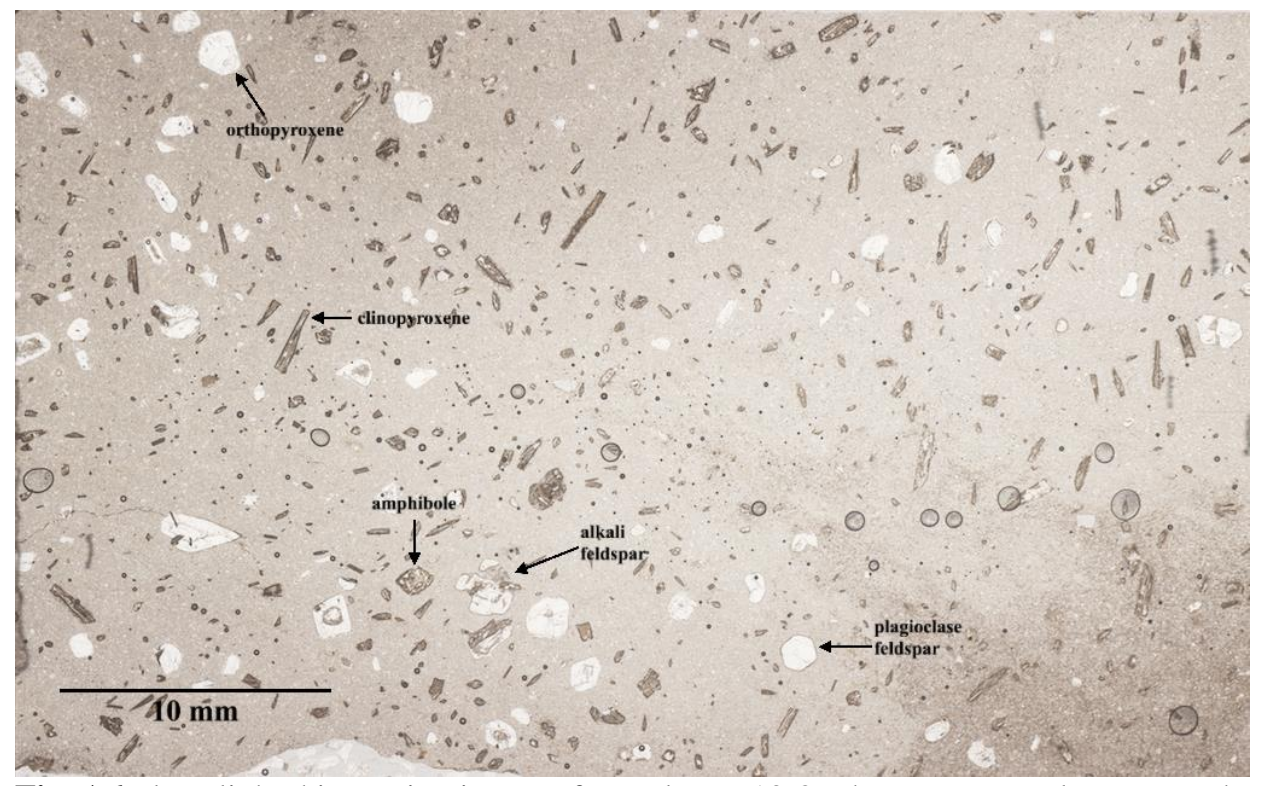

Fig. A6 Plane light thin section image of sample TM13-8. Phenocrysts are large enough to be seen with the naked eye and account for $\sim 20 \%$ of the total area. The fine-grained groundmass is composed of quartz and plagioclase laths. Clinopyroxene are subhedral and lath-like, highly fractured and resorbed, and account for $\sim 35 \%$ of phenocrysts. Alkali feldspar, accounting for $\sim 25 \%$ of phenocrysts, are subhedral, rounded, and often twinned and found in aggregates. Plagioclase feldspar, $\sim 20 \%$ of phenocrysts, are subhedral with distinct compositional zoning. Amphibole, $\sim 15 \%$, are euhedral to subhedral, but highly fractured, while orthopyroxene, $\sim 5 \%$, are euhedral but fractured. 


\section{$\underline{\text { Intermediate Rocks }}$}

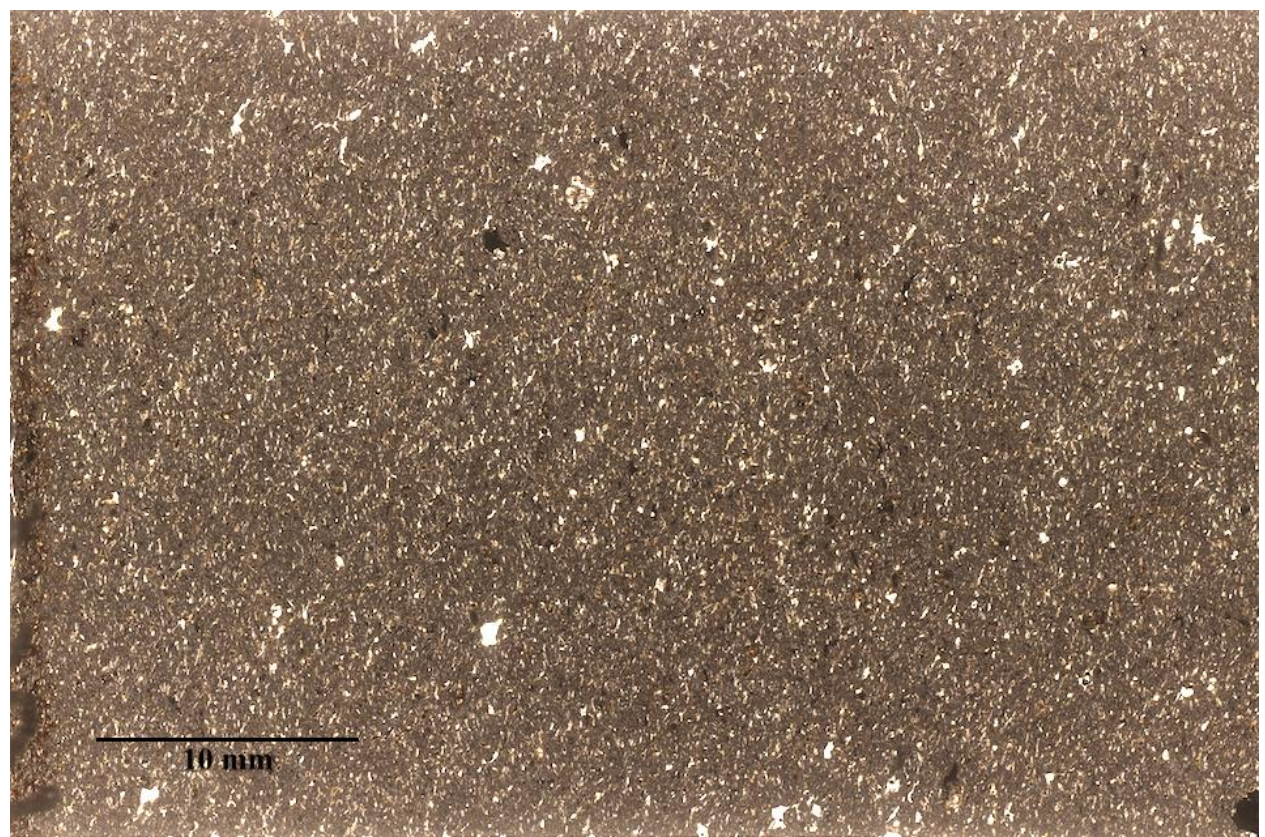

Fig. A7 Plane light thin section image of sample TM6; dacite. The groundmass is primarily of plagioclase laths, which are aligned in the direction of flow, with quartz in the interstices. Phenocrysts, all of which are subhedral to anhedral and fractured, account for $\sim 8 \%$ of the total area. The light, irregularly shaped areas are quartz, which account for $\sim 80 \%$ of phenocrysts. Clinopyroxene, biotite, and othropyroxene are also present as phenocrysts at $\sim 10 \%, \sim 8 \%$ and $\sim 2 \%$, respectively.

\section{$\underline{\text { Basaltic }}$}

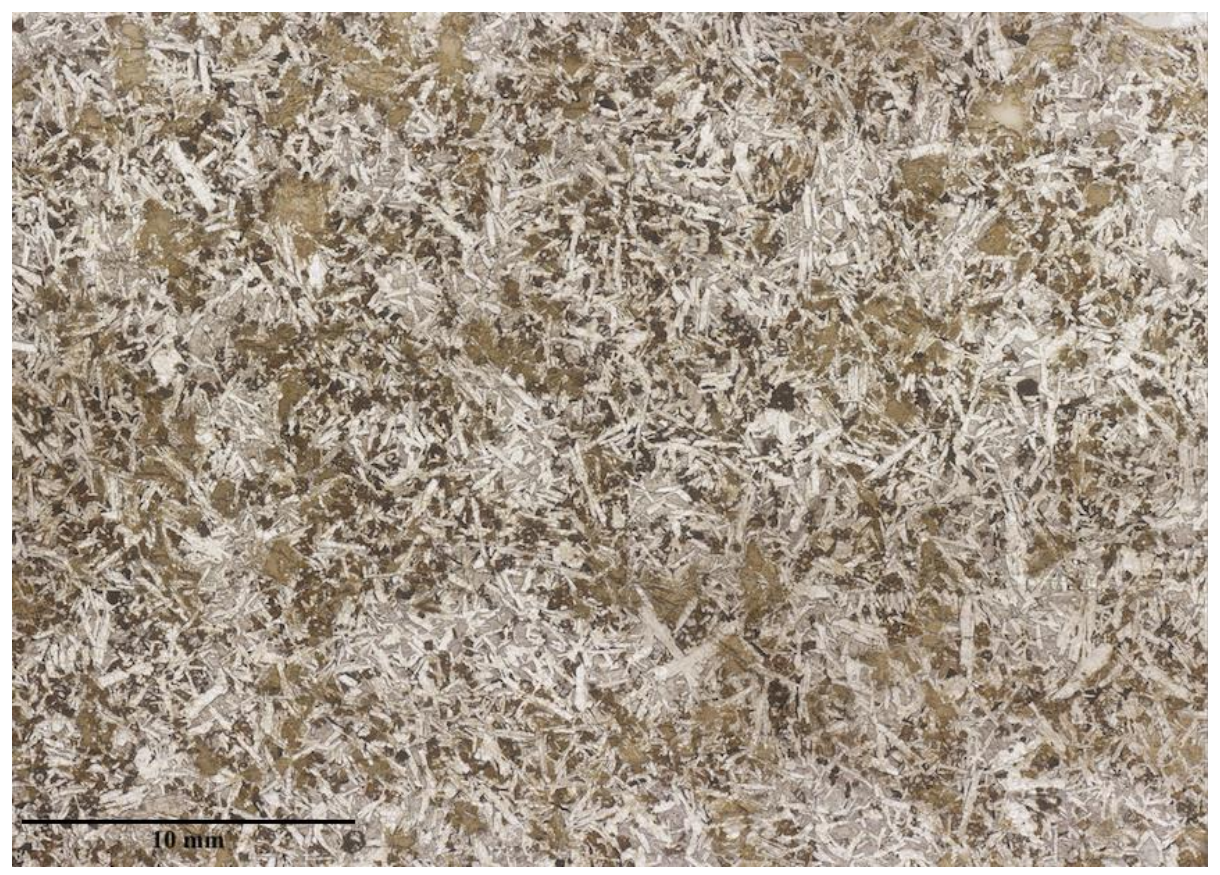

Fig. A8 Plane light thin section image of sample G314$09-2$. It is intergranular in texture; the angular interstices between plagioclase grains are occupied by grains of ferromagnesium minerals; olivine, pyroxene, and iron titanium oxides. Phenocryst modal abundance is as follows: $~ 55 \%$ plagioclase, $\sim 40 \%$ pyroxene, $\sim 3 \%$ olivine, and $2 \%$ Fe-Ti oxides. 


\section{CRUSTAL ROCKS}

\section{Metamorphic Rocks}

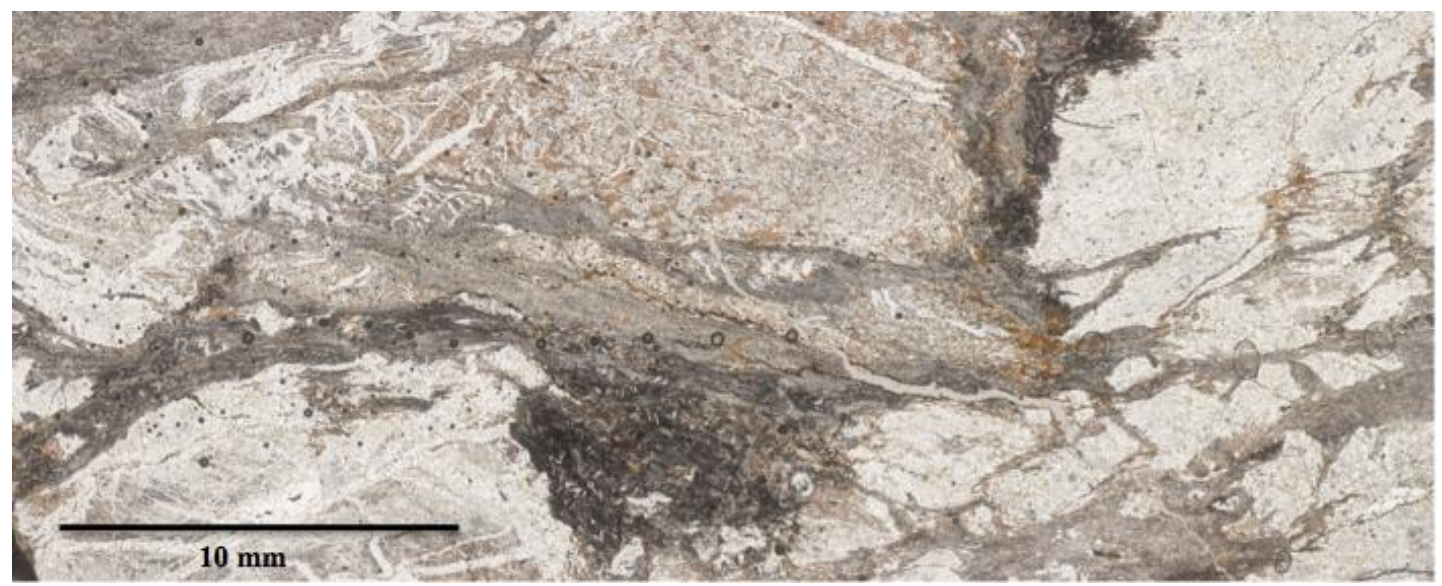

Fig. A9 Plane light thin section image of sample MS12-47; biotite fels, which is a metasedimentary rock found in abundance in the study area. The texture is partially lepidoblastic and partially granoblastic with a pure quartz groundmass. Mineral phase abundance is as follows: $~ 70 \%$ quartz, $\sim 13 \%$ biotite, $\sim 7 \%$ plagioclase (albite), $\sim 8 \%$ alkali feldspar (orthoclase), and $\sim 2 \%$ muscovite. Feldspars show alteration to sericite.

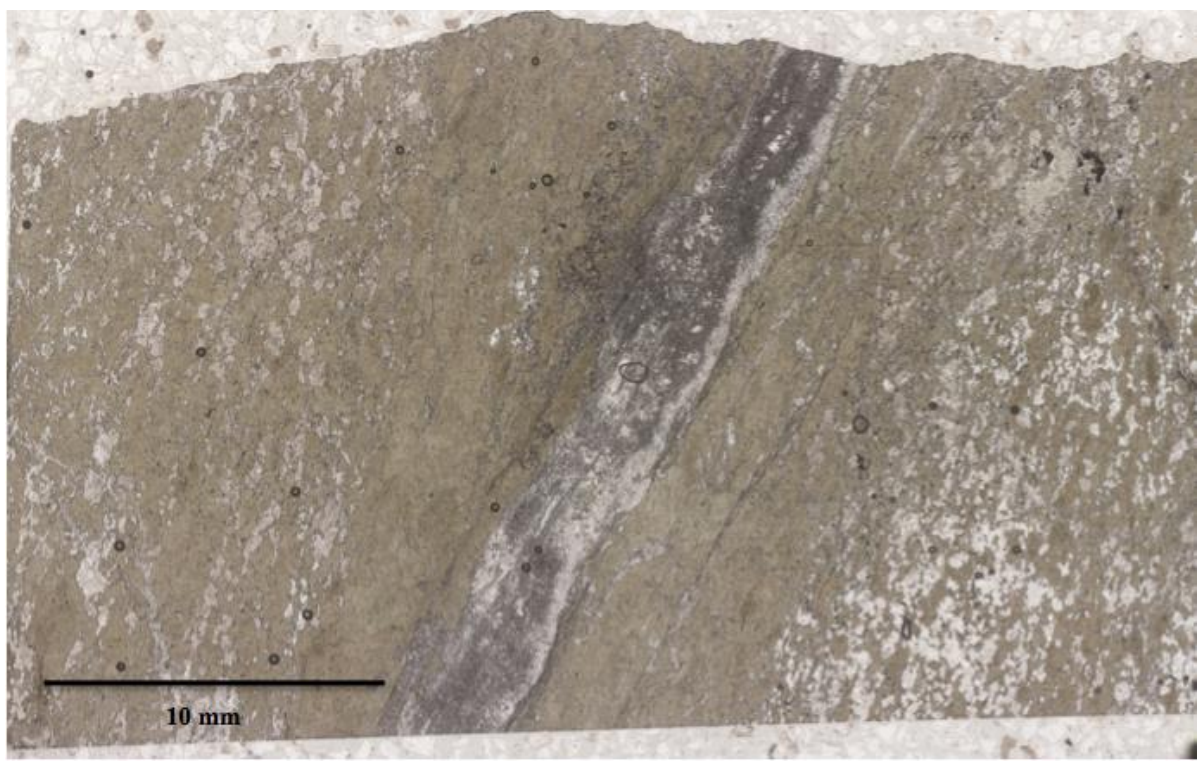

Fig. A10 Plane light thin section image of sample TM13-5; amphibolite. It is medium-grained in texture, shows weak to moderate foliation/incipient schistosity, and contains primarily amphibole and plagioclase feldspar with biotite and chlorite as accessory phases. Large hornblende porphyroblasts and actinolite laths make up over $50 \%$ of the sample by area. Plagioclase accounts for $\sim 40 \%$; oxides, $\sim 10 \%$, and biotite and chlorite, $<1 \%$. 


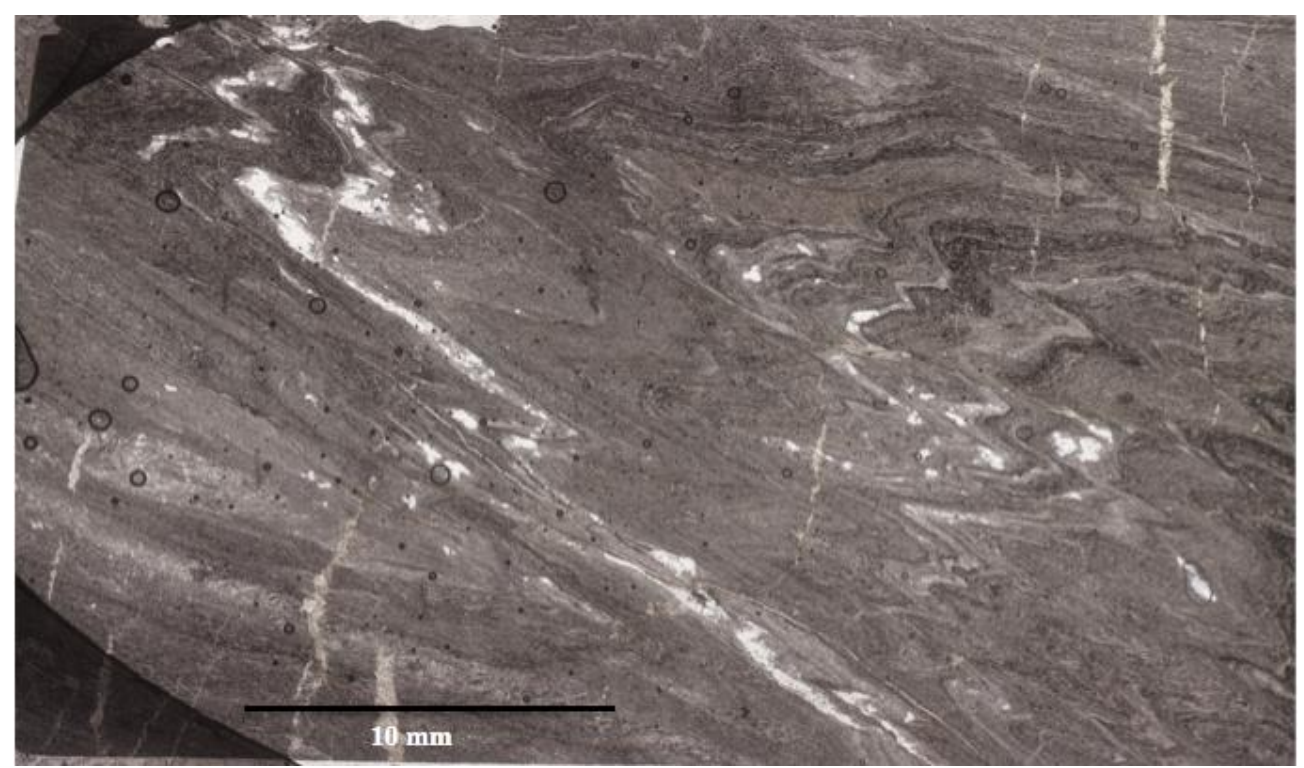

Fig. A11 Plane light thin section image of sample TM13-7; mica schist. It is medium-grained with strong foliation and contains mainly quartz and biotite, and to a lesser extent, muscovite and chlorite. There are zones that contain only quartz and here, the grains are interlocking and show strong undulatory extinction. Chevron-like folds can be seen overprinting the original schistose orientation of the grains.

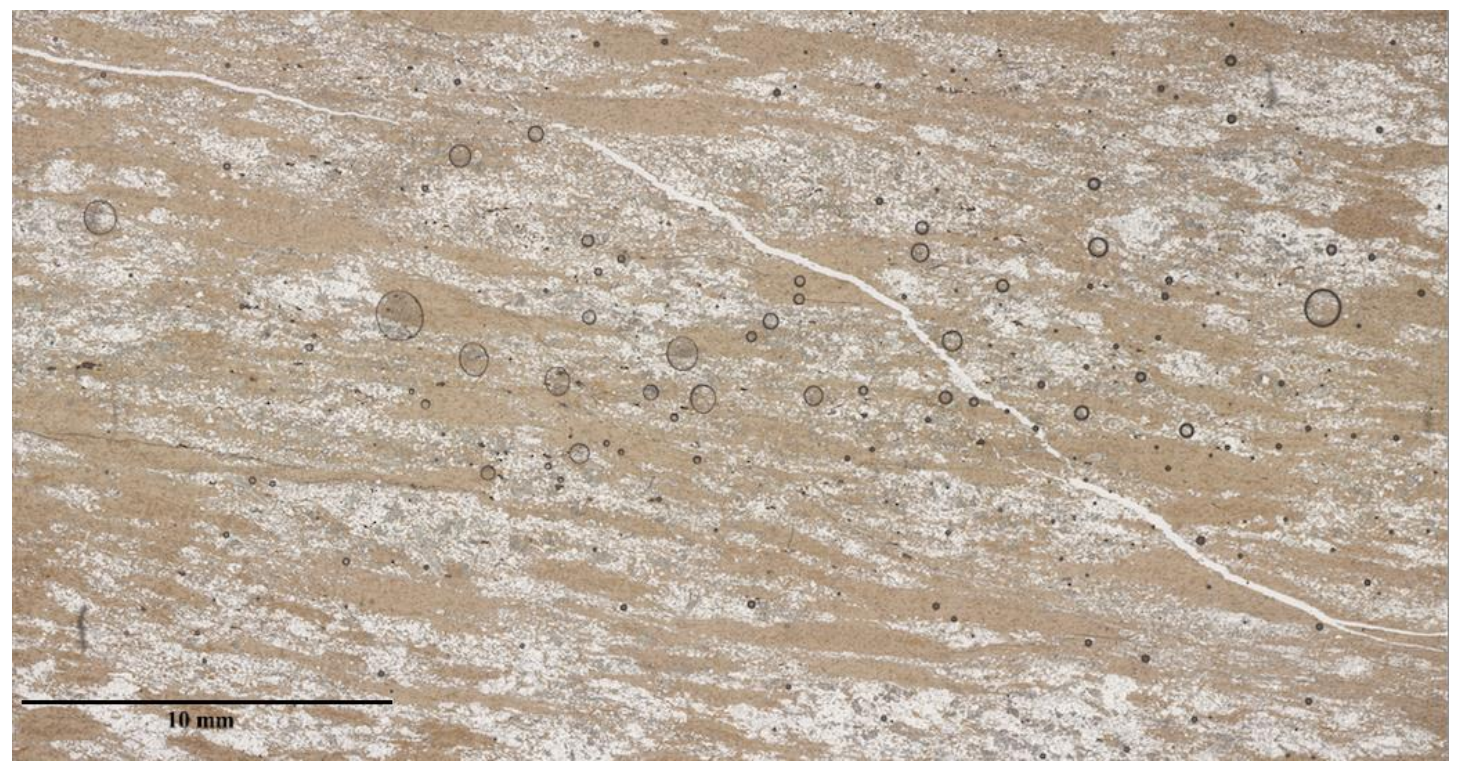

Fig. A12 Plane light thin section image of sample TM13-1; biotite schist. It is fine-grained and shows weak to moderate foliation. Quartz show sutured boundaries, and some grains show undulating extinction. Biotite displays moderate parallelism with amphibole porphyroblasts found among biotite laths. Segregation of minerals into mica-rich and quartz-rich domains parallel to the schistosity is observed. There are few ( 1$2 \%$ ) oxides. 


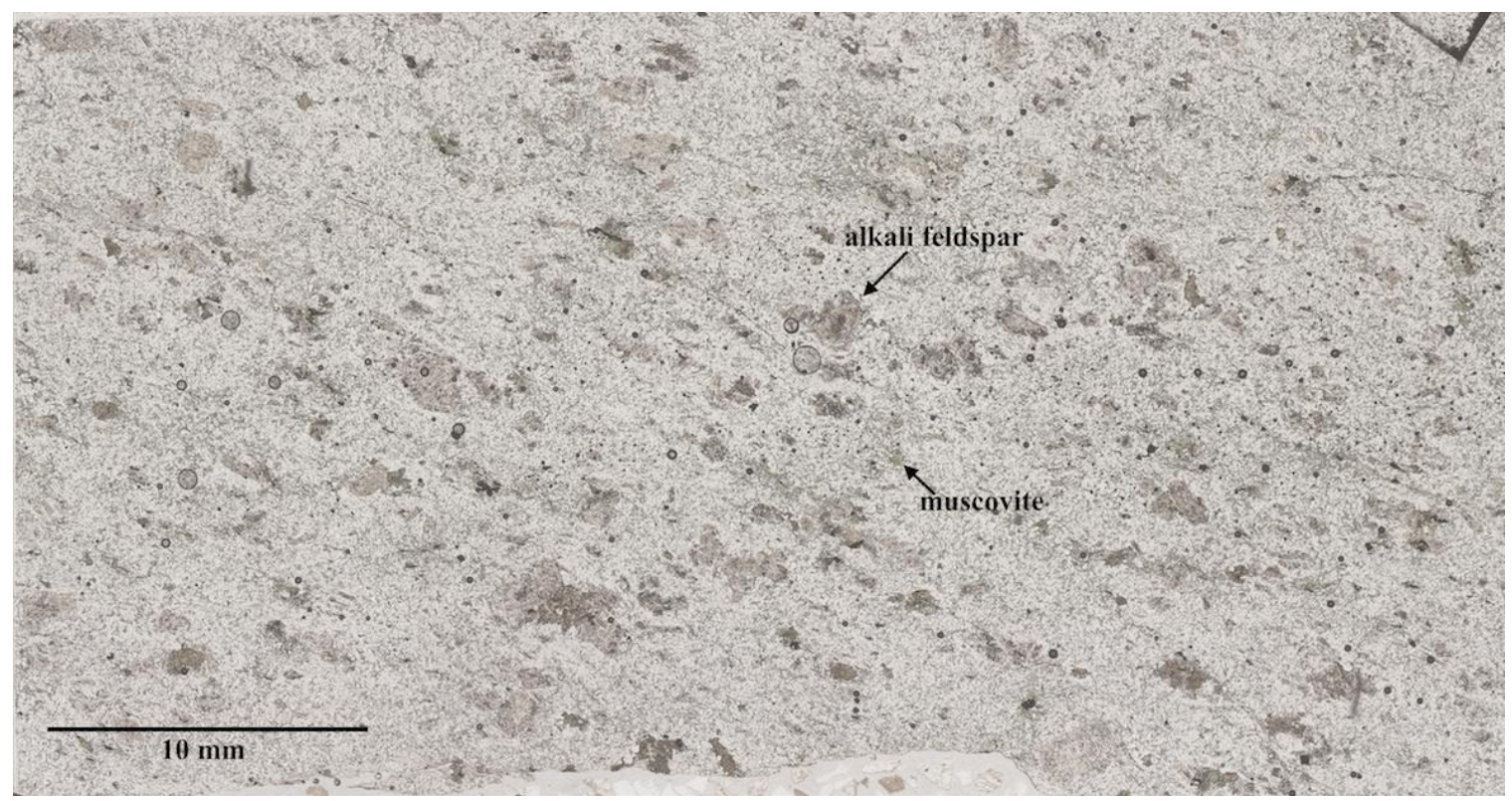

Fig. A13 Plane light thin section image of sample TM13-6; micaceous quartzite. This sample is dominated by interlocking quartz with alkali feldspar and muscovite porphyroblasts. The incipient alteration of the feldspar gives it a cloudy appearance while muscovite has a greenish hue. All mineral grains are elongated and define a diagonal fabric. This texture is a form of schistosity called grain-flattening fabric.

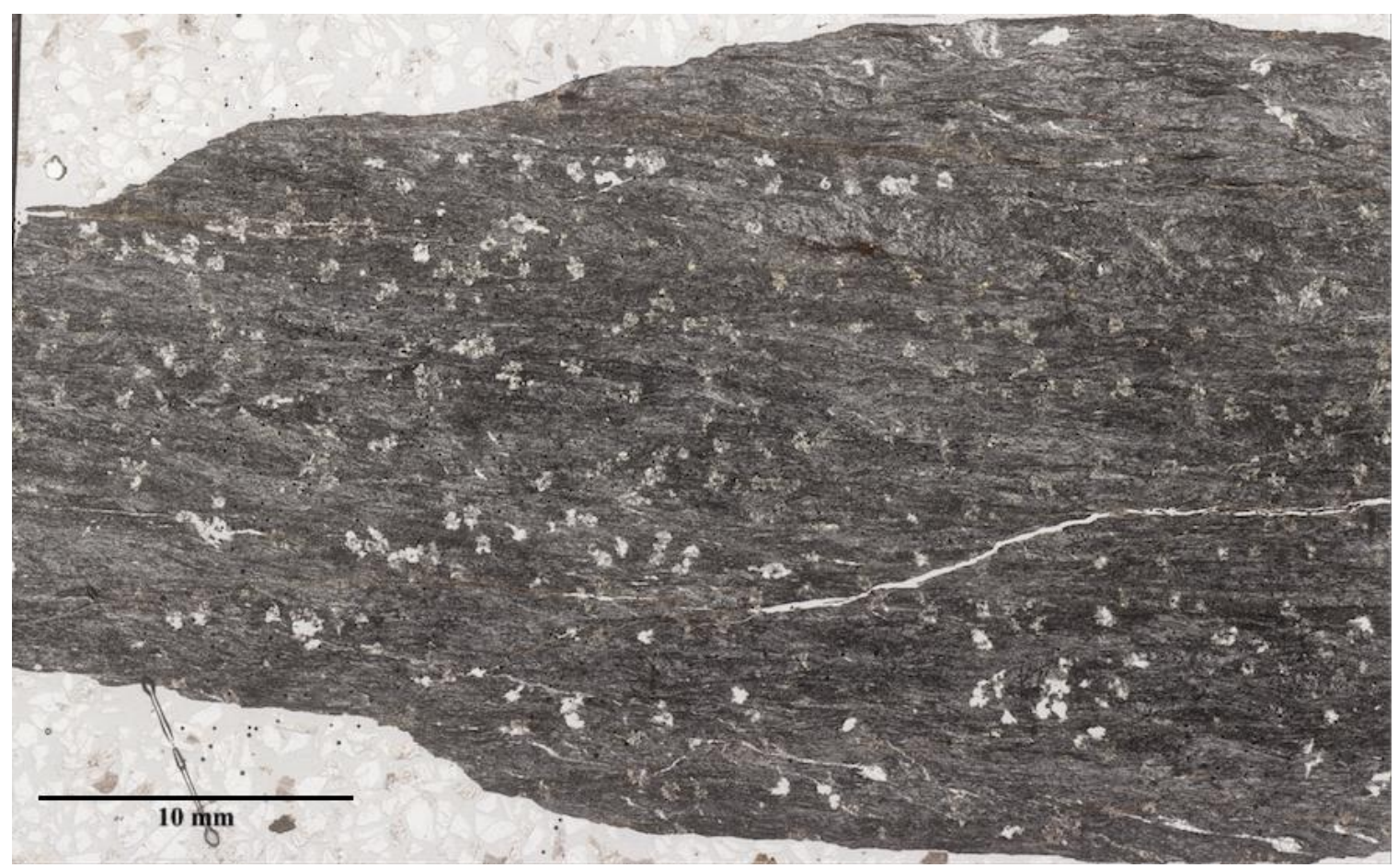

Fig. A14 Plane light thin section image of sample MS12-48; argillite. Argillite is considered as metasedimentary, so is included here among the other metamorphics. Quartz is the dominate phase and displays two populations; a fine-grained equigranular groundmass and larger porphyroblasts, which can be seen as the grey, irregularly shaped patches in the image. Biotite accounts for $\sim 15 \%$ of the area. The textured is incipiently lepidoblastic. 


\section{$\underline{\text { Granites }}$}

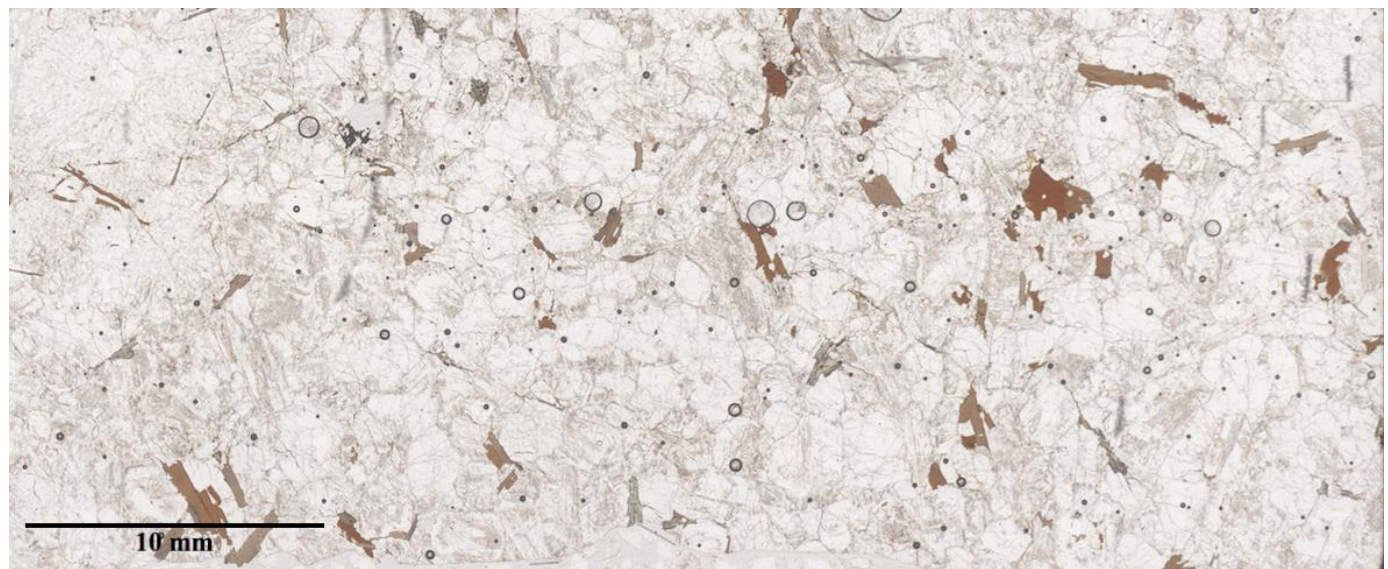

Fig. A15 Plane light thin section image of sample TM13-4; granite. It displays large, interlocking phenocrysts of quartz, alkali feldspar, plagioclase, and biotite. All are euhedral to subhedral. Feldspars show strong compositional zoning. There are excellent examples of graphic texture and feldspar exsolution lamellae throughout. Feldspars are weathering to clay minerals. Accessory chlorite and muscovite are subhedral. The modal abundance of minerals present is as follows: $\sim 60 \%$ alkali-feldspar, $\sim 25 \%$ quartz, $\sim 10 \%$ biotite, $<5 \%$ plagioclase feldspar, $\sim 1 \%$ chlorite, and $<1 \%$ muscovite.

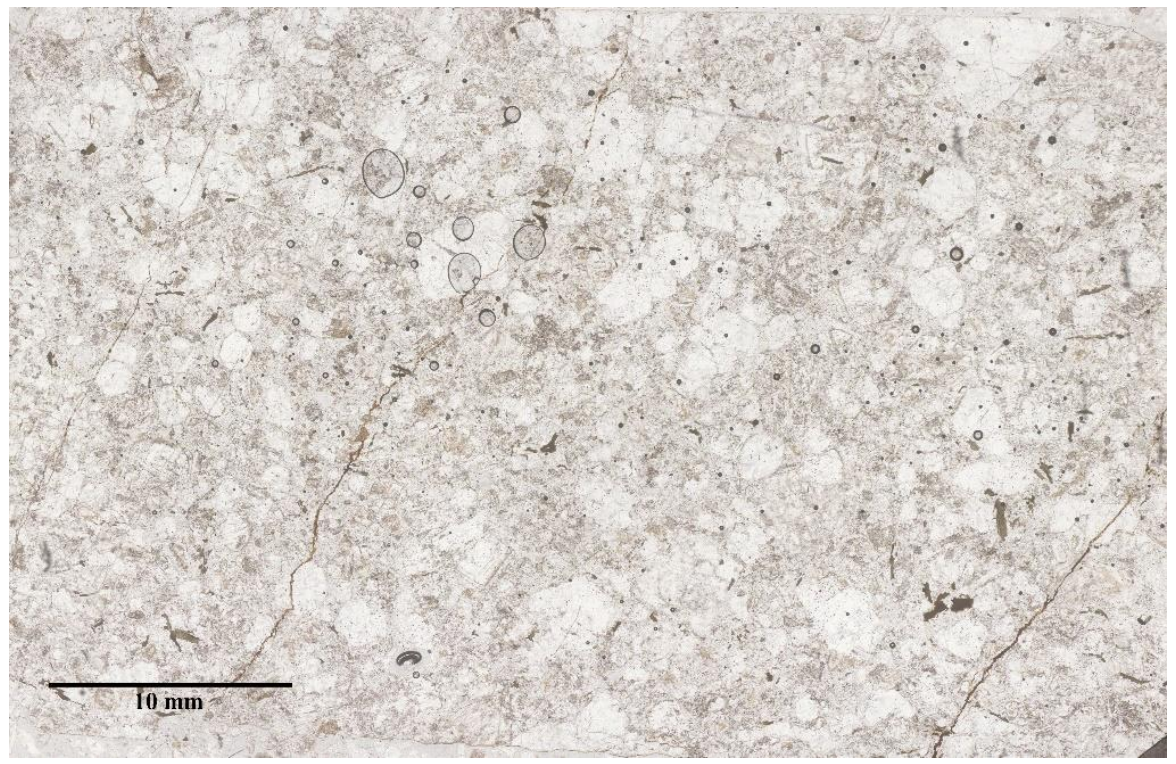

Fig. A16 Plane light thin section image of sample TM13-2; granite. This sample is similar in appearance and mineralogy to sample TM13-4 with two key differences. First, there are two quartz populations. The first are typical subhedral and interlocking quartz phenocrysts, similar in size an appearance to those observed in TM13-4. The second population are fine-

grained, equigranular porphyroblasts. This population also shows undulatory extinction. The darker green/brown areas between quartz and plagioclase grains are biotite and chlorite. Incipient alignment of these platy minerals is seen. This, along with the equigranular quartz population, indicates incipient metamorphism. The modal abundance of minerals present is as follows: $\sim 60 \%$ alkali-feldspar, $\sim 25 \%$ quartz, $\sim 10 \%$ biotite, $<5 \%$ plagioclase feldspar, $\sim 1 \%$ chlorite. 


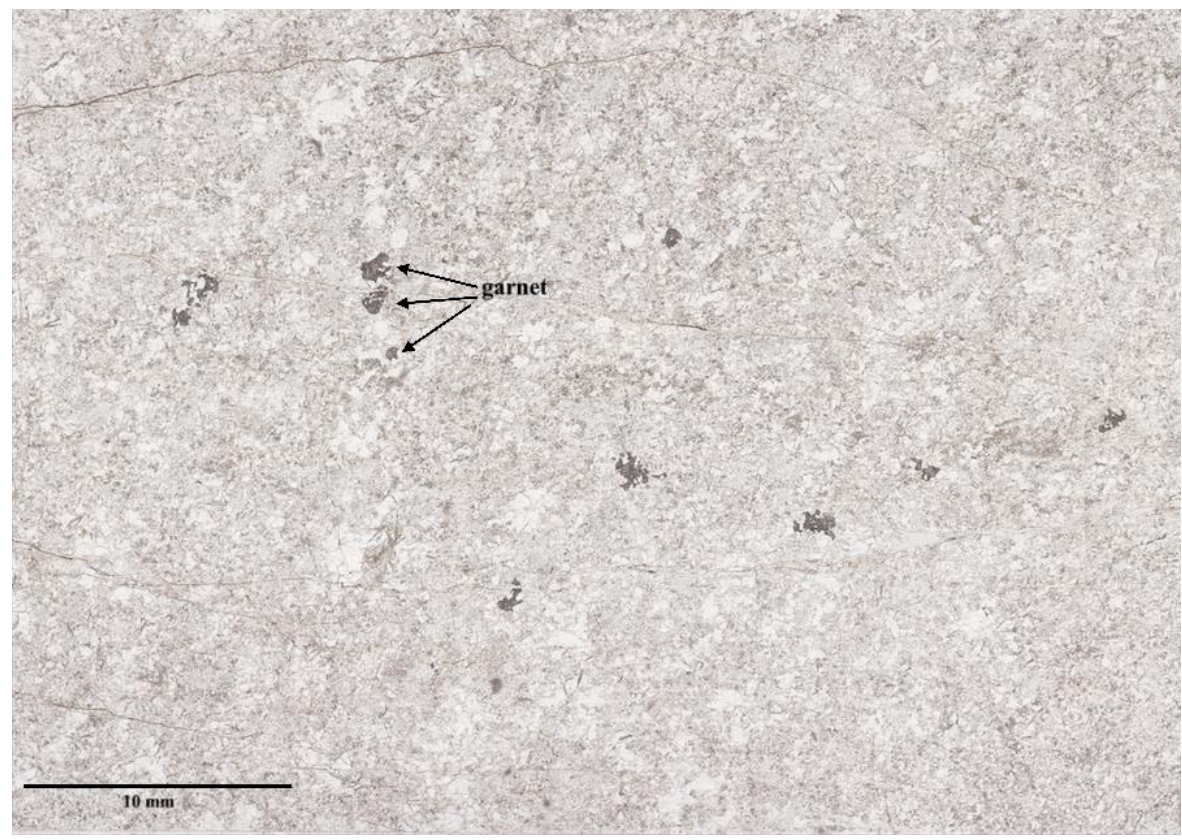

Fig. A17 Plane light thin section image of sample G314-09-4; granite. Mineral modal abundance as follows: $~ 80 \%$ quartz, $\sim 15 \%$ alkali feldspar, $3 \%$ plagioclase feldspar, $\sim 1 \%$ garnet, $\sim 1 \%$ biotite, $<1 \%$ chlorite. The granite displays interlocking grains of quartz with both alkali feldspar and sparse plagioclase feldspar. Some quartz show undulatory extinction. The feldspars are in the processes of breaking down into clay minerals, such as sericite. Graphic texture, the intergrowth of quartz and alkali feldspar, is observed. Sparse, yet large $(\sim 2 \mathrm{~mm})$, and highly fractured garnet phenocrysts are observed. The undulatory extinction of quartz and presence of chlorite indicate low-grade metamorphism.

\section{Granodiorites/Diorites}

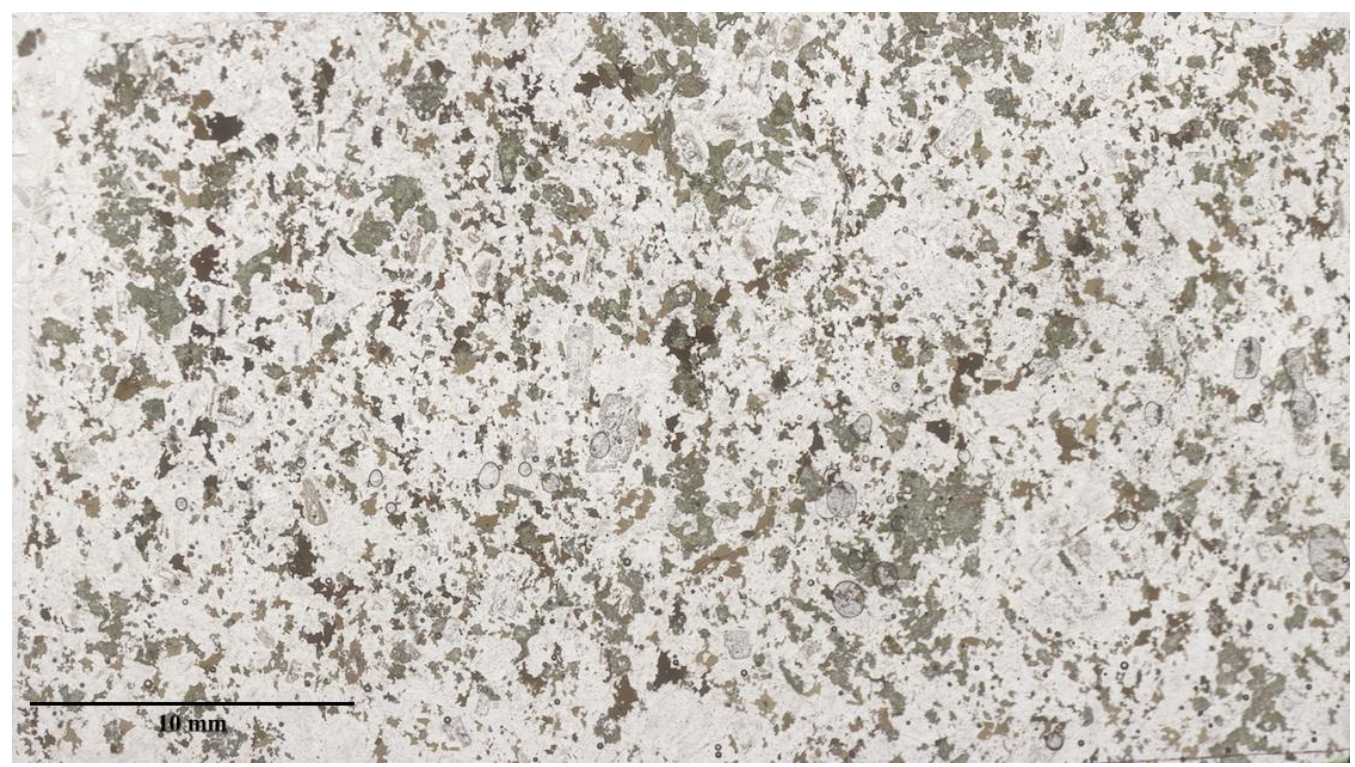

Fig. A18 Plane light thin section image of sample TM13-3; granodiorite. It is medium to large-grained and the texture is phaneritic. Phenocrysts are of plagioclase feldspar, alkali feldspar, amphibole, biotite, and quartz. Feldspars are both euhedral and subhedral with strong compositional zoning and abundant twinning. Subhedral to anhedral amphibole are located in interstitial spaces. The modal abundance of minerals is as follows: $25 \%$ quartz, $20 \%$ plagioclase feldspar, 20\% alkali feldspar, $20 \%$ amphibole, $~ 5 \%$ biotite, $<1 \%$ chlorite, $<1 \%$ apatite. 


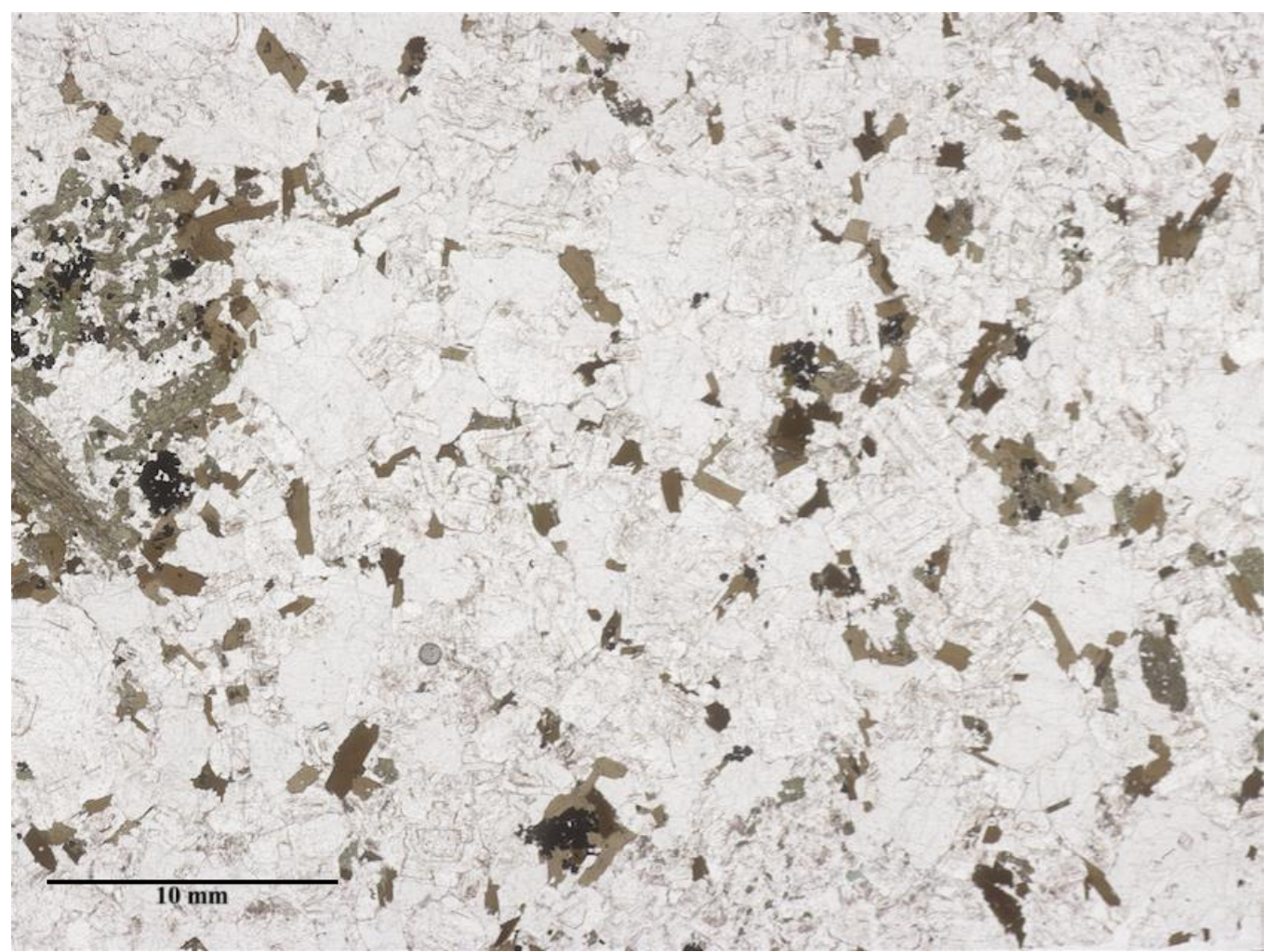

Fig. A19 Plane light thin section image of sample TM13-9; diorite. The mineral modal abundance is as follows: $\sim 50 \%$ plagioclase feldspar, $\sim 20 \%$ biotite, $\sim 10 \%$ quartz, $\sim 10 \%$ amphibole, $\sim 5 \%$ alkali feldspar, $\sim 5 \%$ oxides. The texture is phaneritic with some plagioclase phenocrysts greater than $5 \mathrm{~mm}$. Plagioclase is highly euhedral and shows strong compositional zoning. All other phenocrysts are euhedral to subhedral with oxides being relatively large and squarish. 


\section{APPENDIX B: XRF AND ICP MS GEOCHEMICAL DATA}

\section{METHODS}

\section{$\underline{\text { X-Ray Fluorescence }}$}

Bulk analyses of the rock samples were conducted at the GeoAnalytical Lab at Washington State University (WSU) to determine major and trace element abundances. Fresh portions of the samples were crushed into fine gravel-sized fragments using a Chipmunk Rock Crusher. The samples were then ground into a powder using a tungsten carbide bowl in a swing mill. The powders were homogeneously mixed with dilithium tetraborate $\left(\mathrm{Li}_{2} \mathrm{~B}_{4} \mathrm{O}_{7}\right)$ at a 2:1 flux to rock ratio in a VSM3 vortex mixer. Following the sample preparation and analytical procedures of Johnson et al. (1999), the mixture was then placed in graphite crucibles, fused into glass beads in a muffle furnace at $1000^{\circ} \mathrm{C}$, reground with the swing mill, and fused again prior to analysis to ensure complete homogenization. One duplicate bead was prepared for each nineteen samples to verify accuracy of the analysis. A ThermoARL AdvantXP+ sequential X-ray fluorescence (XRF) spectrometer was used to analyze for ten major elements: $\mathrm{Si}, \mathrm{Ti}, \mathrm{Al}, \mathrm{Fe}, \mathrm{Mn}, \mathrm{Mg}$, $\mathrm{Ca}, \mathrm{Na}, \mathrm{K}$, and $\mathrm{P}$, and twenty-one trace elements: $\mathrm{Ni}, \mathrm{Cr}, \mathrm{Sc}, \mathrm{V}, \mathrm{Ba}, \mathrm{Rb}, \mathrm{Sr}, \mathrm{Zr}, \mathrm{Y}, \mathrm{Nb}$, $\mathrm{Ga}, \mathrm{Cu}, \mathrm{Zn}, \mathrm{Pb}, \mathrm{La}, \mathrm{Ce}$, Th, Nd, U, Cs, and As. The X-ray intensity of each element within the prepared glass bead was compared to beads prepared from known standards to determine the unknown concentrations.

\section{Inductively Coupled Plasma Mass Spectrometry}

An HP45000 inductively coupled plasma mass spectrometer (ICP-MS) was used for further trace element analyses at the GeoAnalytical Lab at WSU. Analyses were conducted using a 1:1 dilithium tetraborate to rock ratio. The trace elements analyzed were $\mathrm{Ba}, \mathrm{Rb}, \mathrm{Y}, \mathrm{Nb}, \mathrm{Cs}, \mathrm{Hf}, \mathrm{Ta}, \mathrm{Pb}, \mathrm{Th}, \mathrm{U}, \mathrm{Sr}, \mathrm{Zr}$, and the fourteen naturally occurring rare earth elements (REE): La, Ce, Pr, Nd, Sm, Eu, Gd, Tb, Dy, Ho, Er, Tm, Yb, and Lu. The sample preparation and analytical procedures are after Knaack and Hooper (1994) 
Table B1. Whole rock geochemical analysis of rhyolites

\begin{tabular}{|c|c|c|c|c|c|c|c|c|}
\hline & Low-sili & & A-like & & & & & \\
\hline \multicolumn{9}{|l|}{$\begin{array}{l}\text { XRF } \\
\text { wt\% }\end{array}$} \\
\hline $\mathrm{SiO}_{2}$ & 73.5 & 74 & 77.5 & 79.6 & 78.7 & 77 & 77.7 & 75.2 \\
\hline $\mathrm{TiO}_{2}$ & 0.3 & 0.3 & 0.1 & 0.1 & 0.1 & 0.1 & 0.1 & 0.2 \\
\hline $\mathrm{Al}_{2} \mathrm{O}_{3}$ & 14.4 & 14.2 & 12.4 & 11.6 & 11.8 & 12.5 & 12.3 & 13 \\
\hline $\mathrm{FeO}^{*}$ & 1.9 & 1.7 & 1.3 & 0.8 & 1 & 1.4 & 1 & 1.7 \\
\hline $\mathrm{MnO}$ & 0 & 0 & 0 & 0 & 0 & 0 & 0 & 0 \\
\hline $\mathrm{MgO}$ & 0.3 & 0.3 & 0 & 0 & 0 & 0 & 0 & 0 \\
\hline $\mathrm{CaO}$ & 1.7 & 1.5 & 0.3 & 0.2 & 0.2 & 0.2 & 0.4 & 0.7 \\
\hline $\mathrm{Na}_{2} \mathrm{O}$ & 3.1 & 3.6 & 2.4 & 3.4 & 3.7 & 4.1 & 3.6 & 2.9 \\
\hline $\mathrm{K}_{2} \mathrm{O}$ & 4.7 & 4.1 & 6 & 4.3 & 4.5 & 4.8 & 4.8 & 6.3 \\
\hline $\mathrm{P}_{2} \mathrm{O}_{5}$ & 0.1 & 0.1 & 0 & 0 & 0 & 0 & 0 & 0 \\
\hline Total & 100 & 100 & 100 & 100 & 100 & 100 & 100 & 100 \\
\hline \multicolumn{9}{|l|}{$\begin{array}{l}\text { XRF } \\
\text { ppm }\end{array}$} \\
\hline $\mathrm{Ni}$ & 5.4 & 5.3 & 4.3 & 5 & 5.9 & 4.8 & 7.7 & 0 \\
\hline $\mathrm{Cr}$ & 6.4 & 9.2 & 3.9 & 4.6 & 5.9 & 5.1 & 7.1 & 1.1 \\
\hline $\mathrm{Sc}$ & 2.8 & 3.2 & 0 & 0.3 & 0.2 & 1.3 & 1.1 & 1 \\
\hline $\mathrm{V}$ & 17.1 & 22.4 & 1.5 & 3.3 & 11.1 & 3.4 & 5.1 & 0.1 \\
\hline $\mathrm{Ba}$ & 864 & 828.4 & 78.7 & 85.6 & 98.5 & 109.6 & 221.7 & 1261.9 \\
\hline $\mathrm{Rb}$ & 82 & 82.1 & 116.7 & 100.8 & 104.4 & 106.2 & 117.3 & 358.7 \\
\hline $\mathrm{Sr}$ & 213.6 & 173.3 & 12.1 & 15.2 & 21.5 & 20.1 & 25.1 & 84.4 \\
\hline $\mathrm{Zr}$ & 186.9 & 181.9 & 228.9 & 207.8 & 215.2 & 246.3 & 133.4 & 348.2 \\
\hline $\mathrm{Y}$ & 16.4 & 15.2 & 41.4 & 33.8 & 34.7 & 34.5 & 25.6 & 51.1 \\
\hline $\mathrm{Nb}$ & 11.2 & 12.9 & 32.5 & 30.1 & 30.3 & 32.3 & 26.2 & 41 \\
\hline $\mathrm{Ga}$ & 16 & 17.6 & 22.9 & 25.3 & 22.8 & 26.8 & 22.2 & 25.2 \\
\hline $\mathrm{Cu}$ & 5.7 & 8 & 6.8 & 5.1 & 5 & 4.8 & 5 & 1.3 \\
\hline $\mathrm{Zn}$ & 36.6 & 40.4 & 92.2 & 70.2 & 47.3 & 79.8 & 42.6 & 130.2 \\
\hline $\mathrm{Pb}$ & 9.6 & 10.1 & 14.7 & 14 & 12.8 & 11.1 & 13.7 & 10.6 \\
\hline $\mathrm{La}$ & 26.6 & 23.9 & 40.2 & 39 & 43.4 & 23.9 & 37.8 & 56.7 \\
\hline $\mathrm{Ce}$ & 39.7 & 45.2 & 77.4 & 65.5 & 80 & 59.4 & 66.4 & 118 \\
\hline Th & 6.4 & 7.4 & 11.6 & 10.7 & 10.1 & 10.8 & 11.7 & 10 \\
\hline $\mathrm{Nd}$ & 15.6 & 20 & 32.7 & 32.8 & 39 & 22 & 29.1 & 52.8 \\
\hline
\end{tabular}




\begin{tabular}{|c|c|c|c|c|c|c|c|c|}
\hline $\begin{array}{l}\text { XRF } \\
\text { ratios }\end{array}$ & & & & & & & & \\
\hline $\mathrm{AP}$ & 0.7 & 0.7 & 0.8 & 0.9 & 0.9 & 0.9 & 0.9 & 0.4 \\
\hline $\mathrm{Fe} / \mathrm{Fe}+\mathrm{Mg}$ & 0.9 & 0.8 & 1 & 1 & 1 & 1 & 1 & 1 \\
\hline $\mathrm{Ce} / \mathrm{Y}$ & 2.4 & 3 & 1.9 & 1.9 & 2.3 & 1.7 & 2.6 & 2.3 \\
\hline \multicolumn{9}{|l|}{$\begin{array}{l}\text { ICPMS } \\
\text { ppm }\end{array}$} \\
\hline $\mathrm{La}$ & 26.4 & 28.6 & 41 & 40.8 & 46.4 & 24.9 & 38.7 & 57.1 \\
\hline $\mathrm{Ce}$ & 42.7 & 46 & 81.17 & 70.6 & 84.3 & 62.4 & 67.8 & 117.3 \\
\hline $\operatorname{Pr}$ & 5 & 5.5 & 9.64 & 9.4 & 10.7 & 5.6 & 8.7 & 14.2 \\
\hline $\mathrm{Nd}$ & 16.9 & 19.1 & 35.63 & 35.2 & 39.7 & 20.7 & 31 & 55.2 \\
\hline $\mathrm{Sm}$ & 3.2 & 3.6 & 7.93 & 7.4 & 8.4 & 4.8 & 6.3 & 11.7 \\
\hline $\mathrm{Eu}$ & 0.7 & 0.7 & 0.29 & 0.3 & 0.3 & 0.3 & 0.3 & 1.5 \\
\hline $\mathrm{Gd}$ & 2.8 & 2.9 & 7.34 & 6.6 & 7.3 & 4.9 & 5.2 & 10.5 \\
\hline $\mathrm{Tb}$ & 0.5 & 0.5 & 1.29 & 1.1 & 1.2 & 1 & 0.9 & 1.7 \\
\hline Dy & 2.8 & 2.7 & 7.92 & 6.6 & 7 & 6.5 & 5.2 & 10.3 \\
\hline Но & 0.6 & 0.5 & 1.55 & 1.3 & 1.3 & 1.4 & 1 & 2 \\
\hline $\mathrm{Er}$ & 1.5 & 1.4 & 4.22 & 3.3 & 3.5 & 3.8 & 2.7 & 5.3 \\
\hline $\mathrm{Tm}$ & 0.2 & 0.2 & 0.61 & 0.5 & 0.5 & 0.6 & 0.4 & 0.8 \\
\hline $\mathrm{Yb}$ & 1.5 & 1.4 & 3.81 & 2.9 & 3.2 & 3.7 & 2.3 & 4.5 \\
\hline $\mathrm{Lu}$ & 0.3 & 0.2 & 0.56 & 0.4 & 0.5 & 0.6 & 0.3 & 0.7 \\
\hline $\mathrm{Ba}$ & 868.9 & 844.6 & 79.92 & 84.3 & 95.2 & 110.1 & 220 & 1299.3 \\
\hline Th & 8.1 & 8.5 & 11.03 & 10.7 & 10.8 & 11.6 & 12.4 & 8.6 \\
\hline $\mathrm{Nb}$ & 11.5 & 12.9 & 34.13 & 30.9 & 31.7 & 33.8 & 26.4 & 42.1 \\
\hline $\mathrm{Y}$ & 15.8 & 14.6 & 41.36 & 32.6 & 33.9 & 33.2 & 24.8 & 50.7 \\
\hline $\mathrm{Hf}$ & 5 & 4.8 & 7.71 & 6.9 & 7.1 & 7.9 & 5.3 & 9.9 \\
\hline $\mathrm{Ta}$ & 1.2 & 1.3 & 2.63 & 2.5 & 2.5 & 2.7 & 2.4 & 3 \\
\hline $\mathrm{U}$ & 2.8 & 2.9 & 3.86 & 3.1 & 3.9 & 2.9 & 3.3 & 3.2 \\
\hline $\mathrm{Pb}$ & 11.4 & 11.3 & 13.94 & 14.4 & 13.1 & 11.2 & 14.2 & 12 \\
\hline $\mathrm{Rb}$ & 77 & 78.7 & 112.51 & 97.3 & 99.2 & 102.6 & 111.8 & 360.4 \\
\hline Cs & 2.7 & 2 & 3.76 & 2.5 & 3.5 & 2 & 2.3 & 6.2 \\
\hline $\mathrm{Sr}$ & 206.2 & 169.7 & 13.74 & 15.4 & 19.2 & 19.2 & 24.2 & 86.1 \\
\hline $\mathrm{Sc}$ & 3.2 & 3.7 & 0.69 & 0.7 & 1 & 0.9 & 1.3 & 1.1 \\
\hline $\mathrm{Zr}$ & 187.3 & 180 & 239.41 & 207.6 & 216.1 & 248.1 & 132.5 & 366.5 \\
\hline
\end{tabular}

\section{ICPMS}

ratios

\begin{tabular}{lrrrrrrr}
$\mathrm{La} / \mathrm{Sm}$ & 8.2 & 8 & 5.5 & 5.5 & 5.2 & 6.1 & 4.9 \\
$\mathrm{La} / \mathrm{Yb}$ & 17.5 & 20.3 & 14.1 & 14.6 & 6.7 & 16.5 & 12.6 \\
$\mathrm{Ce} / \mathrm{Yb}$ & 28.3 & 32.7 & 24.5 & 26.5 & 16.8 & 28.9 & 25.9 \\
$\mathrm{Ba} / \mathrm{Rb}$ & 11.3 & 10.7 & 0.9 & 1 & 1.1 & 2 & 3.6 \\
\hline
\end{tabular}




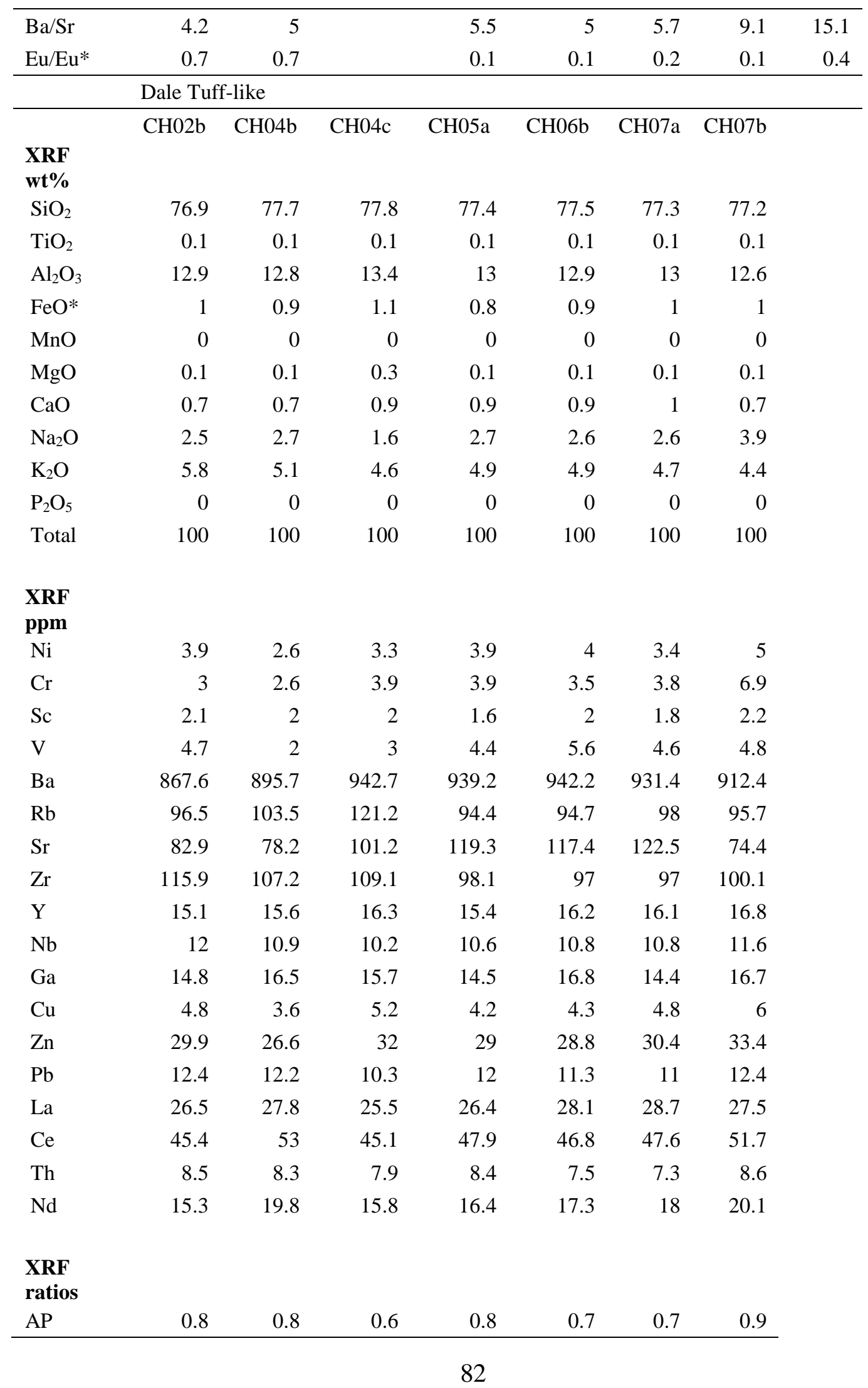




\begin{tabular}{|c|c|c|c|c|c|c|c|}
\hline $\mathrm{Fe} / \mathrm{Fe}+\mathrm{Mg}$ & 0.9 & 0.9 & 0.8 & 0.9 & 0.9 & 0.9 & 0.9 \\
\hline $\mathrm{Ce} / \mathrm{Y}$ & 3 & 3.4 & 2.8 & 3.1 & 2.9 & 3 & 3.1 \\
\hline \multicolumn{8}{|l|}{$\begin{array}{l}\text { ICPMS } \\
\text { ppm }\end{array}$} \\
\hline $\mathrm{La}$ & 28.6 & & & 27.9 & & 28.1 & 30.2 \\
\hline $\mathrm{Ce}$ & 49.9 & & & 49.5 & & 48.9 & 53.3 \\
\hline $\operatorname{Pr}$ & 5.3 & & & 5.2 & & 5.3 & 5.6 \\
\hline $\mathrm{Nd}$ & 17.3 & & & 17.5 & & 17.6 & 18.8 \\
\hline $\mathrm{Sm}$ & 3.2 & & & 3.1 & & 3.2 & 3.4 \\
\hline $\mathrm{Eu}$ & 0.4 & & & 0.4 & & 0.5 & 0.4 \\
\hline Gd & 2.6 & & & 2.6 & & 2.7 & 2.9 \\
\hline $\mathrm{Tb}$ & 0.4 & & & 0.4 & & 0.4 & 0.5 \\
\hline Dy & 2.5 & & & 2.6 & & 2.6 & 2.9 \\
\hline Ho & 0.5 & & & 0.5 & & 0.5 & 0.6 \\
\hline $\mathrm{Er}$ & 1.4 & & & 1.4 & & 1.5 & 1.6 \\
\hline $\mathrm{Tm}$ & 0.2 & & & 0.2 & & 0.2 & 0.2 \\
\hline $\mathrm{Yb}$ & 1.5 & & & 1.4 & & 1.5 & 1.6 \\
\hline $\mathrm{Lu}$ & 0.2 & & & 0.2 & & 0.2 & 0.3 \\
\hline $\mathrm{Ba}$ & 899.9 & & & 937.5 & & 928.8 & 927 \\
\hline Th & 9.5 & & & 8.5 & & 8.4 & 9.6 \\
\hline $\mathrm{Nb}$ & 12.2 & & & 11 & & 10.8 & 12.1 \\
\hline $\mathrm{Y}$ & 14.6 & & & 14.5 & & 15.1 & 16 \\
\hline $\mathrm{Hf}$ & 3.9 & & & 3.2 & & 3.2 & 3.5 \\
\hline $\mathrm{Ta}$ & 1.4 & & & 1.3 & & 1.3 & 1.4 \\
\hline $\mathrm{U}$ & 3.3 & & & 3 & & 3 & 3.4 \\
\hline $\mathrm{Pb}$ & 13.4 & & & 13.5 & & 13.3 & 13.8 \\
\hline $\mathrm{Rb}$ & 93.8 & & & 89.2 & & 93 & 91.3 \\
\hline Cs & 3.3 & & & 3.6 & & 3.5 & 3.4 \\
\hline $\mathrm{Sr}$ & 82.5 & & & 116.4 & & 119 & 72.6 \\
\hline $\mathrm{Sc}$ & 1.9 & & & 2 & & 2 & 2 \\
\hline $\mathrm{Zr}$ & 117.2 & & & 95.1 & & 94.4 & 98.5 \\
\hline
\end{tabular}

ICPMS

ratios

\begin{tabular}{lrrrr}
$\mathrm{La} / \mathrm{Sm}$ & 8.9 & 8.9 & 8.8 & 8.8 \\
$\mathrm{La} / \mathrm{Yb}$ & 19.7 & 19.3 & 18.9 & 18.9 \\
$\mathrm{Ce} / \mathrm{Yb}$ & 34.3 & 34.2 & 32.9 & 33.3 \\
$\mathrm{Ba} / \mathrm{Rb}$ & 9.6 & 10.5 & 10 & 10.2 \\
$\mathrm{Ba} / \mathrm{Sr}$ & 10.9 & 8.1 & 7.8 & 12.8 \\
$\mathrm{Eu} / \mathrm{Eu}^{*}$ & 0.4 & 0.5 & 0.5 & 0.4 \\
\hline
\end{tabular}




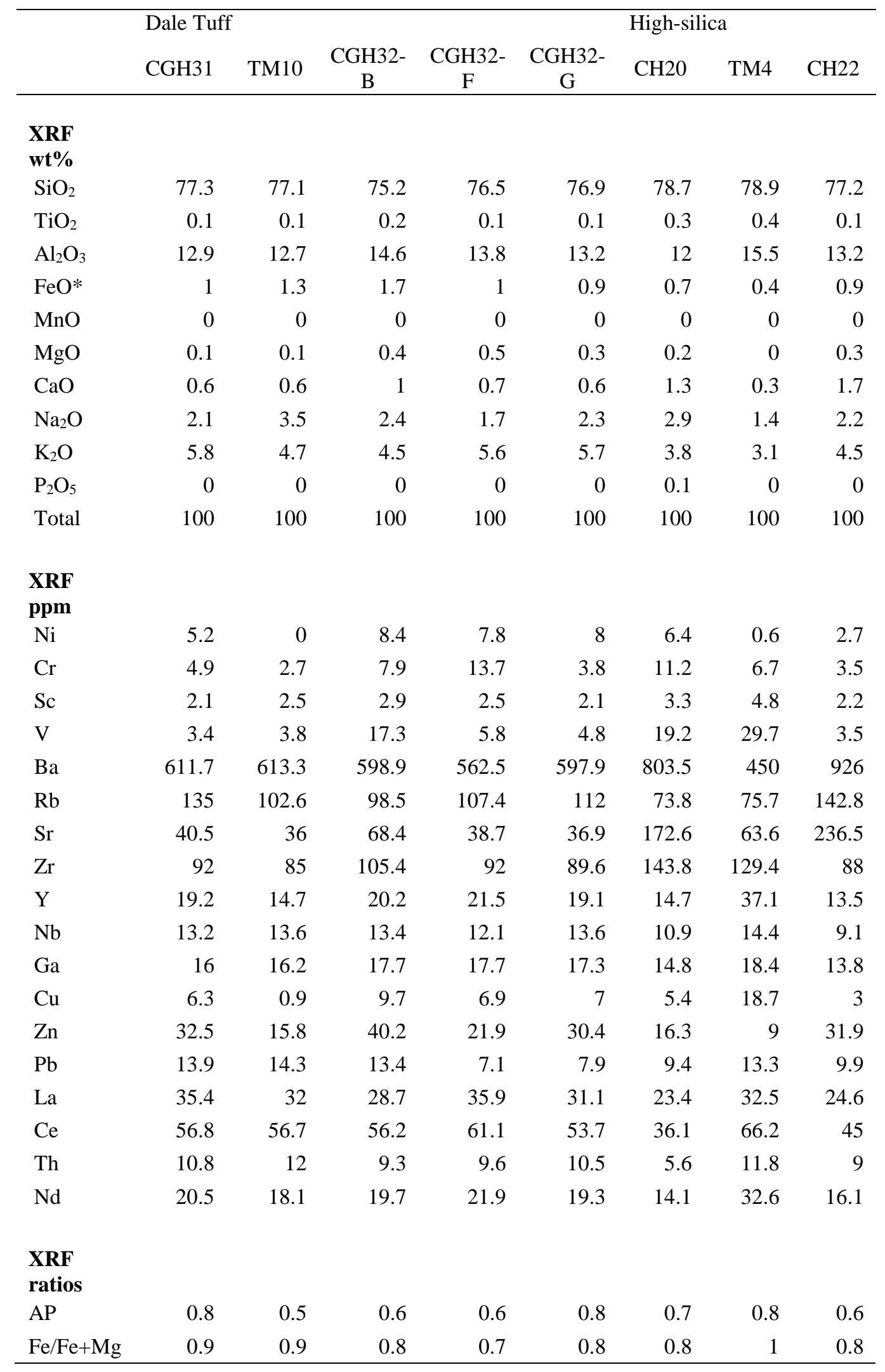




\begin{tabular}{|c|c|c|c|c|c|c|c|c|}
\hline $\mathrm{Ce} / \mathrm{Y}$ & 3 & 3.9 & 2.8 & 2.8 & 2.8 & 2.5 & 1.8 & 3.3 \\
\hline \multicolumn{9}{|l|}{$\begin{array}{l}\text { ICPMS } \\
\text { ppm }\end{array}$} \\
\hline $\mathrm{La}$ & 33.7 & 32.1 & 31.9 & & 32.1 & & 32.3 & 25.5 \\
\hline $\mathrm{Ce}$ & 58.8 & 56.3 & 55.9 & & 57.4 & & 64.2 & 43.4 \\
\hline $\operatorname{Pr}$ & 6.4 & 6 & 6.1 & & 6.2 & & 8.6 & 4.5 \\
\hline $\mathrm{Nd}$ & 21.2 & 20 & 20.9 & & 20.6 & & 32 & 14.8 \\
\hline $\mathrm{Sm}$ & 3.9 & 3.5 & 3.9 & & 3.9 & & 7.3 & 2.7 \\
\hline $\mathrm{Eu}$ & 0.3 & 0.3 & 0.4 & & 0.3 & & 1.1 & 0.4 \\
\hline $\mathrm{Gd}$ & 3.4 & 2.9 & 3.4 & & 3.3 & & 6.1 & 2.3 \\
\hline $\mathrm{Tb}$ & 0.5 & 0.5 & 0.6 & & 0.5 & & 1.1 & 0.4 \\
\hline Dy & 3.3 & 2.7 & 3.5 & & 3.3 & & 6.8 & 2.2 \\
\hline Ho & 0.7 & 0.5 & 0.7 & & 0.7 & & 1.4 & 0.5 \\
\hline $\mathrm{Er}$ & 1.9 & 1.4 & 1.9 & & 1.8 & & 3.9 & 1.3 \\
\hline $\mathrm{Tm}$ & 0.3 & 0.2 & 0.3 & & 0.3 & & 0.6 & 0.2 \\
\hline $\mathrm{Yb}$ & 1.9 & 1.4 & 2 & & 1.9 & & 3.9 & 1.3 \\
\hline $\mathrm{Lu}$ & 0.3 & 0.2 & 0.3 & & 0.3 & & 0.6 & 0.2 \\
\hline $\mathrm{Ba}$ & 615.5 & 623.3 & 602.5 & & 603.8 & & 455.3 & 937.9 \\
\hline Th & 11 & 10.8 & 10.2 & & 11 & & 9.4 & 9.3 \\
\hline $\mathrm{Nb}$ & 13.9 & 13.5 & 13.6 & & 14.1 & & 14.5 & 9.6 \\
\hline Y & 18.7 & 14.5 & 19.2 & & 18.8 & & 35.6 & 13.2 \\
\hline $\mathrm{Hf}$ & 3.5 & 3.4 & 3.6 & & 3.4 & & 4.1 & 3.1 \\
\hline $\mathrm{Ta}$ & 1.6 & 1.5 & 1.5 & & 1.6 & & 1.4 & 1.1 \\
\hline $\mathrm{U}$ & 3.9 & 2.7 & 3.5 & & 3.9 & & 4.8 & 3.2 \\
\hline $\mathrm{Pb}$ & 15 & 14.7 & 13.8 & & 9.1 & & 12.1 & 12 \\
\hline $\mathrm{Rb}$ & 126.9 & 102.8 & 94.1 & & 106.2 & & 75.6 & 135.9 \\
\hline Cs & 4.4 & 3.4 & 4.8 & & 4.1 & & 3.1 & 5.3 \\
\hline $\mathrm{Sr}$ & 39.2 & 36.9 & 67.1 & & 36.2 & & 63.3 & 230.4 \\
\hline $\mathrm{Sc}$ & 2.5 & 2.1 & 4.3 & & 3.1 & & 4.1 & 1.7 \\
\hline $\mathrm{Zr}$ & 91 & 89.9 & 102.5 & & 88.3 & & 129.4 & 87.7 \\
\hline \multicolumn{9}{|l|}{$\begin{array}{l}\text { ICPMS } \\
\text { ratios }\end{array}$} \\
\hline $\mathrm{La} / \mathrm{Sm}$ & 8.6 & 9.1 & 8.2 & & 8.3 & & 4.4 & 9.5 \\
\hline $\mathrm{La} / \mathrm{Yb}$ & 18.1 & 23.1 & 16.3 & & 17.2 & & 2.7 & 6 \\
\hline $\mathrm{Ce} / \mathrm{Yb}$ & 31.6 & 40.5 & 28.6 & & 30.8 & & 16.3 & 33.1 \\
\hline $\mathrm{Ba} / \mathrm{Rb}$ & 4.9 & 6.1 & 6.4 & & 5.7 & & 6 & 6.9 \\
\hline $\mathrm{Ba} / \mathrm{Sr}$ & 15.7 & 16.9 & 9 & & 16.7 & & 7.2 & 4.1 \\
\hline $\mathrm{Eu} / \mathrm{Eu}^{*}$ & 0.2 & 0.3 & 0.4 & & 0.2 & & 0.5 & 0.5 \\
\hline
\end{tabular}


Table B2. Whole rock geochemical analysis of basalt and dacites

\begin{tabular}{|c|c|c|c|}
\hline & basalt & dacite & dacite \\
\hline & $\begin{array}{c}\text { G314-09- } \\
2\end{array}$ & $\mathrm{CH} 01$ & TM6 \\
\hline \multicolumn{4}{|c|}{ XRF wt\% } \\
\hline $\mathrm{SiO} 2$ & 48.05 & 64.88 & 68 \\
\hline $\mathrm{TiO} 2$ & 1.937 & 0.808 & 0.612 \\
\hline $\mathrm{A} 12 \mathrm{O} 3$ & 16.61 & 16.74 & 17.05 \\
\hline $\mathrm{FeO}^{*}$ & 12.07 & 4.01 & 2.72 \\
\hline $\mathrm{MnO}$ & 0.171 & 0.044 & 0.039 \\
\hline $\mathrm{MgO}$ & 7.85 & 1.84 & 0.94 \\
\hline $\mathrm{CaO}$ & 10.33 & 4.80 & 4.83 \\
\hline $\mathrm{Na} 2 \mathrm{O}$ & 2.48 & 4.51 & 3.74 \\
\hline $\mathrm{K} 2 \mathrm{O}$ & 0.34 & 2.05 & 1.9 \\
\hline $\mathrm{P} 2 \mathrm{O} 5$ & 0.168 & 0.327 & 0.158 \\
\hline Total & 100 & 100.00 & 100 \\
\hline \multicolumn{4}{|c|}{ XRF ppm } \\
\hline $\mathrm{Ni}$ & 134 & 30 & 8 \\
\hline $\mathrm{Cr}$ & 177 & 47 & 23 \\
\hline $\mathrm{Sc}$ & 29 & 11 & 10 \\
\hline $\mathrm{V}$ & 221 & 79 & 75 \\
\hline $\mathrm{Ba}$ & 135 & 696 & 827 \\
\hline $\mathrm{Rb}$ & 8 & 48 & 39 \\
\hline $\mathrm{Sr}$ & 281 & 505 & 485 \\
\hline $\mathrm{Zr}$ & 83 & 198 & 121 \\
\hline $\mathrm{Y}$ & 22 & 21 & 15 \\
\hline $\mathrm{Nb}$ & 7.4 & 17.7 & 7 \\
\hline $\mathrm{Ga}$ & 14 & 20 & 16 \\
\hline $\mathrm{Cu}$ & 147 & 20 & 13 \\
\hline $\mathrm{Zn}$ & 94 & 54 & 50 \\
\hline $\mathrm{Pb}$ & 0 & 6 & 8 \\
\hline $\mathrm{La}$ & 6 & 25 & 18 \\
\hline $\mathrm{Ce}$ & 16 & 47 & 29 \\
\hline $\mathrm{Th}$ & 2 & 3 & 3 \\
\hline $\mathrm{Nd}$ & 14 & 23 & 16 \\
\hline $\mathrm{U}$ & 0 & & \\
\hline
\end{tabular}

\begin{tabular}{lrrr} 
ICPMS ppm & & & \\
$\mathrm{La}$ & 6.35 & 28.04 & 15.92 \\
$\mathrm{Ce}$ & 15.79 & 47.35 & 29.34 \\
\hline
\end{tabular}




\begin{tabular}{lrrr}
\hline $\mathrm{Pr}$ & 2.45 & 6.11 & 3.64 \\
$\mathrm{Nd}$ & 12.09 & 22.71 & 14.17 \\
$\mathrm{Sm}$ & 3.68 & 4.56 & 3.05 \\
$\mathrm{Eu}$ & 1.37 & 1.27 & 0.92 \\
$\mathrm{Gd}$ & & 4.03 & 2.73 \\
$\mathrm{~Tb}$ & & 0.64 & 0.45 \\
$\mathrm{Dy}$ & 4.36 & 3.72 & 2.7 \\
$\mathrm{Ho}$ & & 0.73 & 0.52 \\
$\mathrm{Er}$ & & 1.99 & 1.42 \\
$\mathrm{Tm}$ & & 0.28 & 0.2 \\
$\mathrm{Yb}$ & 2.1 & 1.82 & 1.23 \\
$\mathrm{Lu}$ & 0.33 & 0.28 & 0.21 \\
$\mathrm{Ba}$ & 137 & 695 & 836.92 \\
$\mathrm{Th}$ & & 4.72 & 3.07 \\
$\mathrm{Nb}$ & & 18.47 & 7.16 \\
$\mathrm{Y}$ & 22.05 & 19.87 & 13.61 \\
$\mathrm{Hf}$ & 2.14 & 4.60 & 3.16 \\
$\mathrm{Ta}$ & 0.53 & 1.27 & 0.51 \\
$\mathrm{U}$ & 0.16 & 1.78 & 1.29 \\
$\mathrm{~Pb}$ & 0.65 & 8.04 & 8.13 \\
$\mathrm{Rb}$ & 8 & 45.2 & 38.65 \\
$\mathrm{Cs}$ & 1.53 & 0.95 & 1.92 \\
$\mathrm{Sr}$ & 297 & 496 & 480.22 \\
$\mathrm{Sc}$ & & 10.7 & 8.82 \\
$\mathrm{Zr}$ & 83 & 196 & 121.43 \\
\hline
\end{tabular}

Table B3. Whole rock geochemical analysis of crustal rocks

\begin{tabular}{cccccccc}
\hline \hline & $\begin{array}{c}\text { biotite } \\
\text { schist }\end{array}$ & amphibolite & $\begin{array}{c}\text { mica } \\
\text { schist }\end{array}$ & mica schist & $\begin{array}{c}\text { biotite } \\
\text { fels }\end{array}$ & argillite & $\begin{array}{c}\text { micaceous } \\
\text { quartzite }\end{array}$ \\
\hline & $\begin{array}{c}\text { TM13- } \\
\text { XRF }\end{array}$ & TM13-5 & TM13-7 & TM13-7b & $\begin{array}{c}\text { MS12- } \\
47\end{array}$ & $\begin{array}{c}\text { MS12- } \\
48\end{array}$ & TM13-6 \\
$\mathbf{w t \%}$ & & & & & & & \\
$\mathrm{SiO} 2$ & 46.63 & 47.22 & 56.7 & 33.58 & 80.28 & 85.31 & 72.78 \\
$\mathrm{TiO} 2$ & 1.564 & 0.932 & 0.763 & 1.01 & 0.471 & 0.507 & 0.477 \\
$\mathrm{Al2O} 3$ & 18.05 & 17.85 & 15.18 & 29.23 & 9.47 & 7.94 & 14.3 \\
$\mathrm{FeO} *$ & 14.98 & 10.21 & 9.18 & 23 & 3.49 & 2.2 & 3.98 \\
$\mathrm{MnO}$ & 0.222 & 0.213 & 0.202 & 0.312 & 0.06 & 0.011 & 0.085 \\
$\mathrm{MgO}$ & 7.54 & 8.76 & 9.39 & 7.58 & 2.02 & 0.81 & 1.59 \\
$\mathrm{CaO}$ & 3.33 & 12.49 & 2.11 & 0.8 & 0.4 & 0.68 & 2.72 \\
$\mathrm{Na2O}$ & 1.91 & 2 & 4.4 & 0.54 & 1.01 & 1.31 & 3.7 \\
$\mathrm{~K} 2 \mathrm{O}$ & 5.62 & 0.14 & 1.96 & 3.73 & 2.72 & 1.18 & 0.25 \\
\hline
\end{tabular}




\begin{tabular}{|c|c|c|c|c|c|c|c|}
\hline $\mathrm{P} 2 \mathrm{O} 5$ & 0.147 & 0.184 & 0.122 & 0.22 & 0.081 & 0.04 & 0.117 \\
\hline Total & 100 & 100 & 100 & 100 & 100 & 97.33 & 100 \\
\hline \multicolumn{8}{|l|}{$\begin{array}{l}\text { XRF } \\
\text { ppm }\end{array}$} \\
\hline $\mathrm{Ni}$ & 106 & 75 & 161 & 11 & 11 & 7 & 3 \\
\hline $\mathrm{Cr}$ & 269 & 265 & 209 & 2 & 51 & 46 & 4 \\
\hline $\mathrm{Sc}$ & 50 & 45 & 25 & 18 & 12 & 11 & 14 \\
\hline $\mathrm{V}$ & 389 & 333 & 182 & 85 & 116 & 200 & 35 \\
\hline $\mathrm{Ba}$ & 3597 & 45 & 532 & 1982 & 2374 & 563 & 291 \\
\hline $\mathrm{Rb}$ & 232 & 1 & 52 & 39 & 66 & 39 & 3 \\
\hline $\mathrm{Sr}$ & 204 & 323 & 38 & 40 & 72 & 105 & 188 \\
\hline $\mathrm{Zr}$ & 87 & 39 & 81 & 223 & 89 & 103 & 114 \\
\hline Y & 30 & 18 & 18 & 26 & 17 & 26 & 32 \\
\hline $\mathrm{Nb}$ & 10.4 & 2.7 & 3.2 & 7.2 & 7.4 & 9.3 & 3.8 \\
\hline $\mathrm{Ga}$ & 25 & 18 & 17 & 32 & 16 & 12 & 15 \\
\hline $\mathrm{Cu}$ & 60 & 24 & 18 & 4 & 58 & 19 & 7 \\
\hline $\mathrm{Zn}$ & 186 & 81 & 113 & 295 & 78 & 53 & 52 \\
\hline $\mathrm{Pb}$ & 6 & 2 & 2 & 2 & 7 & 11 & 2 \\
\hline $\mathrm{La}$ & 6 & 5 & 7 & 0 & 22 & 22 & 9 \\
\hline $\mathrm{Ce}$ & 18 & 16 & 18 & 3 & 38 & 40 & 19 \\
\hline Th & 1 & 0 & 1 & 3 & 7 & 7 & 1 \\
\hline $\mathrm{Nd}$ & 13 & 12 & 11 & 4 & 18 & 22 & 11 \\
\hline $\mathrm{U}$ & 1 & 0 & 1 & 1 & 3 & 4 & 0 \\
\hline \multicolumn{8}{|c|}{$\begin{array}{c}\text { ICPMS } \\
\text { ppm }\end{array}$} \\
\hline $\mathrm{La}$ & 5.18 & 16.81 & 6.76 & 0.91 & 19.87 & 22.44 & 8.21 \\
\hline $\mathrm{Ce}$ & 12.74 & 32.93 & 15.49 & 3.23 & 40.26 & 40.42 & 18.22 \\
\hline $\operatorname{Pr}$ & 1.99 & 4.13 & 2.23 & 0.59 & 4.68 & 5.39 & 2.62 \\
\hline $\mathrm{Nd}$ & 10.01 & 16.61 & 10.18 & 3.23 & 17.23 & 20.83 & 11.98 \\
\hline $\mathrm{Sm}$ & 3.21 & 3.54 & 2.73 & 1.16 & 3.34 & 4.33 & 3.51 \\
\hline $\mathrm{Eu}$ & 1.03 & 1.07 & 0.81 & 0.47 & 0.6 & 1.1 & 1.1 \\
\hline $\mathrm{Gd}$ & 4.25 & 3.15 & 3.01 & 2.12 & 2.87 & 4.02 & 4.36 \\
\hline $\mathrm{Tb}$ & 0.78 & 0.48 & 0.53 & 0.41 & 0.47 & 0.64 & 0.78 \\
\hline Dy & 5.23 & 2.86 & 3.42 & 3.1 & 2.96 & 3.99 & 5.28 \\
\hline Ho & 1.13 & 0.57 & 0.72 & 0.82 & 0.61 & 0.84 & 1.13 \\
\hline $\mathrm{Er}$ & 3.1 & 1.58 & 2.03 & 2.85 & 1.71 & 2.34 & 3.35 \\
\hline $\mathrm{Tm}$ & 0.45 & 0.22 & 0.3 & 0.5 & 0.26 & 0.34 & 0.5 \\
\hline $\mathrm{Yb}$ & 2.66 & 1.4 & 1.97 & 3.81 & 1.69 & 2.14 & 3.23 \\
\hline $\mathrm{Lu}$ & 0.42 & 0.23 & 0.3 & 0.72 & 0.28 & 0.36 & 0.52 \\
\hline $\mathrm{Ba}$ & 3559 & 734 & 535 & 1935 & 2483 & 578 & 297 \\
\hline
\end{tabular}




\begin{tabular}{|c|c|c|c|c|c|c|c|}
\hline $\mathrm{Th}$ & 0.33 & 3.78 & 1.74 & 2.46 & 7.06 & 6.68 & 1.41 \\
\hline $\mathrm{Nb}$ & 8.56 & 5.68 & 3.26 & 5.26 & 7.23 & 8.39 & 2.68 \\
\hline $\mathrm{Y}$ & 27.82 & 14.99 & 17.43 & 26.45 & 15.47 & 23.75 & 29.84 \\
\hline $\mathrm{Hf}$ & 2.23 & 2.69 & 2.34 & 6.08 & 2.39 & 2.88 & 3.29 \\
\hline $\mathrm{Ta}$ & 0.33 & 0.47 & 0.21 & 0.35 & 0.63 & 0.64 & 0.19 \\
\hline $\mathrm{U}$ & 0.21 & 1.47 & 0.69 & 0.78 & 2.29 & 3.24 & 0.5 \\
\hline $\mathrm{Pb}$ & 5.35 & 6.63 & 0.39 & 1.29 & 5.46 & 8.83 & 1.53 \\
\hline $\mathrm{Rb}$ & 220.9 & 43.7 & 51.2 & 38.4 & 65.7 & 40 & 2.8 \\
\hline Cs & 22.22 & 3.8 & 1.65 & 0.55 & 3.87 & 3.17 & 0.1 \\
\hline $\mathrm{Sr}$ & 209 & 574 & 39 & 43 & 73 & 111 & 196 \\
\hline $\mathrm{Sc}$ & 50.1 & 14.9 & 25.6 & 19.3 & 12 & 11.6 & 13.1 \\
\hline \multirow[t]{3}{*}{$\mathrm{Zr}$} & 77 & 99 & 82 & 216 & 89 & 106 & 116 \\
\hline & granite & granite & granite & granodiorite & diorite & & \\
\hline & $\begin{array}{c}\text { TM13- } \\
2 \\
\end{array}$ & TM13-4 & $\begin{array}{c}\text { G314- } \\
09-4 \\
\end{array}$ & TM13-3 & $\begin{array}{c}\text { TM13- } \\
9 \\
\end{array}$ & & \\
\hline \multicolumn{8}{|l|}{$\begin{array}{l}\text { XRF } \\
\text { wt\% }\end{array}$} \\
\hline $\mathrm{SiO} 2$ & 77.14 & 72.91 & 75.87 & 62.52 & 62 & & \\
\hline $\mathrm{TiO} 2$ & 0.095 & 0.23 & 0.022 & 0.703 & 0.667 & & \\
\hline $\mathrm{A} 12 \mathrm{O} 3$ & 13.39 & 15.28 & 14.74 & 16.82 & 16.39 & & \\
\hline $\mathrm{FeO} *$ & 0.36 & 1.53 & 0.73 & 5.39 & 5.53 & & \\
\hline $\mathrm{MnO}$ & 0.013 & 0.029 & 0.033 & 0.098 & 0.104 & & \\
\hline $\mathrm{MgO}$ & 0.1 & 0.58 & 0.06 & 3.02 & 3.73 & & \\
\hline $\mathrm{CaO}$ & 1.09 & 2.71 & 1.23 & 5.7 & 6.42 & & \\
\hline $\mathrm{Na} 2 \mathrm{O}$ & 3.79 & 4.23 & 6.37 & 3.69 & 3.47 & & \\
\hline $\mathrm{K} 2 \mathrm{O}$ & 3.98 & 2.39 & 0.91 & 1.85 & 1.53 & & \\
\hline $\mathrm{P} 2 \mathrm{O} 5$ & 0.046 & 0.108 & 0.037 & 0.2 & 0.16 & & \\
\hline Total & 100 & 100 & 100 & 100 & 100 & & \\
\hline \multicolumn{8}{|l|}{$\begin{array}{l}\text { XRF } \\
\text { ppm }\end{array}$} \\
\hline $\mathrm{Ni}$ & 4 & 3 & 0 & 18 & 35 & & \\
\hline $\mathrm{Cr}$ & 5 & 4 & 2 & 40 & 73 & & \\
\hline $\mathrm{Sc}$ & 3 & 3 & 3 & 15 & 18 & & \\
\hline V & 8 & 13 & 3 & 135 & 147 & & \\
\hline $\mathrm{Ba}$ & 1203 & 1063 & 195 & 736 & 487 & & \\
\hline $\mathrm{Rb}$ & 78 & 41 & 26 & 46 & 40 & & \\
\hline $\mathrm{Sr}$ & 201 & 488 & 76 & 567 & 501 & & \\
\hline $\mathrm{Zr}$ & 54 & 105 & 51 & 101 & 112 & & \\
\hline $\mathrm{Y}$ & 13 & 10 & 20 & 16 & 17 & & \\
\hline $\mathrm{Nb}$ & 6.7 & 4.5 & 6.2 & 6.4 & 4.6 & & \\
\hline $\mathrm{Ga}$ & 14 & 16 & 17 & 19 & 17 & & \\
\hline
\end{tabular}




\begin{tabular}{|c|c|c|c|c|c|}
\hline $\mathrm{Cu}$ & 2 & 7 & 16 & 24 & 40 \\
\hline $\mathrm{Zn}$ & 5 & 27 & 11 & 69 & 70 \\
\hline $\mathrm{Pb}$ & 23 & 13 & 16 & 7 & 6 \\
\hline $\mathrm{La}$ & 23 & 11 & 16 & 16 & 11 \\
\hline $\mathrm{Ce}$ & 35 & 20 & 29 & 32 & 28 \\
\hline Th & 8 & 2 & 8 & 5 & 2 \\
\hline $\mathrm{Nd}$ & 11 & 9 & 12 & 15 & 16 \\
\hline $\mathrm{U}$ & 4 & 1 & 4 & 3 & 1 \\
\hline \multicolumn{6}{|c|}{$\begin{array}{c}\text { ICPMS } \\
\text { ppm }\end{array}$} \\
\hline $\mathrm{La}$ & 19.55 & 11.95 & 16.71 & 16.81 & 12.18 \\
\hline $\mathrm{Ce}$ & 33.85 & 20.6 & 32.4 & 32.93 & 26.17 \\
\hline $\operatorname{Pr}$ & 3.57 & 2.3 & 3.65 & 4.13 & 3.55 \\
\hline $\mathrm{Nd}$ & 11.88 & 7.89 & 12.96 & 16.61 & 15 \\
\hline $\mathrm{Sm}$ & 2.23 & 1.56 & 2.84 & 3.54 & 3.34 \\
\hline $\mathrm{Eu}$ & 0.46 & 0.7 & 0.16 & 1.07 & 0.96 \\
\hline $\mathrm{Gd}$ & 1.89 & 1.36 & 2.81 & 3.15 & 3.2 \\
\hline $\mathrm{Tb}$ & 0.32 & 0.23 & 0.52 & 0.48 & 0.51 \\
\hline Dy & 2.03 & 1.4 & 3.36 & 2.86 & 2.95 \\
\hline Ho & 0.41 & 0.29 & 0.68 & 0.57 & 0.6 \\
\hline $\mathrm{Er}$ & 1.18 & 0.85 & 1.88 & 1.58 & 1.64 \\
\hline $\mathrm{Tm}$ & 0.19 & 0.13 & 0.28 & 0.22 & 0.23 \\
\hline $\mathrm{Yb}$ & 1.21 & 0.92 & 1.81 & 1.4 & 1.48 \\
\hline $\mathrm{Lu}$ & 0.19 & 0.15 & 0.27 & 0.23 & 0.23 \\
\hline $\mathrm{Ba}$ & 1235 & 1083 & 200 & 734 & 483 \\
\hline Th & 8.87 & 2.72 & 6.17 & 3.78 & 2.71 \\
\hline $\mathrm{Nb}$ & 6.27 & 4.29 & 5.78 & 5.68 & 4.02 \\
\hline $\mathrm{Y}$ & 12.37 & 8.55 & 19.69 & 14.99 & 15.73 \\
\hline $\mathrm{Hf}$ & 1.93 & 2.66 & 2.39 & 2.69 & 2.98 \\
\hline $\mathrm{Ta}$ & 0.94 & 0.37 & 0.96 & 0.47 & 0.3 \\
\hline $\mathrm{U}$ & 2.94 & 1.74 & 3.45 & 1.47 & 0.91 \\
\hline $\mathrm{Pb}$ & 20.61 & 12.06 & 16.7 & 6.63 & 5.19 \\
\hline $\mathrm{Rb}$ & 79.9 & 40.9 & 26.8 & 43.7 & 38.2 \\
\hline Cs & 2.31 & 2.09 & 0.43 & 3.8 & 1.74 \\
\hline $\mathrm{Sr}$ & 215 & 506 & 79 & 574 & 503 \\
\hline $\mathrm{Sc}$ & 2 & 1.8 & 2.2 & 14.9 & 16.3 \\
\hline $\mathrm{Zr}$ & 58 & 108 & 55 & 99 & 109 \\
\hline
\end{tabular}


NOTES

\section{APPENDIX C: EXPERIMENTAL MELTS}

The label "magnetite" on EDS phase maps is used generally to refer to FeTi oxides.

Figure C2: Due to the limitations of the AZtec software, some of the phase which is labeled as "clinopyroxene" likely should belong to the "melt" phase. This discrepancy can be seen by comparing the EDS map with the BSE image, i.e. the darker shade of grey surrounding the lighter phenocrysts of clinopyroxene.

Figure C11: Due to the limitations of the AZtec software, some of the phase which is labeled as "melt" likely is biotite. This is based on the reflectivity of the BSE image and compositional analysis of this phase.

Figure C47: The BSE image and EDS map do not correspond, however, these are representative of the same sample (MS65). This BSE image was selected because it was the clearest and most representative of all those obtained. 

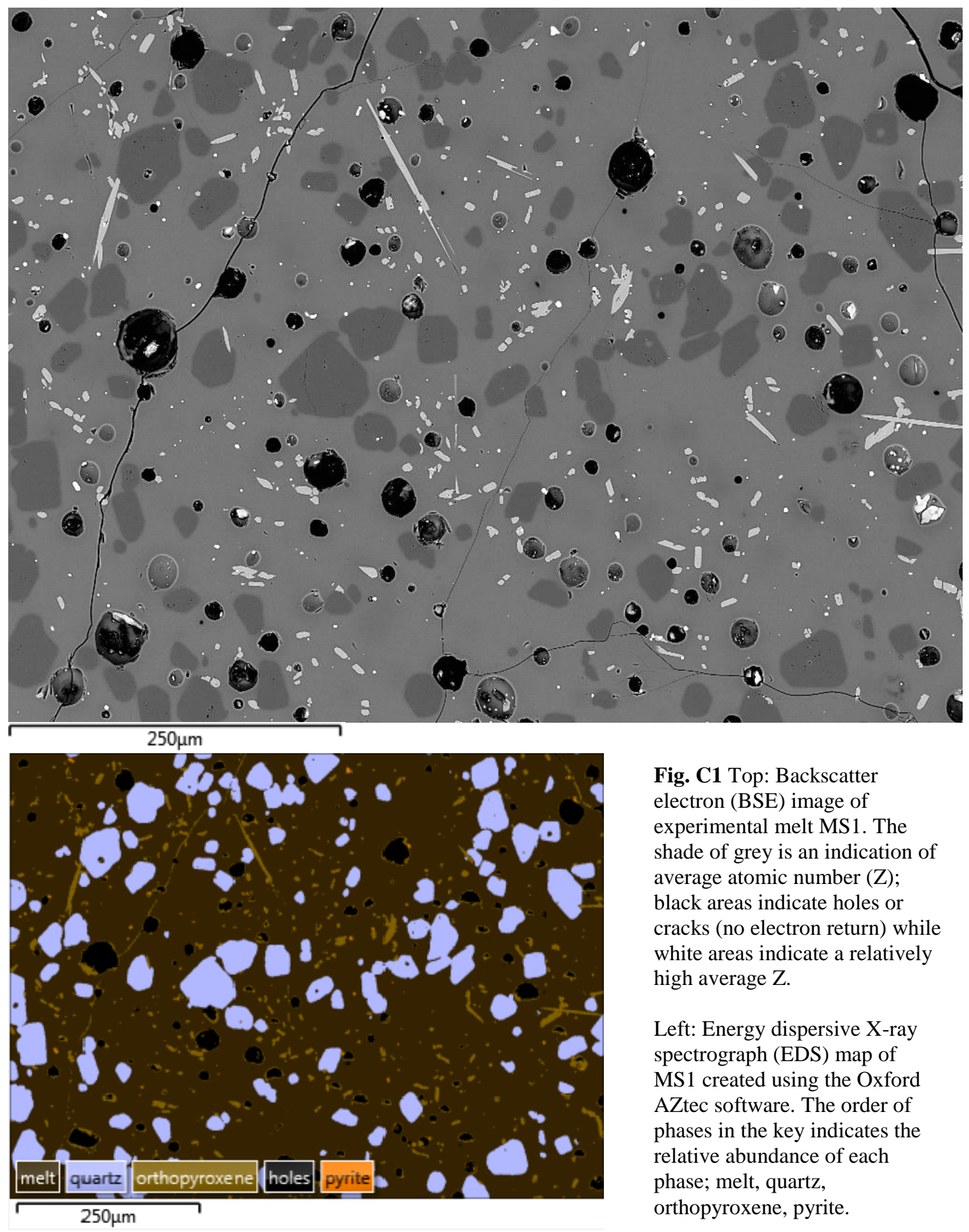

Fig. C1 Top: Backscatter electron (BSE) image of experimental melt MS1. The shade of grey is an indication of average atomic number $(\mathrm{Z})$; black areas indicate holes or cracks (no electron return) while white areas indicate a relatively high average $Z$.

Left: Energy dispersive X-ray spectrograph (EDS) map of MS1 created using the Oxford AZtec software. The order of phases in the key indicates the relative abundance of each phase; melt, quartz, orthopyroxene, pyrite. 

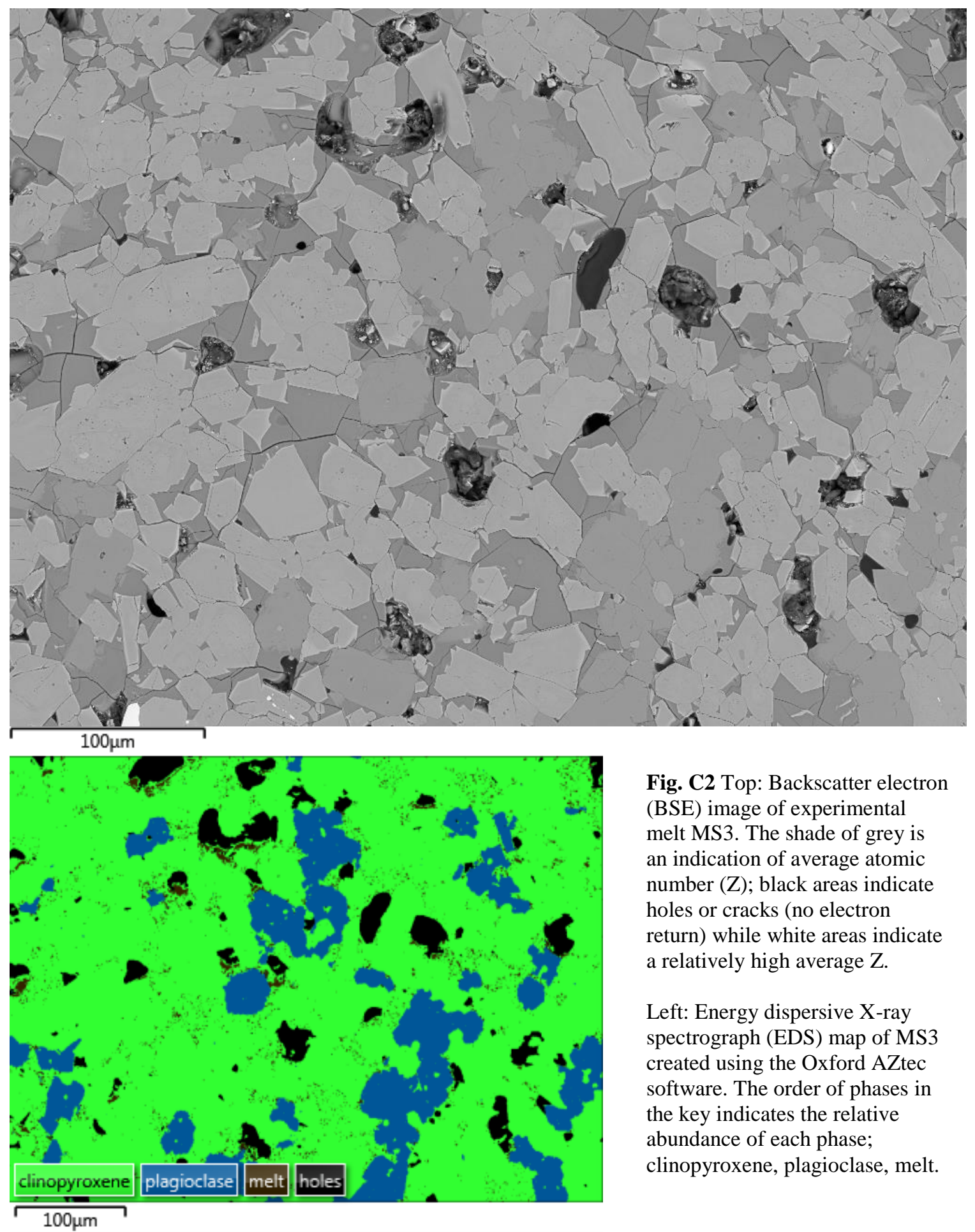

Fig. C2 Top: Backscatter electron (BSE) image of experimental melt MS3. The shade of grey is an indication of average atomic number (Z); black areas indicate holes or cracks (no electron return) while white areas indicate a relatively high average $\mathrm{Z}$.

Left: Energy dispersive X-ray spectrograph (EDS) map of MS3 created using the Oxford AZtec software. The order of phases in the key indicates the relative abundance of each phase; clinopyroxene, plagioclase, melt. 

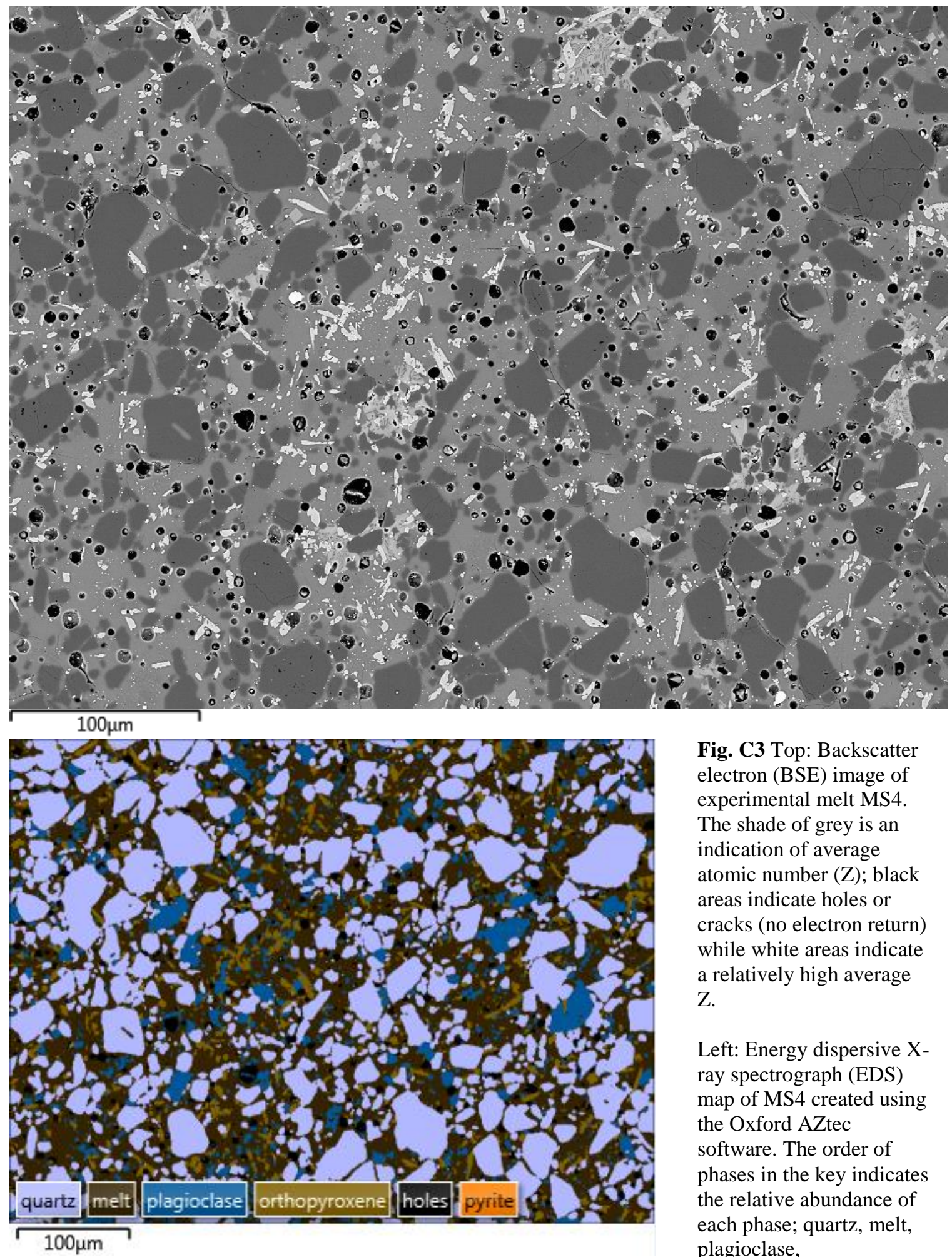

Fig. C3 Top: Backscatter electron (BSE) image of experimental melt MS4. The shade of grey is an indication of average atomic number (Z); black areas indicate holes or cracks (no electron return) while white areas indicate a relatively high average $\mathrm{Z}$.

Left: Energy dispersive Xray spectrograph (EDS) map of MS4 created using the Oxford AZtec software. The order of phases in the key indicates the relative abundance of each phase; quartz, melt, plagioclase, orthopyroxene, pyrite. 

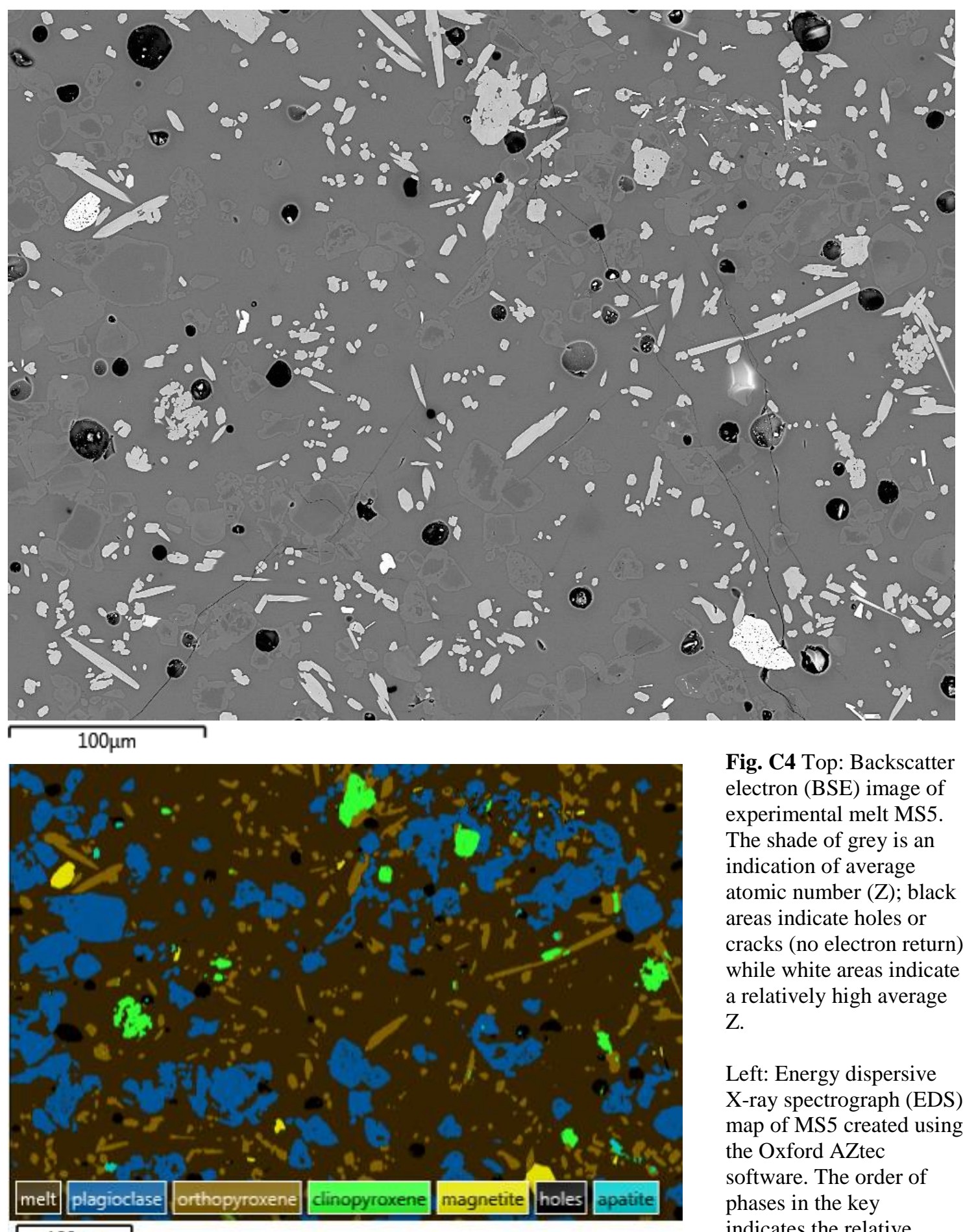

Fig. C4 Top: Backscatter electron (BSE) image of experimental melt MS5. The shade of grey is an indication of average atomic number (Z); black areas indicate holes or cracks (no electron return) while white areas indicate a relatively high average $\mathrm{Z}$.

Left: Energy dispersive X-ray spectrograph (EDS) map of MS5 created using the Oxford AZtec software. The order of phases in the key indicates the relative abundance of each phase; melt, plagioclase, orthopyroxene, clinopyroxene, FeTi oxides, apatite. 

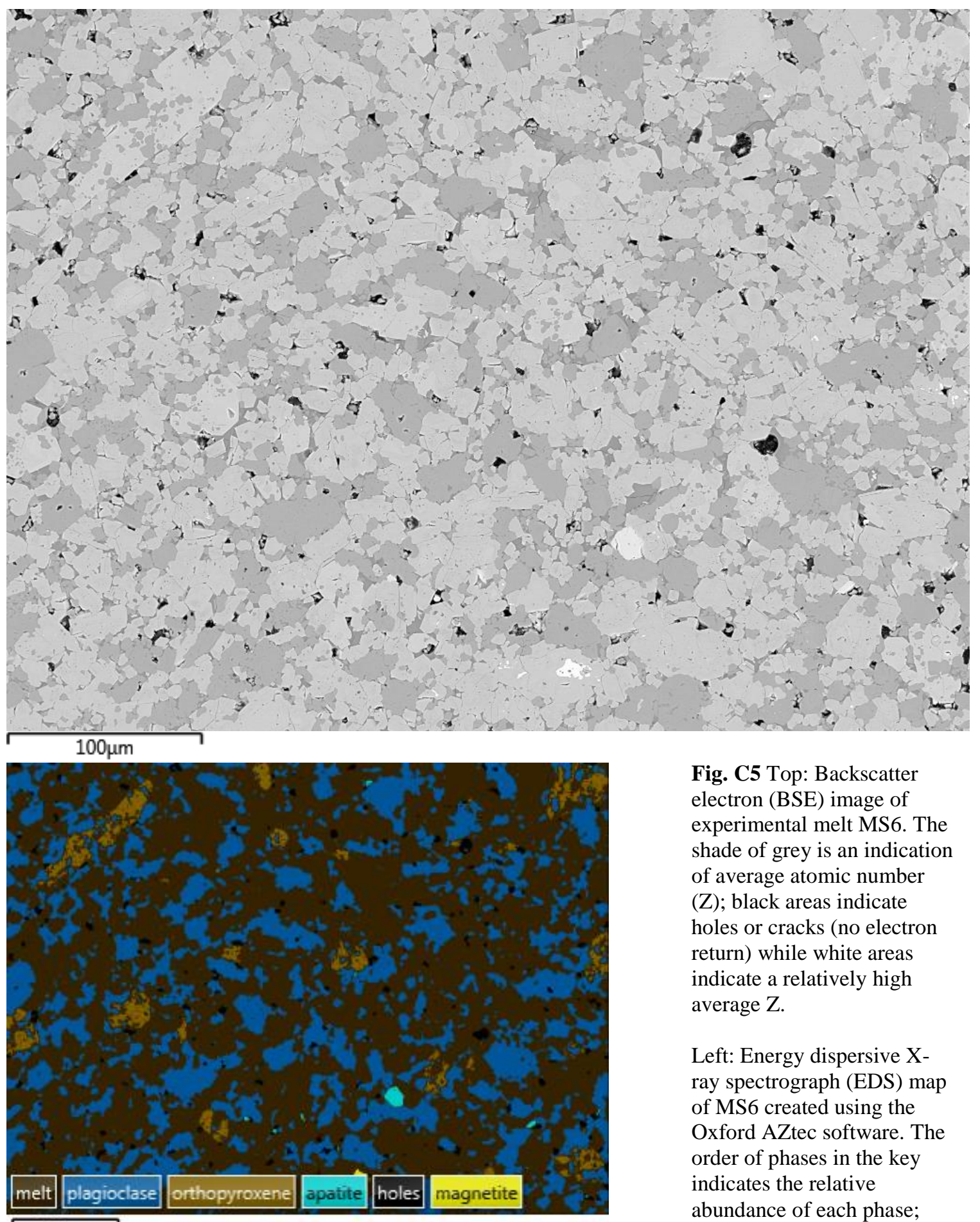

Fig. C5 Top: Backscatter electron (BSE) image of experimental melt MS6. The shade of grey is an indication of average atomic number (Z); black areas indicate holes or cracks (no electron return) while white areas indicate a relatively high average $\mathrm{Z}$.

Left: Energy dispersive Xray spectrograph (EDS) map of MS6 created using the Oxford AZtec software. The order of phases in the key indicates the relative abundance of each phase; melt, plagioclase, orthopyroxene, apatite, FeTi oxides. 

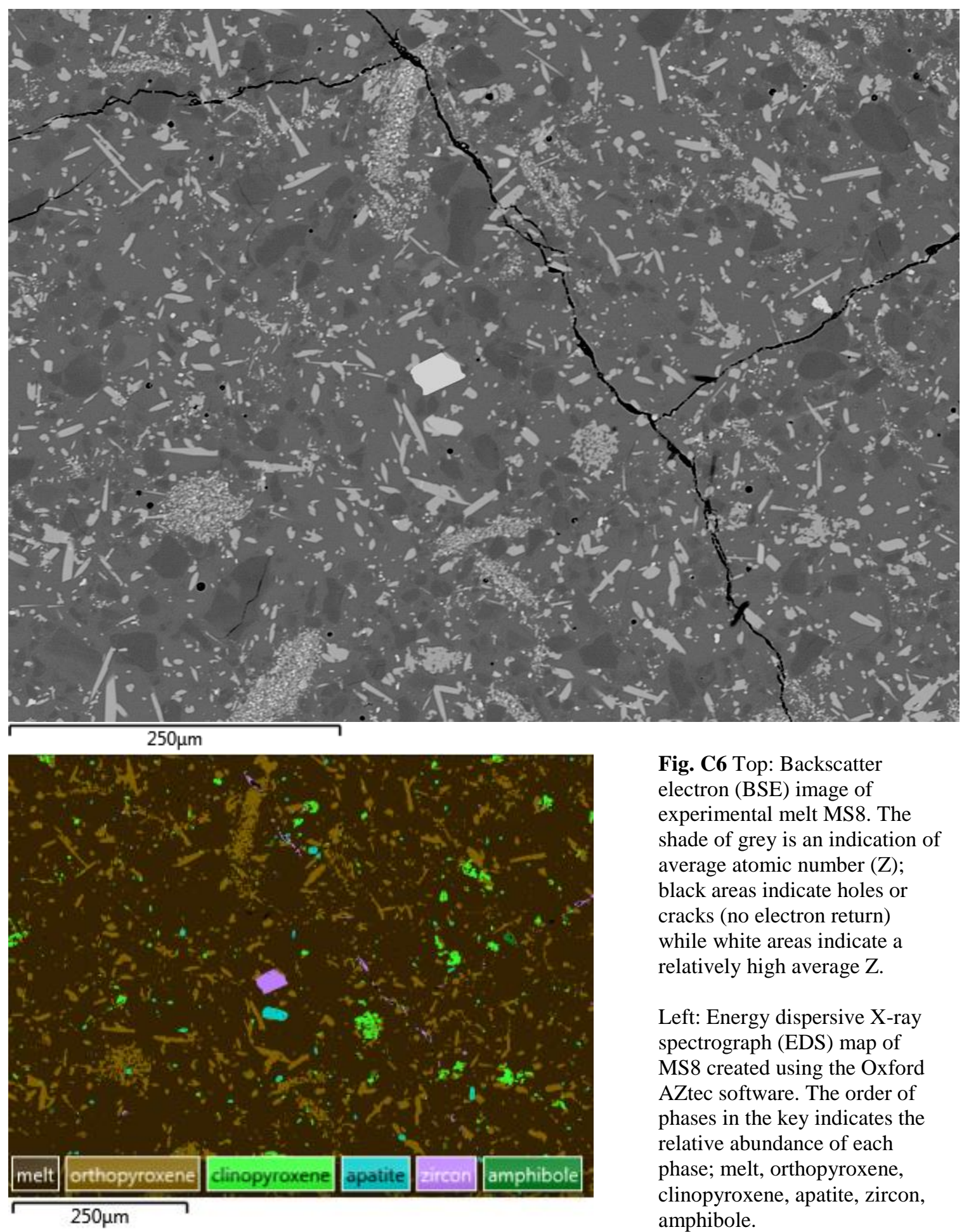

Fig. C6 Top: Backscatter electron (BSE) image of experimental melt MS8. The shade of grey is an indication of average atomic number $(\mathrm{Z})$; black areas indicate holes or cracks (no electron return) while white areas indicate a relatively high average $\mathrm{Z}$.

Left: Energy dispersive X-ray spectrograph (EDS) map of MS8 created using the Oxford AZtec software. The order of phases in the key indicates the relative abundance of each phase; melt, orthopyroxene, clinopyroxene, apatite, zircon, amphibole. 

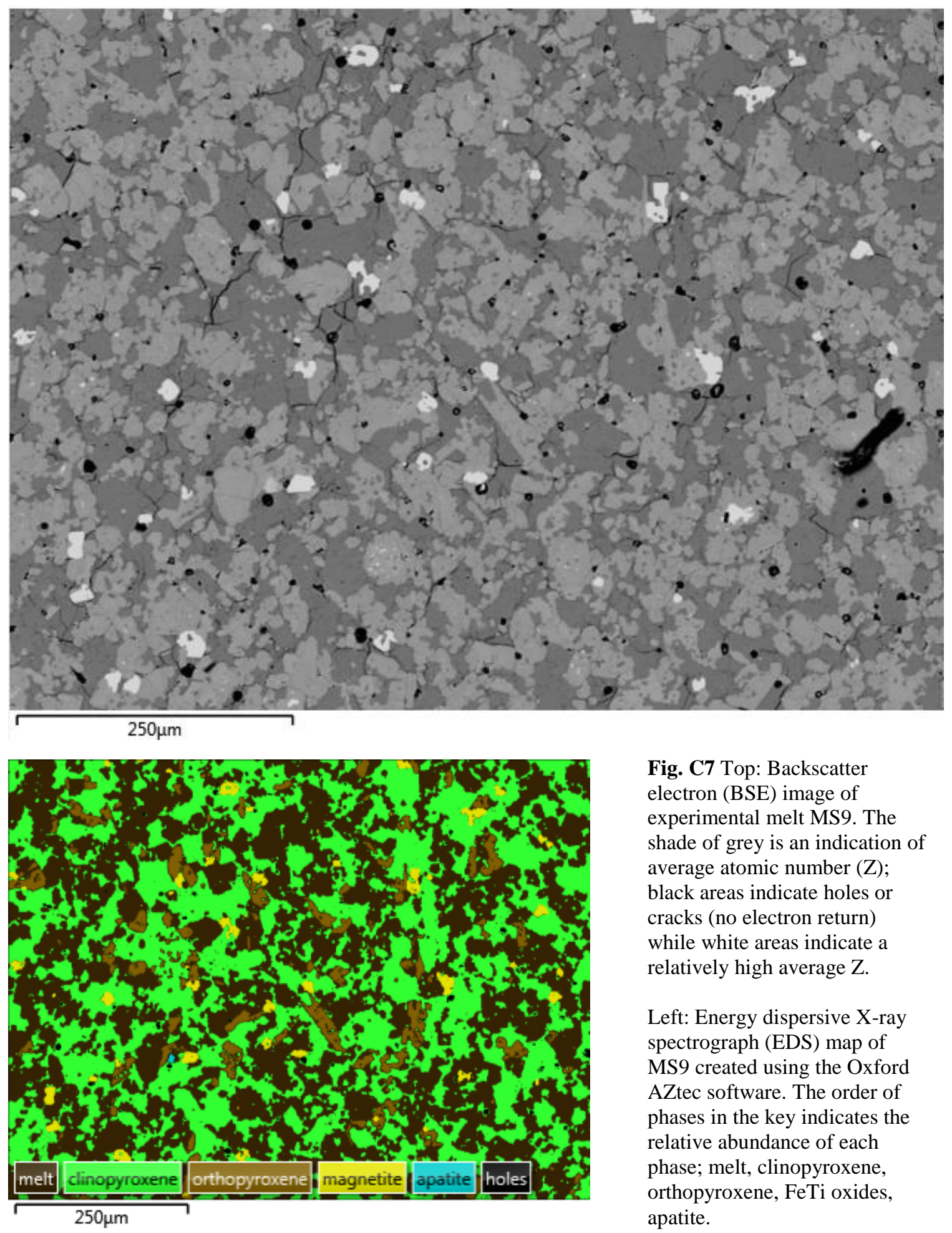

Fig. C7 Top: Backscatter electron (BSE) image of experimental melt MS9. The shade of grey is an indication of average atomic number $(\mathrm{Z})$; black areas indicate holes or cracks (no electron return) while white areas indicate a relatively high average $\mathrm{Z}$.

Left: Energy dispersive X-ray spectrograph (EDS) map of MS9 created using the Oxford AZtec software. The order of phases in the key indicates the relative abundance of each phase; melt, clinopyroxene, orthopyroxene, FeTi oxides, apatite. 

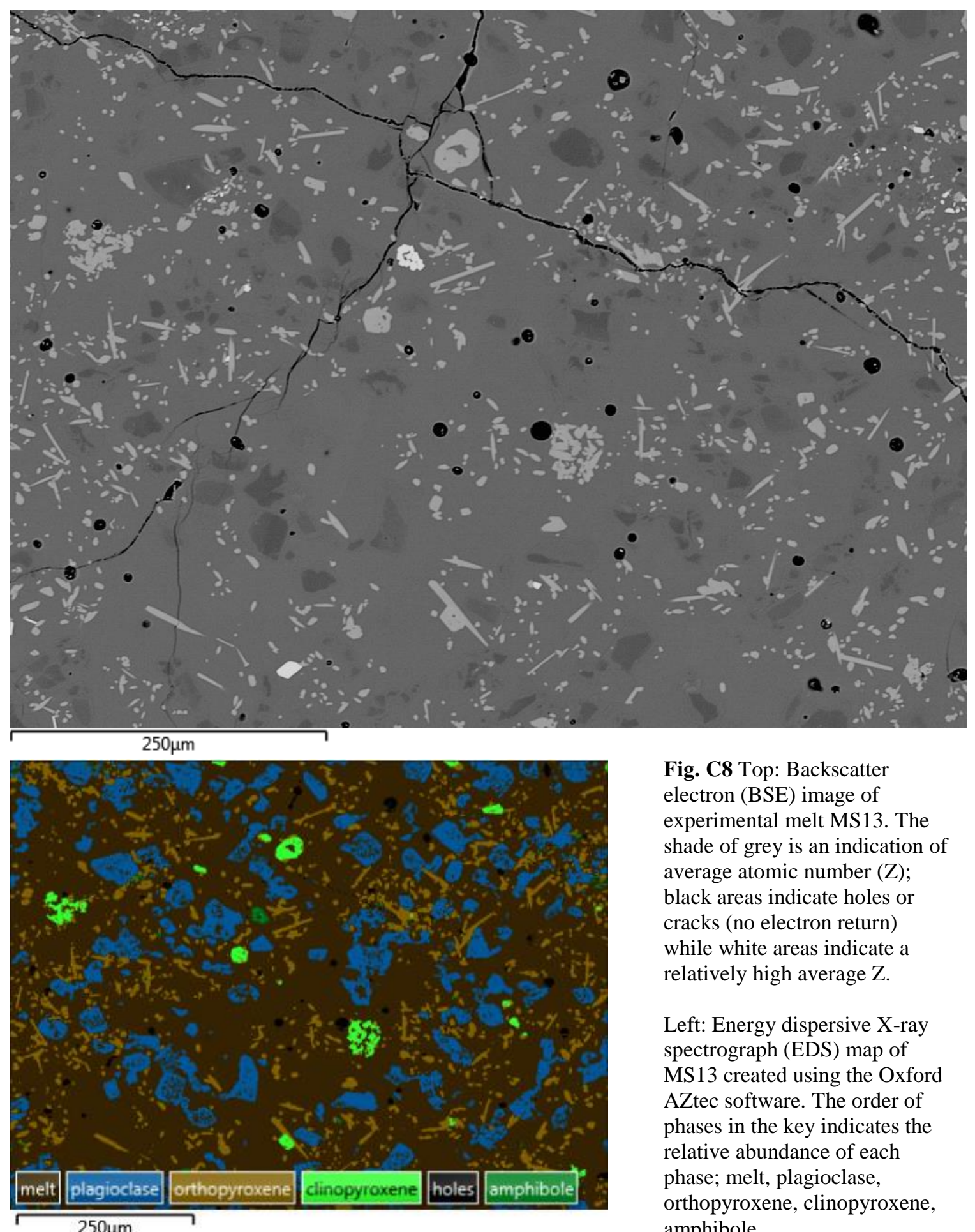

Fig. C8 Top: Backscatter electron (BSE) image of experimental melt MS13. The shade of grey is an indication of average atomic number (Z); black areas indicate holes or cracks (no electron return) while white areas indicate a relatively high average $\mathrm{Z}$.

Left: Energy dispersive X-ray spectrograph (EDS) map of MS13 created using the Oxford AZtec software. The order of phases in the key indicates the relative abundance of each phase; melt, plagioclase, orthopyroxene, clinopyroxene, amphibole. 


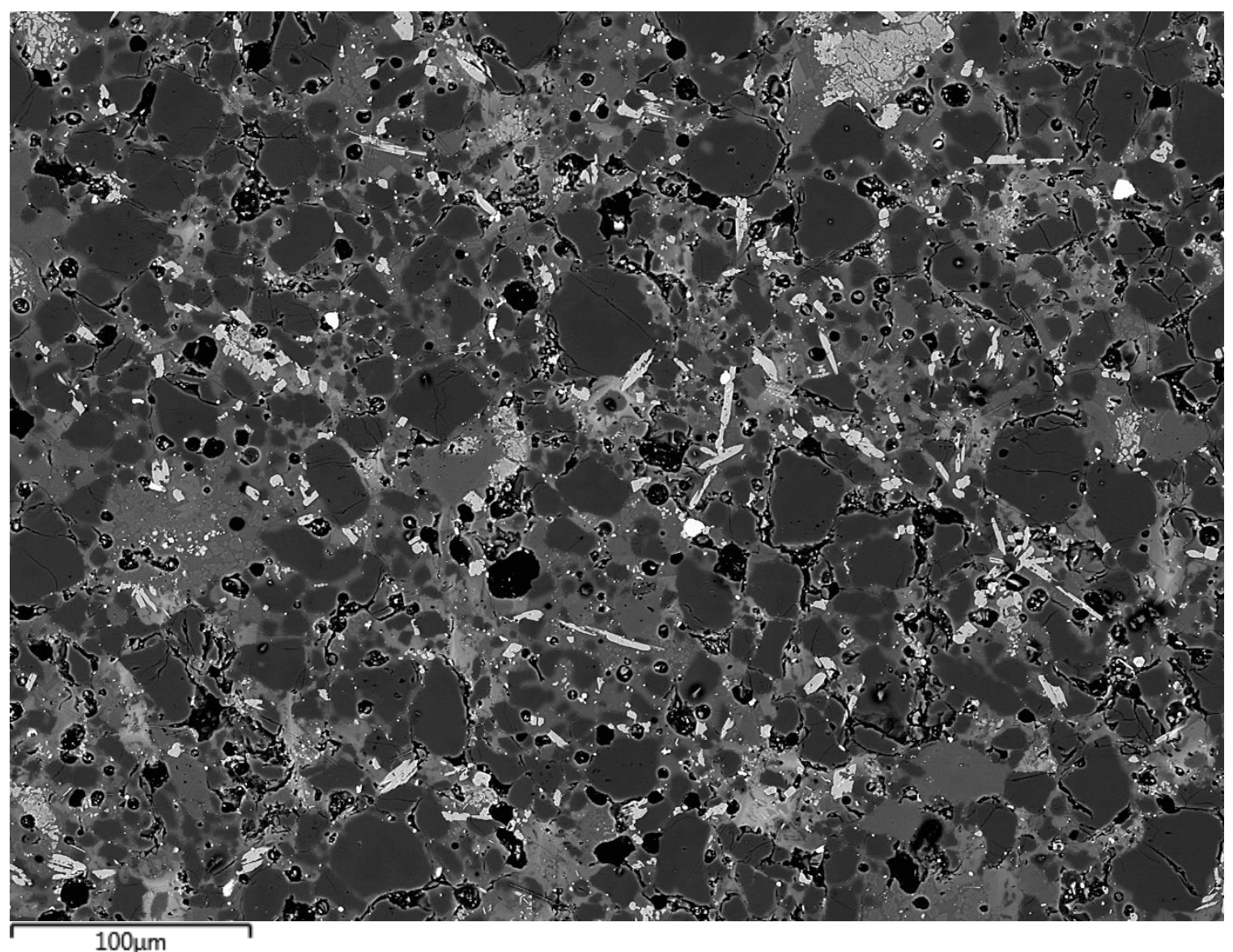

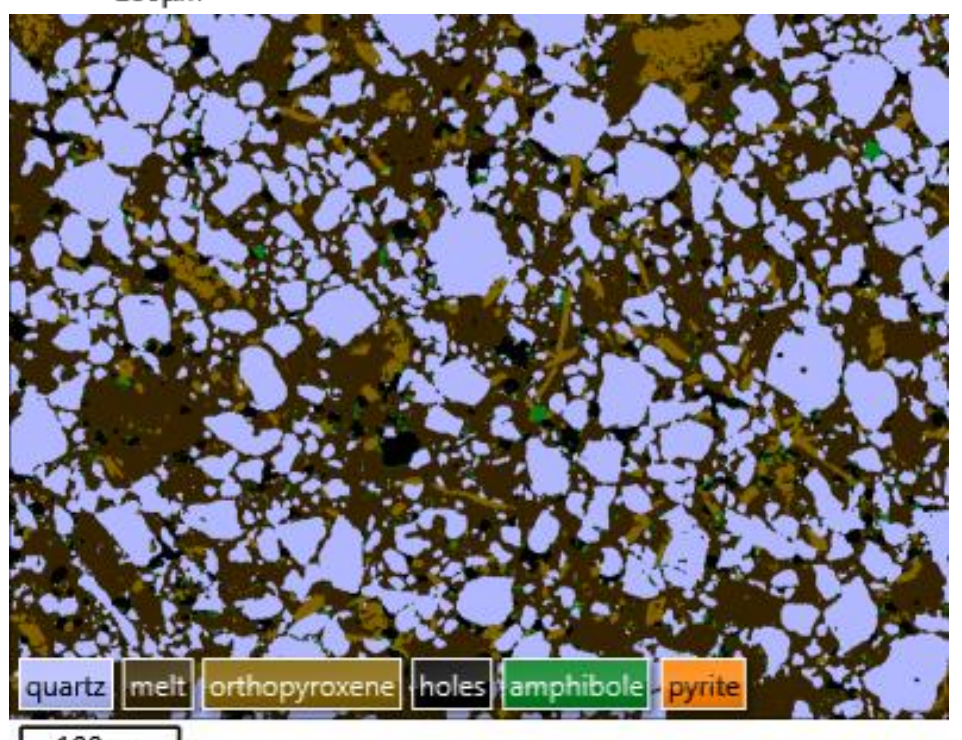

$100 \mu \mathrm{m}$
Fig. C9 Top: Backscatter electron (BSE) image of experimental melt MS15. The shade of grey is an indication of average atomic number $(\mathrm{Z})$; black areas indicate holes or cracks (no electron return) while white areas indicate a relatively high average $Z$.

Left: Energy dispersive X-ray spectrograph (EDS) map of MS15 created using the Oxford AZtec software. The order of phases in the key indicates the relative abundance of each phase; quartz, melt, orthopyroxene, FeTi oxides, pyrite. 

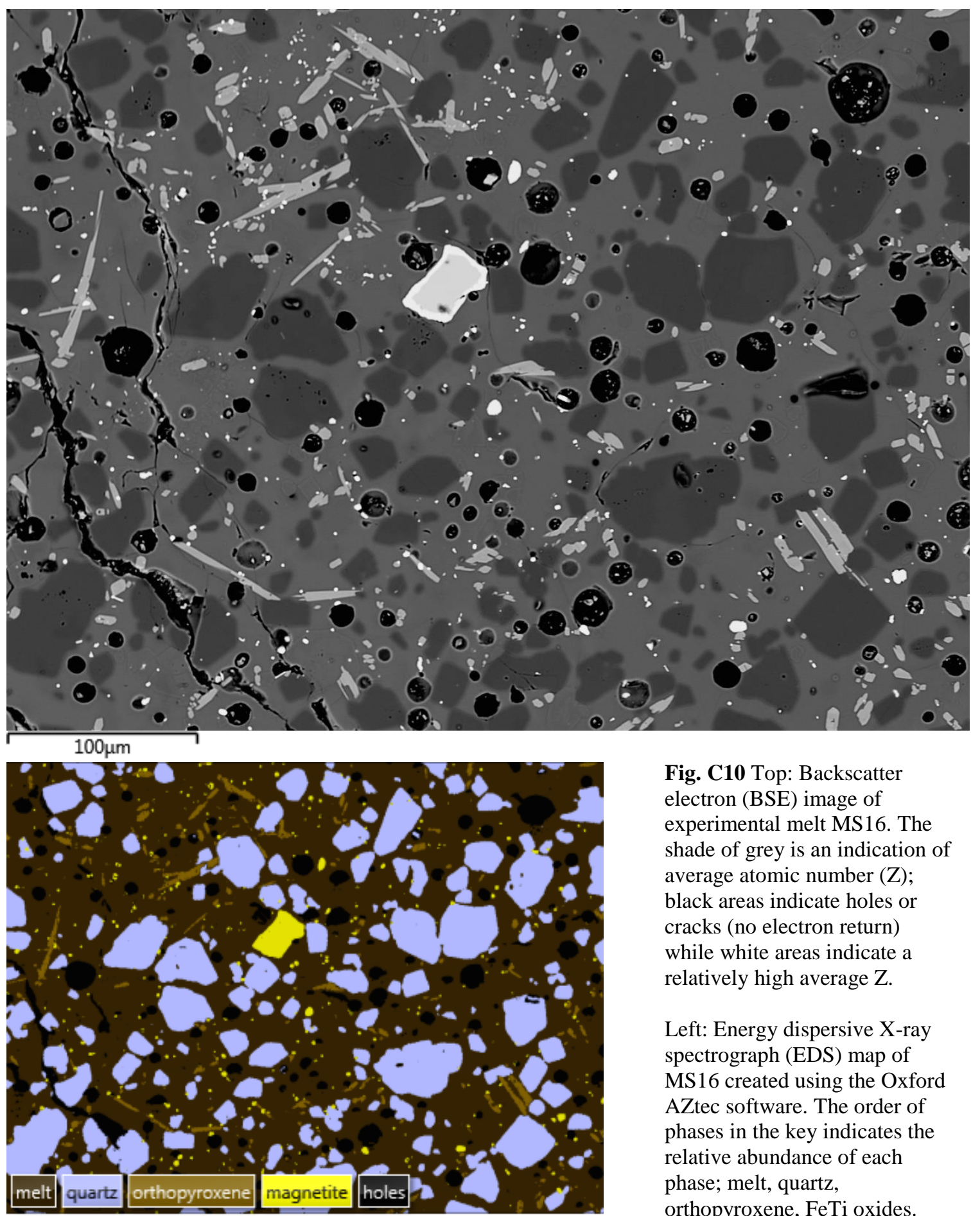

Fig. C10 Top: Backscatter electron (BSE) image of experimental melt MS16. The shade of grey is an indication of average atomic number $(\mathrm{Z})$; black areas indicate holes or cracks (no electron return) while white areas indicate a relatively high average $\mathrm{Z}$.

Left: Energy dispersive X-ray spectrograph (EDS) map of MS16 created using the Oxford AZtec software. The order of phases in the key indicates the relative abundance of each phase; melt, quartz, orthopyroxene, FeTi oxides. 

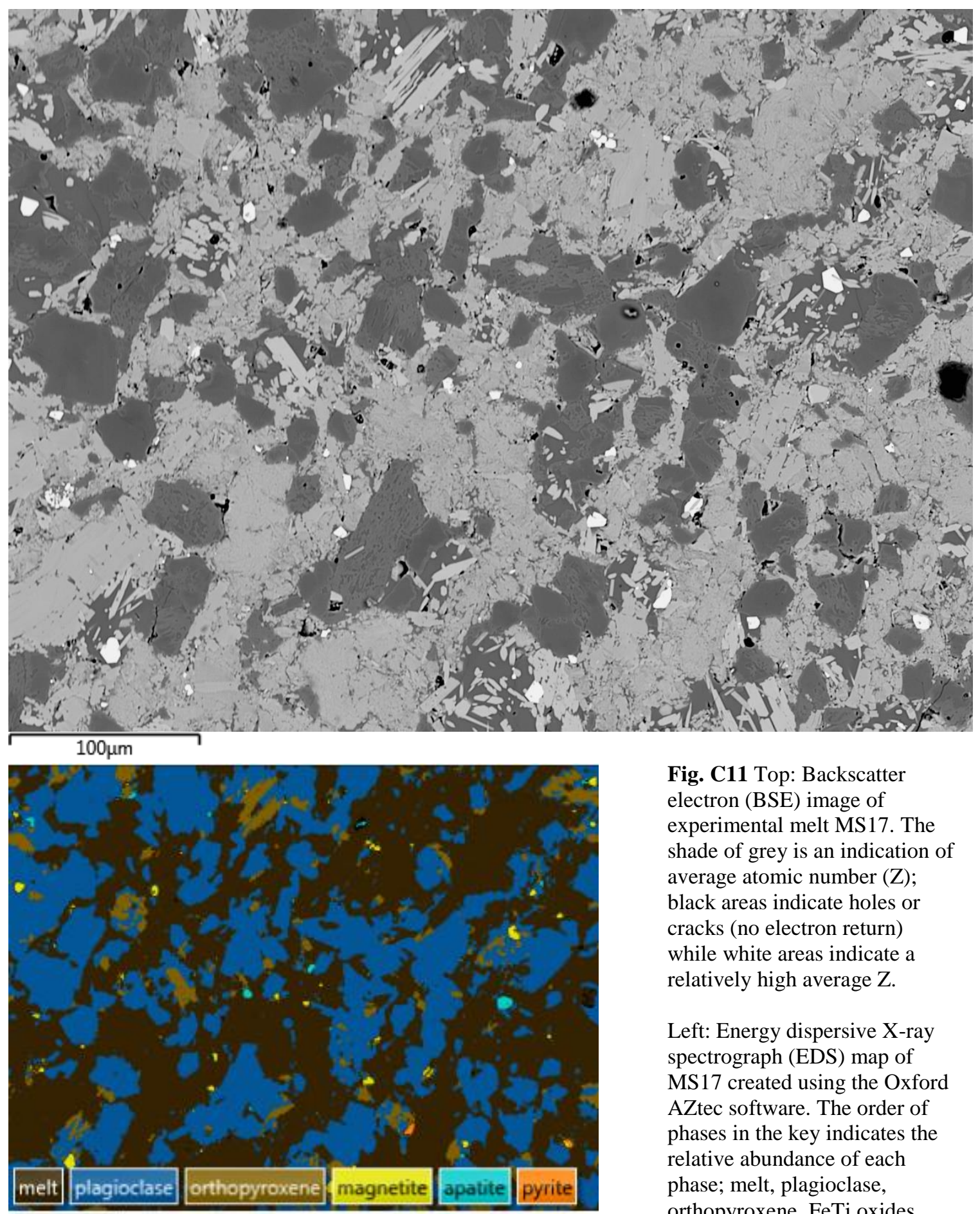

Fig. C11 Top: Backscatter electron (BSE) image of experimental melt MS17. The shade of grey is an indication of average atomic number (Z); black areas indicate holes or cracks (no electron return) while white areas indicate a relatively high average $\mathrm{Z}$.

Left: Energy dispersive X-ray spectrograph (EDS) map of MS17 created using the Oxford AZtec software. The order of phases in the key indicates the relative abundance of each phase; melt, plagioclase, orthopyroxene, FeTi oxides, apatite, pyrite. 

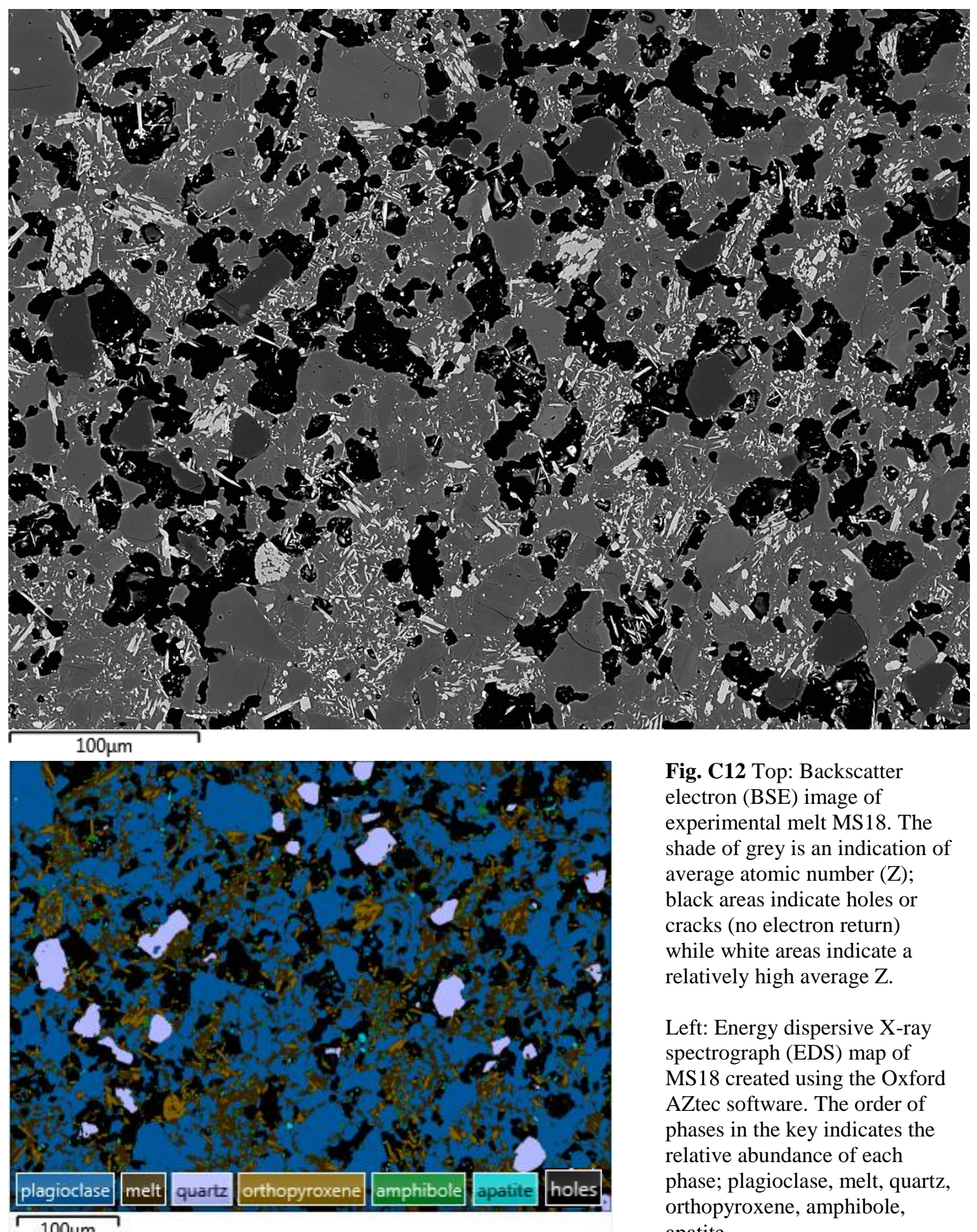

Fig. C12 Top: Backscatter electron (BSE) image of experimental melt MS18. The shade of grey is an indication of average atomic number $(\mathrm{Z})$; black areas indicate holes or cracks (no electron return) while white areas indicate a relatively high average $\mathrm{Z}$.

Left: Energy dispersive X-ray spectrograph (EDS) map of MS18 created using the Oxford AZtec software. The order of phases in the key indicates the relative abundance of each phase; plagioclase, melt, quartz, orthopyroxene, amphibole, apatite. 

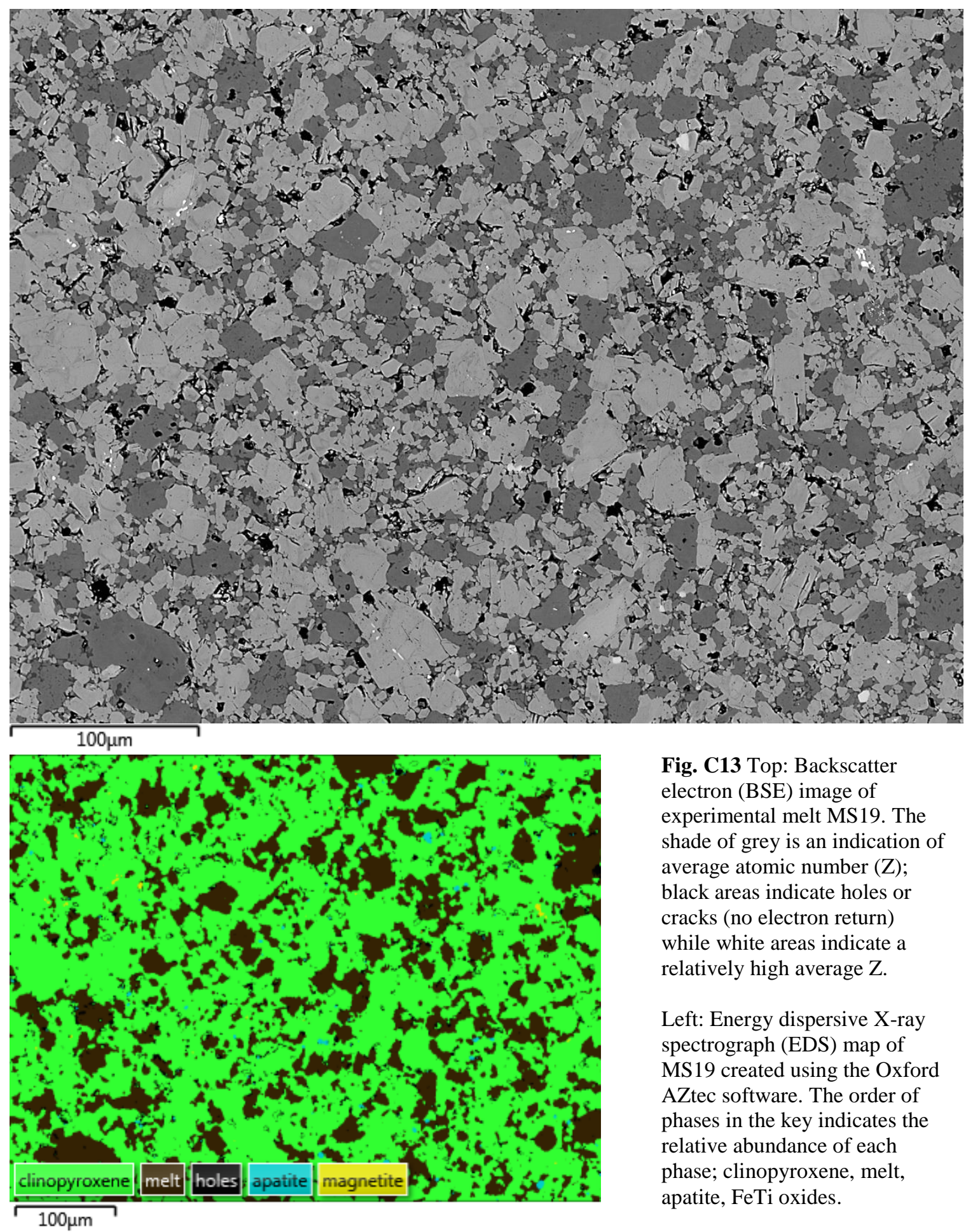

Fig. C13 Top: Backscatter electron (BSE) image of experimental melt MS19. The shade of grey is an indication of average atomic number $(\mathrm{Z})$; black areas indicate holes or cracks (no electron return) while white areas indicate a relatively high average $\mathrm{Z}$.

Left: Energy dispersive X-ray spectrograph (EDS) map of MS19 created using the Oxford AZtec software. The order of phases in the key indicates the relative abundance of each phase; clinopyroxene, melt, apatite, FeTi oxides. 

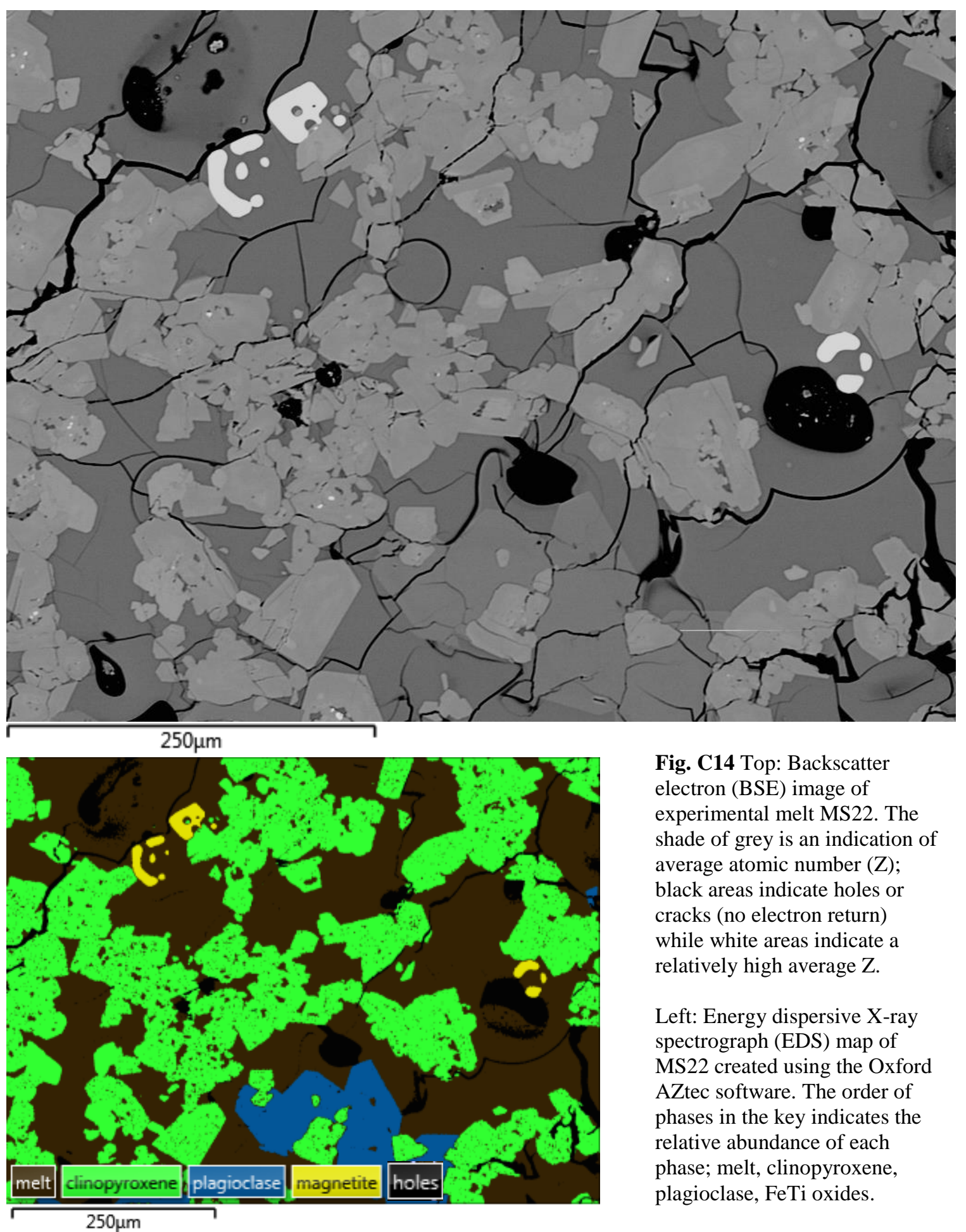

Fig. C14 Top: Backscatter electron (BSE) image of experimental melt MS22. The shade of grey is an indication of average atomic number $(\mathrm{Z})$; black areas indicate holes or cracks (no electron return) while white areas indicate a relatively high average $\mathrm{Z}$.

Left: Energy dispersive X-ray spectrograph (EDS) map of MS22 created using the Oxford AZtec software. The order of phases in the key indicates the relative abundance of each phase; melt, clinopyroxene, plagioclase, FeTi oxides. 

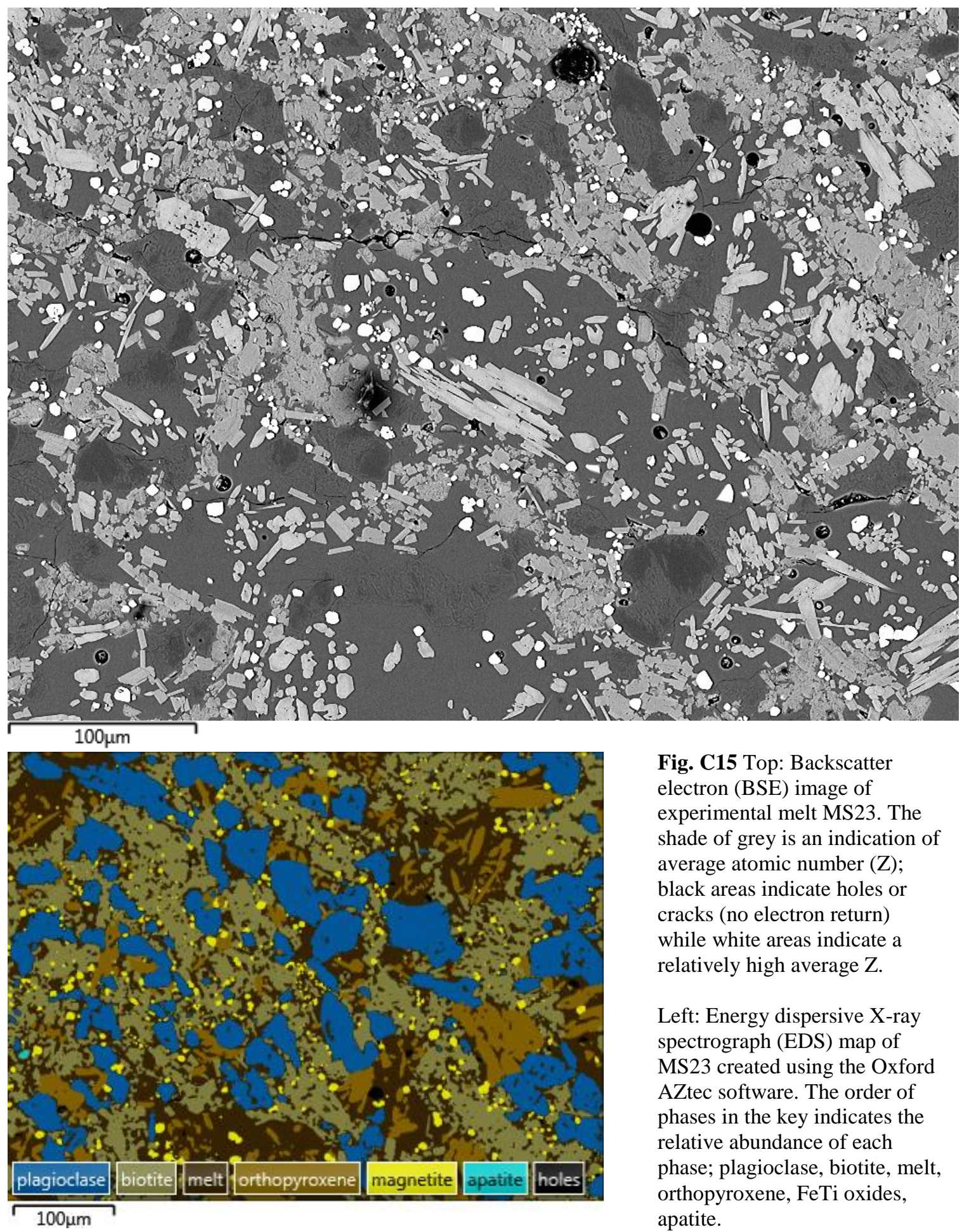

Fig. C15 Top: Backscatter electron (BSE) image of experimental melt MS23. The shade of grey is an indication of average atomic number (Z); black areas indicate holes or cracks (no electron return) while white areas indicate a relatively high average $\mathrm{Z}$.

Left: Energy dispersive X-ray spectrograph (EDS) map of MS23 created using the Oxford AZtec software. The order of phases in the key indicates the relative abundance of each phase; plagioclase, biotite, melt, orthopyroxene, FeTi oxides, apatite. 

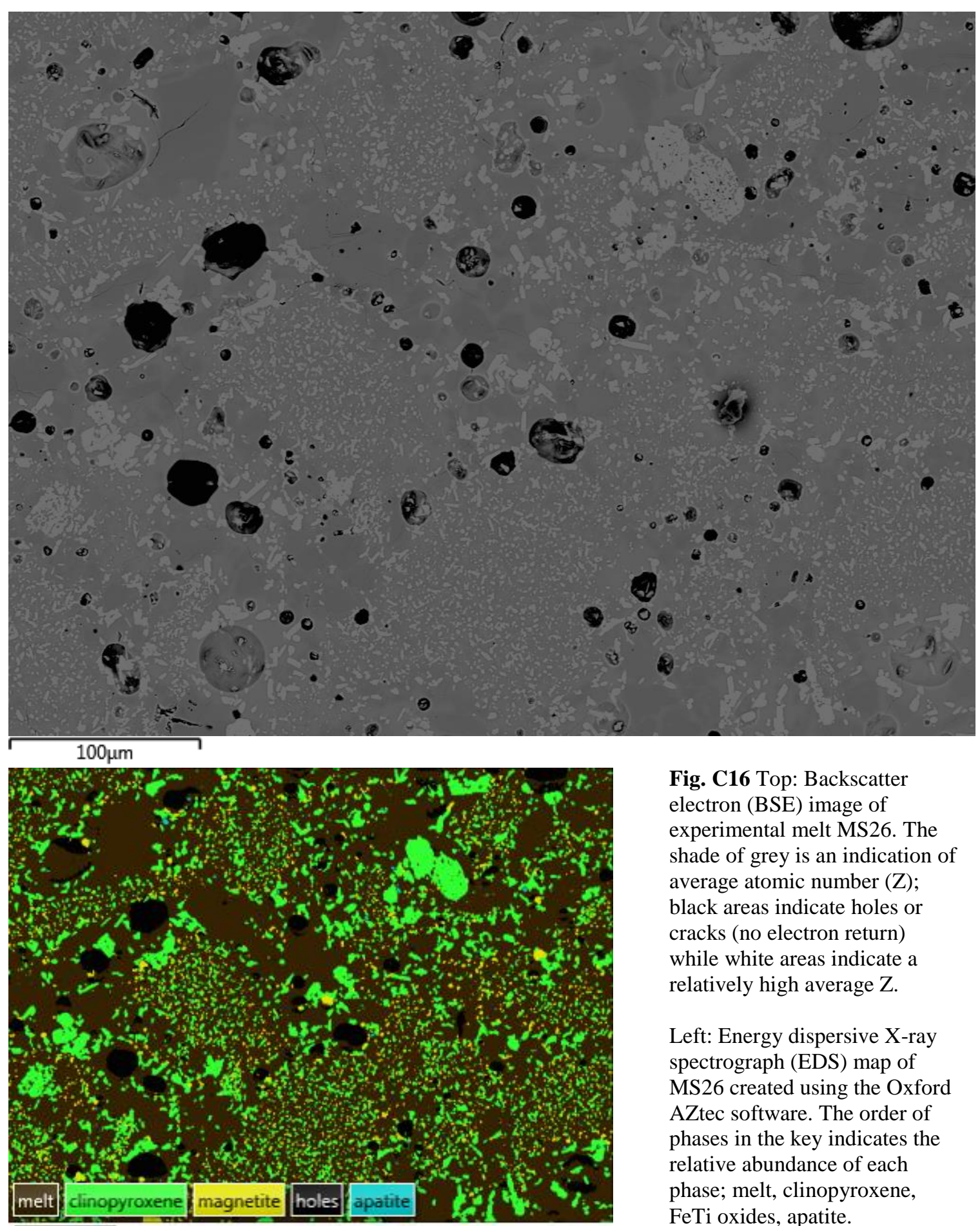

$100 \mu \mathrm{m}$

Fig. C16 Top: Backscatter electron (BSE) image of experimental melt MS26. The shade of grey is an indication of average atomic number $(\mathrm{Z})$; black areas indicate holes or cracks (no electron return) while white areas indicate a relatively high average $\mathrm{Z}$.

Left: Energy dispersive X-ray spectrograph (EDS) map of MS26 created using the Oxford AZtec software. The order of phases in the key indicates the relative abundance of each phase; melt, clinopyroxene, FeTi oxides, apatite. 

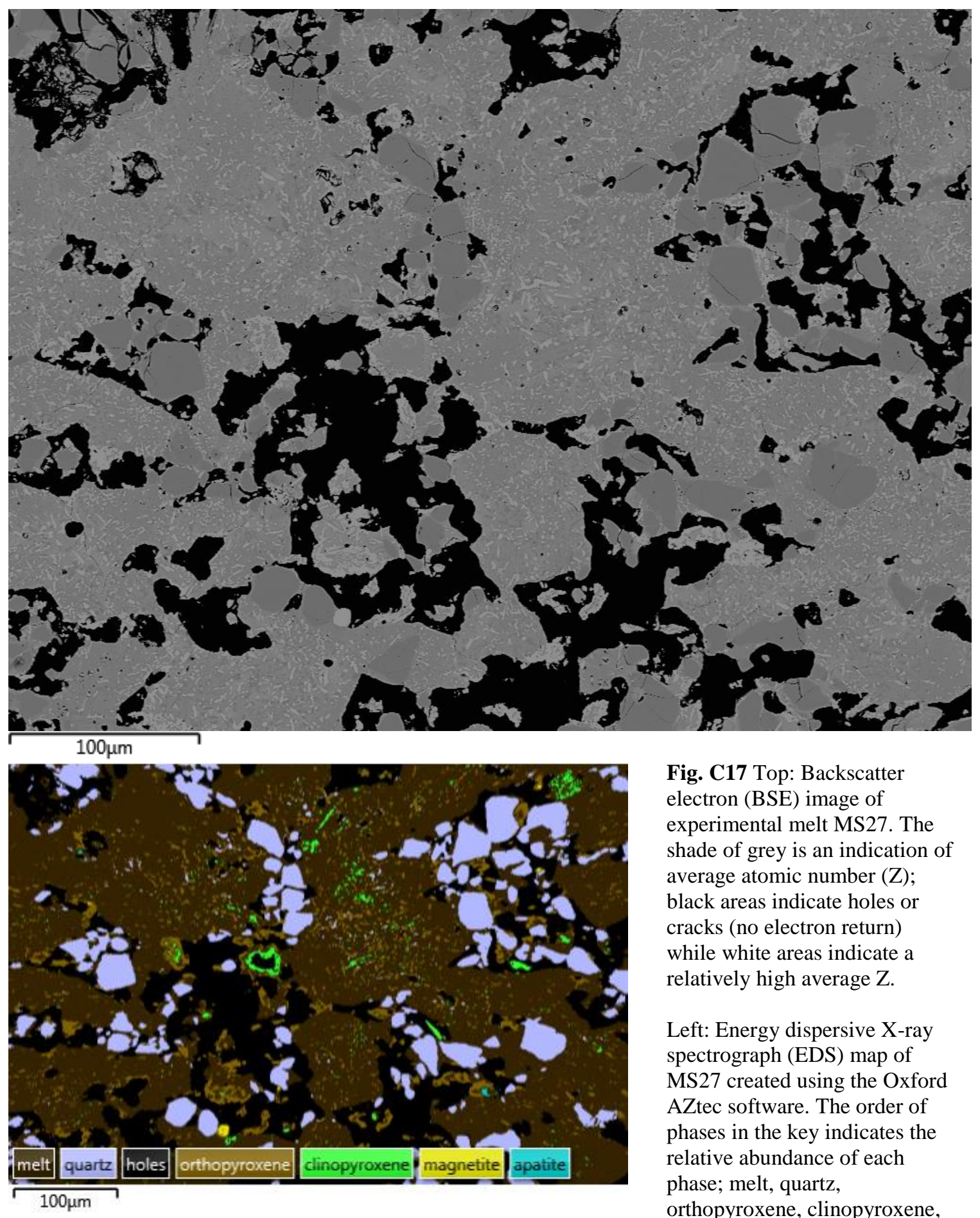

Fig. C17 Top: Backscatter electron (BSE) image of experimental melt MS27. The shade of grey is an indication of average atomic number $(\mathrm{Z})$; black areas indicate holes or cracks (no electron return) while white areas indicate a relatively high average $\mathrm{Z}$.

Left: Energy dispersive X-ray spectrograph (EDS) map of MS27 created using the Oxford AZtec software. The order of phases in the key indicates the relative abundance of each phase; melt, quartz, orthopyroxene, clinopyroxene, FeTi oxides, apatite. 

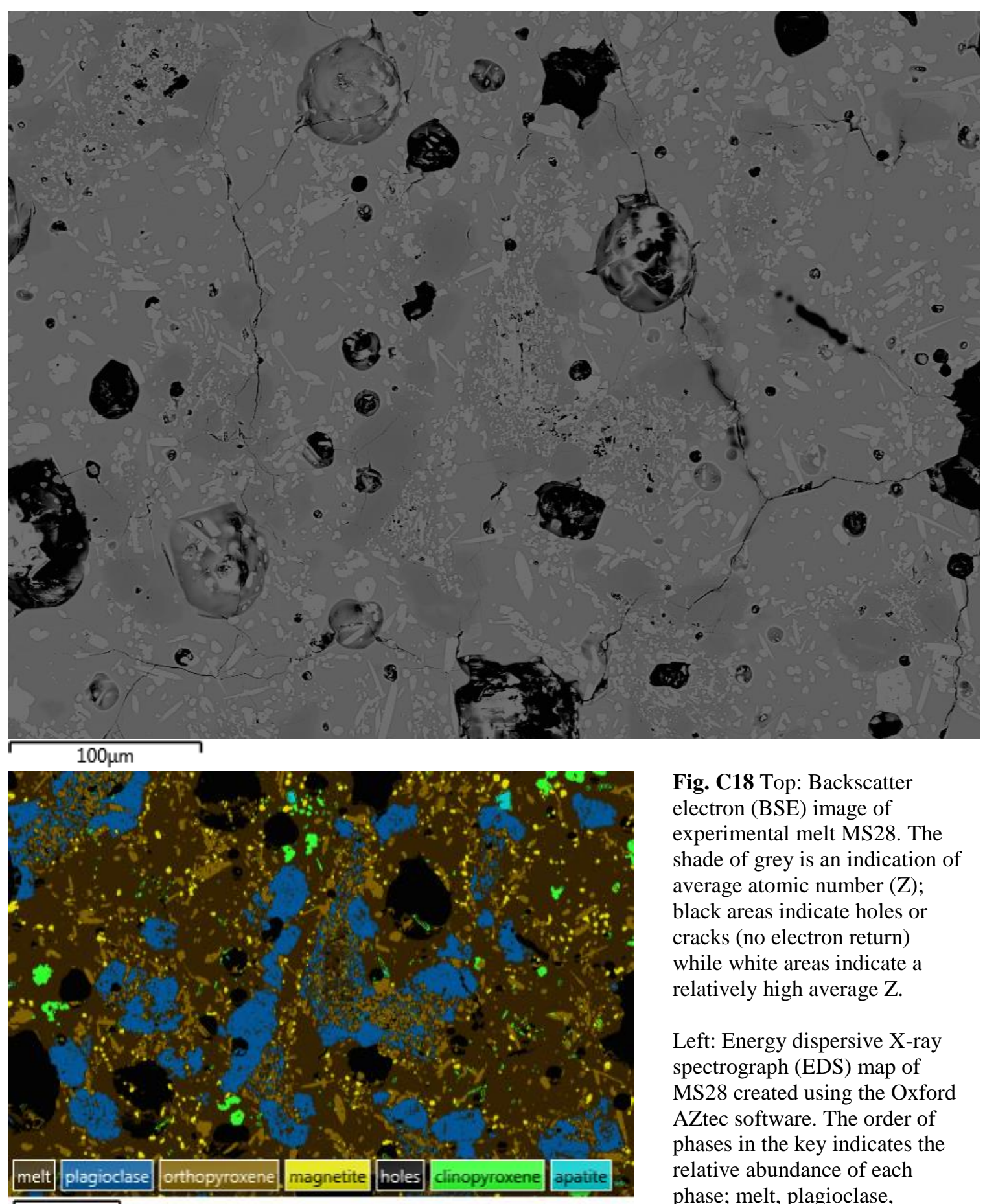

Fig. C18 Top: Backscatter electron (BSE) image of experimental melt MS28. The shade of grey is an indication of average atomic number (Z); black areas indicate holes or cracks (no electron return) while white areas indicate a relatively high average $\mathrm{Z}$.

Left: Energy dispersive X-ray spectrograph (EDS) map of MS28 created using the Oxford AZtec software. The order of phases in the key indicates the relative abundance of each phase; melt, plagioclase, orthopyroxene, FeTi oxides, clinopyroxene, apatite. 

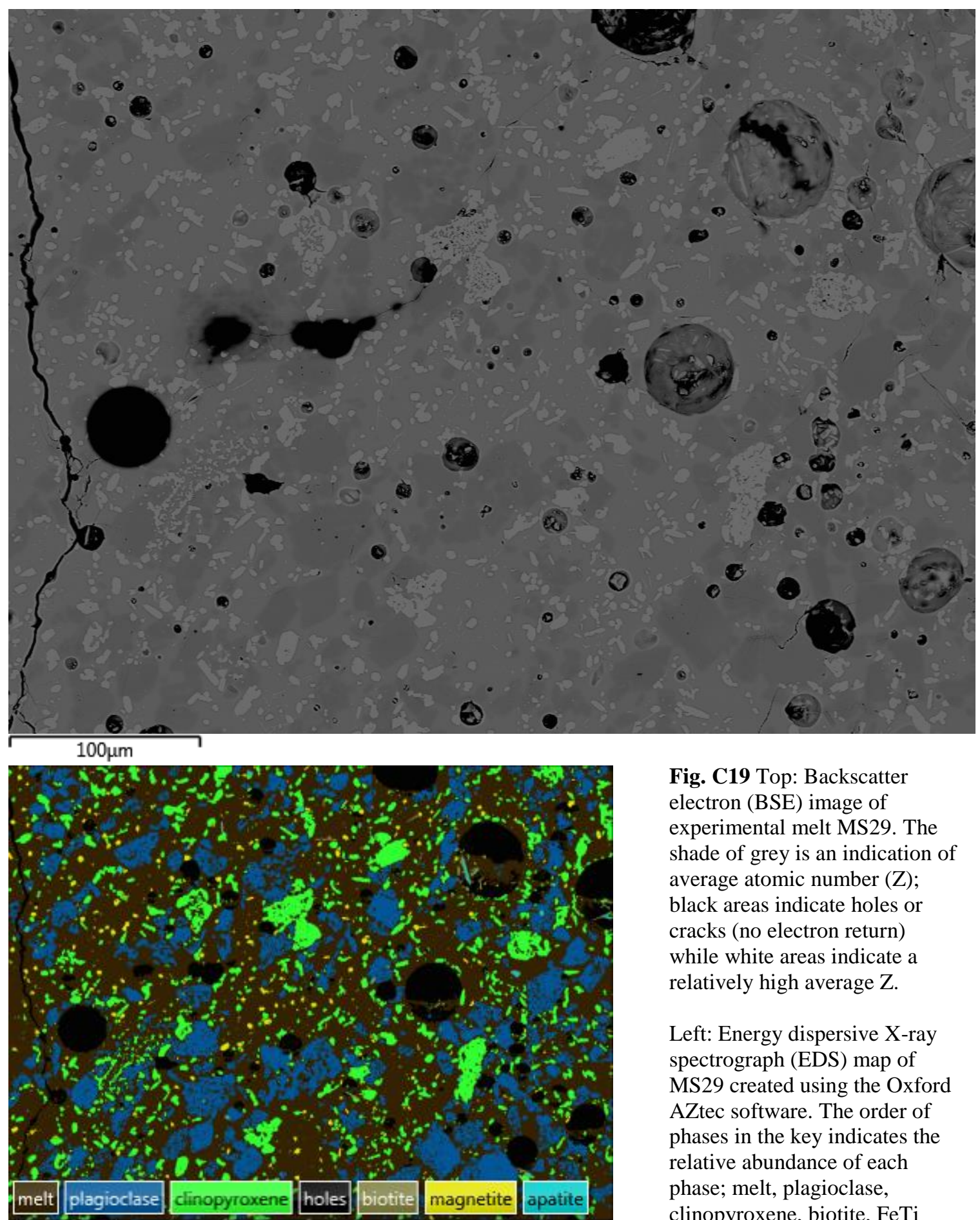

Fig. C19 Top: Backscatter electron (BSE) image of experimental melt MS29. The shade of grey is an indication of average atomic number $(\mathrm{Z})$; black areas indicate holes or cracks (no electron return) while white areas indicate a relatively high average $\mathrm{Z}$.

Left: Energy dispersive X-ray spectrograph (EDS) map of MS29 created using the Oxford AZtec software. The order of phases in the key indicates the relative abundance of each phase; melt, plagioclase, clinopyroxene, biotite, FeTi oxides, apatite. 


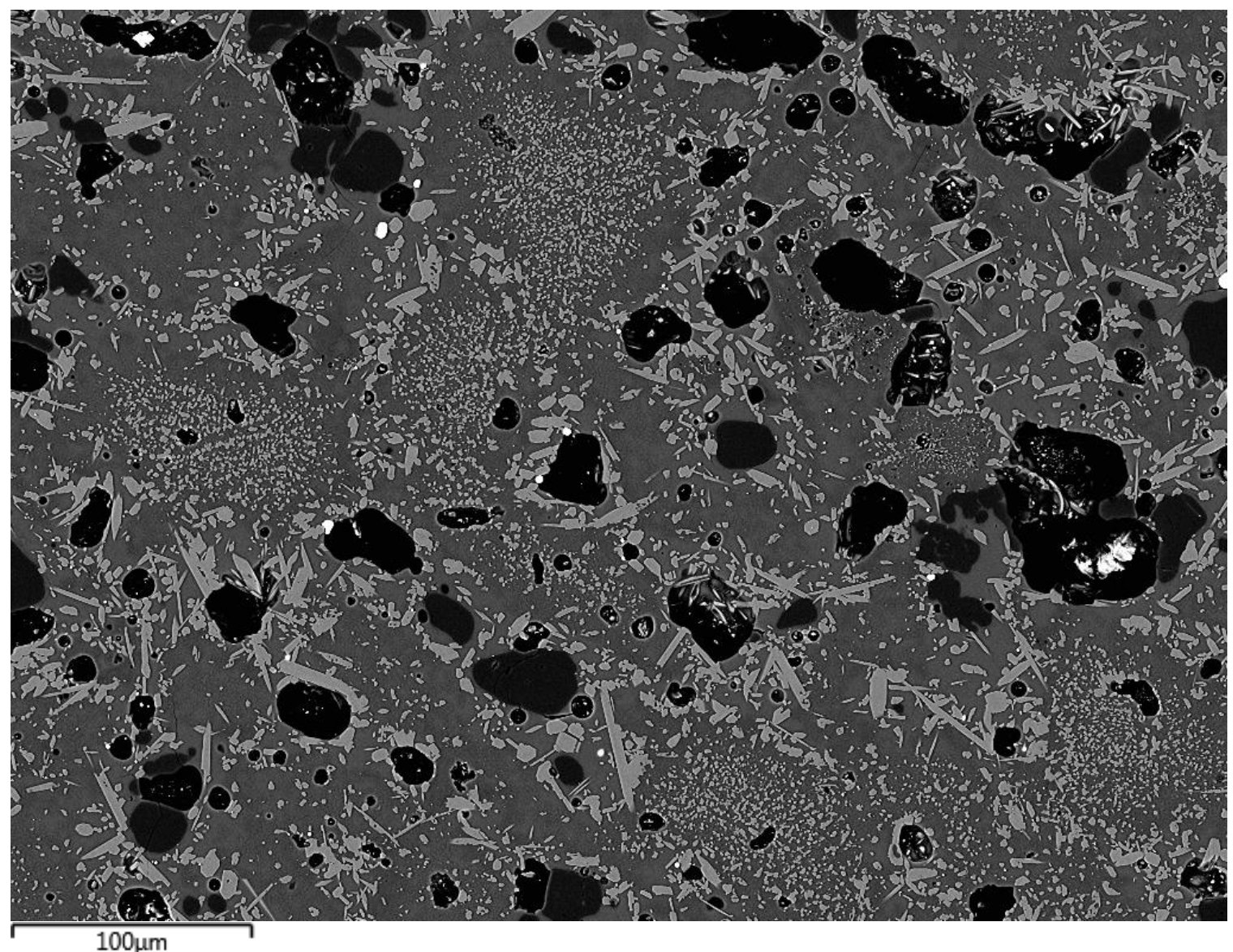

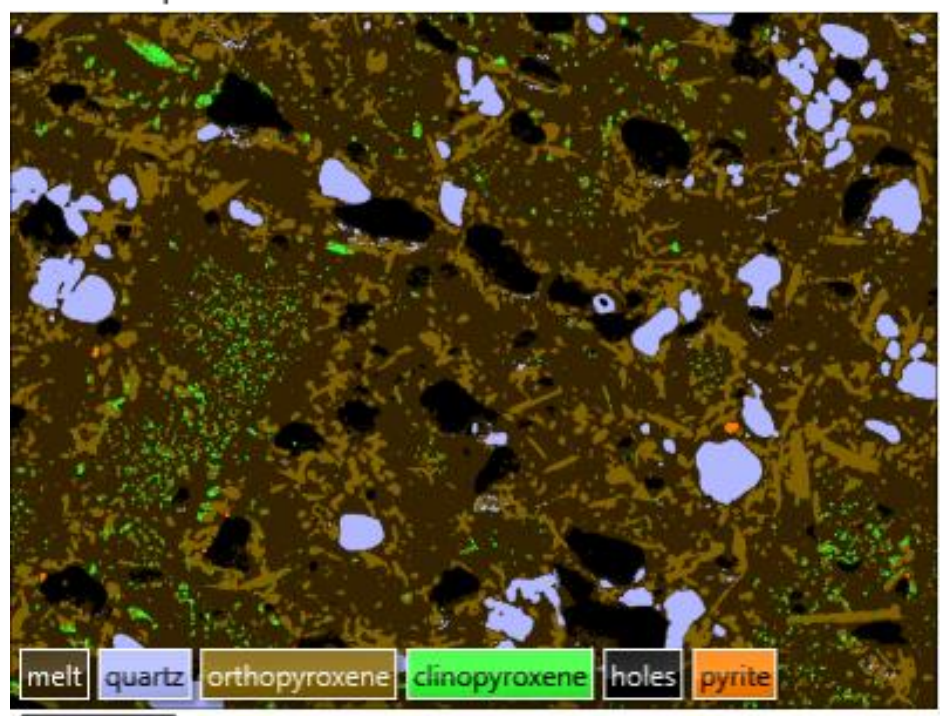

$100 \mu \mathrm{m}$
Fig. C20 Top: Backscatter electron (BSE) image of experimental melt MS30. The shade of grey is an indication of average atomic number $(\mathrm{Z})$; black areas indicate holes or cracks (no electron return) while white areas indicate a relatively high average $\mathrm{Z}$.

Left: Energy dispersive X-ray spectrograph (EDS) map of MS30 created using the Oxford AZtec software. The order of phases in the key indicates the relative abundance of each phase; melt, quartz, orthopyroxene, clinopyroxene, pyrite. 

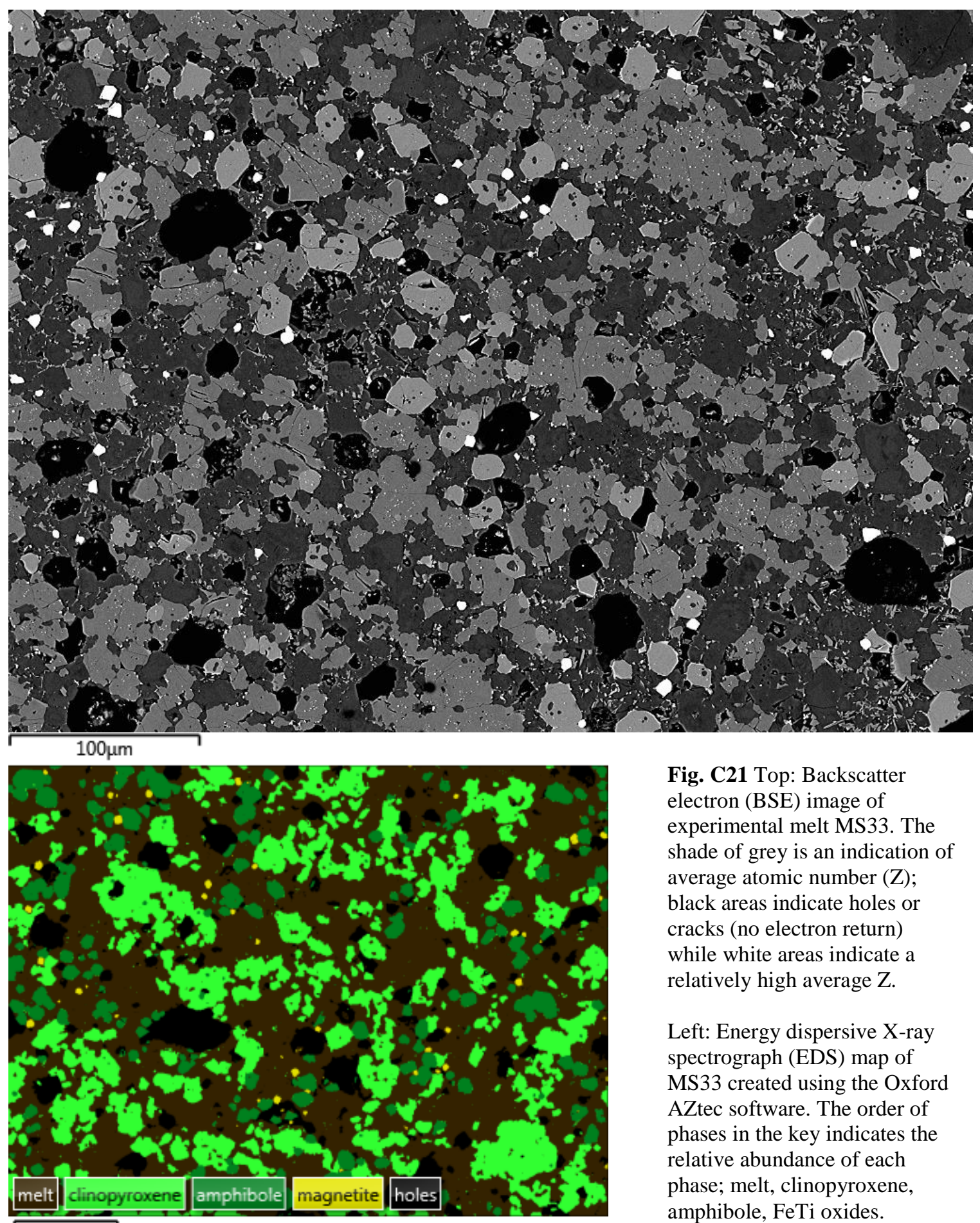

Fig. C21 Top: Backscatter electron (BSE) image of experimental melt MS33. The shade of grey is an indication of average atomic number $(\mathrm{Z})$; black areas indicate holes or cracks (no electron return) while white areas indicate a relatively high average $\mathrm{Z}$.

Left: Energy dispersive X-ray spectrograph (EDS) map of MS33 created using the Oxford AZtec software. The order of phases in the key indicates the relative abundance of each phase; melt, clinopyroxene, amphibole, FeTi oxides. 

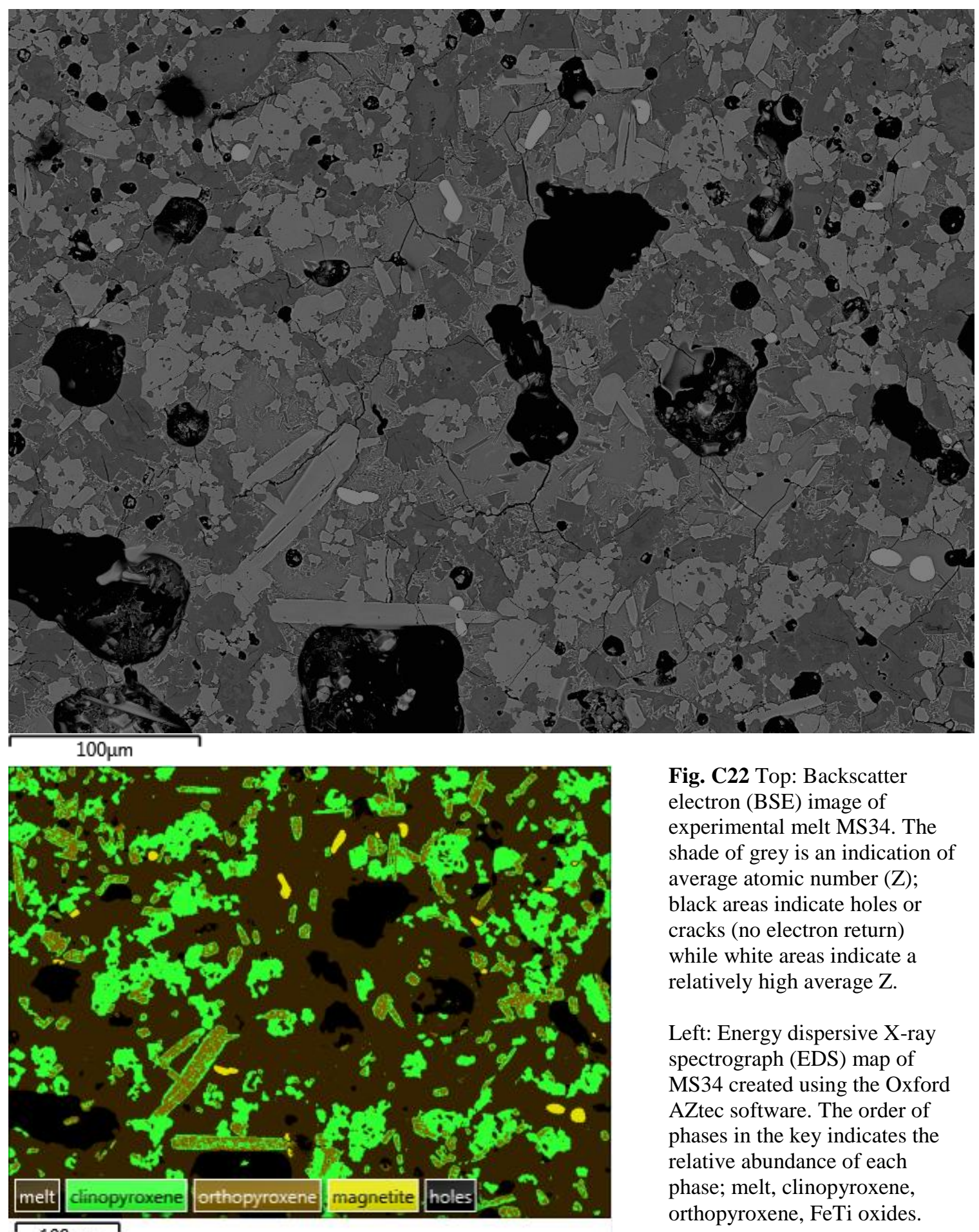

Fig. C22 Top: Backscatter electron (BSE) image of experimental melt MS34. The shade of grey is an indication of average atomic number $(\mathrm{Z})$; black areas indicate holes or cracks (no electron return) while white areas indicate a relatively high average $\mathrm{Z}$.

Left: Energy dispersive X-ray spectrograph (EDS) map of MS34 created using the Oxford AZtec software. The order of phases in the key indicates the relative abundance of each phase; melt, clinopyroxene, orthopyroxene, FeTi oxides. 

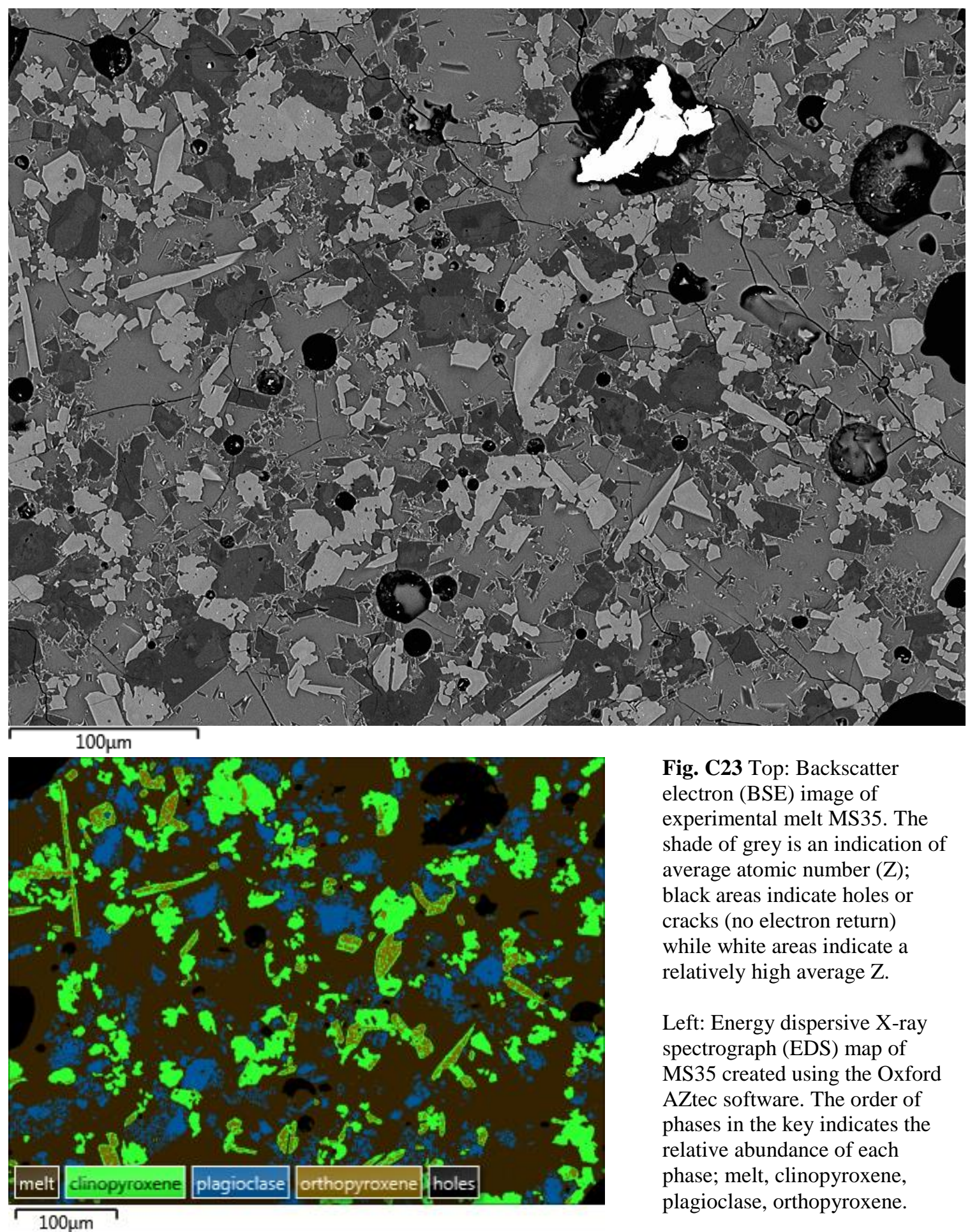

Fig. C23 Top: Backscatter electron (BSE) image of experimental melt MS35. The shade of grey is an indication of average atomic number (Z); black areas indicate holes or cracks (no electron return) while white areas indicate a relatively high average $\mathrm{Z}$.

Left: Energy dispersive X-ray spectrograph (EDS) map of MS35 created using the Oxford AZtec software. The order of phases in the key indicates the relative abundance of each phase; melt, clinopyroxene, plagioclase, orthopyroxene. 

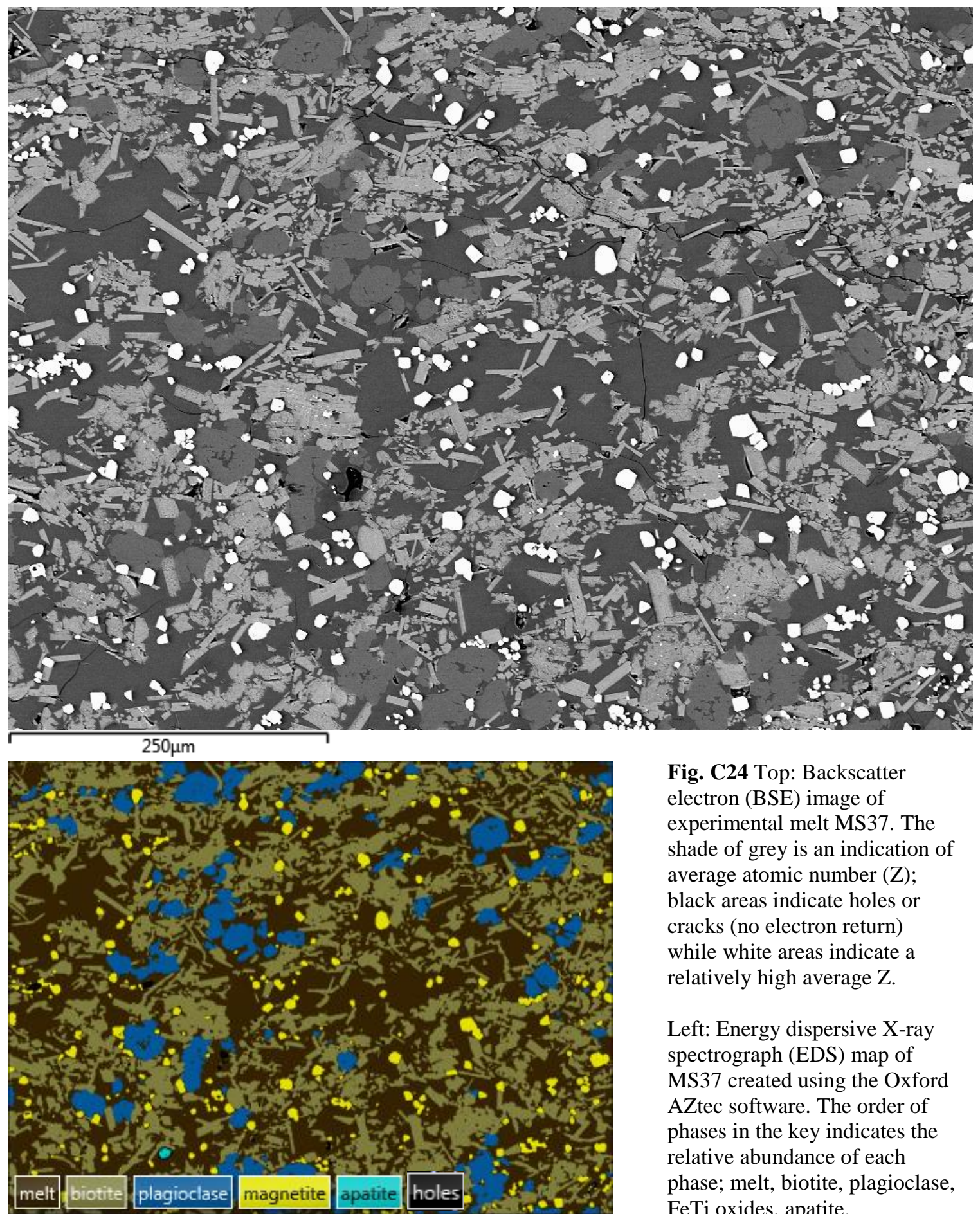

Fig. C24 Top: Backscatter electron (BSE) image of experimental melt MS37. The shade of grey is an indication of average atomic number (Z); black areas indicate holes or cracks (no electron return) while white areas indicate a relatively high average $\mathrm{Z}$.

Left: Energy dispersive X-ray spectrograph (EDS) map of MS37 created using the Oxford AZtec software. The order of phases in the key indicates the relative abundance of each phase; melt, biotite, plagioclase, FeTi oxides, apatite. 

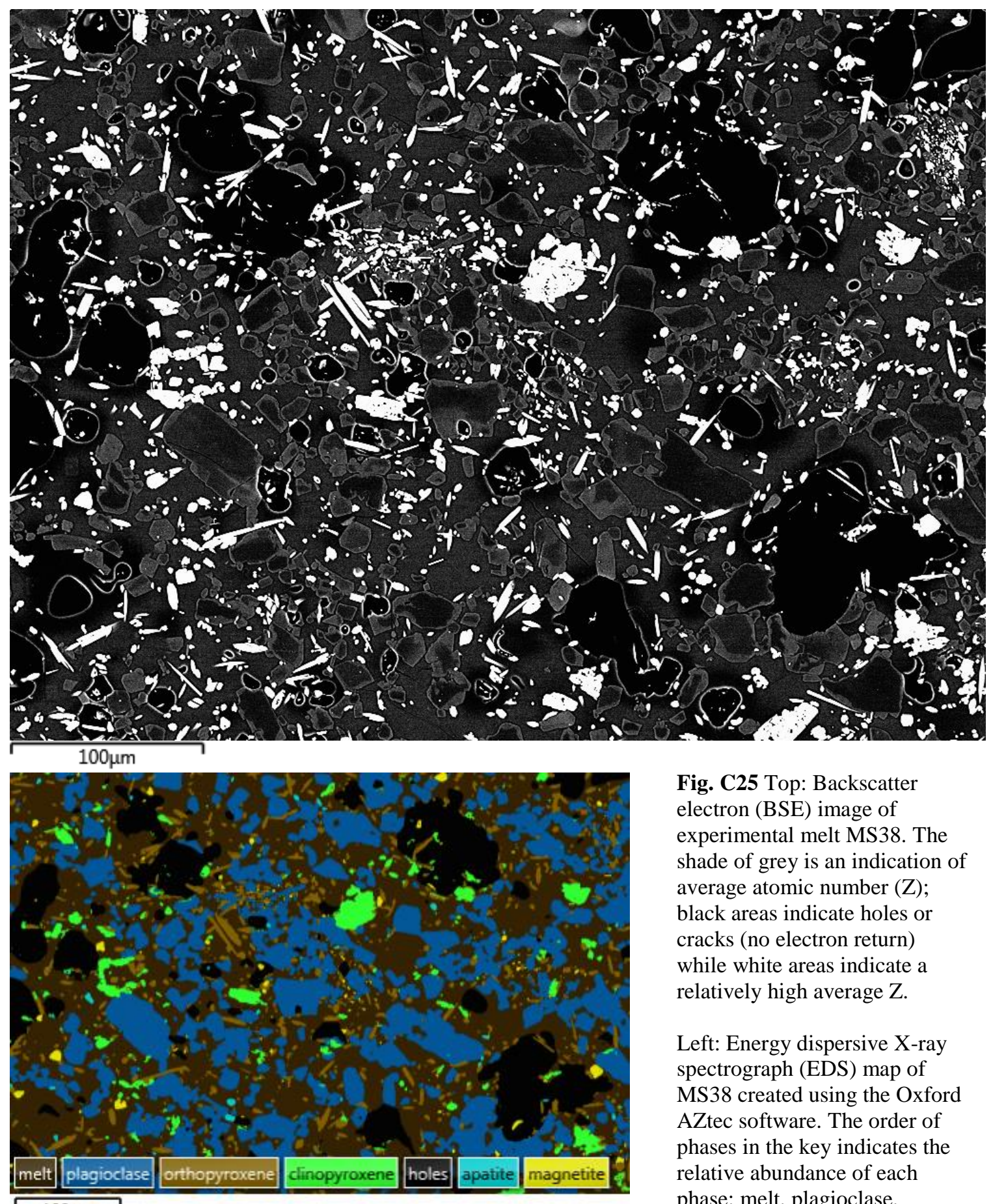

Fig. C25 Top: Backscatter electron (BSE) image of experimental melt MS38. The shade of grey is an indication of average atomic number $(\mathrm{Z})$; black areas indicate holes or cracks (no electron return) while white areas indicate a relatively high average $Z$.

Left: Energy dispersive X-ray spectrograph (EDS) map of MS38 created using the Oxford AZtec software. The order of phases in the key indicates the relative abundance of each phase; melt, plagioclase, orthopyroxene, clinopyroxene, apatite, FeTi oxides. 

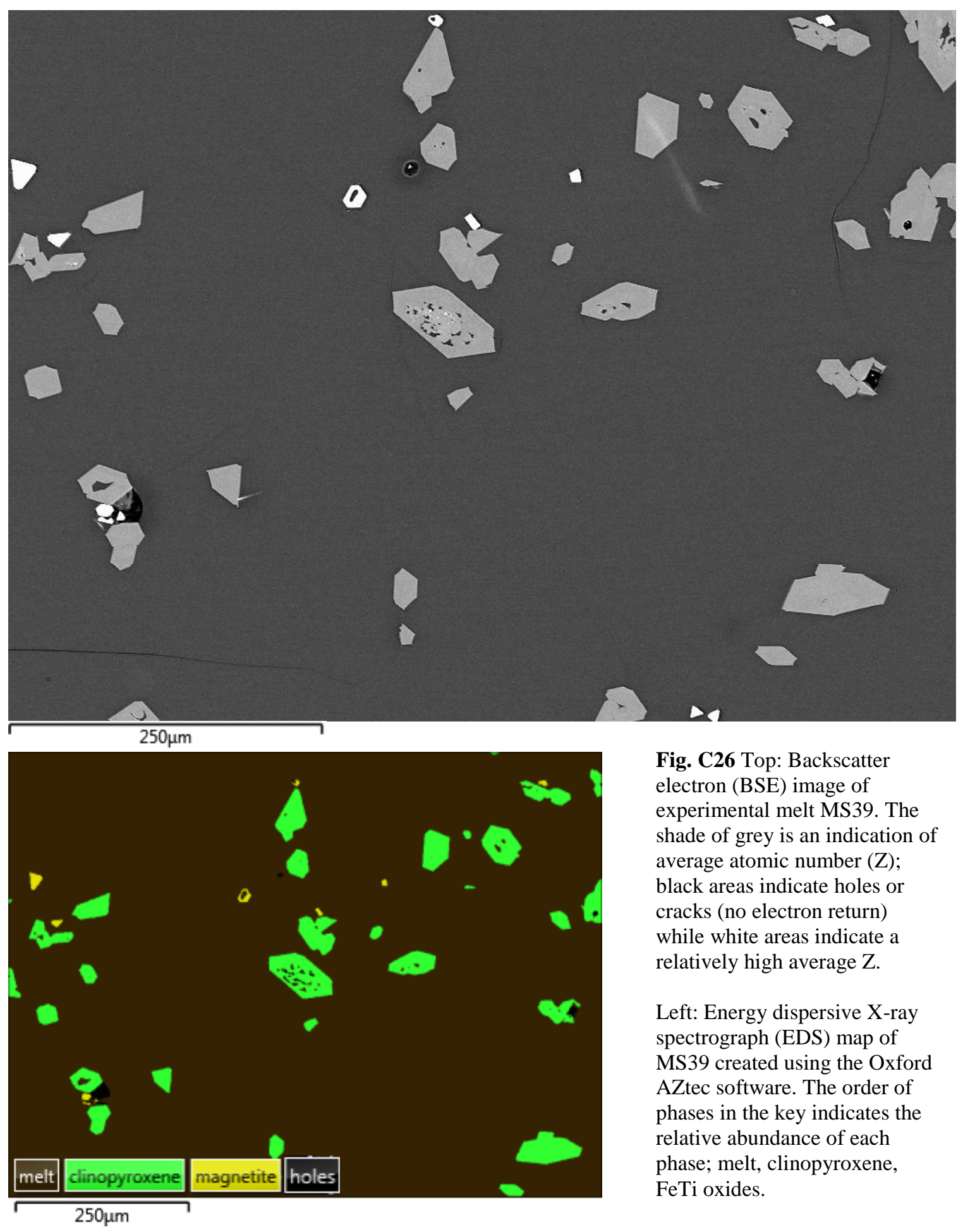

Fig. C26 Top: Backscatter electron (BSE) image of experimental melt MS39. The shade of grey is an indication of average atomic number $(\mathrm{Z})$; black areas indicate holes or cracks (no electron return) while white areas indicate a relatively high average $\mathrm{Z}$.

Left: Energy dispersive X-ray spectrograph (EDS) map of MS39 created using the Oxford AZtec software. The order of phases in the key indicates the relative abundance of each phase; melt, clinopyroxene, FeTi oxides. 

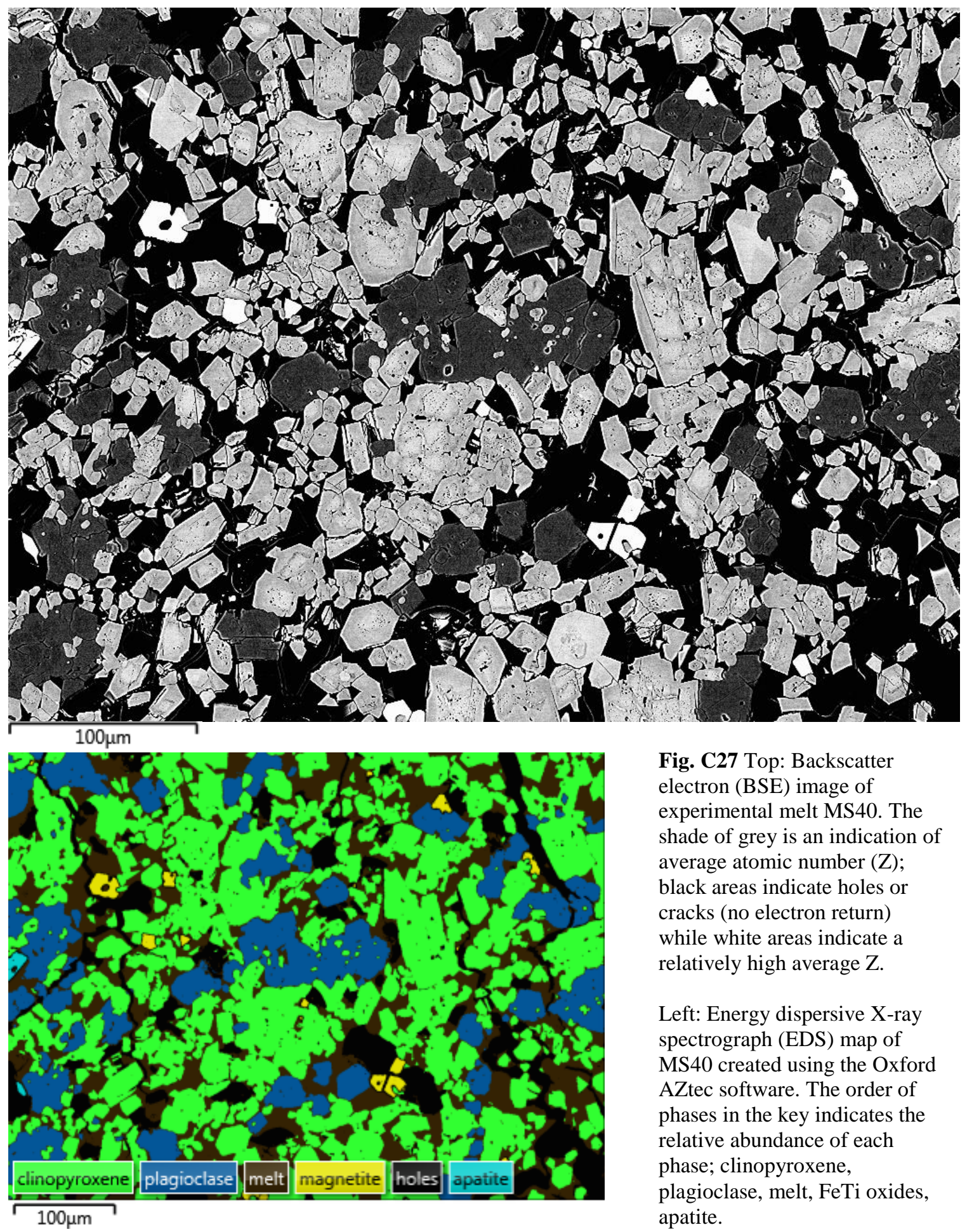

Fig. C27 Top: Backscatter electron (BSE) image of experimental melt MS40. The shade of grey is an indication of average atomic number (Z); black areas indicate holes or cracks (no electron return) while white areas indicate a relatively high average $\mathrm{Z}$.

Left: Energy dispersive X-ray spectrograph (EDS) map of MS40 created using the Oxford AZtec software. The order of phases in the key indicates the relative abundance of each phase; clinopyroxene, plagioclase, melt, FeTi oxides, apatite. 


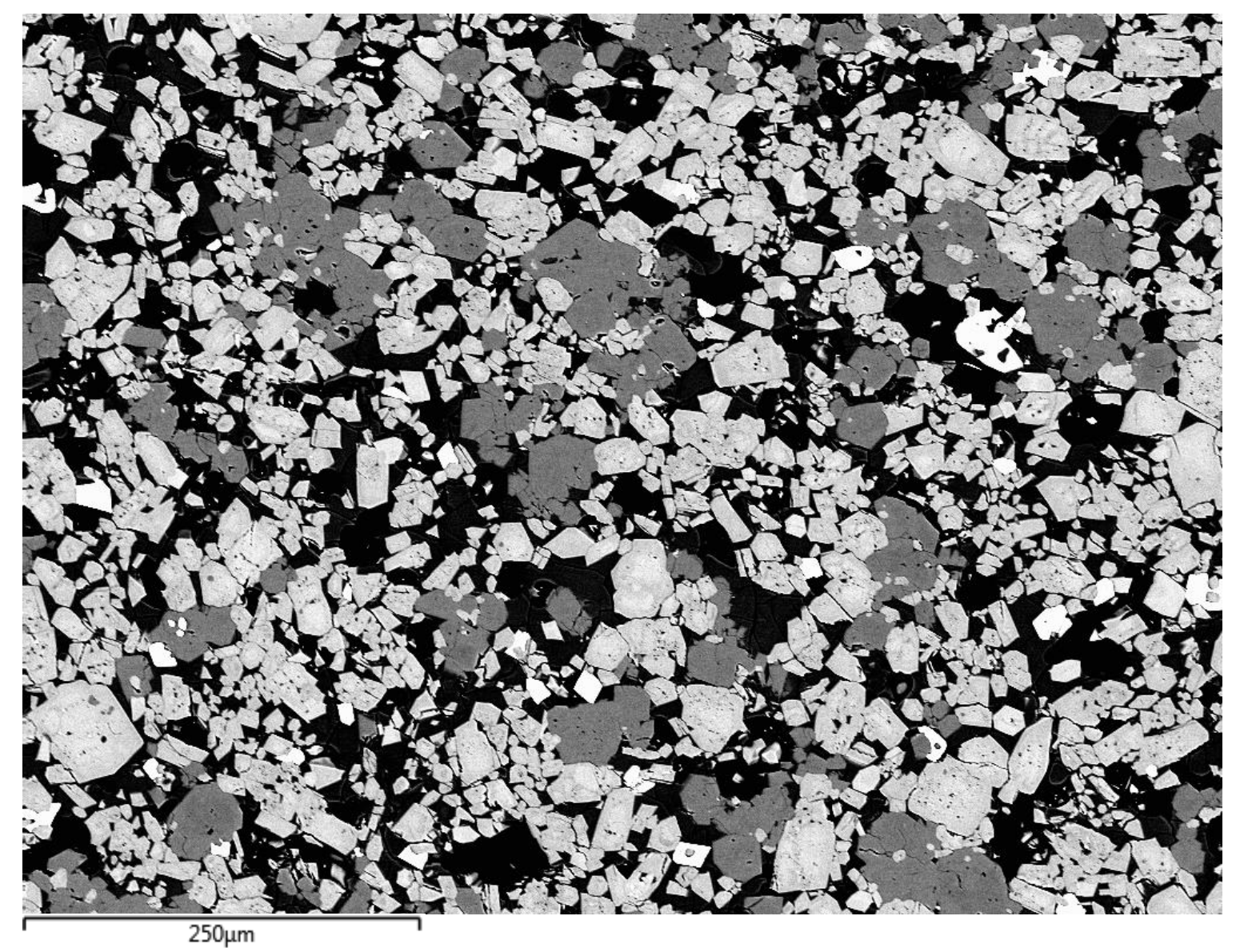

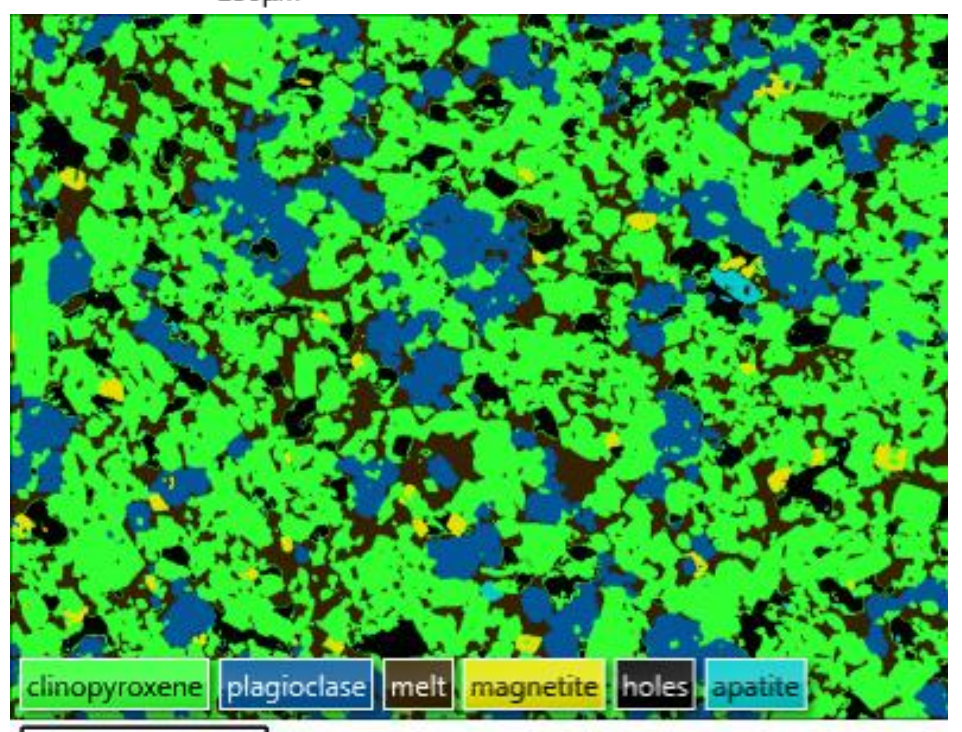

$250 \mu \mathrm{m}$
Fig. C28 Top: Backscatter electron (BSE) image of experimental melt MS41. The shade of grey is an indication of average atomic number $(\mathrm{Z})$; black areas indicate holes or cracks (no electron return) while white areas indicate a relatively high average $Z$.

Left: Energy dispersive X-ray spectrograph (EDS) map of MS41 created using the Oxford AZtec software. The order of phases in the key indicates the relative abundance of each phase; clinopyroxene, plagioclase, melt, FeTi oxides, apatite. 

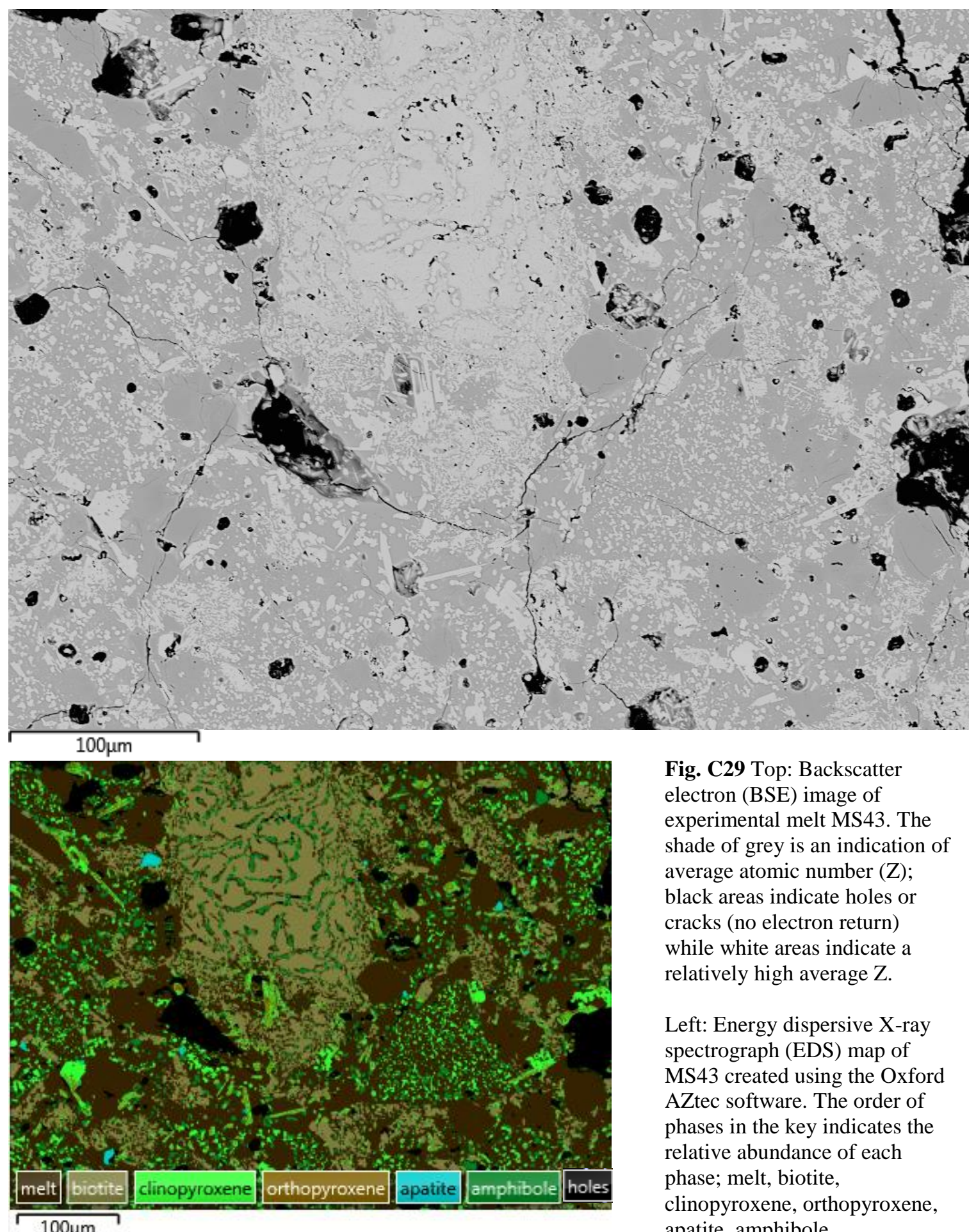

Fig. C29 Top: Backscatter electron (BSE) image of experimental melt MS43. The shade of grey is an indication of average atomic number (Z); black areas indicate holes or cracks (no electron return) while white areas indicate a relatively high average $\mathrm{Z}$.

Left: Energy dispersive X-ray spectrograph (EDS) map of MS43 created using the Oxford AZtec software. The order of phases in the key indicates the relative abundance of each phase; melt, biotite, clinopyroxene, orthopyroxene, apatite, amphibole. 

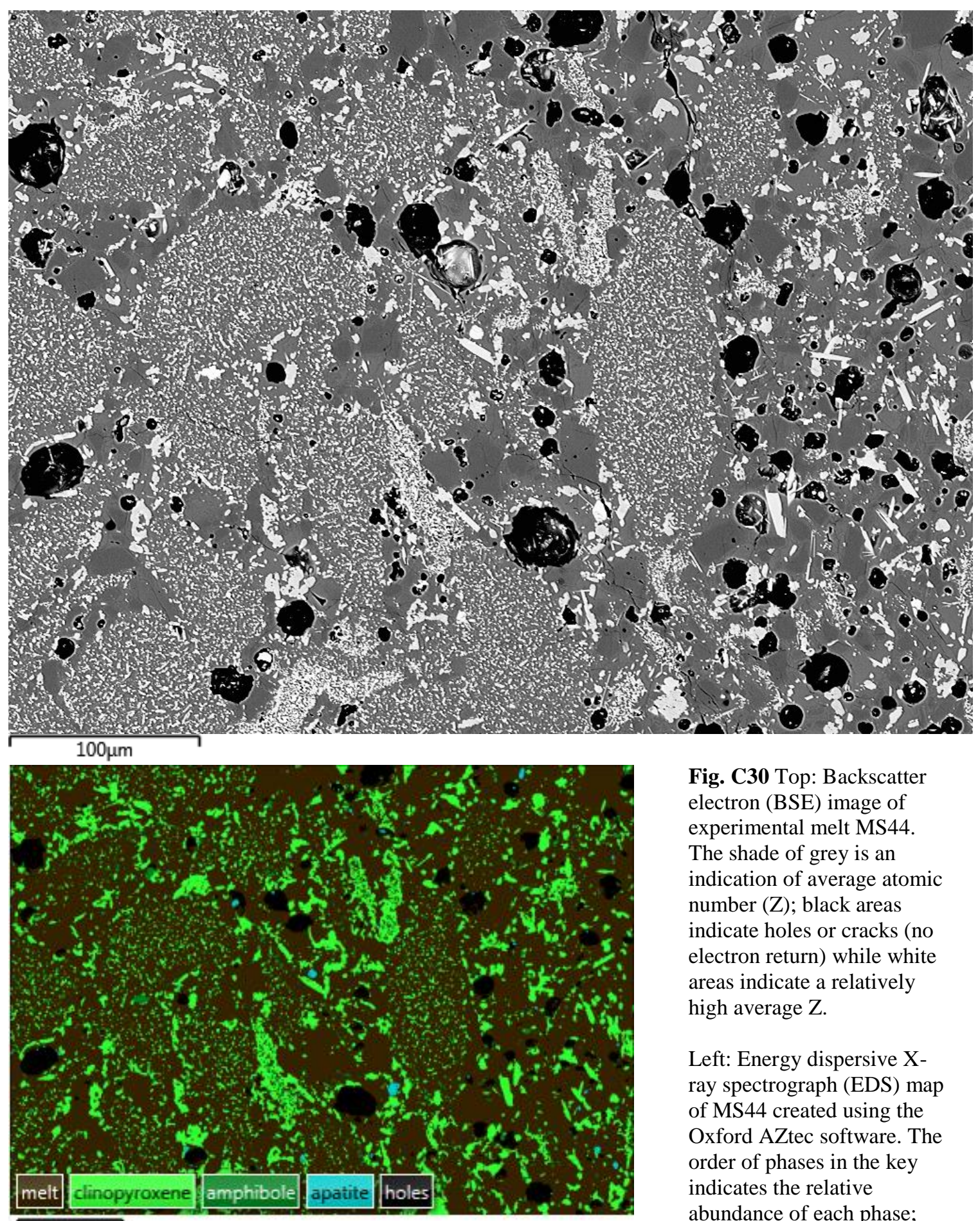

$100 \mu \mathrm{m}$

Fig. C30 Top: Backscatter electron (BSE) image of experimental melt MS44. The shade of grey is an indication of average atomic number (Z); black areas indicate holes or cracks (no electron return) while white areas indicate a relatively high average $Z$.

Left: Energy dispersive Xray spectrograph (EDS) map of MS44 created using the Oxford AZtec software. The order of phases in the key indicates the relative abundance of each phase; melt, clinopyroxene, amphibole, apatite. 

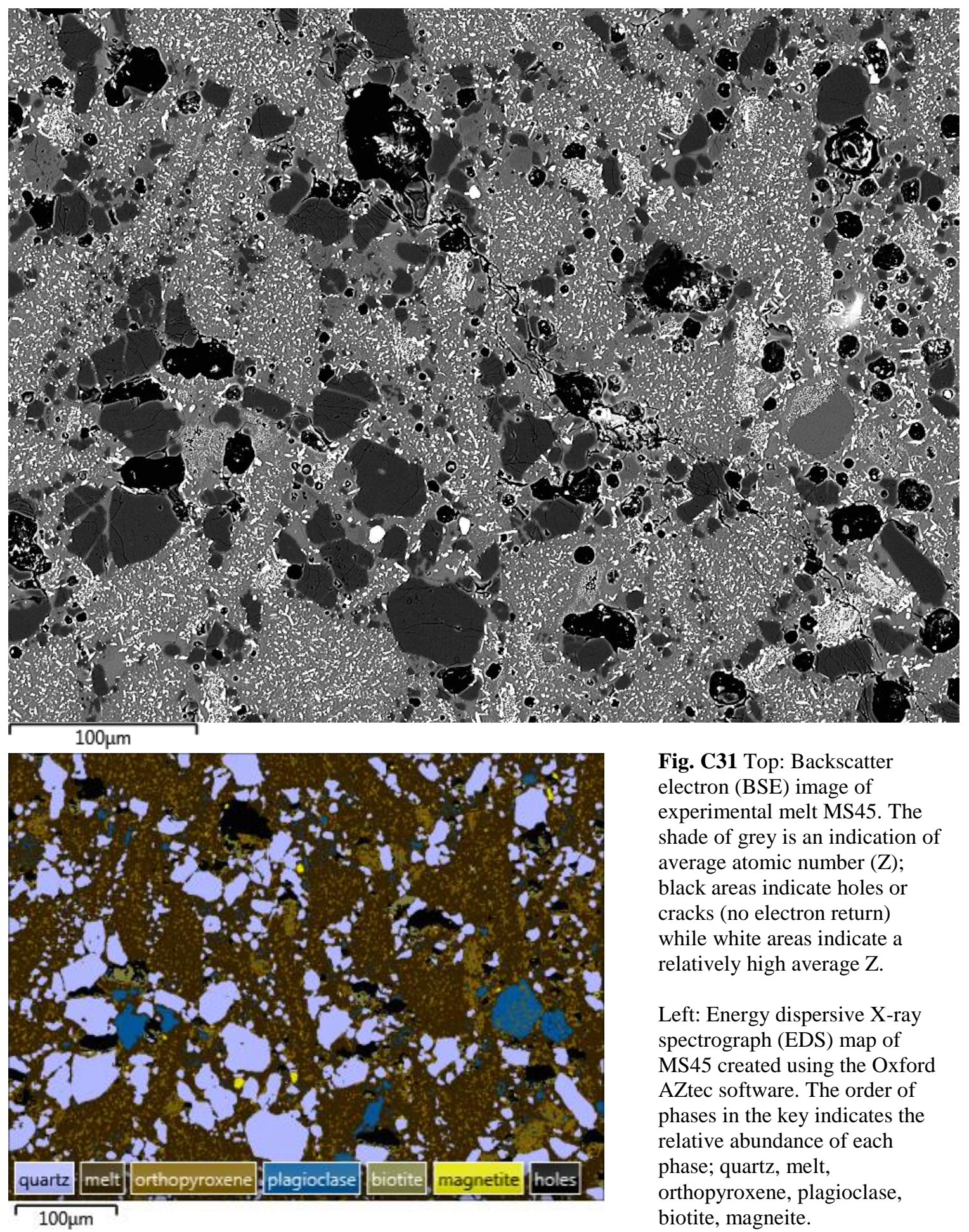

Fig. C31 Top: Backscatter electron (BSE) image of experimental melt MS45. The shade of grey is an indication of average atomic number (Z); black areas indicate holes or cracks (no electron return) while white areas indicate a relatively high average $Z$.

Left: Energy dispersive X-ray spectrograph (EDS) map of MS45 created using the Oxford AZtec software. The order of phases in the key indicates the relative abundance of each phase; quartz, melt, orthopyroxene, plagioclase, biotite, magneite. 

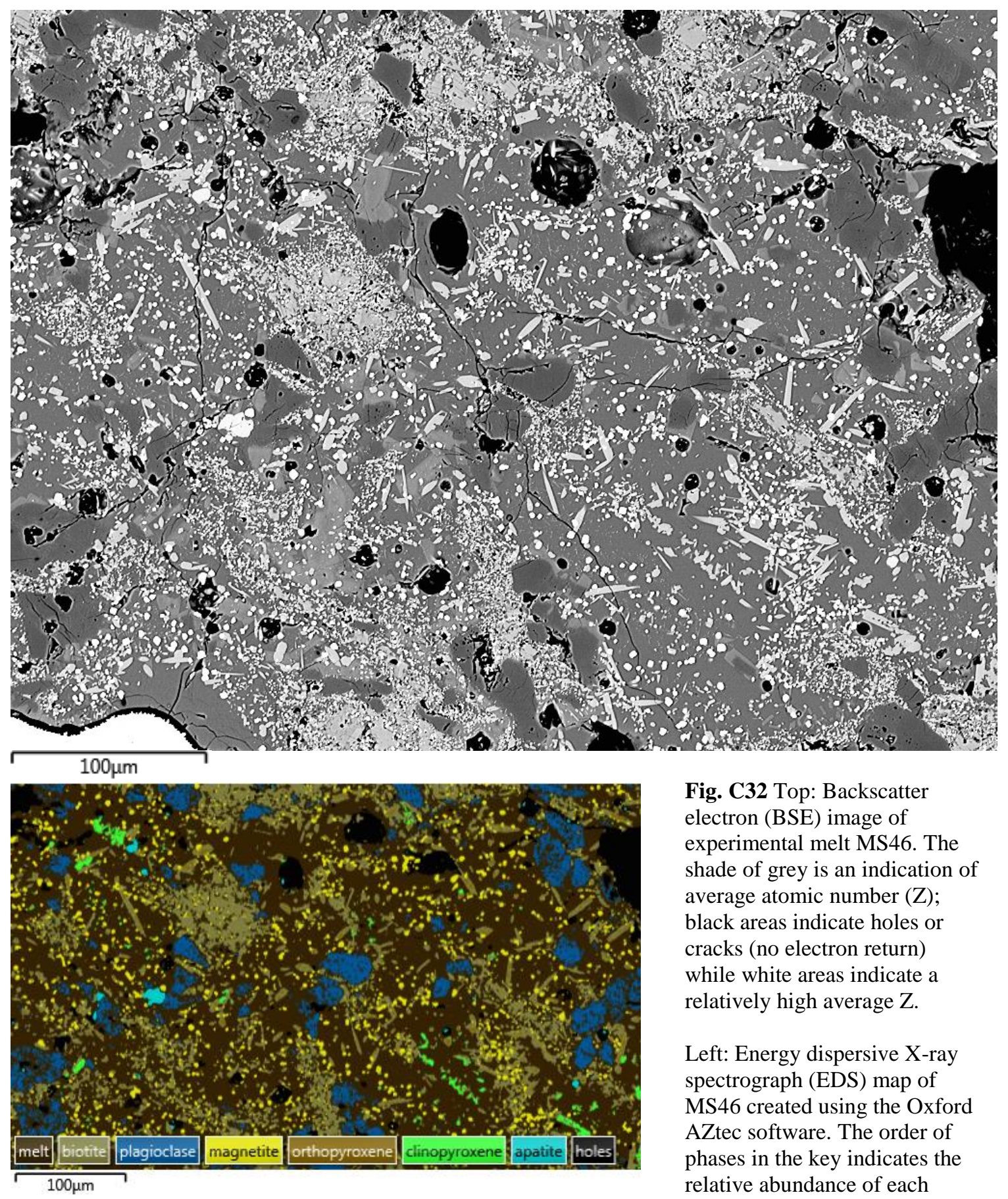

Fig. C32 Top: Backscatter electron (BSE) image of experimental melt MS46. The shade of grey is an indication of average atomic number (Z); black areas indicate holes or cracks (no electron return) while white areas indicate a relatively high average $\mathrm{Z}$.

Left: Energy dispersive X-ray spectrograph (EDS) map of MS46 created using the Oxford AZtec software. The order of phases in the key indicates the relative abundance of each phase; melt, biotite, plagioclase, FeTi oxides, orthopyroxene, clinopyroxene, apatite. 


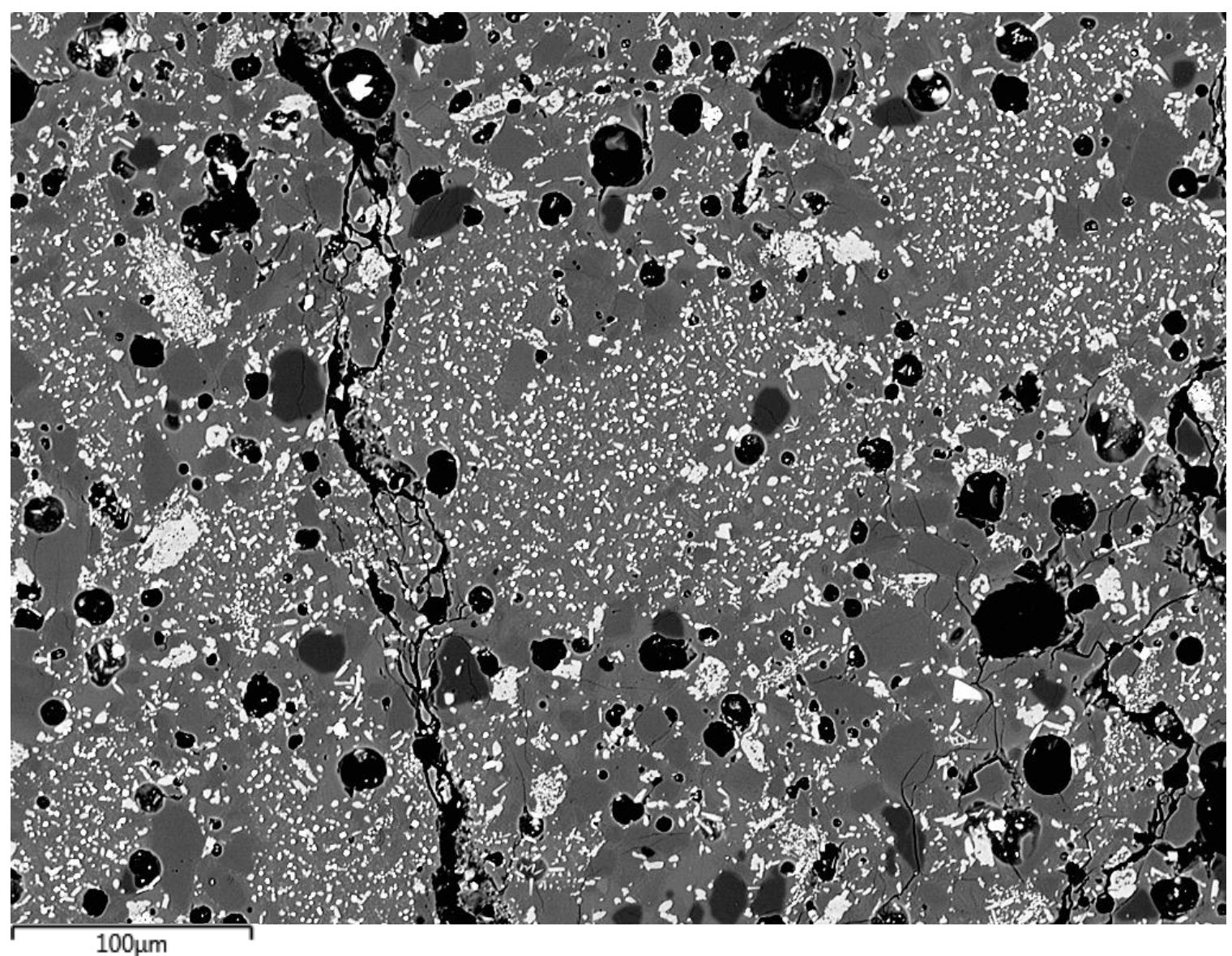

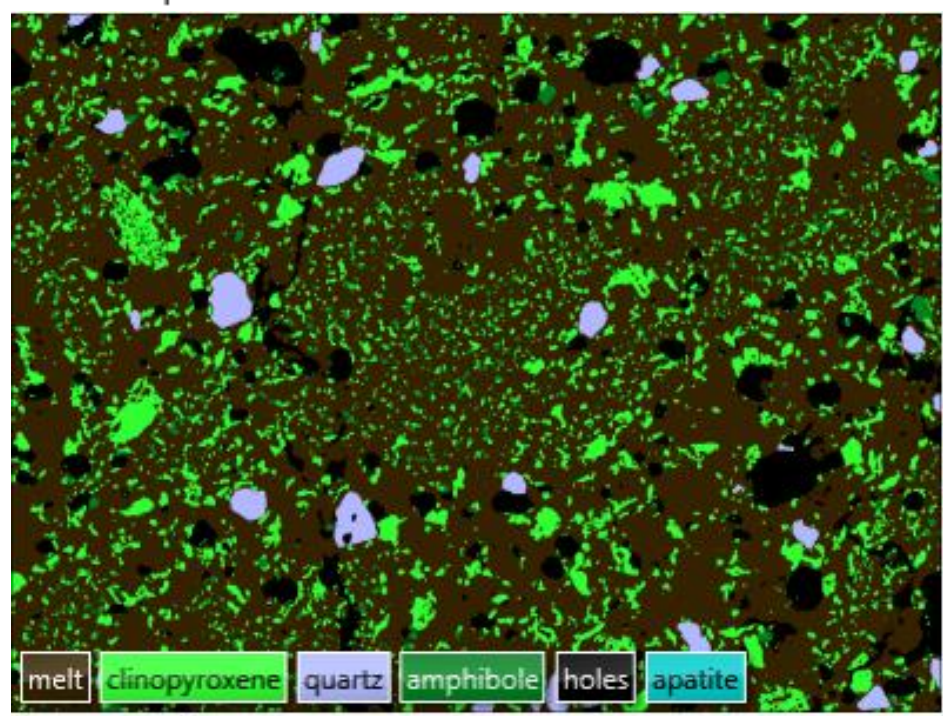

$100 \mu \mathrm{m}$
Fig. C33 Top: Backscatter electron (BSE) image of experimental melt MS47. The shade of grey is an indication of average atomic number $(\mathrm{Z})$; black areas indicate holes or cracks (no electron return) while white areas indicate a relatively high average $\mathrm{Z}$.

Left: Energy dispersive X-ray spectrograph (EDS) map of MS47 created using the Oxford AZtec software. The order of phases in the key indicates the relative abundance of each phase; melt, clinopyroxene, quartz, amphibole, apatite. 

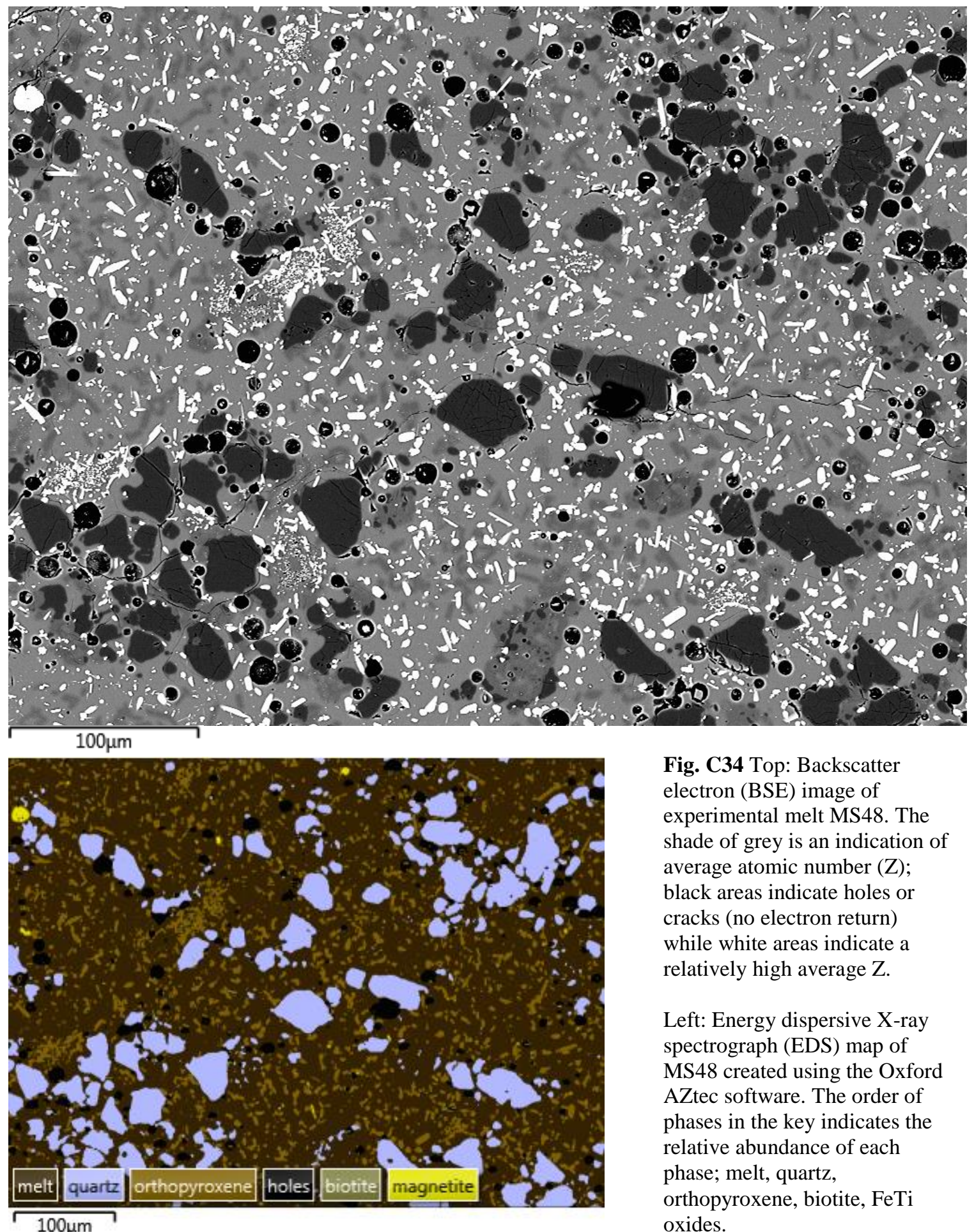

Fig. C34 Top: Backscatter electron (BSE) image of experimental melt MS48. The shade of grey is an indication of average atomic number $(\mathrm{Z})$; black areas indicate holes or cracks (no electron return) while white areas indicate a relatively high average $\mathrm{Z}$.

Left: Energy dispersive X-ray spectrograph (EDS) map of MS48 created using the Oxford AZtec software. The order of phases in the key indicates the relative abundance of each phase; melt, quartz, orthopyroxene, biotite, FeTi oxides. 

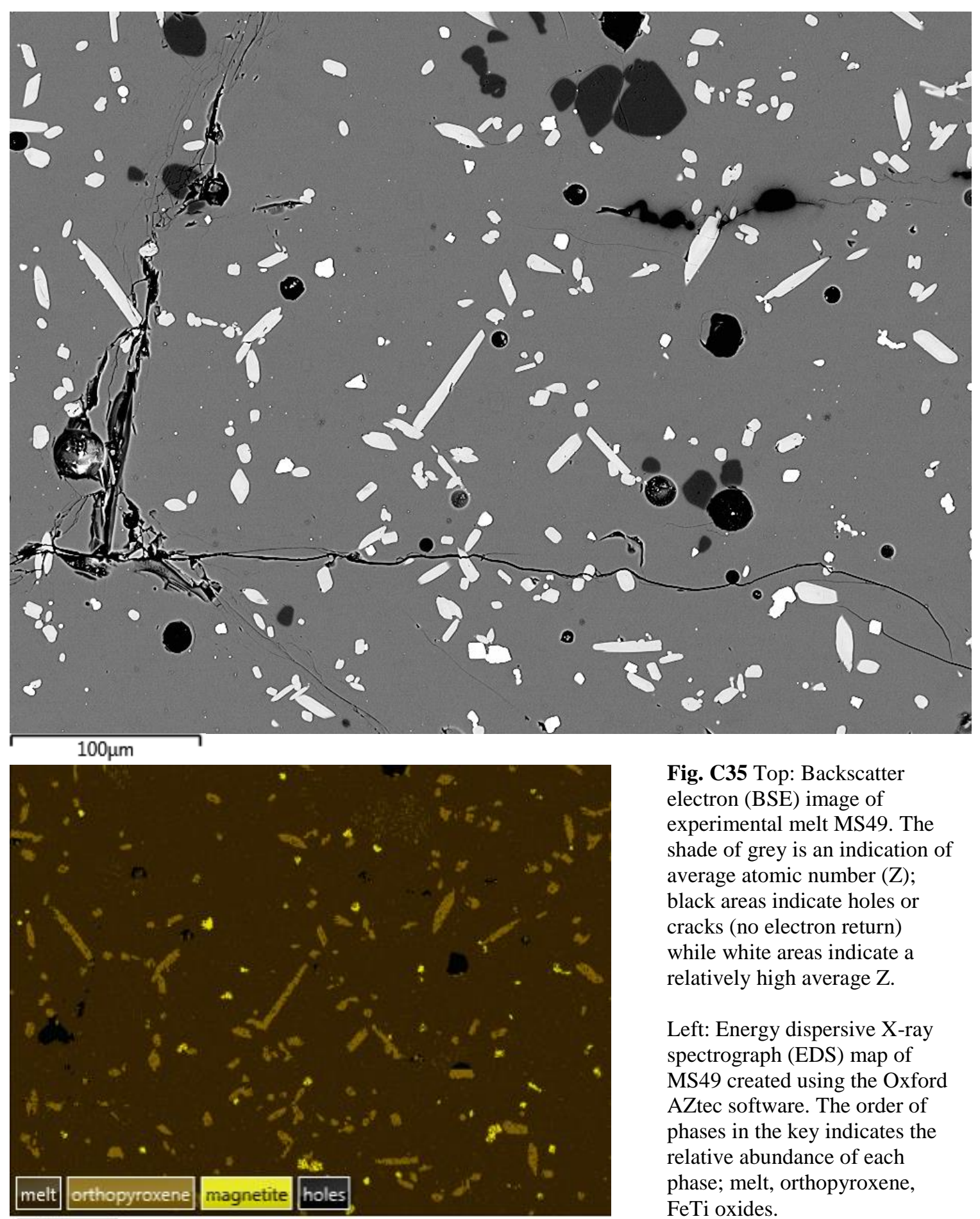

Fig. C35 Top: Backscatter electron (BSE) image of experimental melt MS49. The shade of grey is an indication of average atomic number (Z); black areas indicate holes or cracks (no electron return) while white areas indicate a relatively high average $\mathrm{Z}$.

Left: Energy dispersive X-ray spectrograph (EDS) map of MS49 created using the Oxford AZtec software. The order of phases in the key indicates the relative abundance of each phase; melt, orthopyroxene, FeTi oxides. 

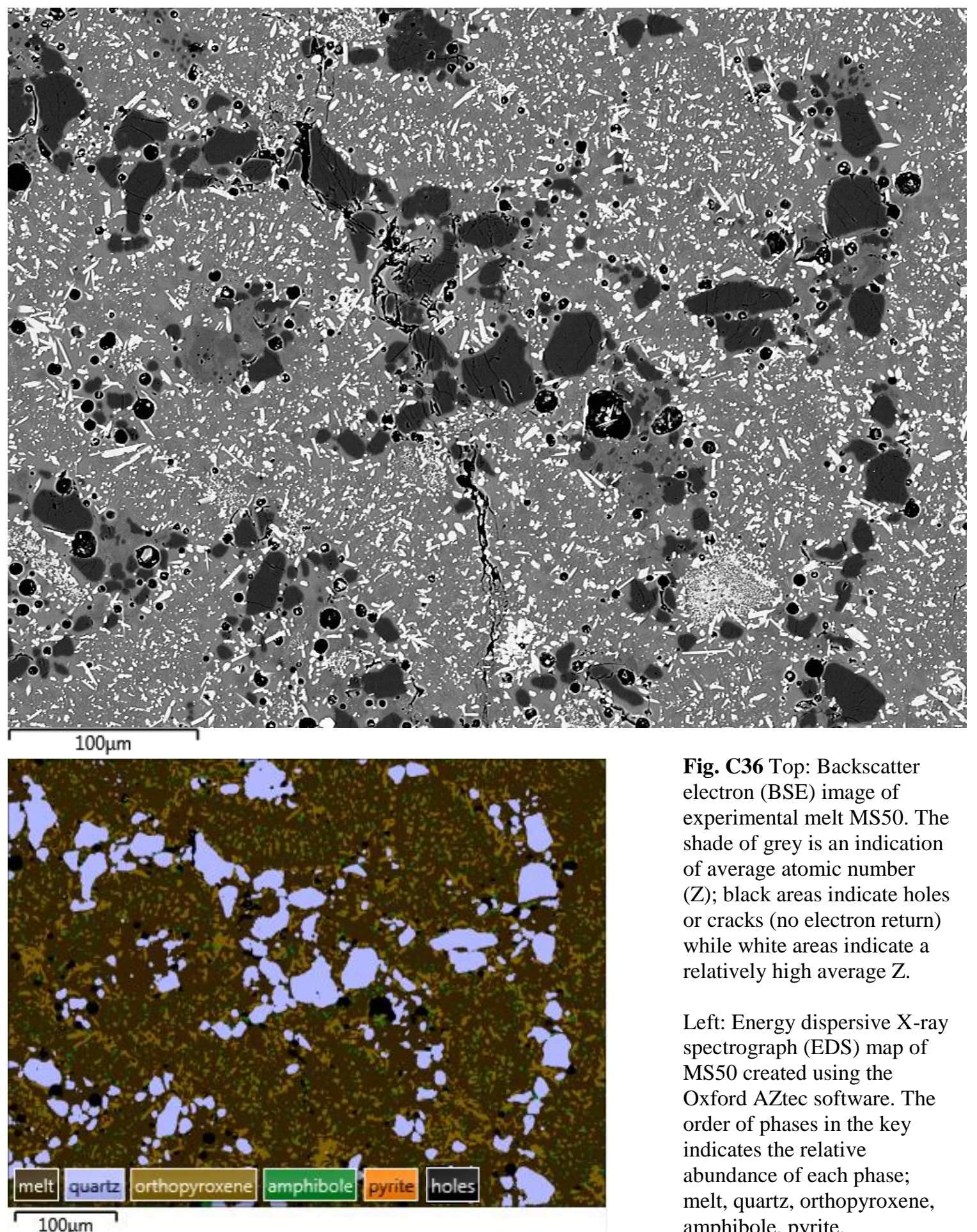

Fig. C36 Top: Backscatter electron (BSE) image of experimental melt MS50. The shade of grey is an indication of average atomic number (Z); black areas indicate holes or cracks (no electron return) while white areas indicate a relatively high average $\mathrm{Z}$.

Left: Energy dispersive X-ray spectrograph (EDS) map of MS50 created using the Oxford AZtec software. The order of phases in the key indicates the relative abundance of each phase; melt, quartz, orthopyroxene, amphibole, pyrite. 

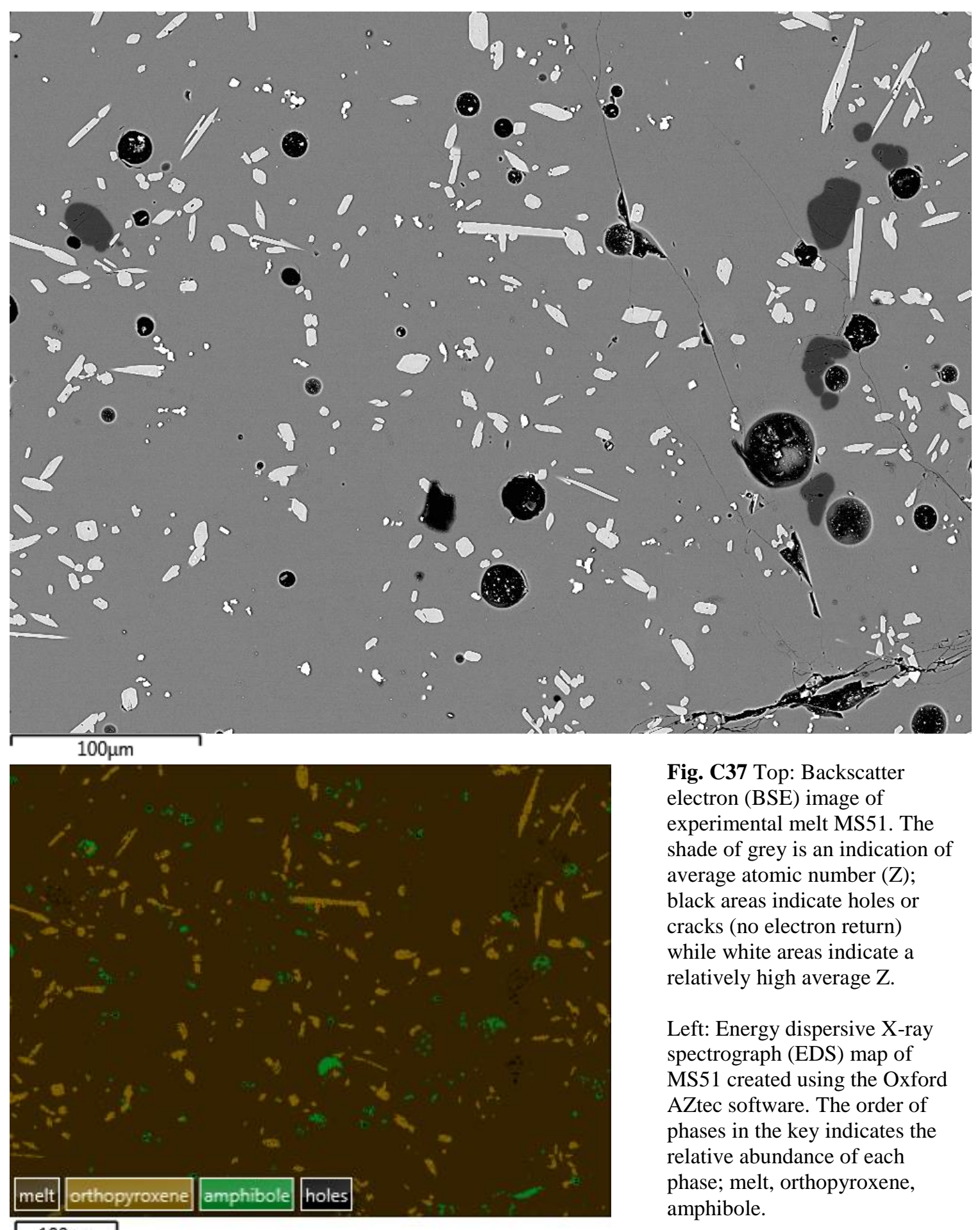

Fig. C37 Top: Backscatter electron (BSE) image of experimental melt MS51. The shade of grey is an indication of average atomic number (Z); black areas indicate holes or cracks (no electron return) while white areas indicate a relatively high average $\mathrm{Z}$.

Left: Energy dispersive X-ray spectrograph (EDS) map of MS51 created using the Oxford AZtec software. The order of phases in the key indicates the relative abundance of each phase; melt, orthopyroxene, amphibole. 

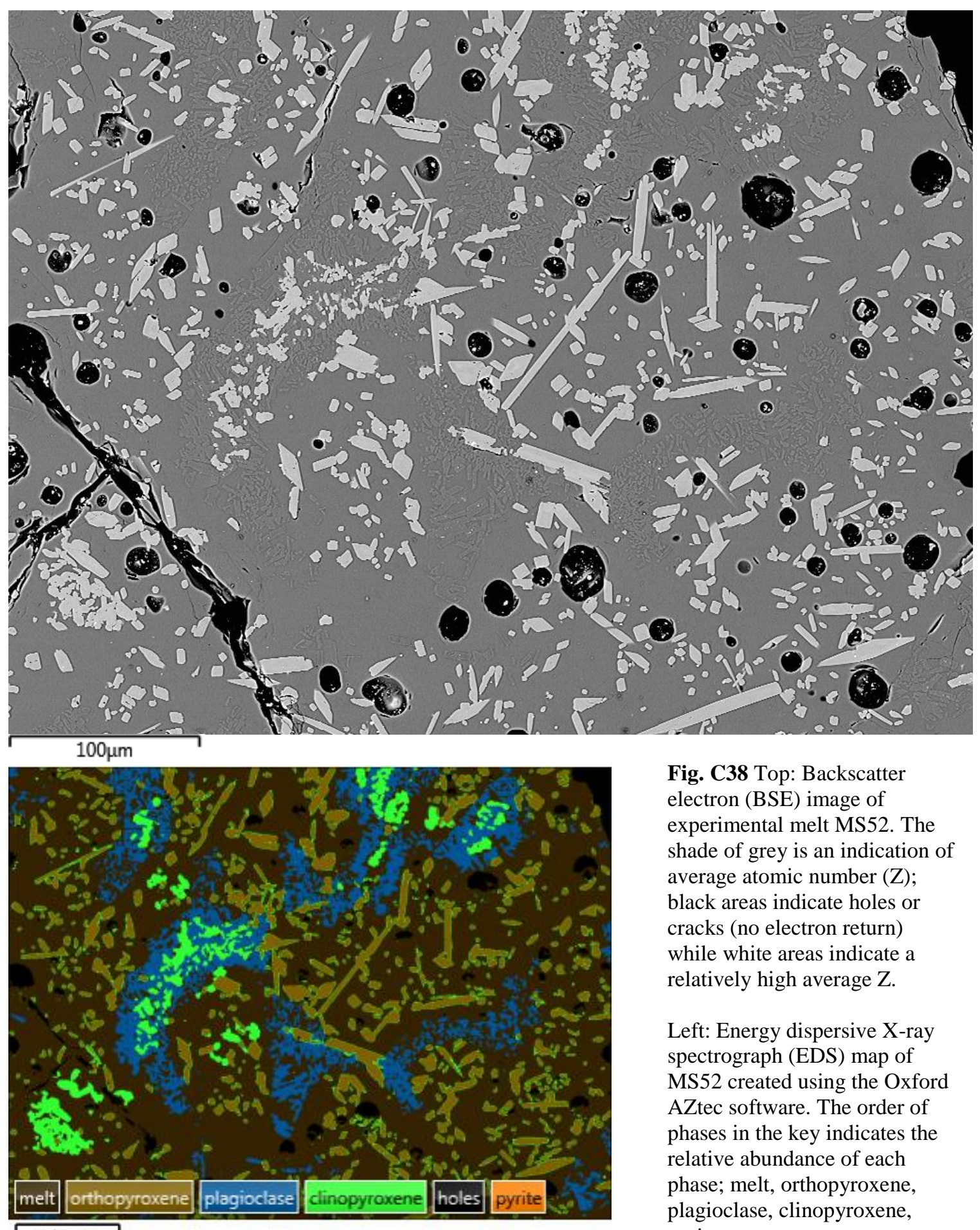

$100 \mu \mathrm{m}$

Fig. C38 Top: Backscatter electron (BSE) image of experimental melt MS52. The shade of grey is an indication of average atomic number (Z); black areas indicate holes or cracks (no electron return) while white areas indicate a relatively high average $\mathrm{Z}$.

Left: Energy dispersive X-ray spectrograph (EDS) map of MS52 created using the Oxford AZtec software. The order of phases in the key indicates the relative abundance of each phase; melt, orthopyroxene, plagioclase, clinopyroxene, pyrite. 

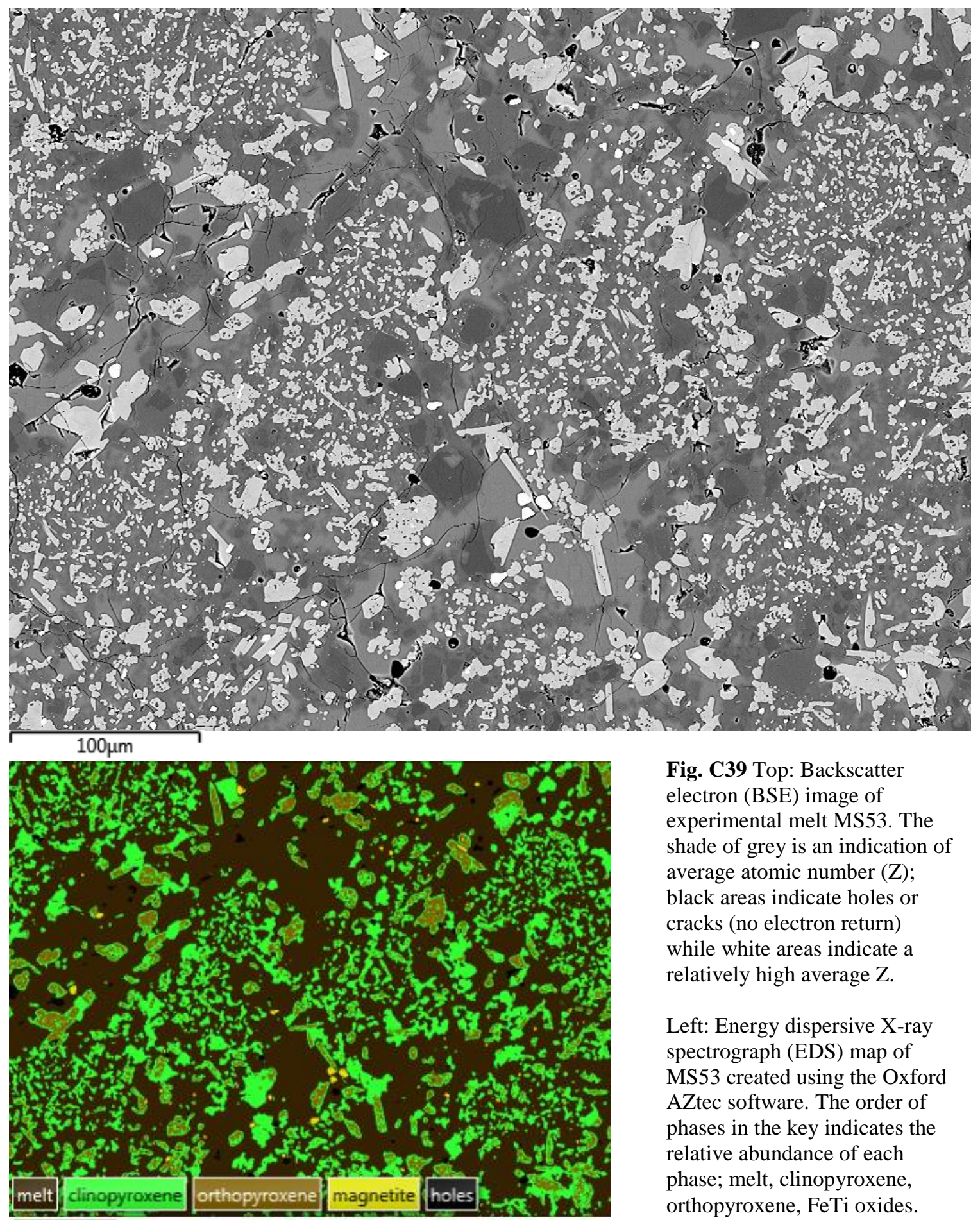

Fig. C39 Top: Backscatter electron (BSE) image of experimental melt MS53. The shade of grey is an indication of average atomic number (Z); black areas indicate holes or cracks (no electron return) while white areas indicate a relatively high average $\mathrm{Z}$.

Left: Energy dispersive X-ray spectrograph (EDS) map of MS53 created using the Oxford AZtec software. The order of phases in the key indicates the relative abundance of each phase; melt, clinopyroxene, orthopyroxene, FeTi oxides.

$100 \mu \mathrm{m}$ 


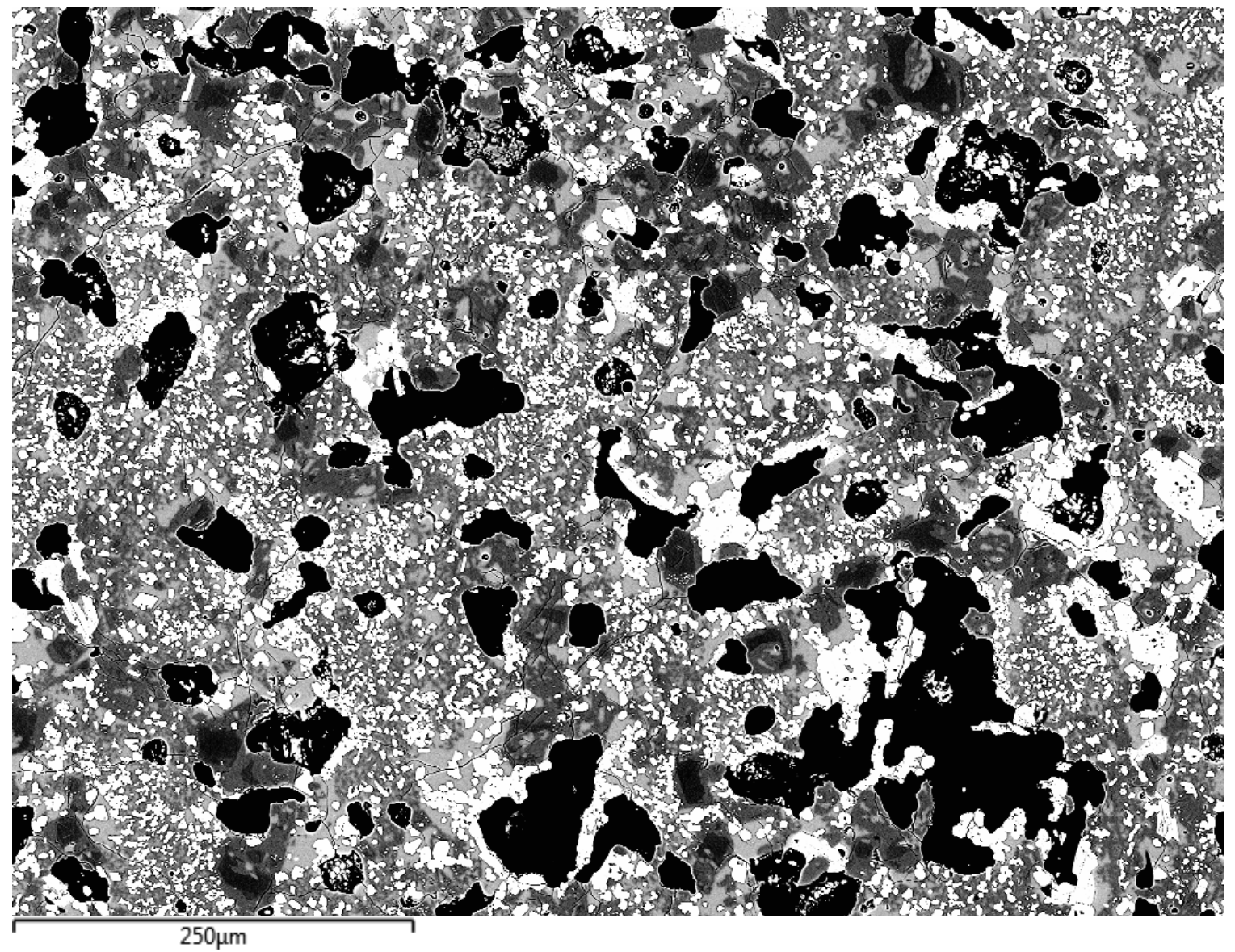

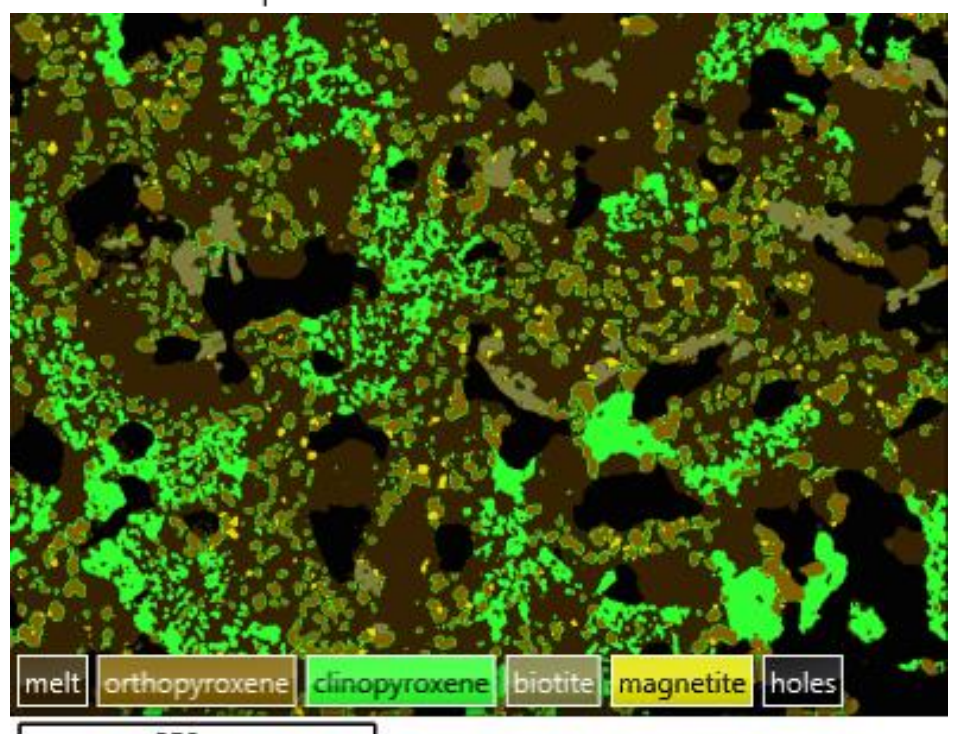

$250 \mu \mathrm{m}$
Fig. C40 Top: Backscatter electron (BSE) image of experimental melt MS55. The shade of grey is an indication of average atomic number (Z); black areas indicate holes or cracks (no electron return) while white areas indicate a relatively high average $\mathrm{Z}$.

Left: Energy dispersive X-ray spectrograph (EDS) map of MS55 created using the Oxford AZtec software. The order of phases in the key indicates the relative abundance of each phase; melt, orthopyroxene, clinopyroxene, biotite, FeTi oxides. 

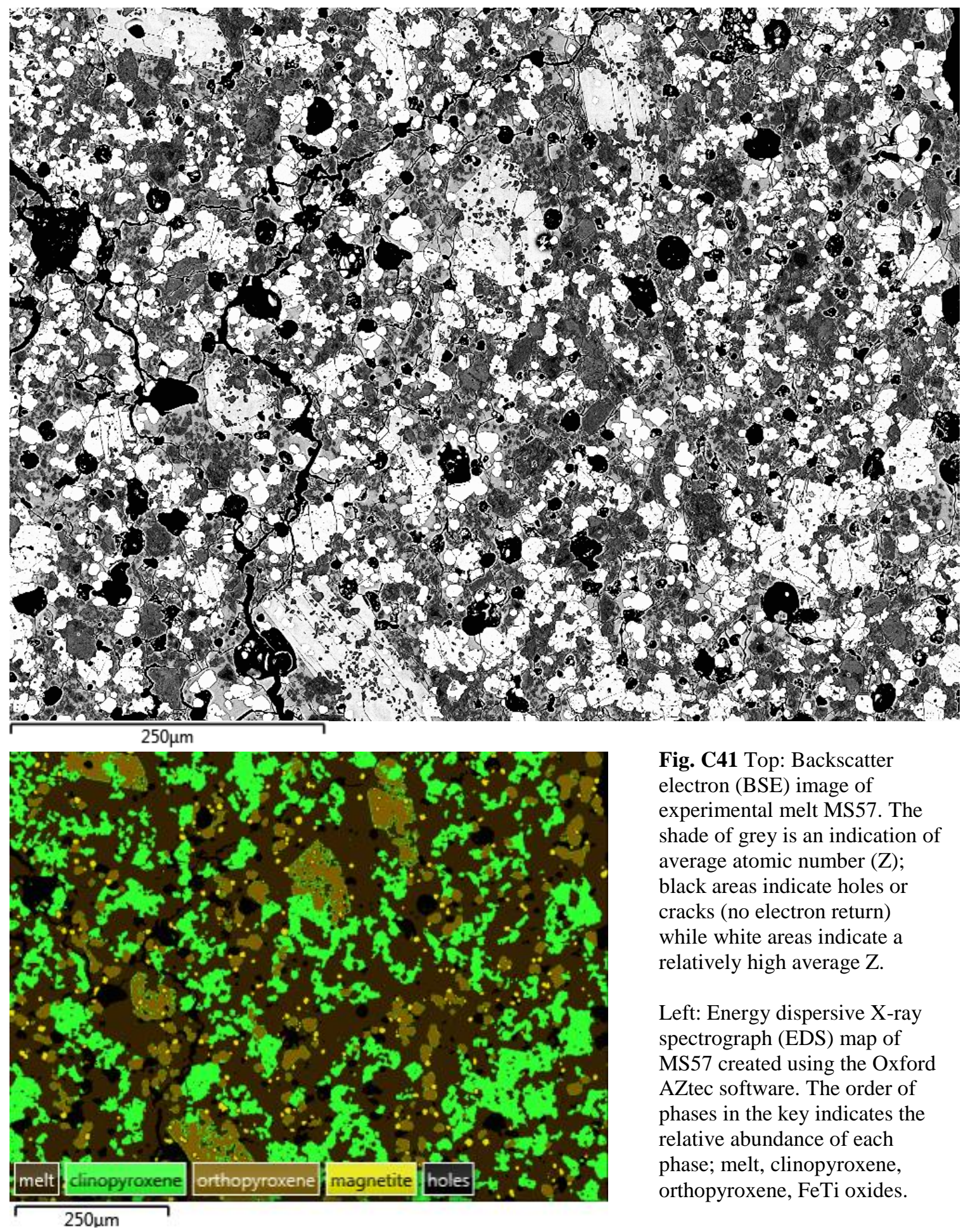

Fig. C41 Top: Backscatter electron (BSE) image of experimental melt MS57. The shade of grey is an indication of average atomic number (Z); black areas indicate holes or cracks (no electron return) while white areas indicate a relatively high average $\mathrm{Z}$.

Left: Energy dispersive X-ray spectrograph (EDS) map of MS57 created using the Oxford AZtec software. The order of phases in the key indicates the relative abundance of each phase; melt, clinopyroxene, orthopyroxene, FeTi oxides. 

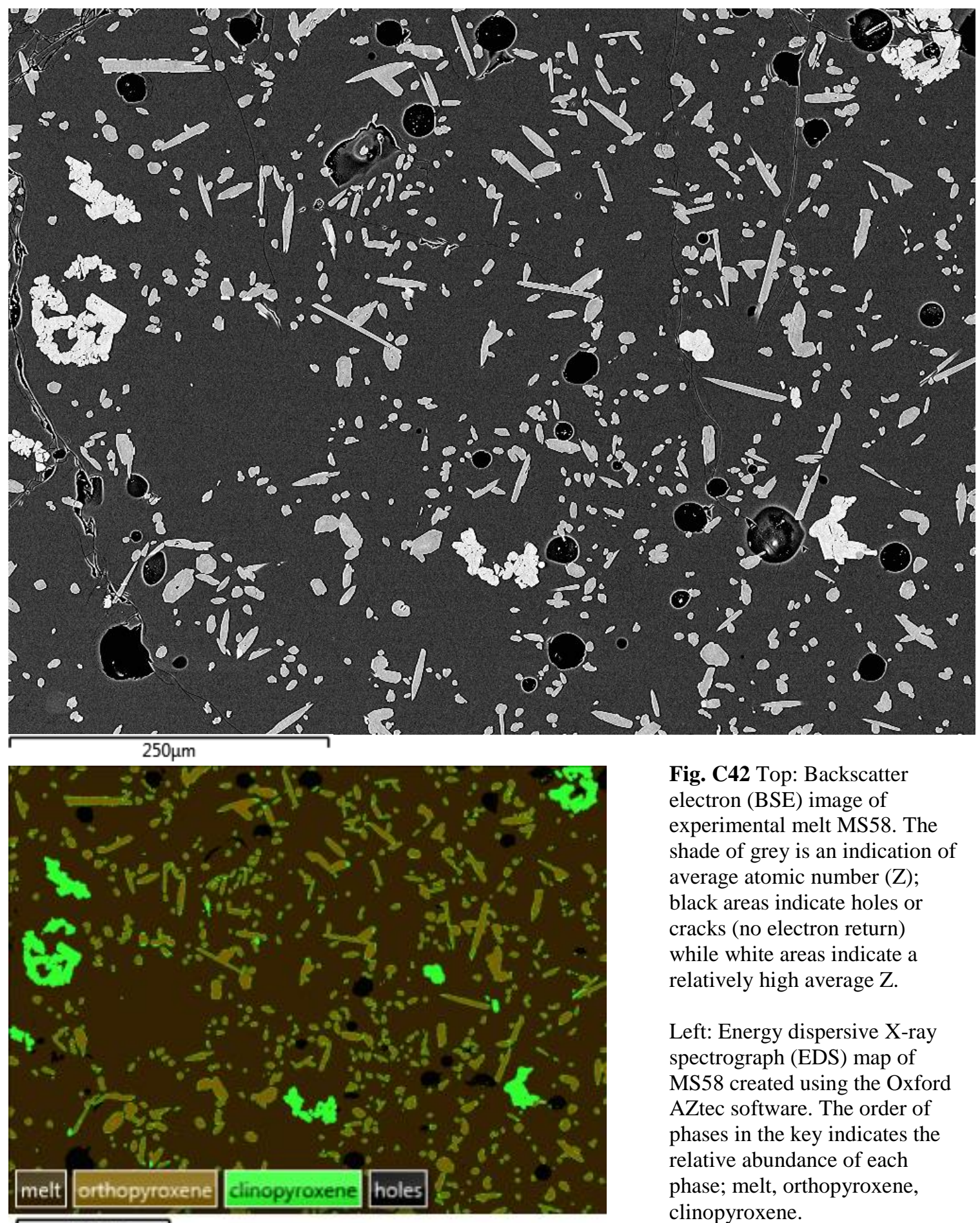

Fig. C42 Top: Backscatter electron (BSE) image of experimental melt MS58. The shade of grey is an indication of average atomic number (Z); black areas indicate holes or cracks (no electron return) while white areas indicate a relatively high average $\mathrm{Z}$.

Left: Energy dispersive X-ray spectrograph (EDS) map of MS58 created using the Oxford AZtec software. The order of phases in the key indicates the relative abundance of each phase; melt, orthopyroxene, clinopyroxene. 

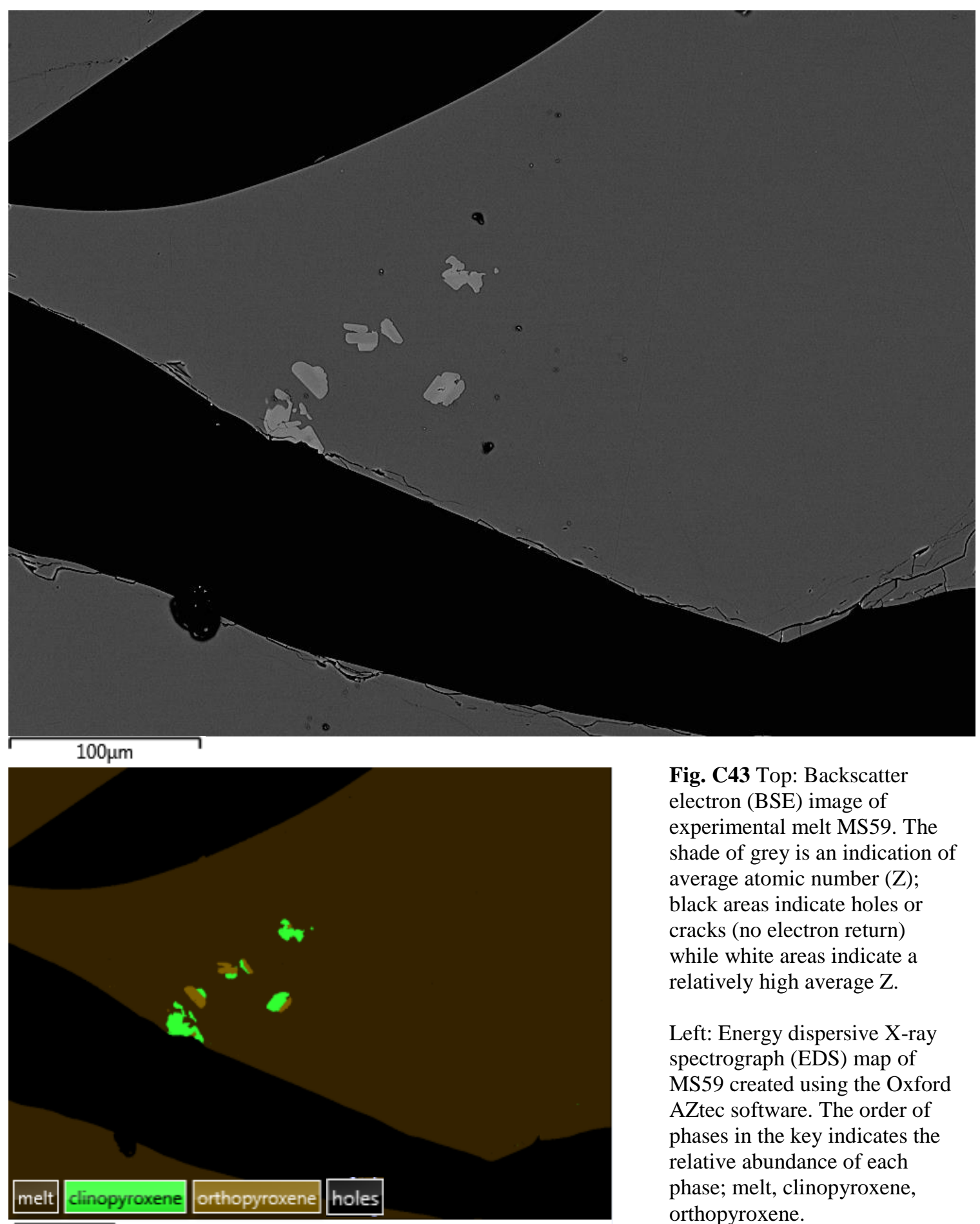

Fig. C43 Top: Backscatter electron (BSE) image of experimental melt MS59. The shade of grey is an indication of average atomic number (Z); black areas indicate holes or cracks (no electron return) while white areas indicate a relatively high average $\mathrm{Z}$.

Left: Energy dispersive X-ray spectrograph (EDS) map of MS59 created using the Oxford AZtec software. The order of phases in the key indicates the relative abundance of each phase; melt, clinopyroxene, orthopyroxene.

$100 \mu \mathrm{m}$ 

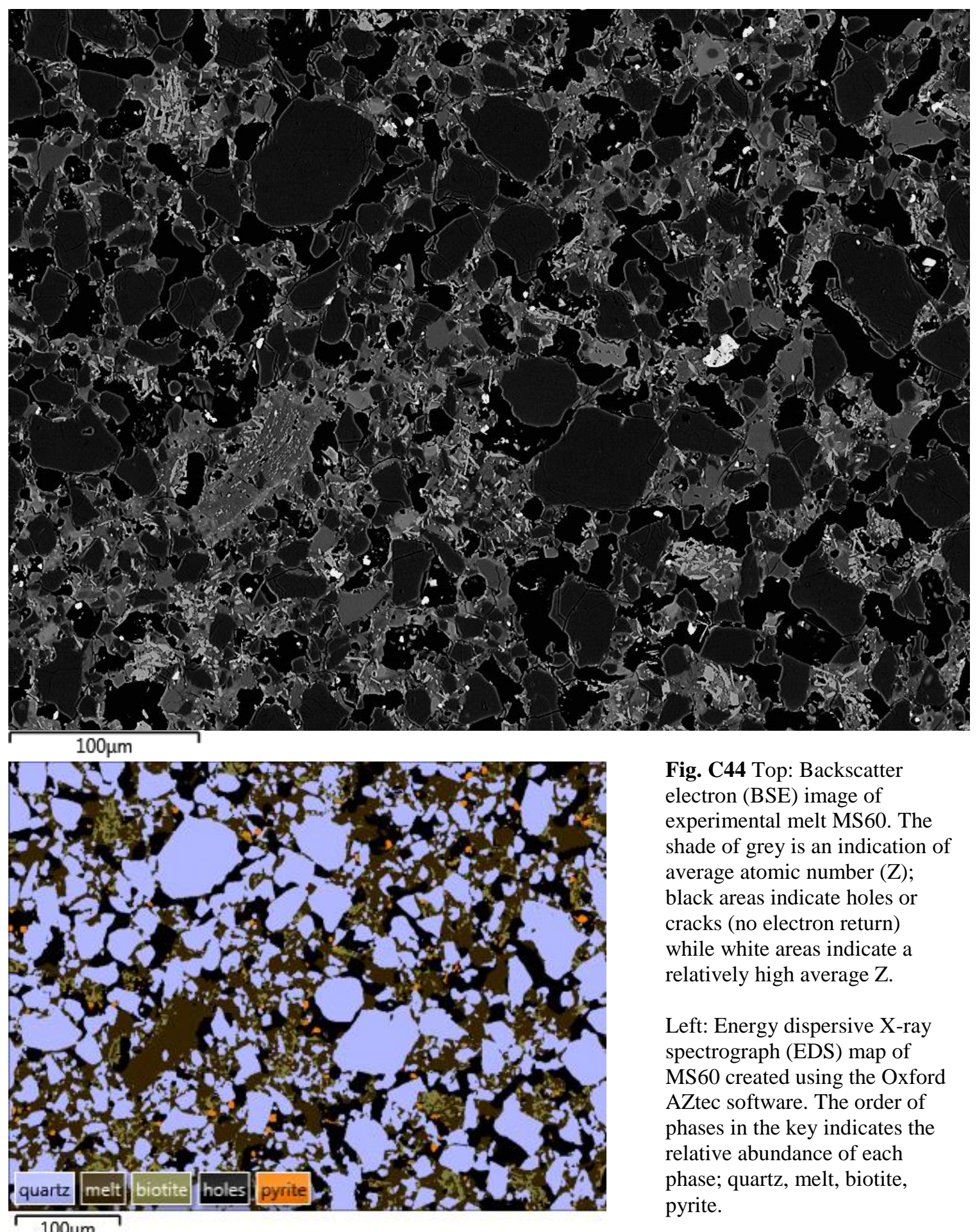

Fig. C44 Top: Backscatter electron (BSE) image of experimental melt MS60. The shade of grey is an indication of average atomic number (Z); black areas indicate holes or cracks (no electron return) while white areas indicate a relatively high average $\mathrm{Z}$.

Left: Energy dispersive X-ray spectrograph (EDS) map of MS60 created using the Oxford AZtec software. The order of phases in the key indicates the relative abundance of each phase; quartz, melt, biotite, pyrite. 

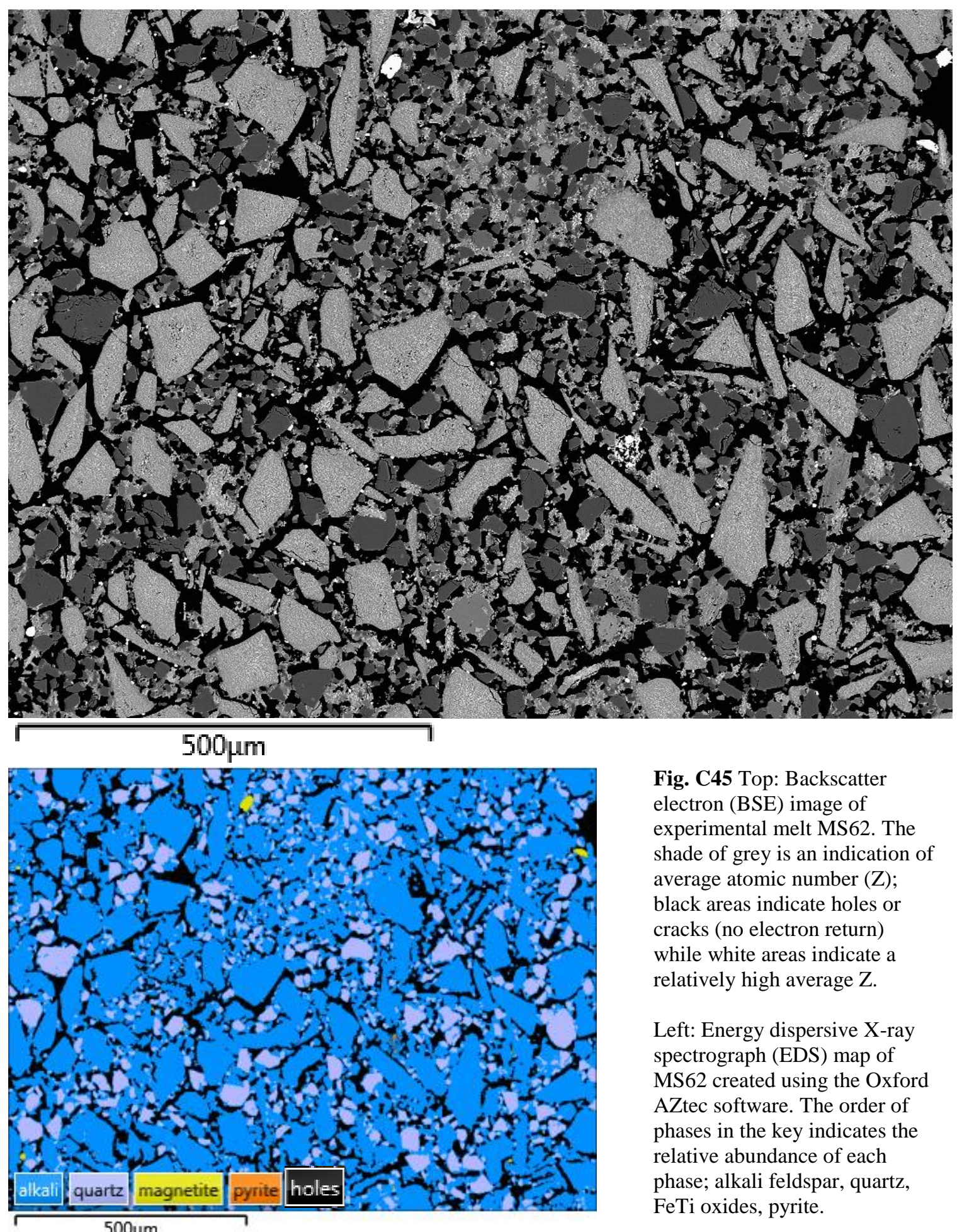

Fig. C45 Top: Backscatter electron (BSE) image of experimental melt MS62. The shade of grey is an indication of average atomic number $(\mathrm{Z})$; black areas indicate holes or cracks (no electron return) while white areas indicate a relatively high average $\mathrm{Z}$.

Left: Energy dispersive X-ray spectrograph (EDS) map of MS62 created using the Oxford AZtec software. The order of phases in the key indicates the relative abundance of each phase; alkali feldspar, quartz, FeTi oxides, pyrite. 

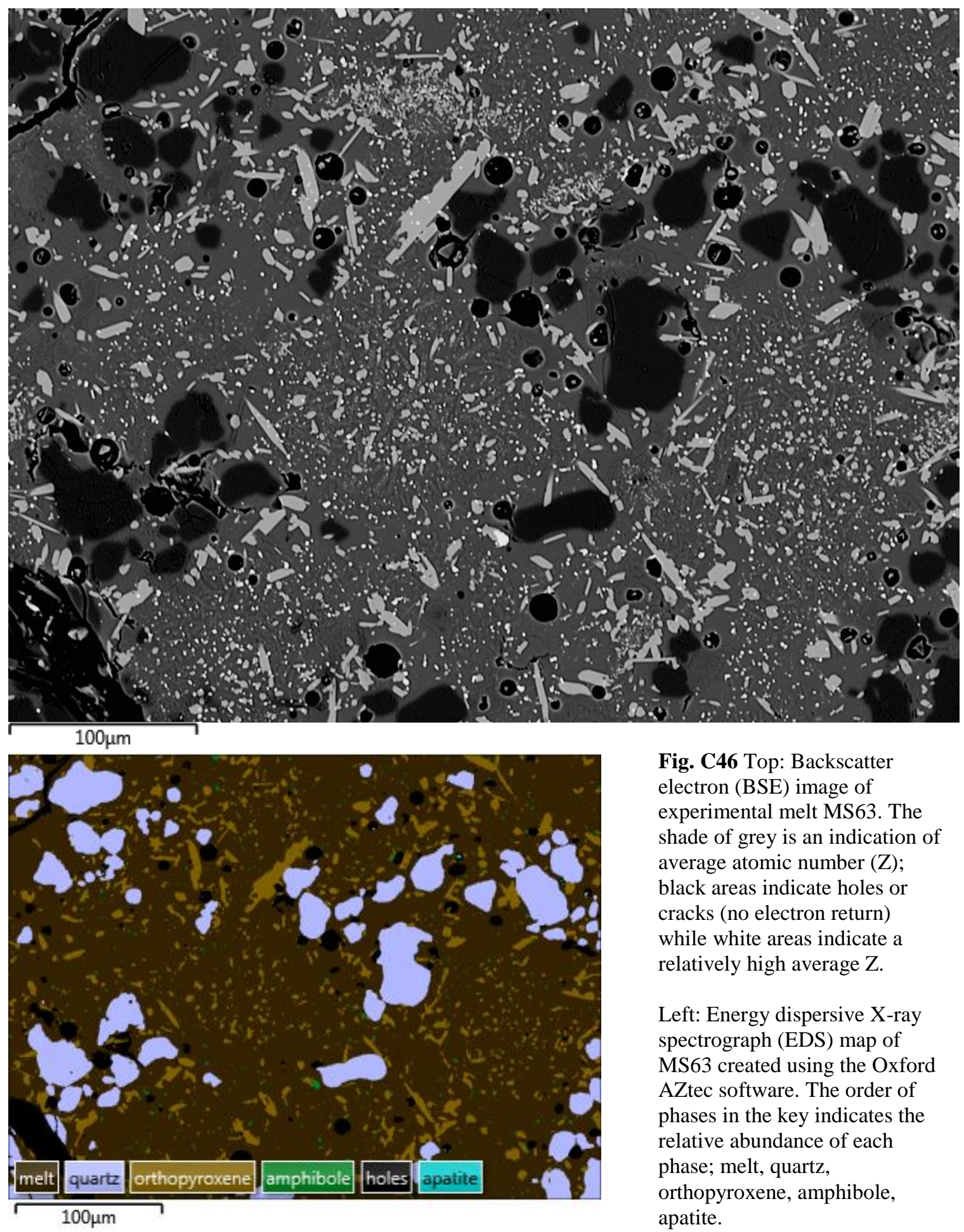

Fig. C46 Top: Backscatter electron (BSE) image of experimental melt MS63. The shade of grey is an indication of average atomic number (Z); black areas indicate holes or cracks (no electron return) while white areas indicate a relatively high average $\mathrm{Z}$.

Left: Energy dispersive X-ray spectrograph (EDS) map of MS63 created using the Oxford AZtec software. The order of phases in the key indicates the relative abundance of each phase; melt, quartz, orthopyroxene, amphibole, apatite. 

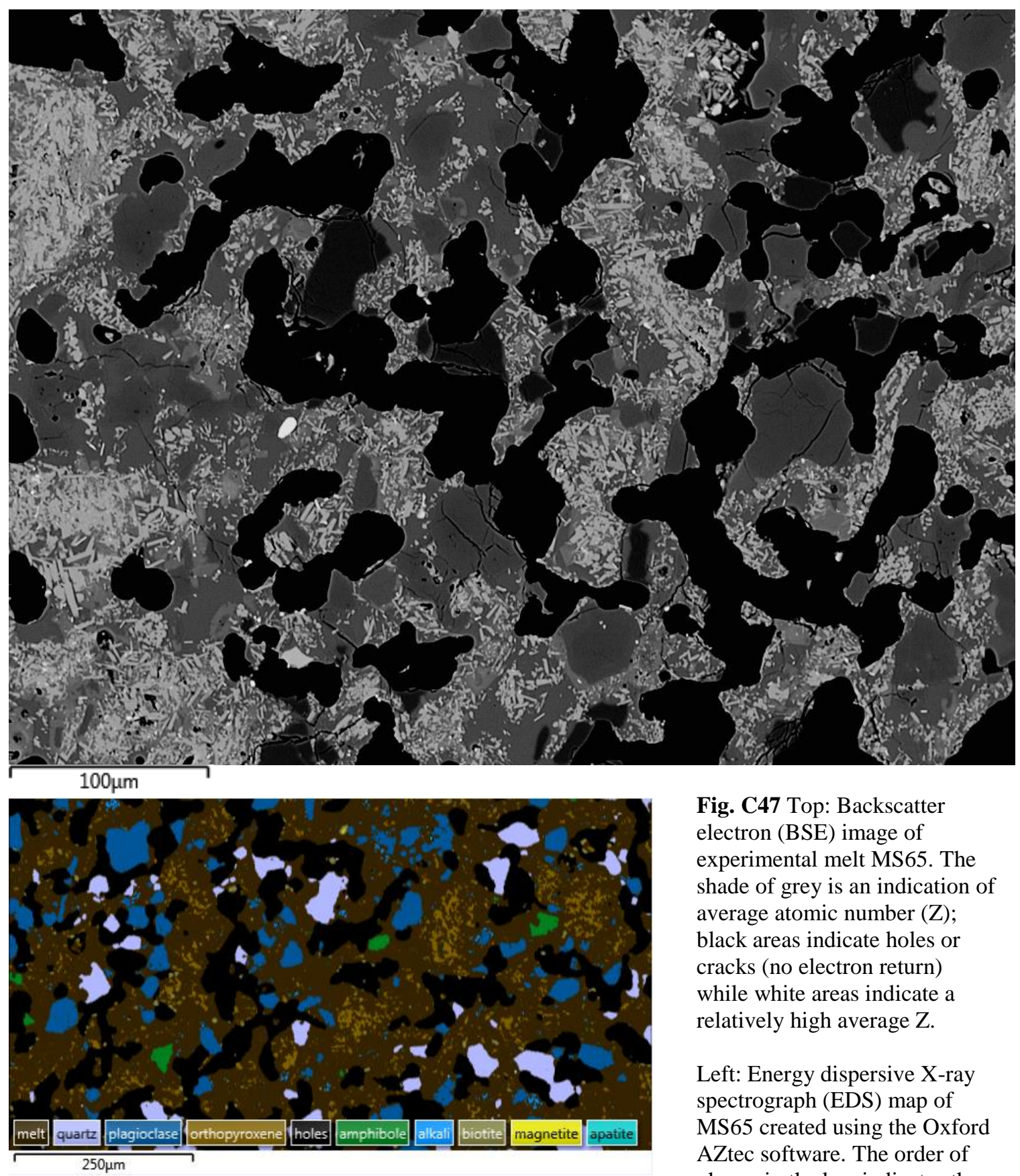

Fig. C47 Top: Backscatter electron (BSE) image of experimental melt MS65. The shade of grey is an indication of average atomic number (Z); black areas indicate holes or cracks (no electron return) while white areas indicate a relatively high average $\mathrm{Z}$.

Left: Energy dispersive X-ray spectrograph (EDS) map of MS65 created using the Oxford AZtec software. The order of phases in the key indicates the relative abundance of each phase; melt, quartz, plagioclase, orthopyroxene, amphibole, alkali feldspar, biotite, FeTi oxides, apatite. 
Table C1: Experimental melt compositions, capsules, and melting conditions

\begin{tabular}{|c|c|c|c|c|c|c|c|c|c|c|c|c|c|c|c|}
\hline \multirow[b]{2}{*}{ Conditions } & \multirow[b]{2}{*}{ Capsule } & \multirow[b]{2}{*}{$\begin{array}{c}\text { Parent } \\
\text { Material }\end{array}$} & \multirow[b]{2}{*}{$\begin{array}{l}\text { Capsule } \\
\text { Material }\end{array}$} & \multirow[b]{2}{*}{$\begin{array}{l}\text { Fluid } \\
\mathrm{X}_{\mathrm{H} 2 \mathrm{O}}\end{array}$} & \multicolumn{11}{|c|}{ Mass relations in charges } \\
\hline & & & & & $\begin{array}{l}\mathrm{H}_{2} \mathrm{O} \\
\mathrm{wt} \%\end{array}$ & $\begin{array}{c}\mathrm{H}_{2} \mathrm{O} \\
\mathrm{mg}\end{array}$ & $\begin{array}{c}\mathrm{Ag}_{2} \mathrm{C}_{2} \mathrm{O}_{4} \\
\mathrm{mg}\end{array}$ & $\begin{array}{l}\text { glass } \\
\text { powder } \\
\mathrm{mg}\end{array}$ & $\begin{array}{c}\text { capsule } \\
\text { mg }\end{array}$ & $\begin{array}{c}\text { capsule } \\
+\mathrm{H}_{2} \mathrm{O}\end{array}$ & $\begin{array}{c}\text { capsule } \\
+\mathrm{H}_{2} \mathrm{O}+ \\
\text { glass }+ \\
\mathrm{Ag}_{2} \mathrm{C}_{2} \mathrm{O}_{4}\end{array}$ & $\begin{array}{l}\text { capsule } \\
+\mathrm{H}_{2} \mathrm{O} \\
+ \text { glass } \\
\end{array}$ & $\begin{array}{c}\text { the } \\
\text { same } \\
\text { after } \\
\text { welding } \\
\end{array}$ & $\begin{array}{c}\text { the } \\
\text { same } \\
\text { after } \\
\text { drying }\end{array}$ & $\begin{array}{l}\text { after } \\
\text { run }\end{array}$ \\
\hline \multirow{6}{*}{ 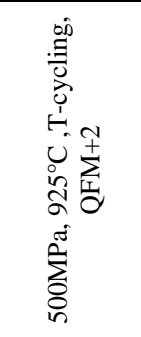 } & MS1 & $\begin{array}{c}\text { MS-12- } \\
47\end{array}$ & $\mathrm{Au}$ & dry & & & & 39.55 & 567.78 & & & 607.33 & & & \\
\hline & MS2 & TM13-3 & $\mathrm{Au}$ & dry & & & & 39.46 & 450.03 & & & 489.76 & & & \\
\hline & MS3 & TM13-5 & $\mathrm{Au}$ & dry & & & & 39.93 & 499.47 & & & 539.40 & & & \\
\hline & MS4 & $\begin{array}{c}\text { MS-12- } \\
47\end{array}$ & $\mathrm{Au}$ & $<1$ & 5.19 & 2.09 & & 40.25 & 574.53 & 576.62 & & 616.87 & & 616.39 & \\
\hline & MS5 & TM13-3 & $\mathrm{Au}$ & $<1$ & 3.40 & 1.39 & & 40.85 & 627.96 & 629.35 & & 670.20 & & 669.92 & \\
\hline & MS6 & TM13-5 & $\mathrm{Au}$ & $<1$ & 2.94 & 1.20 & & 40.79 & 632.63 & 633.83 & & 674.62 & & 674.46 & \\
\hline \multirow{6}{*}{ 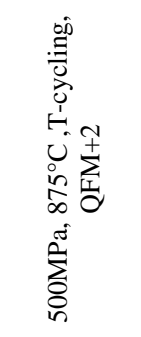 } & MS15 & $\begin{array}{c}\text { MS-12- } \\
47\end{array}$ & $\mathrm{Au}$ & dry & 0 & 0 & & 39.63 & 432.92 & 432.92 & & 472.55 & 472.49 & & 472.41 \\
\hline & MS16 & TM13-3 & $\mathrm{Au}$ & $\approx 3 \%$ & 3.13 & 1.2 & & 38.28 & 579.52 & 580.72 & & 619.00 & 618.93 & & 618.81 \\
\hline & MS17 & TM13-5 & $\mathrm{Au}$ & dry & 0 & 0 & & 39.96 & 488.74 & 488.74 & & 528.70 & 528.59 & & 528.54 \\
\hline & MS18 & $\begin{array}{c}\text { MS-12- } \\
47\end{array}$ & $\mathrm{Au}$ & $\approx 3 \%$ & 3.27 & 1.32 & & 40.40 & 553.94 & 555.26 & & 594.34 & 594.18 & & 595.15 \\
\hline & MS19 & TM13-3 & $\mathrm{Au}$ & $\approx 1 \%$ & 1.06 & 0.42 & & 39.44 & 573.23 & 573.65 & & 613.09 & 612.40 & & 612.33 \\
\hline & MS20 & TM13-5 & $\mathrm{Au}$ & $\approx 4 \%$ & 4.10 & 1.66 & & 40.50 & 731.7 & 733.36 & & 773.86 & 773.82 & & 773.03 \\
\hline \multirow{6}{*}{ 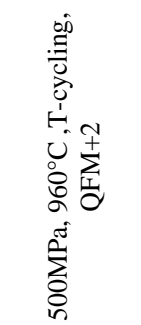 } & MS7 & $\begin{array}{c}\text { MS-12- } \\
47\end{array}$ & $\mathrm{Au}$ & dry & & & & 40.20 & 559.67 & & & 592.44 & & 592.29 & 614.94 \\
\hline & MS8 & TM13-3 & $\mathrm{Au}$ & dry & & & & 40.75 & 516.09 & & & 555.87 & & 555.86 & 555.62 \\
\hline & MS9 & TM13-5 & $\mathrm{Au}$ & dry & & & & 40.45 & 519.67 & & & 559.01 & & 522.86 & 522.70 \\
\hline & MS13 & TM13-3 & $\mathrm{Au}$ & $\approx 3 \%$ & 1.95 & 0.78 & & 40.02 & 483.05 & 483.83 & & 522.14 & 522.08 & 522.12 & 521.89 \\
\hline & MS22 & TM13-5 & $\mathrm{Au}$ & $\approx 3 \%$ & 2.95 & 1.20 & & 40.68 & 549.98 & 552.51 & & 589.10 & & 589.12 & 588.86 \\
\hline & MS23 & TM13-1 & $\mathrm{Au}$ & dry & & & & 39.55 & 562.06 & & & 599.99 & & 599.96 & 599.71 \\
\hline
\end{tabular}




\begin{tabular}{|c|c|c|c|c|c|c|c|c|c|c|c|c|c|c|}
\hline \multirow{6}{*}{ 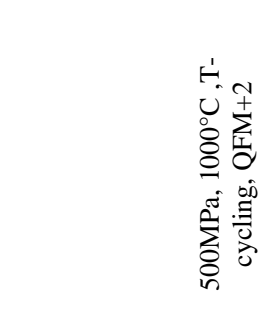 } & \multirow{2}{*}{$\begin{array}{l}\text { MS24 } \\
\text { MS25 }\end{array}$} & $* 50 \mathrm{M} 2 \mathrm{~A}$ & $\mathrm{Au}$ & dry & & 7 & 40 & 519.77 & & 526.23 & 566.90 & & 520.87 & 517.76 \\
\hline & & $* 50 \mathrm{M} 2 \mathrm{~B}$ & $\mathrm{Au}$ & dry & & 7 & 40 & 545.86 & & 553.24 & 597.18 & 597.18 & 596.91 & 593.83 \\
\hline & MS26 & $* 50 \mathrm{M} 2 \mathrm{C}$ & $\mathrm{Au}$ & dry & & 7 & 40 & 520.02 & & 527.23 & 566.67 & 533.43 & 533.37 & 532.92 \\
\hline & MS27 & $* 50 \mathrm{M} 3 \mathrm{~A}$ & $\mathrm{Au}$ & dry & & 7 & 40 & 537.6 & & 544.45 & 583.84 & 583.74 & 583.58 & 583.02 \\
\hline & MS28 & $* 50 \mathrm{M} 3 \mathrm{~B}$ & $\mathrm{Au}$ & dry & & 7 & 40 & 534.85 & & 541.38 & 583.89 & 583.9 & 583.89 & 583.05 \\
\hline & MS29 & $* 50 \mathrm{M} 3 \mathrm{C}$ & $\mathrm{Au}$ & dry & & 7 & 40 & 491.42 & & 497.99 & 539.07 & 539.07 & 538.89 & 538.88 \\
\hline \multirow{6}{*}{$\vec{A}$} & MS30 & $* 50 \mathrm{M} 1 \mathrm{~A}$ & $\mathrm{Au}$ & dry & & 7 & 40 & 529.86 & & 536.20 & 576.20 & 562.69 & 562.68 & 561.19 \\
\hline & MS31 & $* 50 \mathrm{M} 1 \mathrm{~B}$ & $\mathrm{Au}$ & dry & & 7 & 40 & 511.02 & & 517.98 & 559.52 & 558.28 & 558.15 & 554.87 \\
\hline & MS32 & *50M1C & $\mathrm{Au}$ & dry & & 7 & 40 & 513.90 & & 520.88 & 562.38 & 546.39 & 546.31 & 543.87 \\
\hline & MS33 & *50M1D & $\mathrm{Au}$ & dry & & 7 & 40 & 515.97 & & 522.99 & 562.24 & 560.52 & 560.54 & 558.17 \\
\hline & MS34 & $* 50 \mathrm{M} 2 \mathrm{D}$ & $\mathrm{Au}$ & dry & & 7 & 40 & 538.36 & & 545.63 & 586.31 & 586.11 & 586.17 & 585.63 \\
\hline & MS35 & *50M3D & $\mathrm{Au}$ & dry & & 7 & 40 & 512.56 & & 519.58 & 559.45 & 557.72 & 557.7 & 557.99 \\
\hline \multirow{6}{*}{ 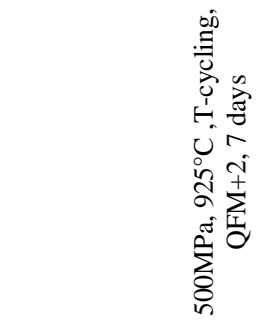 } & MS36 & TM13-1 & $\mathrm{Au}$ & dry & 0 & - & 40.48 & 528.31 & & & 569.90 & 568.95 & 568.86 & 568.13 \\
\hline & MS37 & TM13-1 & $\mathrm{Au}$ & $\sim 2 \%$ & 0.8 & - & 40.51 & 537.49 & 538.38 & & 577.38 & 577.22 & 577.09 & 577.00 \\
\hline & MS38 & TM13-3 & $\mathrm{Au}$ & $\sim 4 \%$ & 1.6 & - & 40.22 & 639.26 & 640.94 & & 680.45 & 680.36 & 680.34 & 684.45 \\
\hline & MS39 & TM13-3 & $\mathrm{Au}$ & $\sim 6 \%$ & 2.4 & - & 40.58 & 621.09 & 623.62 & & 663.91 & 663.66 & 663.64 & 663.47 \\
\hline & MS40 & TM13-5 & $\mathrm{Au}$ & $\sim 4 \%$ & 1.6 & - & 40.15 & 563.54 & 565.17 & & 604.28 & 604.14 & 604.06 & 603.91 \\
\hline & MS41 & TM13-5 & $\mathrm{Au}$ & $\sim 6 \%$ & 2.4 & - & 40.52 & 505.1 & 507.51 & & 547.24 & 547.05 & 547.02 & 546.92 \\
\hline \multirow{6}{*}{ 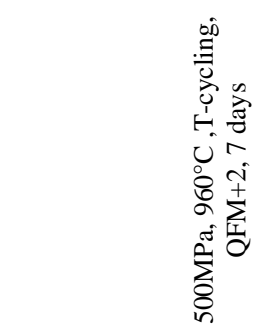 } & MS42 & $* 50 \mathrm{M} 2 \mathrm{~A}$ & $\mathrm{Au}$ & dry & & 4.02 & 40.61 & 542.82 & & 587.23 & & 586.93 & 586.9 & 584.88 \\
\hline & MS43 & $* 50 \mathrm{M} 2 \mathrm{~B}$ & $\mathrm{Au}$ & dry & & 4.09 & 40.41 & 526.58 & & 570.16 & & 569.45 & 569.44 & 569.35 \\
\hline & MS44 & $* 50 \mathrm{M} 2 \mathrm{C}$ & $\mathrm{Au}$ & dry & & 4.01 & 40.43 & 512.3 & & 556.44 & & 556.29 & 556.27 & 555.90 \\
\hline & MS45 & $* 50 \mathrm{M} 3 \mathrm{~A}$ & $\mathrm{Au}$ & dry & & 4.01 & 40.46 & 532.27 & & 576.67 & & 576.41 & 576.37 & 576.18 \\
\hline & MS46 & $* 50 \mathrm{M} 3 \mathrm{~B}$ & $\mathrm{Au}$ & dry & & 4.06 & 40.44 & 536.15 & & 580.09 & & 580.01 & 579.97 & 579.82 \\
\hline & MS47 & *50M3C & $\mathrm{Au}$ & dry & & 4.22 & 40.54 & 527.03 & & 570.99 & & 570.88 & 570.84 & 570.62 \\
\hline
\end{tabular}




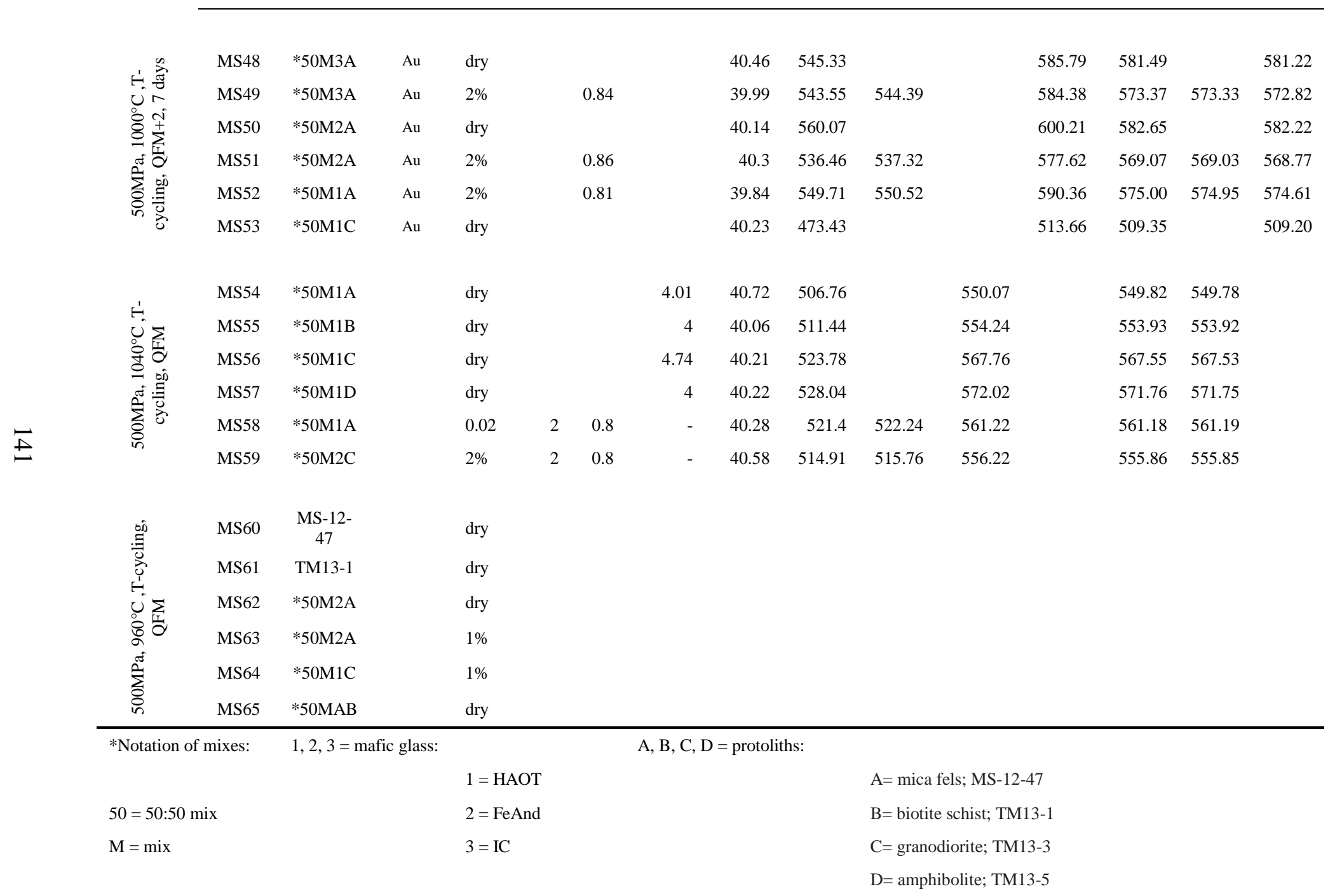


Table C2: Unormalized phase proportions and phase compositions

\begin{tabular}{|c|c|c|c|c|c|c|c|c|c|c|c|c|}
\hline \multirow[t]{13}{*}{ MS1 } & \multicolumn{3}{|c|}{ melt: $78.7 \%$} & \multicolumn{3}{|c|}{ quartz: $16.9 \%$} & \multicolumn{3}{|c|}{ orthopyroxene: $4.5 \%$} & \multicolumn{3}{|c|}{ pyrite: $0.01 \%$} \\
\hline & element & $\begin{array}{c}\text { atomic } \\
\%\end{array}$ & Wt $\%$ & element & $\begin{array}{c}\text { atomic } \\
\%\end{array}$ & $\mathrm{Wt} \%$ & element & $\begin{array}{c}\text { atomic } \\
\%\end{array}$ & Wt $\%$ & element & $\begin{array}{c}\text { atomic } \\
\%\end{array}$ & Wt \% \\
\hline & $\mathrm{O}$ & 64.61 & 49.74 & $\mathrm{O}$ & 66.56 & 52.90 & $\mathrm{O}$ & 62.20 & 45.42 & $\mathrm{O}$ & 66.64 & 42.24 \\
\hline & $\mathrm{Al}$ & 5.01 & 6.50 & $\mathrm{Si}$ & 33.24 & 46.39 & $\mathrm{Na}$ & 0.43 & 0.45 & $\mathrm{Si}$ & 0.62 & 0.69 \\
\hline & $\mathrm{Si}$ & 27.38 & 37.01 & $\mathrm{Fe}$ & 0.07 & 0.20 & $\mathrm{Mg}$ & 6.62 & 7.35 & $\mathrm{~S}$ & 16.32 & 20.74 \\
\hline & K & 1.66 & 3.13 & $\mathrm{Br}$ & 0.13 & 0.51 & $\mathrm{Al}$ & 2.95 & 3.63 & $\mathrm{Fe}$ & 16.42 & 36.33 \\
\hline & $\mathrm{Ca}$ & 0.24 & 0.46 & & & & $\mathrm{Si}$ & 19.85 & 25.44 & & & \\
\hline & $\mathrm{Ti}$ & 0.16 & 0.37 & & & & $\mathrm{~S}$ & 1.70 & 2.48 & & & \\
\hline & $\mathrm{Fe}$ & 0.89 & 2.39 & & & & K & 0.68 & 1.21 & & & \\
\hline & $\mathrm{Ba}$ & 0.06 & 0.40 & & & & $\mathrm{Ca}$ & 0.16 & 0.29 & & & \\
\hline & & & & & & & $\mathrm{Ti}$ & 0.18 & 0.40 & & & \\
\hline & & & & & & & V & 0.04 & 0.09 & & & \\
\hline & & & & & & & $\mathrm{Fe}$ & 5.20 & 13.24 & & & \\
\hline \multirow[t]{12}{*}{ MS3 } & \multicolumn{3}{|c|}{ clinopyroxene: $76.9 \%$} & \multicolumn{3}{|c|}{ plagioclase: $14.6 \%$} & \multicolumn{3}{|c|}{ melt: $2.2 \%$} & & & \\
\hline & element & $\begin{array}{c}\text { atomic } \\
\%\end{array}$ & Wt $\%$ & element & $\begin{array}{c}\text { atomic } \\
\%\end{array}$ & Wt $\%$ & element & $\begin{array}{c}\text { atomic } \\
\%\end{array}$ & Wt $\%$ & & & \\
\hline & $\mathrm{O}$ & 60.36 & 43.79 & $\mathrm{O}$ & 54.26 & 38.81 & $\mathrm{O}$ & 61.76 & 46.97 & & & \\
\hline & $\mathrm{Na}$ & 1.49 & 1.55 & $\mathrm{Na}$ & 0.83 & 0.85 & $\mathrm{Na}$ & 2.30 & 2.51 & & & \\
\hline & $\mathrm{Mg}$ & 5.16 & 5.69 & $\mathrm{Al}$ & 16.93 & 20.41 & $\mathrm{Al}$ & 8.70 & 11.16 & & & \\
\hline & $\mathrm{Al}$ & 6.59 & 8.06 & $\mathrm{Si}$ & 19.42 & 24.38 & $\mathrm{Si}$ & 23.14 & 30.89 & & & \\
\hline & $\mathrm{Si}$ & 17.93 & 22.84 & $\mathrm{Ca}$ & 8.28 & 14.83 & K & 0.34 & 0.63 & & & \\
\hline & K & 0.11 & 0.20 & $\mathrm{Fe}$ & 0.29 & 0.73 & $\mathrm{Ca}$ & 2.83 & 5.40 & & & \\
\hline & $\mathrm{Ca}$ & 4.45 & 8.09 & & & & $\mathrm{Ti}$ & 0.12 & 0.27 & & & \\
\hline & $\mathrm{Ti}$ & 0.30 & 0.64 & & & & $\mathrm{Mn}$ & 0.13 & 0.33 & & & \\
\hline & $\mathrm{Mn}$ & 0.08 & 0.19 & & & & $\mathrm{Fe}$ & 0.70 & 1.85 & & & \\
\hline & $\mathrm{Fe}$ & 3.53 & 8.94 & & & & & & & & & \\
\hline \multirow[t]{9}{*}{ MS4 } & \multicolumn{3}{|c|}{ quartz: $41.9 \%$} & \multicolumn{3}{|c|}{ melt: $40.6 \%$} & \multicolumn{3}{|c|}{ plagioclase: $7.7 \%$} & \multicolumn{3}{|c|}{ orthopyroxene: $6.1 \%$} \\
\hline & element & $\begin{array}{c}\text { atomic } \\
\%\end{array}$ & Wt $\%$ & element & $\begin{array}{c}\text { atomic } \\
\%\end{array}$ & Wt $\%$ & element & $\begin{array}{c}\text { atomic } \\
\%\end{array}$ & Wt $\%$ & element & $\begin{array}{c}\text { atomic } \\
\%\end{array}$ & Wt $\%$ \\
\hline & $\mathrm{O}$ & 66.26 & 52.21 & $\mathrm{O}$ & 63.27 & 48.28 & $\mathrm{O}$ & 62.18 & 47.65 & $\mathrm{O}$ & 61.52 & 43.95 \\
\hline & $\mathrm{Si}$ & 32.99 & 45.63 & $\mathrm{Na}$ & 1.47 & 1.61 & $\mathrm{Na}$ & 0.34 & 0.37 & $\mathrm{Na}$ & 0.58 & 0.59 \\
\hline & K & 0.32 & 0.61 & $\mathrm{Al}$ & 5.99 & 7.71 & $\mathrm{Mg}$ & 3.97 & 4.63 & $\mathrm{Mg}$ & 5.80 & 6.30 \\
\hline & $\mathrm{Fe}$ & 0.13 & 0.35 & $\mathrm{Si}$ & 25.22 & 33.78 & $\mathrm{Al}$ & 12.12 & 15.67 & $\mathrm{Al}$ & 4.41 & 5.31 \\
\hline & $\mathrm{Br}$ & 0.30 & 1.19 & S & 0.11 & 0.17 & $\mathrm{Si}$ & 18.73 & 25.19 & $\mathrm{Si}$ & 18.65 & 23.39 \\
\hline & & & & K & 2.57 & 4.79 & K & 0.65 & 1.21 & S & 1.09 & 1.56 \\
\hline & & & & $\mathrm{Ca}$ & 0.36 & 0.69 & $\mathrm{Ca}$ & 0.09 & 0.18 & K & 0.80 & 1.39 \\
\hline
\end{tabular}




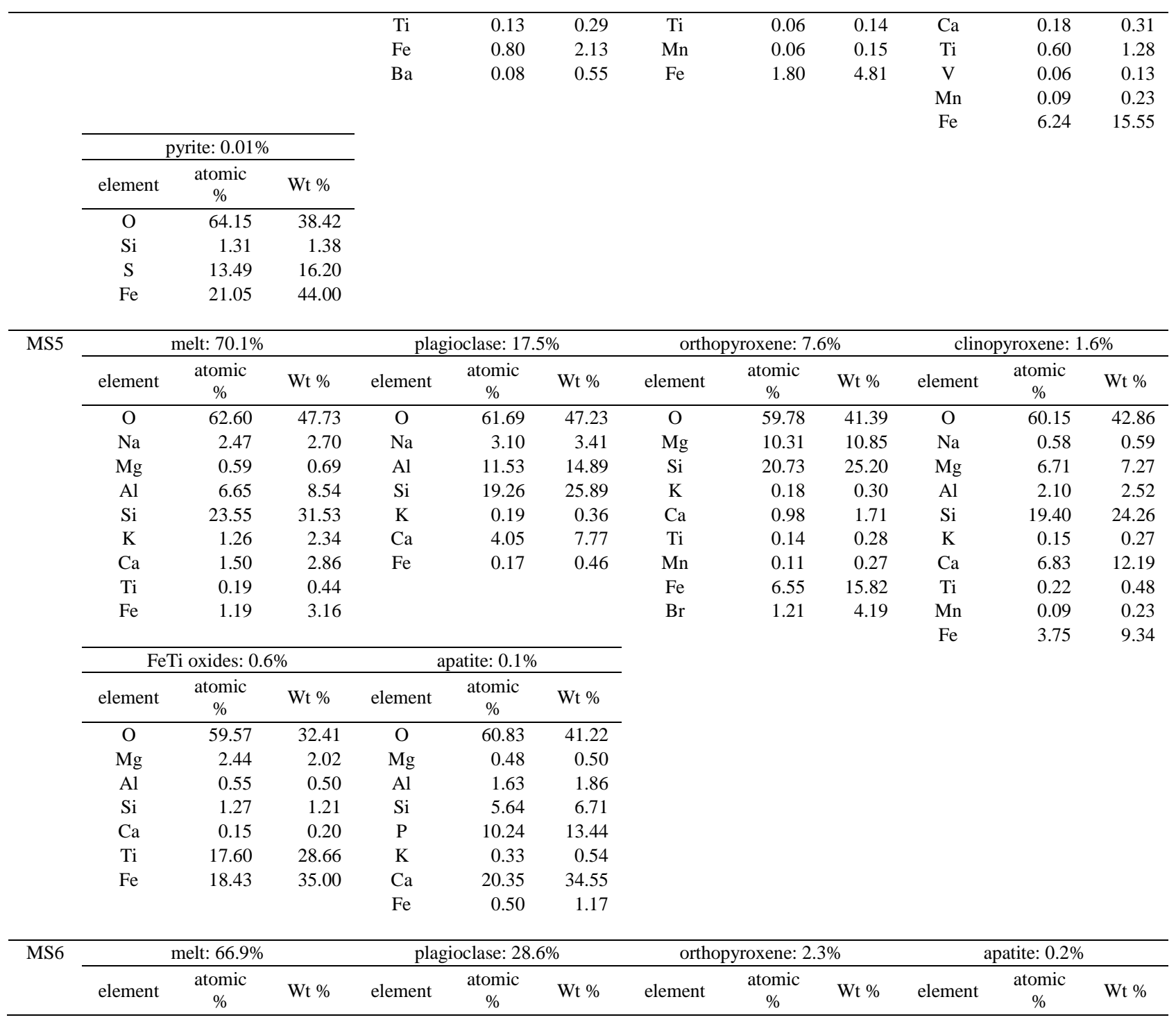




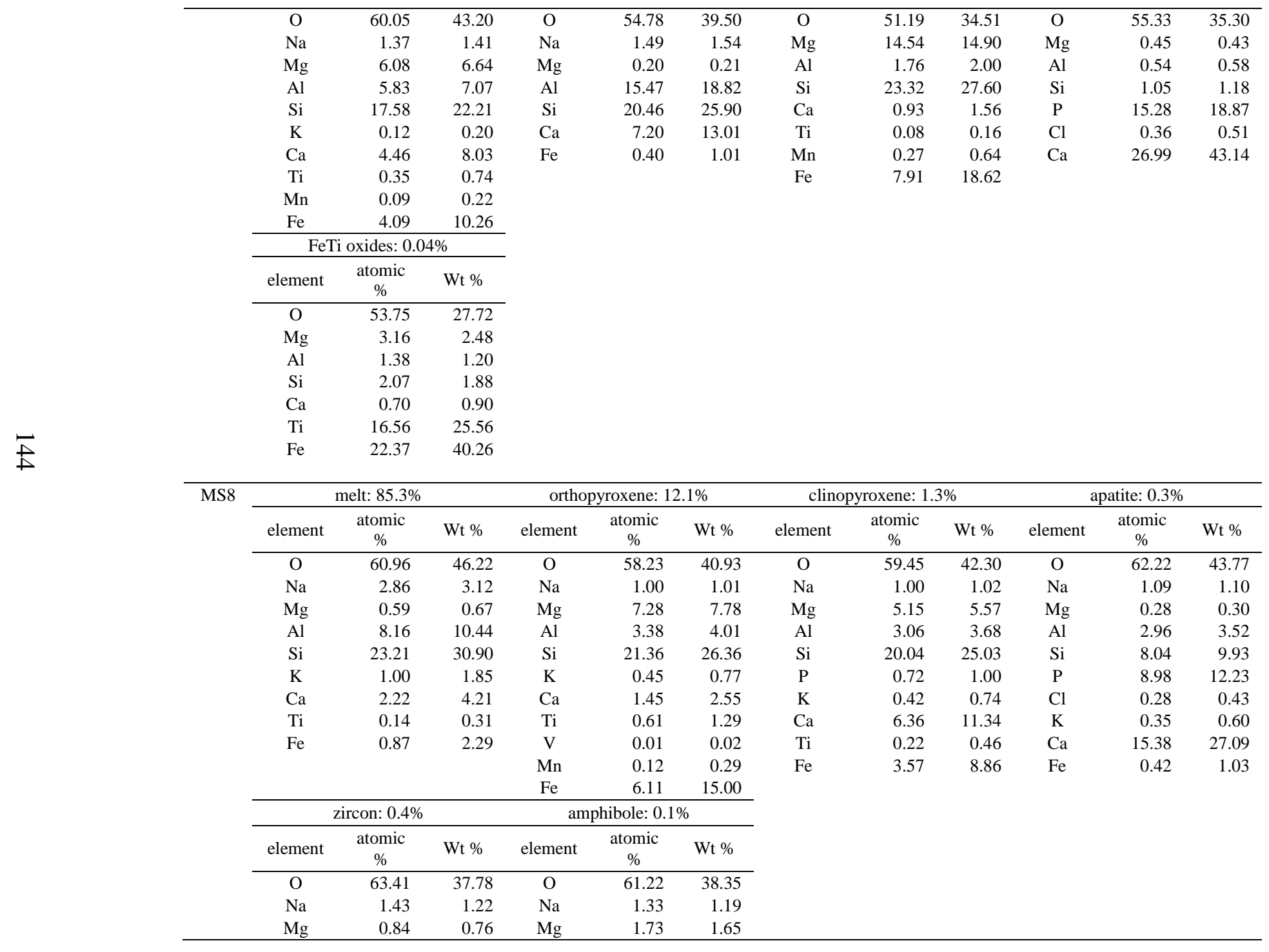




\begin{tabular}{|c|c|c|c|c|c|c|c|c|c|c|c|c|}
\hline & $\mathrm{Al}$ & 2.28 & 2.29 & $\mathrm{Al}$ & 2.86 & 3.02 & & & & & & \\
\hline & $\mathrm{Si}$ & 19.55 & 20.45 & $\mathrm{Si}$ & 11.31 & 12.43 & & & & & & \\
\hline & $\mathrm{K}$ & 0.75 & 1.09 & $\mathrm{~K}$ & 0.64 & 0.99 & & & & & & \\
\hline & $\mathrm{Ca}$ & 1.47 & 2.19 & $\mathrm{Ca}$ & 0.58 & 0.91 & & & & & & \\
\hline & $\mathrm{Fe}$ & 0.53 & 1.11 & $\mathrm{Ti}$ & 9.61 & 18.02 & & & & & & \\
\hline & $\mathrm{Zr}$ & 9.75 & 33.12 & $\mathrm{Fe}$ & 10.72 & 23.44 & & & & & & \\
\hline \multirow[t]{18}{*}{ MS9 } & \multicolumn{3}{|c|}{ melt: $50.3 \%$} & \multicolumn{3}{|c|}{ clinopyroxene: $42.6 \%$} & \multicolumn{3}{|c|}{ orthopyroxene: $4.5 \%$} & \multicolumn{3}{|c|}{ FeTi oxides: $1.6 \%$} \\
\hline & element & $\begin{array}{c}\text { atomic } \\
\%\end{array}$ & Wt $\%$ & element & $\begin{array}{c}\text { atomic } \\
\%\end{array}$ & Wt $\%$ & element & $\begin{array}{c}\text { atomic } \\
\%\end{array}$ & Wt $\%$ & element & $\begin{array}{c}\text { atomic } \\
\%\end{array}$ & Wt $\%$ \\
\hline & $\mathrm{O}$ & 60.75 & 44.90 & $\mathrm{O}$ & 60.00 & 43.00 & $\mathrm{O}$ & 58.92 & 42.34 & $\mathrm{O}$ & 53.33 & 27.11 \\
\hline & $\mathrm{Na}$ & 1.48 & 1.58 & $\mathrm{Na}$ & 1.00 & 1.00 & $\mathrm{Mg}$ & 15.20 & 16.59 & $\mathrm{Mg}$ & 2.77 & 2.14 \\
\hline & $\mathrm{Mg}$ & 4.21 & 4.72 & $\mathrm{Mg}$ & 5.54 & 5.62 & $\mathrm{Al}$ & 1.51 & 1.83 & $\mathrm{Al}$ & 5.00 & 4.29 \\
\hline & $\mathrm{Al}$ & 8.58 & 10.70 & $\mathrm{Al}$ & 3.10 & 3.82 & $\mathrm{Si}$ & 16.90 & 21.31 & $\mathrm{Si}$ & 1.92 & 1.71 \\
\hline & $\mathrm{Si}$ & 17.81 & 23.11 & $\mathrm{Si}$ & 20.04 & 25.03 & $\mathrm{Ca}$ & 1.00 & 1.80 & $\mathrm{Ca}$ & 0.60 & 0.76 \\
\hline & $\mathrm{K}$ & 0.08 & 0.14 & $\mathrm{Ca}$ & 6.70 & 12.53 & $\mathrm{Ti}$ & 0.19 & 0.42 & $\mathrm{Ti}$ & 2.24 & 3.41 \\
\hline & $\mathrm{Ca}$ & 4.63 & 8.57 & $\mathrm{Fe}$ & 3.62 & 9.00 & $\mathrm{Mn}$ & 0.85 & 2.11 & $\mathrm{Fe}$ & 34.13 & 60.57 \\
\hline & $\mathrm{Ti}$ & 0.19 & 0.41 & & & & $\mathrm{Fe}$ & 5.42 & 13.60 & & & \\
\hline & $\mathrm{Mn}$ & 0.05 & 0.11 & & & & & & & & & \\
\hline & $\mathrm{Fe}$ & 2.23 & 5.75 & & & & & & & & & \\
\hline & \multicolumn{3}{|c|}{ apatite: $0.02 \%$} & & & & & & & & & \\
\hline & element & $\begin{array}{c}\text { atomic } \\
\%\end{array}$ & Wt $\%$ & & & & & & & & & \\
\hline & $\mathrm{O}$ & 59.52 & 38.95 & & & & & & & & & \\
\hline & $\mathrm{Si}$ & 2.34 & 2.68 & & & & & & & & & \\
\hline & $\mathrm{P}$ & 11.14 & 14.11 & & & & & & & & & \\
\hline & $\mathrm{Ca}$ & 27.00 & 44.25 & & & & & & & & & \\
\hline \multirow[t]{11}{*}{ MS13 } & \multicolumn{3}{|c|}{ melt: $73.3 \%$} & \multicolumn{3}{|c|}{ plagioclase: $15.0 \%$} & \multicolumn{3}{|c|}{ orthopyroxene: $9.4 \%$} & \multicolumn{3}{|c|}{ clinopyroxene: $1.0 \%$} \\
\hline & element & $\begin{array}{c}\text { atomic } \\
\%\end{array}$ & Wt $\%$ & element & $\begin{array}{c}\text { atomic } \\
\%\end{array}$ & Wt $\%$ & element & $\begin{array}{c}\text { atomic } \\
\%\end{array}$ & Wt $\%$ & element & $\begin{array}{c}\text { atomic } \\
\%\end{array}$ & Wt $\%$ \\
\hline & $\mathrm{O}$ & 62.46 & 47.52 & $\mathrm{O}$ & 59.59 & 45.07 & $\mathrm{O}$ & 59.27 & 42.93 & $\mathrm{O}$ & 59.37 & 40.78 \\
\hline & $\mathrm{Na}$ & 2.58 & 2.82 & $\mathrm{Na}$ & 3.44 & 3.74 & $\mathrm{Na}$ & 1.07 & 1.11 & $\mathrm{Na}$ & 0.50 & 0.49 \\
\hline & $\mathrm{Mg}$ & 0.67 & 0.78 & $\mathrm{Al}$ & 12.26 & 15.64 & $\mathrm{Mg}$ & 8.11 & 8.93 & $\mathrm{Mg}$ & 7.23 & 7.55 \\
\hline & $\mathrm{Al}$ & 6.81 & 8.74 & $\mathrm{Si}$ & 20.08 & 26.67 & $\mathrm{Al}$ & 3.32 & 4.06 & $\mathrm{Si}$ & 20.13 & 24.28 \\
\hline & $\mathrm{Si}$ & 23.15 & 30.93 & K & 0.17 & 0.32 & $\mathrm{Si}$ & 21.59 & 27.45 & K & 0.15 & 0.26 \\
\hline & K & 1.17 & 2.17 & $\mathrm{Ca}$ & 4.27 & 8.09 & K & 0.47 & 0.84 & $\mathrm{Ca}$ & 7.30 & 12.55 \\
\hline & $\mathrm{Ca}$ & 1.68 & 3.19 & $\mathrm{Fe}$ & 0.18 & 0.48 & $\mathrm{Ca}$ & 1.17 & 2.11 & $\mathrm{Ti}$ & 0.23 & 0.47 \\
\hline & $\mathrm{Ti}$ & 0.21 & 0.49 & & & & $\mathrm{Ti}$ & 0.16 & 0.36 & $\mathrm{Fe}$ & 3.71 & 8.89 \\
\hline & $\mathrm{Fe}$ & 1.27 & 3.37 & & & & $\mathrm{Mn}$ & 0.10 & 0.24 & $\mathrm{Br}$ & 1.38 & 4.73 \\
\hline
\end{tabular}




\begin{tabular}{|c|c|c|c|c|c|c|c|c|c|c|c|c|}
\hline \multirow{2}{*}{\multicolumn{4}{|c|}{ amphibole: $0.2 \%$}} & & & & \multirow[t]{3}{*}{$\mathrm{Fe}$} & \multirow[t]{3}{*}{4.74} & \multirow[t]{3}{*}{11.98} & & & \\
\hline & & & & & & & & & & & & \\
\hline & element & $\begin{array}{c}\text { atomic } \\
\%\end{array}$ & Wt $\%$ & & & & & & & & & \\
\hline & $\mathrm{O}$ & 59.93 & 38.00 & & & & & & & & & \\
\hline & $\mathrm{Na}$ & 1.68 & 1.53 & & & & & & & & & \\
\hline & $\mathrm{Mg}$ & 2.38 & 2.29 & & & & & & & & & \\
\hline & $\mathrm{Al}$ & 3.92 & 4.19 & & & & & & & & & \\
\hline & $\mathrm{Si}$ & 12.50 & 13.91 & & & & & & & & & \\
\hline & $\mathrm{K}$ & 0.45 & 0.69 & & & & & & & & & \\
\hline & $\mathrm{Ca}$ & 0.89 & 1.42 & & & & & & & & & \\
\hline & $\mathrm{Ti}$ & 7.55 & 14.34 & & & & & & & & & \\
\hline & $\mathrm{V}$ & 0.30 & 0.61 & & & & & & & & & \\
\hline & $\mathrm{Fe}$ & 10.40 & 23.02 & & & & & & & & & \\
\hline \multirow[t]{22}{*}{ MS15 } & \multicolumn{3}{|c|}{ quartz: $43.6 \%$} & \multicolumn{3}{|c|}{ melt: $44.3 \%$} & \multicolumn{3}{|c|}{ orthopyroxene: $5.6 \%$} & \multicolumn{3}{|c|}{ amphibole: $1.6 \%$} \\
\hline & element & $\begin{array}{c}\text { atomic } \\
\%\end{array}$ & Wt $\%$ & element & $\begin{array}{c}\text { atomic } \\
\%\end{array}$ & Wt $\%$ & element & $\begin{array}{c}\text { atomic } \\
\%\end{array}$ & Wt $\%$ & element & $\begin{array}{c}\text { atomic } \\
\%\end{array}$ & Wt $\%$ \\
\hline & $\mathrm{O}$ & 66.43 & 51.81 & $\mathrm{O}$ & 62.72 & 47.94 & $\mathrm{O}$ & 60.54 & 43.13 & $\mathrm{O}$ & 63.23 & 44.48 \\
\hline & $\mathrm{Al}$ & 0.42 & 0.56 & $\mathrm{Na}$ & 1.40 & 1.54 & $\mathrm{Na}$ & 0.61 & 0.63 & $\mathrm{Na}$ & 1.29 & 1.31 \\
\hline & $\mathrm{Si}$ & 32.52 & 44.52 & $\mathrm{Mg}$ & 1.31 & 1.53 & $\mathrm{Mg}$ & 6.21 & 6.73 & $\mathrm{Mg}$ & 1.24 & 1.33 \\
\hline & $\mathrm{K}$ & 0.21 & 0.39 & $\mathrm{Al}$ & 7.67 & 9.89 & $\mathrm{Al}$ & 5.63 & 6.77 & $\mathrm{Al}$ & 4.34 & 5.15 \\
\hline & $\mathrm{Ca}$ & 0.06 & 0.12 & $\mathrm{Si}$ & 23.32 & 31.30 & $\mathrm{Si}$ & 18.63 & 23.30 & $\mathrm{Si}$ & 16.45 & 20.32 \\
\hline & $\mathrm{Fe}$ & 0.11 & 0.31 & $\mathrm{~K}$ & 2.20 & 4.11 & $\mathrm{~S}$ & 0.12 & 0.17 & $\mathrm{~S}$ & 3.62 & 5.11 \\
\hline & Os & 0.15 & 1.40 & $\mathrm{Ca}$ & 0.32 & 0.62 & $\mathrm{~K}$ & 1.61 & 2.81 & $\mathrm{~K}$ & 1.15 & 1.97 \\
\hline & \multirow[t]{6}{*}{$\mathrm{Pt}$} & 0.09 & 0.88 & $\mathrm{Ti}$ & 0.08 & 0.19 & $\mathrm{Ca}$ & 0.16 & 0.29 & $\mathrm{Ca}$ & 0.44 & 0.77 \\
\hline & & & & $\mathrm{Fe}$ & 0.88 & 2.36 & $\mathrm{Ti}$ & 0.42 & 0.91 & $\mathrm{Ti}$ & 1.82 & 3.84 \\
\hline & & & & $\mathrm{Ba}$ & 0.08 & 0.53 & V & 0.05 & 0.11 & $\mathrm{Fe}$ & 6.40 & 15.72 \\
\hline & & & & & & & $\mathrm{Mn}$ & 0.10 & 0.25 & & & \\
\hline & & & & & & & $\mathrm{Fe}$ & 5.83 & 14.49 & & & \\
\hline & & & & & & & $\mathrm{Ba}$ & 0.07 & 0.43 & & & \\
\hline & \multicolumn{3}{|c|}{ pyrite: $0.02 \%$} & & & & & & & & & \\
\hline & element & $\begin{array}{c}\text { atomic } \\
\%\end{array}$ & $\mathrm{Wt} \%$ & & & & & & & & & \\
\hline & $\mathrm{O}$ & 66.62 & 42.50 & & & & & & & & & \\
\hline & $\mathrm{Si}$ & 1.94 & 2.17 & & & & & & & & & \\
\hline & S & 15.84 & 20.25 & & & & & & & & & \\
\hline & $\mathrm{Fe}$ & 15.23 & 33.91 & & & & & & & & & \\
\hline & $\mathrm{Br}$ & 0.37 & 1.18 & & & & & & & & & \\
\hline
\end{tabular}




\begin{tabular}{|c|c|c|c|c|c|c|c|c|c|c|c|c|}
\hline \multirow[t]{13}{*}{ MS16 } & \multicolumn{3}{|c|}{ melt: $62.9 \%$} & \multicolumn{3}{|c|}{ quartz: $26.4 \%$} & \multicolumn{3}{|c|}{ orthopyroxene: $2.3 \%$} & \multicolumn{3}{|c|}{ FeTi oxides: $1.5 \%$} \\
\hline & element & $\begin{array}{c}\text { atomic } \\
\%\end{array}$ & $\mathrm{Wt} \%$ & element & $\begin{array}{c}\text { atomic } \\
\%\end{array}$ & $\mathrm{Wt} \%$ & element & $\begin{array}{c}\text { atomic } \\
\%\end{array}$ & Wt $\%$ & element & $\begin{array}{c}\text { atomic } \\
\%\end{array}$ & Wt $\%$ \\
\hline & $\mathrm{O}$ & 63.52 & 48.75 & $\mathrm{O}$ & 66.52 & 52.03 & $\mathrm{O}$ & 60.10 & 43.02 & $\mathrm{O}$ & 57.73 & 35.21 \\
\hline & $\mathrm{Na}$ & 1.07 & 1.18 & $\mathrm{Si}$ & 32.86 & 45.12 & $\mathrm{Mg}$ & 11.08 & 12.05 & $\mathrm{Na}$ & 0.60 & 0.53 \\
\hline & $\mathrm{Mg}$ & 0.96 & 1.12 & K & 0.15 & 0.28 & $\mathrm{Al}$ & 2.97 & 3.58 & $\mathrm{Mg}$ & 0.78 & 0.73 \\
\hline & $\mathrm{Al}$ & 5.61 & 7.27 & $\mathrm{Fe}$ & 0.06 & 0.16 & $\mathrm{Si}$ & 18.60 & 23.38 & $\mathrm{Al}$ & 2.33 & 2.40 \\
\hline & $\mathrm{Si}$ & 25.59 & 34.48 & $\mathrm{Y}$ & 0.31 & 1.33 & K & 0.10 & 0.17 & $\mathrm{Si}$ & 11.56 & 12.38 \\
\hline & $\mathrm{K}$ & 1.88 & 3.53 & & & & $\mathrm{Ti}$ & 0.17 & 0.36 & S & 5.34 & 6.52 \\
\hline & $\mathrm{Ca}$ & 0.26 & 0.50 & & & & Mn & 0.11 & 0.28 & K & 0.62 & 0.92 \\
\hline & $\mathrm{Ti}$ & 0.12 & 0.27 & & & & $\mathrm{Fe}$ & 6.87 & 17.15 & $\mathrm{Ca}$ & 0.10 & 0.15 \\
\hline & $\mathrm{Fe}$ & 0.92 & 2.45 & & & & & & & $\mathrm{Ti}$ & 11.15 & 20.37 \\
\hline & $\mathrm{Ba}$ & 0.07 & 0.46 & & & & & & & $\mathrm{~V}$ & 0.20 & 0.39 \\
\hline & & & & & & & & & & $\mathrm{Fe}$ & 9.59 & 20.42 \\
\hline \multirow[t]{23}{*}{ MS17 } & \multicolumn{3}{|c|}{ melt: $55.2 \%$} & \multicolumn{3}{|c|}{ plagioclase: $38.3 \%$} & \multicolumn{3}{|c|}{ orthopyroxene: $5.3 \%$} & \multicolumn{3}{|c|}{ FeTi oxides: $0.4 \%$} \\
\hline & element & $\begin{array}{c}\text { atomic } \\
\%\end{array}$ & $\mathrm{Wt} \%$ & element & $\begin{array}{c}\text { atomic } \\
\%\end{array}$ & $\mathrm{Wt} \%$ & element & $\begin{array}{c}\text { atomic } \\
\%\end{array}$ & Wt $\%$ & element & $\begin{array}{c}\text { atomic } \\
\%\end{array}$ & $\mathrm{Wt} \%$ \\
\hline & $\mathrm{O}$ & 58.95 & 40.77 & $\mathrm{O}$ & 61.77 & 46.95 & $\mathrm{O}$ & 60.35 & 41.89 & $\mathrm{O}$ & 53.64 & 26.87 \\
\hline & $\mathrm{Na}$ & 0.62 & 0.61 & $\mathrm{Na}$ & 2.87 & 3.13 & $\mathrm{Mg}$ & 7.68 & 8.10 & $\mathrm{Mg}$ & 1.32 & 1.00 \\
\hline & $\mathrm{Mg}$ & 5.95 & 6.25 & $\mathrm{Al}$ & 10.27 & 13.16 & $\mathrm{Al}$ & 2.62 & 3.06 & $\mathrm{Al}$ & 3.98 & 3.36 \\
\hline & $\mathrm{Al}$ & 7.40 & 8.63 & $\mathrm{Si}$ & 20.32 & 27.11 & $\mathrm{Si}$ & 19.54 & 23.81 & $\mathrm{Si}$ & 2.53 & 2.22 \\
\hline & $\mathrm{Si}$ & 15.76 & 19.13 & $\mathrm{~K}$ & 0.96 & 1.79 & K & 0.39 & 0.67 & S & 0.50 & 0.50 \\
\hline & $\mathrm{K}$ & 4.10 & 6.93 & $\mathrm{Ca}$ & 3.10 & 5.89 & $\mathrm{Ca}$ & 0.46 & 0.81 & $\mathrm{Ti}$ & 1.78 & 2.66 \\
\hline & $\mathrm{Ca}$ & 0.23 & 0.40 & $\mathrm{Fe}$ & 0.71 & 1.88 & $\mathrm{Ti}$ & 0.06 & 0.13 & $\mathrm{Fe}$ & 36.26 & 63.39 \\
\hline & $\mathrm{Ti}$ & 0.71 & 1.47 & $\mathrm{~Tb}$ & 0.01 & 0.08 & $\mathrm{Mn}$ & 0.34 & 0.82 & & & \\
\hline & V & 0.05 & 0.11 & & & & $\mathrm{Fe}$ & 8.55 & 20.71 & & & \\
\hline & $\mathrm{Fe}$ & 6.04 & 14.59 & & & & & & & & & \\
\hline & In & 0.06 & 0.27 & & & & & & & & & \\
\hline & $\mathrm{Ba}$ & 0.14 & 0.83 & & & & & & & & & \\
\hline & \multicolumn{3}{|c|}{ apatite: $0.2 \%$} & \multicolumn{3}{|c|}{ pyrite: $0.04 \%$} & & & & & & \\
\hline & element & $\begin{array}{c}\text { atomic } \\
\%\end{array}$ & $\mathrm{Wt} \%$ & element & $\begin{array}{c}\text { atomic } \\
\%\end{array}$ & $\mathrm{Wt} \%$ & & & & & & \\
\hline & $\mathrm{O}$ & 60.69 & 41.19 & $\mathrm{O}$ & 66.82 & 41.95 & & & & & & \\
\hline & $\mathrm{Na}$ & 0.55 & 0.53 & S & 16.68 & 20.99 & & & & & & \\
\hline & $\mathrm{Mg}$ & 0.94 & 0.97 & $\mathrm{Fe}$ & 16.32 & 35.77 & & & & & & \\
\hline & $\mathrm{Al}$ & 2.11 & 2.41 & $\mathrm{Ta}$ & 0.18 & 1.29 & & & & & & \\
\hline & $\mathrm{Si}$ & 5.71 & 6.80 & & & & & & & & & \\
\hline & $\mathrm{P}$ & 10.29 & 13.51 & & & & & & & & & \\
\hline & K & 1.06 & 1.77 & & & & & & & & & \\
\hline
\end{tabular}




\begin{tabular}{|c|c|c|c|c|c|c|c|c|c|c|c|c|}
\hline & $\begin{array}{l}\mathrm{Ca} \\
\mathrm{Fe}\end{array}$ & $\begin{array}{r}17.03 \\
1.63\end{array}$ & $\begin{array}{r}28.95 \\
3.87\end{array}$ & & & & & & & & & \\
\hline \multirow[t]{25}{*}{ MS18 } & \multicolumn{3}{|c|}{ plagioclase: $42.7 \%$} & \multicolumn{3}{|c|}{ melt: $20.2 \%$} & \multicolumn{3}{|c|}{ quartz: $4.0 \%$} & \multicolumn{3}{|c|}{ orthopyroxene: $5.6 \%$} \\
\hline & element & $\begin{array}{c}\text { atomic } \\
\% \\
\end{array}$ & Wt $\%$ & element & $\begin{array}{c}\text { atomic } \\
\%\end{array}$ & Wt $\%$ & element & $\begin{array}{c}\text { atomic } \\
\%\end{array}$ & Wt $\%$ & element & $\begin{array}{c}\text { atomic } \\
\%\end{array}$ & Wt $\%$ \\
\hline & $\mathrm{O}$ & 61.83 & 47.47 & $\mathrm{O}$ & 62.17 & 46.46 & $\mathrm{O}$ & 66.40 & 52.10 & $\mathrm{O}$ & 60.27 & 42.59 \\
\hline & $\mathrm{Na}$ & 3.55 & 3.91 & $\mathrm{Na}$ & 1.53 & 1.64 & $\mathrm{Si}$ & 32.89 & 45.29 & $\mathrm{Na}$ & 0.60 & 0.61 \\
\hline & $\mathrm{Al}$ & 9.89 & 12.80 & $\mathrm{Mg}$ & 2.44 & 2.77 & $\mathrm{~K}$ & 0.12 & 0.24 & $\mathrm{Mg}$ & 8.05 & 8.64 \\
\hline & $\mathrm{Si}$ & 20.85 & 28.10 & $\mathrm{Al}$ & 4.64 & 5.85 & $\mathrm{Ca}$ & 0.14 & 0.27 & $\mathrm{Al}$ & 1.74 & 2.07 \\
\hline & $\mathrm{K}$ & 0.70 & 1.32 & $\mathrm{Si}$ & 23.55 & 30.89 & $\mathrm{Fe}$ & 0.14 & 0.39 & $\mathrm{Si}$ & 19.96 & 24.76 \\
\hline & $\mathrm{Ca}$ & 2.83 & 5.45 & $\mathrm{Ar}$ & 0.10 & 0.18 & $\mathrm{Br}$ & 0.14 & 0.53 & $\mathrm{~K}$ & 0.40 & 0.68 \\
\hline & \multirow[t]{5}{*}{$\mathrm{Fe}$} & 0.35 & 0.94 & $\mathrm{~K}$ & 1.75 & 3.20 & $\mathrm{Sb}$ & 0.10 & 0.58 & $\mathrm{Ca}$ & 2.09 & 3.70 \\
\hline & & & & $\mathrm{Ca}$ & 1.22 & 2.28 & $\mathrm{Te}$ & 0.05 & 0.28 & $\mathrm{Ti}$ & 0.17 & 0.35 \\
\hline & & & & $\mathrm{Ti}$ & 0.21 & 0.46 & & & & $\mathrm{~V}$ & 0.03 & 0.07 \\
\hline & & & & $\mathrm{Fe}$ & 2.40 & 6.26 & & & & $\mathrm{Mn}$ & 0.14 & 0.35 \\
\hline & & & & & & & & & & $\mathrm{Fe}$ & 6.55 & 16.17 \\
\hline & \multicolumn{3}{|c|}{ amphibole: $1.2 \%$} & \multicolumn{3}{|c|}{ apatite: $0.4 \%$} & & & & & & \\
\hline & element & $\begin{array}{c}\text { atomic } \\
\%\end{array}$ & Wt $\%$ & element & $\begin{array}{c}\text { atomic } \\
\%\end{array}$ & $\mathrm{Wt} \%$ & & & & & & \\
\hline & $\mathrm{O}$ & 61.35 & 41.25 & $\mathrm{O}$ & 58.96 & 40.52 & & & & & & \\
\hline & $\mathrm{Na}$ & 1.68 & 1.63 & $\mathrm{Na}$ & 1.23 & 0.53 & & & & & & \\
\hline & $\mathrm{Mg}$ & 1.73 & 1.77 & $\mathrm{Mg}$ & 0.78 & 0.97 & & & & & & \\
\hline & $\mathrm{Al}$ & 4.20 & 4.76 & $\mathrm{Al}$ & 2.07 & 2.41 & & & & & & \\
\hline & $\mathrm{Si}$ & 16.13 & 19.03 & $\mathrm{Si}$ & 7.83 & 6.80 & & & & & & \\
\hline & $\mathrm{K}$ & 1.08 & 1.77 & $\mathrm{P}$ & 6.92 & 13.51 & & & & & & \\
\hline & $\mathrm{Ca}$ & 1.05 & 1.77 & $\mathrm{Cl}$ & 0.36 & 0.67 & & & & & & \\
\hline & $\mathrm{Ti}$ & 5.86 & 11.80 & $\mathrm{~K}$ & 0.68 & 1.77 & & & & & & \\
\hline & $\mathrm{Fe}$ & 6.91 & 16.23 & $\mathrm{Ca}$ & 20.57 & 28.95 & & & & & & \\
\hline & & & & $\mathrm{Fe}$ & 0.59 & 3.87 & & & & & & \\
\hline \multirow[t]{8}{*}{ MS19 } & \multicolumn{3}{|c|}{ clinopyroxene: $68.2 \%$} & \multicolumn{3}{|c|}{ melt: $27.8 \%$} & \multicolumn{3}{|c|}{ apatite: $0.3 \%$} & \multicolumn{3}{|c|}{ FeTi oxides: $0.1 \%$} \\
\hline & element & $\begin{array}{c}\text { atomic } \\
\%\end{array}$ & Wt $\%$ & element & $\begin{array}{c}\text { atomic } \\
\%\end{array}$ & $\mathrm{Wt} \%$ & element & $\begin{array}{c}\text { atomic } \\
\%\end{array}$ & Wt $\%$ & element & $\begin{array}{c}\text { atomic } \\
\%\end{array}$ & Wt $\%$ \\
\hline & $\mathrm{O}$ & 59.98 & 43.16 & $\mathrm{O}$ & 61.60 & 46.37 & $\mathrm{O}$ & 60.18 & 41.17 & $\mathrm{O}$ & 58.95 & 35.71 \\
\hline & $\mathrm{Na}$ & 1.42 & 1.47 & $\mathrm{Na}$ & 1.46 & 1.58 & $\mathrm{Na}$ & 0.82 & 0.81 & $\mathrm{Na}$ & 1.05 & 0.91 \\
\hline & $\mathrm{Mg}$ & 6.18 & 6.76 & $\mathrm{Al}$ & 13.26 & 16.84 & $\mathrm{Mg}$ & 1.80 & 1.87 & $\mathrm{Mg}$ & 4.61 & 4.25 \\
\hline & $\mathrm{Al}$ & 5.99 & 7.27 & $\mathrm{Si}$ & 17.31 & 22.87 & $\mathrm{Al}$ & 2.84 & 3.28 & $\mathrm{Al}$ & 3.02 & 3.09 \\
\hline & $\mathrm{Si}$ & 17.37 & 21.95 & $\mathrm{Ca}$ & 5.94 & 11.20 & $\mathrm{Si}$ & 6.92 & 8.31 & $\mathrm{Si}$ & 7.94 & 8.45 \\
\hline & $\mathrm{K}$ & 0.11 & 0.19 & $\mathrm{Fe}$ & 0.41 & 1.08 & $\mathrm{P}$ & 8.39 & 11.11 & $\mathrm{Ca}$ & 2.25 & 3.42 \\
\hline
\end{tabular}




\begin{tabular}{|c|c|c|c|c|c|c|c|c|c|c|c|c|}
\hline & $\mathrm{Ca}$ & 4.43 & 7.98 & \multirow[t]{5}{*}{$\mathrm{Tb}$} & \multirow[t]{5}{*}{0.01} & \multirow[t]{5}{*}{0.06} & $\mathrm{Cl}$ & 0.15 & 0.23 & \multirow{5}{*}{$\begin{array}{l}\mathrm{Ti} \\
\mathrm{Fe}\end{array}$} & \multirow{5}{*}{$\begin{array}{r}8.98 \\
13.19\end{array}$} & \multirow{5}{*}{$\begin{array}{l}16.28 \\
27.89\end{array}$} \\
\hline & $\mathrm{Ti}$ & 0.33 & 0.71 & & & & $\mathrm{Ca}$ & 17.68 & 30.31 & & & \\
\hline & $\mathrm{V}$ & 0.02 & 0.05 & & & & $\mathrm{Fe}$ & 1.22 & 2.91 & & & \\
\hline & $\mathrm{Mn}$ & 0.10 & 0.24 & & & & & & & & & \\
\hline & $\mathrm{Fe}$ & 4.08 & 10.25 & & & & & & & & & \\
\hline \multirow[t]{12}{*}{ MS22 } & \multicolumn{3}{|c|}{ melt: $47.7 \%$} & \multicolumn{3}{|c|}{ clinopyroxene: $40.0 \%$} & \multicolumn{3}{|c|}{ plagioclase: $5.8 \%$} & \multicolumn{3}{|c|}{ FeTi oxides: $0.7 \%$} \\
\hline & element & $\begin{array}{c}\text { atomic } \\
\%\end{array}$ & Wt $\%$ & element & $\begin{array}{c}\text { atomic } \\
\%\end{array}$ & Wt \% & element & $\begin{array}{c}\text { atomic } \\
\%\end{array}$ & Wt $\%$ & element & $\begin{array}{c}\text { atomic } \\
\%\end{array}$ & Wt $\%$ \\
\hline & $\mathrm{O}$ & 63.61 & 47.80 & $\mathrm{O}$ & 58.56 & 42.17 & $\mathrm{O}$ & 59.59 & 44.01 & $\mathrm{O}$ & 53.67 & 27.49 \\
\hline & $\mathrm{Na}$ & 1.51 & 1.63 & $\mathrm{Na}$ & 1.41 & 1.46 & $\mathrm{Na}$ & 0.52 & 0.55 & $\mathrm{Mg}$ & 3.57 & 2.78 \\
\hline & $\mathrm{Mg}$ & 2.39 & 2.73 & $\mathrm{Mg}$ & 8.24 & 9.02 & $\mathrm{Al}$ & 15.50 & 19.31 & $\mathrm{Al}$ & 6.00 & 5.18 \\
\hline & $\mathrm{Al}$ & 8.11 & 10.28 & $\mathrm{Al}$ & 6.32 & 7.68 & $\mathrm{Si}$ & 16.58 & 21.50 & $\mathrm{Si}$ & 0.80 & 0.72 \\
\hline & $\mathrm{Si}$ & 17.96 & 23.70 & $\mathrm{Si}$ & 16.19 & 20.46 & $\mathrm{Ca}$ & 7.54 & 13.96 & $\mathrm{Ca}$ & 0.23 & 0.29 \\
\hline & K & 0.11 & 0.21 & K & 0.08 & 0.13 & $\mathrm{Fe}$ & 0.26 & 0.67 & $\mathrm{Ti}$ & 1.29 & 1.97 \\
\hline & $\mathrm{Ca}$ & 3.83 & 7.21 & $\mathrm{Ca}$ & 5.49 & 9.91 & & & & V & 0.21 & 0.34 \\
\hline & $\mathrm{Ti}$ & 0.17 & 0.39 & $\mathrm{Ti}$ & 0.36 & 0.78 & & & & $\mathrm{Fe}$ & 34.19 & 61.13 \\
\hline & $\mathrm{Mn}$ & 0.08 & 0.20 & $\mathrm{Fe}$ & 3.34 & 8.38 & & & & Co & 0.06 & 0.10 \\
\hline & $\mathrm{Fe}$ & 2.23 & 5.86 & & & & & & & & & \\
\hline \multirow[t]{18}{*}{ MS23 } & \multicolumn{3}{|c|}{ plagioclase: $21.8 \%$} & \multicolumn{3}{|c|}{ biotite: $30.6 \%$} & \multicolumn{3}{|c|}{ melt: $33.3 \%$} & \multicolumn{3}{|c|}{ orthopyroxene: $10.2 \%$} \\
\hline & element & $\begin{array}{c}\text { atomic } \\
\%\end{array}$ & Wt $\%$ & element & $\begin{array}{c}\text { atomic } \\
\%\end{array}$ & Wt \% & element & $\begin{array}{c}\text { atomic } \\
\%\end{array}$ & Wt $\%$ & element & $\begin{array}{c}\text { atomic } \\
\%\end{array}$ & Wt $\%$ \\
\hline & $\mathrm{O}$ & 60.00 & 45.15 & $\mathrm{O}$ & 57.23 & 39.47 & $\mathrm{O}$ & 60.13 & 44.11 & $\mathrm{O}$ & 58.36 & 40.79 \\
\hline & $\mathrm{Na}$ & 2.69 & 2.91 & $\mathrm{Na}$ & 0.53 & 0.53 & $\mathrm{Na}$ & 1.90 & 2.01 & $\mathrm{Mg}$ & 9.33 & 9.91 \\
\hline & $\mathrm{Al}$ & 12.15 & 15.41 & $\mathrm{Mg}$ & 7.70 & 8.07 & $\mathrm{Mg}$ & 1.70 & 1.90 & $\mathrm{Al}$ & 3.35 & 3.95 \\
\hline & $\mathrm{Si}$ & 19.80 & 26.15 & $\mathrm{Al}$ & 7.76 & 9.02 & $\mathrm{Al}$ & 7.62 & 9.43 & $\mathrm{Si}$ & 20.16 & 24.74 \\
\hline & K & 0.73 & 1.35 & $\mathrm{Si}$ & 15.53 & 18.80 & $\mathrm{Si}$ & 21.88 & 28.17 & K & 0.55 & 0.95 \\
\hline & $\mathrm{Ca}$ & 4.24 & 8.00 & $\mathrm{Cl}$ & 0.04 & 0.06 & K & 3.15 & 5.64 & $\mathrm{Ca}$ & 0.55 & 0.96 \\
\hline & \multirow[t]{7}{*}{$\mathrm{Fe}$} & 0.39 & 1.04 & K & 4.50 & 7.58 & $\mathrm{Ca}$ & 0.93 & 1.70 & $\mathrm{Ti}$ & 0.09 & 0.20 \\
\hline & & & & $\mathrm{Ca}$ & 0.15 & 0.26 & $\mathrm{Ti}$ & 0.20 & 0.44 & $\mathrm{Mn}$ & 0.27 & 0.65 \\
\hline & & & & $\mathrm{Ti}$ & 0.89 & 1.83 & V & 0.02 & 0.05 & $\mathrm{Fe}$ & 7.32 & 17.85 \\
\hline & & & & V & 0.05 & 0.11 & $\mathrm{Mn}$ & 0.05 & 0.13 & & & \\
\hline & & & & $\mathrm{Fe}$ & 5.41 & 13.04 & $\mathrm{Fe}$ & 2.35 & 6.02 & & & \\
\hline & & & & In & 0.05 & 0.25 & $\mathrm{Ba}$ & 0.06 & 0.39 & & & \\
\hline & & & & $\mathrm{Ba}$ & 0.17 & 0.99 & & & & & & \\
\hline & \multicolumn{3}{|c|}{ FeTi oxides: $3.2 \%$} & \multicolumn{3}{|c|}{ apatite: $0.4 \%$} & & & & & & \\
\hline & element & $\begin{array}{c}\text { atomic } \\
\%\end{array}$ & Wt $\%$ & element & $\begin{array}{c}\text { atomic } \\
\%\end{array}$ & Wt $\%$ & & & & & & \\
\hline & $\mathrm{O}$ & 54.25 & 28.82 & $\mathrm{O}$ & 61.48 & 41.14 & & & & & & \\
\hline
\end{tabular}




\begin{tabular}{|c|c|c|c|c|c|c|c|c|c|c|c|c|}
\hline & $\mathrm{Mg}$ & 2.11 & 1.70 & $\mathrm{Si}$ & 0.63 & 0.74 & & & & & & \\
\hline & $\mathrm{Al}$ & 6.14 & 5.50 & $\mathrm{P}$ & 14.16 & 18.34 & & & & & & \\
\hline & $\mathrm{Si}$ & 4.86 & 4.53 & $\mathrm{Ca}$ & 23.74 & 39.79 & & & & & & \\
\hline & $\mathrm{S}$ & 0.27 & 0.29 & & & & & & & & & \\
\hline & $\mathrm{K}$ & 0.65 & 0.85 & & & & & & & & & \\
\hline & $\mathrm{Ca}$ & 0.19 & 0.26 & & & & & & & & & \\
\hline & $\mathrm{Ti}$ & 1.40 & 2.22 & & & & & & & & & \\
\hline & V & 0.17 & 0.28 & & & & & & & & & \\
\hline & $\mathrm{Fe}$ & 29.96 & 55.55 & & & & & & & & & \\
\hline \multirow[t]{12}{*}{ MS26 } & \multicolumn{3}{|c|}{ melt: $73.7 \%$} & \multicolumn{3}{|c|}{ clinopyroxene: $17.0 \%$} & \multicolumn{3}{|c|}{ FeTi oxides: $3.9 \%$} & \multicolumn{3}{|c|}{ apatite: $0.1 \%$} \\
\hline & element & $\begin{array}{c}\text { atomic } \\
\%\end{array}$ & Wt $\%$ & element & $\begin{array}{c}\text { atomic } \\
\%\end{array}$ & Wt $\%$ & element & $\begin{array}{c}\text { atomic } \\
\%\end{array}$ & Wt $\%$ & element & $\begin{array}{c}\text { atomic } \\
\%\end{array}$ & Wt $\%$ \\
\hline & $\mathrm{O}$ & 62.12 & 47.23 & $\mathrm{O}$ & 60.62 & 42.91 & $\mathrm{O}$ & 37.58 & 57.14 & $\mathrm{O}$ & 61.73 & 42.13 \\
\hline & $\mathrm{Na}$ & 2.92 & 3.19 & $\mathrm{Na}$ & 0.90 & 0.92 & $\mathrm{Na}$ & 1.93 & 2.04 & $\mathrm{Al}$ & 1.45 & 1.67 \\
\hline & $\mathrm{Mg}$ & 0.44 & 0.51 & $\mathrm{Mg}$ & 5.70 & 6.14 & $\mathrm{Mg}$ & 1.30 & 1.30 & $\mathrm{Si}$ & 5.13 & 6.14 \\
\hline & $\mathrm{Al}$ & 7.86 & 10.08 & $\mathrm{Al}$ & 2.53 & 3.02 & $\mathrm{Al}$ & 5.34 & 4.82 & $P$ & 11.74 & 15.51 \\
\hline & $\mathrm{Si}$ & 22.09 & 29.48 & $\mathrm{Si}$ & 20.36 & 25.30 & $\mathrm{Si}$ & 22.37 & 19.38 & $\mathrm{Ca}$ & 19.33 & 33.05 \\
\hline & $\mathrm{K}$ & 1.09 & 2.02 & $\mathrm{~K}$ & 0.46 & 0.80 & $\mathrm{P}$ & 0.25 & 0.19 & $\mathrm{Fe}$ & 0.63 & 1.49 \\
\hline & $\mathrm{Ca}$ & 2.24 & 4.27 & $\mathrm{Ca}$ & 3.25 & 5.76 & $\mathrm{~K}$ & 1.94 & 1.21 & & & \\
\hline & $\mathrm{Ti}$ & 0.22 & 0.49 & $\mathrm{Ti}$ & 0.29 & 0.62 & $\mathrm{Ca}$ & 2.25 & 1.37 & & & \\
\hline & \multirow[t]{2}{*}{$\mathrm{Fe}$} & 1.02 & 2.72 & $\mathrm{Mn}$ & 0.15 & 0.35 & $\mathrm{Ti}$ & 10.69 & 5.43 & & & \\
\hline & & & & $\mathrm{Fe}$ & 5.74 & 14.19 & $\mathrm{Fe}$ & 16.35 & 7.12 & & & \\
\hline \multirow[t]{14}{*}{ MS27 } & \multicolumn{3}{|c|}{ melt: $55.0 \%$} & \multicolumn{3}{|c|}{ quartz: $13.0 \%$} & \multicolumn{3}{|c|}{ orthopyroxene: $6.5 \%$} & \multicolumn{3}{|c|}{ clinopyroxene: $0.8 \%$} \\
\hline & element & $\begin{array}{c}\text { atomic } \\
\%\end{array}$ & Wt $\%$ & element & $\begin{array}{c}\text { atomic } \\
\%\end{array}$ & Wt \% & element & $\begin{array}{c}\text { atomic } \\
\%\end{array}$ & Wt $\%$ & element & $\begin{array}{c}\text { atomic } \\
\%\end{array}$ & Wt $\%$ \\
\hline & $\mathrm{O}$ & 62.79 & 47.55 & $\mathrm{O}$ & 66.38 & 51.77 & $\mathrm{O}$ & 61.05 & 41.96 & $\mathrm{O}$ & 61.55 & 46.93 \\
\hline & $\mathrm{Na}$ & 2.1 & 2.28 & $\mathrm{Al}$ & 0.47 & 0.62 & $\mathrm{Na}$ & 1 & 0.99 & $\mathrm{Na}$ & 3.31 & 3.63 \\
\hline & $\mathrm{Mg}$ & 0.51 & 0.59 & $\mathrm{Si}$ & 32.4 & 44.35 & $\mathrm{Mg}$ & 3.24 & 3.39 & $\mathrm{Mg}$ & 0.34 & 0.39 \\
\hline & $\mathrm{Al}$ & 5.78 & 7.38 & $\mathrm{~K}$ & 0.18 & 0.34 & $\mathrm{Al}$ & 3.18 & 3.69 & $\mathrm{Al}$ & 10.61 & 13.64 \\
\hline & $\mathrm{Si}$ & 24.32 & 32.33 & $\mathrm{Ca}$ & 0.1 & 0.2 & $\mathrm{Si}$ & 20.43 & 24.66 & $\mathrm{Si}$ & 19.77 & 26.46 \\
\hline & $\mathrm{K}$ & 1.55 & 2.88 & $\mathrm{Fe}$ & 0.25 & 0.68 & $\mathrm{~K}$ & 0.79 & 1.33 & $\mathrm{~K}$ & 0.81 & 1.51 \\
\hline & $\mathrm{Ca}$ & 1.19 & 2.26 & Os & 0.14 & 1.33 & $\mathrm{Ca}$ & 0.86 & 1.48 & $\mathrm{Ca}$ & 2.83 & 5.41 \\
\hline & $\mathrm{Ti}$ & 0.19 & 0.44 & $\mathrm{Pt}$ & 0.07 & 0.71 & $\mathrm{Ti}$ & 0.96 & 1.98 & $\mathrm{Ti}$ & 0.1 & 0.23 \\
\hline & $\mathrm{Fe}$ & 1.52 & 4.02 & & & & $\mathrm{Mn}$ & 0.18 & 0.42 & $\mathrm{Fe}$ & 0.67 & 1.79 \\
\hline & \multirow{2}{*}{$\mathrm{Ba}$} & 0.04 & 0.28 & & & & $\mathrm{Fe}$ & 8.26 & 19.82 & & & \\
\hline & & & & & & & $\mathrm{Ba}$ & 0.05 & 0.29 & & & \\
\hline & \multicolumn{3}{|c|}{ FeTi oxides: $0.04 \%$} & \multicolumn{3}{|c|}{ apatite: $0.02 \%$} & & & & & & \\
\hline
\end{tabular}




\begin{tabular}{|c|c|c|c|c|c|c|c|c|c|c|c|c|}
\hline & element & $\begin{array}{c}\text { atomic } \\
\%\end{array}$ & Wt \% & element & $\begin{array}{c}\text { atomic } \\
\%\end{array}$ & $\mathrm{Wt} \%$ & & & & & & \\
\hline & $\mathrm{O}$ & 59.98 & 32.37 & $\mathrm{O}$ & 61.5 & 41.74 & & & & & & \\
\hline & $\mathrm{Mg}$ & 1.37 & 1.13 & $\mathrm{Al}$ & 1.32 & 1.51 & & & & & & \\
\hline & $\mathrm{Si}$ & 1.38 & 1.31 & $\mathrm{Si}$ & 5.52 & 6.58 & & & & & & \\
\hline & $\mathrm{Ti}$ & 18.59 & 30.03 & $\mathrm{P}$ & 11.44 & 15.03 & & & & & & \\
\hline & $\mathrm{Fe}$ & 18.67 & 35.17 & K & 0.67 & 1.1 & & & & & & \\
\hline & & & & $\mathrm{Ca}$ & 18.39 & 31.27 & & & & & & \\
\hline & & & & $\mathrm{Fe}$ & 1.17 & 2.77 & & & & & & \\
\hline \multirow[t]{25}{*}{ MS28 } & \multicolumn{3}{|c|}{ melt: $60.7 \%$} & \multicolumn{3}{|c|}{ plagioclase: $12.1 \%$} & \multicolumn{3}{|c|}{ orthopyroxene: $11.1 \%$} & \multicolumn{3}{|c|}{ FeTi oxides: $2.1 \%$} \\
\hline & element & $\begin{array}{c}\text { atomic } \\
\%\end{array}$ & $\mathrm{Wt} \%$ & element & $\begin{array}{c}\text { atomic } \\
\%\end{array}$ & $\mathrm{Wt} \%$ & element & $\begin{array}{c}\text { atomic } \\
\%\end{array}$ & Wt $\%$ & element & $\begin{array}{c}\text { atomic } \\
\%\end{array}$ & $\mathrm{Wt} \%$ \\
\hline & $\mathrm{O}$ & 61.40 & 45.02 & $\mathrm{O}$ & 61.50 & 46.93 & $\mathrm{O}$ & 60.07 & 42.34 & $\mathrm{O}$ & 54.50 & 27.74 \\
\hline & $\mathrm{Na}$ & 2.20 & 2.32 & $\mathrm{Na}$ & 3.28 & 3.59 & $\mathrm{Na}$ & 0.70 & 0.71 & $\mathrm{Mg}$ & 2.06 & 1.60 \\
\hline & $\mathrm{Mg}$ & 1.10 & 1.22 & $\mathrm{Al}$ & 11.21 & 14.43 & $\mathrm{Mg}$ & 8.08 & 8.66 & $\mathrm{Al}$ & 3.63 & 3.11 \\
\hline & $\mathrm{Al}$ & 7.06 & 8.73 & $\mathrm{Si}$ & 19.41 & 26.00 & $\mathrm{Al}$ & 2.93 & 3.48 & $\mathrm{Si}$ & 1.89 & 1.69 \\
\hline & $\mathrm{Si}$ & 21.33 & 27.46 & $\mathrm{~K}$ & 0.73 & 1.36 & $\mathrm{Si}$ & 19.04 & 23.55 & K & 0.22 & 0.28 \\
\hline & $\mathrm{K}$ & 2.67 & 4.79 & $\mathrm{Ca}$ & 3.49 & 6.68 & $\mathrm{~K}$ & 0.64 & 1.11 & $\mathrm{Ca}$ & 0.13 & 0.16 \\
\hline & $\mathrm{Ca}$ & 1.25 & 2.31 & $\mathrm{Fe}$ & 0.38 & 1.01 & $\mathrm{Ca}$ & 1.02 & 1.80 & $\mathrm{Ti}$ & 5.22 & 7.95 \\
\hline & $\mathrm{Ti}$ & 0.30 & 0.66 & & & & $\mathrm{Ti}$ & 0.32 & 0.68 & V & 0.12 & 0.20 \\
\hline & $\mathrm{Fe}$ & 2.56 & 6.57 & & & & $\mathrm{Mn}$ & 0.20 & 0.50 & $\mathrm{Fe}$ & 32.24 & 57.28 \\
\hline & $\mathrm{Ba}$ & 0.06 & 0.36 & & & & $\mathrm{Fe}$ & 6.98 & 17.18 & & & \\
\hline & $\mathrm{Ir}$ & 0.06 & 0.56 & & & & & & & & & \\
\hline & \multicolumn{3}{|c|}{ clinopyroxene: $1.7 \%$} & \multicolumn{3}{|c|}{ apatite: $0.1 \%$} & & & & & & \\
\hline & element & $\begin{array}{c}\text { atomic } \\
\%\end{array}$ & Wt \% & element & $\begin{array}{c}\text { atomic } \\
\%\end{array}$ & Wt \% & & & & & & \\
\hline & $\mathrm{O}$ & 60.39 & 42.95 & $\mathrm{O}$ & 59.01 & 39.83 & & & & & & \\
\hline & $\mathrm{Na}$ & 0.91 & 0.93 & F & 3.41 & 2.73 & & & & & & \\
\hline & $\mathrm{Mg}$ & 5.02 & 5.42 & $\mathrm{Al}$ & 0.52 & 0.59 & & & & & & \\
\hline & $\mathrm{Al}$ & 3.25 & 3.90 & $\mathrm{Si}$ & 1.6 & 1.89 & & & & & & \\
\hline & $\mathrm{Si}$ & 18.64 & 23.28 & $\mathrm{P}$ & 13.04 & 17.04 & & & & & & \\
\hline & $\mathrm{P}$ & 0.77 & 1.06 & $\mathrm{Ca}$ & 22.43 & 37.92 & & & & & & \\
\hline & K & 0.79 & 1.38 & & & & & & & & & \\
\hline & $\mathrm{Ca}$ & 6.08 & 10.84 & & & & & & & & & \\
\hline & $\mathrm{Ti}$ & 0.20 & 0.43 & & & & & & & & & \\
\hline & $\mathrm{Fe}$ & 3.95 & 9.81 & & & & & & & & & \\
\hline MS29 & & eelt: $62.0 \%$ & & pla & oclase: 17 & & clin & roxene: 10 & & & ides: & \\
\hline
\end{tabular}




\begin{tabular}{|c|c|c|c|c|c|c|c|c|c|c|c|c|}
\hline & element & $\begin{array}{c}\text { atomic } \\
\%\end{array}$ & Wt $\%$ & element & $\begin{array}{c}\text { atomic } \\
\%\end{array}$ & Wt \% & element & $\begin{array}{c}\text { atomic } \\
\%\end{array}$ & Wt $\%$ & element & $\begin{array}{c}\text { atomic } \\
\%\end{array}$ & $\mathrm{Wt} \%$ \\
\hline & $\mathrm{O}$ & 62.38 & 47.06 & $\mathrm{O}$ & 61.58 & 47.38 & $\mathrm{O}$ & 60.46 & 41.67 & $\mathrm{O}$ & 52.57 & 26.37 \\
\hline & $\mathrm{Na}$ & 2.58 & 2.79 & $\mathrm{Na}$ & 3.97 & 4.39 & $\mathrm{Mg}$ & 5.96 & 6.25 & $\mathrm{Mg}$ & 1.27 & 0.96 \\
\hline & $\mathrm{Mg}$ & 0.47 & 0.54 & $\mathrm{Al}$ & 10.85 & 14.08 & $\mathrm{Al}$ & 1.75 & 2.04 & $\mathrm{Al}$ & 2.23 & 1.89 \\
\hline & $\mathrm{Al}$ & 6.18 & 7.86 & $\mathrm{Si}$ & 19.89 & 26.86 & $\mathrm{Si}$ & 19.98 & 24.17 & $\mathrm{Si}$ & 3.86 & 3.40 \\
\hline & $\mathrm{Si}$ & 23.43 & 31.03 & $\mathrm{~K}$ & 0.33 & 0.62 & K & 0.23 & 0.39 & K & 0.21 & 0.26 \\
\hline & K & 1.47 & 2.70 & $\mathrm{Ca}$ & 3.16 & 6.09 & $\mathrm{Ca}$ & 3.54 & 6.11 & $\mathrm{Ca}$ & 0.28 & 0.35 \\
\hline & $\mathrm{Ca}$ & 1.47 & 2.78 & $\mathrm{Fe}$ & 0.21 & 0.58 & $\mathrm{Ti}$ & 0.18 & 0.37 & $\mathrm{Ti}$ & 10.14 & 15.23 \\
\hline & $\mathrm{Ti}$ & 0.26 & 0.58 & & & & $\mathrm{Mn}$ & 0.22 & 0.52 & $\mathrm{Fe}$ & 29.43 & 51.53 \\
\hline & $\mathrm{Fe}$ & 1.77 & 4.65 & & & & $\mathrm{Fe}$ & 7.68 & 18.48 & & & \\
\hline & \multicolumn{3}{|c|}{ biotite: $0.6 \%$} & \multicolumn{3}{|c|}{ apatite: $0.2 \%$} & & & & & & \\
\hline & element & $\begin{array}{c}\text { atomic } \\
\%\end{array}$ & Wt $\%$ & element & $\begin{array}{c}\text { atomic } \\
\%\end{array}$ & Wt \% & & & & & & \\
\hline & $\mathrm{O}$ & 62.09 & 45.47 & $\mathrm{O}$ & 61.30 & 42.25 & & & & & & \\
\hline & $\mathrm{Na}$ & 1.99 & 2.09 & $\mathrm{Na}$ & 0.89 & 0.88 & & & & & & \\
\hline & $\mathrm{Mg}$ & 0.58 & 0.65 & $\mathrm{Mg}$ & 0.32 & 0.33 & & & & & & \\
\hline & $\mathrm{Al}$ & 4.60 & 5.68 & $\mathrm{Al}$ & 1.87 & 2.18 & & & & & & \\
\hline & $\mathrm{Si}$ & 17.75 & 22.82 & $\mathrm{Si}$ & 6.71 & 8.12 & & & & & & \\
\hline & $\mathrm{P}$ & 3.78 & 5.36 & $P$ & 10.53 & 14.05 & & & & & & \\
\hline & K & 1.10 & 1.98 & $\mathrm{Cl}$ & 0.20 & 0.31 & & & & & & \\
\hline & $\mathrm{Ca}$ & 6.63 & 12.16 & K & 0.39 & 0.65 & & & & & & \\
\hline \multirow{2}{*}{\multicolumn{2}{|c|}{$\mathrm{Fe}$}} & 1.49 & 3.80 & $\mathrm{Ca}$ & 17.00 & 29.35 & & & & & & \\
\hline & & & & $\mathrm{Fe}$ & 0.78 & 1.89 & & & & & & \\
\hline \multirow[t]{14}{*}{ MS30 } & \multicolumn{3}{|c|}{ melt: $65.5 \%$} & \multicolumn{3}{|c|}{ quartz: $6.7 \%$} & \multicolumn{3}{|c|}{ orthopyroxene: $17.8 \%$} & \multicolumn{3}{|c|}{ clinopyroxene: $1.6 \%$} \\
\hline & element & $\begin{array}{c}\text { atomic } \\
\%\end{array}$ & Wt $\%$ & element & $\begin{array}{c}\text { atomic } \\
\%\end{array}$ & Wt \% & element & $\begin{array}{c}\text { atomic } \\
\%\end{array}$ & Wt $\%$ & element & $\begin{array}{c}\text { atomic } \\
\%\end{array}$ & Wt $\%$ \\
\hline & $\mathrm{O}$ & 62.39 & 47.11 & $\mathrm{O}$ & 66.37 & 52.23 & $\mathrm{O}$ & 60.70 & 44.09 & $\mathrm{O}$ & 60.56 & 43.87 \\
\hline & $\mathrm{Na}$ & 1.68 & 1.82 & $\mathrm{Al}$ & 0.43 & 0.57 & $\mathrm{Na}$ & 0.54 & 0.56 & $\mathrm{Na}$ & 0.79 & 0.82 \\
\hline & $\mathrm{Mg}$ & 1.22 & 1.40 & $\mathrm{Si}$ & 32.56 & 44.97 & $\mathrm{Mg}$ & 8.75 & 9.66 & $\mathrm{Mg}$ & 6.00 & 6.61 \\
\hline & $\mathrm{Al}$ & 7.34 & 9.34 & K & 0.14 & 0.27 & $\mathrm{Al}$ & 2.37 & 2.90 & $\mathrm{Al}$ & 4.19 & 5.13 \\
\hline & $\mathrm{Si}$ & 22.12 & 29.32 & $\mathrm{Ca}$ & 0.20 & 0.40 & $\mathrm{Si}$ & 20.45 & 26.07 & $\mathrm{Si}$ & 19.30 & 24.55 \\
\hline & K & 0.89 & 1.64 & $\mathrm{Fe}$ & 0.16 & 0.43 & K & 0.34 & 0.61 & K & 0.23 & 0.41 \\
\hline & $\mathrm{Ca}$ & 2.76 & 5.23 & $\mathrm{Sb}$ & 0.06 & 0.34 & $\mathrm{Ca}$ & 1.63 & 2.96 & $\mathrm{Ca}$ & 5.44 & 9.88 \\
\hline & $\mathrm{Ti}$ & 0.27 & 0.61 & $\mathrm{Au}$ & 0.03 & 0.31 & $\mathrm{Ti}$ & 0.19 & 0.42 & $\mathrm{Ti}$ & 0.22 & 0.48 \\
\hline & \multirow[t]{4}{*}{$\mathrm{Fe}$} & 1.34 & 3.54 & $\mathrm{~Pb}$ & 0.05 & 0.48 & V & 0.02 & 0.04 & $\mathrm{Fe}$ & 3.27 & 8.26 \\
\hline & & & & & & & $\mathrm{Mn}$ & 0.09 & 0.24 & & & \\
\hline & & & & & & & $\mathrm{Fe}$ & 4.91 & 12.46 & & & \\
\hline & & e: $0.1 \%$ & & & & & & & & & & \\
\hline
\end{tabular}




\begin{tabular}{|c|c|c|c|c|c|c|c|c|c|c|c|c|}
\hline & element & $\begin{array}{c}\text { atomic } \\
\%\end{array}$ & $\mathrm{Wt} \%$ & & & & & & & & & \\
\hline & $\mathrm{O}$ & 66.77 & 42.46 & & & & & & & & & \\
\hline & $\mathrm{Si}$ & 0.60 & 0.67 & & & & & & & & & \\
\hline & $\mathrm{S}$ & 16.47 & 20.99 & & & & & & & & & \\
\hline & $\mathrm{Fe}$ & 16.16 & 35.88 & & & & & & & & & \\
\hline \multirow[t]{11}{*}{ MS33 } & \multicolumn{3}{|c|}{ melt: $55.9 \%$} & \multicolumn{3}{|c|}{ clinopyroxene: $25.4 \%$} & \multicolumn{3}{|c|}{ amphibole: $8.8 \%$} & \multicolumn{3}{|c|}{ FeTi oxides: $0.5 \%$} \\
\hline & element & $\begin{array}{c}\text { atomic } \\
\%\end{array}$ & $\mathrm{Wt} \%$ & element & $\begin{array}{c}\text { atomic } \\
\%\end{array}$ & Wt $\%$ & element & $\begin{array}{c}\text { atomic } \\
\%\end{array}$ & $\mathrm{Wt} \%$ & element & $\begin{array}{c}\text { atomic } \\
\%\end{array}$ & Wt $\%$ \\
\hline & $\mathrm{O}$ & 61.27 & 45.90 & $\mathrm{O}$ & 59.91 & 42.79 & $\mathrm{O}$ & 57.53 & 39.97 & $\mathrm{O}$ & 54.82 & 28.71 \\
\hline & $\mathrm{Na}$ & 2.61 & 2.82 & $\mathrm{Na}$ & 0.58 & 0.60 & $\mathrm{Mg}$ & 17.57 & 18.55 & $\mathrm{Mg}$ & 3.31 & 2.63 \\
\hline & $\mathrm{Mg}$ & 0.62 & 0.71 & $\mathrm{Mg}$ & 7.07 & 7.68 & $\mathrm{Al}$ & 0.85 & 1.00 & $\mathrm{Al}$ & 5.37 & 4.74 \\
\hline & $\mathrm{Al}$ & 11.16 & 14.10 & $\mathrm{Al}$ & 3.38 & 4.07 & $\mathrm{Si}$ & 14.56 & 17.76 & $\mathrm{Si}$ & 1.48 & 1.36 \\
\hline & $\mathrm{Si}$ & 18.16 & 23.89 & $\mathrm{Si}$ & 18.07 & 22.66 & $\mathrm{Ca}$ & 0.39 & 0.68 & $\mathrm{Ca}$ & 0.28 & 0.36 \\
\hline & $\mathrm{K}$ & 0.20 & 0.36 & $\mathrm{Ca}$ & 7.20 & 12.88 & $\mathrm{Ti}$ & 0.07 & 0.15 & $\mathrm{Ti}$ & 4.65 & 7.28 \\
\hline & $\mathrm{Ca}$ & 4.49 & 8.44 & $\mathrm{Ti}$ & 0.35 & 0.74 & Mn & 0.16 & 0.37 & $\mathrm{~V}$ & 0.40 & 0.67 \\
\hline & $\mathrm{Ti}$ & 0.20 & 0.45 & $\mathrm{Mn}$ & 0.10 & 0.25 & $\mathrm{Fe}$ & 8.87 & 21.51 & $\mathrm{Cr}$ & 0.43 & 0.74 \\
\hline & $\mathrm{Fe}$ & 1.28 & 3.34 & $\mathrm{Fe}$ & 3.34 & 8.32 & & & & $\mathrm{Fe}$ & 29.27 & 53.50 \\
\hline \multirow[t]{12}{*}{ MS34 } & \multicolumn{3}{|c|}{ melt: $63.6 \%$} & \multicolumn{3}{|c|}{ clinopyroxene: $20.6 \%$} & \multicolumn{3}{|c|}{ orthopyroxene: $5.2 \%$} & \multicolumn{3}{|c|}{ FeTi oxides: $0.5 \%$} \\
\hline & element & $\begin{array}{c}\text { atomic } \\
\%\end{array}$ & Wt $\%$ & element & $\begin{array}{c}\text { atomic } \\
\%\end{array}$ & Wt $\%$ & element & $\begin{array}{c}\text { atomic } \\
\%\end{array}$ & Wt $\%$ & element & $\begin{array}{c}\text { atomic } \\
\%\end{array}$ & $\mathrm{Wt} \%$ \\
\hline & $\mathrm{O}$ & 61.38 & 42.67 & $\mathrm{O}$ & 60.04 & 42.67 & $\mathrm{O}$ & 59.88 & 42.86 & $\mathrm{O}$ & 59.33 & 32.20 \\
\hline & $\mathrm{Na}$ & 2.67 & 0.55 & $\mathrm{Na}$ & 0.54 & 0.55 & $\mathrm{Mg}$ & 12.12 & 13.19 & $\mathrm{Mg}$ & 3.12 & 2.57 \\
\hline & $\mathrm{Mg}$ & 0.76 & 8.27 & $\mathrm{Mg}$ & 7.66 & 8.27 & $\mathrm{Al}$ & 1.50 & 1.81 & $\mathrm{Al}$ & 0.51 & 0.47 \\
\hline & $\mathrm{Al}$ & 9.44 & 3.19 & $\mathrm{Al}$ & 2.66 & 3.19 & $\mathrm{Si}$ & 18.82 & 23.65 & $\mathrm{Si}$ & 0.60 & 0.58 \\
\hline & $\mathrm{Si}$ & 19.31 & 23.34 & $\mathrm{Si}$ & 18.71 & 23.34 & $\mathrm{Ca}$ & 0.89 & 1.60 & $\mathrm{Ca}$ & 0.17 & 0.23 \\
\hline & $\mathrm{K}$ & 0.71 & 0.20 & $\mathrm{~K}$ & 0.12 & 0.20 & $\mathrm{Ti}$ & 0.18 & 0.39 & $\mathrm{Ti}$ & 17.46 & 28.37 \\
\hline & $\mathrm{Ca}$ & 3.45 & 9.16 & $\mathrm{Ca}$ & 5.14 & 9.16 & $\mathrm{Mn}$ & 0.13 & 0.33 & V & 0.23 & 0.40 \\
\hline & $\mathrm{Ti}$ & 0.37 & 0.78 & $\mathrm{Ti}$ & 0.37 & 0.78 & $\mathrm{Fe}$ & 6.47 & 16.16 & $\mathrm{Fe}$ & 18.57 & 35.18 \\
\hline & $\mathrm{Fe}$ & 1.85 & 0.28 & $\mathrm{Mn}$ & 0.12 & 0.28 & & & & & & \\
\hline & Ir & 0.06 & 11.55 & $\mathrm{Fe}$ & 4.66 & 11.55 & & & & & & \\
\hline \multirow[t]{5}{*}{ MS35 } & \multicolumn{3}{|c|}{ melt: $66.5 \%$} & \multicolumn{3}{|c|}{ clinopyroxene: $15.3 \%$} & \multicolumn{3}{|c|}{ plagioclase: $10.7 \%$} & \multicolumn{3}{|c|}{ orthopyroxene: $2.6 \%$} \\
\hline & element & $\begin{array}{c}\text { atomic } \\
\%\end{array}$ & Wt $\%$ & element & $\begin{array}{c}\text { atomic } \\
\%\end{array}$ & Wt $\%$ & element & $\begin{array}{c}\text { atomic } \\
\%\end{array}$ & Wt $\%$ & element & $\begin{array}{c}\text { atomic } \\
\%\end{array}$ & $\mathrm{Wt} \%$ \\
\hline & $\mathrm{O}$ & 61.43 & 44.97 & $\mathrm{O}$ & 60.13 & 42.36 & $\mathrm{O}$ & 61.43 & 46.56 & $\mathrm{O}$ & 59.84 & 42.37 \\
\hline & $\mathrm{Na}$ & 2.55 & 2.68 & $\mathrm{Mg}$ & 7.76 & 8.31 & $\mathrm{Na}$ & 2.39 & 2.60 & $\mathrm{Mg}$ & 11.57 & 12.45 \\
\hline & $\mathrm{Mg}$ & 0.97 & 1.08 & $\mathrm{Al}$ & 2.15 & 2.55 & $\mathrm{Al}$ & 12.93 & 16.53 & $\mathrm{Al}$ & 1.22 & 1.46 \\
\hline
\end{tabular}




\begin{tabular}{|c|c|c|c|c|c|c|c|c|c|c|c|c|}
\hline & $\mathrm{Al}$ & 7.64 & 9.43 & $\mathrm{Si}$ & 18.96 & 23.45 & $\mathrm{Si}$ & 17.68 & 23.52 & $\mathrm{Si}$ & 18.94 & 23.55 \\
\hline & $\mathrm{Si}$ & 20.30 & 26.08 & $\mathrm{~K}$ & 0.10 & 0.17 & $\mathrm{~K}$ & 0.16 & 0.31 & $\mathrm{Ca}$ & 0.87 & 1.54 \\
\hline & $\mathrm{K}$ & 0.99 & 1.77 & $\mathrm{Ca}$ & 5.08 & 8.96 & $\mathrm{Ca}$ & 5.11 & 9.71 & $\mathrm{Ti}$ & 0.12 & 0.25 \\
\hline & $\mathrm{Ca}$ & 2.71 & 4.97 & $\mathrm{Ti}$ & 0.28 & 0.59 & $\mathrm{Fe}$ & 0.29 & 0.78 & Mn & 0.16 & 0.38 \\
\hline & $\mathrm{Ti}$ & 0.44 & 0.97 & Mn & 0.16 & 0.40 & & & & $\mathrm{Fe}$ & 7.28 & 17.99 \\
\hline & Mn & 0.06 & 0.15 & $\mathrm{Fe}$ & 5.38 & 13.22 & & & & & & \\
\hline & $\mathrm{Fe}$ & 2.83 & 7.22 & & & & & & & & & \\
\hline & Ir & 0.08 & 0.68 & & & & & & & & & \\
\hline \multirow[t]{21}{*}{ MS37 } & \multicolumn{3}{|c|}{ melt: $50.9 \%$} & \multicolumn{3}{|c|}{ biotite: $34.8 \%$} & \multicolumn{3}{|c|}{ plagioclase: $9.3 \%$} & \multicolumn{3}{|c|}{ FeTi oxides: $4.1 \%$} \\
\hline & element & $\begin{array}{c}\text { atomic } \\
\%\end{array}$ & Wt $\%$ & element & $\begin{array}{c}\text { atomic } \\
\%\end{array}$ & Wt $\%$ & element & $\begin{array}{c}\text { atomic } \\
\%\end{array}$ & Wt $\%$ & element & $\begin{array}{c}\text { atomic } \\
\%\end{array}$ & Wt $\%$ \\
\hline & $\mathrm{O}$ & 61.96 & 46.63 & $\mathrm{O}$ & 58.60 & 41.56 & $\mathrm{O}$ & 61.56 & 46.50 & $\mathrm{O}$ & 53.16 & 26.69 \\
\hline & $\mathrm{Na}$ & 2.12 & 2.30 & $\mathrm{Na}$ & 0.59 & 0.61 & $\mathrm{Na}$ & 1.72 & 1.86 & $\mathrm{Mg}$ & 2.00 & 1.53 \\
\hline & $\mathrm{Mg}$ & 1.16 & 1.32 & $\mathrm{Mg}$ & 8.73 & 9.41 & $\mathrm{Al}$ & 13.11 & 16.70 & $\mathrm{Al}$ & 4.41 & 3.73 \\
\hline & $\mathrm{Al}$ & 7.33 & 9.31 & $\mathrm{Al}$ & 7.53 & 9.01 & $\mathrm{Si}$ & 17.56 & 23.28 & $\mathrm{Si}$ & 2.55 & 2.25 \\
\hline & $\mathrm{Si}$ & 22.24 & 29.38 & $\mathrm{Si}$ & 15.04 & 18.73 & K & 0.26 & 0.49 & K & 0.26 & 0.32 \\
\hline & $\mathrm{K}$ & 2.14 & 3.94 & K & 4.21 & 7.30 & $\mathrm{Ca}$ & 5.50 & 10.41 & $\mathrm{Ca}$ & 0.16 & 0.20 \\
\hline & $\mathrm{Ca}$ & 1.32 & 2.48 & $\mathrm{Ca}$ & 0.16 & 0.29 & $\mathrm{Fe}$ & 0.29 & 0.77 & $\mathrm{Ti}$ & 1.44 & 2.17 \\
\hline & $\mathrm{Ti}$ & 0.13 & 0.30 & $\mathrm{Ti}$ & 0.70 & 1.49 & & & & V & 0.17 & 0.26 \\
\hline & V & 0.01 & 0.03 & V & 0.04 & 0.09 & & & & Mn & 0.33 & 0.57 \\
\hline & $\mathrm{Mn}$ & 0.07 & 0.17 & $\mathrm{Mn}$ & 0.06 & 0.15 & & & & $\mathrm{Fe}$ & 35.53 & 62.28 \\
\hline & $\mathrm{Fe}$ & 1.47 & 3.87 & $\mathrm{Fe}$ & 4.13 & 10.23 & & & & & & \\
\hline & \multirow[t]{2}{*}{$\mathrm{Ba}$} & 0.04 & 0.27 & In & 0.04 & 0.23 & & & & & & \\
\hline & \multirow{2}{*}{\multicolumn{3}{|c|}{ apatite: $0.02 \%$}} & $\mathrm{Ba}$ & 0.15 & 0.91 & & & & & & \\
\hline & & & & & & & & & & & & \\
\hline & element & $\begin{array}{c}\text { atomic } \\
\%\end{array}$ & Wt $\%$ & & & & & & & & & \\
\hline & $\mathrm{O}$ & 60.77 & 40.31 & & & & & & & & & \\
\hline & $\mathrm{Si}$ & 0.39 & 0.46 & & & & & & & & & \\
\hline & $\mathrm{P}$ & 14.09 & 18.10 & & & & & & & & & \\
\hline & $\mathrm{Ca}$ & 24.75 & 41.13 & & & & & & & & & \\
\hline \multirow[t]{7}{*}{ MS38 } & \multicolumn{3}{|c|}{ melt: $47.7 \%$} & \multicolumn{3}{|c|}{ plagioclase: $28.0 \%$} & \multicolumn{3}{|c|}{ orthopyroxene: $6.7 \%$} & \multicolumn{3}{|c|}{ clinopyroxene: $3.2 \%$} \\
\hline & element & $\begin{array}{c}\text { atomic } \\
\%\end{array}$ & Wt $\%$ & element & $\begin{array}{c}\text { atomic } \\
\%\end{array}$ & $\mathrm{Wt} \%$ & element & $\begin{array}{c}\text { atomic } \\
\%\end{array}$ & Wt $\%$ & element & $\begin{array}{c}\text { atomic } \\
\%\end{array}$ & Wt $\%$ \\
\hline & $\mathrm{O}$ & 63.47 & 48.71 & $\mathrm{O}$ & 60.38 & 45.98 & $\mathrm{O}$ & 59.11 & 42.13 & $\mathrm{O}$ & 60.38 & 43.36 \\
\hline & $\mathrm{Na}$ & 2.30 & 2.53 & $\mathrm{Na}$ & 3.62 & 3.96 & $\mathrm{Na}$ & 0.71 & 0.73 & $\mathrm{Na}$ & 0.89 & 0.91 \\
\hline & $\mathrm{Mg}$ & 0.56 & 0.66 & $\mathrm{Al}$ & 11.02 & 14.16 & $\mathrm{Mg}$ & 9.14 & 9.90 & $\mathrm{Mg}$ & 6.02 & 6.57 \\
\hline & $\mathrm{Al}$ & 5.81 & 7.53 & $\mathrm{Si}$ & 20.91 & 27.96 & $\mathrm{Al}$ & 2.41 & 2.90 & $\mathrm{Al}$ & 2.74 & 3.32 \\
\hline & $\mathrm{Si}$ & 24.07 & 32.44 & $\mathrm{~K}$ & 0.33 & 0.62 & $\mathrm{Si}$ & 20.93 & 26.19 & $\mathrm{Si}$ & 19.81 & 24.97 \\
\hline
\end{tabular}




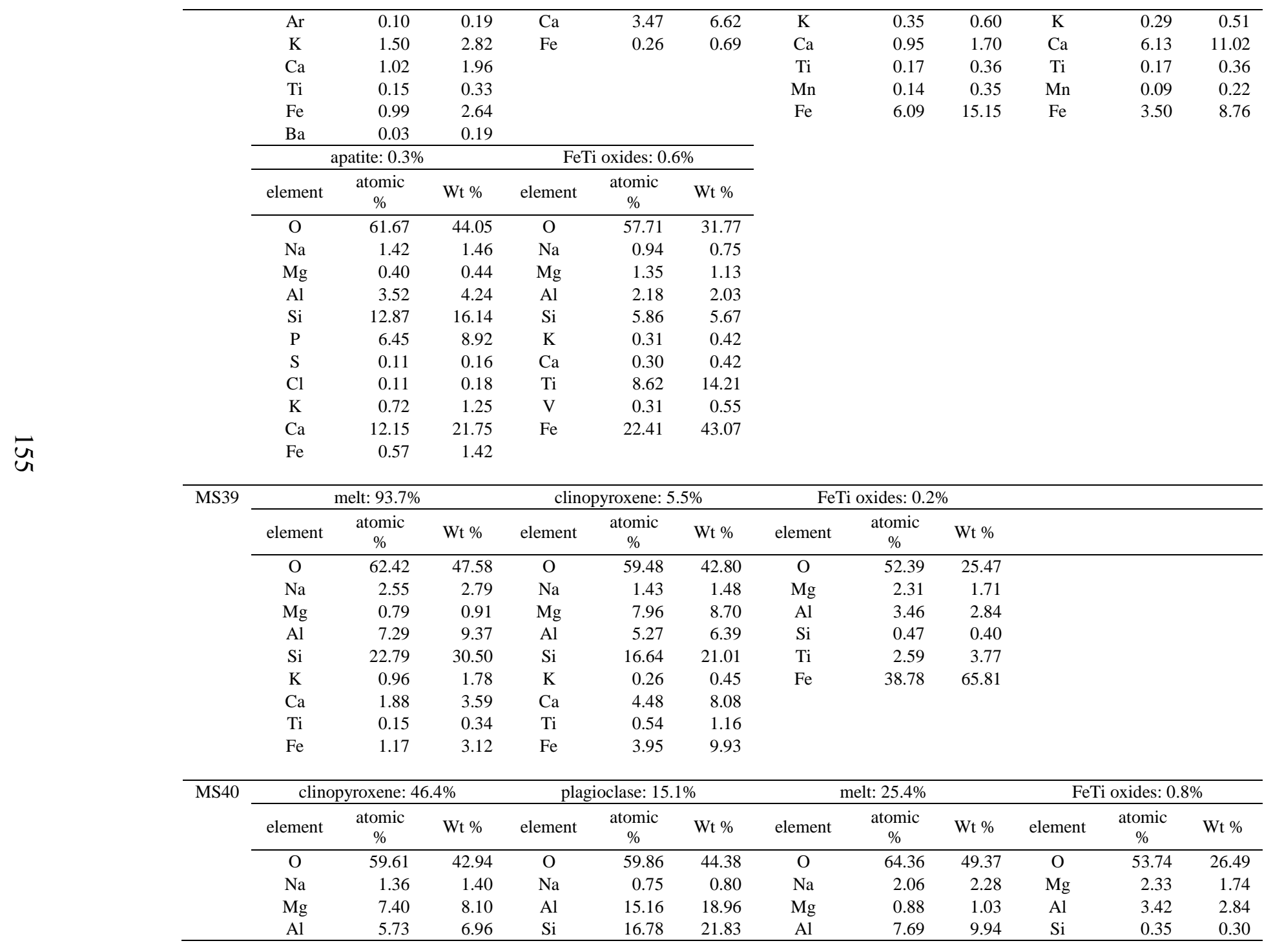




\begin{tabular}{|c|c|c|c|c|c|c|c|c|c|c|c|c|}
\hline & $\mathrm{Si}$ & 16.71 & 21.14 & $\mathrm{Ca}$ & 7.20 & 13.37 & $\mathrm{Si}$ & 20.48 & 27.58 & $\mathrm{Ca}$ & 0.18 & 0.23 \\
\hline & $\mathrm{K}$ & 0.09 & 0.16 & $\mathrm{Fe}$ & 0.26 & 0.66 & $\mathrm{P}$ & 0.11 & 0.16 & $\mathrm{Ti}$ & 1.51 & 2.23 \\
\hline & $\mathrm{Ca}$ & 4.88 & 8.81 & & & & $\mathrm{Cl}$ & 0.03 & 0.05 & V & 0.20 & 0.31 \\
\hline & $\mathrm{Ti}$ & 0.36 & 0.77 & & & & K & 0.20 & 0.38 & $\mathrm{Fe}$ & 38.21 & 65.73 \\
\hline & $\mathrm{Mn}$ & 0.09 & 0.22 & & & & $\mathrm{Ca}$ & 2.95 & 5.67 & Co & 0.07 & 0.13 \\
\hline & $\mathrm{Fe}$ & 3.78 & 9.50 & & & & $\mathrm{Ti}$ & 0.10 & 0.24 & & & \\
\hline & & & & & & & $\mathrm{Mn}$ & 0.05 & 0.14 & & & \\
\hline & & & & & & & $\mathrm{Fe}$ & 1.04 & 2.80 & & & \\
\hline & & & & & & & Os & 0.04 & 0.37 & & & \\
\hline & & atite: $0.1^{\prime}$ & & & & & & & & & & \\
\hline & element & $\begin{array}{c}\text { atomic } \\
\%\end{array}$ & Wt $\%$ & & & & & & & & & \\
\hline & $\mathrm{O}$ & 58.91 & 37.89 & & & & & & & & & \\
\hline & $\mathrm{Si}$ & 0.37 & 0.41 & & & & & & & & & \\
\hline & $\mathrm{P}$ & 14.95 & 18.61 & & & & & & & & & \\
\hline & $\mathrm{Cl}$ & 0.19 & 0.27 & & & & & & & & & \\
\hline & $\mathrm{Ca}$ & 25.11 & 40.46 & & & & & & & & & \\
\hline & $\mathrm{Sb}$ & 0.48 & 2.37 & & & & & & & & & \\
\hline MS41 & clin & yroxene: 5 & & & clase: 16 & & & lt: $18.8 \%$ & & & oxides: 1. & \\
\hline & element & $\begin{array}{c}\text { atomic } \\
\%\end{array}$ & Wt $\%$ & element & $\begin{array}{c}\text { atomic } \\
\%\end{array}$ & Wt $\%$ & element & $\begin{array}{c}\text { atomic } \\
\%\end{array}$ & Wt $\%$ & element & $\begin{array}{c}\text { atomic } \\
\%\end{array}$ & Wt $\%$ \\
\hline & $\mathrm{O}$ & 59.29 & 42.69 & $\mathrm{O}$ & 60.06 & 44.59 & $\mathrm{O}$ & 65.31 & 50.78 & $\mathrm{O}$ & 53.96 & 27.61 \\
\hline & $\mathrm{Na}$ & 1.47 & 1.52 & $\mathrm{Na}$ & 0.87 & 0.93 & $\mathrm{Na}$ & 2.14 & 2.4 & $\mathrm{Mg}$ & 2.62 & 2.04 \\
\hline & $\mathrm{Mg}$ & 7.01 & 7.67 & $\mathrm{Al}$ & 14.81 & 18.55 & $\mathrm{Mg}$ & 0.49 & 0.58 & $\mathrm{Al}$ & 4.05 & 3.5 \\
\hline & $\mathrm{Al}$ & 5.94 & 7.21 & $\mathrm{Si}$ & 16.91 & 22.03 & $\mathrm{Al}$ & 7.49 & 9.83 & $\mathrm{Si}$ & 3.11 & 2.79 \\
\hline & $\mathrm{Si}$ & 17.25 & 21.81 & $\mathrm{Ca}$ & 7.02 & 13.05 & $\mathrm{Si}$ & 20.65 & 28.18 & $\mathrm{Ca}$ & 0.66 & 0.84 \\
\hline & K & 0.12 & 0.2 & $\mathrm{Fe}$ & 0.33 & 0.85 & $\mathrm{P}$ & 0.13 & 0.19 & $\mathrm{Ti}$ & 1.32 & 2.03 \\
\hline & $\mathrm{Ca}$ & 4.8 & 8.65 & & & & K & 0.23 & 0.44 & V & 0.18 & 0.29 \\
\hline & $\mathrm{Ti}$ & 0.35 & 0.75 & & & & $\mathrm{Ca}$ & 2.6 & 5.06 & $\mathrm{Fe}$ & 34.09 & 60.89 \\
\hline & $\mathrm{Mn}$ & 0.09 & 0.22 & & & & $\mathrm{Ti}$ & 0.09 & 0.21 & & & \\
\hline & $\mathrm{Fe}$ & 3.69 & 9.28 & & & & $\mathrm{Mn}$ & 0.05 & 0.14 & & & \\
\hline & & & & & & & $\mathrm{Fe}$ & 0.81 & 2.2 & & & \\
\hline & & atite: 0.39 & & & & & & & & & & \\
\hline & element & $\begin{array}{c}\text { atomic } \\
\%\end{array}$ & Wt $\%$ & & & & & & & & & \\
\hline & $\mathrm{O}$ & 63.8 & 43.88 & & & & & & & & & \\
\hline & $\mathrm{Na}$ & 0.45 & 0.44 & & & & & & & & & \\
\hline & $\mathrm{Mg}$ & 0.53 & 0.56 & & & & & & & & & \\
\hline & $\mathrm{Al}$ & 0.92 & 1.07 & & & & & & & & & \\
\hline & $\mathrm{Si}$ & 2.39 & 2.88 & & & & & & & & & \\
\hline
\end{tabular}




\begin{tabular}{|c|c|c|c|c|c|c|c|c|c|c|c|c|}
\hline & $\begin{array}{l}\mathrm{P} \\
\mathrm{Ca} \\
\mathrm{Fe}\end{array}$ & $\begin{array}{r}10.53 \\
20.95 \\
0.43\end{array}$ & $\begin{array}{r}14.03 \\
36.11 \\
1.03\end{array}$ & & & & & & & & & \\
\hline \multirow[t]{25}{*}{ MS43 } & \multicolumn{3}{|c|}{ melt: $54.1 \%$} & \multicolumn{3}{|c|}{ biotite: $22.2 \%$} & \multicolumn{3}{|c|}{ amphibole: $7.9 \%$} & \multicolumn{3}{|c|}{ clinopyroxene: $7.4 \%$} \\
\hline & element & $\begin{array}{c}\text { atomic } \\
\%\end{array}$ & Wt $\%$ & element & $\begin{array}{c}\text { atomic } \\
\%\end{array}$ & $\mathrm{Wt} \%$ & element & $\begin{array}{c}\text { atomic } \\
\%\end{array}$ & Wt $\%$ & element & $\begin{array}{c}\text { atomic } \\
\%\end{array}$ & $\mathrm{Wt} \%$ \\
\hline & $\mathrm{O}$ & 61.59 & 46.38 & $\mathrm{O}$ & 59.29 & 41.57 & $\mathrm{O}$ & 46.76 & 25.90 & $\mathrm{O}$ & 60.31 & 43.11 \\
\hline & $\mathrm{Na}$ & 2.55 & 2.75 & $\mathrm{Na}$ & 0.73 & 0.74 & $\mathrm{Na}$ & 1.79 & 1.43 & $\mathrm{Na}$ & 0.75 & 0.77 \\
\hline & $\mathrm{Mg}$ & 0.87 & 1.00 & $\mathrm{Mg}$ & 6.99 & 7.45 & $\mathrm{Mg}$ & 2.78 & 2.34 & $\mathrm{Mg}$ & 7.03 & 7.64 \\
\hline & $\mathrm{Al}$ & 8.44 & 10.72 & $\mathrm{Al}$ & 6.50 & 7.68 & $\mathrm{Al}$ & 6.82 & 6.37 & $\mathrm{Al}$ & 2.89 & 3.48 \\
\hline & $\mathrm{Si}$ & 20.99 & 27.75 & $\mathrm{Si}$ & 16.30 & 20.07 & $\mathrm{Si}$ & 14.86 & 14.45 & $\mathrm{Si}$ & 19.61 & 24.61 \\
\hline & $\mathrm{K}$ & 1.95 & 3.59 & K & 3.30 & 5.65 & $\mathrm{P}$ & 0.39 & 0.42 & K & 0.60 & 1.05 \\
\hline & $\mathrm{Ca}$ & 2.18 & 4.11 & $\mathrm{Ca}$ & 0.51 & 0.89 & K & 1.39 & 1.88 & $\mathrm{Ca}$ & 3.63 & 6.50 \\
\hline & $\mathrm{Ti}$ & 0.21 & 0.47 & $\mathrm{Ti}$ & 0.95 & 2.00 & $\mathrm{Ca}$ & 1.60 & 2.23 & $\mathrm{Ti}$ & 0.23 & 0.50 \\
\hline & \multirow[t]{4}{*}{$\mathrm{Fe}$} & 1.22 & 3.22 & V & 0.05 & 0.10 & $\mathrm{Ti}$ & 2.79 & 4.63 & $\mathrm{Mn}$ & 0.17 & 0.42 \\
\hline & & & & $\mathrm{Fe}$ & 5.18 & 12.68 & V & 0.11 & 0.20 & $\mathrm{Fe}$ & 4.77 & 11.91 \\
\hline & & & & In & 0.05 & 0.24 & $\mathrm{Fe}$ & 20.64 & 39.91 & & & 0.24 \\
\hline & & & & $\mathrm{Ba}$ & 0.15 & 0.92 & In & 0.06 & 0.25 & & & 0.92 \\
\hline & \multicolumn{3}{|c|}{ orthopyroxene: $1.2 \%$} & \multicolumn{3}{|c|}{ apatite: $0.2 \%$} & & & & & & \\
\hline & element & $\begin{array}{c}\text { atomic } \\
\%\end{array}$ & Wt $\%$ & element & $\begin{array}{c}\text { atomic } \\
\%\end{array}$ & Wt $\%$ & & & & & & \\
\hline & $\mathrm{O}$ & 59.69 & 42.23 & $\mathrm{O}$ & 60.85 & 40.82 & & & & & & \\
\hline & $\mathrm{Mg}$ & 11.76 & 12.64 & $\mathrm{Mg}$ & 0.39 & 0.40 & & & & & & \\
\hline & $\mathrm{Al}$ & 1.62 & 1.93 & $\mathrm{Al}$ & 0.90 & 1.01 & & & & & & \\
\hline & $\mathrm{Si}$ & 18.46 & 22.93 & $\mathrm{Si}$ & 2.53 & 2.99 & & & & & & \\
\hline & $\mathrm{K}$ & 0.20 & 0.34 & $\mathrm{P}$ & 12.74 & 16.54 & & & & & & \\
\hline & $\mathrm{Ca}$ & 0.61 & 1.08 & $\mathrm{Cl}$ & 0.25 & 0.37 & & & & & & \\
\hline & $\mathrm{Ti}$ & 0.20 & 0.43 & $\mathrm{~K}$ & 0.26 & 0.43 & & & & & & \\
\hline & $\mathrm{Mn}$ & 0.25 & 0.61 & $\mathrm{Ca}$ & 21.56 & 36.24 & & & & & & \\
\hline & $\mathrm{Fe}$ & 7.21 & 17.82 & $\mathrm{Fe}$ & 0.52 & 1.21 & & & & & & \\
\hline \multirow[t]{7}{*}{ MS44 } & \multicolumn{3}{|c|}{ melt: $76.3 \%$} & \multicolumn{3}{|c|}{ clinopyroxene: $16.7 \%$} & \multicolumn{3}{|c|}{ amphibole: $2.3 \%$} & \multicolumn{3}{|c|}{ apatite: $0.23 \%$} \\
\hline & element & $\begin{array}{c}\text { atomic } \\
\%\end{array}$ & Wt $\%$ & element & $\begin{array}{c}\text { atomic } \\
\%\end{array}$ & Wt $\%$ & element & $\begin{array}{c}\text { atomic } \\
\%\end{array}$ & Wt $\%$ & element & $\begin{array}{c}\text { atomic } \\
\%\end{array}$ & Wt $\%$ \\
\hline & $\mathrm{O}$ & 62.06 & 47.03 & $\mathrm{O}$ & 60.76 & 43.27 & $\mathrm{O}$ & 60.88 & 40.78 & $\mathrm{O}$ & 60.03 & 40.24 \\
\hline & $\mathrm{Na}$ & 2.87 & 3.13 & $\mathrm{Na}$ & 1.12 & 1.14 & $\mathrm{Na}$ & 1.85 & 1.78 & $\mathrm{Na}$ & 0.61 & 0.59 \\
\hline & $\mathrm{Mg}$ & 0.69 & 0.79 & $\mathrm{Mg}$ & 5.31 & 5.75 & $\mathrm{Mg}$ & 1.38 & 1.41 & $\mathrm{Mg}$ & 0.36 & 0.36 \\
\hline & $\mathrm{Al}$ & 7.56 & 9.66 & $\mathrm{Al}$ & 3.12 & 3.74 & $\mathrm{Al}$ & 4.59 & 5.18 & $\mathrm{Al}$ & 1.24 & 1.41 \\
\hline & $\mathrm{Si}$ & 22.03 & 29.31 & $\mathrm{Si}$ & 20.38 & 25.48 & $\mathrm{Si}$ & 16.68 & 19.61 & $\mathrm{Si}$ & 4.62 & 5.43 \\
\hline
\end{tabular}




\begin{tabular}{|c|c|c|c|c|c|c|c|c|c|c|c|c|}
\hline & $\mathrm{K}$ & 1.05 & 1.95 & $\mathrm{~K}$ & 0.56 & 0.97 & $\mathrm{P}$ & 0.12 & 0.16 & $P$ & 10.34 & 13.42 \\
\hline & $\mathrm{Ca}$ & 2.24 & 4.25 & $\mathrm{Ca}$ & 2.80 & 4.99 & K & 0.90 & 1.47 & $\mathrm{Cl}$ & 0.21 & 0.31 \\
\hline & $\mathrm{Ti}$ & 0.27 & 0.62 & $\mathrm{Ti}$ & 0.42 & 0.90 & $\mathrm{Ca}$ & 1.35 & 2.27 & $\mathrm{~K}$ & 0.37 & 0.60 \\
\hline & $\mathrm{Fe}$ & 1.23 & 3.25 & Mn & 0.13 & 0.32 & $\mathrm{Ti}$ & 3.98 & 7.97 & $\mathrm{Ca}$ & 21.75 & 36.52 \\
\hline & & & & $\mathrm{Fe}$ & 5.41 & 13.44 & $\mathrm{Fe}$ & 8.28 & 19.36 & $\mathrm{Fe}$ & 0.47 & 1.11 \\
\hline \multirow[t]{27}{*}{ MS45 } & \multicolumn{3}{|c|}{ quartz: $22.4 \%$} & \multicolumn{3}{|c|}{ melt: $54.8 \%$} & \multicolumn{3}{|c|}{ orthopyroxene: $11.9 \%$} & \multicolumn{3}{|c|}{ plagioclase: $3.0 \%$} \\
\hline & element & $\begin{array}{c}\text { atomic } \\
\%\end{array}$ & Wt $\%$ & element & $\begin{array}{c}\text { atomic } \\
\%\end{array}$ & Wt $\%$ & element & $\begin{array}{c}\text { atomic } \\
\%\end{array}$ & Wt $\%$ & element & $\begin{array}{c}\text { atomic } \\
\%\end{array}$ & Wt $\%$ \\
\hline & $\mathrm{O}$ & 66.28 & 52.53 & $\mathrm{O}$ & 62.26 & 46.59 & $\mathrm{O}$ & 60.83 & 42.23 & $\mathrm{O}$ & 61.26 & 46.25 \\
\hline & $\mathrm{Al}$ & 0.60 & 0.80 & $\mathrm{Na}$ & 2.40 & 2.58 & $\mathrm{Na}$ & 1.22 & 1.22 & $\mathrm{Na}$ & 2.62 & 2.84 \\
\hline & $\mathrm{Si}$ & 32.43 & 45.12 & $\mathrm{Mg}$ & 0.52 & 0.59 & $\mathrm{Mg}$ & 3.60 & 3.79 & $\mathrm{Mg}$ & 1.03 & 1.18 \\
\hline & $\mathrm{K}$ & 0.34 & 0.66 & $\mathrm{Al}$ & 6.73 & 8.49 & $\mathrm{Al}$ & 3.85 & 4.51 & $\mathrm{Al}$ & 12.06 & 15.36 \\
\hline & $\mathrm{Ca}$ & 0.11 & 0.23 & $\mathrm{Si}$ & 22.92 & 30.11 & $\mathrm{Si}$ & 19.87 & 24.22 & $\mathrm{Si}$ & 18.05 & 23.92 \\
\hline & \multirow[t]{6}{*}{$\mathrm{Fe}$} & 0.24 & 0.66 & $\mathrm{~K}$ & 1.71 & 3.13 & $\mathrm{P}$ & 0.11 & 0.15 & $\mathrm{~K}$ & 0.52 & 0.95 \\
\hline & & & & $\mathrm{Ca}$ & 1.46 & 2.73 & $\mathrm{~K}$ & 0.91 & 1.54 & $\mathrm{Ca}$ & 3.07 & 5.81 \\
\hline & & & & $\mathrm{Ti}$ & 0.22 & 0.49 & $\mathrm{Ca}$ & 0.98 & 1.71 & $\mathrm{Fe}$ & 1.40 & 3.68 \\
\hline & & & & $\mathrm{Fe}$ & 1.67 & 4.37 & $\mathrm{Ti}$ & 0.76 & 1.59 & & & \\
\hline & & & & $\mathrm{Ba}$ & 0.05 & 0.31 & $\mathrm{Mn}$ & 0.17 & 0.41 & & & \\
\hline & & & & Ir & 0.07 & 0.60 & $\mathrm{Fe}$ & 7.69 & 18.63 & & & \\
\hline & \multicolumn{3}{|c|}{ biotite: $1.1 \%$} & \multicolumn{3}{|c|}{ FeTi oxides: $0.2 \%$} & & & & & & \\
\hline & element & $\begin{array}{c}\text { atomic } \\
\%\end{array}$ & Wt $\%$ & element & $\begin{array}{c}\text { atomic } \\
\%\end{array}$ & Wt $\%$ & & & & & & \\
\hline & $\mathrm{O}$ & 58.29 & 42.17 & $\mathrm{O}$ & 58.04 & 31.51 & & & & & & \\
\hline & $\mathrm{Na}$ & 5.67 & 5.89 & $\mathrm{Mg}$ & 1.25 & 1.03 & & & & & & \\
\hline & $\mathrm{Mg}$ & 2.78 & 3.05 & $\mathrm{Al}$ & 1.90 & 1.74 & & & & & & \\
\hline & $\mathrm{Al}$ & 3.28 & 4.00 & $\mathrm{Si}$ & 4.98 & 4.75 & & & & & & \\
\hline & $\mathrm{Si}$ & 19.90 & 25.27 & $\mathrm{~K}$ & 0.34 & 0.45 & & & & & & \\
\hline & $\mathrm{S}$ & 0.60 & 0.87 & $\mathrm{Ca}$ & 0.28 & 0.38 & & & & & & \\
\hline & $\mathrm{Cl}$ & 2.47 & 3.97 & $\mathrm{Ti}$ & 10.32 & 16.78 & & & & & & \\
\hline & $\mathrm{K}$ & 2.94 & 5.21 & $\mathrm{Fe}$ & 22.89 & 43.37 & & & & & & \\
\hline & $\mathrm{Ca}$ & 1.57 & 2.85 & & & & & & & & & \\
\hline & $\mathrm{Ti}$ & 0.30 & 0.66 & & & & & & & & & \\
\hline & $\mathrm{Fe}$ & 1.88 & 4.75 & & & & & & & & & \\
\hline & $\mathrm{Zr}$ & 0.32 & 1.31 & & & & & & & & & \\
\hline \multirow[t]{3}{*}{ MS46 } & \multicolumn{3}{|c|}{ melt: $60.5 \%$} & \multicolumn{3}{|c|}{ biotite: $19.7 \%$} & \multicolumn{3}{|c|}{ plagioclase: $6.9 \%$} & \multicolumn{3}{|c|}{ FeTi oxides: $5.6 \%$} \\
\hline & element & $\begin{array}{c}\text { atomic } \\
\%\end{array}$ & Wt $\%$ & element & $\begin{array}{c}\text { atomic } \\
\%\end{array}$ & Wt $\%$ & element & $\begin{array}{c}\text { atomic } \\
\%\end{array}$ & Wt $\%$ & element & $\begin{array}{c}\text { atomic } \\
\%\end{array}$ & Wt $\%$ \\
\hline & $\mathrm{O}$ & 61.74 & 46.23 & $\mathrm{O}$ & 60.03 & 42.37 & $\mathrm{O}$ & 61.41 & 47.00 & $\mathrm{O}$ & 44.97 & 23.78 \\
\hline
\end{tabular}




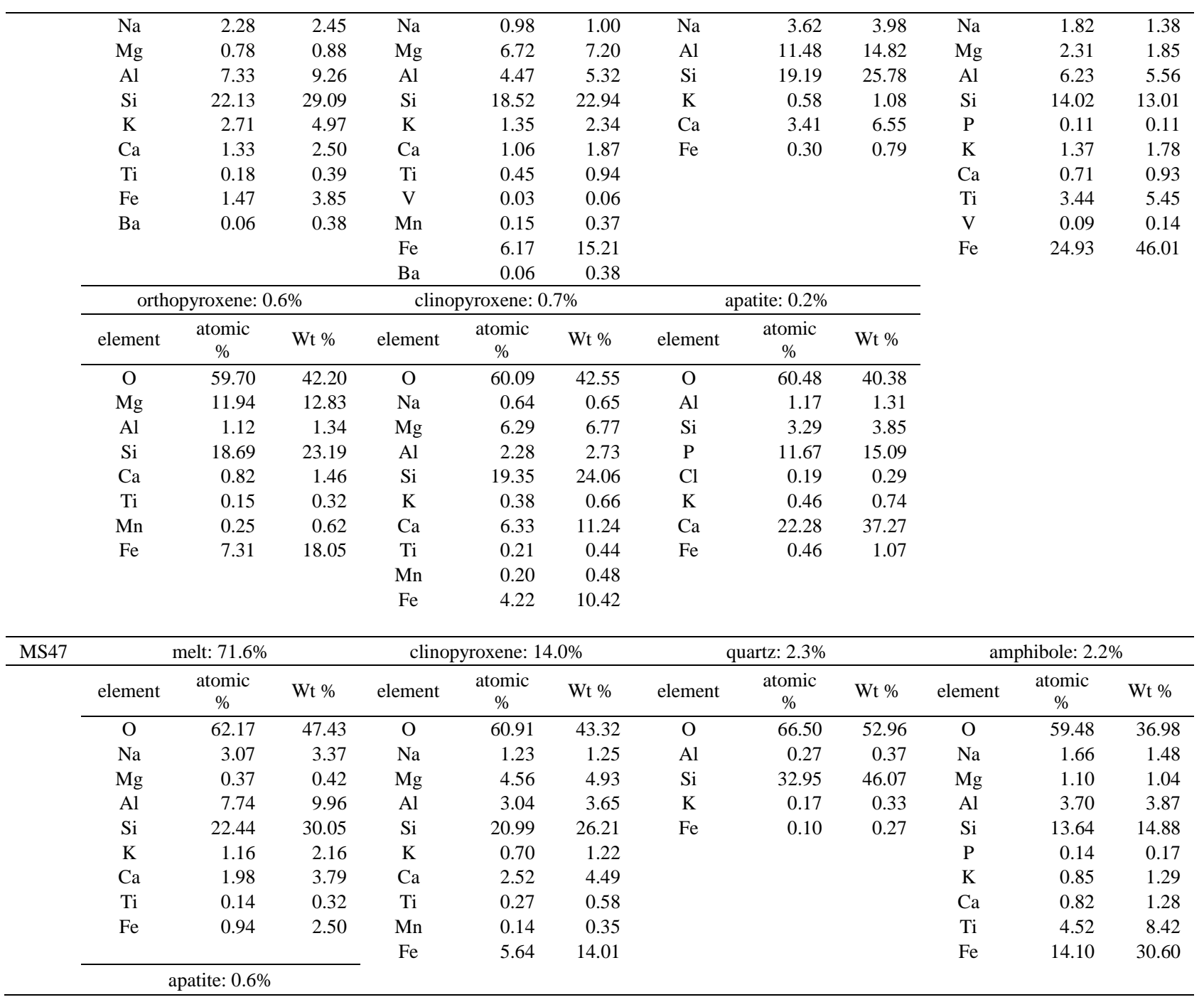




\begin{tabular}{|c|c|c|c|c|c|c|c|c|c|c|c|c|}
\hline & element & $\begin{array}{c}\text { atomic } \\
\%\end{array}$ & Wt $\%$ & & & & & & & & & \\
\hline & $\mathrm{O}$ & 59.91 & 39.21 & & & & & & & & & \\
\hline & $\mathrm{P}$ & 13.22 & 16.75 & & & & & & & & & \\
\hline & $\mathrm{Ca}$ & 26.87 & 44.05 & & & & & & & & & \\
\hline \multirow[t]{23}{*}{ MS48 } & \multicolumn{3}{|c|}{ melt: $67.1 \%$} & \multicolumn{3}{|c|}{ quartz: $16.6 \%$} & \multicolumn{3}{|c|}{ orthopyroxene: $10.9 \%$} & \multicolumn{3}{|c|}{ biotite: $0.7 \%$} \\
\hline & element & $\begin{array}{c}\text { atomic } \\
\%\end{array}$ & Wt $\%$ & element & $\begin{array}{c}\text { atomic } \\
\%\end{array}$ & Wt $\%$ & element & $\begin{array}{c}\text { atomic } \\
\%\end{array}$ & Wt $\%$ & element & $\begin{array}{c}\text { atomic } \\
\%\end{array}$ & Wt $\%$ \\
\hline & $\mathrm{O}$ & 62.68 & 47.21 & $\mathrm{O}$ & 66.36 & 52.43 & $\mathrm{O}$ & 60.95 & 41.69 & $\mathrm{O}$ & 61.99 & 45.57 \\
\hline & $\mathrm{Na}$ & 2.12 & 2.29 & $\mathrm{Si}$ & 33.05 & 45.84 & $\mathrm{Na}$ & 0.85 & 0.83 & $\mathrm{Na}$ & 1.88 & 1.99 \\
\hline & $\mathrm{Mg}$ & 0.32 & 0.37 & K & 0.19 & 0.37 & $\mathrm{Mg}$ & 3.97 & 4.13 & $\mathrm{Mg}$ & 0.93 & 1.04 \\
\hline & $\mathrm{Al}$ & 6.28 & 7.98 & $\mathrm{Fe}$ & 0.16 & 0.45 & $\mathrm{Al}$ & 2.64 & 3.05 & $\mathrm{Al}$ & 5.02 & 6.23 \\
\hline & $\mathrm{Si}$ & 23.91 & 31.62 & $\mathrm{Br}$ & 0.23 & 0.92 & $\mathrm{Si}$ & 19.54 & 23.47 & $\mathrm{Si}$ & 20.15 & 26.01 \\
\hline & K & 1.69 & 3.12 & & & & $\mathrm{P}$ & 0.11 & 0.15 & $\mathrm{P}$ & 1.76 & 2.51 \\
\hline & $\mathrm{Ca}$ & 1.30 & 2.46 & & & & S & 0.26 & 0.36 & S & 0.09 & 0.14 \\
\hline & $\mathrm{Ti}$ & 0.15 & 0.33 & & & & K & 0.64 & 1.08 & K & 1.15 & 2.07 \\
\hline & $\mathrm{Fe}$ & 1.44 & 3.79 & & & & $\mathrm{Ca}$ & 1.06 & 1.81 & $\mathrm{Ca}$ & 4.90 & 9.02 \\
\hline & $\mathrm{Ba}$ & 0.05 & 0.31 & & & & $\mathrm{Ti}$ & 1.10 & 2.25 & $\mathrm{Fe}$ & 2.12 & 5.44 \\
\hline & \multirow[t]{2}{*}{$\mathrm{Ir}$} & 0.06 & 0.52 & & & & $\mathrm{Mn}$ & 0.20 & 0.46 & & & \\
\hline & & & & & & & $\mathrm{Fe}$ & 8.68 & 20.73 & & & \\
\hline & \multicolumn{3}{|c|}{ FeTi oxides: $0.2 \%$} & & & & & & & & & \\
\hline & element & $\begin{array}{c}\text { atomic } \\
\%\end{array}$ & Wt $\%$ & & & & & & & & & \\
\hline & $\mathrm{O}$ & 59.37 & 31.94 & & & & & & & & & \\
\hline & $\mathrm{Mg}$ & 1.00 & 0.82 & & & & & & & & & \\
\hline & $\mathrm{Al}$ & 0.87 & 0.79 & & & & & & & & & \\
\hline & $\mathrm{Si}$ & 2.09 & 1.97 & & & & & & & & & \\
\hline & $\mathrm{S}$ & 0.26 & 0.28 & & & & & & & & & \\
\hline & $\mathrm{Ti}$ & 15.69 & 25.27 & & & & & & & & & \\
\hline & $\mathrm{Fe}$ & 20.73 & 38.93 & & & & & & & & & \\
\hline \multirow[t]{7}{*}{ MS49 } & \multicolumn{3}{|c|}{ melt: $91.9 \%$} & \multicolumn{3}{|c|}{ orthopyroxene: $6.3 \%$} & \multicolumn{3}{|c|}{ FeTi oxides: $0.4 \%$} & & & \\
\hline & element & $\begin{array}{c}\text { atomic } \\
\%\end{array}$ & $\mathrm{Wt} \%$ & element & $\begin{array}{c}\text { atomic } \\
\%\end{array}$ & Wt $\%$ & element & $\begin{array}{c}\text { atomic } \\
\%\end{array}$ & $\mathrm{Wt} \%$ & & & \\
\hline & $\mathrm{O}$ & 51.73 & 36.48 & $\mathrm{O}$ & 59.69 & 40.84 & $\mathrm{O}$ & 59.46 & 34.44 & & & \\
\hline & $\mathrm{Na}$ & 2.08 & 2.1 & $\mathrm{Mg}$ & 10.01 & 10.41 & $\mathrm{Na}$ & 0.98 & 0.82 & & & \\
\hline & $\mathrm{Mg}$ & 0.67 & 0.71 & $\mathrm{Al}$ & 0.93 & 1.08 & $\mathrm{Mg}$ & 0.68 & 0.6 & & & \\
\hline & $\mathrm{Al}$ & 6.51 & 7.74 & $\mathrm{Si}$ & 18.41 & 22.11 & $\mathrm{Al}$ & 2.44 & 2.39 & & & \\
\hline & $\mathrm{Si}$ & 33.26 & 41.18 & $\mathrm{~S}$ & 0.16 & 0.23 & $\mathrm{Si}$ & 7.32 & 7.44 & & & \\
\hline
\end{tabular}




\begin{tabular}{|c|c|c|c|c|c|c|c|c|c|c|c|c|}
\hline & $\mathrm{K}$ & 1.73 & 2.98 & $\mathrm{Ca}$ & 0.53 & 0.91 & $\mathrm{~S}$ & 2.66 & 3.08 & & & \\
\hline & $\mathrm{Ca}$ & 1.49 & 2.63 & $\mathrm{Ti}$ & 0.18 & 0.38 & K & 0.43 & 0.6 & & & \\
\hline & $\mathrm{Ti}$ & 0.22 & 0.46 & $\mathrm{Mn}$ & 0.38 & 0.9 & $\mathrm{Ca}$ & 0.54 & 0.78 & & & \\
\hline & $\mathrm{Fe}$ & 2.32 & 5.72 & $\mathrm{Fe}$ & 9.70 & 23.16 & $\mathrm{Ti}$ & 5.77 & 10.01 & & & \\
\hline & & & & & & & $\mathrm{Mn}$ & 0.92 & 1.83 & & & \\
\hline & & & & & & & $\mathrm{Fe}$ & 18.80 & 38.01 & & & \\
\hline MS50 & & elt: $65.6 \%$ & & & Irtz: $14.5 \%$ & & orthc & oxene: 13 & & & iibole: 2 & \\
\hline & element & $\begin{array}{c}\text { atomic } \\
\%\end{array}$ & Wt $\%$ & element & $\begin{array}{c}\text { atomic } \\
\%\end{array}$ & Wt $\%$ & element & $\begin{array}{c}\text { atomic } \\
\%\end{array}$ & Wt $\%$ & element & $\begin{array}{c}\text { atomic } \\
\%\end{array}$ & Wt $\%$ \\
\hline & $\mathrm{O}$ & 62.48 & 52.61 & $\mathrm{O}$ & 66.32 & 52.61 & $\mathrm{O}$ & 60.93 & 43.28 & $\mathrm{O}$ & 61.97 & 42.65 \\
\hline & $\mathrm{Na}$ & 2.16 & 0.71 & $\mathrm{Al}$ & 0.53 & 0.71 & $\mathrm{Na}$ & 0.84 & 0.85 & $\mathrm{Na}$ & 1.48 & 1.46 \\
\hline & $\mathrm{Mg}$ & 0.53 & 45.29 & $\mathrm{Si}$ & 32.52 & 45.29 & $\mathrm{Mg}$ & 5.85 & 6.31 & $\mathrm{Mg}$ & 1.08 & 1.13 \\
\hline & $\mathrm{Al}$ & 7.00 & 0.55 & K & 0.28 & 0.55 & $\mathrm{Al}$ & 2.80 & 3.35 & $\mathrm{Al}$ & 4.32 & 5.02 \\
\hline & $\mathrm{Si}$ & 22.83 & 0.26 & $\mathrm{Ca}$ & 0.13 & 0.26 & $\mathrm{Si}$ & 20.84 & 25.99 & $\mathrm{Si}$ & 18.00 & 21.74 \\
\hline & $\mathrm{P}$ & 0.10 & 0.58 & $\mathrm{Fe}$ & 0.21 & 0.58 & K & 0.66 & 1.15 & $\mathrm{P}$ & 0.17 & 0.23 \\
\hline & K & 1.46 & 52.61 & & & & $\mathrm{Ca}$ & 1.25 & 2.22 & $\mathrm{~S}$ & 0.17 & 0.23 \\
\hline & $\mathrm{Ca}$ & 1.89 & 0.71 & & & & $\mathrm{Ti}$ & 0.32 & 0.69 & K & 1.31 & 2.20 \\
\hline & $\mathrm{Ti}$ & 0.26 & 45.29 & & & & V & 0.02 & 0.05 & $\mathrm{Ca}$ & 1.10 & 1.89 \\
\hline & $\mathrm{Fe}$ & 1.21 & 0.55 & & & & $\mathrm{Mn}$ & 0.14 & 0.34 & $\mathrm{Ti}$ & 4.58 & 9.44 \\
\hline & $\mathrm{Ba}$ & 0.05 & 0.26 & & & & $\mathrm{Fe}$ & 6.36 & 15.77 & $\mathrm{Fe}$ & 5.83 & 14.00 \\
\hline & Os & 0.03 & 0.58 & & & & & & & & & \\
\hline & & rite: 0.019 & & & & & & & & & & \\
\hline & element & $\underset{\%}{\text { atomic }}$ & Wt \% & & & & & & & & & \\
\hline & $\mathrm{O}$ & 64.13 & 39.19 & & & & & & & & & \\
\hline & $\mathrm{Al}$ & 0.80 & 0.82 & & & & & & & & & \\
\hline & $\mathrm{Si}$ & 3.56 & 3.82 & & & & & & & & & \\
\hline & $\mathrm{S}$ & 12.15 & 14.88 & & & & & & & & & \\
\hline & $\mathrm{Fe}$ & 19.36 & 41.29 & & & & & & & & & \\
\hline MS51 & & elt: $94.0 \%$ & & orth & yroxene: & & & ibole: 1.1 & & & & \\
\hline & element & $\begin{array}{c}\text { atomic } \\
\%\end{array}$ & Wt $\%$ & element & $\begin{array}{c}\text { atomic } \\
\%\end{array}$ & Wt $\%$ & element & $\begin{array}{c}\text { atomic } \\
\%\end{array}$ & Wt $\%$ & & & \\
\hline & $\mathrm{O}$ & 63.14 & 47.82 & $\mathrm{O}$ & 59.96 & 42.50 & $\mathrm{O}$ & 61.19 & 38.39 & & & \\
\hline & $\mathrm{Na}$ & 1.60 & 1.75 & $\mathrm{Mg}$ & 11.29 & 12.17 & $\mathrm{Na}$ & 1.02 & 0.92 & & & \\
\hline & $\mathrm{Mg}$ & 0.70 & 0.80 & $\mathrm{Al}$ & 1.09 & 1.31 & $\mathrm{Mg}$ & 1.20 & 1.15 & & & \\
\hline & $\mathrm{Al}$ & 5.24 & 6.70 & $\mathrm{Si}$ & 19.25 & 23.96 & $\mathrm{Al}$ & 2.73 & 2.88 & & & \\
\hline & $\mathrm{Si}$ & 24.76 & 32.91 & K & 0.12 & 0.22 & $\mathrm{Si}$ & 10.96 & 12.07 & & & \\
\hline & $\mathrm{K}$ & 1.16 & 2.14 & $\mathrm{Ca}$ & 0.80 & 1.43 & $\mathrm{~S}$ & 2.28 & 2.87 & & & \\
\hline
\end{tabular}




\begin{tabular}{|c|c|c|c|c|c|c|c|c|c|c|c|c|}
\hline & $\mathrm{Ca}$ & 1.50 & 2.85 & $\mathrm{Ti}$ & 0.18 & 0.38 & $\mathrm{~K}$ & 0.65 & 0.99 & & & \\
\hline & $\mathrm{Ti}$ & 0.29 & 0.65 & $\mathrm{Mn}$ & 0.24 & 0.57 & $\mathrm{Ca}$ & 1.57 & 2.47 & & & \\
\hline & $\mathrm{Fe}$ & 1.57 & 4.16 & $\mathrm{Fe}$ & 7.06 & 17.47 & $\mathrm{Ti}$ & 6.32 & 11.88 & & & \\
\hline & $\mathrm{Ba}$ & 0.04 & 0.23 & & & & $\mathrm{Mn}$ & 1.79 & 3.85 & & & \\
\hline & & & & & & & $\mathrm{Fe}$ & 10.29 & 22.53 & & & \\
\hline \multirow[t]{18}{*}{ MS52 } & \multicolumn{3}{|c|}{ melt: $66.8 \%$} & \multicolumn{3}{|c|}{ orthopyroxene: $12.1 \%$} & \multicolumn{3}{|c|}{ plagioclase: $10.4 \%$} & \multicolumn{3}{|c|}{ clinopyroxene: $7.7 \%$} \\
\hline & element & $\begin{array}{c}\text { atomic } \\
\%\end{array}$ & Wt $\%$ & element & $\begin{array}{c}\text { atomic } \\
\%\end{array}$ & Wt $\%$ & element & $\begin{array}{c}\text { atomic } \\
\%\end{array}$ & Wt $\%$ & element & $\begin{array}{c}\text { atomic } \\
\%\end{array}$ & Wt $\%$ \\
\hline & $\mathrm{O}$ & 62.93 & 47.66 & $\mathrm{O}$ & 59.76 & 42.30 & $\mathrm{O}$ & 61.83 & 46.69 & $\mathrm{O}$ & 61.08 & 44.69 \\
\hline & $\mathrm{Na}$ & 1.54 & 1.68 & $\mathrm{Mg}$ & 12.13 & 13.04 & $\mathrm{Na}$ & 1.47 & 1.59 & $\mathrm{Na}$ & 0.73 & 0.77 \\
\hline & $\mathrm{Mg}$ & 0.90 & 1.03 & $\mathrm{Si}$ & 20.40 & 25.34 & $\mathrm{Mg}$ & 0.31 & 0.36 & $\mathrm{Mg}$ & 6.15 & 6.84 \\
\hline & $\mathrm{Al}$ & 6.03 & 7.71 & K & 0.10 & 0.17 & $\mathrm{Al}$ & 11.82 & 15.06 & $\mathrm{Al}$ & 3.45 & 4.25 \\
\hline & $\mathrm{Si}$ & 23.80 & 31.64 & $\mathrm{Ca}$ & 0.90 & 1.59 & $\mathrm{Si}$ & 18.61 & 24.66 & $\mathrm{Si}$ & 20.79 & 26.70 \\
\hline & $\mathrm{K}$ & 0.92 & 1.71 & $\mathrm{Ti}$ & 0.10 & 0.22 & K & 0.26 & 0.47 & K & 0.35 & 0.62 \\
\hline & $\mathrm{Ca}$ & 2.13 & 4.03 & $\mathrm{Mn}$ & 0.11 & 0.27 & $\mathrm{Ca}$ & 5.20 & 9.83 & $\mathrm{Ca}$ & 3.96 & 7.27 \\
\hline & $\mathrm{Ti}$ & 0.27 & 0.60 & $\mathrm{Fe}$ & 5.57 & 13.77 & $\mathrm{Fe}$ & 0.51 & 1.34 & $\mathrm{Ti}$ & 0.18 & 0.39 \\
\hline & V & 0.00 & 0.01 & $\mathrm{Br}$ & 0.93 & 3.30 & & & & $\mathrm{Mn}$ & 0.09 & 0.21 \\
\hline & $\mathrm{Fe}$ & 1.49 & 3.93 & & & & & & & $\mathrm{Fe}$ & 3.23 & 8.25 \\
\hline & \multicolumn{3}{|c|}{ pyrite: $0.8 \%$} & & & & & & & & & \\
\hline & element & $\underset{\%}{\text { atomic }}$ & Wt \% & & & & & & & & & \\
\hline & $\mathrm{O}$ & 65.67 & 40.95 & & & & & & & & & \\
\hline & $\mathrm{Si}$ & 1.87 & 2.04 & & & & & & & & & \\
\hline & $\mathrm{S}$ & 14.73 & 18.41 & & & & & & & & & \\
\hline & $\mathrm{Fe}$ & 17.73 & 38.60 & & & & & & & & & \\
\hline \multirow[t]{12}{*}{ MS53 } & \multicolumn{3}{|c|}{ melt: $68.0 \%$} & \multicolumn{3}{|c|}{ clinopyroxene: $25.3 \%$} & \multicolumn{3}{|c|}{ orthopyroxene: $5.1 \%$} & \multicolumn{3}{|c|}{ FeTi oxides: $0.4 \%$} \\
\hline & element & $\begin{array}{c}\text { atomic } \\
\%\end{array}$ & Wt $\%$ & element & $\begin{array}{c}\text { atomic } \\
\%\end{array}$ & Wt $\%$ & element & $\begin{array}{c}\text { atomic } \\
\%\end{array}$ & Wt $\%$ & element & $\begin{array}{c}\text { atomic } \\
\%\end{array}$ & Wt $\%$ \\
\hline & $\mathrm{O}$ & 61.66 & 46.66 & $\mathrm{O}$ & 60.42 & 43.55 & $\mathrm{O}$ & 59.83 & 42.68 & $\mathrm{O}$ & 57.89 & 31.17 \\
\hline & $\mathrm{Na}$ & 2.87 & 3.12 & $\mathrm{Na}$ & 0.89 & 0.93 & $\mathrm{Mg}$ & 12.08 & 13.10 & $\mathrm{Mg}$ & 2.50 & 2.05 \\
\hline & $\mathrm{Mg}$ & 0.58 & 0.67 & $\mathrm{Mg}$ & 6.86 & 7.51 & $\mathrm{Al}$ & 1.41 & 1.70 & $\mathrm{Al}$ & 1.68 & 1.53 \\
\hline & $\mathrm{Al}$ & 9.73 & 12.41 & $\mathrm{Al}$ & 3.47 & 4.22 & $\mathrm{Si}$ & 18.80 & 23.55 & $\mathrm{Si}$ & 2.72 & 2.57 \\
\hline & $\mathrm{Si}$ & 20.01 & 26.58 & $\mathrm{Si}$ & 19.49 & 24.66 & $\mathrm{Ca}$ & 0.80 & 1.42 & $\mathrm{Ca}$ & 0.42 & 0.56 \\
\hline & $\mathrm{K}$ & 0.61 & 1.13 & $\mathrm{~K}$ & 0.30 & 0.52 & $\mathrm{Ti}$ & 0.15 & 0.32 & $\mathrm{Ti}$ & 12.21 & 19.68 \\
\hline & $\mathrm{Ca}$ & 3.38 & 6.40 & $\mathrm{Ca}$ & 4.08 & 7.36 & $\mathrm{Mn}$ & 0.13 & 0.33 & $\mathrm{Fe}$ & 22.58 & 42.44 \\
\hline & $\mathrm{Ti}$ & 0.18 & 0.41 & $\mathrm{Ti}$ & 0.21 & 0.45 & $\mathrm{Fe}$ & 6.79 & 16.90 & & & \\
\hline & $\mathrm{Fe}$ & 1.00 & 2.63 & $\mathrm{Mn}$ & 0.10 & 0.25 & & & & & & \\
\hline & & & & $\mathrm{Fe}$ & 4.19 & 10.55 & & & & & & \\
\hline
\end{tabular}




\begin{tabular}{|c|c|c|c|c|c|c|c|c|c|c|c|c|}
\hline \multirow[t]{24}{*}{ MS55 } & \multicolumn{3}{|c|}{ melt: $53.9 \%$} & \multicolumn{3}{|c|}{ orthopyroxene: $10.0 \%$} & \multicolumn{3}{|c|}{ clinopyroxene: $16.0 \%$} & \multicolumn{3}{|c|}{ biotite: $2.5 \%$} \\
\hline & element & $\begin{array}{c}\text { atomic } \\
\%\end{array}$ & Wt $\%$ & element & $\begin{array}{c}\text { atomic } \\
\%\end{array}$ & Wt $\%$ & element & $\begin{array}{c}\text { atomic } \\
\%\end{array}$ & Wt $\%$ & element & $\begin{array}{c}\text { atomic } \\
\%\end{array}$ & $\mathrm{Wt} \%$ \\
\hline & $\mathrm{O}$ & 61.22 & 45.74 & $\mathrm{O}$ & 57.49 & 39.28 & $\mathrm{O}$ & 60.03 & 43.11 & $\mathrm{O}$ & 58.80 & 40.36 \\
\hline & $\mathrm{Na}$ & 2.34 & 2.51 & $\mathrm{Mg}$ & 16.21 & 16.83 & $\mathrm{Na}$ & 1.01 & 1.04 & $\mathrm{Na}$ & 0.59 & 0.58 \\
\hline & $\mathrm{Mg}$ & 0.64 & 0.73 & $\mathrm{Al}$ & 1.02 & 1.18 & $\mathrm{Mg}$ & 6.04 & 6.60 & $\mathrm{Mg}$ & 7.17 & 7.48 \\
\hline & $\mathrm{Al}$ & 10.33 & 13.01 & $\mathrm{Si}$ & 14.47 & 17.35 & $\mathrm{Al}$ & 4.63 & 5.61 & $\mathrm{Al}$ & 7.17 & 8.31 \\
\hline & $\mathrm{Si}$ & 19.04 & 24.97 & $\mathrm{~K}$ & 0.21 & 0.36 & $\mathrm{Si}$ & 18.43 & 23.24 & $\mathrm{Si}$ & 14.15 & 17.05 \\
\hline & $\mathrm{K}$ & 1.54 & 2.82 & $\mathrm{Ca}$ & 0.32 & 0.55 & $\mathrm{~K}$ & 0.91 & 1.60 & $\mathrm{~K}$ & 4.22 & 7.08 \\
\hline & $\mathrm{Ca}$ & 3.36 & 6.29 & $\mathrm{Ti}$ & 0.11 & 0.22 & $\mathrm{Ca}$ & 4.97 & 8.94 & $\mathrm{Ca}$ & 0.19 & 0.32 \\
\hline & $\mathrm{Ti}$ & 0.18 & 0.41 & $\mathrm{Mn}$ & 0.19 & 0.44 & $\mathrm{Ti}$ & 0.27 & 0.58 & $\mathrm{Ti}$ & 2.27 & 4.66 \\
\hline & $\mathrm{Fe}$ & 1.31 & 3.42 & $\mathrm{Fe}$ & 9.98 & 23.80 & $\mathrm{Mn}$ & 0.10 & 0.24 & $\mathrm{Fe}$ & 5.13 & 12.30 \\
\hline & $\mathrm{Cu}$ & 0.03 & 0.10 & & & & $\mathrm{Fe}$ & 3.61 & 9.05 & $\mathrm{Ba}$ & 0.32 & 1.87 \\
\hline & \multicolumn{3}{|c|}{ amphibole: $1.1 \%$} & & & & & & & & & \\
\hline & element & $\begin{array}{c}\text { atomic } \\
\%\end{array}$ & Wt $\%$ & & & & & & & & & \\
\hline & $\mathrm{O}$ & 54.63 & 28.53 & & & & & & & & & \\
\hline & $\mathrm{Mg}$ & 3.25 & 2.58 & & & & & & & & & \\
\hline & $\mathrm{Al}$ & 5.21 & 4.58 & & & & & & & & & \\
\hline & $\mathrm{Si}$ & 1.66 & 1.52 & & & & & & & & & \\
\hline & $\mathrm{K}$ & 0.16 & 0.20 & & & & & & & & & \\
\hline & $\mathrm{Ca}$ & 0.24 & 0.31 & & & & & & & & & \\
\hline & $\mathrm{Ti}$ & 4.59 & 7.18 & & & & & & & & & \\
\hline & $\mathrm{V}$ & 0.22 & 0.37 & & & & & & & & & \\
\hline & $\mathrm{Cr}$ & 0.31 & 0.52 & & & & & & & & & \\
\hline & $\mathrm{Fe}$ & 29.74 & 54.21 & & & & & & & & & \\
\hline \multirow[t]{12}{*}{ MS57 } & \multicolumn{3}{|c|}{ melt: $57.3 \%$} & \multicolumn{3}{|c|}{ clinopyroxene: $24.0 \%$} & \multicolumn{3}{|c|}{ orthopyroxene: $9.2 \%$} & \multicolumn{3}{|c|}{ FeTi oxides: $1.6 \%$} \\
\hline & element & $\begin{array}{c}\text { atomic } \\
\%\end{array}$ & $\mathrm{Wt} \%$ & element & $\begin{array}{c}\text { atomic } \\
\%\end{array}$ & Wt $\%$ & element & $\begin{array}{c}\text { atomic } \\
\%\end{array}$ & Wt $\%$ & element & $\begin{array}{c}\text { atomic } \\
\%\end{array}$ & $\mathrm{Wt} \%$ \\
\hline & $\mathrm{O}$ & 59.88 & 44.28 & $\mathrm{O}$ & 58.40 & 41.52 & $\mathrm{O}$ & 56.32 & 39.91 & $\mathrm{O}$ & 53.92 & 27.84 \\
\hline & $\mathrm{Na}$ & 2.48 & 2.64 & $\mathrm{Na}$ & 0.69 & 0.70 & $\mathrm{Mg}$ & 17.50 & 18.84 & $\mathrm{Mg}$ & 3.96 & 3.11 \\
\hline & $\mathrm{Mg}$ & 1.10 & 1.24 & $\mathrm{Mg}$ & 7.76 & 8.38 & $\mathrm{Al}$ & 1.46 & 1.75 & $\mathrm{Al}$ & 5.47 & 4.77 \\
\hline & $\mathrm{Al}$ & 11.24 & 14.02 & $\mathrm{Al}$ & 3.51 & 4.21 & $\mathrm{Si}$ & 17.25 & 21.46 & $\mathrm{Si}$ & 0.70 & 0.63 \\
\hline & $\mathrm{Si}$ & 18.60 & 24.15 & $\mathrm{Si}$ & 18.96 & 23.66 & $\mathrm{Ca}$ & 0.57 & 1.01 & $\mathrm{Ca}$ & 0.23 & 0.30 \\
\hline & $\mathrm{K}$ & 0.17 & 0.31 & $\mathrm{Ca}$ & 6.97 & 12.42 & $\mathrm{Ti}$ & 0.08 & 0.17 & $\mathrm{Ti}$ & 3.58 & 5.53 \\
\hline & $\mathrm{Ca}$ & 4.65 & 8.62 & $\mathrm{Ti}$ & 0.28 & 0.60 & $\mathrm{Mn}$ & 0.17 & 0.42 & V & 0.29 & 0.47 \\
\hline & $\mathrm{Ti}$ & 0.20 & 0.45 & $\mathrm{Mn}$ & 0.10 & 0.25 & $\mathrm{Fe}$ & 6.65 & 16.46 & $\mathrm{Cr}$ & 0.31 & 0.53 \\
\hline & $\mathrm{Mn}$ & 0.03 & 0.09 & $\mathrm{Fe}$ & 3.32 & 8.24 & & & & $\mathrm{Fe}$ & 31.54 & 56.83 \\
\hline & $\mathrm{Fe}$ & 1.63 & 4.21 & & & & & & & & & \\
\hline
\end{tabular}




\begin{tabular}{|c|c|c|c|c|c|c|c|c|c|c|c|c|}
\hline \multirow[t]{12}{*}{ MS58 } & \multicolumn{3}{|c|}{ melt: $82.8 \%$} & \multicolumn{3}{|c|}{ orthopyroxene: $8.6 \%$} & \multicolumn{3}{|c|}{ clinopyroxene: $6.1 \%$} & & & \\
\hline & element & $\begin{array}{c}\text { atomic } \\
\%\end{array}$ & Wt $\%$ & element & $\begin{array}{c}\text { atomic } \\
\%\end{array}$ & Wt \% & element & $\begin{array}{c}\text { atomic } \\
\%\end{array}$ & Wt \% & & & \\
\hline & $\mathrm{O}$ & 62.47 & 46.70 & $\mathrm{O}$ & 58.11 & 42.18 & $\mathrm{O}$ & 59.74 & 43.48 & & & \\
\hline & $\mathrm{Na}$ & 1.43 & 1.54 & $\mathrm{Mg}$ & 14.14 & 15.60 & $\mathrm{Na}$ & 0.57 & 0.60 & & & \\
\hline & $\mathrm{Mg}$ & 1.32 & 1.50 & $\mathrm{Al}$ & 1.23 & 1.50 & $\mathrm{Mg}$ & 7.68 & 8.50 & & & \\
\hline & $\mathrm{Al}$ & 6.46 & 8.14 & $\mathrm{Si}$ & 20.43 & 26.03 & $\mathrm{Al}$ & 3.11 & 3.82 & & & \\
\hline & $\mathrm{Si}$ & 22.84 & 29.98 & $\mathrm{~K}$ & 0.04 & 0.08 & $\mathrm{Si}$ & 20.98 & 26.81 & & & \\
\hline & $\mathrm{K}$ & 0.77 & 1.40 & $\mathrm{Ca}$ & 0.94 & 1.71 & $\mathrm{~K}$ & 0.27 & 0.47 & & & \\
\hline & $\mathrm{Ca}$ & 2.62 & 4.91 & $\mathrm{Ti}$ & 0.07 & 0.16 & $\mathrm{Ca}$ & 4.25 & 7.75 & & & \\
\hline & $\mathrm{Ti}$ & 0.25 & 0.56 & $\mathrm{Cr}$ & 0.04 & 0.10 & $\mathrm{Ti}$ & 0.15 & 0.32 & & & \\
\hline & $\mathrm{Fe}$ & 1.77 & 4.62 & Mn & 0.11 & 0.27 & $\mathrm{Mn}$ & 0.09 & 0.23 & & & \\
\hline & & & & $\mathrm{Fe}$ & 4.89 & 12.38 & $\mathrm{Fe}$ & 3.16 & 8.03 & & & \\
\hline \multirow[t]{11}{*}{ MS59 } & \multicolumn{3}{|c|}{ melt: $66.1 \%$} & \multicolumn{3}{|c|}{ clinopyroxene: $0.5 \%$} & \multicolumn{3}{|c|}{ orthopyroxene: $0.2 \%$} & & & \\
\hline & element & $\begin{array}{c}\text { atomic } \\
\%\end{array}$ & $\mathrm{Wt} \%$ & element & $\begin{array}{c}\text { atomic } \\
\%\end{array}$ & Wt $\%$ & element & $\begin{array}{c}\text { atomic } \\
\%\end{array}$ & Wt $\%$ & & & \\
\hline & $\mathrm{O}$ & 61.86 & 46.16 & $\mathrm{O}$ & 59.94 & 42.75 & $\mathrm{O}$ & 60.07 & 43.69 & & & \\
\hline & $\mathrm{Na}$ & 2.48 & 2.65 & $\mathrm{Na}$ & 0.44 & 0.45 & $\mathrm{Mg}$ & 12.52 & 13.84 & & & \\
\hline & $\mathrm{Mg}$ & 1.34 & 1.52 & $\mathrm{Mg}$ & 7.77 & 8.42 & $\mathrm{Al}$ & 1.23 & 1.51 & & & \\
\hline & $\mathrm{Al}$ & 6.83 & 8.59 & $\mathrm{Al}$ & 2.31 & 2.77 & $\mathrm{Si}$ & 19.53 & 24.93 & & & \\
\hline & $\mathrm{Si}$ & 21.59 & 28.28 & $\mathrm{Si}$ & 18.57 & 23.25 & $\mathrm{Ca}$ & 1.14 & 2.07 & & & \\
\hline & $\mathrm{K}$ & 0.96 & 1.75 & $\mathrm{Ca}$ & 6.89 & 12.31 & $\mathrm{Fe}$ & 5.50 & 13.96 & & & \\
\hline & $\mathrm{Ca}$ & 2.27 & 4.25 & $\mathrm{Ti}$ & 0.37 & 0.79 & & & & & & \\
\hline & $\mathrm{Ti}$ & 0.44 & 0.98 & $\mathrm{Fe}$ & 3.72 & 9.26 & & & & & & \\
\hline & $\mathrm{Fe}$ & 2.23 & 5.81 & & & & & & & & & \\
\hline \multirow[t]{12}{*}{ MS60 } & \multicolumn{3}{|c|}{ quartz: $45.4 \%$} & \multicolumn{3}{|c|}{ melt: $27.4 \%$} & \multicolumn{3}{|c|}{ biotite: $5.2 \%$} & \multicolumn{3}{|c|}{ pyrite: $0.9 \%$} \\
\hline & element & $\begin{array}{c}\text { atomic } \\
\%\end{array}$ & $\mathrm{Wt} \%$ & element & $\begin{array}{c}\text { atomic } \\
\%\end{array}$ & Wt $\%$ & element & $\begin{array}{c}\text { atomic } \\
\%\end{array}$ & Wt $\%$ & element & $\begin{array}{c}\text { atomic } \\
\%\end{array}$ & $\mathrm{Wt} \%$ \\
\hline & $\mathrm{O}$ & 66.31 & 52.63 & $\mathrm{O}$ & 62.10 & 47.10 & $\mathrm{O}$ & 61.01 & 44.06 & $\mathrm{O}$ & 64.07 & 41.91 \\
\hline & $\mathrm{Al}$ & 0.73 & 0.98 & $\mathrm{Na}$ & 1.61 & 1.76 & $\mathrm{Na}$ & 0.72 & 0.75 & $\mathrm{Mg}$ & 2.59 & 2.57 \\
\hline & $\mathrm{Si}$ & 32.38 & 45.12 & $\mathrm{Mg}$ & 1.31 & 1.51 & $\mathrm{Mg}$ & 5.40 & 5.92 & $\mathrm{Al}$ & 2.20 & 2.43 \\
\hline & $\mathrm{K}$ & 0.30 & 0.58 & $\mathrm{Al}$ & 8.26 & 10.56 & $\mathrm{Al}$ & 6.63 & 8.08 & $\mathrm{Si}$ & 7.33 & 8.42 \\
\hline & $\mathrm{Ca}$ & 0.07 & 0.14 & $\mathrm{Si}$ & 22.32 & 29.71 & $\mathrm{Si}$ & 19.04 & 24.15 & $S$ & 9.46 & 12.41 \\
\hline & $\mathrm{Ti}$ & 0.03 & 0.08 & $\mathrm{Ar}$ & 0.22 & 0.42 & $S$ & 0.12 & 0.17 & $\mathrm{~K}$ & 0.27 & 0.43 \\
\hline & $\mathrm{Fe}$ & 0.17 & 0.47 & $\mathrm{~K}$ & 2.72 & 5.05 & $\mathrm{Ar}$ & 0.10 & 0.18 & $\mathrm{Ti}$ & 0.92 & 1.79 \\
\hline & & & & $\mathrm{Ca}$ & 0.36 & 0.67 & $\mathrm{~K}$ & 1.06 & 1.87 & $\mathrm{Fe}$ & 13.16 & 30.04 \\
\hline & & & & $\mathrm{Ti}$ & 0.12 & 0.26 & $\mathrm{Ca}$ & 0.16 & 0.30 & & & \\
\hline & & & & $\mathrm{V}$ & 0.02 & 0.05 & $\mathrm{Ti}$ & 0.36 & 0.77 & & & \\
\hline
\end{tabular}




\begin{tabular}{|c|c|c|c|c|c|c|c|c|c|c|c|c|}
\hline & & & & $\begin{array}{l}\mathrm{Fe} \\
\mathrm{Ba}\end{array}$ & $\begin{array}{l}0.87 \\
0.09\end{array}$ & $\begin{array}{l}2.31 \\
0.60\end{array}$ & $\begin{array}{c}\mathrm{V} \\
\mathrm{Mn} \\
\mathrm{Fe} \\
\mathrm{Ba}\end{array}$ & $\begin{array}{l}0.03 \\
0.07 \\
5.26 \\
0.04\end{array}$ & $\begin{array}{r}0.08 \\
0.18 \\
13.26 \\
0.24\end{array}$ & & & \\
\hline \multirow[t]{14}{*}{ MS62 } & \multicolumn{3}{|c|}{ alkali feldspar: $63.5 \%$} & \multicolumn{3}{|c|}{ quartz: $15.4 \%$} & \multicolumn{3}{|c|}{ FeTi oxides: $0.1 \%$} & \multicolumn{3}{|c|}{ pyrite: $0.01 \%$} \\
\hline & element & $\begin{array}{c}\text { atomic } \\
\%\end{array}$ & Wt $\%$ & element & $\begin{array}{c}\text { atomic } \\
\%\end{array}$ & $\mathrm{Wt} \%$ & element & $\begin{array}{c}\text { atomic } \\
\%\end{array}$ & $\mathrm{Wt} \%$ & element & $\begin{array}{c}\text { atomic } \\
\%\end{array}$ & Wt $\%$ \\
\hline & $\mathrm{O}$ & 59.48 & 43.53 & $\mathrm{O}$ & 64.70 & 50.96 & $\mathrm{O}$ & 64.60 & 38.21 & $\mathrm{O}$ & 10.27 & 4.04 \\
\hline & $\mathrm{Na}$ & 2.02 & 2.12 & $\mathrm{Si}$ & 35.02 & 48.42 & $\mathrm{Mg}$ & 0.76 & 0.68 & $\mathrm{Si}$ & 1.87 & 1.29 \\
\hline & $\mathrm{Mg}$ & 1.59 & 1.77 & K & 0.19 & 0.37 & $\mathrm{Al}$ & 0.64 & 0.64 & S & 44.31 & 34.92 \\
\hline & $\mathrm{Al}$ & 6.64 & 8.20 & $\mathrm{Fe}$ & 0.09 & 0.26 & $\mathrm{Si}$ & 2.84 & 2.94 & $\mathrm{Fe}$ & 43.54 & 59.75 \\
\hline & $\mathrm{Si}$ & 24.08 & 30.94 & & & & K & 0.28 & 0.40 & & & \\
\hline & $\mathrm{K}$ & 1.51 & 2.70 & & & & $\mathrm{Ti}$ & 22.56 & 39.94 & & & \\
\hline & $\mathrm{Ca}$ & 1.74 & 3.20 & & & & $\mathrm{Fe}$ & 8.32 & 17.18 & & & \\
\hline & $\mathrm{Ti}$ & 0.43 & 0.94 & & & & & & & & & \\
\hline & V & 0.02 & 0.05 & & & & & & & & & \\
\hline & $\mathrm{Mn}$ & 0.05 & 0.13 & & & & & & & & & \\
\hline & $\mathrm{Fe}$ & 2.36 & 6.04 & & & & & & & & & \\
\hline & $\mathrm{Ba}$ & 0.06 & 0.39 & & & & & & & & & \\
\hline \multirow[t]{17}{*}{ MS63 } & \multicolumn{3}{|c|}{ melt: $71.9 \%$} & \multicolumn{3}{|c|}{ quartz: $13.7 \%$} & \multicolumn{3}{|c|}{ orthopyroxene: $9.1 \%$} & \multicolumn{3}{|c|}{ amphibole: $0.6 \%$} \\
\hline & element & $\begin{array}{c}\text { atomic } \\
\%\end{array}$ & Wt \% & element & $\begin{array}{c}\text { atomic } \\
\%\end{array}$ & Wt $\%$ & element & $\begin{array}{c}\text { atomic } \\
\%\end{array}$ & Wt $\%$ & element & $\begin{array}{c}\text { atomic } \\
\%\end{array}$ & Wt $\%$ \\
\hline & $\mathrm{O}$ & 62.61 & 47.32 & $\mathrm{O}$ & 66.43 & 52.75 & $\mathrm{O}$ & 60.6 & 42.49 & $\mathrm{O}$ & 61.04 & 38.55 \\
\hline & $\mathrm{Na}$ & 1.95 & 2.11 & $\mathrm{Al}$ & 0.36 & 0.48 & $\mathrm{Na}$ & 0.47 & 0.48 & $\mathrm{Na}$ & 1.14 & 1.04 \\
\hline & $\mathrm{Mg}$ & 0.67 & 0.77 & $\mathrm{Si}$ & 32.77 & 45.68 & $\mathrm{Mg}$ & 7.39 & 7.87 & $\mathrm{Mg}$ & 1.49 & 1.43 \\
\hline & $\mathrm{Al}$ & 6.56 & 8.36 & K & 0.19 & 0.36 & $\mathrm{Al}$ & 1.93 & 2.29 & $\mathrm{Al}$ & 2.96 & 3.16 \\
\hline & $\mathrm{Si}$ & 23.21 & 30.8 & $\mathrm{Ca}$ & 0.1 & 0.19 & $\mathrm{Si}$ & 19.93 & 24.53 & $\mathrm{Si}$ & 12.79 & 14.18 \\
\hline & K & 1.37 & 2.52 & $\mathrm{Fe}$ & 0.15 & 0.41 & S & 0.22 & 0.31 & S & 0.27 & 0.34 \\
\hline & $\mathrm{Ca}$ & 1.8 & 3.41 & & & 0.12 & K & 0.34 & 0.58 & K & 0.73 & 1.13 \\
\hline & $\mathrm{Ti}$ & 0.38 & 0.86 & & & & $\mathrm{Ca}$ & 1.11 & 1.96 & $\mathrm{Ca}$ & 0.65 & 1.03 \\
\hline & \multirow[t]{3}{*}{$\mathrm{Fe}$} & 1.46 & 3.85 & & & & $\mathrm{Ti}$ & 0.26 & 0.54 & $\mathrm{Ti}$ & 8.2 & 15.51 \\
\hline & & & & & & & $\mathrm{Mn}$ & 0.17 & 0.42 & $\mathrm{Fe}$ & 10.73 & 23.65 \\
\hline & & & & & & & $\mathrm{Fe}$ & 7.57 & 18.54 & & & \\
\hline & \multicolumn{3}{|c|}{ apatite: $0.01 \%$} & & & & & & & & & \\
\hline & element & $\begin{array}{c}\text { atomic } \\
\%\end{array}$ & Wt $\%$ & & & & & & & & & \\
\hline & $\mathrm{O}$ & 56.41 & 35.54 & & & & & & & & & \\
\hline & $\mathrm{Si}$ & 5.37 & 5.93 & & & & & & & & & \\
\hline
\end{tabular}




\begin{tabular}{|c|c|c|c|c|c|c|c|c|c|c|c|c|}
\hline & $\begin{array}{c}\mathrm{P} \\
\mathrm{Ca}\end{array}$ & $\begin{array}{r}4.97 \\
33.25\end{array}$ & $\begin{array}{r}6.06 \\
52.47\end{array}$ & & & & & & & & & \\
\hline \multirow[t]{33}{*}{ MS65 } & \multicolumn{3}{|c|}{ melt: $51.2 \%$} & \multicolumn{3}{|c|}{ quartz: $6.0 \%$} & \multicolumn{3}{|c|}{ plagioclase: $7.7 \%$} & \multicolumn{3}{|c|}{ orthopyroxene: $3.7 \%$} \\
\hline & element & $\begin{array}{c}\text { atomic } \\
\%\end{array}$ & Wt \% & element & $\begin{array}{c}\text { atomic } \\
\%\end{array}$ & $\mathrm{Wt} \%$ & element & $\begin{array}{c}\text { atomic } \\
\%\end{array}$ & Wt $\%$ & element & $\begin{array}{c}\text { atomic } \\
\%\end{array}$ & $\mathrm{Wt} \%$ \\
\hline & $\mathrm{O}$ & 61.94 & 45.76 & $\mathrm{O}$ & 66.45 & 51.81 & $\mathrm{O}$ & 61.71 & 47.29 & $\mathrm{O}$ & 60.20 & 42.11 \\
\hline & $\mathrm{Na}$ & 1.07 & 1.14 & $\mathrm{Al}$ & 0.32 & 0.42 & $\mathrm{Na}$ & 3.20 & 3.52 & $\mathrm{Mg}$ & 7.43 & 7.90 \\
\hline & $\mathrm{Mg}$ & 2.32 & 2.60 & $\mathrm{Si}$ & 32.61 & 44.64 & $\mathrm{Al}$ & 10.99 & 14.21 & $\mathrm{Al}$ & 5.26 & 6.20 \\
\hline & $\mathrm{Al}$ & 5.95 & 7.41 & $\mathrm{~K}$ & 0.22 & 0.42 & $\mathrm{Si}$ & 19.85 & 26.71 & $\mathrm{Si}$ & 17.66 & 21.69 \\
\hline & $\mathrm{Si}$ & 22.50 & 29.19 & $\mathrm{Fe}$ & 0.15 & 0.41 & $\mathrm{~K}$ & 0.66 & 1.24 & K & 0.80 & 1.37 \\
\hline & $\mathrm{Ar}$ & 0.09 & 0.17 & Os & 0.16 & 1.48 & $\mathrm{Ca}$ & 3.39 & 6.50 & $\mathrm{Ca}$ & 0.27 & 0.47 \\
\hline & K & 2.51 & 4.53 & $\mathrm{Pt}$ & 0.09 & 0.83 & $\mathrm{Fe}$ & 0.19 & 0.52 & $\mathrm{Ti}$ & 0.51 & 1.07 \\
\hline & $\mathrm{Ca}$ & 0.46 & 0.86 & & & & & & & $\mathrm{Mn}$ & 0.12 & 0.30 \\
\hline & $\mathrm{Ti}$ & 0.24 & 0.54 & & & & & & & $\mathrm{Fe}$ & 7.74 & 18.90 \\
\hline & $\mathrm{V}$ & 0.02 & 0.05 & & & & & & & & & \\
\hline & $\mathrm{Mn}$ & 0.04 & 0.11 & & & & & & & & & \\
\hline & $\mathrm{Fe}$ & 2.78 & 7.16 & & & & & & & & & \\
\hline & $\mathrm{Ba}$ & 0.08 & 0.49 & & & & & & & & & \\
\hline & \multicolumn{3}{|c|}{ amphibole: $0.5 \%$} & \multicolumn{3}{|c|}{ alkali feldspar: $0.4 \%$} & \multicolumn{3}{|c|}{ biotite: $0.7 \%$} & \multicolumn{3}{|c|}{ FeTi oxides: $0.03 \%$} \\
\hline & element & $\begin{array}{c}\text { atomic } \\
\%\end{array}$ & $\mathrm{Wt} \%$ & element & $\begin{array}{c}\text { atomic } \\
\%\end{array}$ & $\mathrm{Wt} \%$ & element & $\begin{array}{c}\text { atomic } \\
\%\end{array}$ & Wt $\%$ & element & $\begin{array}{c}\text { atomic } \\
\%\end{array}$ & $\mathrm{Wt} \%$ \\
\hline & $\mathrm{O}$ & 61.18 & 46.31 & $\mathrm{O}$ & 61.23 & 45.24 & $\mathrm{O}$ & 61.56 & 40.89 & $\mathrm{O}$ & 60.26 & 32.71 \\
\hline & $\mathrm{Mg}$ & 4.66 & 5.36 & $\mathrm{Na}$ & 1.31 & 1.39 & $\mathrm{Na}$ & 0.78 & 0.74 & $\mathrm{Mg}$ & 2.31 & 1.91 \\
\hline & $\mathrm{Al}$ & 13.53 & 17.27 & $\mathrm{Al}$ & 8.42 & 10.49 & $\mathrm{Mg}$ & 2.09 & 2.11 & $\mathrm{Ti}$ & 20.52 & 33.34 \\
\hline & $\mathrm{Si}$ & 16.97 & 22.55 & $\mathrm{Si}$ & 21.96 & 28.48 & $\mathrm{Al}$ & 3.81 & 4.27 & $\mathrm{Fe}$ & 16.91 & 32.04 \\
\hline & $\mathrm{Ar}$ & 1.22 & 2.31 & K & 6.12 & 11.05 & $\mathrm{Si}$ & 15.85 & 18.48 & & & \\
\hline & $\mathrm{K}$ & 0.31 & 0.58 & $\mathrm{Ca}$ & 0.44 & 0.81 & $\mathrm{~K}$ & 1.53 & 2.48 & & & \\
\hline & $\mathrm{Mn}$ & 0.23 & 0.61 & $\mathrm{Fe}$ & 0.22 & 0.56 & $\mathrm{Ca}$ & 0.24 & 0.40 & & & \\
\hline & \multirow[t]{2}{*}{$\mathrm{Fe}$} & 1.90 & 5.02 & $\mathrm{Ba}$ & 0.31 & 1.98 & $\mathrm{Ti}$ & 6.52 & 12.97 & & & \\
\hline & & & & & & & $\mathrm{Fe}$ & 7.62 & 17.66 & & & \\
\hline & \multicolumn{3}{|c|}{ apatite: $0.02 \%$} & & & & & & & & & \\
\hline & element & $\begin{array}{c}\text { atomic } \\
\%\end{array}$ & Wt $\%$ & & & & & & & & & \\
\hline & $\mathrm{O}$ & 61.40 & 41.04 & & & & & & & & & \\
\hline & $\mathrm{Si}$ & 2.07 & 2.43 & & & & & & & & & \\
\hline & $\mathrm{P}$ & 14.13 & 18.28 & & & & & & & & & \\
\hline & $\mathrm{Ca}$ & 21.94 & 36.73 & & & & & & & & & \\
\hline & $\mathrm{Br}$ & 0.46 & 1.52 & & & & & & & & & \\
\hline
\end{tabular}




\section{APPENDIX D: PARTIAL MELT MODELING}

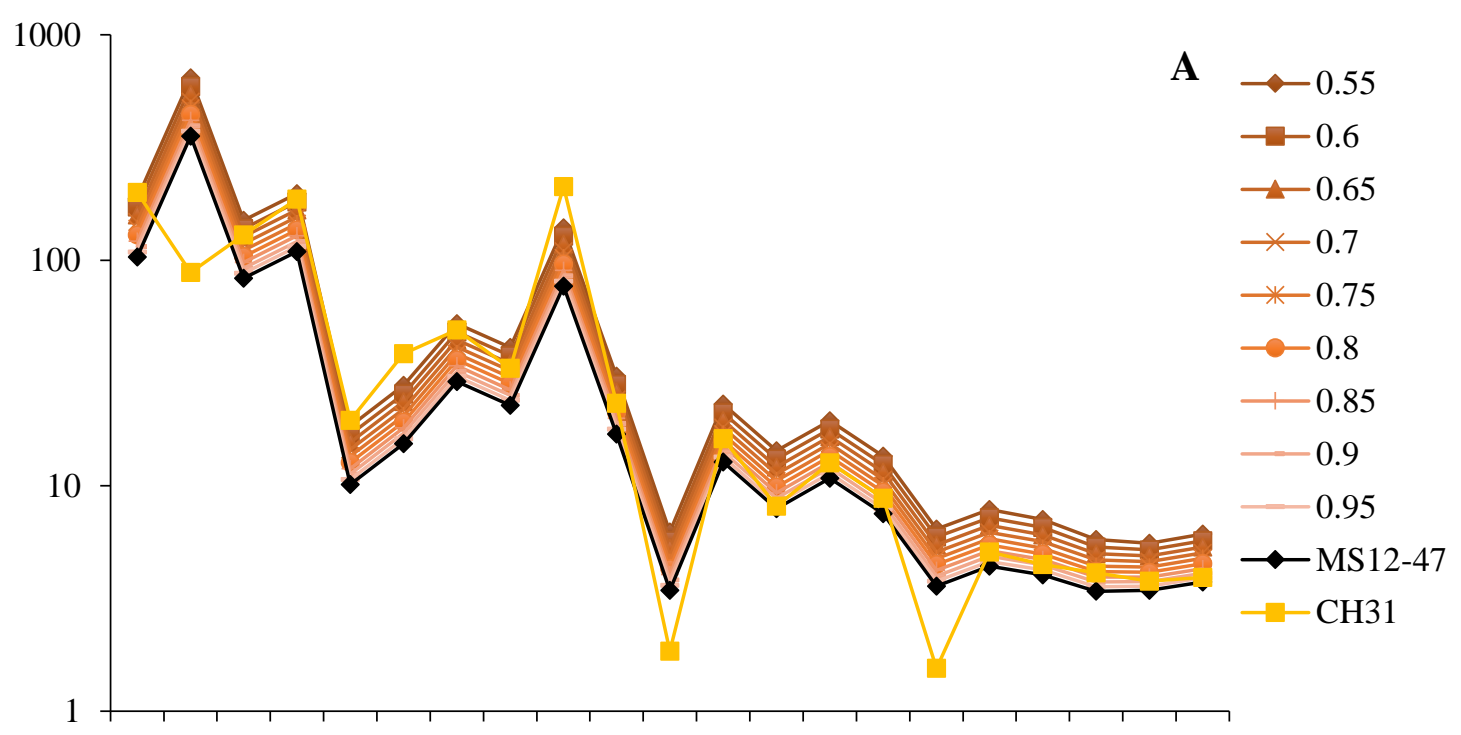

$\mathrm{Rb} \mathrm{Ba}$ Th $\mathrm{U}$ Nb Ta $\mathrm{La} \mathrm{Ce} \mathrm{Pb}$ Pr $\mathrm{Sr} \mathrm{Nd} \mathrm{Zr} \mathrm{Hf} \mathrm{Sm} \mathrm{Eu} \mathrm{Tb} \mathrm{Dy} \mathrm{Y} \mathrm{Yb} \mathrm{Lu}$

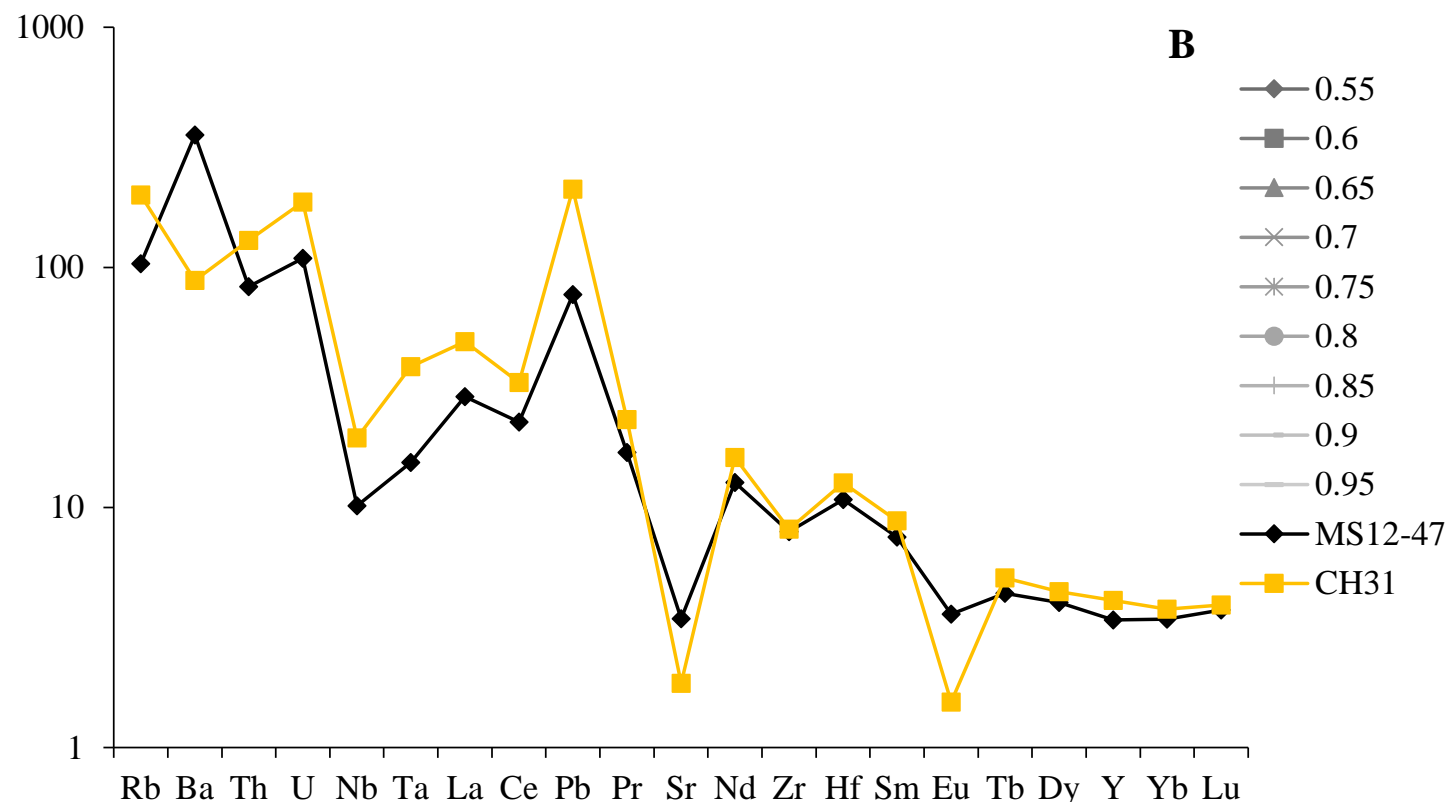

Fig. D1 Primitive mantle normalized (Sun and McDonough, 1989) spider diagram for the partial melting of experimental melt MS1. A shows the results of the batch melt model; B shows the results of the fractional melt model. The melt percentages are approximately $\pm 20 \%$ that observed in the experimental melt. The trace element concentrations of the parent material, sample MS12-47 (biotite fels), are indicated by the black diamonds. The trace element concentrations of the Dale Tuff, sample CH31, which is included for comparison, are indicated by the yellow squares. For experimental melt MS1, it can be seen that the batch melt model (A) approximates the data. The fit increases as melt percent increases. 


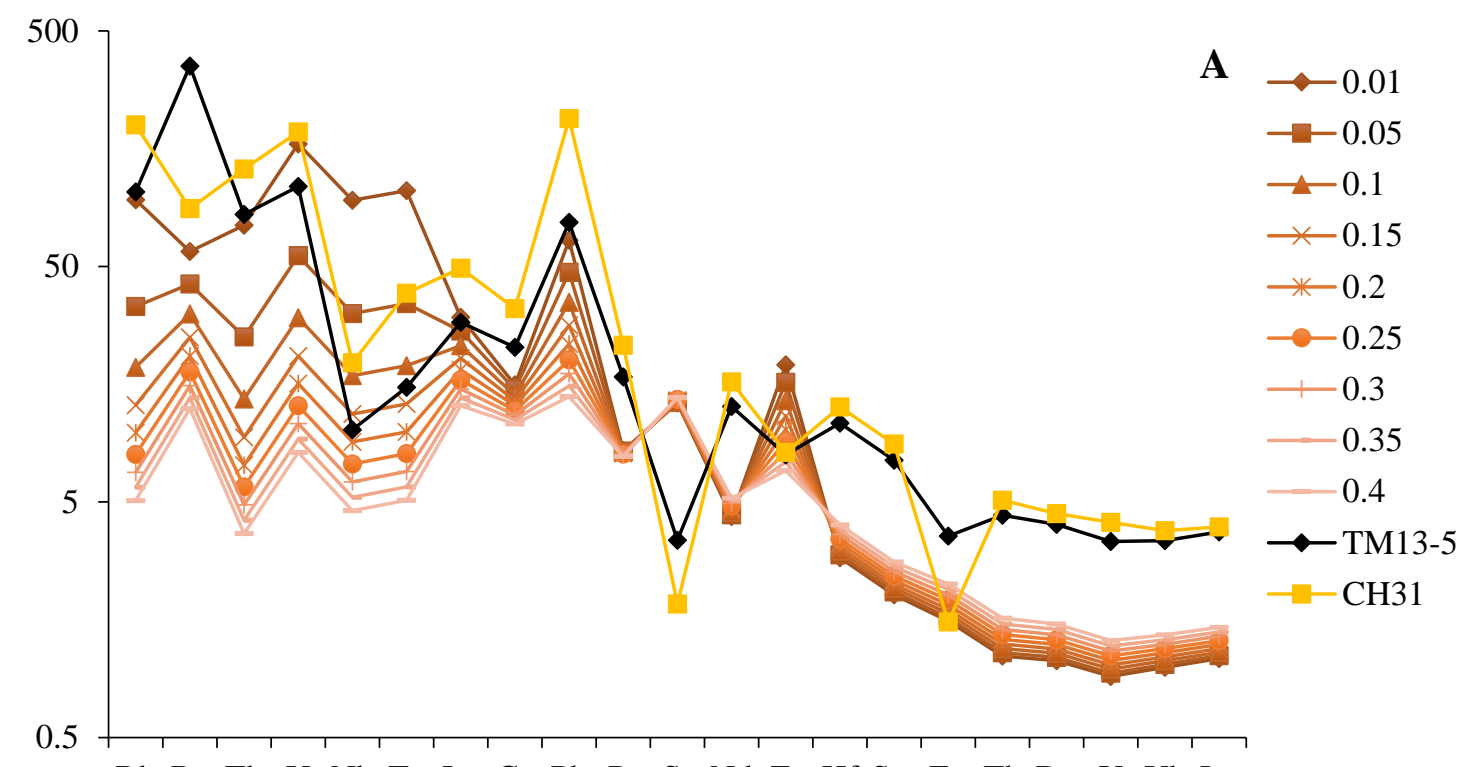

$\mathrm{Rb} \mathrm{Ba}$ Th $\mathrm{U}$ Nb Ta $\mathrm{La} \mathrm{Ce} \mathrm{Pb}$ Pr $\mathrm{Sr} \mathrm{Nd} \mathrm{Zr} \mathrm{Hf} \mathrm{Sm} \mathrm{Eu} \mathrm{Tb} \mathrm{Dy} \mathrm{Y} \mathrm{Yb} \mathrm{Lu}$

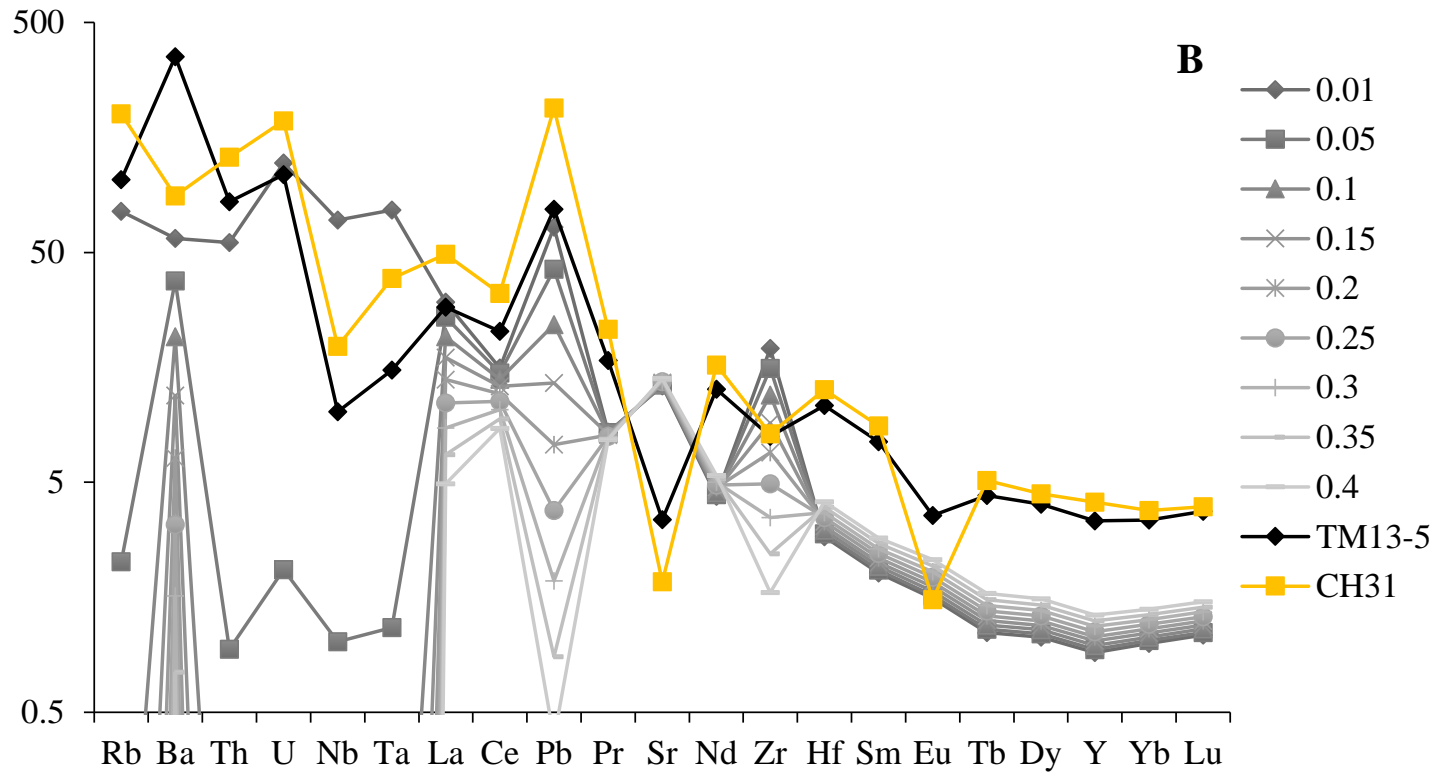

Fig. D2 Primitive mantle normalized (Sun and McDonough, 1989) spider diagram for the partial melting of experimental melt MS3. A shows the results of the batch melt model; B shows the results of the fractional melt model. The melt percentages are approximately $\pm 20 \%$ that observed in the experimental melt. The trace element concentrations of the parent material, sample TM13-5 (amphibolite), are indicated by the black diamonds. The trace element concentrations of the Dale Tuff, sample CH31, which is included for comparison, are indicated by the yellow squares. Neither model is a satisfactory fit for experimental melt MS3. 


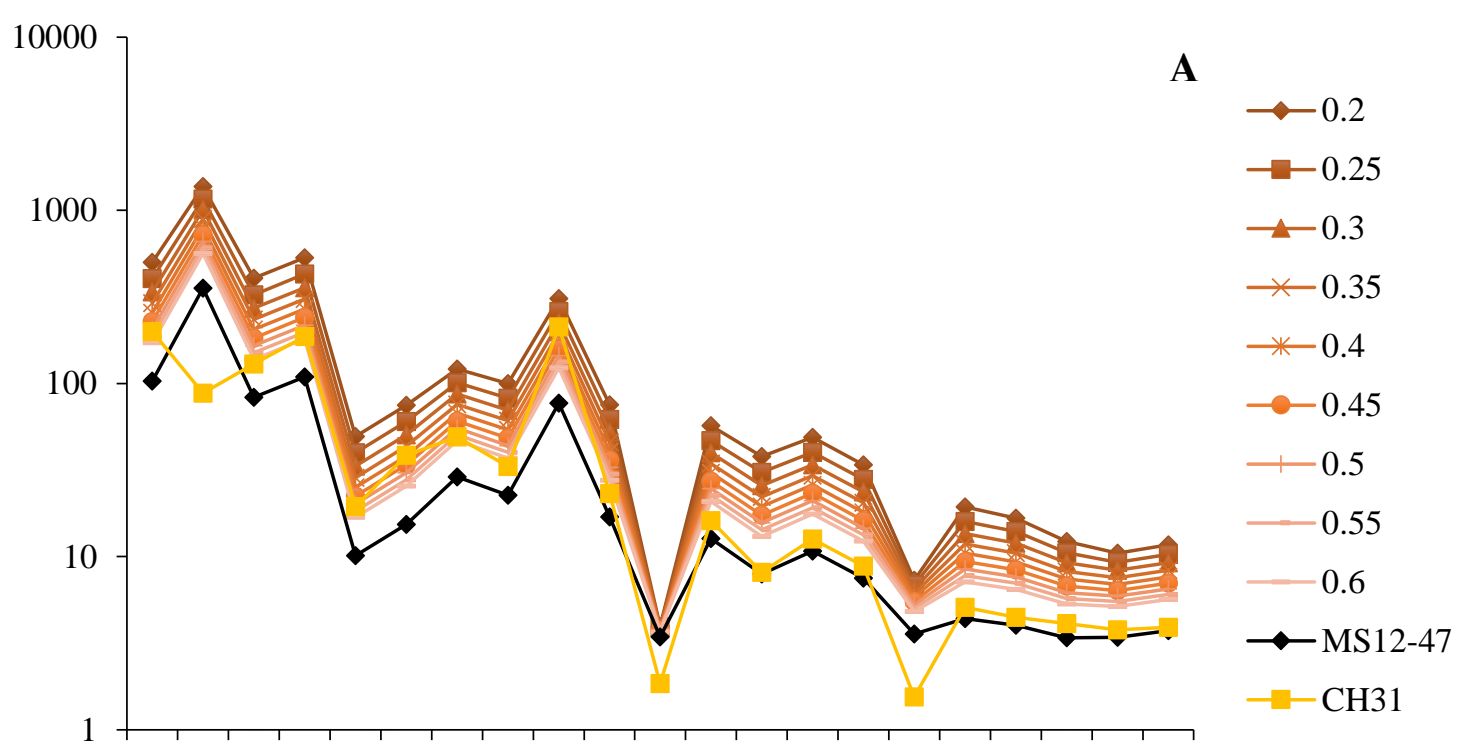

$\mathrm{Rb}$ Ba Th U Nb Ta La Ce Pb Pr Sr Nd Zr Hf Sm Eu Tb Dy Y Yb Lu

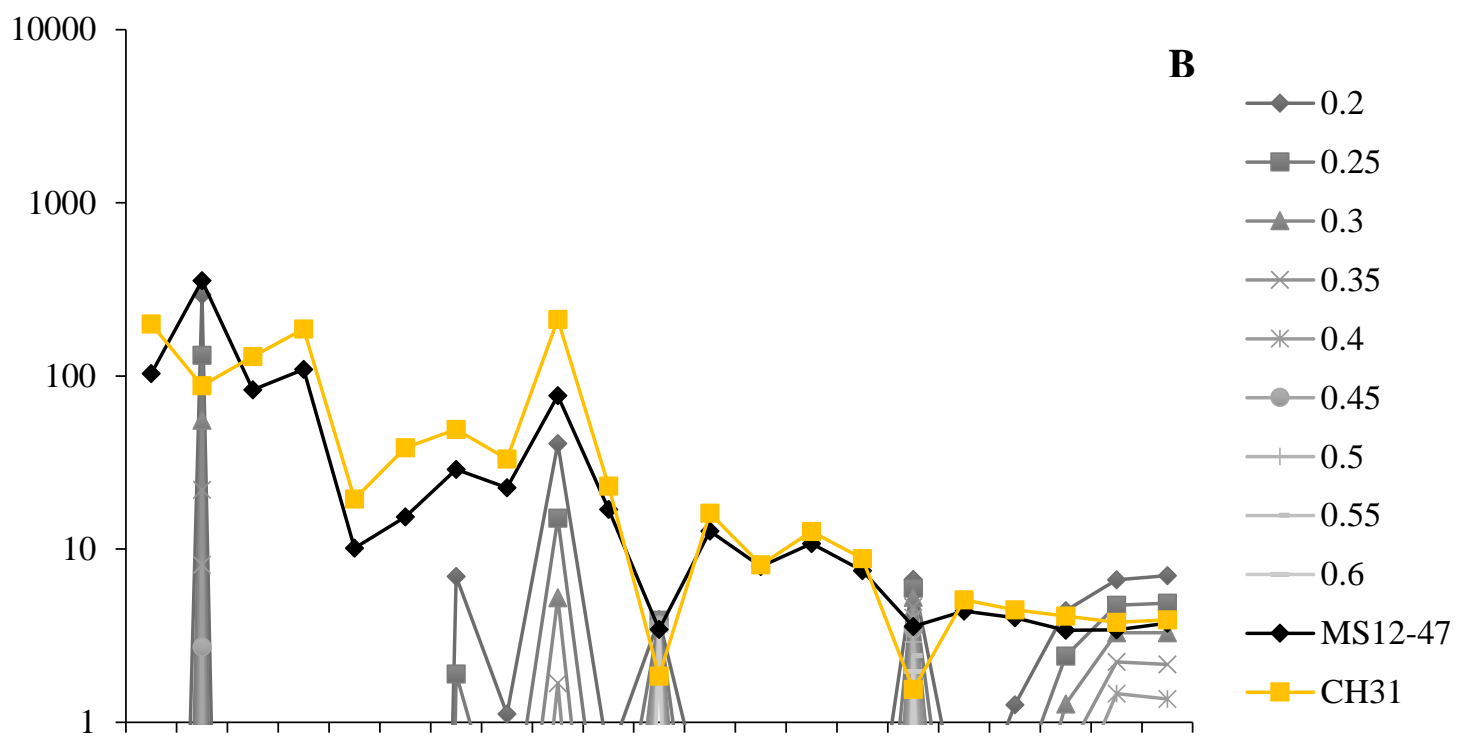

$\mathrm{Rb} \mathrm{Ba}$ Th U Nb Ta La Ce Pb Pr Sr Nd Zr Hf Sm Eu Tb Dy Y Yb Lu

Fig. D3 Primitive mantle normalized (Sun and McDonough, 1989) spider diagram for the partial melting of experimental melt MS4. A shows the results of the batch melt model; B shows the results of the fractional melt model. The melt percentages are approximately $\pm 20 \%$ that observed in the experimental melt. The trace element concentrations of the parent material, sample MS12-47 (biotite fels), are indicated by the black diamonds. The trace element concentrations of the Dale Tuff, sample CH31, which is included for comparison, are indicated by the yellow squares. For experimental melt MS4, it can be seen that the batch melt model (A) approximates the data. The fit increases as melt percent increases. 


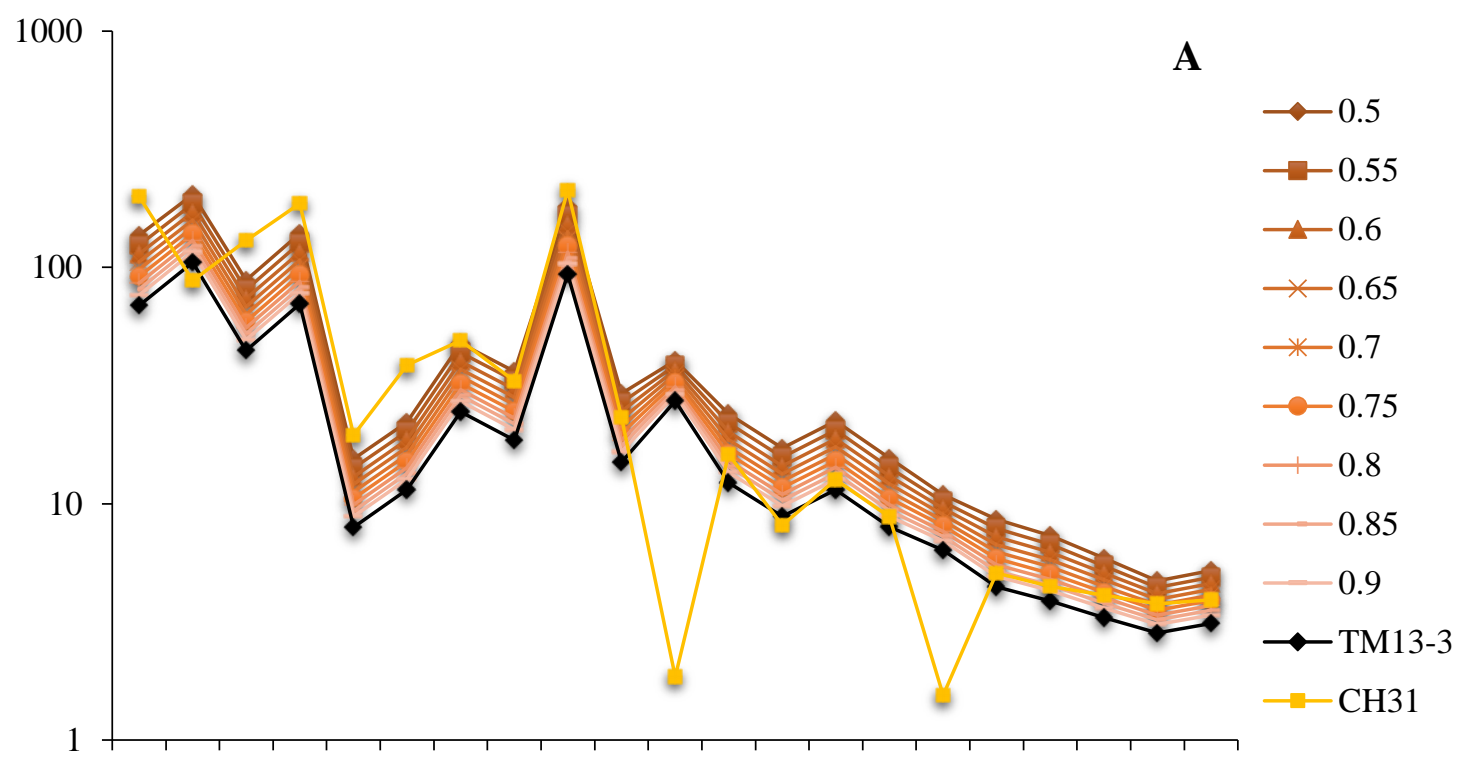

$\mathrm{Rb} \mathrm{Ba}$ Th $\mathrm{U}$ Nb Ta La Ce Pb Pr Sr Nd Zr Hf Sm Eu Tb Dy Y Yb Lu

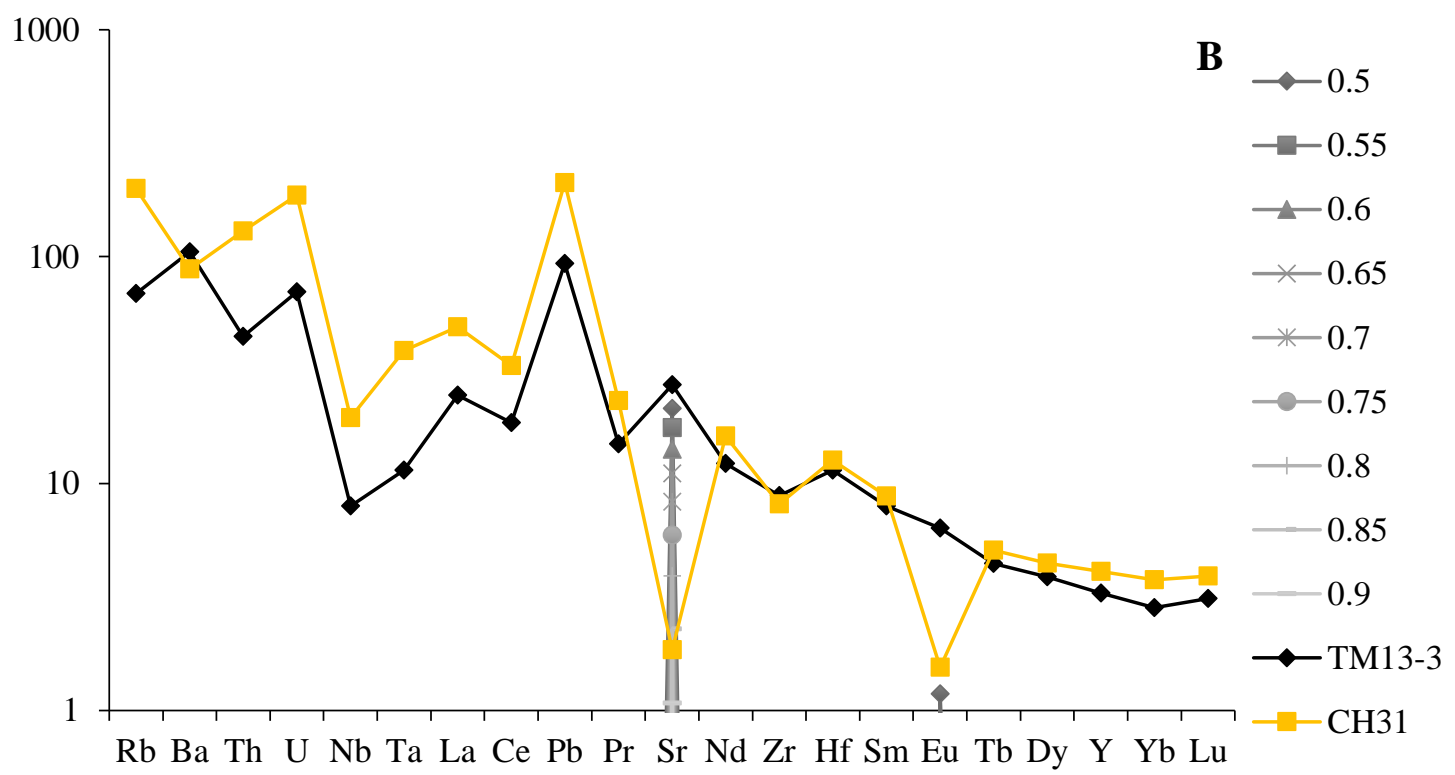

Fig. D4 Primitive mantle normalized (Sun and McDonough, 1989) spider diagram for the partial melting of experimental melt MS5. A shows the results of the batch melt model; B shows the results of the fractional melt model. The melt percentages are approximately $\pm 20 \%$ that observed in the experimental melt. The trace element concentrations of the parent material, sample TM13-3 (granodiorite), are indicated by the black diamonds. The trace element concentrations of the Dale Tuff, sample CH31, which is included for comparison, are indicated by the yellow squares. For experimental melt MS5, it can be seen that the batch melt model (A) approximates the data. The fit increases as melt percent increases. 

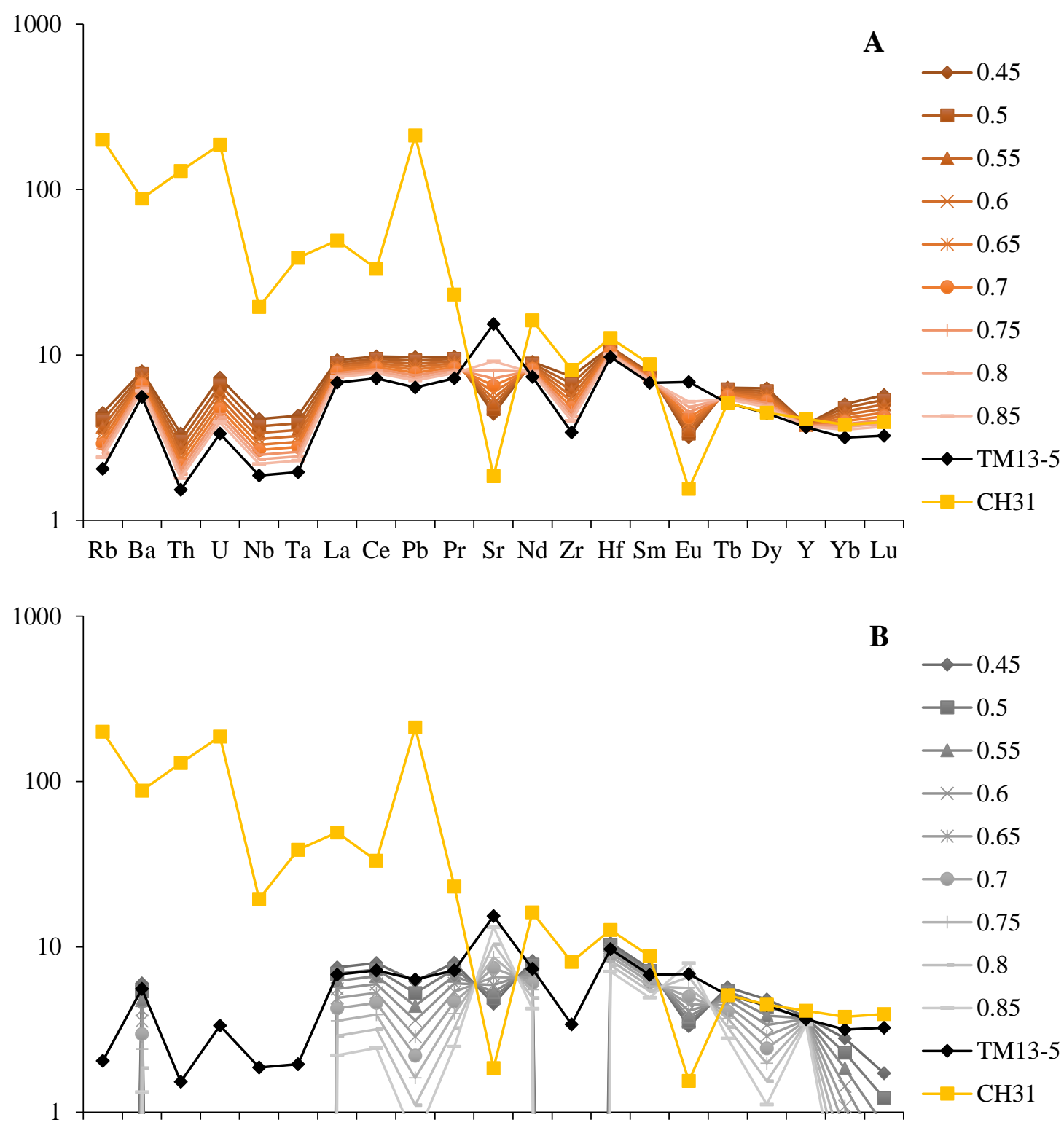

$\mathrm{Rb} \mathrm{Ba}$ Th $\mathrm{U}$ Nb Ta $\mathrm{La} \mathrm{Ce} \mathrm{Pb}$ Pr $\mathrm{Sr} \mathrm{Nd} \mathrm{Zr}$ Hf Sm Eu Tb Dy Y Yb Lu

Fig. D5 Primitive mantle normalized (Sun and McDonough, 1989) spider diagram for the partial melting of experimental melt MS6. A shows the results of the batch melt model; B shows the results of the fractional melt model. The melt percentages are approximately $\pm 20 \%$ that observed in the experimental melt. The trace element concentrations of the parent material, sample TM13-5 (amphibolite), are indicated by the black diamonds. The trace element concentrations of the Dale Tuff, sample CH31, which is included for comparison, are indicated by the yellow squares. For experimental melt MS6, it can be seen that the batch melt model (A) approximates the data, although there are notable exceptions at $\mathrm{Sr}$ and Eu. In general, the fit increases as melt percent increases. 


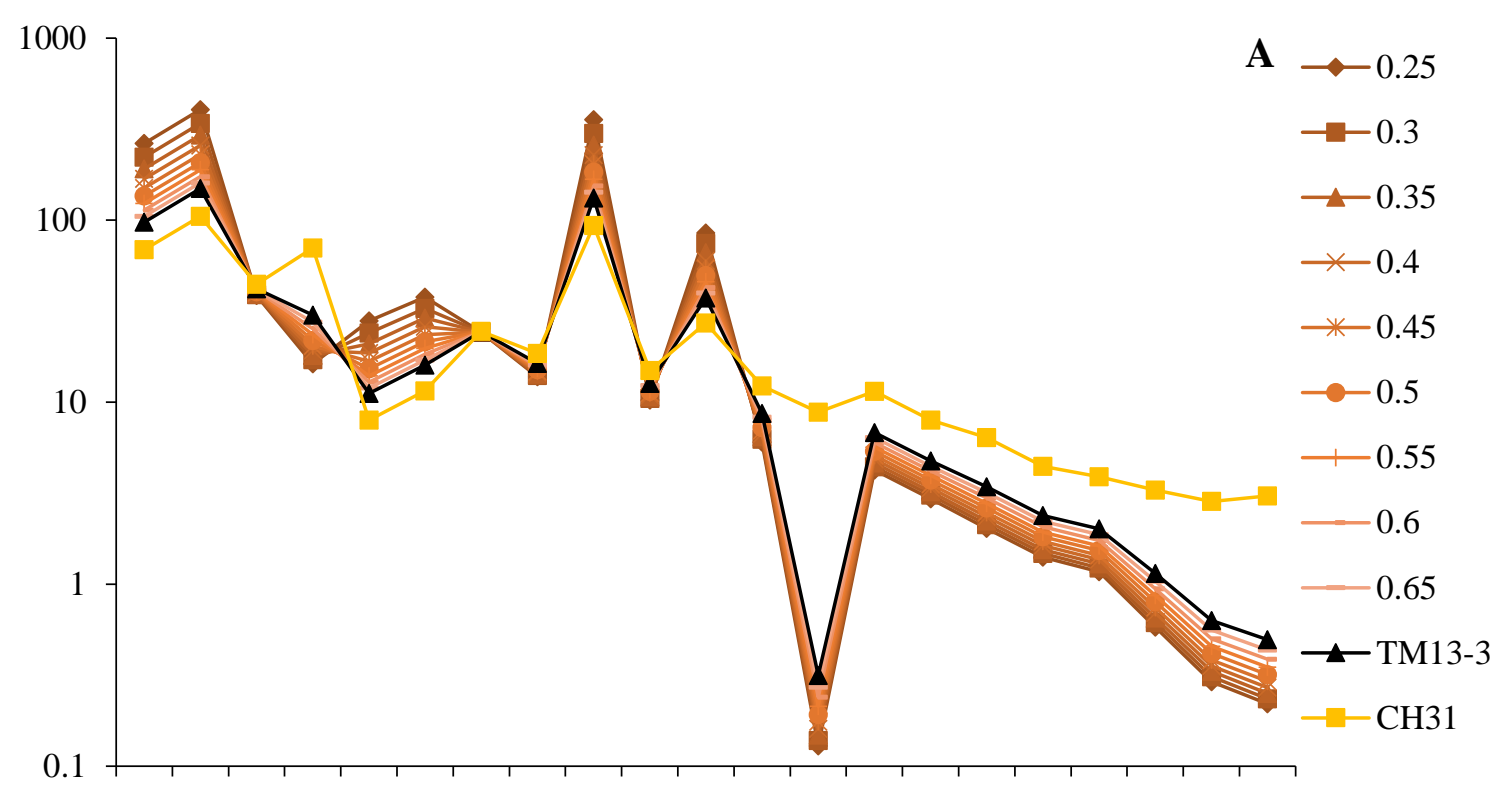

$\mathrm{Rb} \mathrm{Ba}$ Th $\mathrm{U}$ Nb Ta $\mathrm{La} \mathrm{Ce} \mathrm{Pb}$ Pr $\mathrm{Sr} \mathrm{Nd} \mathrm{Zr}$ Hf Sm Eu Tb Dy Y Yb Lu

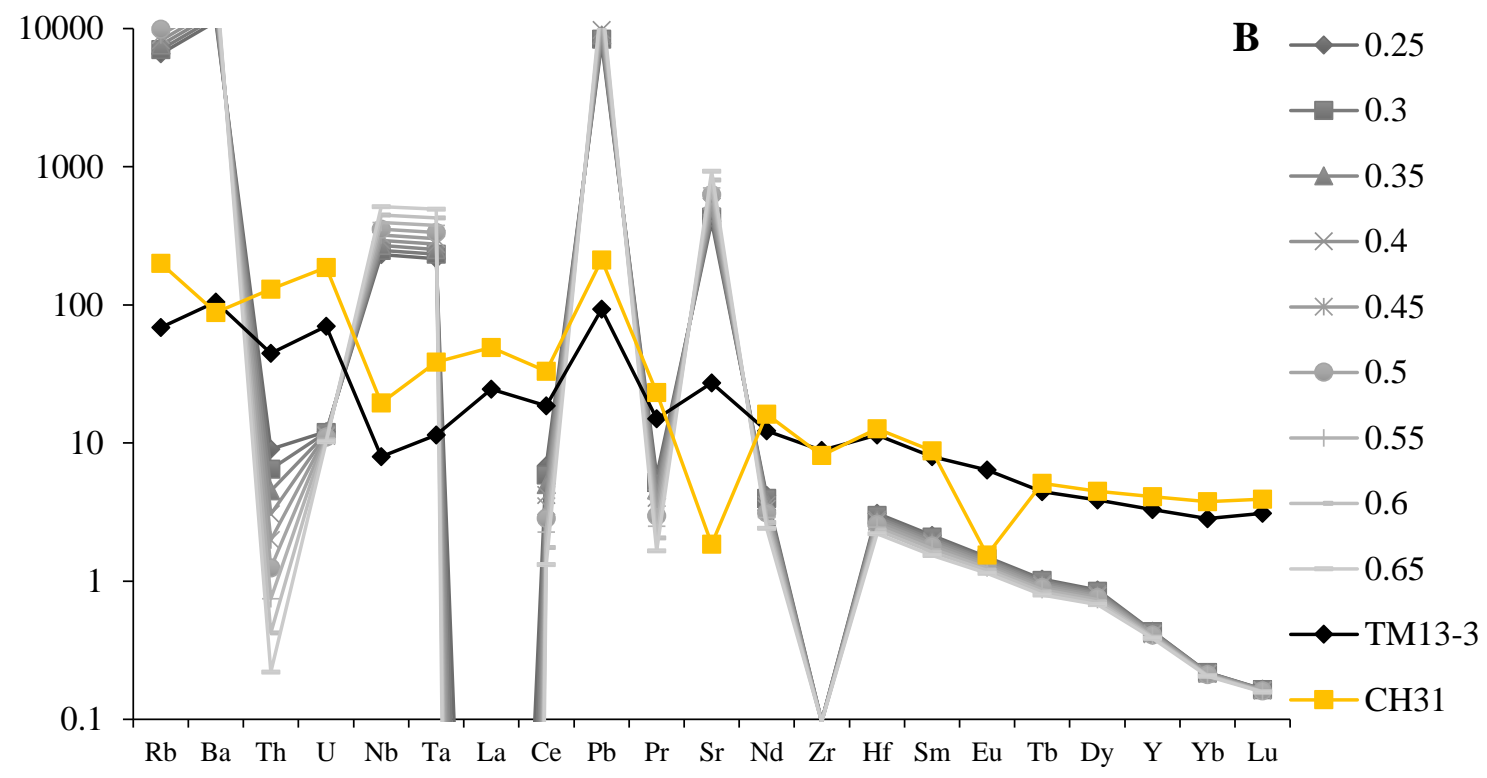

Fig. D6 Primitive mantle normalized (Sun and McDonough, 1989) spider diagram for the partial melting of experimental melt MS8. A shows the results of the batch melt model; B shows the results of the fractional melt model. The melt percentages are approximately $\pm 20 \%$ that observed in the experimental melt. The trace element concentrations of the parent material, sample TM13-3 (granodiorite), are indicated by the black diamonds. The trace element concentrations of the Dale Tuff, sample CH31, which is included for comparison, are indicated by the yellow squares. For experimental melt MS8, it can be seen that batch melt model (A) approximates the data. The fit increases as melt percent increases. 


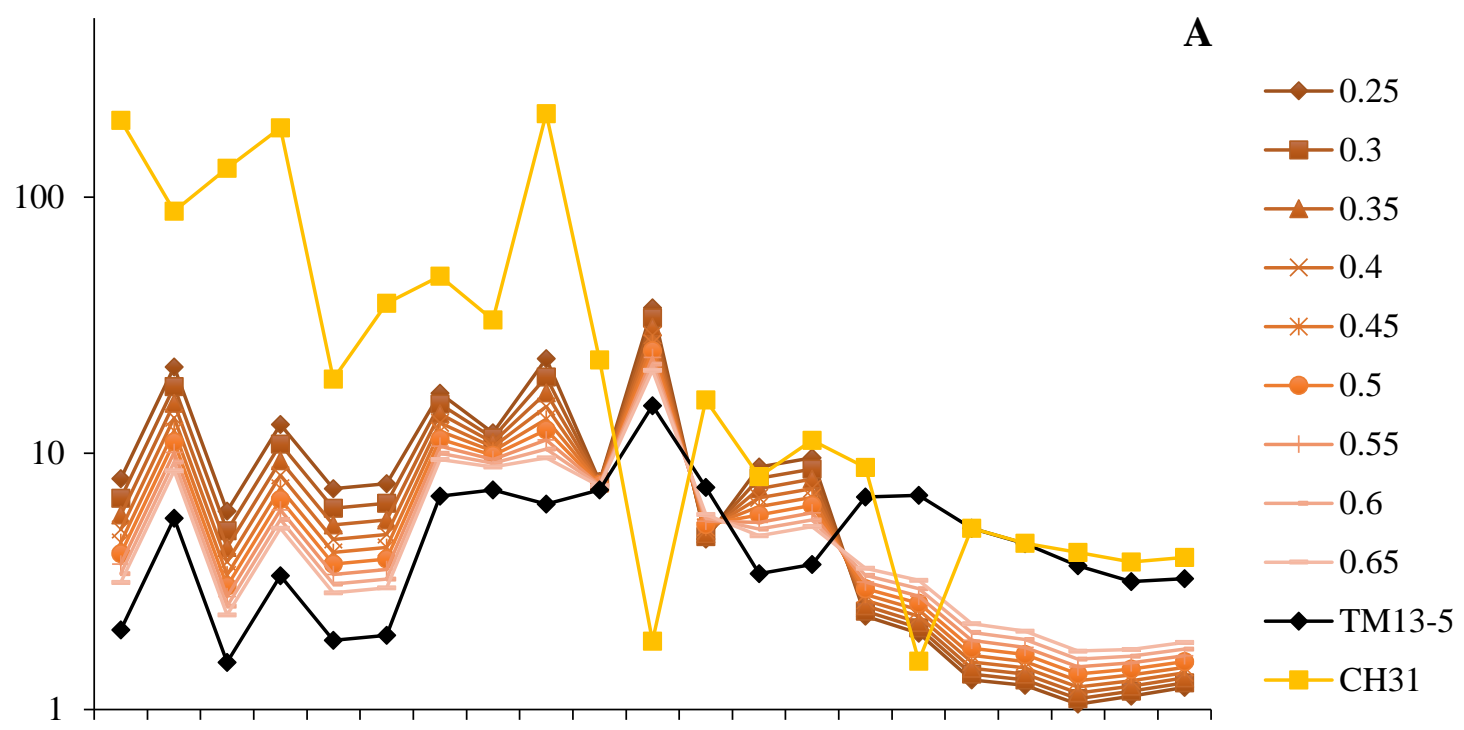

$\mathrm{Rb} \mathrm{Ba}$ Th $\mathrm{U}$ Nb Ta La Ce Pb Pr Sr Nd Zr Hf Sm Eu Tb Dy Y Yb Lu

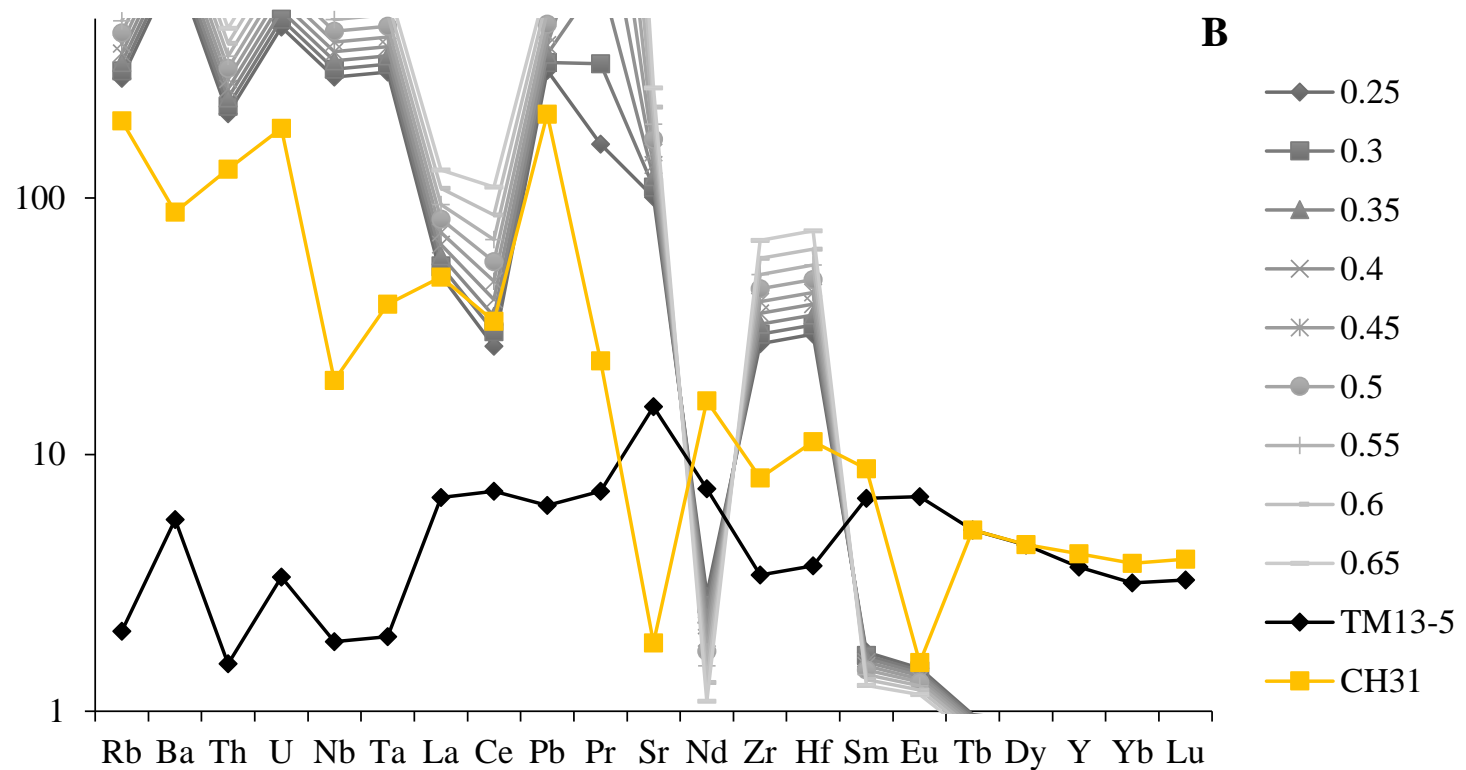

Fig. D7 Primitive mantle normalized (Sun and McDonough, 1989) spider diagram for the partial melting of experimental melt MS9. A shows the results of the batch melt model; B shows the results of the fractional melt model. The melt percentages are approximately $\pm 20 \%$ that observed in the experimental melt. The trace element concentrations of the parent material, sample TM13-5 (amphibolite), are indicated by the black diamonds. The trace element concentrations of the Dale Tuff, sample CH31, which is included for comparison, are indicated by the yellow squares. For experimental melt MS9, it can be seen that batch melt model (A) approximates the data. The fit increases as melt percent increases. 


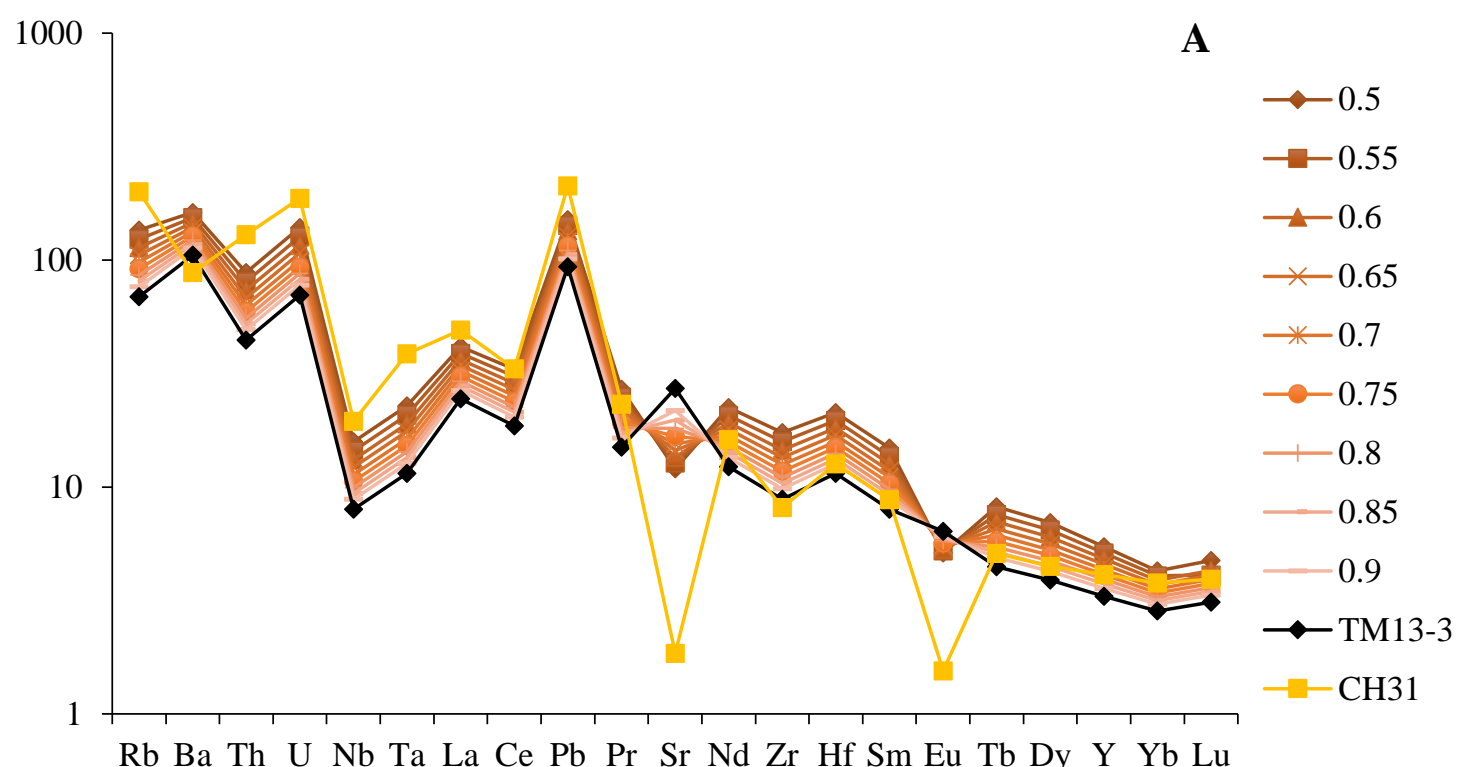

$\mathrm{Rb} \mathrm{Ba}$ Th $\mathrm{U}$ Nb Ta $\mathrm{La} \mathrm{Ce} \mathrm{Pb} \operatorname{Pr} \mathrm{Sr} \mathrm{Nd} \mathrm{Zr} \mathrm{Hf} \mathrm{Sm} \mathrm{Eu} \mathrm{Tb} \mathrm{Dy} \mathrm{Y} \mathrm{Yb} \mathrm{Lu}$

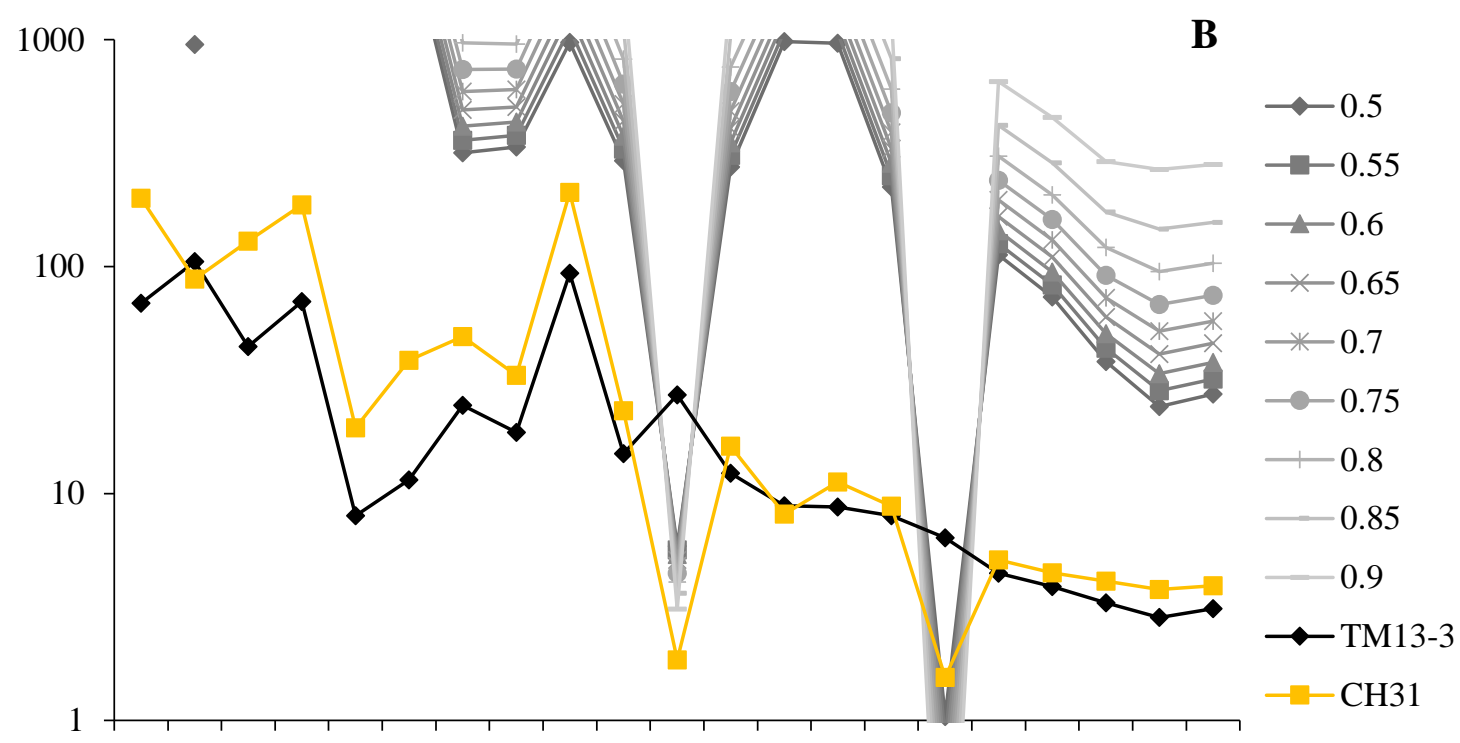

$\mathrm{Rb} \mathrm{Ba}$ Th $\mathrm{U}$ Nb Ta La Ce Pb Pr Sr Nd Zr Hf Sm Eu Tb Dy Y Yb Lu

Fig. D8 Primitive mantle normalized (Sun and McDonough, 1989) spider diagram for the partial melting of experimental melt MS13. A shows the results of the batch melt model; B shows the results of the fractional melt model. The melt percentages are approximately $\pm 20 \%$ that observed in the experimental melt. The trace element concentrations of the parent material, sample TM13-3 (granodiorite), are indicated by the black diamonds. The trace element concentrations of the Dale Tuff, sample CH31, which is included for comparison, are indicated by the yellow squares. For experimental melt MS13, it can be seen that the batch melt model (A) approximates the data. The fit increases as melt percent increases. 

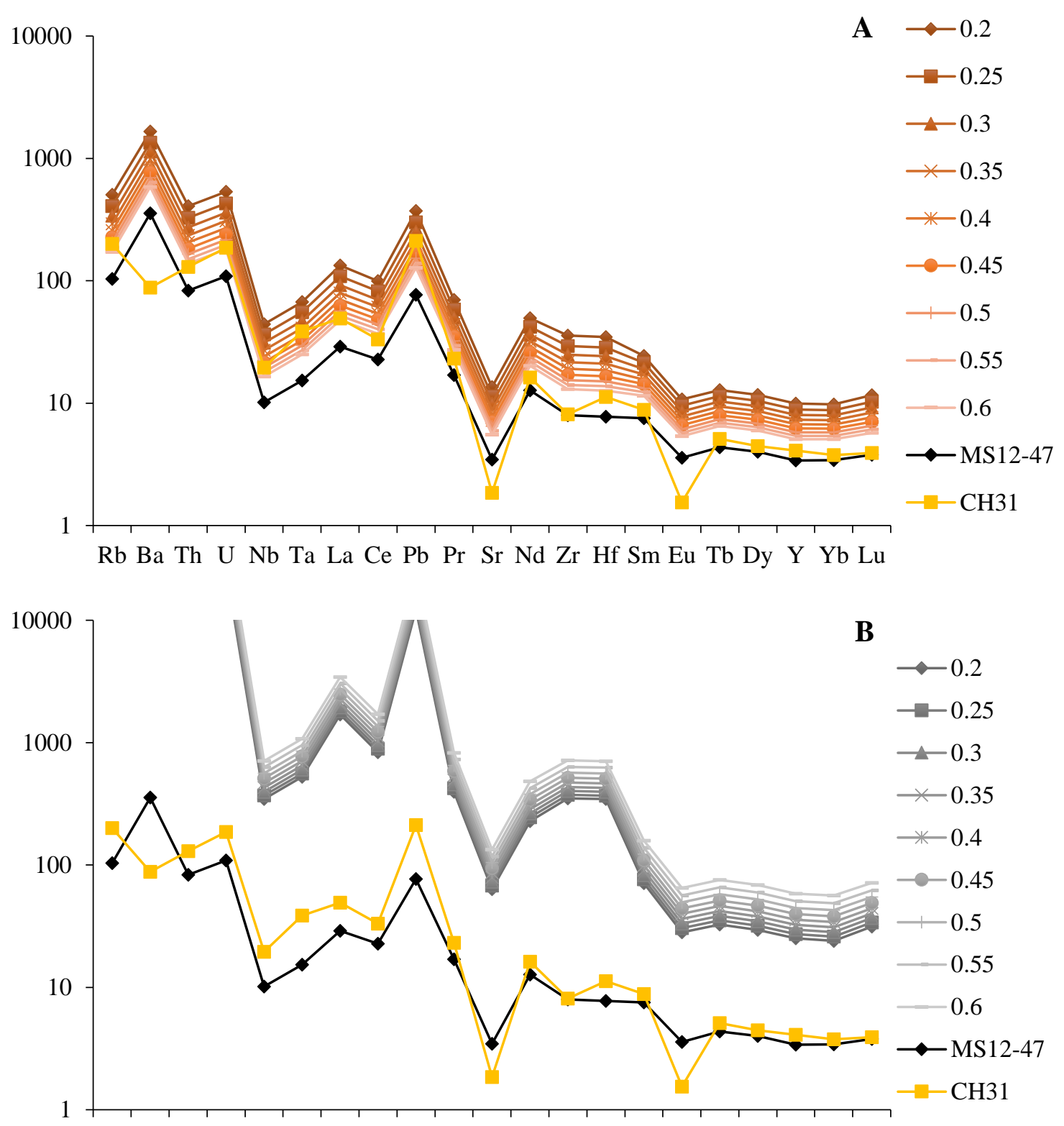

$\mathrm{Rb}$ Ba Th U Nb Ta La Ce Pb Pr Sr Nd Zr Hf Sm Eu Tb Dy Y Yb Lu

Fig. D9 Primitive mantle normalized (Sun and McDonough, 1989) spider diagram for the partial melting of experimental melt MS15. A shows the results of the batch melt model; B shows the results of the fractional melt model. The melt percentages are approximately $\pm 20 \%$ that observed in the experimental melt. The trace element concentrations of the parent material, sample MS12-47 (biotite fels) are indicated by the black diamonds. The trace element concentrations of the Dale Tuff, sample CH31, which is included for comparison, are indicated by the yellow squares. For experimental melt MS15, it can be seen that the batch melt model (A) approximates the data. The fit increases as melt percent increases. 


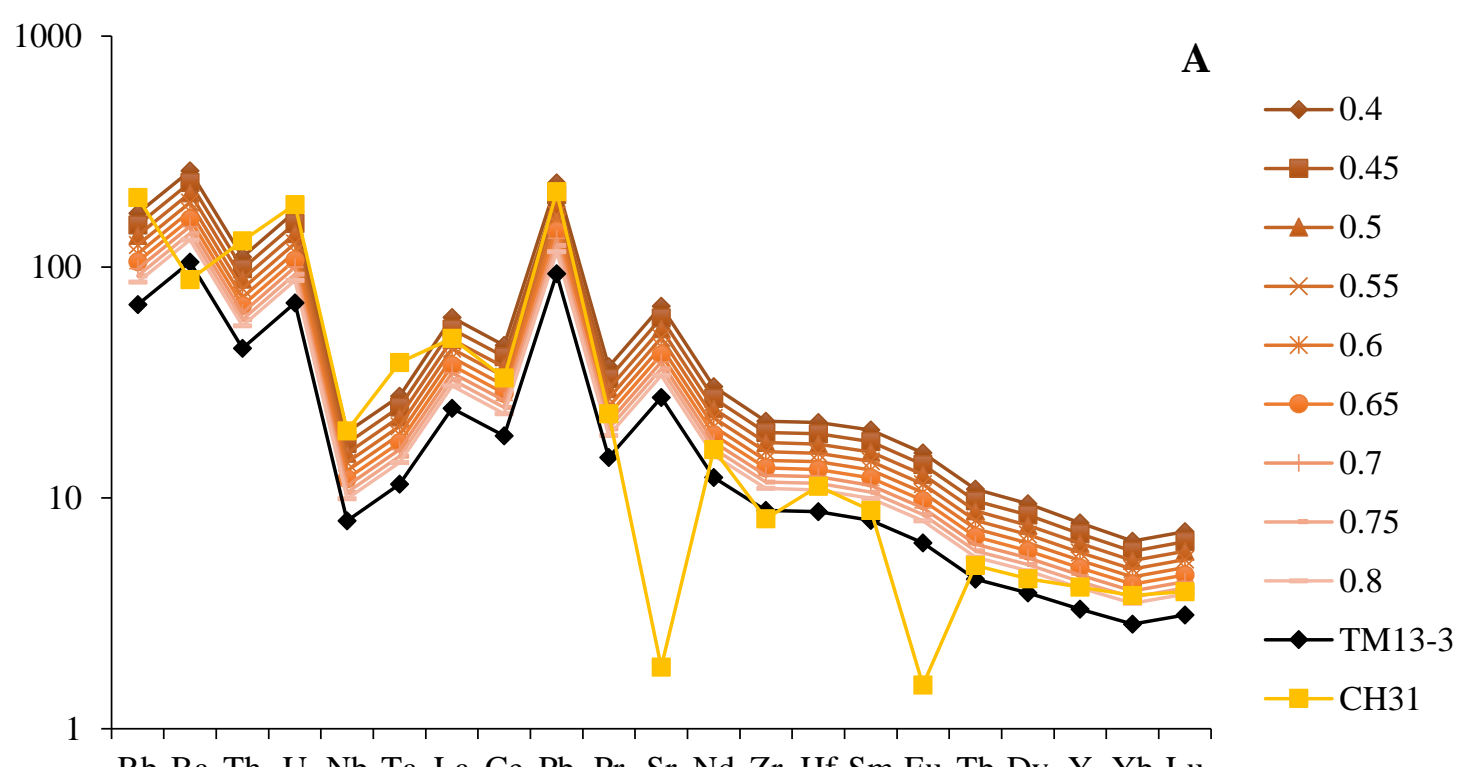

$\mathrm{Rb} \mathrm{Ba}$ Th $\mathrm{U}$ Nb Ta La Ce Pb Pr Sr Nd Zr Hf Sm Eu Tb Dy Y Yb Lu

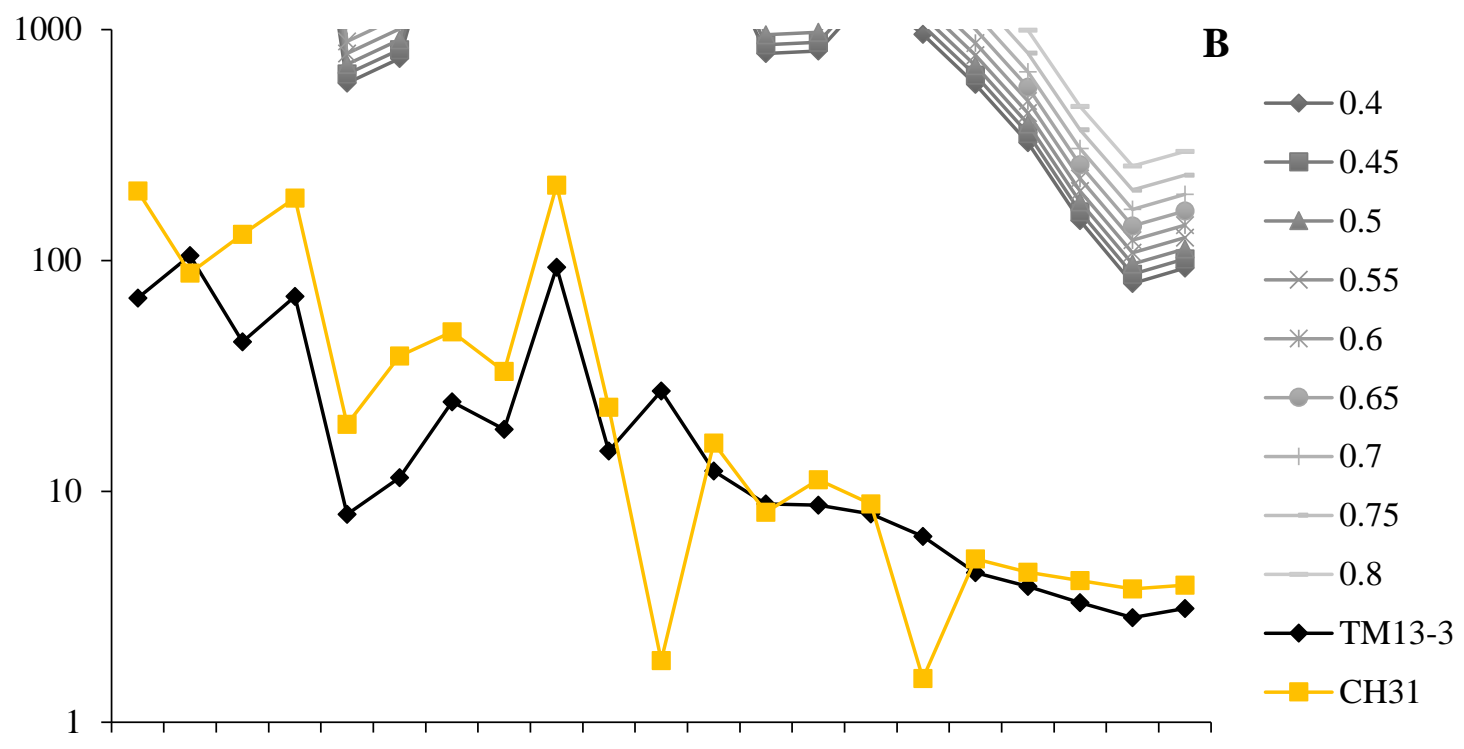

$\mathrm{Rb} \mathrm{Ba}$ Th $\mathrm{U}$ Nb Ta La Ce Pb Pr Sr Nd Zr Hf Sm Eu Tb Dy Y Yb Lu

Fig. D10 Primitive mantle normalized (Sun and McDonough, 1989) spider diagram for the partial melting of experimental melt MS16. A shows the results of the batch melt model; B shows the results of the fractional melt model. The melt percentages are approximately $\pm 20 \%$ that observed in the experimental melt. The trace element concentrations of the parent material, sample TM13-3 (granodiorite), are indicated by the black diamonds. The trace element concentrations of the Dale Tuff, sample CH31, which is included for comparison, are indicated by the yellow squares. For experimental melt MS16, it can be seen that the batch melt model (A) approximates the data. The fit increases as melt percent increases. 

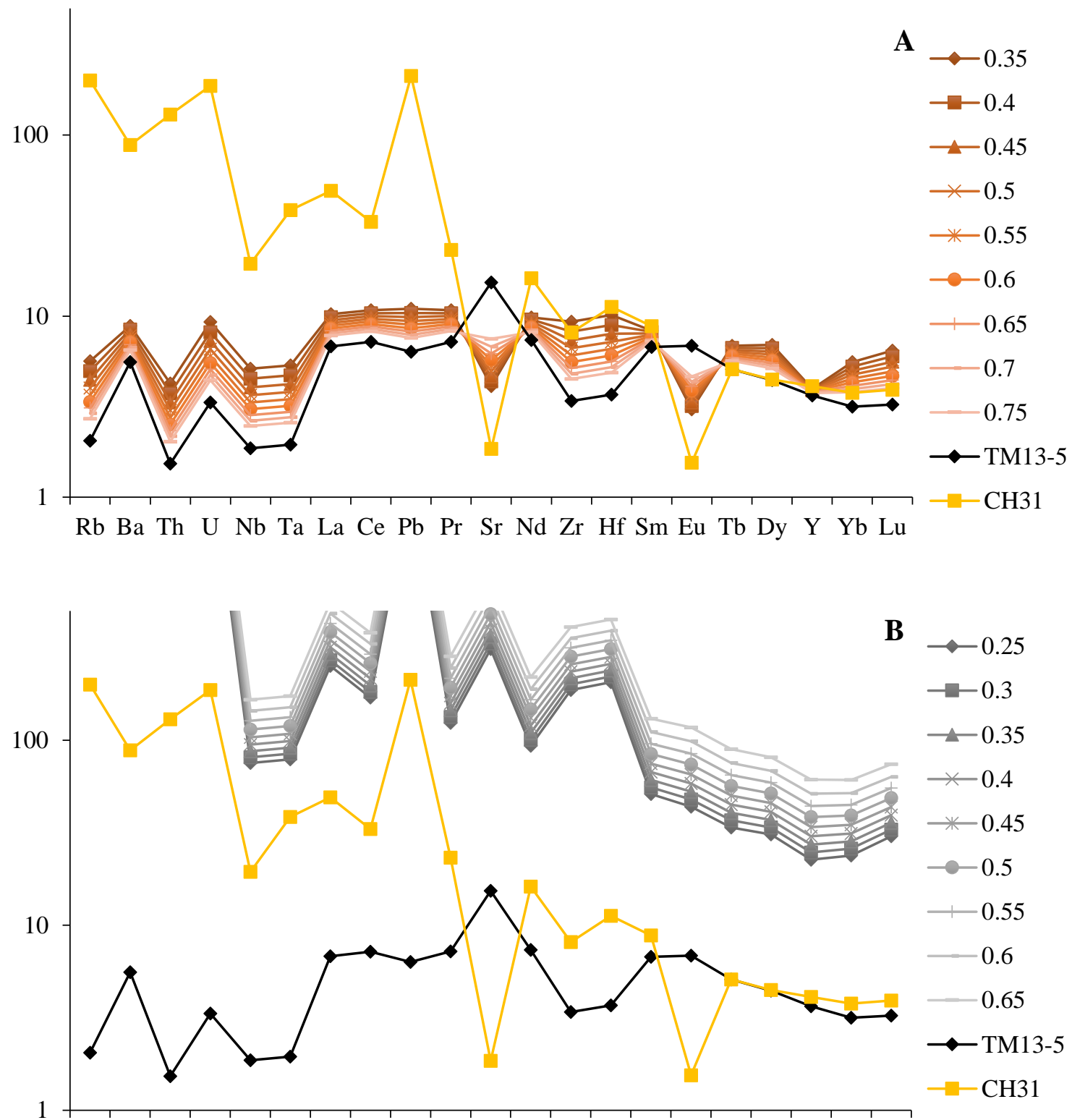

$\mathrm{Rb} \mathrm{Ba}$ Th $\mathrm{U}$ Nb Ta $\mathrm{La} \mathrm{Ce} \mathrm{Pb}$ Pr $\mathrm{Sr} \mathrm{Nd} \mathrm{Zr} \mathrm{Hf} \mathrm{Sm} \mathrm{Eu}$ Tb Dy Y Yb Lu

Fig. D11 Primitive mantle normalized (Sun and McDonough, 1989) spider diagram for the partial melting of experimental melt MS17. A shows the results of the batch melt model; B shows the results of the fractional melt model. The melt percentages are approximately $\pm 20 \%$ that observed in the experimental melt. The trace element concentrations of the parent material, sample TM13-5 (amphibolite), are indicated by the black diamonds. The trace element concentrations of the Dale Tuff, sample CH31, which is included for comparison, are indicated by the yellow squares. For experimental melt MS17, it can be seen that the batch melt model (A) approximates the data with notable exceptions at $\mathrm{Sr}$ and Eu. In general, the fit increases as melt percent increases. 

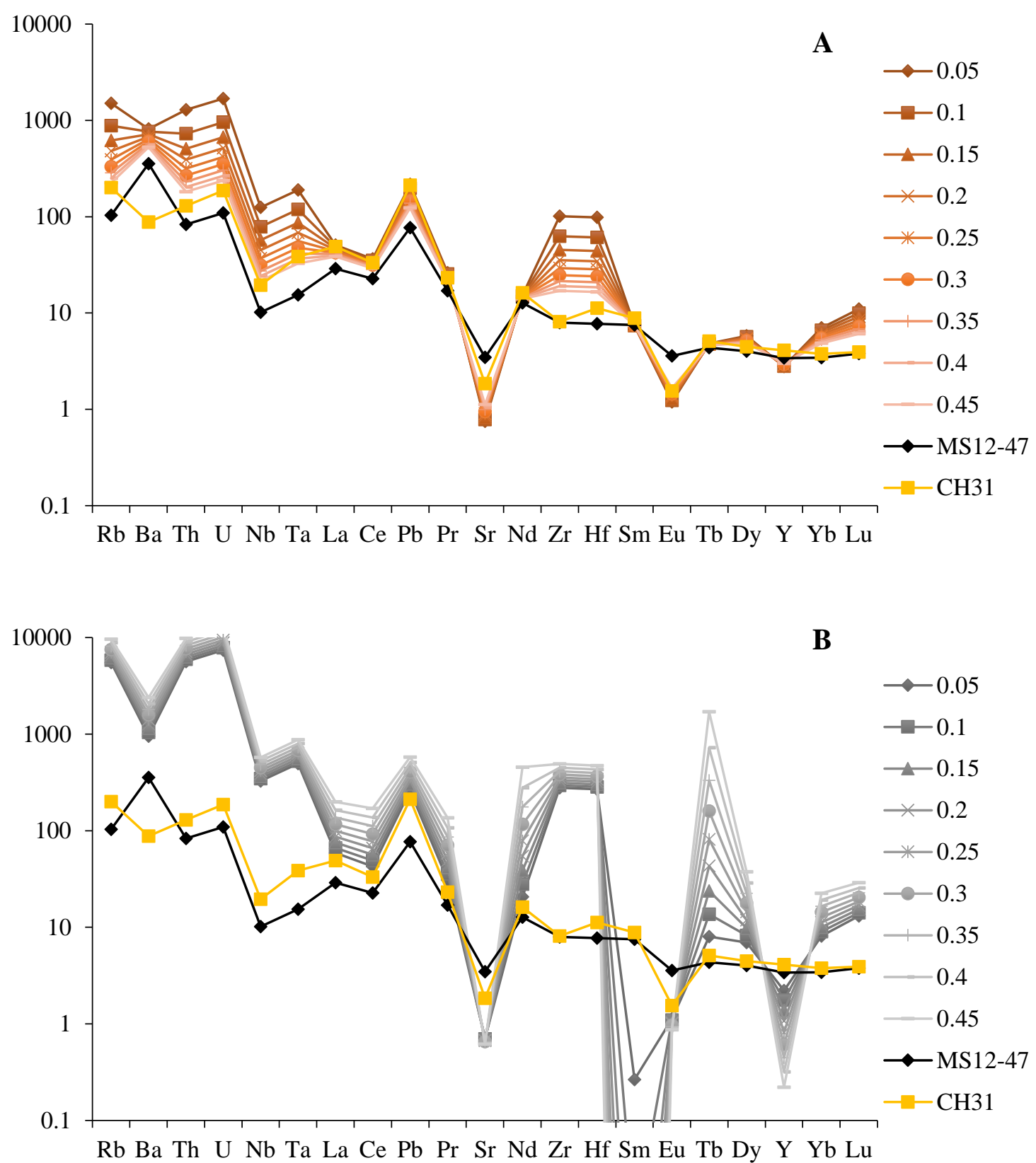

Fig. D12 Primitive mantle normalized (Sun and McDonough, 1989) spider diagram for the partial melting of experimental melt MS18. A shows the results of the batch melt model; B shows the results of the fractional melt model. The melt percentages are approximately $\pm 20 \%$ that observed in the experimental melt. The trace element concentrations of the parent material, sample MS12-47 (biotite fels), are indicated by the black diamonds. The trace element concentrations of the Dale Tuff, sample $\mathrm{CH} 31$, which is included for comparison, are indicated by the yellow squares. For experimental melt MS18, it can be seen that the batch melt model (A) approximates the data, with a notable exception at Eu. In general, the fit increases as melt percent increases. 


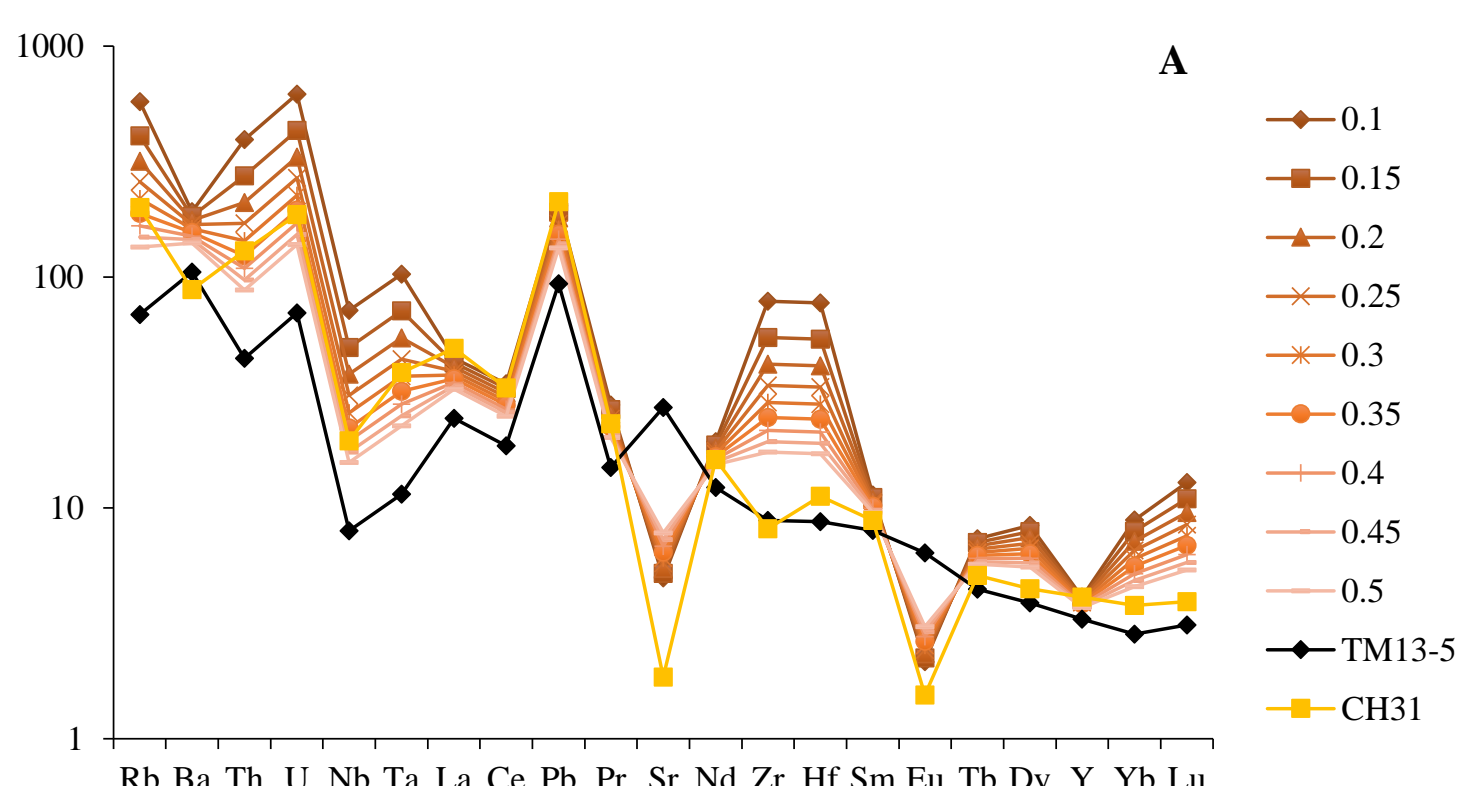

$\mathrm{Rb} \mathrm{Ba}$ Th U Nb Ta La Ce Pb Pr Sr Nd Zr Hf Sm Eu Tb Dy Y Yb Lu

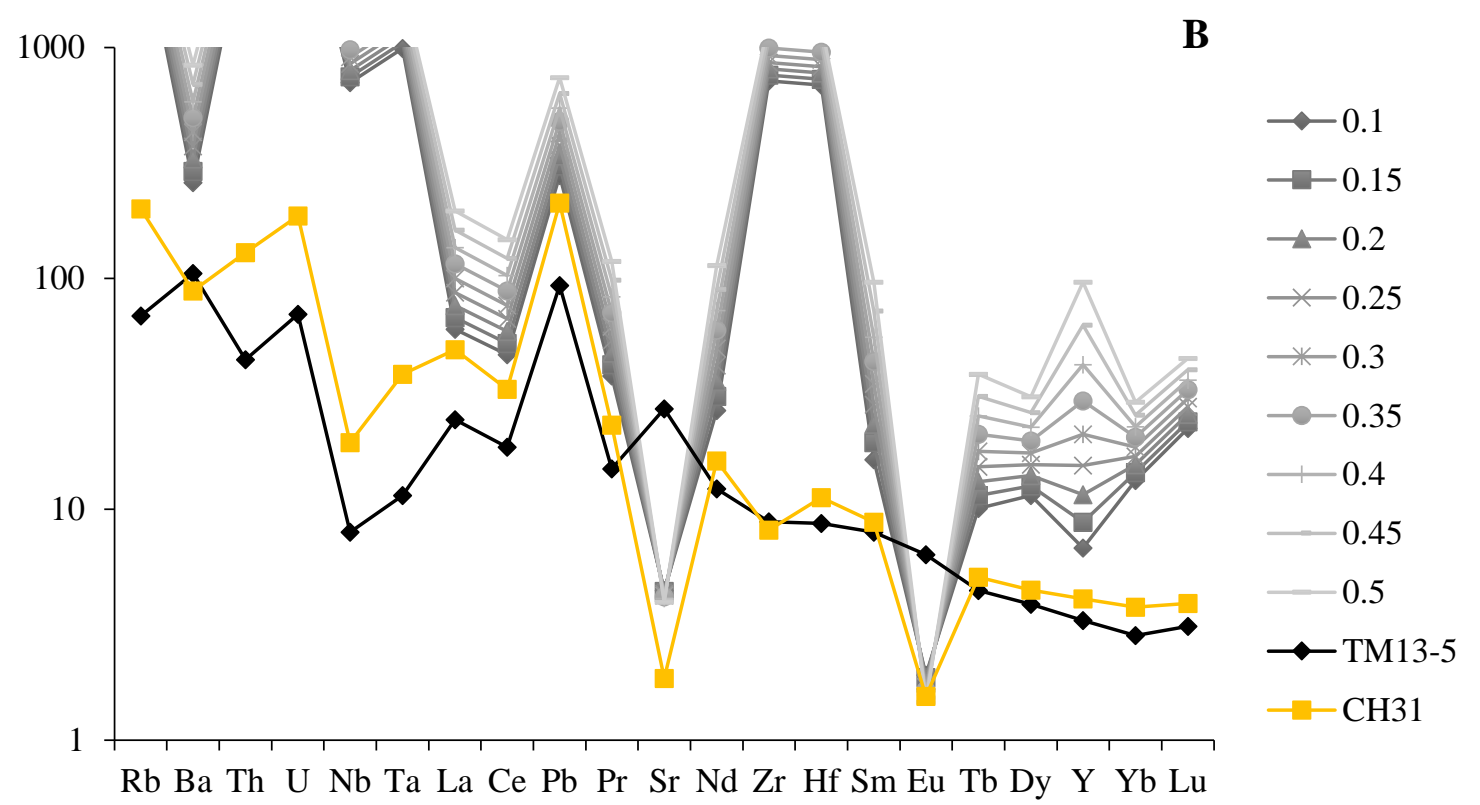

Fig. D13 Primitive mantle normalized (Sun and McDonough, 1989) spider diagram for the partial melting of experimental melt MS19. A shows the results of the batch melt model; B shows the results of the fractional melt model. The melt percentages are approximately $\pm 20 \%$ that observed in the experimental melt. The trace element concentrations of the parent material, sample TM13-5 (amphibolite), are indicated by the black diamonds. The trace element concentrations of the Dale Tuff, sample CH31, which is included for comparison, are indicated by the yellow squares. For experimental melt MS19, it can be seen that the batch melt model (A) approximates the data, although there are notable exceptions at $\mathrm{Sr}$ and Eu. In general, the fit increases as melt percent increases. 


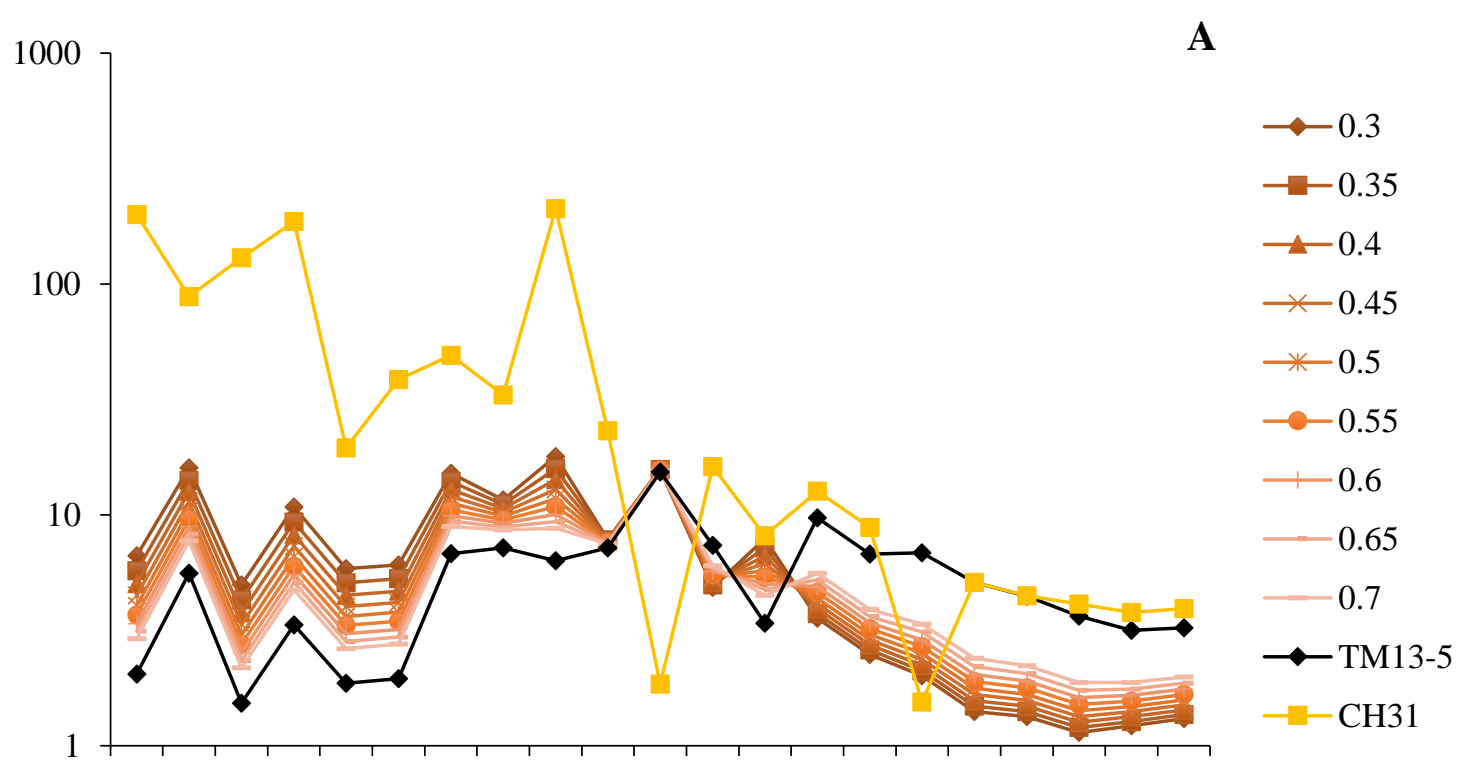

$\mathrm{Rb}$ Ba Th U Nb Ta La Ce Pb Pr Sr Nd Zr Hf Sm Eu Tb Dy Y Yb Lu

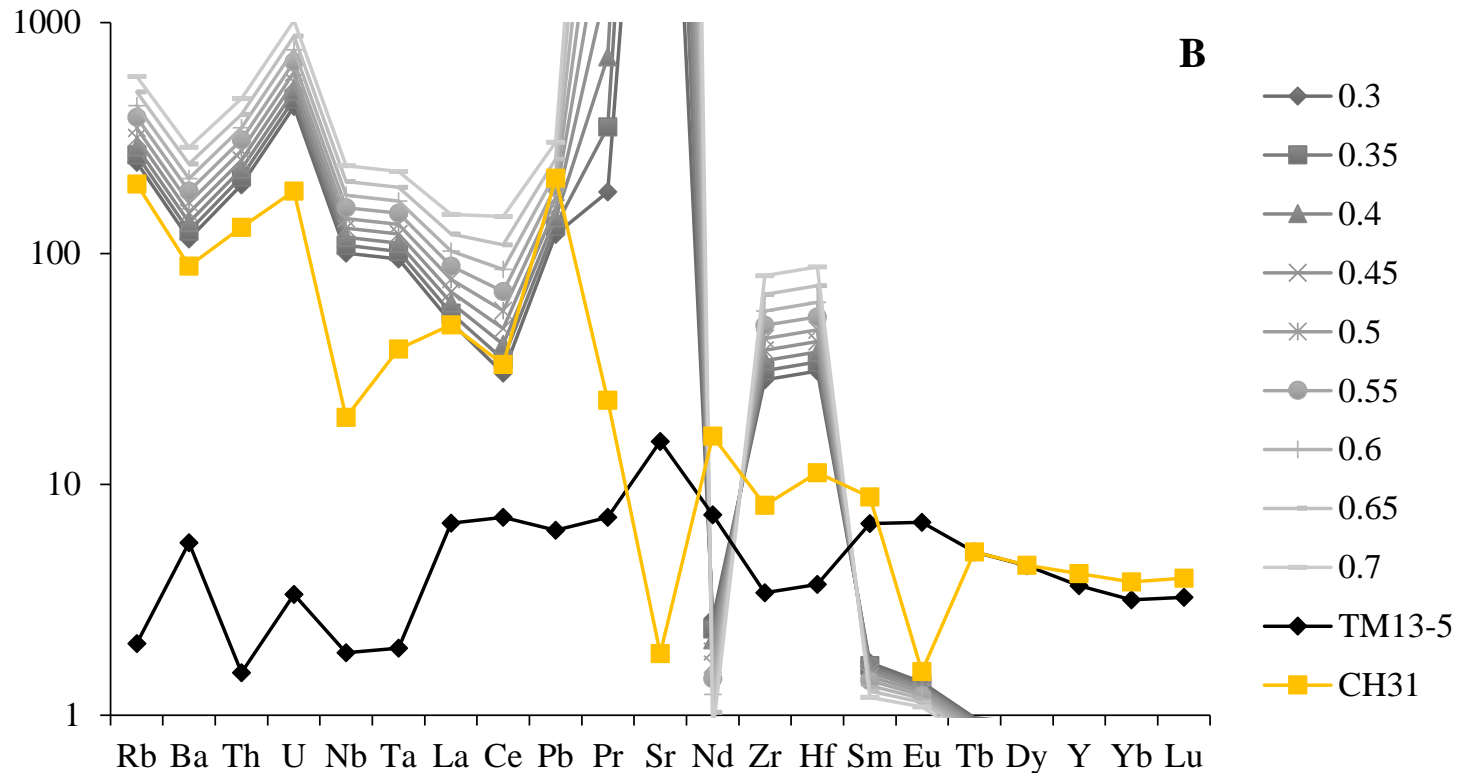

Fig. D14 Primitive mantle normalized (Sun and McDonough, 1989) spider diagram for the partial melting of experimental melt MS22. A shows the results of the batch melt model; B shows the results of the fractional melt model. The melt percentages are approximately $\pm 20 \%$ that observed in the experimental melt. The trace element concentrations of the parent material, sample TM13-5 (amphibolite), are indicated by the black diamonds. The trace element concentrations of the Dale Tuff, sample $\mathrm{CH} 31$, which is included for comparison, are indicated by the yellow squares. For experimental melt MS22, it can be seen that the batch melt model (A) approximates the data. The fit increases as melt percent increases, particularly for LREE. 

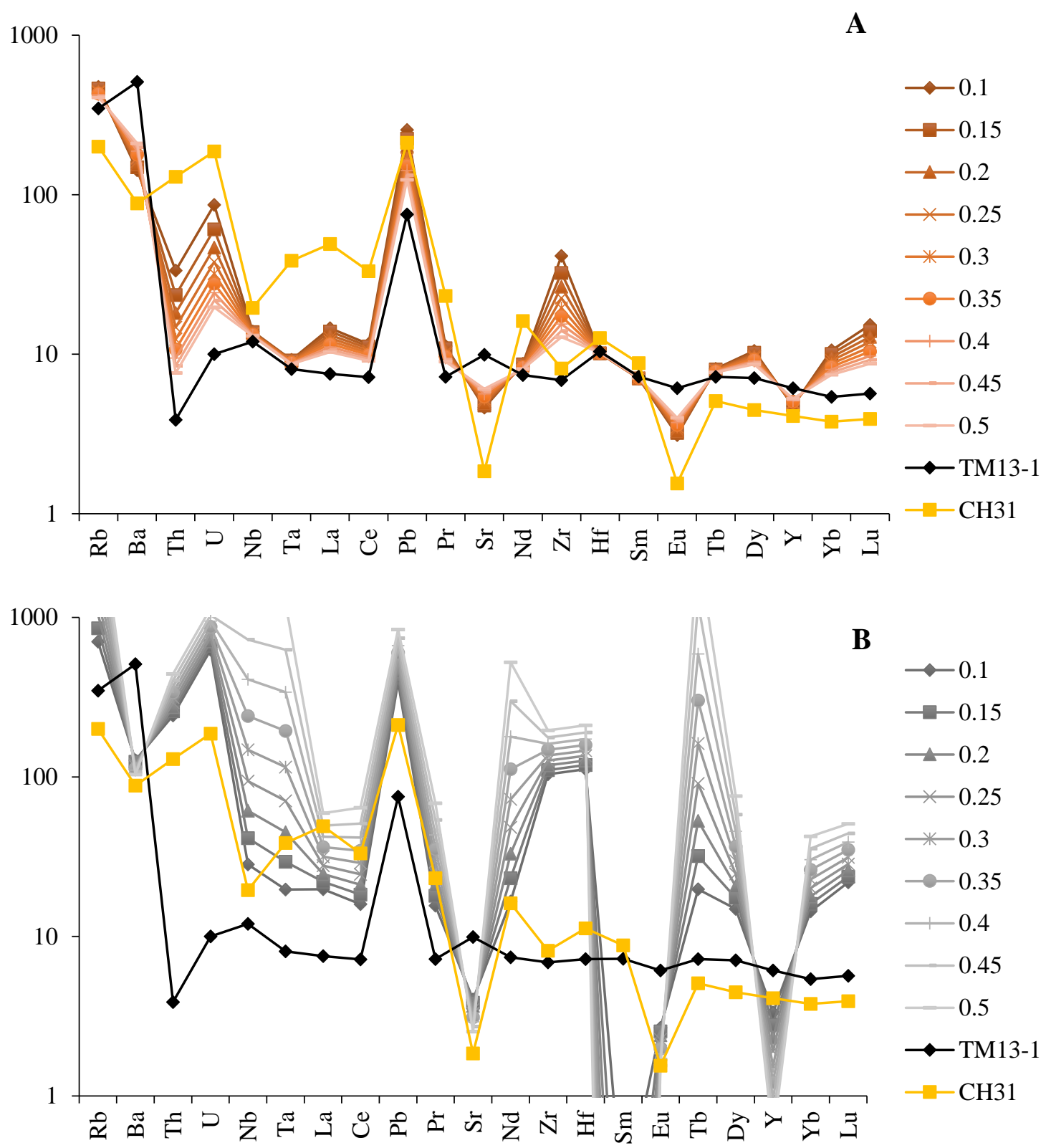

Fig. D15 Primitive mantle normalized (Sun and McDonough, 1989) spider diagram for the partial melting of experimental melt MS23. A shows the results of the batch melt model; B shows the results of the fractional melt model. The melt percentages are approximately $\pm 20 \%$ that observed in the experimental melt. The trace element concentrations of the parent material, sample TM13-1 (biotite schist), are indicated by the black diamonds. The trace element concentrations of the Dale Tuff, sample CH31, which is included for comparison, are indicated by the yellow squares. For experimental melt MS23, it can be seen that the batch melt model (A) approximates the data, although there are notable exceptions at $\mathrm{Ba}, \mathrm{Sr}, \mathrm{Zr}$, and $\mathrm{Eu}$. In general, the fit increases as melt percent increases. 


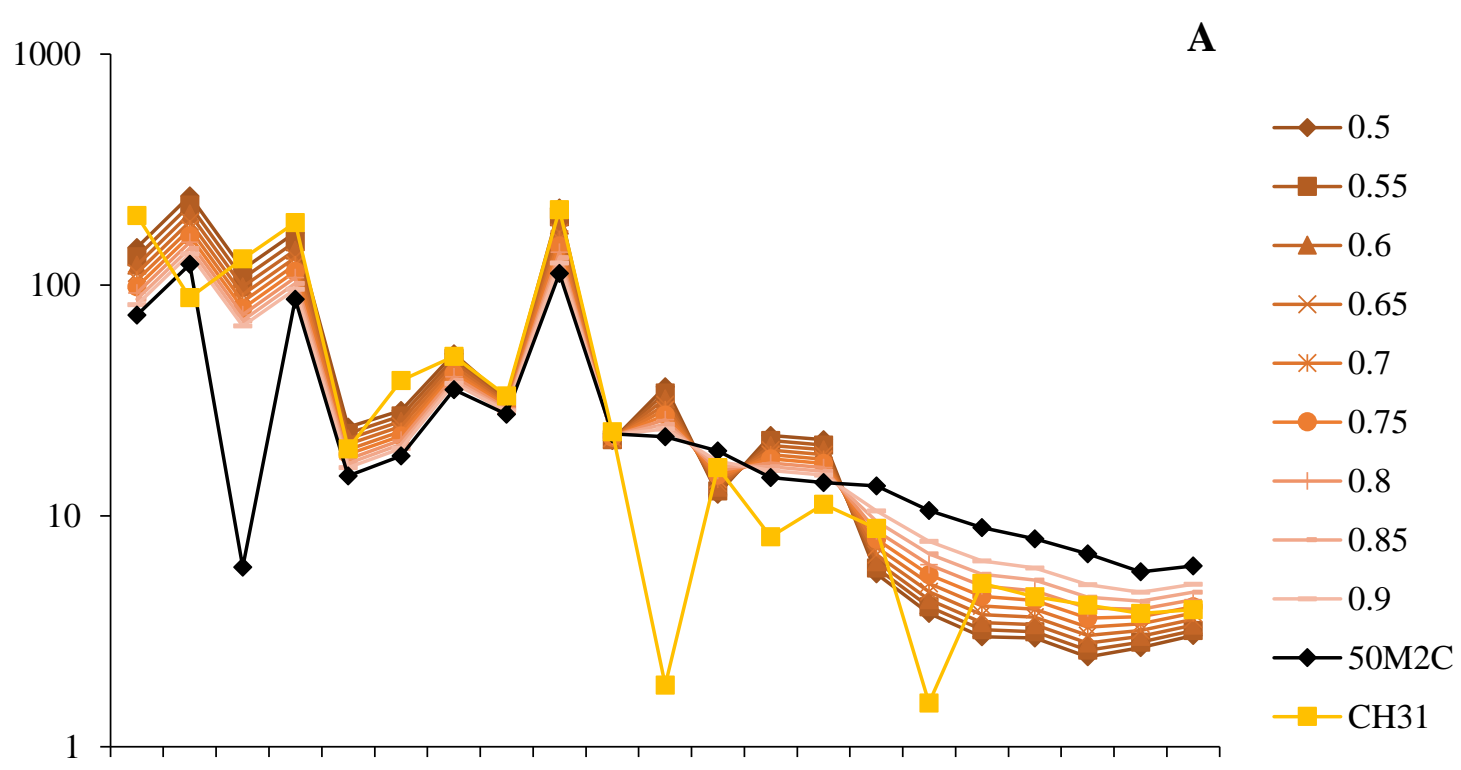

$\mathrm{Rb} \mathrm{Ba}$ Th $\mathrm{U}$ Nb Ta La Ce Pb Pr Sr Nd Zr Hf Sm Eu Tb Dy Y Yb Lu

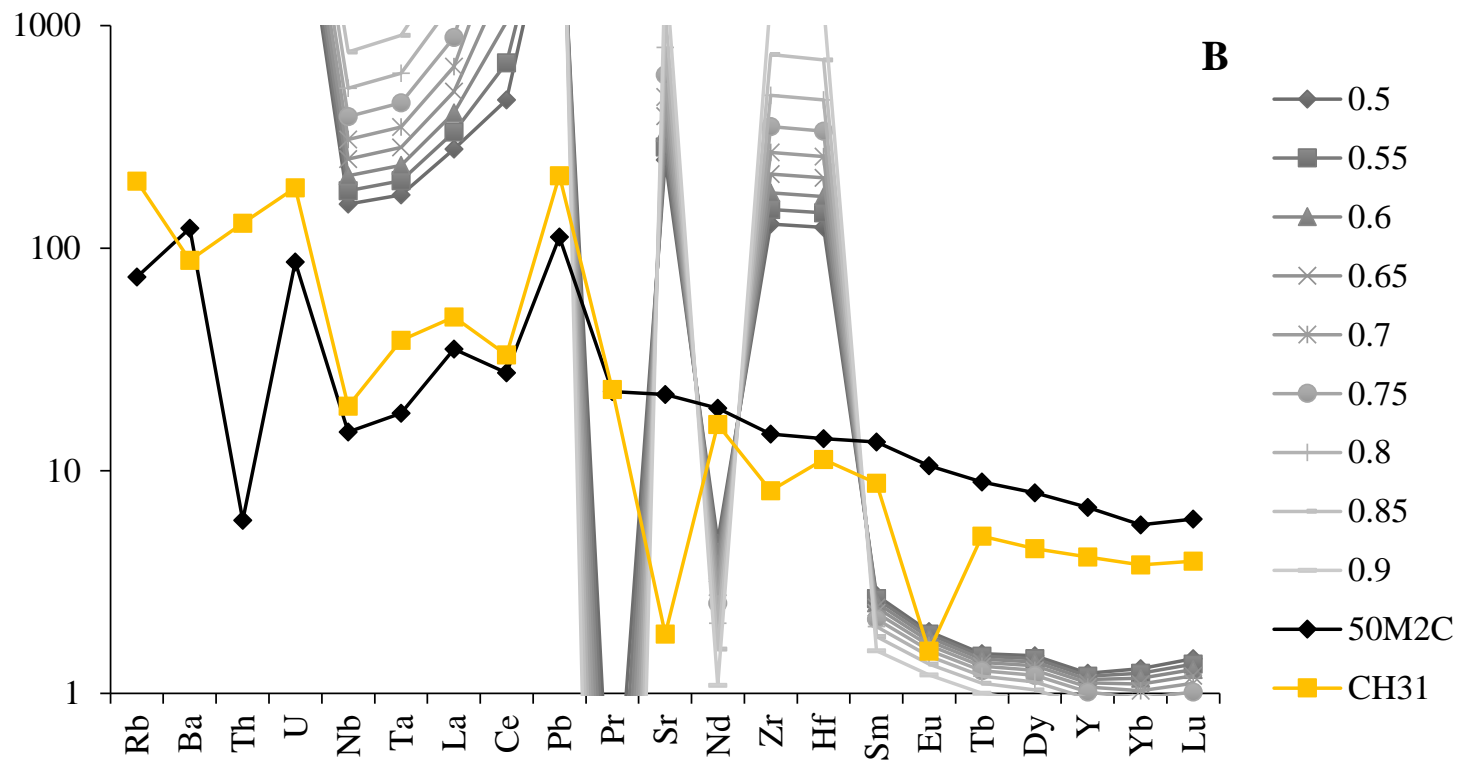

Fig. D16 Primitive mantle normalized (Sun and McDonough, 1989) spider diagram for the partial melting of experimental melt MS26. A shows the results of the batch melt model; B shows the results of the fractional melt model. The melt percentages are approximately $\pm 20 \%$ that observed in the experimental melt. The trace element concentrations of the parent material, sample 50M2C (50/50 mixture of mafic glass and diorite), are indicated by the black diamonds. The trace element concentrations of the Dale Tuff, sample $\mathrm{CH} 31$, which is included for comparison, are indicated by the yellow squares. For experimental melt MS26, it can be seen that the batch melt model (A) approximates the data. The fit increases as melt percent increases, particularly for LREE. 


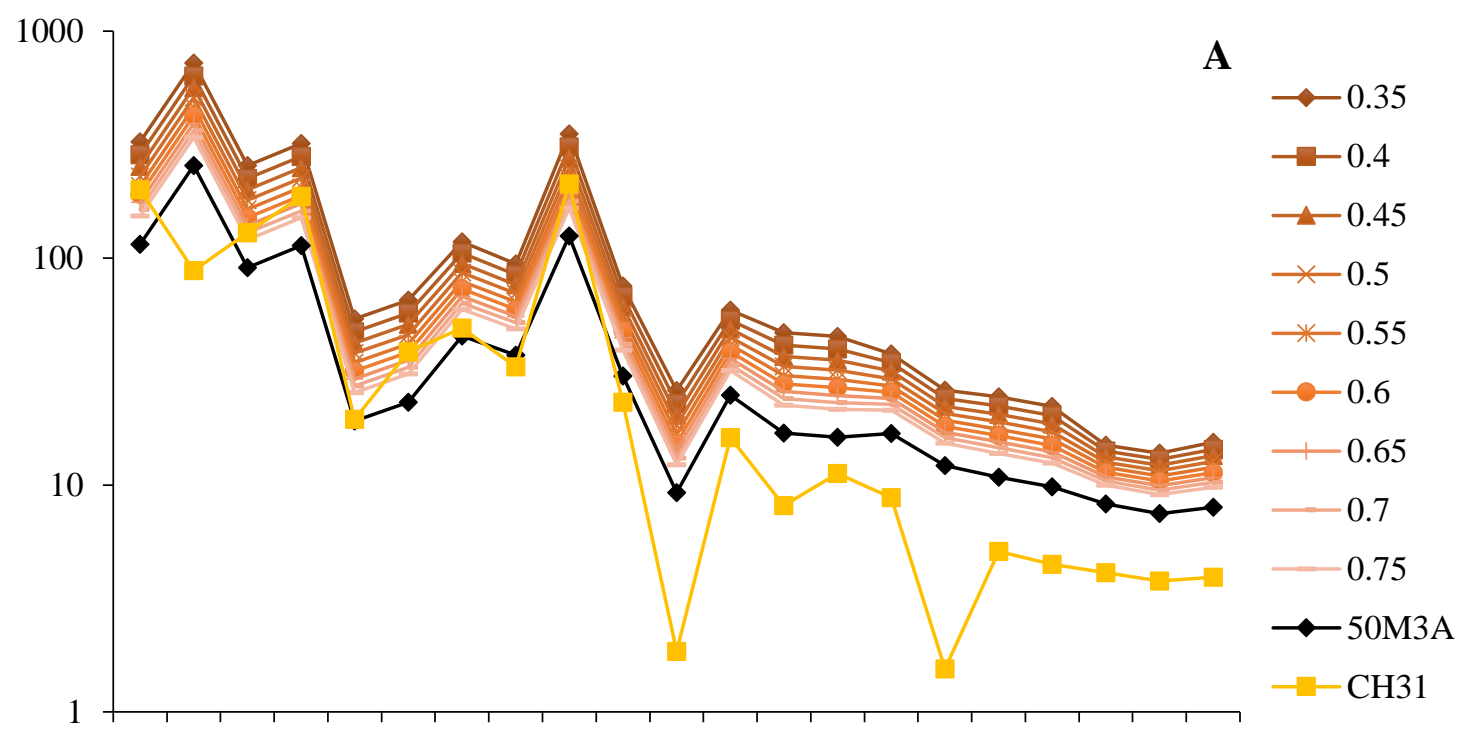

$\mathrm{Rb} \mathrm{Ba}$ Th $\mathrm{U}$ Nb Ta La Ce Pb Pr Sr Nd Zr Hf Sm Eu Tb Dy Y Yb Lu

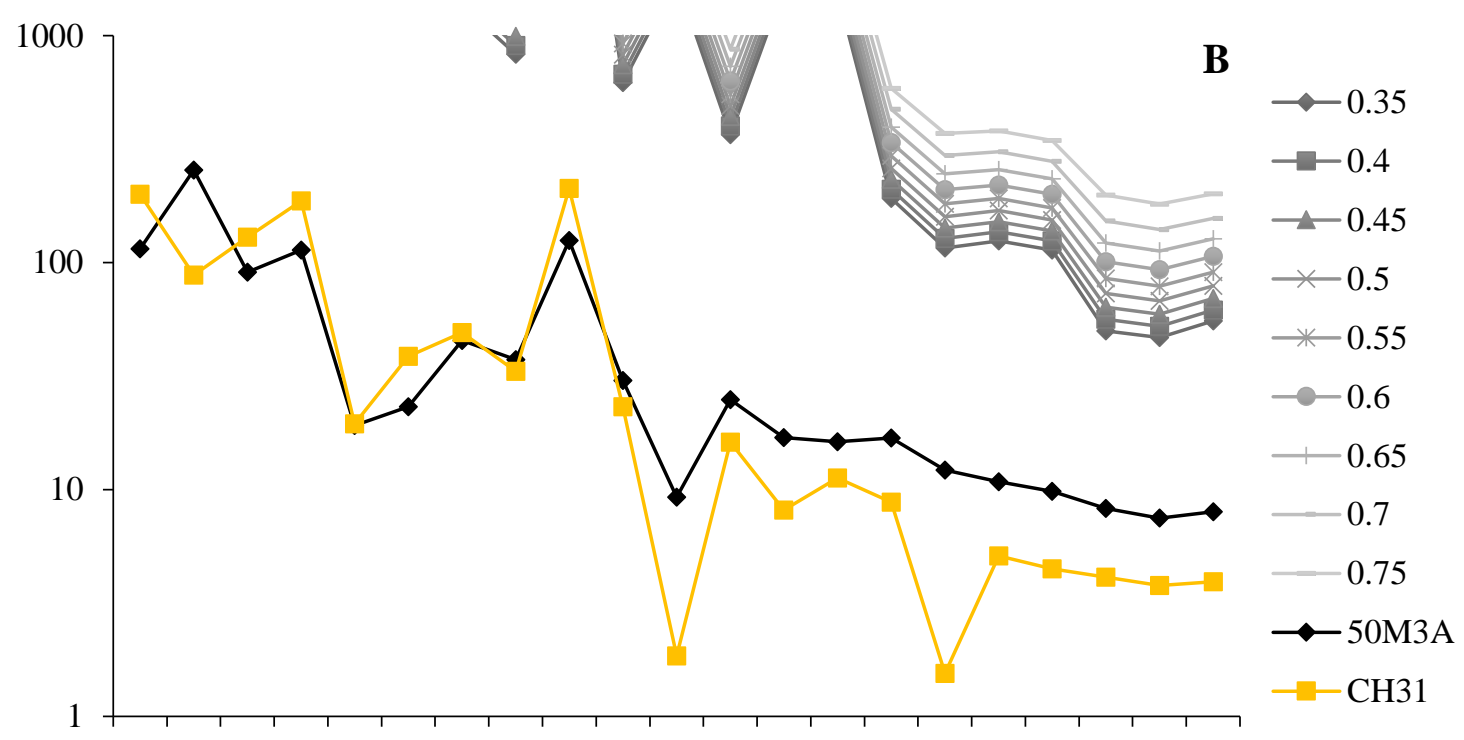

$\mathrm{Rb} \mathrm{Ba}$ Th $\mathrm{U}$ Nb Ta La Ce Pb Pr Sr Nd Zr Hf Sm Eu Tb Dy Y Yb Lu

Fig. D17 Primitive mantle normalized (Sun and McDonough, 1989) spider diagram for the partial melting of experimental melt MS27. A shows the results of the batch melt model; B shows the results of the fractional melt model. The melt percentages are approximately $\pm 20 \%$ that observed in the experimental melt. The trace element concentrations of the parent material, sample 50M3A (50/50 mixture of mafic glass and biotite fels), are indicated by the black diamonds. The trace element concentrations of the Dale Tuff, sample $\mathrm{CH} 31$, which is included for comparison, are indicated by the yellow squares. For experimental melt MS27, it can be seen that the batch melt model (A) approximates the data. The fit increases as melt percent increases. 


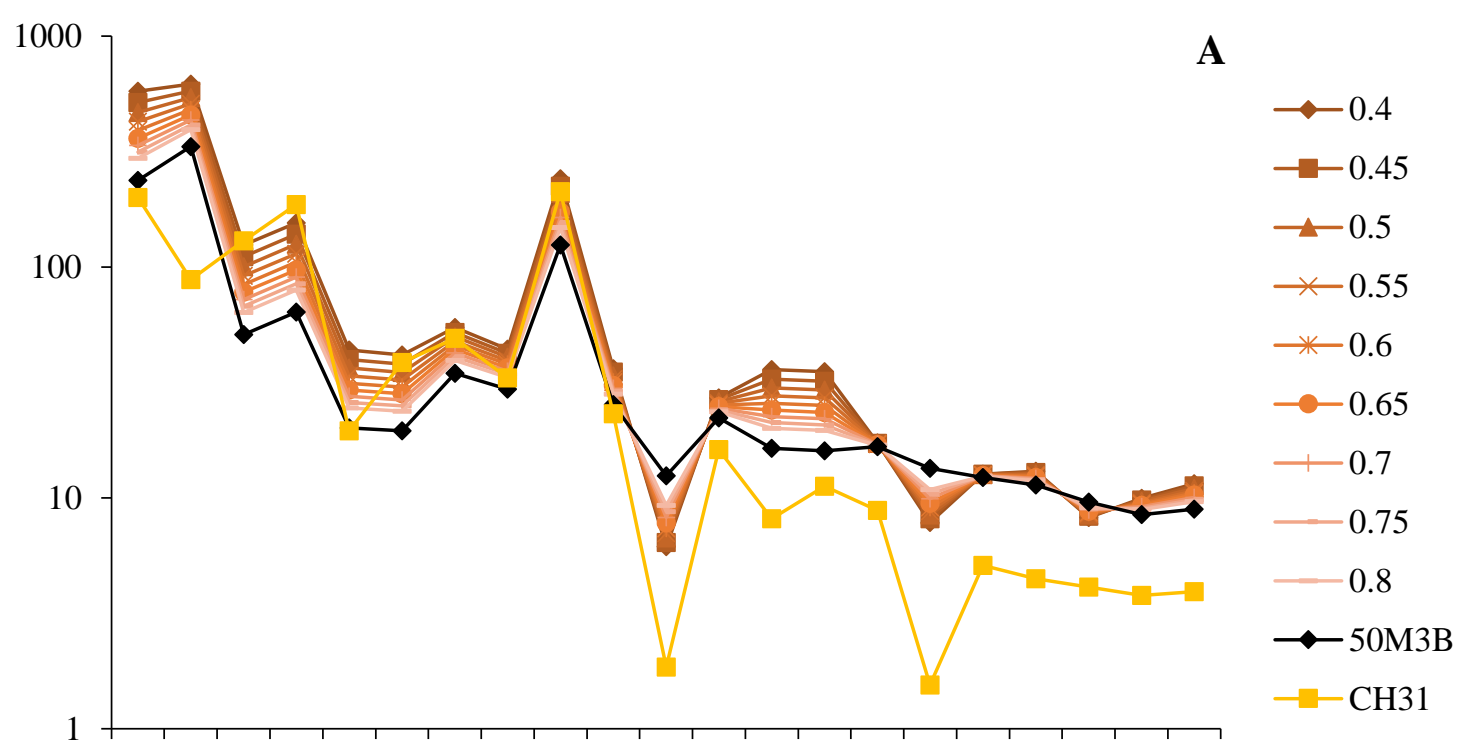

$\mathrm{Rb} \mathrm{Ba}$ Th $\mathrm{U}$ Nb Ta La Ce Pb Pr Sr Nd Zr Hf Sm Eu Tb Dy Y Yb Lu

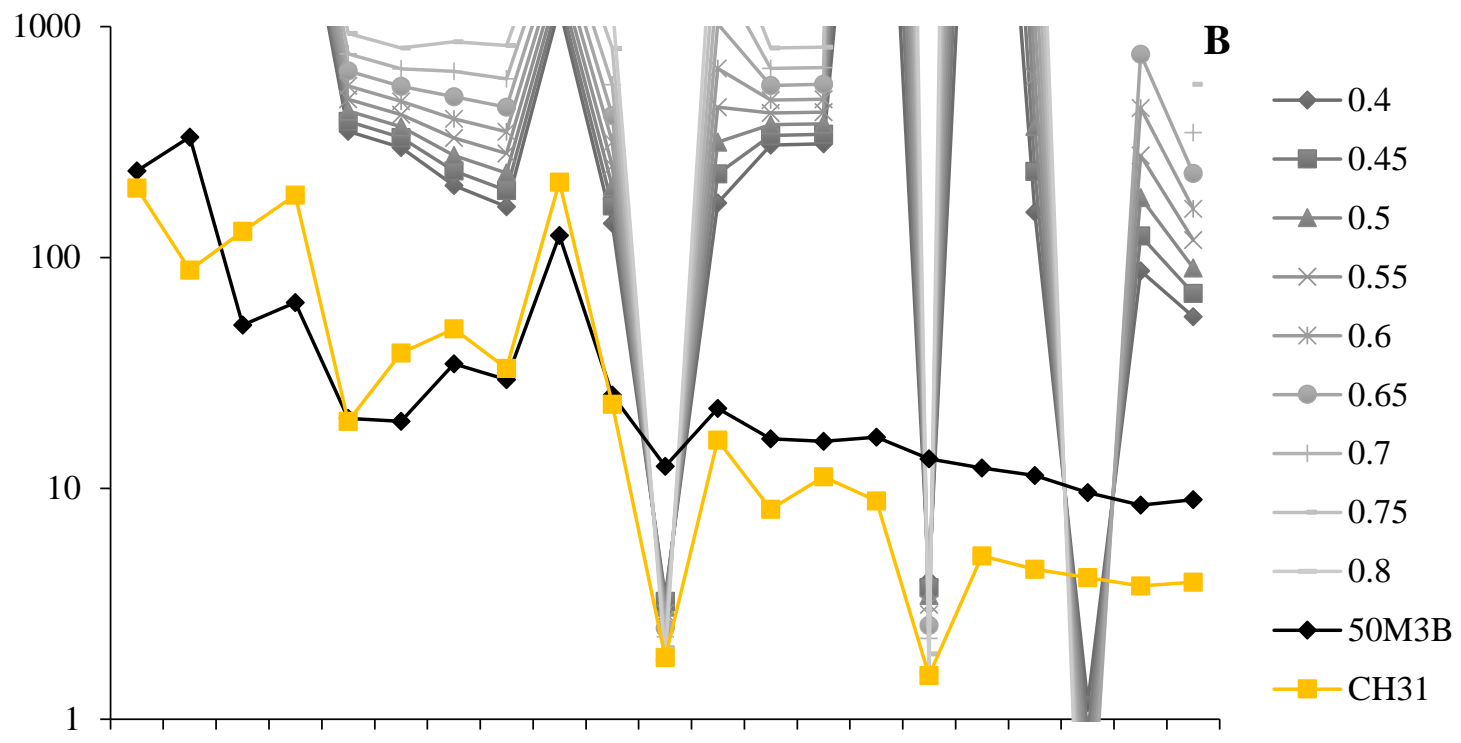

$\mathrm{Rb} \mathrm{Ba}$ Th $\mathrm{U}$ Nb Ta La Ce Pb Pr Sr Nd Zr Hf Sm Eu Tb Dy Y Yb Lu

Fig. D18 Primitive mantle normalized (Sun and McDonough, 1989) spider diagram for the partial melting of experimental melt MS28. A shows the results of the batch melt model; B shows the results of the fractional melt model. The melt percentages are approximately $\pm 20 \%$ that observed in the experimental melt. The trace element concentrations of the parent material, sample 50M3B (50/50 mixture of mafic glass and biotite schist), are indicated by the black diamonds. The trace element concentrations of the Dale Tuff, sample $\mathrm{CH} 31$, which is included for comparison, are indicated by the yellow squares. For experimental melt MS28, it can be seen that the batch melt model (A) approximates the data. The fit increases as melt percent increases. 


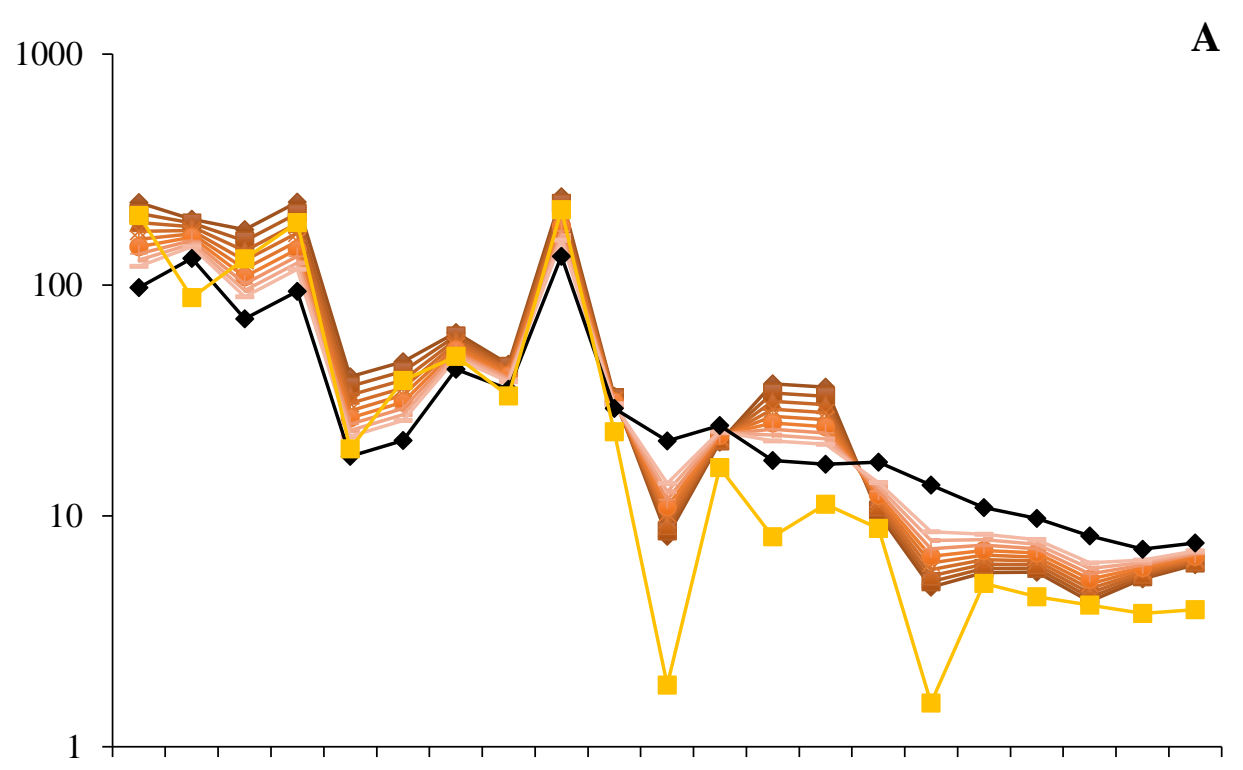

A

$\mathrm{Rb} \mathrm{Ba}$ Th $\mathrm{U}$ Nb Ta La Ce Pb Pr Sr Nd Zr Hf Sm Eu Tb Dy Y Yb Lu

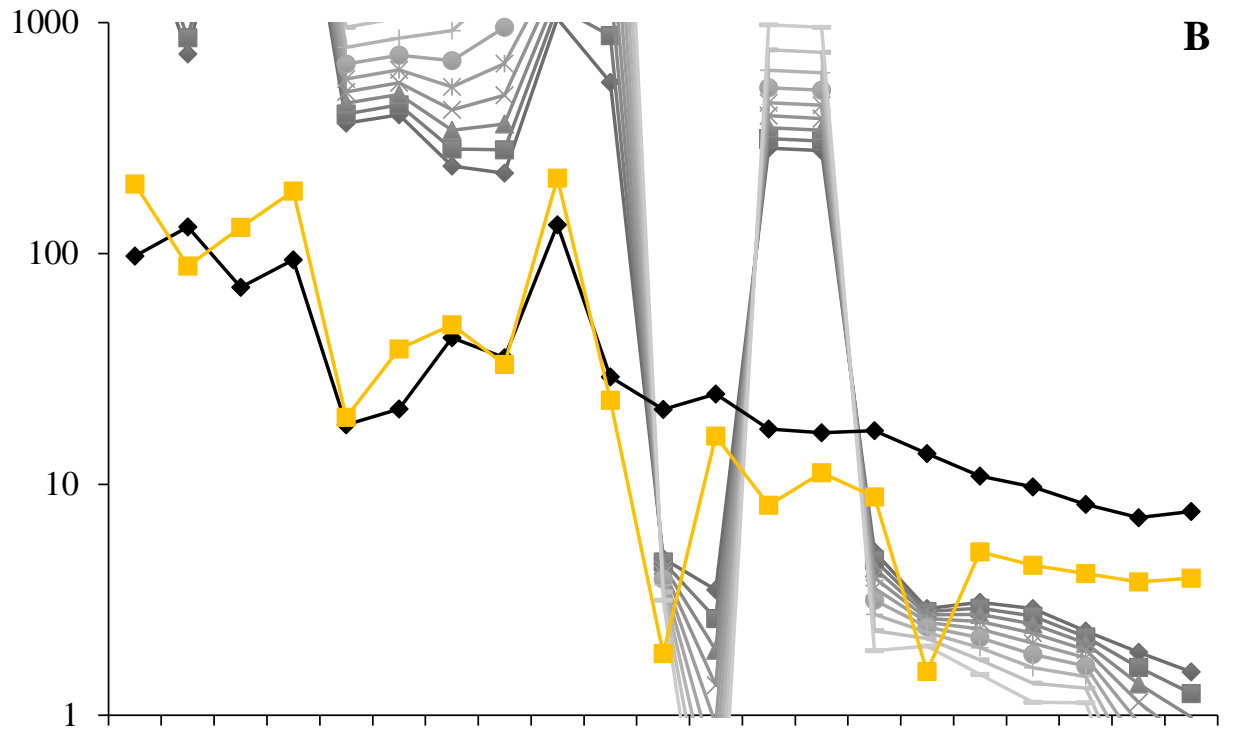

B

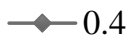

$-0.45$

$-0.5$

$\times 0.55$

- 0.6

$-0.65$

$-0.7$

$-0.75$

$-0.8$

$\rightarrow 50 \mathrm{M} 3 \mathrm{C}$

$\mathrm{Rb}$ Ba Th $\mathrm{U}$ Nb Ta La Ce Pb Pr Sr Nd Zr Hf Sm Eu Tb Dy Y Yb Lu

Fig. D19 Primitive mantle normalized (Sun and McDonough, 1989) spider diagram for the partial melting of experimental melt MS29. A shows the results of the batch melt model; B shows the results of the fractional melt model. The melt percentages are approximately $\pm 20 \%$ that observed in the experimental melt. The trace element concentrations of the parent material, sample 50M3C (50/50 mixture of mafic glass and diorite), are indicated by the black diamonds. The trace element concentrations of the Dale Tuff, sample $\mathrm{CH} 31$, which is included for comparison, are indicated by the yellow squares. For experimental melt MS29, it can be seen that the batch melt model (A) approximates the data. The fit increases as melt percent increases. 


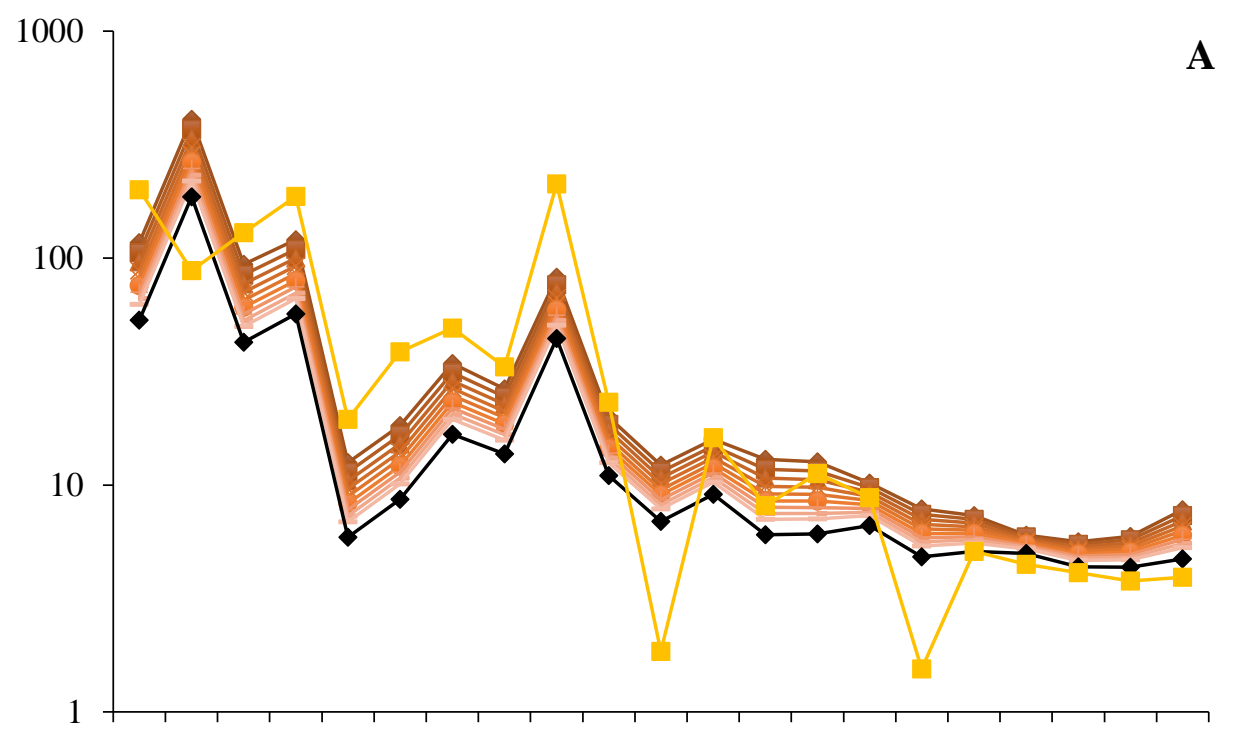

A

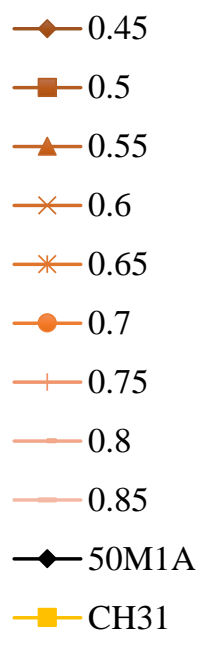

$\mathrm{Rb} \mathrm{Ba}$ Th U Nb Ta La Ce Pb Pr Sr Nd Zr Hf Sm Eu Tb Dy Y Yb Lu

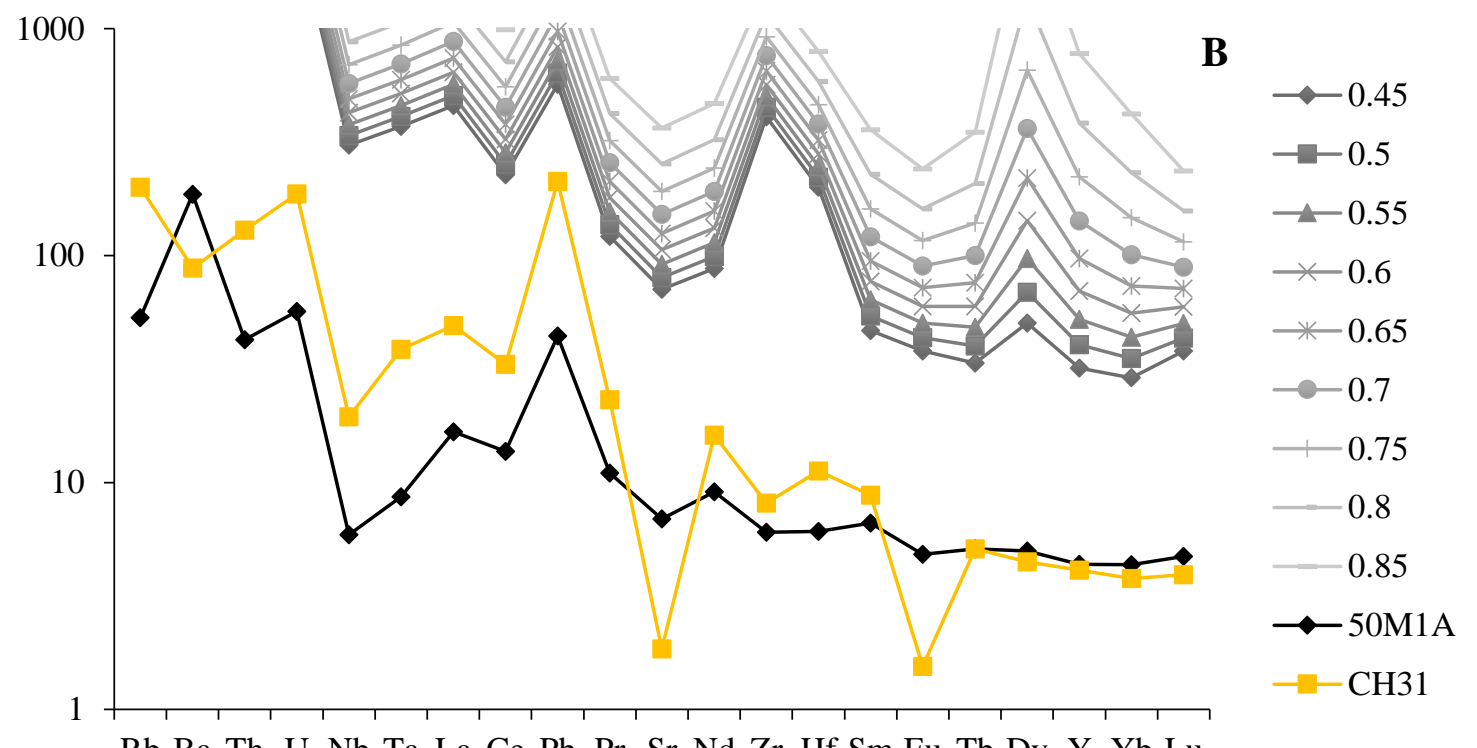

$\mathrm{Rb} \mathrm{Ba}$ Th U Nb Ta La Ce Pb Pr Sr Nd Zr Hf Sm Eu Tb Dy Y Yb Lu

Fig. D20 Primitive mantle normalized (Sun and McDonough, 1989) spider diagram for the partial melting of experimental melt MS30. A shows the results of the batch melt model; B shows the results of the fractional melt model. The melt percentages are approximately $\pm 20 \%$ that observed in the experimental melt. The trace element concentrations of the parent material, sample 50M1A (50/50 mixture of mafic glass and biotite fels), are indicated by the black diamonds. The trace element concentrations of the Dale Tuff, sample $\mathrm{CH} 31$, which is included for comparison, are indicated by the yellow squares. For experimental melt MS30, it can be seen that the batch melt model (A) approximates the data. The fit increases as melt percent increases. 


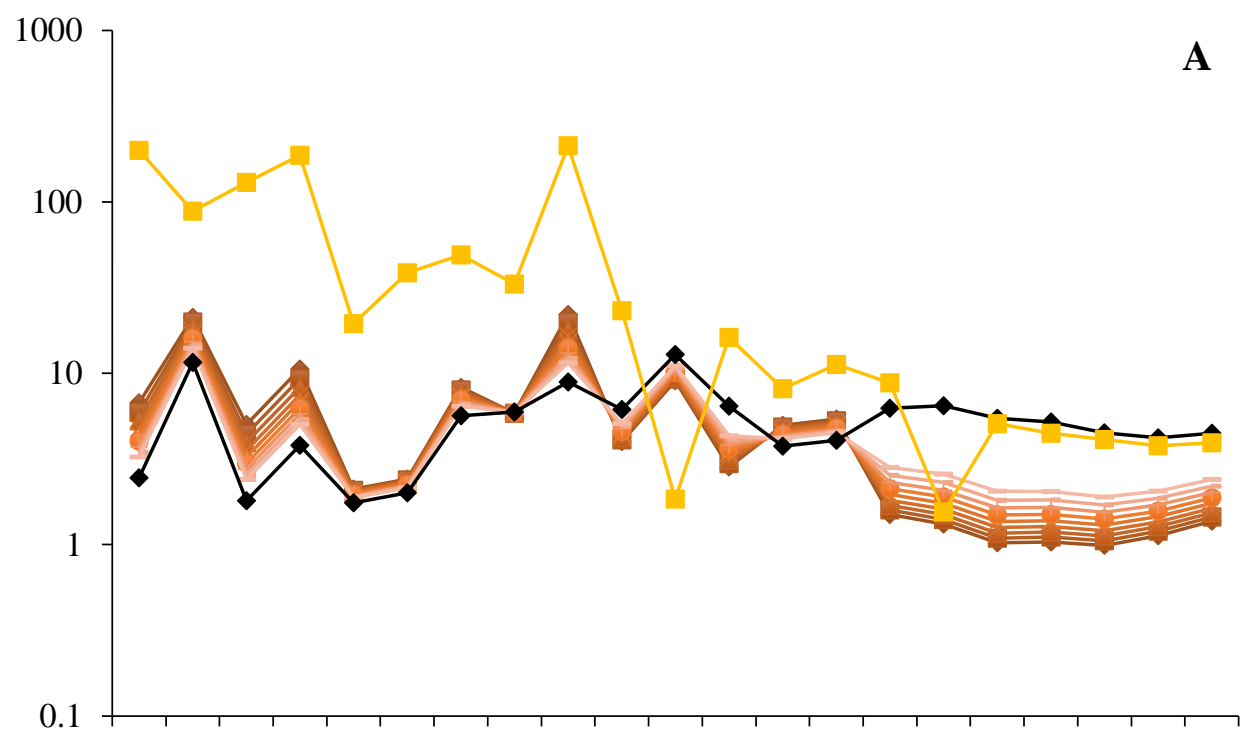

A

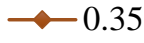

$-0.4$

$-0.45$

$\times 0.5$

* 0.55

$\longrightarrow 0.6$

$-0.65$

$-0.7$

$\multimap 50 \mathrm{M} 1 \mathrm{D}$

$-\mathrm{CH} 31$

$\mathrm{Rb} \mathrm{Ba}$ Th $\mathrm{U}$ Nb Ta $\mathrm{La} \mathrm{Ce} \mathrm{Pb} \operatorname{Pr} \mathrm{Sr} \mathrm{Nd} \mathrm{Zr}$ Hf Sm Eu Tb Dy Y Yb Lu

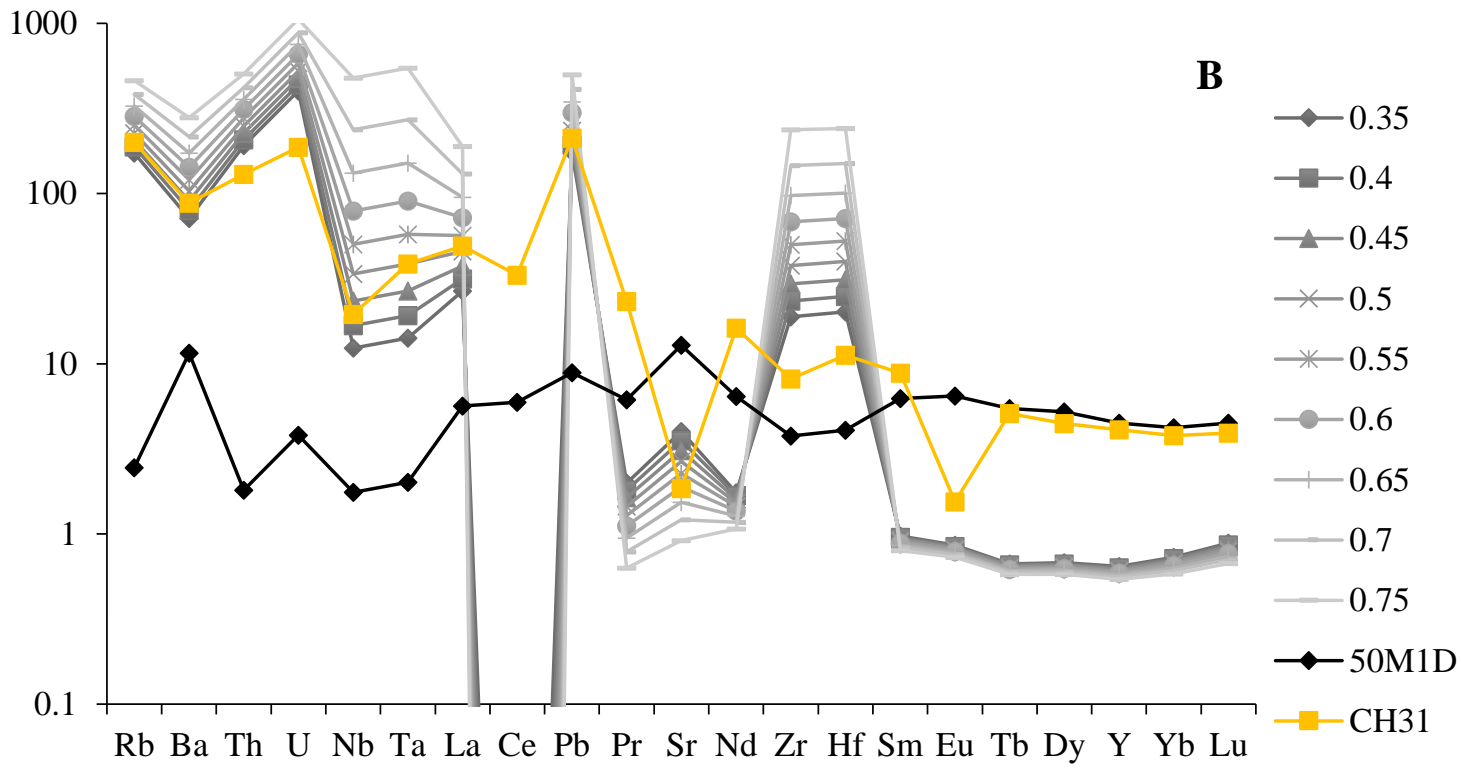

Fig. D21 Primitive mantle normalized (Sun and McDonough, 1989) spider diagram for the partial melting of experimental melt MS33. A shows the results of the batch melt model; B shows the results of the fractional melt model. The melt percentages are approximately $\pm 20 \%$ that observed in the experimental melt. The trace element concentrations of the parent material, sample 50M1D (50/50 mixture of mafic glass and amphibolite), are indicated by the black diamonds. The trace element concentrations of the Dale Tuff, sample $\mathrm{CH} 31$, which is included for comparison, are indicated by the yellow squares. For experimental melt MS33, it can be seen that the batch melt model (A) approximates the data. The fit increases as melt percent increases, particularly for LREE. 


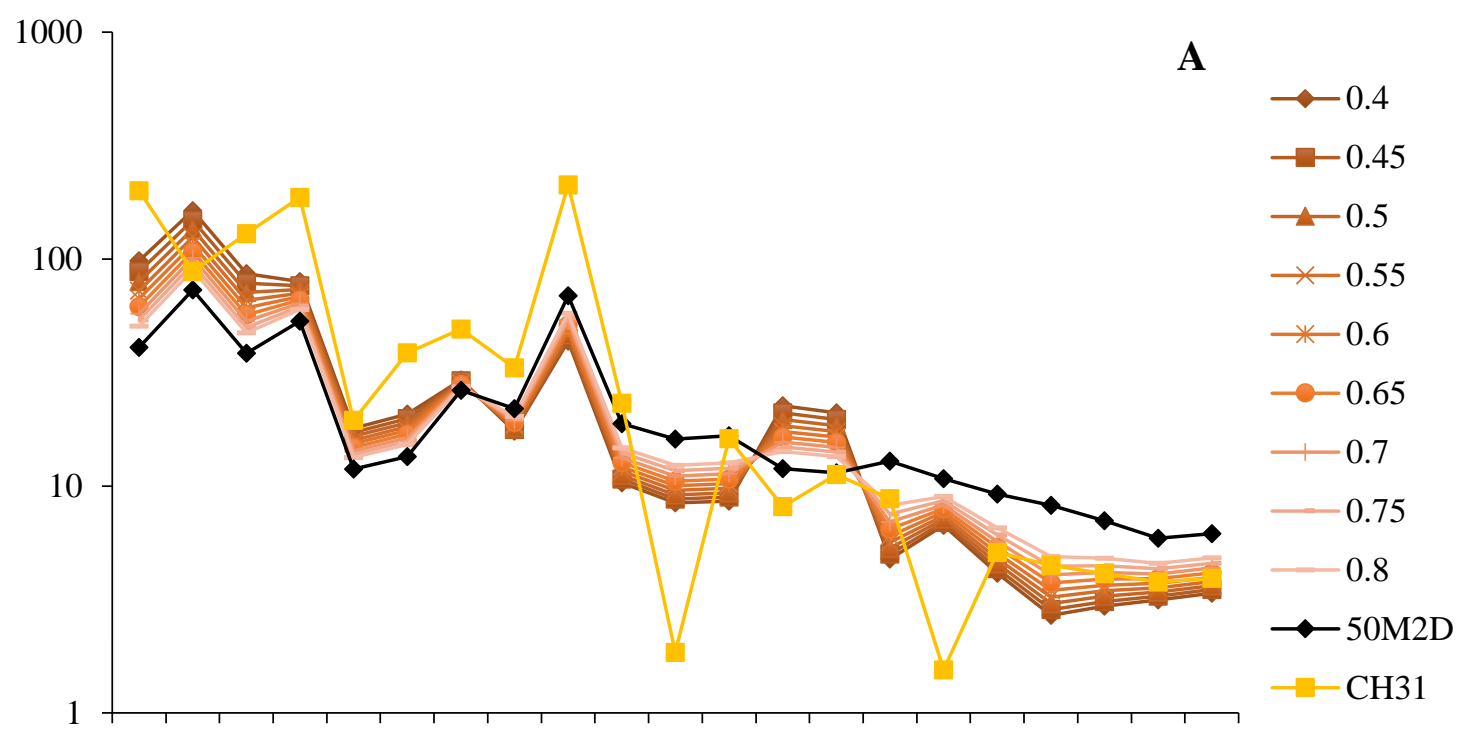

$\mathrm{Rb} \mathrm{Ba}$ Th $\mathrm{U}$ Nb Ta $\mathrm{La} \mathrm{Ce} \mathrm{Pb}$ Pr $\mathrm{Sr} \mathrm{Nd} \mathrm{Zr}$ Hf Sm Eu Tb Dy Y Yb Lu

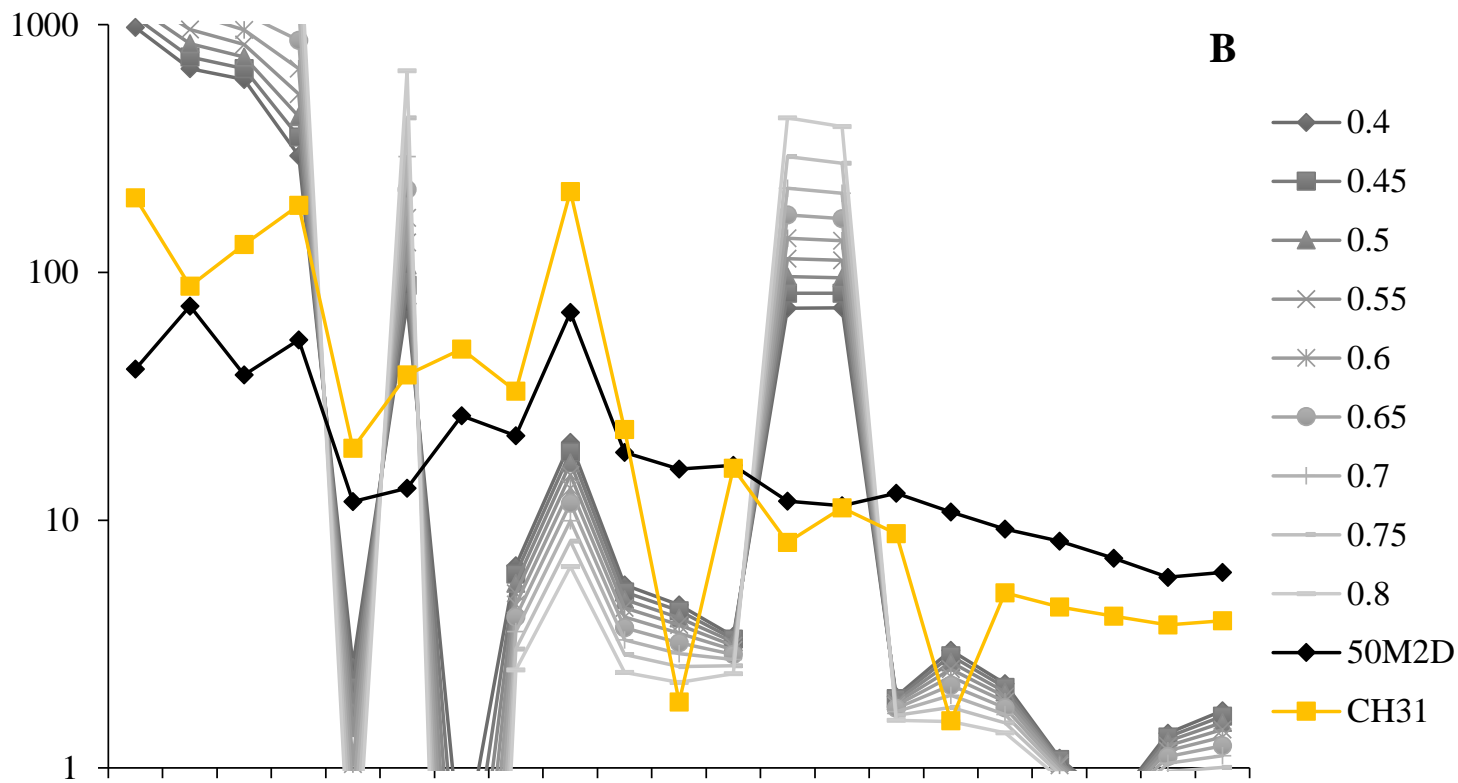

$\mathrm{Rb}$ Ba Th $\mathrm{U}$ Nb Ta La Ce Pb Pr Sr Nd Zr Hf Sm Eu Tb Dy Y Yb Lu

Fig. D22 Primitive mantle normalized (Sun and McDonough, 1989) spider diagram for the partial melting of experimental melt MS34. A shows the results of the batch melt model; B shows the results of the fractional melt model. The melt percentages are approximately $\pm 20 \%$ that observed in the experimental melt. The trace element concentrations of the parent material, sample 50M2D (50/50 mixture of mafic glass and amphibolite), are indicated by the black diamonds. The trace element concentrations of the Dale Tuff, sample $\mathrm{CH} 31$, which is included for comparison, are indicated by the yellow squares. For experimental melt MS34, it can be seen that the batch melt model (A) approximates the data. The fit increases as melt percent increases, particularly for LREE. 


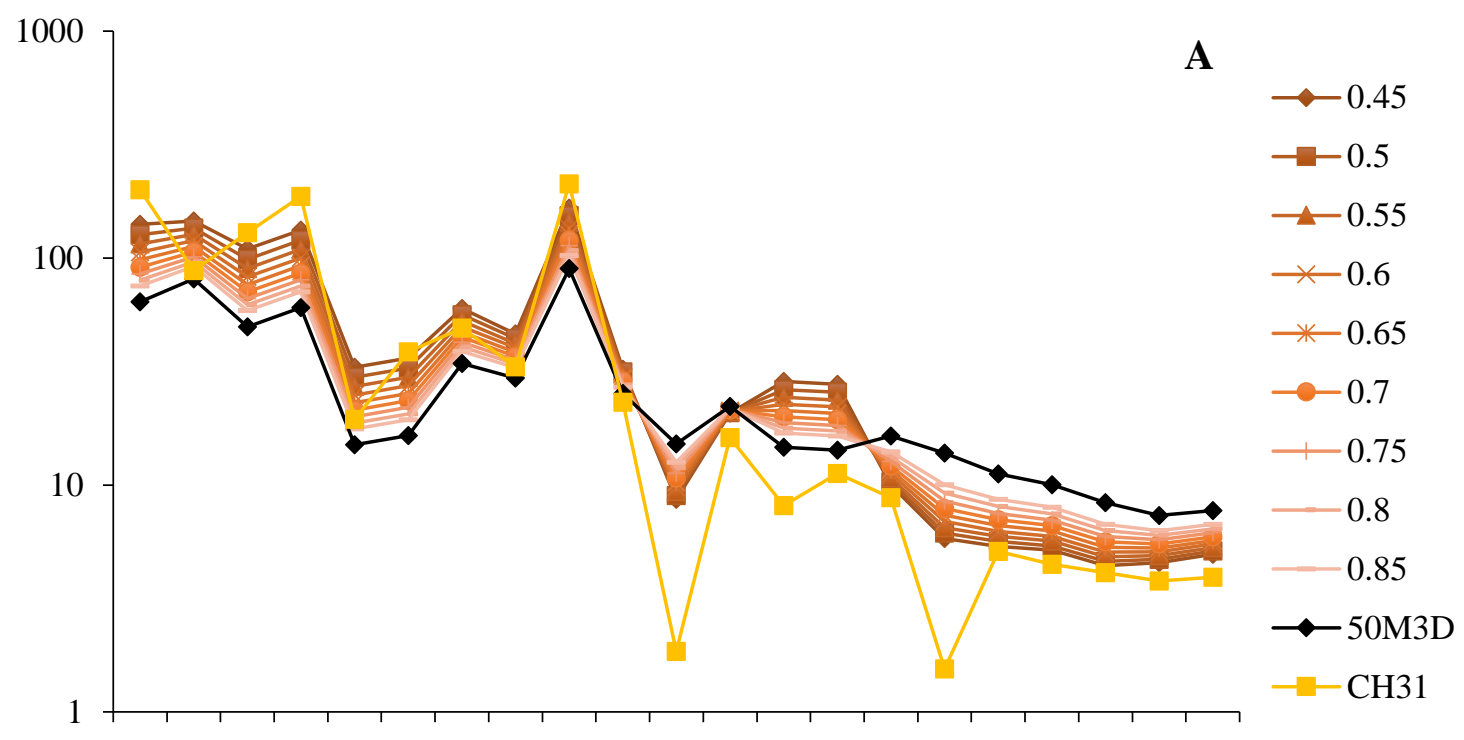

$\mathrm{Rb} \mathrm{Ba}$ Th $\mathrm{U}$ Nb Ta La Ce Pb Pr Sr Nd Zr Hf Sm Eu Tb Dy Y Yb Lu

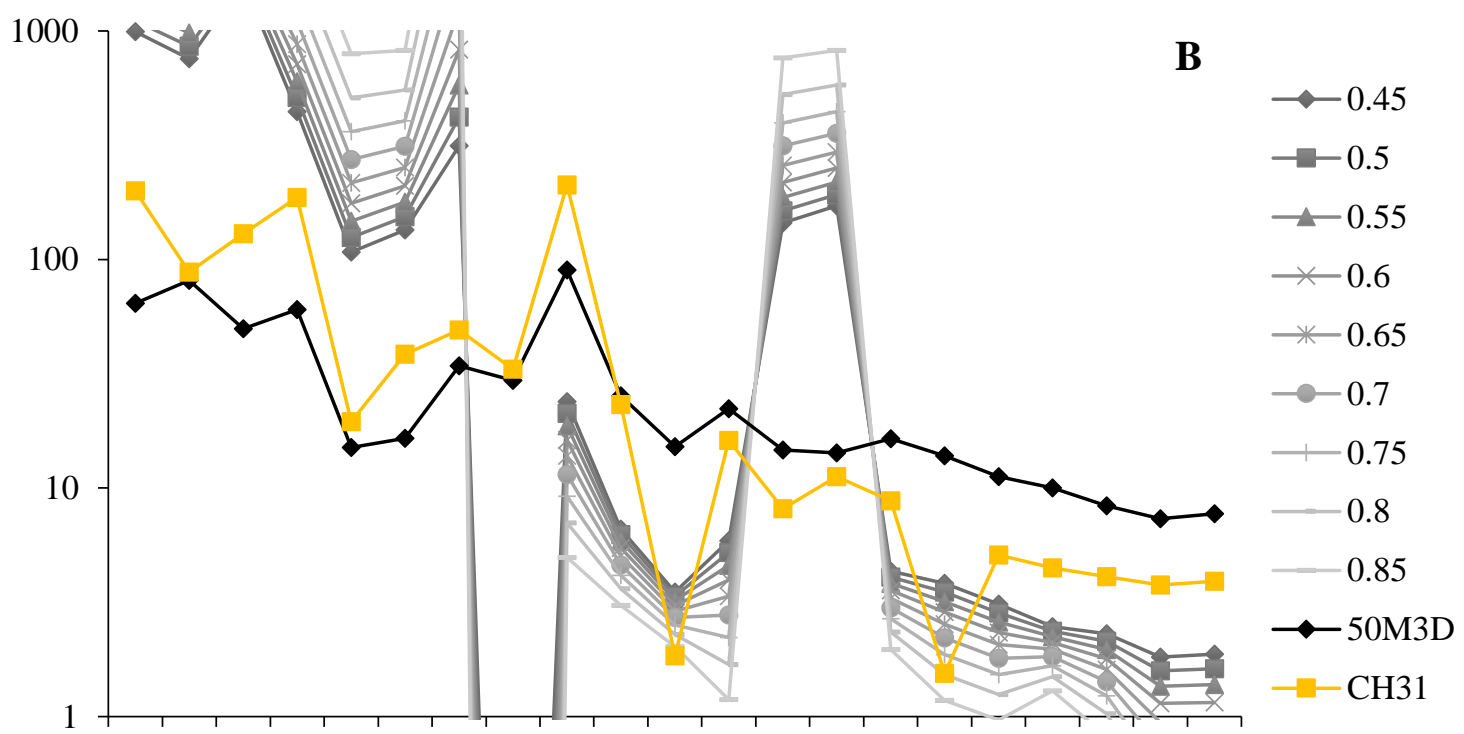

$\mathrm{Rb} \mathrm{Ba}$ Th $\mathrm{U}$ Nb Ta La Ce Pb Pr Sr Nd Zr Hf Sm Eu Tb Dy Y Yb Lu

Fig. D23 Primitive mantle normalized (Sun and McDonough, 1989) spider diagram for the partial melting of experimental melt MS35. A shows the results of the batch melt model; B shows the results of the fractional melt model. The melt percentages are approximately $\pm 20 \%$ that observed in the experimental melt. The trace element concentrations of the parent material, sample 50M3D (50/50 mixture of mafic glass and amphibolite), are indicated by the black diamonds. The trace element concentrations of the Dale Tuff, sample $\mathrm{CH} 31$, which is included for comparison, are indicated by the yellow squares. For experimental melt MS35, it can be seen that the batch melt model (A) approximates the data. The fit increases as melt percent increases, particularly for LREE. 


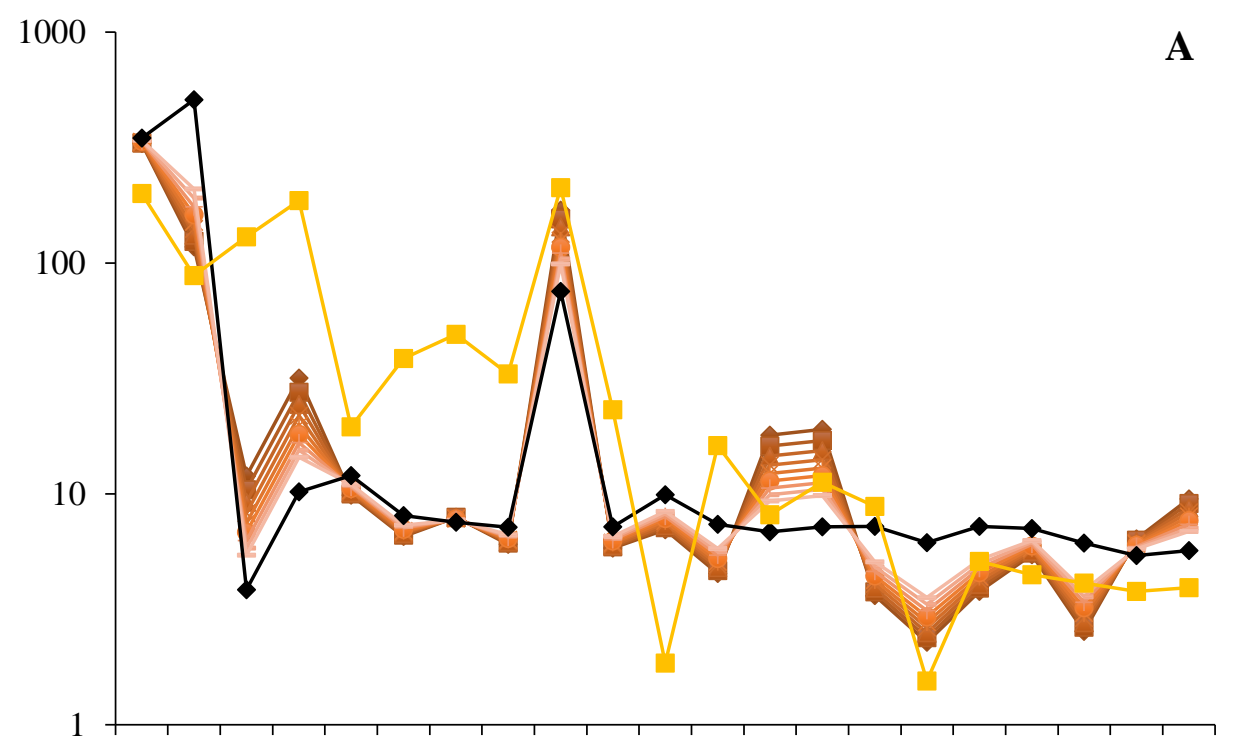

A

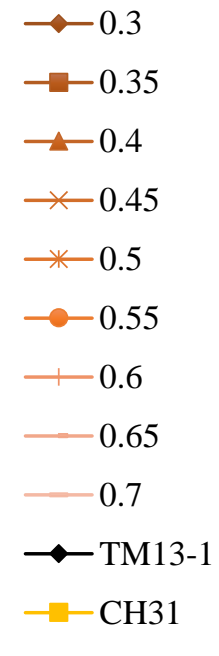

$\mathrm{Rb} \mathrm{Ba}$ Th U Nb Ta La Ce Pb Pr Sr Nd Zr Hf Sm Eu Tb Dy Y Yb Lu

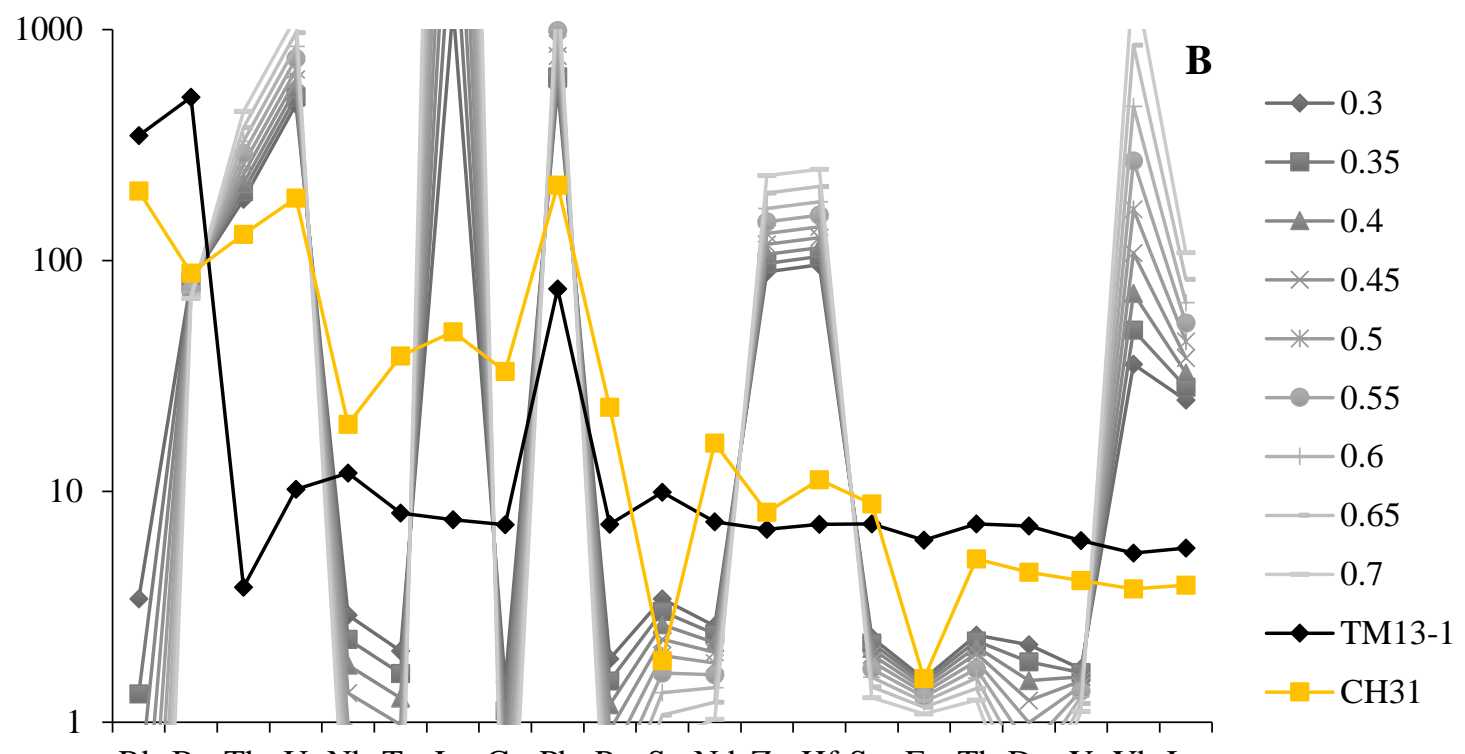

$\mathrm{Rb} \mathrm{Ba}$ Th $\mathrm{U}$ Nb Ta $\mathrm{La} \mathrm{Ce} \mathrm{Pb}$ Pr Sr Nd Zr Hf Sm Eu Tb Dy Y Yb Lu

Fig. D24 Primitive mantle normalized (Sun and McDonough, 1989) spider diagram for the partial melting of experimental melt MS37. A shows the results of the batch melt model; B shows the results of the fractional melt model. The melt percentages are approximately $\pm 20 \%$ that observed in the experimental melt. The trace element concentrations of the parent material, sample TM13-1 (biotite schist) are indicated by the black diamonds. The trace element concentrations of the Dale Tuff, sample CH31, which is included for comparison, are indicated by the yellow squares. For experimental melt MS37, it can be seen that the batch melt model (A) approximates the data with a notable exception at $\mathrm{Ba}$. The fit increases as melt percent increases, particularly for LREE. 


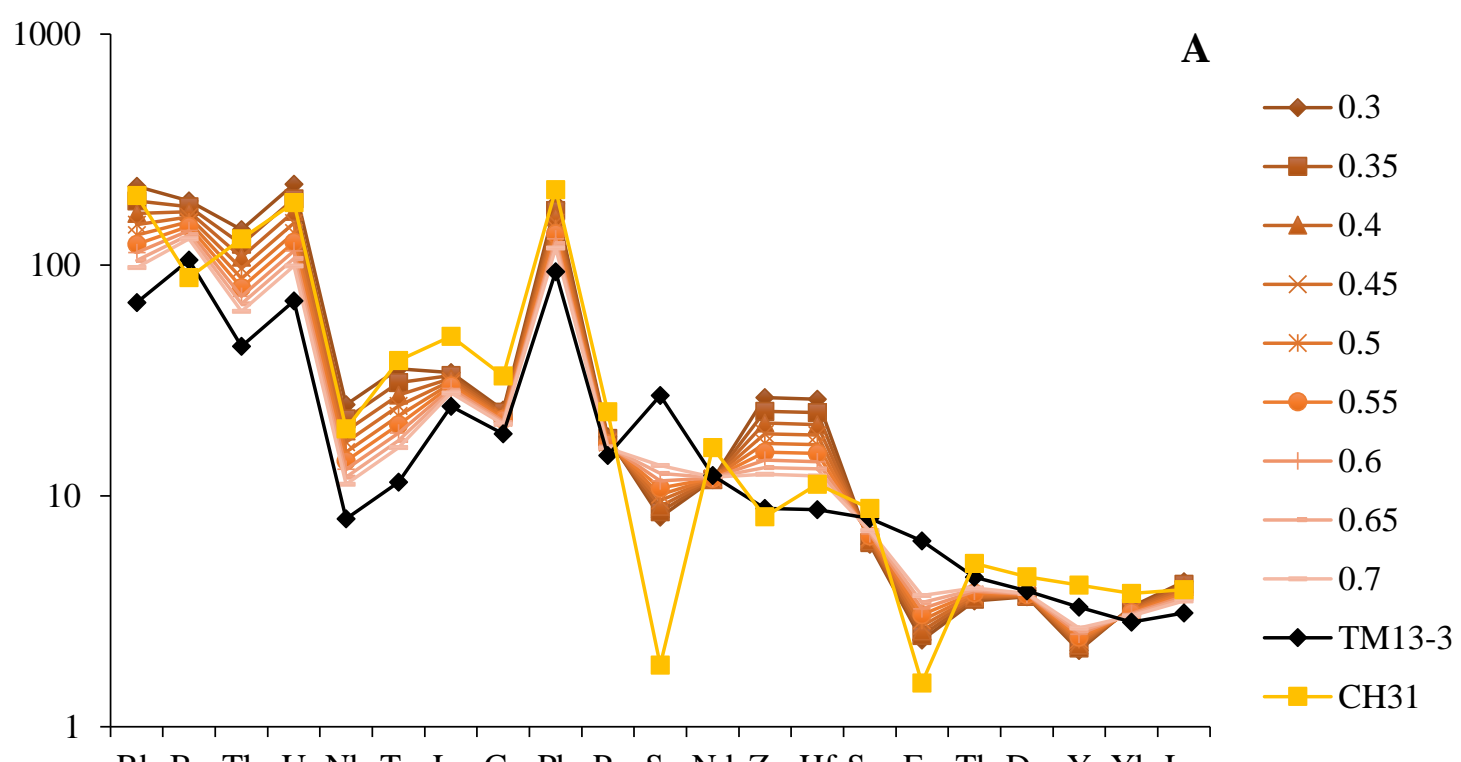

$\mathrm{Rb} \mathrm{Ba}$ Th U Nb Ta La Ce Pb Pr Sr Nd Zr Hf Sm Eu Tb Dy Y Yb Lu

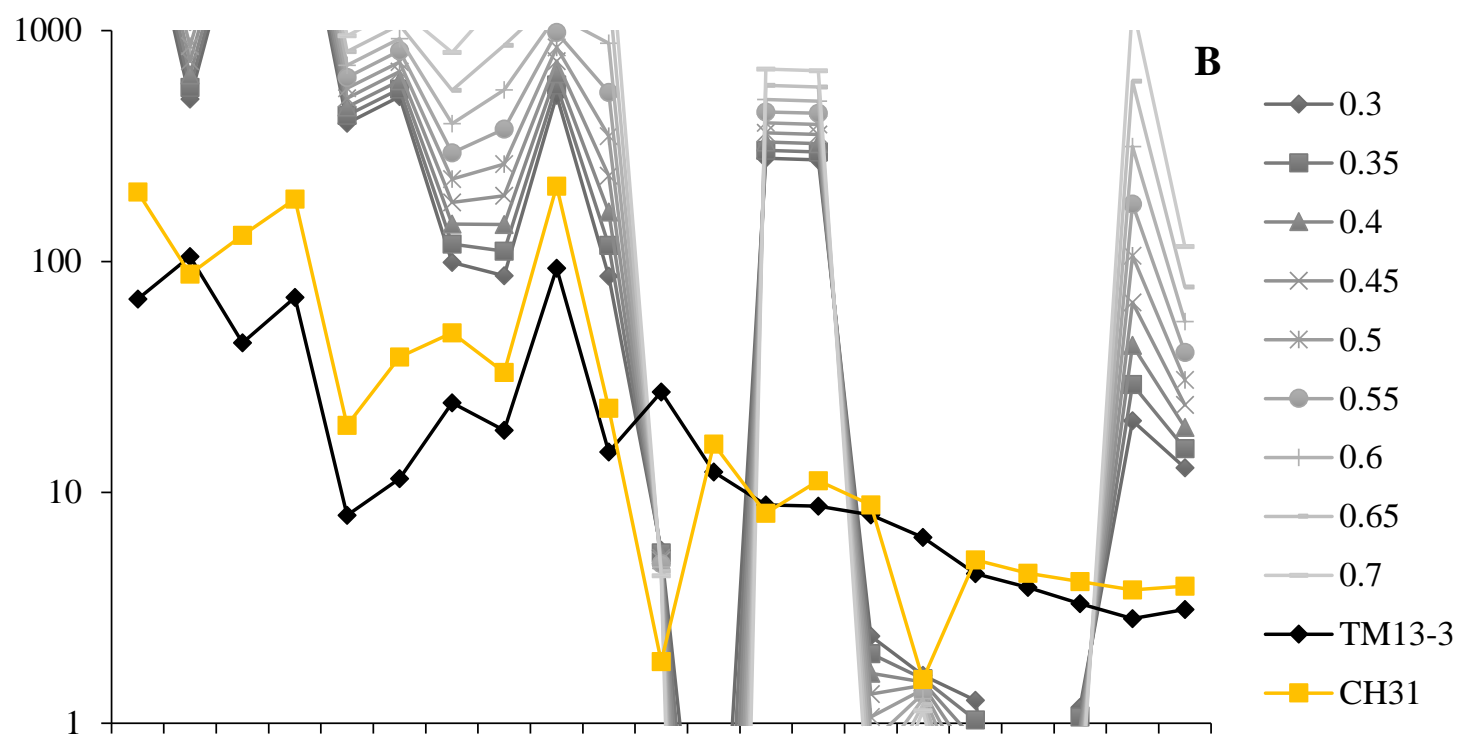

$\mathrm{Rb} \mathrm{Ba}$ Th $\mathrm{U}$ Nb Ta La Ce Pb Pr Sr Nd Zr Hf Sm Eu Tb Dy Y Yb Lu

Fig. D25 Primitive mantle normalized (Sun and McDonough, 1989) spider diagram for the partial melting of experimental melt MS38. A shows the results of the batch melt model; B shows the results of the fractional melt model. The melt percentages are approximately $\pm 20 \%$ that observed in the experimental melt. The trace element concentrations of the parent material, sample TM13-3 (granodiorite), are indicated by the black diamonds. The trace element concentrations of the Dale Tuff, sample CH38, which is included for comparison, are indicated by the yellow squares. For experimental melt MS38, it can be seen that the batch melt model (A) approximates the data with notable exceptions at $\mathrm{Sr}$ and Eu. The fit increases as melt percent increases. 


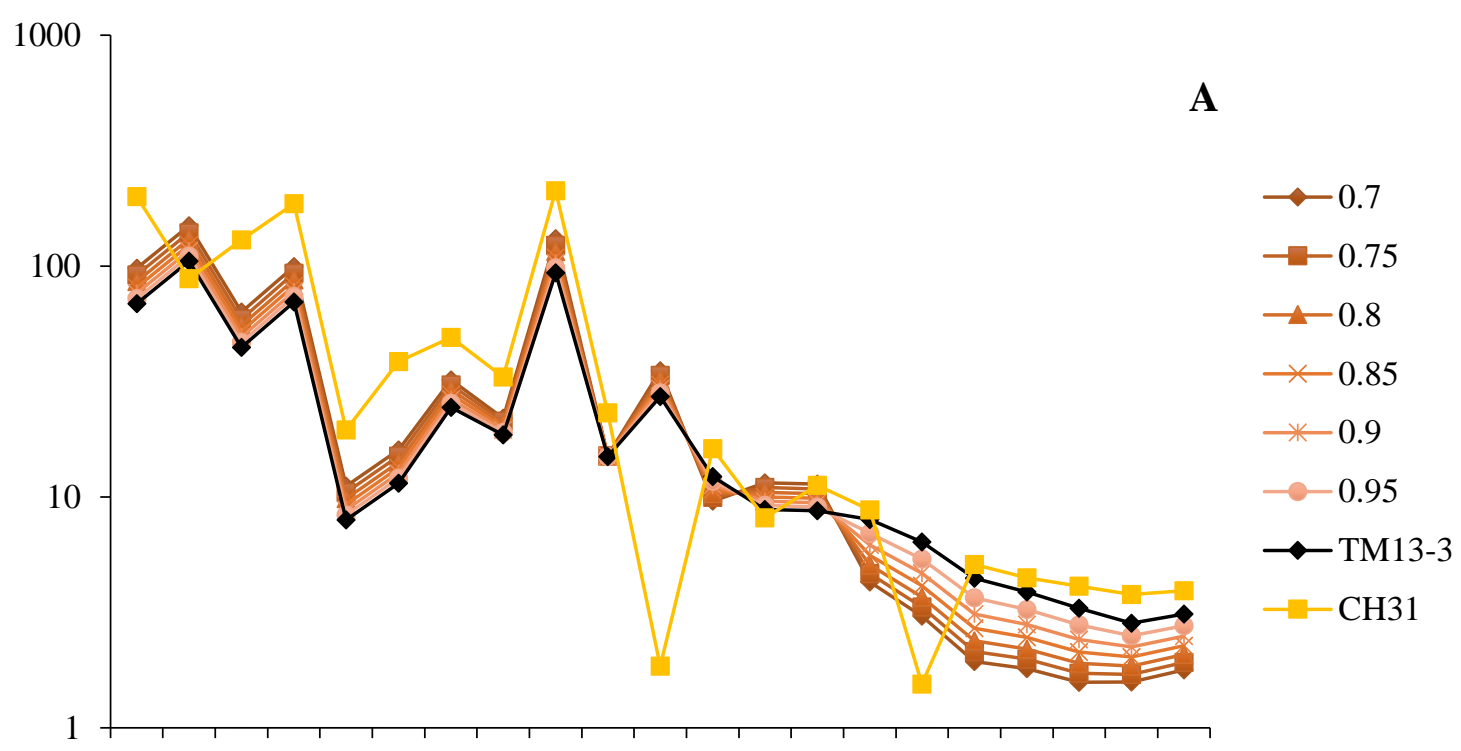

$\mathrm{Rb} \mathrm{Ba}$ Th U Nb Ta La Ce Pb Pr Sr Nd Zr Hf Sm Eu Tb Dy Y Yb Lu

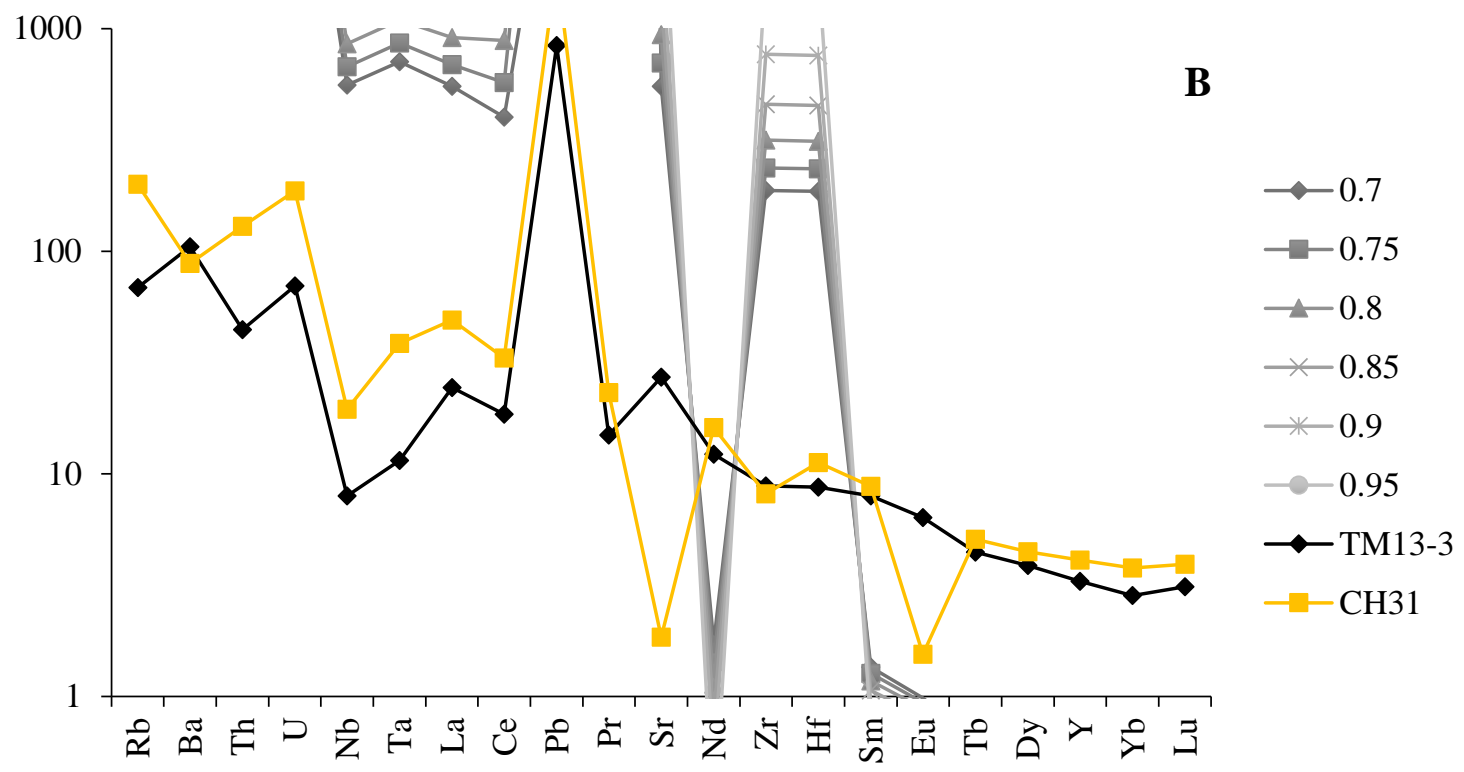

Fig. D26 Primitive mantle normalized (Sun and McDonough, 1989) spider diagram for the partial melting of experimental melt MS39. A shows the results of the batch melt model; B shows the results of the fractional melt model. The melt percentages are approximately $\pm 20 \%$ that observed in the experimental melt. The trace element concentrations of the parent material, sample TM13-3 (granodiorite), are indicated by the black diamonds. The trace element concentrations of the Dale Tuff, sample CH31, which is included for comparison, are indicated by the yellow squares. For experimental melt MS39, it can be seen that the batch melt model (A) approximates the data. The fit increases as melt percent increases. 

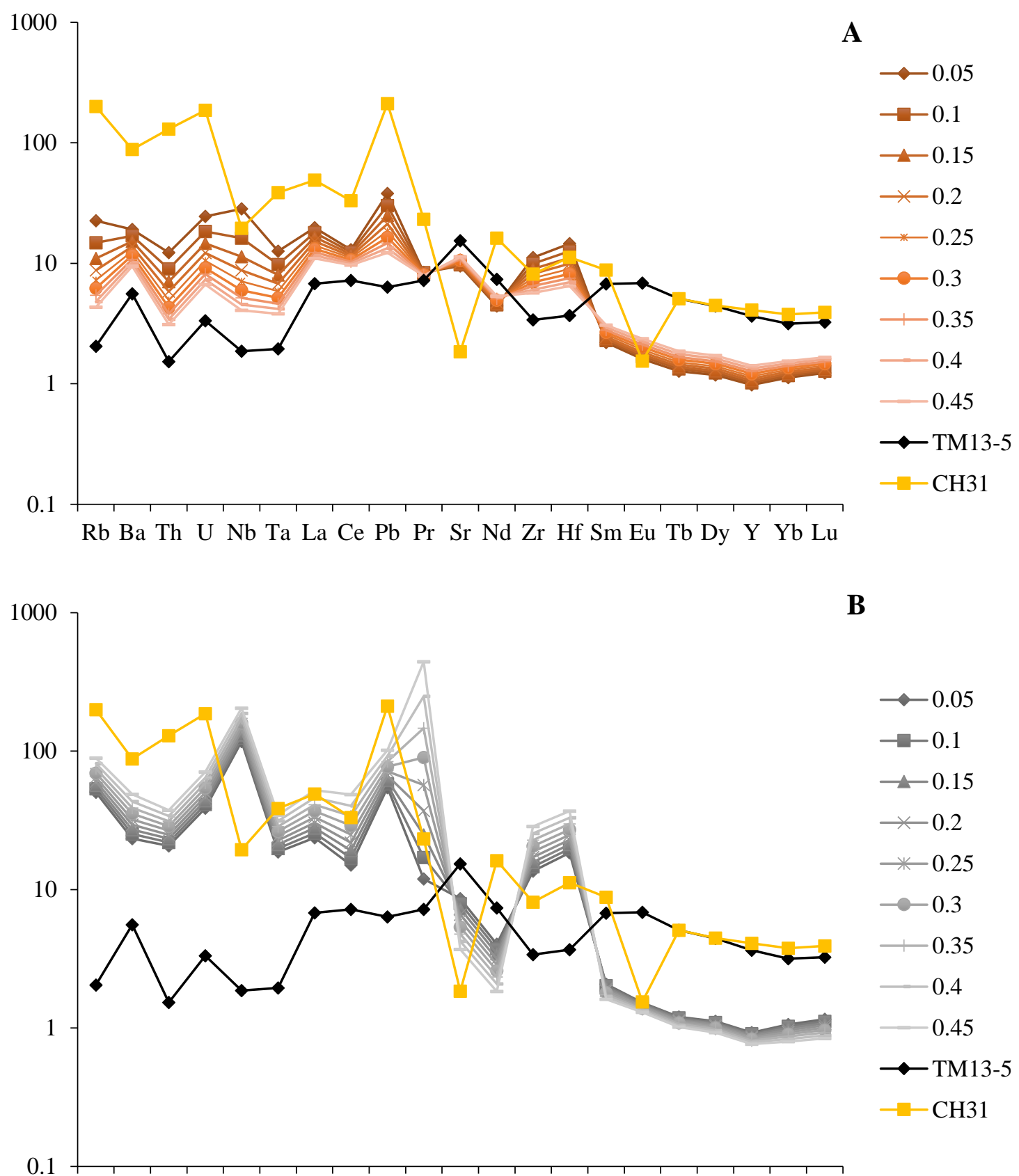

$\mathrm{Rb} \mathrm{Ba}$ Th U Nb Ta La Ce Pb Pr Sr Nd Zr Hf Sm Eu Tb Dy Y Yb Lu

Fig. D27 Primitive mantle normalized (Sun and McDonough, 1989) spider diagram for the partial melting of experimental melt MS40. A shows the results of the batch melt model; B shows the results of the fractional melt model. The melt percentages are approximately $\pm 20 \%$ that observed in the experimental melt. The trace element concentrations of the parent material, sample TM13-5 (amphibolite), are indicated by the black diamonds. The trace element concentrations of the Dale Tuff, sample $\mathrm{CH} 31$, which is included for comparison, are indicated by the yellow squares. For experimental melt MS40, it can be seen that the batch melt model (A) approximates the data with notable exceptions at $\mathrm{Pb}$ and HREE. In general, the fit increases as melt percent increases. 


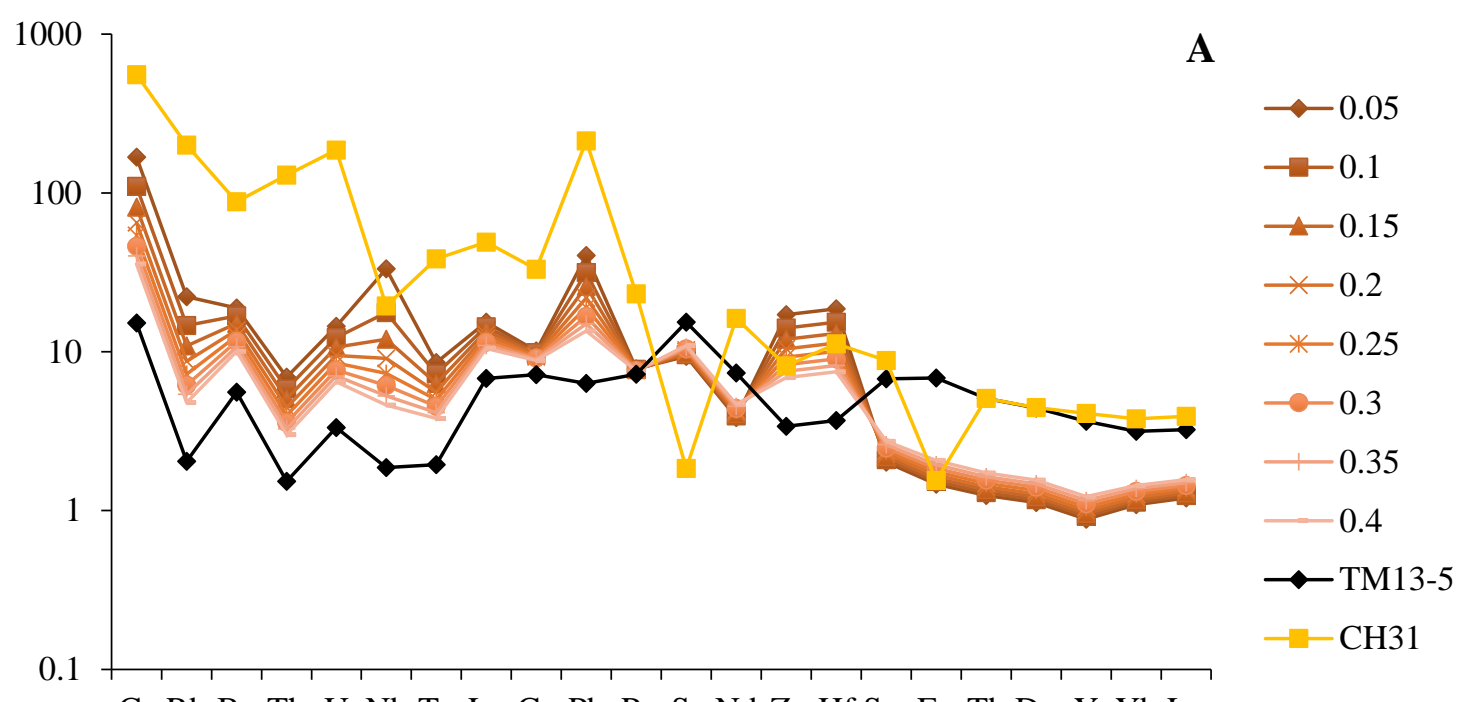

Cs Rb Ba Th U Nb Ta La Ce Pb Pr Sr Nd Zr Hf Sm Eu Tb Dy Y Yb Lu

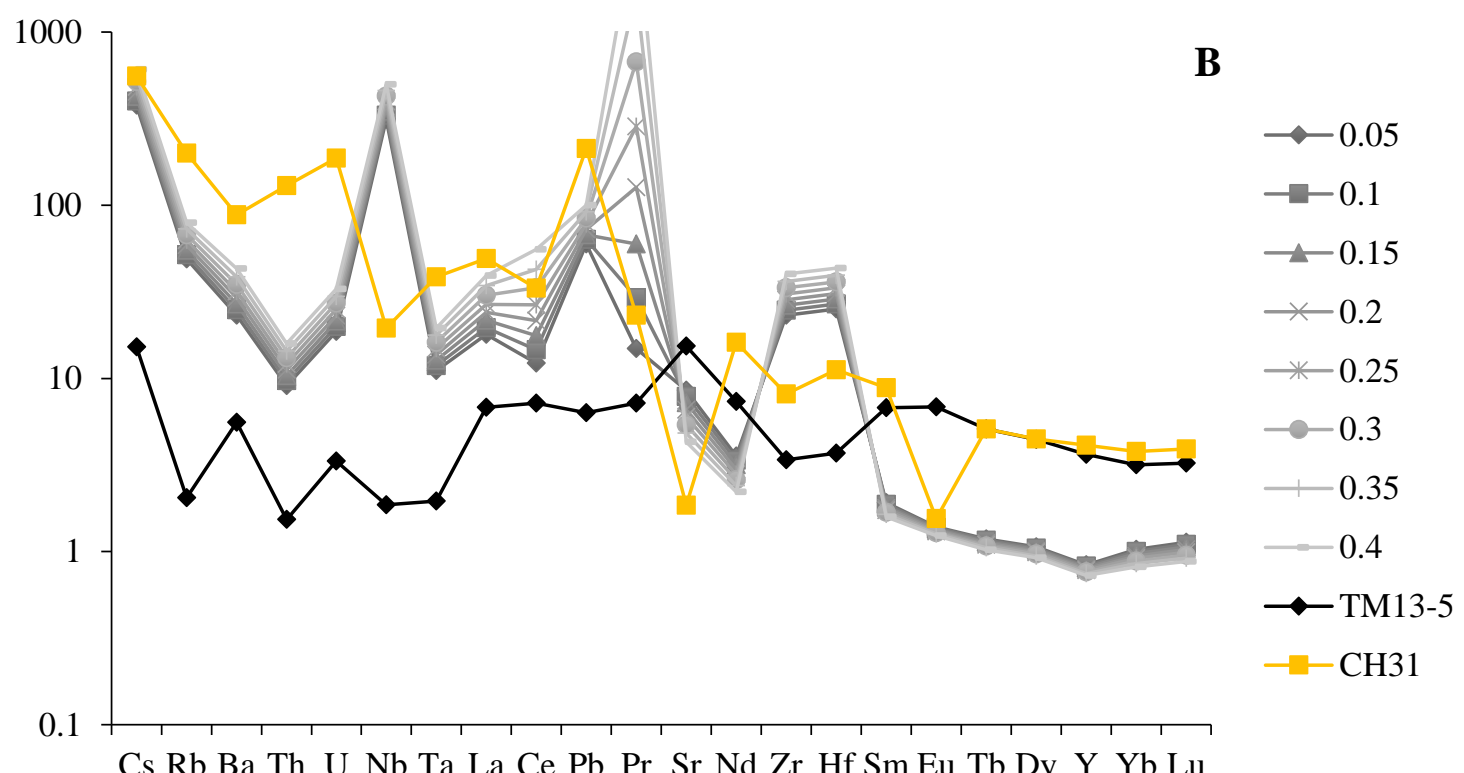

Fig. D28 Primitive mantle normalized (Sun and McDonough, 1989) spider diagram for the partial melting of experimental melt MS41. A shows the results of the batch melt model; B shows the results of the fractional melt model. The melt percentages are approximately $\pm 20 \%$ that observed in the experimental melt. The trace element concentrations of the parent material, sample TM13-5 (amphibolite), are indicated by the black diamonds. The trace element concentrations of the Dale Tuff, sample CH31, which is included for comparison, are indicated by the yellow squares. For experimental melt MS41, it can be seen that the batch melt model (A) approximates the data with notable exceptions at $\mathrm{Pb}, \mathrm{Zr}, \mathrm{Hf}$, and HREE. In general, the fit increases as melt percent increases. 


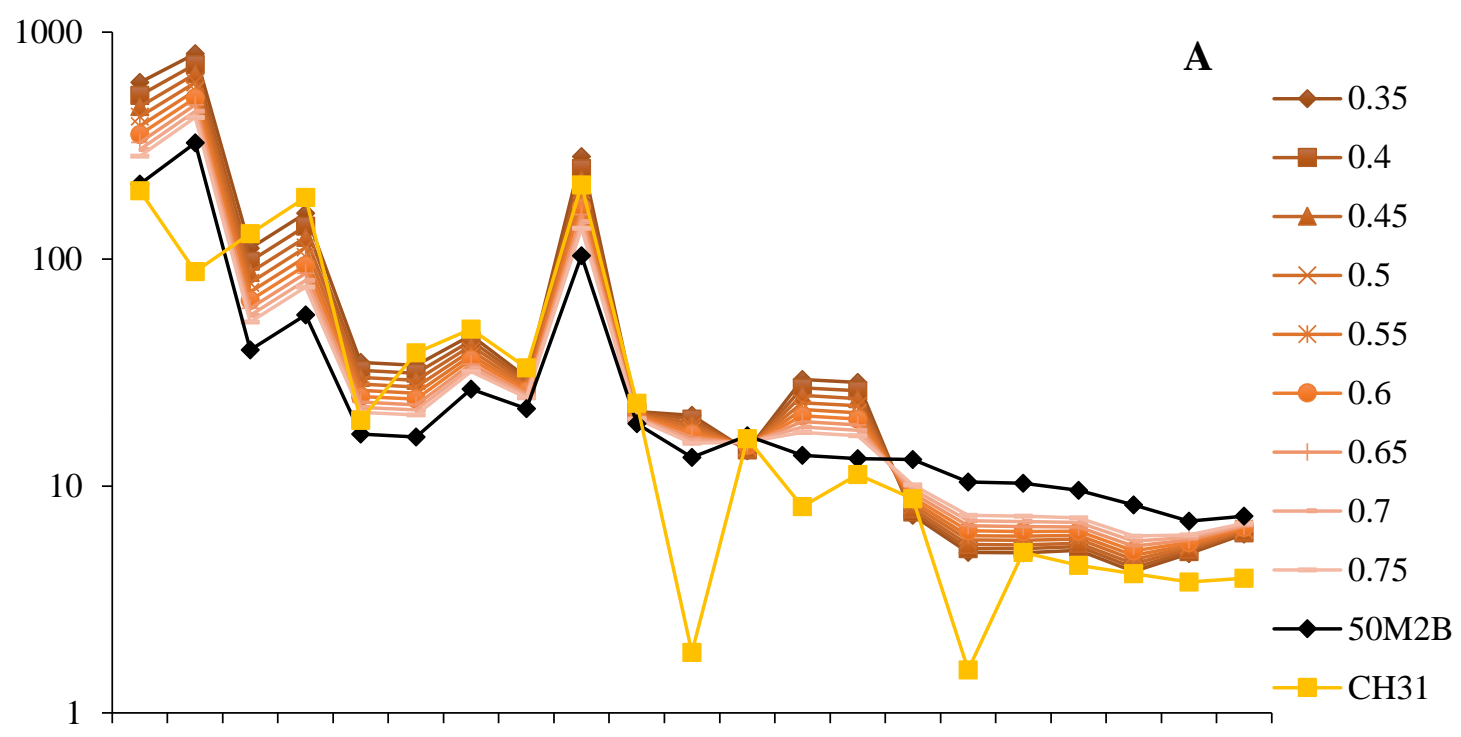

$\mathrm{Rb} \mathrm{Ba}$ Th $\mathrm{U}$ Nb Ta La Ce Pb Pr Sr Nd Zr Hf Sm Eu Tb Dy Y Yb Lu

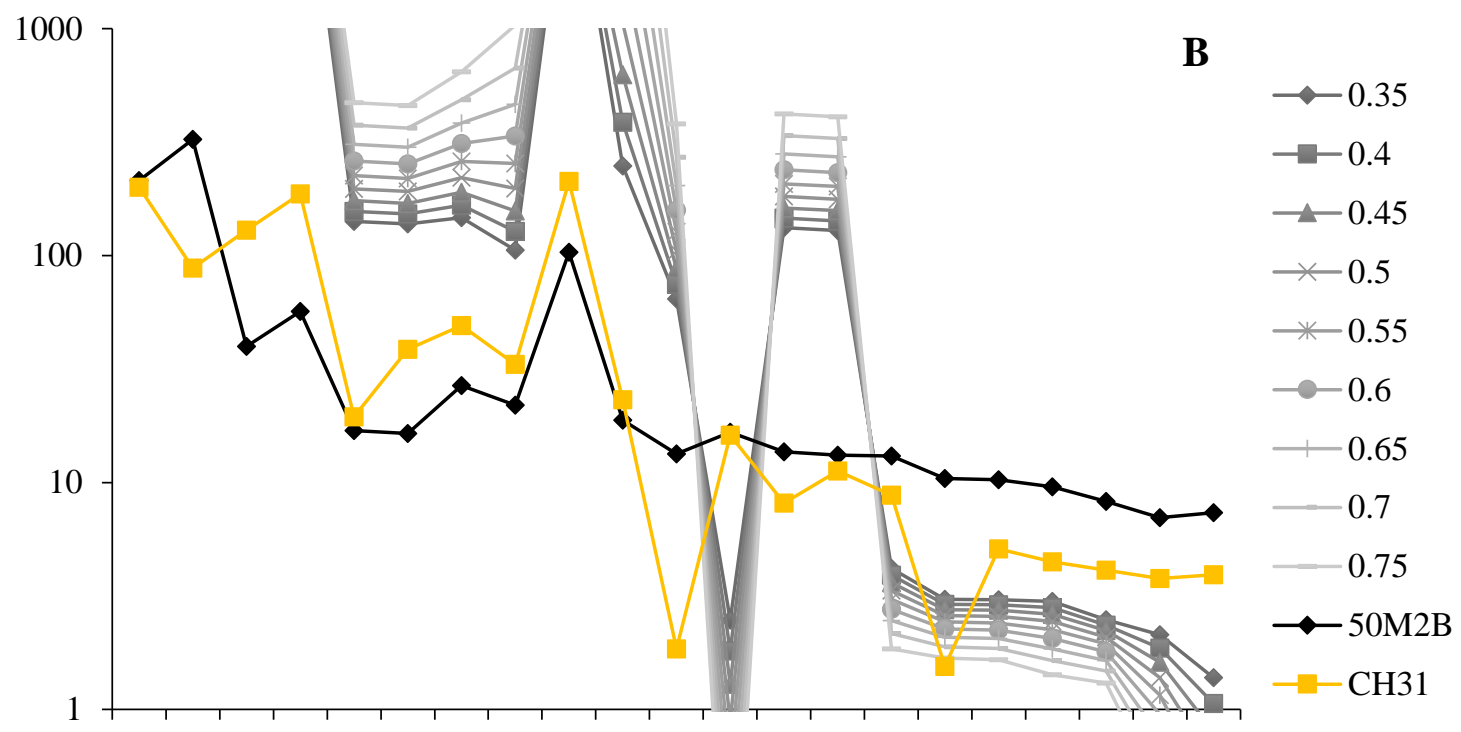

$\mathrm{Rb} \mathrm{Ba}$ Th $\mathrm{U}$ Nb Ta $\mathrm{La} \mathrm{Ce} \mathrm{Pb}$ Pr $\mathrm{Sr} \mathrm{Nd} \mathrm{Zr}$ Hf Sm Eu Tb Dy Y Yb Lu

Fig. D29 Primitive mantle normalized (Sun and McDonough, 1989) spider diagram for the partial melting of experimental melt MS43. A shows the results of the batch melt model; B shows the results of the fractional melt model. The melt percentages are approximately $\pm 20 \%$ that observed in the experimental melt. The trace element concentrations of the parent material, sample 50M2B (50/50 mixture of mafic glass and biotite schist), are indicated by the black diamonds. The trace element concentrations of the Dale Tuff, sample $\mathrm{CH} 31$, which is included for comparison, are indicated by the yellow squares. For experimental melt MS43, it can be seen that the batch melt model (A) approximates the data. Fit increases as melt percent increases. 


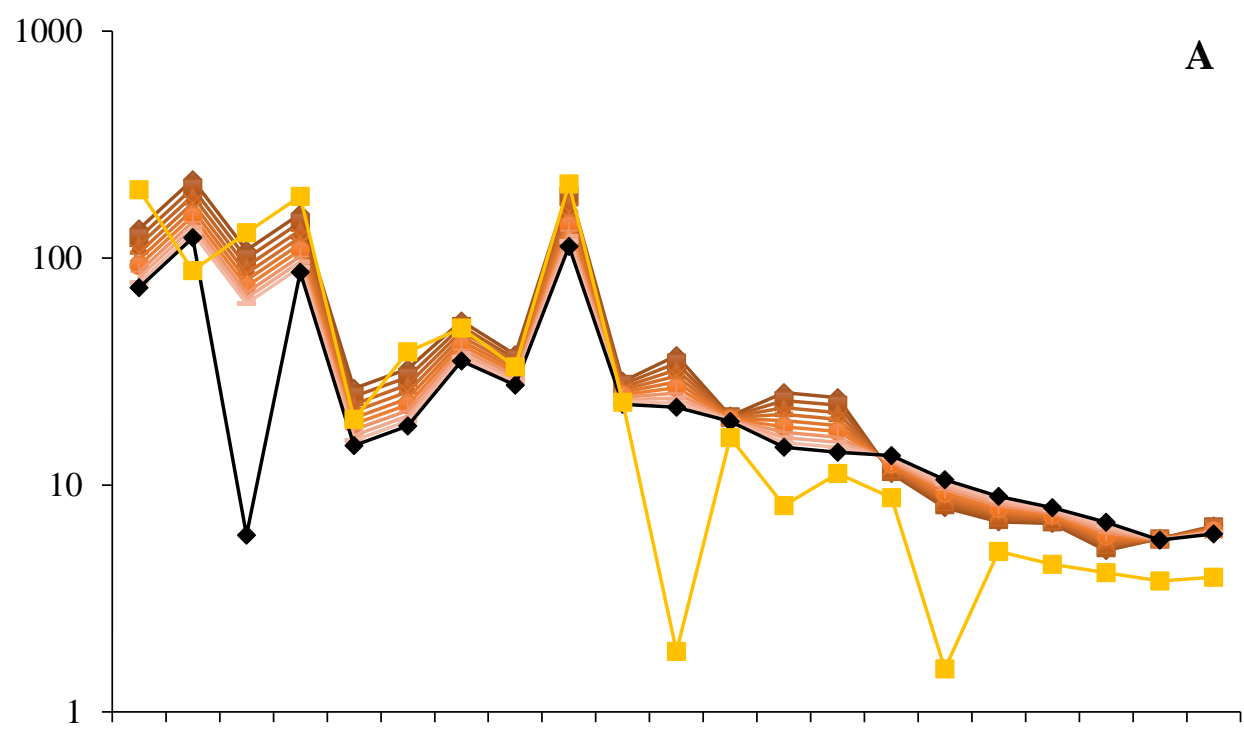

$\mathbf{A}$

$\longrightarrow 0.55$

$-0.6$

$-0.65$

$\times 0.7$

* -0.75

$-0.8$

$-0.85$

0.9

$-0.95$

$\rightarrow 50 \mathrm{M} 2 \mathrm{C}$

$-\mathrm{CH} 31$

$\mathrm{Rb} \mathrm{Ba}$ Th $\mathrm{U}$ Nb Ta $\mathrm{La} \mathrm{Ce} \mathrm{Pb}$ Pr $\mathrm{Sr} \mathrm{Nd} \mathrm{Zr}$ Hf Sm Eu Tb Dy Y Yb Lu

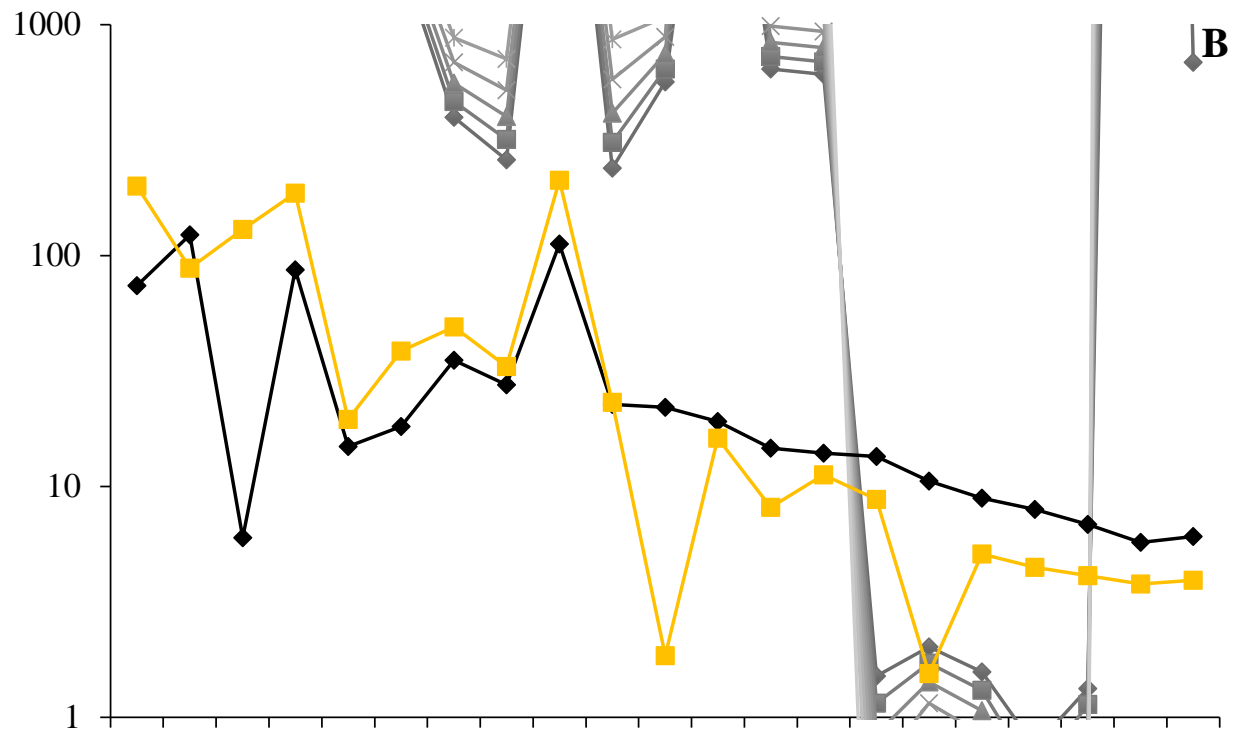

B

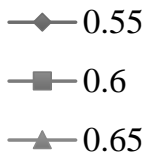

$\leftarrow 0.7$

* 0.75

$-0.8$

$-0.85$

$-0.9$

$-0.95$

$\rightarrow 50 \mathrm{M} 2 \mathrm{C}$

$-\mathrm{CH} 31$

$\mathrm{Rb} \mathrm{Ba}$ Th U Nb Ta La Ce Pb Pr Sr Nd Zr Hf Sm Eu Tb Dy Y Yb Lu

Fig. D30 Primitive mantle normalized (Sun and McDonough, 1989) spider diagram for the partial melting of experimental melt MS44. A shows the results of the batch melt model; B shows the results of the fractional melt model. The melt percentages are approximately $\pm 20 \%$ that observed in the experimental melt. The trace element concentrations of the parent material, sample 50M2C (50/50 mixture of mafic glass and diorite), are indicated by the black diamonds. The trace element concentrations of the Dale Tuff, sample $\mathrm{CH} 31$, which is included for comparison, are indicated by the yellow squares. For experimental melt MS44, it can be seen that the batch melt model (A) approximates the data with a notable exception at Th. In general, the fit increases as melt percent increases. 


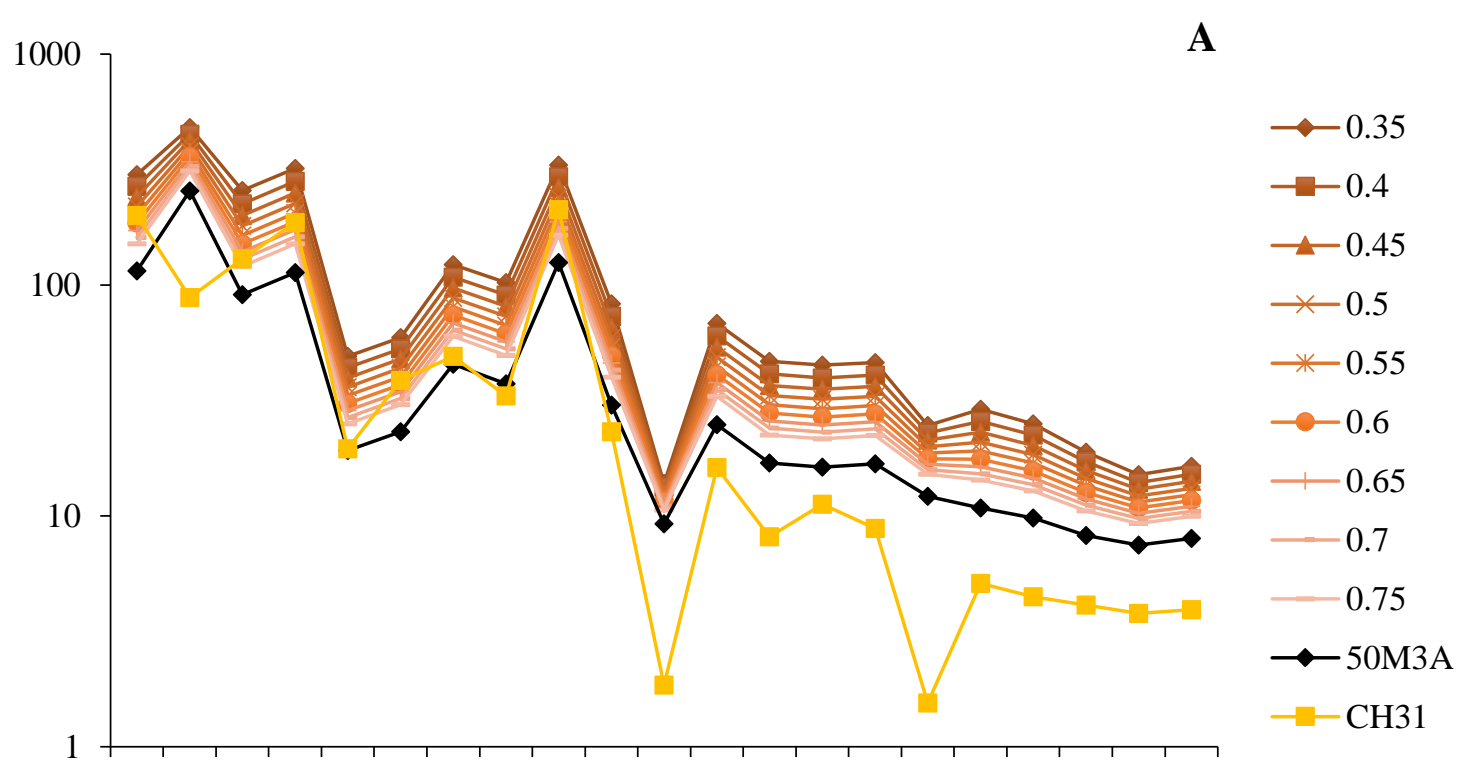

$\mathrm{Rb}$ Ba Th U Nb Ta La Ce Pb Pr Sr Nd Zr Hf Sm Eu Tb Dy Y Yb Lu

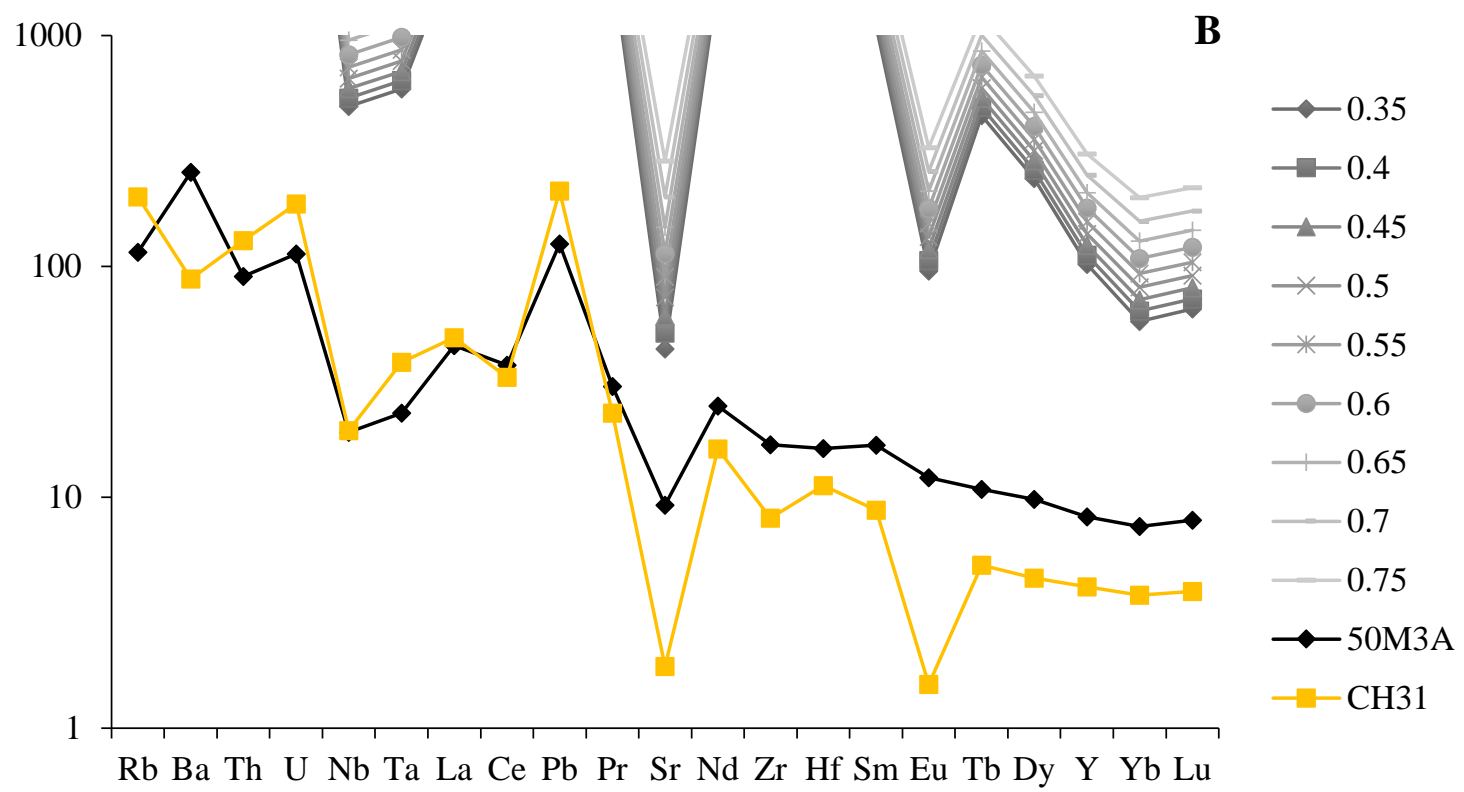

Fig. D31 Primitive mantle normalized (Sun and McDonough, 1989) spider diagram for the partial melting of experimental melt MS45. A shows the results of the batch melt model; B shows the results of the fractional melt model. The melt percentages are approximately $\pm 20 \%$ that observed in the experimental melt. The trace element concentrations of the parent material, sample 50M3A (50/50 mixture of mafic glass and biotite fels), are indicated by the black diamonds. The trace element concentrations of the Dale Tuff, sample $\mathrm{CH} 31$, which is included for comparison, are indicated by the yellow squares. For experimental melt MS45, it can be seen that the batch melt model (A) approximates the data. The fit increases as melt percent increases. 


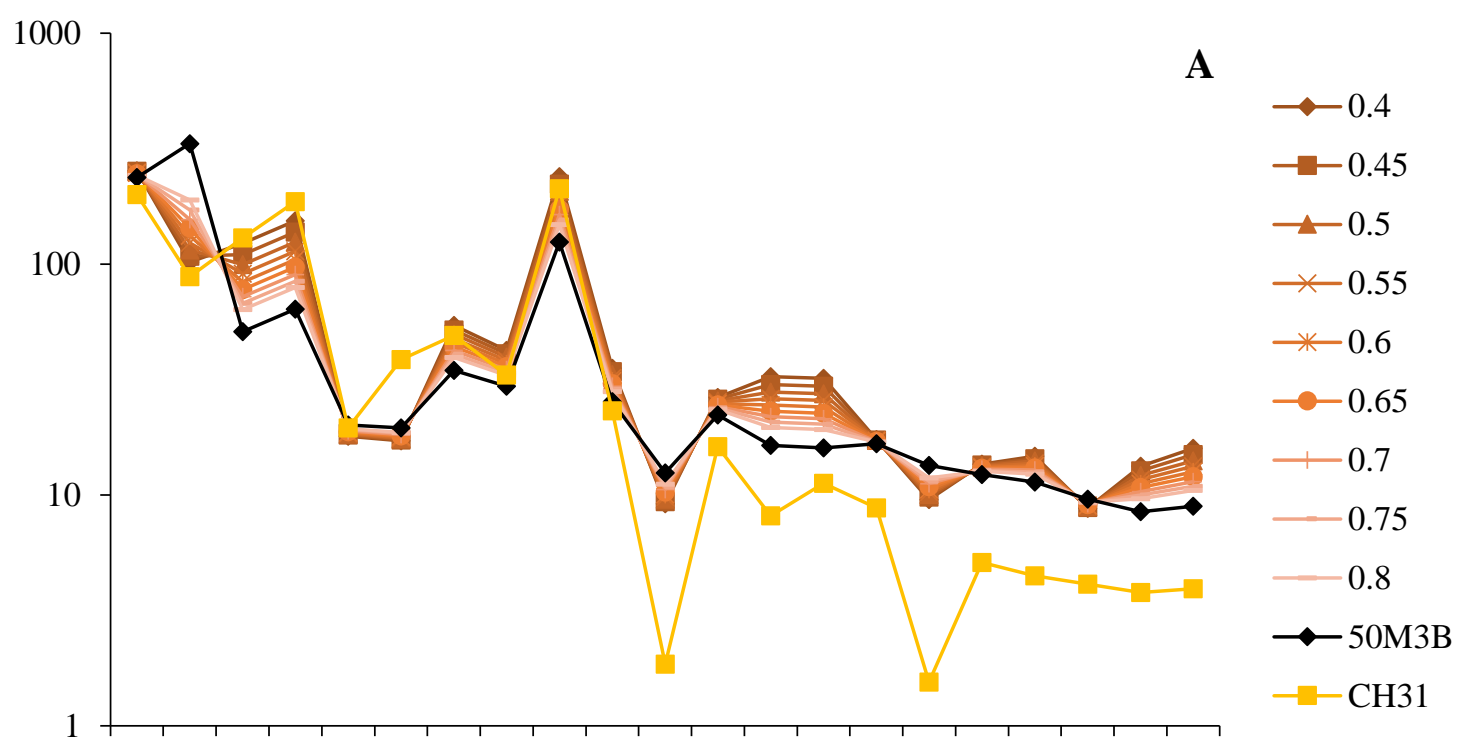

$\mathrm{Rb} \mathrm{Ba}$ Th U Nb Ta La Ce Pb Pr Sr Nd Zr Hf Sm Eu Tb Dy Y Yb Lu

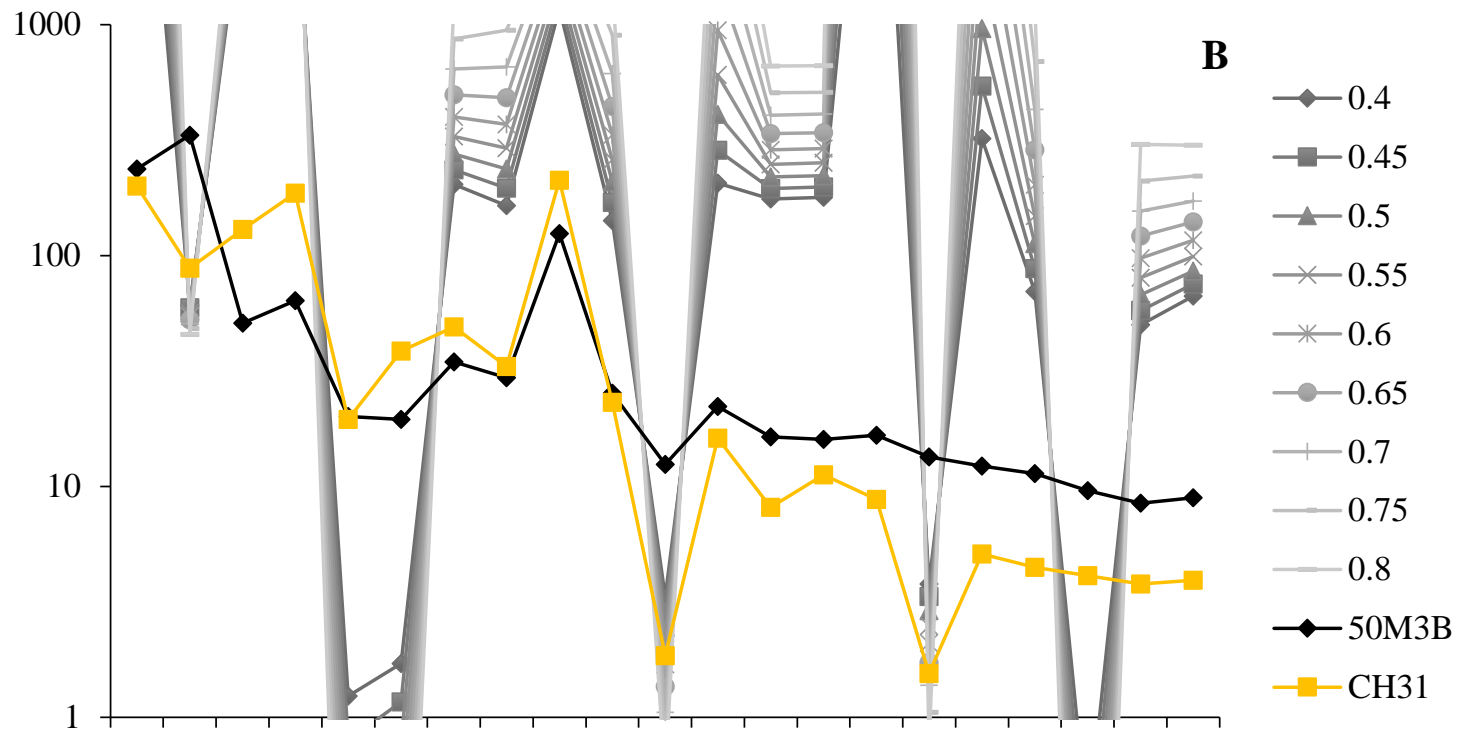

$\mathrm{Rb} \mathrm{Ba}$ Th U Nb Ta La Ce Pb Pr Sr Nd Zr Hf Sm Eu Tb Dy Y Yb Lu

Fig. D32 Primitive mantle normalized (Sun and McDonough, 1989) spider diagram for the partial melting of experimental melt MS46. A shows the results of the batch melt model; B shows the results of the fractional melt model. The melt percentages are approximately $\pm 20 \%$ that observed in the experimental melt. The trace element concentrations of the parent material, sample 50M3B (50/50 mixture of mafic glass and biotite schist), are indicated by the black diamonds. The trace element concentrations of the Dale Tuff, sample $\mathrm{CH} 31$, which is included for comparison, are indicated by the yellow squares. For experimental melt MS46, it can be seen that the batch melt model (A) approximates the data with a notable exception at $\mathrm{Ba}$. The fit increases as melt percent increases. 


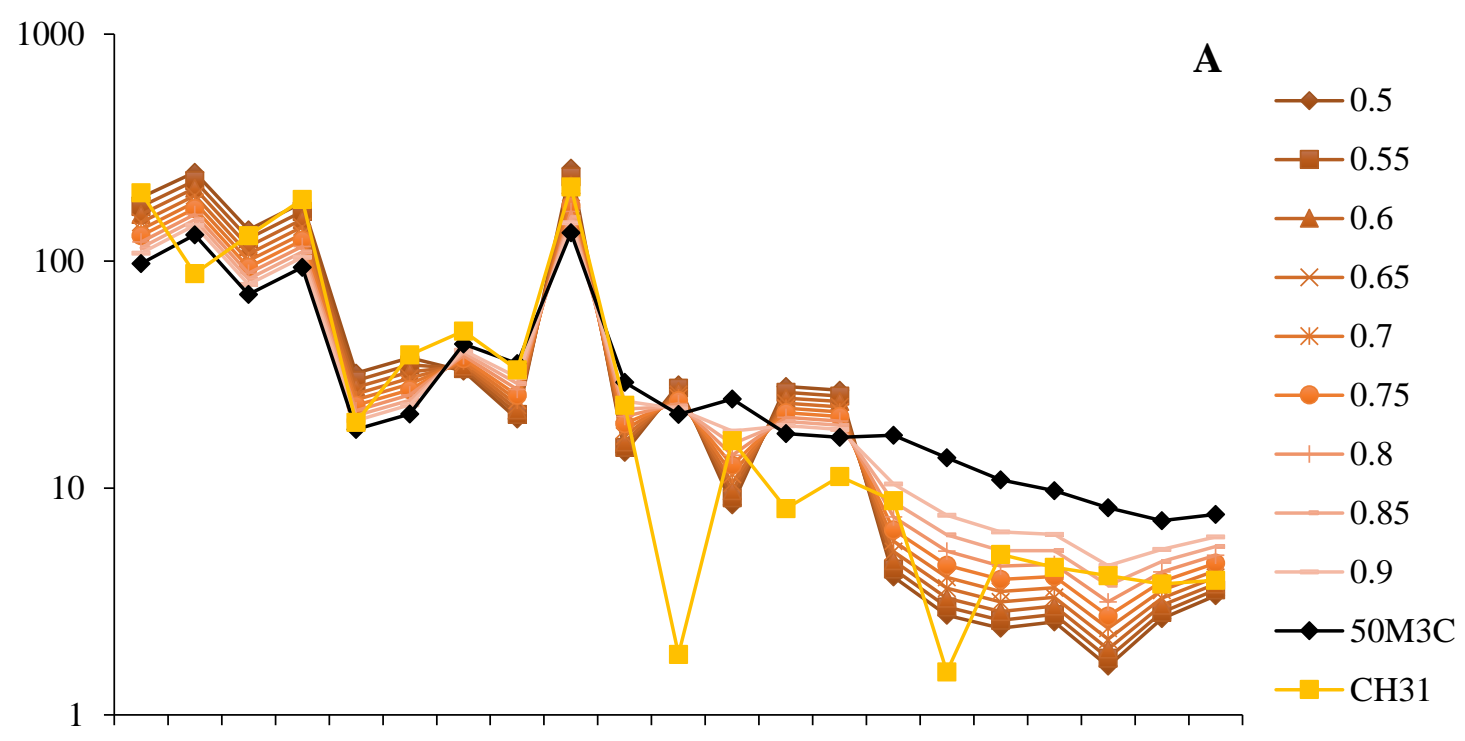

$\mathrm{Rb} \mathrm{Ba} \mathrm{Th} \mathrm{U}$ Nb Ta La Ce Pb Pr Sr Nd Zr Hf Sm Eu Tb Dy Y Yb Lu

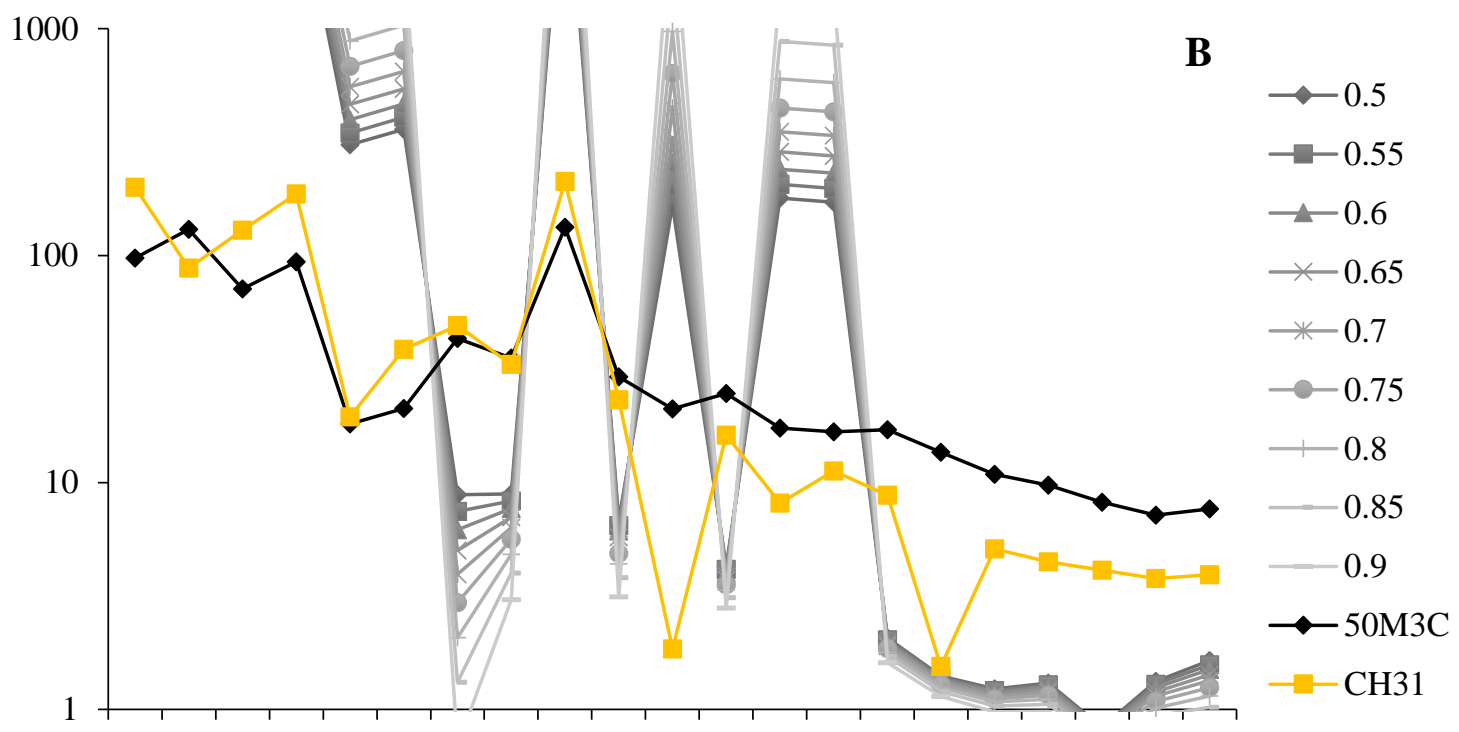

$\mathrm{Rb} \mathrm{Ba}$ Th $\mathrm{U}$ Nb Ta $\mathrm{La} \mathrm{Ce} \mathrm{Pb}$ Pr Sr Nd Zr Hf Sm Eu Tb Dy Y Yb Lu

Fig. D33 Primitive mantle normalized (Sun and McDonough, 1989) spider diagram for the partial melting of experimental melt MS47. A shows the results of the batch melt model; B shows the results of the fractional melt model. The melt percentages are approximately $\pm 20 \%$ that observed in the experimental melt. The trace element concentrations of the parent material, sample 50M3C (50/50 mixture of mafic glass and diorite), are indicated by the black diamonds. The trace element concentrations of the Dale Tuff, sample $\mathrm{CH} 31$, which is included for comparison, are indicated by the yellow squares. For experimental melt MS47, it can be seen that the batch melt model (A) approximates the data, with a notable exception at $\mathrm{Nd}$. In general, the fit increases as melt percent increases, particularly for LREE. 


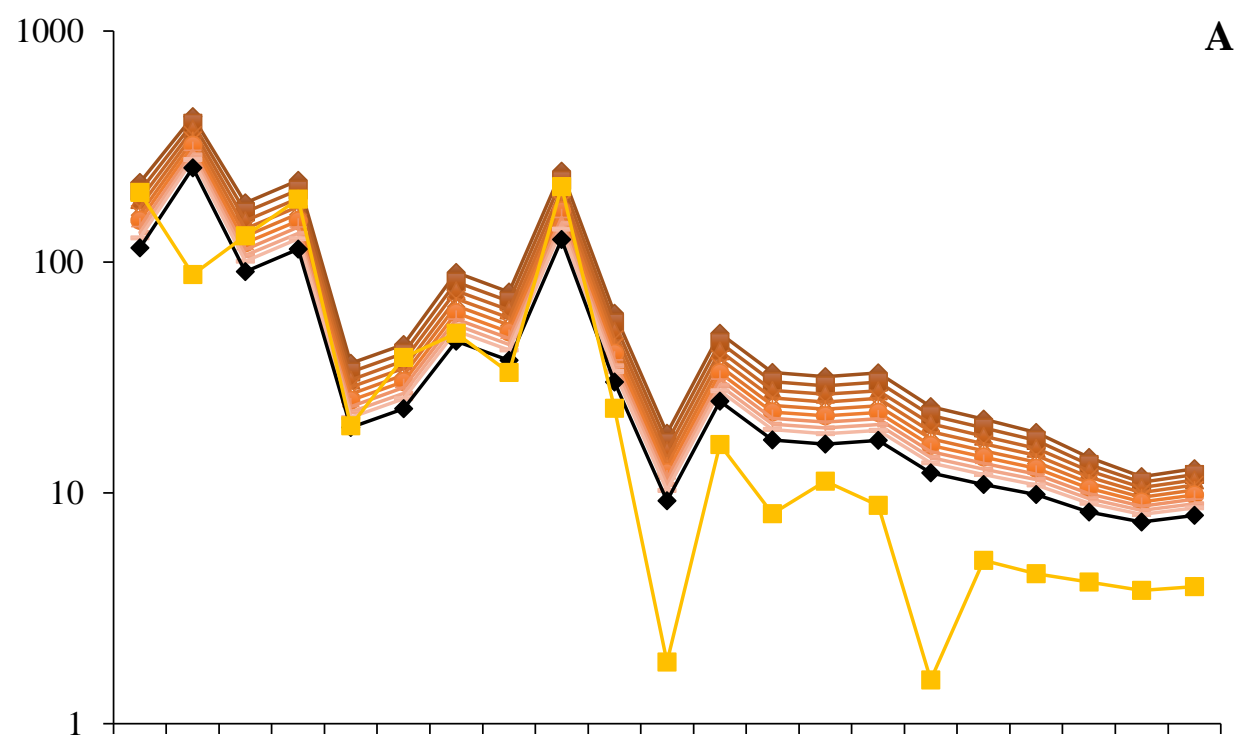

A

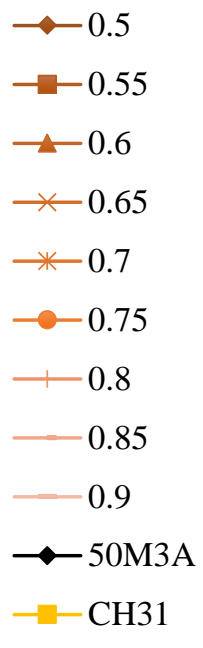

$\mathrm{Rb} \mathrm{Ba}$ Th U Nb Ta La Ce Pb Pr Sr Nd Zr Hf Sm Eu Tb Dy Y Yb Lu

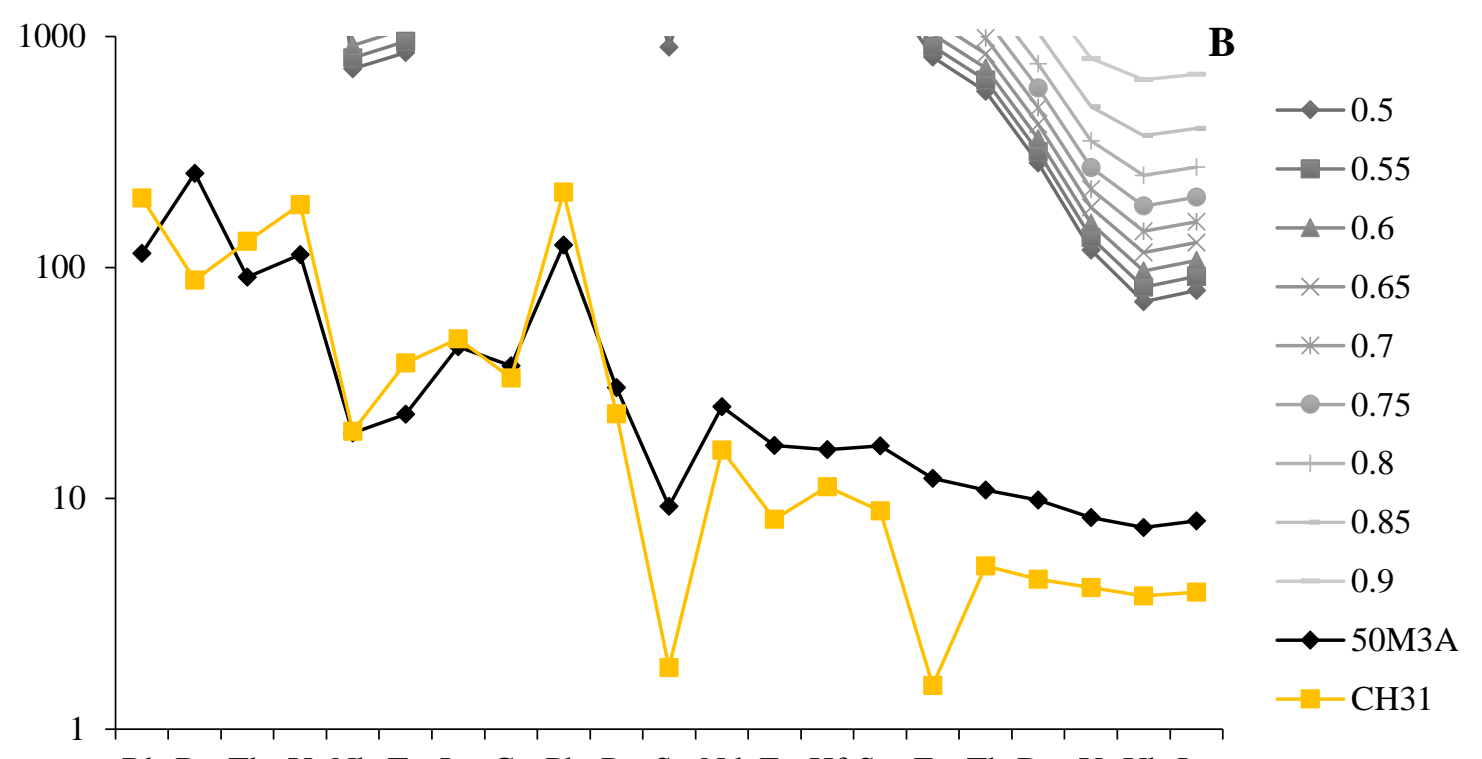

$\mathrm{Rb} \mathrm{Ba}$ Th $\mathrm{U}$ Nb Ta La Ce Pb Pr Sr Nd Zr Hf Sm Eu Tb Dy Y Yb Lu

Fig. D34 Primitive mantle normalized (Sun and McDonough, 1989) spider diagram for the partial melting of experimental melt MS48. A shows the results of the batch melt model; B shows the results of the fractional melt model. The melt percentages are approximately $\pm 20 \%$ that observed in the experimental melt. The trace element concentrations of the parent material, sample 50M3A (50/50 mixture of mafic glass and biotite fels), are indicated by the black diamonds. The trace element concentrations of the Dale Tuff, sample $\mathrm{CH} 31$, which is included for comparison, are indicated by the yellow squares. For experimental melt MS48, it can be seen that the batch melt model (A) approximates the data. The fit increases as melt percent increases. 


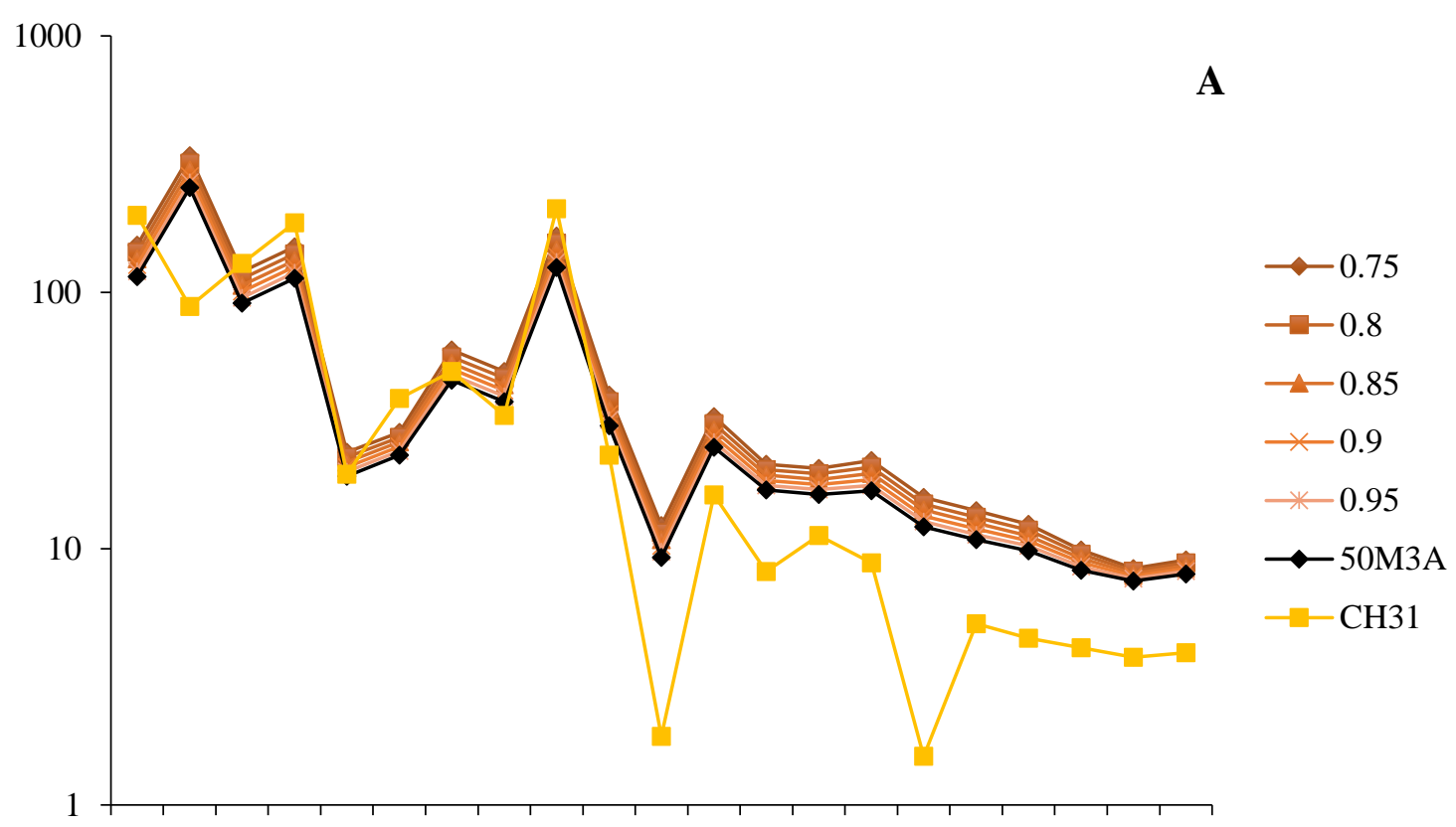

$\mathrm{Rb} \mathrm{Ba}$ Th $\mathrm{U}$ Nb Ta La Ce Pb Pr Sr Nd Zr Hf Sm Eu Tb Dy Y Yb Lu

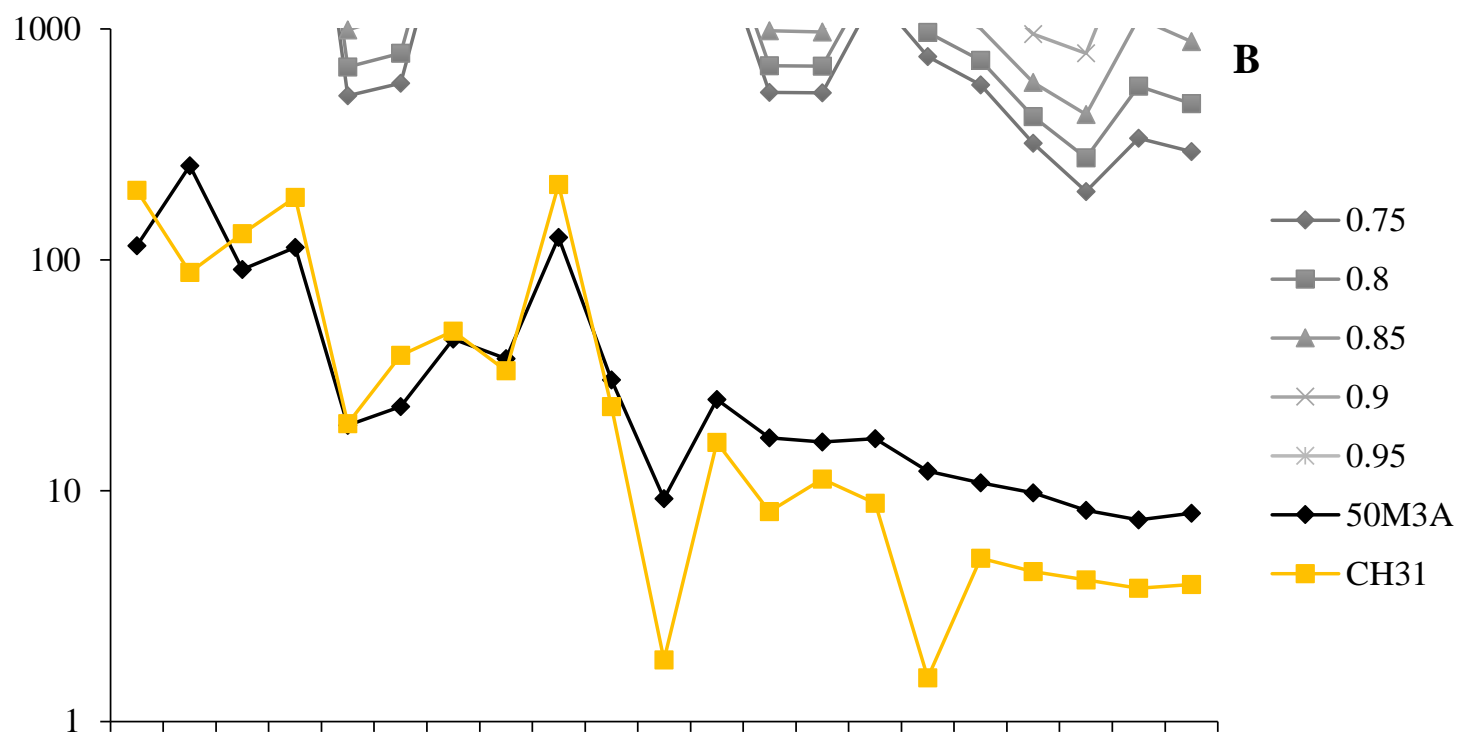

$\mathrm{Rb} \mathrm{Ba}$ Th U Nb Ta La Ce Pb Pr Sr Nd Zr Hf Sm Eu Tb Dy Y Yb Lu

Fig. D35 Primitive mantle normalized (Sun and McDonough, 1989) spider diagram for the partial melting of experimental melt MS49. A shows the results of the batch melt model; B shows the results of the fractional melt model. The melt percentages are approximately - 20\% that observed in the experimental melt. The trace element concentrations of the parent material, sample 50M3A (50/50 mixture of mafic glass and biotite fels), are indicated by the black diamonds. The trace element concentrations of the Dale Tuff, sample $\mathrm{CH} 31$, which is included for comparison, are indicated by the yellow squares. For experimental melt MS49, it can be seen that the batch model (A) approximates the data. 


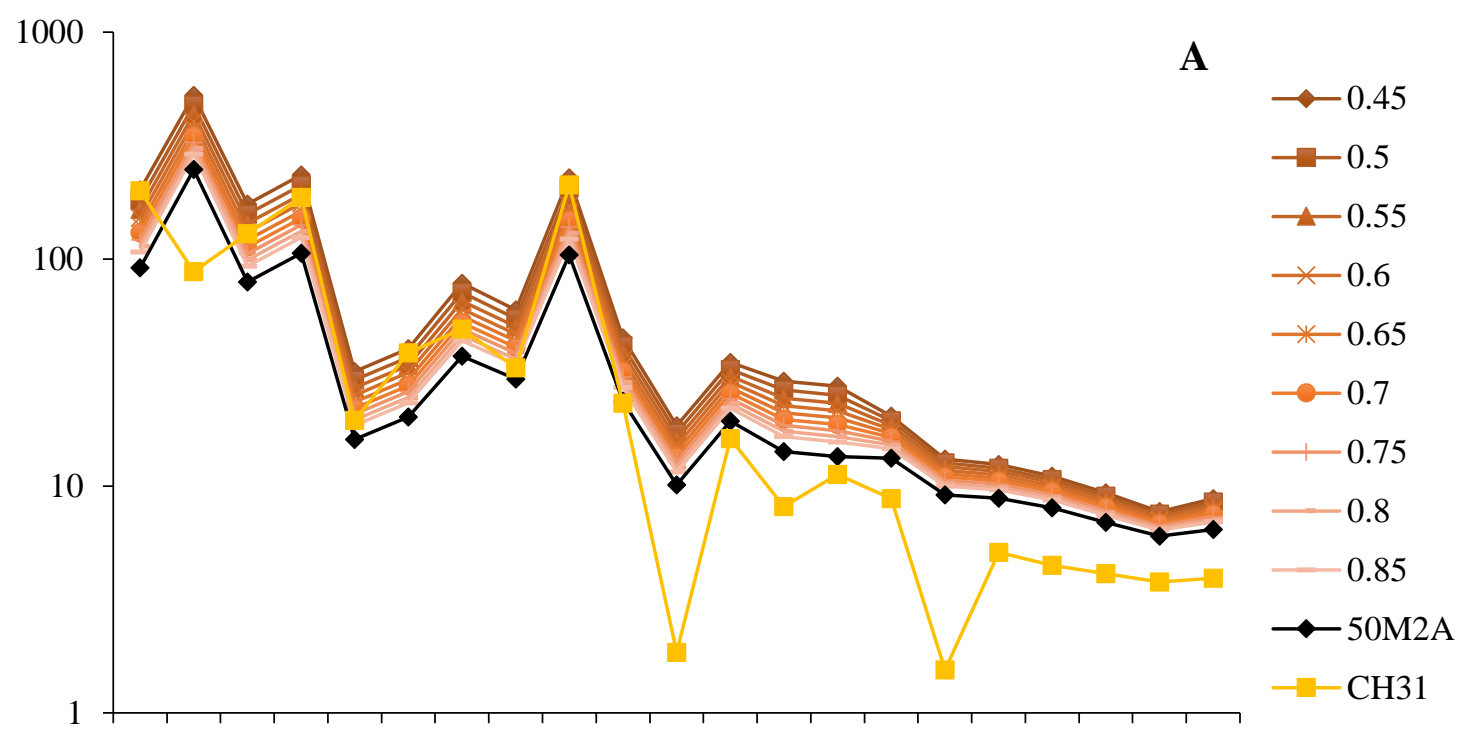

$\mathrm{Rb} \mathrm{Ba}$ Th $\mathrm{U}$ Nb Ta La Ce Pb Pr Sr Nd Zr Hf Sm Eu Tb Dy Y Yb Lu

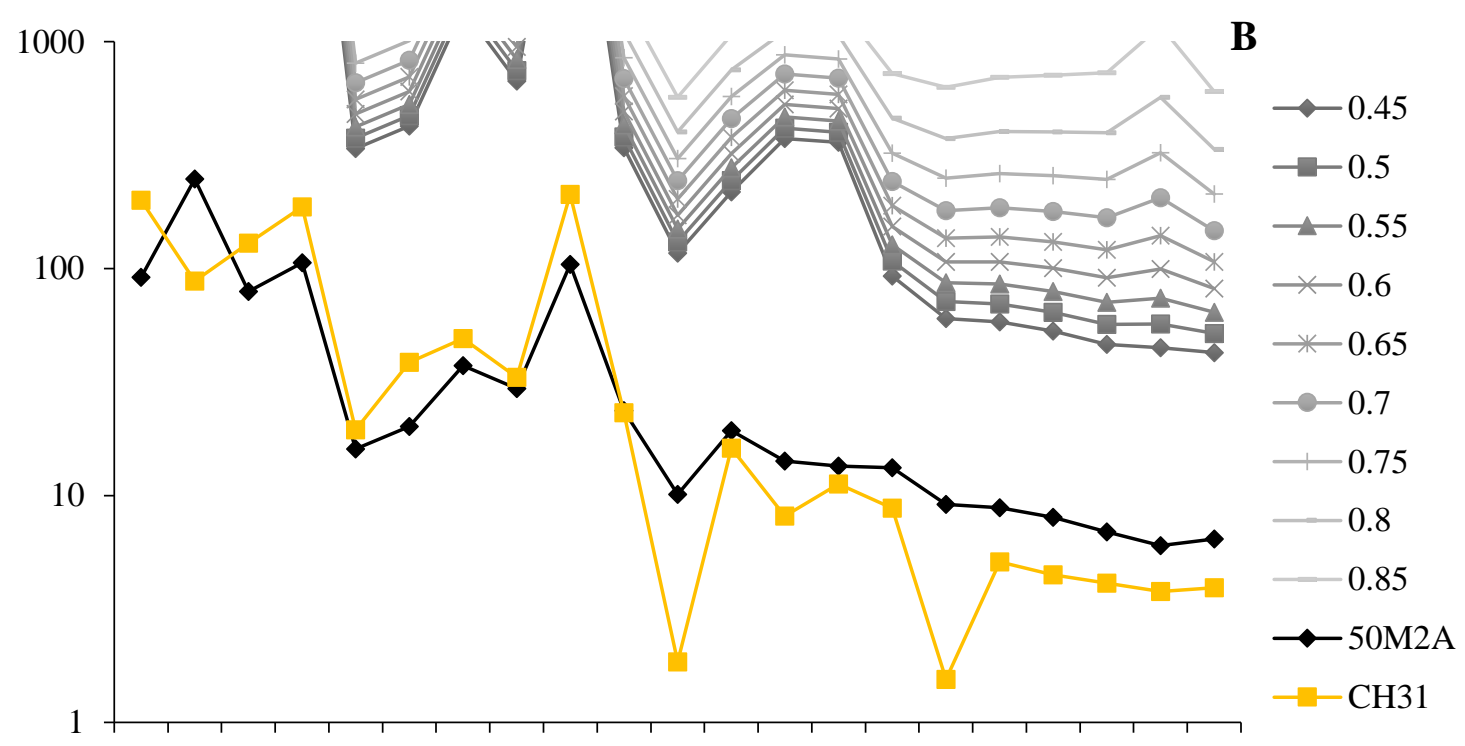

$\mathrm{Rb} \mathrm{Ba}$ Th $\mathrm{U}$ Nb Ta La Ce Pb Pr Sr Nd Zr Hf Sm Eu Tb Dy Y Yb Lu

Fig. D36 Primitive mantle normalized (Sun and McDonough, 1989) spider diagram for the partial melting of experimental melt MS50. A shows the results of the batch melt model; B shows the results of the fractional melt model. The melt percentages are approximately $\pm 20 \%$ that observed in the experimental melt. The trace element concentrations of the parent material, sample 50M2A (50/50 mixture of mafic glass and biotite fels), are indicated by the black diamonds. The trace element concentrations of the Dale Tuff, sample $\mathrm{CH} 31$, which is included for comparison, are indicated by the yellow squares. For experimental melt MS50, it can be seen that the batch melt model (A) approximates the data. The fit increases as melt percent increases. 


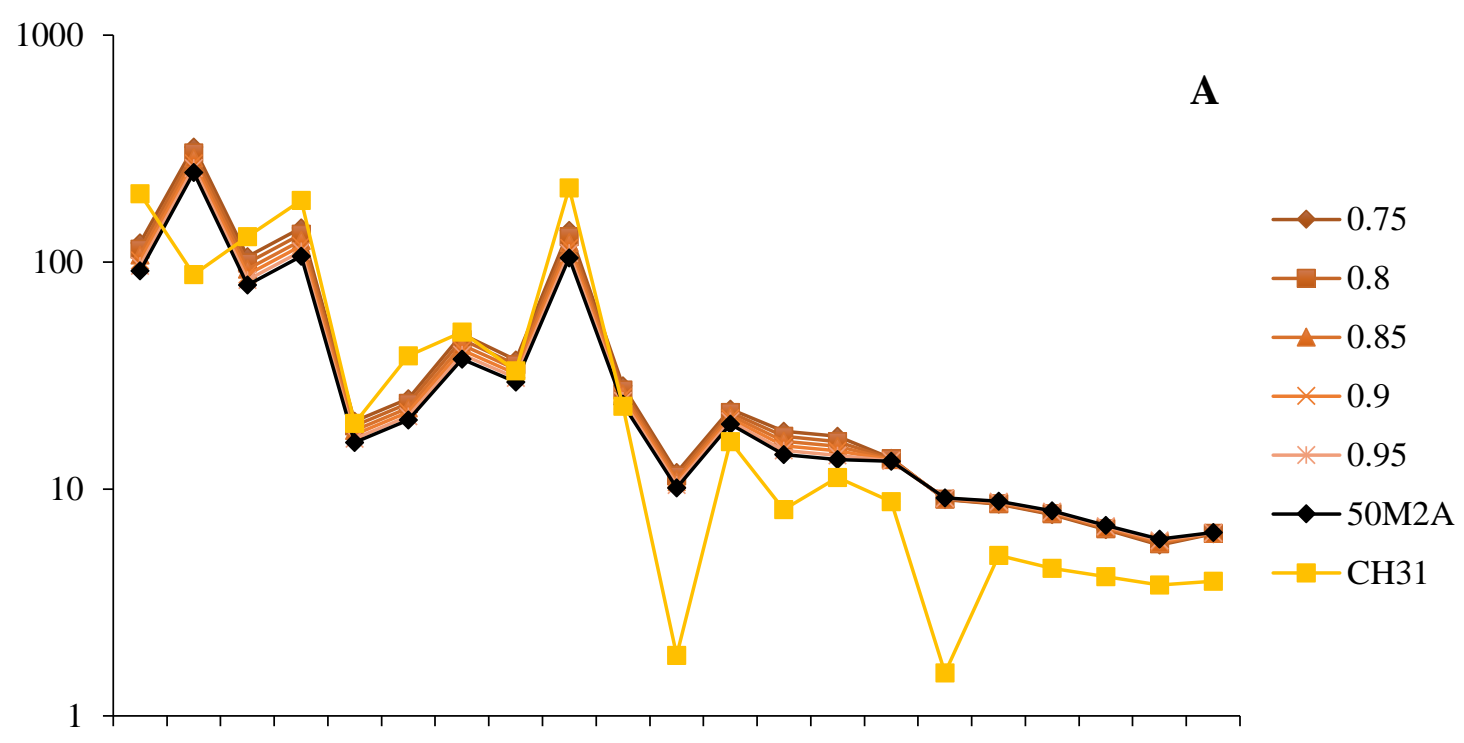

$\mathrm{Rb} \mathrm{Ba}$ Th $\mathrm{U}$ Nb Ta La Ce Pb Pr Sr Nd Zr Hf Sm Eu Tb Dy Y Yb Lu

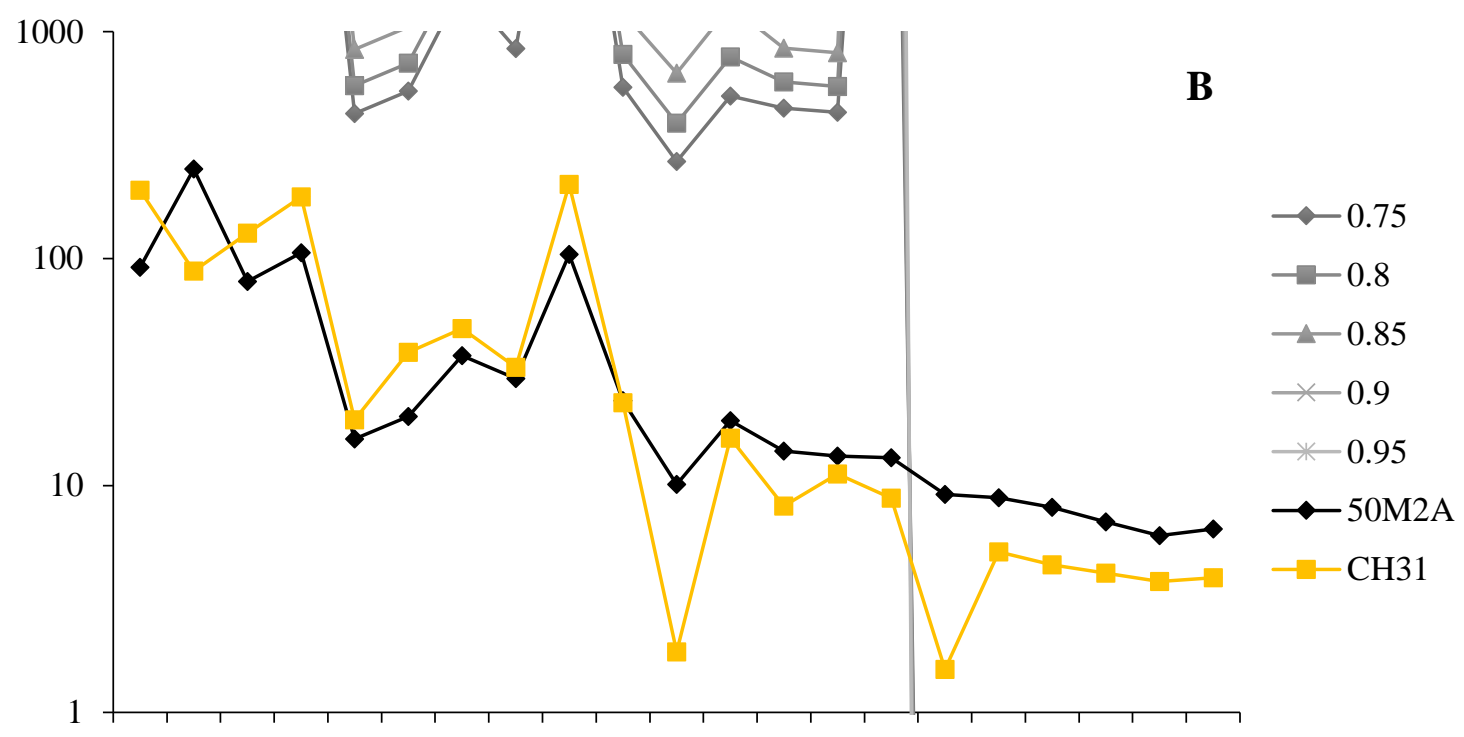

$\mathrm{Rb} \mathrm{Ba}$ Th U Nb Ta La Ce Pb Pr Sr Nd Zr Hf Sm Eu Tb Dy Y Yb Lu

Fig. D37 Primitive mantle normalized (Sun and McDonough, 1989) spider diagram for the partial melting of experimental melt MS51. A shows the results of the batch melt model; B shows the results of the fractional melt model. The melt percentages are approximately $\pm 20 \%$ that observed in the experimental melt. The trace element concentrations of the parent material, sample 50M2A (50/50 mixture of mafic glass and biotite fels), are indicated by the black diamonds. The trace element concentrations of the Dale Tuff, sample $\mathrm{CH} 31$, which is included for comparison, are indicated by the yellow squares. For experimental melt MS51, it can be seen that the batch melt model (A) approximates the data. The fit increases as melt percent increases. 


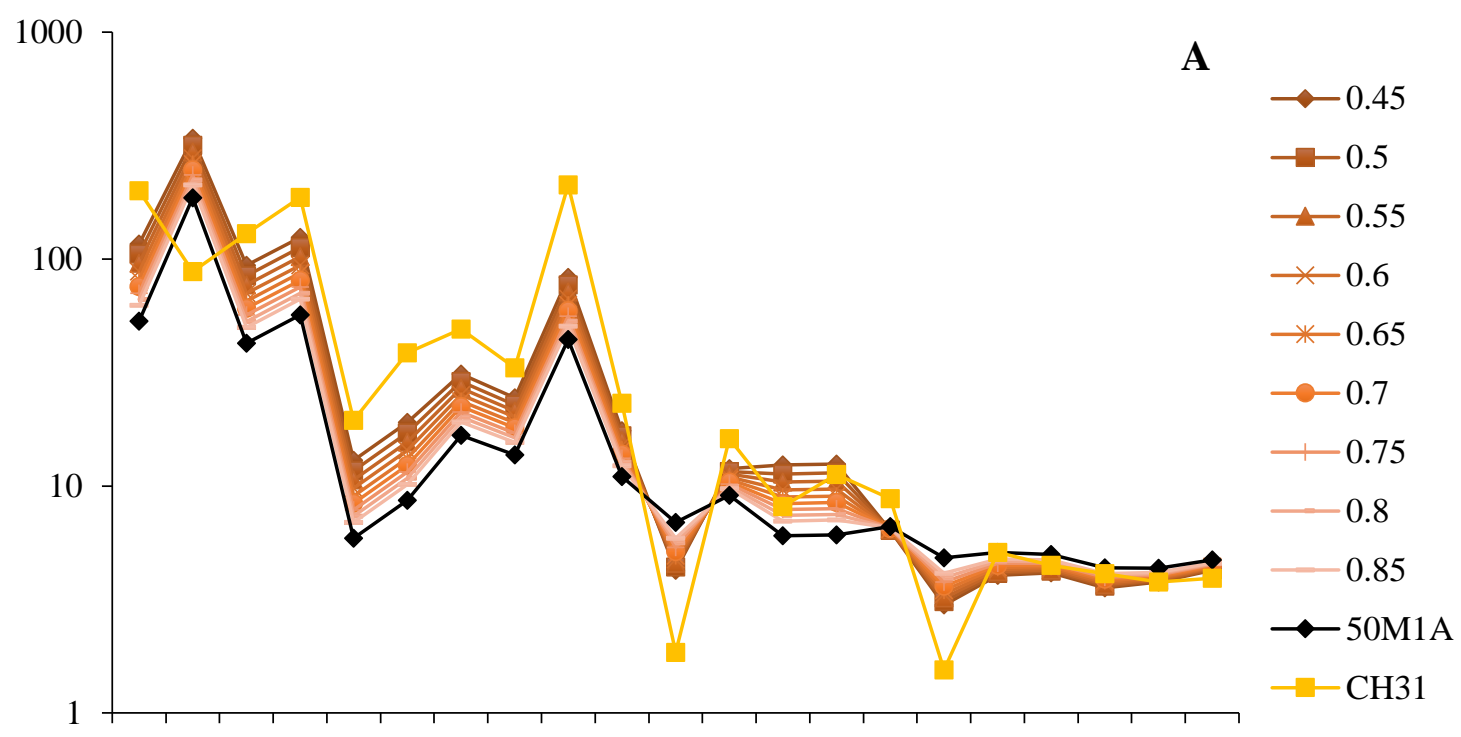

$\mathrm{Rb} \mathrm{Ba}$ Th $\mathrm{U}$ Nb Ta La Ce Pb Pr Sr Nd Zr Hf Sm Eu Tb Dy Y Yb Lu

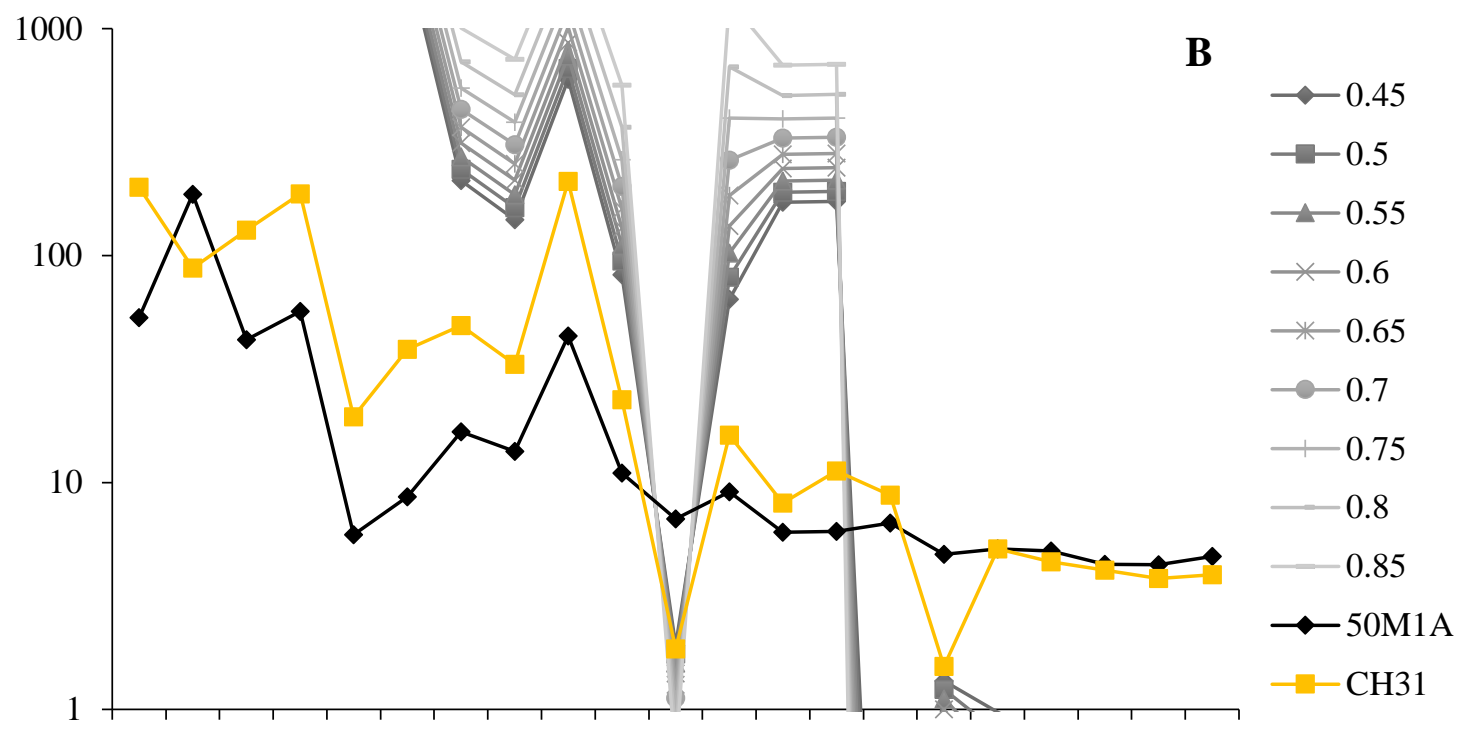

$\mathrm{Rb} \mathrm{Ba}$ Th $\mathrm{U}$ Nb Ta $\mathrm{La} \mathrm{Ce} \mathrm{Pb}$ Pr Sr Nd Zr Hf Sm Eu Tb Dy Y Yb Lu

Fig. D38 Primitive mantle normalized (Sun and McDonough, 1989) spider diagram for the partial melting of experimental melt MS52. A shows the results of the batch melt model; B shows the results of the fractional melt model. The melt percentages are approximately $\pm 20 \%$ that observed in the experimental melt. The trace element concentrations of the parent material, sample 50M1A (50/50 mixture of mafic glass and biotite fels), are indicated by the black diamonds. The trace element concentrations of the Dale Tuff, sample $\mathrm{CH} 31$, which is included for comparison, are indicated by the yellow squares. For experimental melt MS52, it can be seen that the batch melt model (A) approximates the data. The fit increases as melt percent increases. 


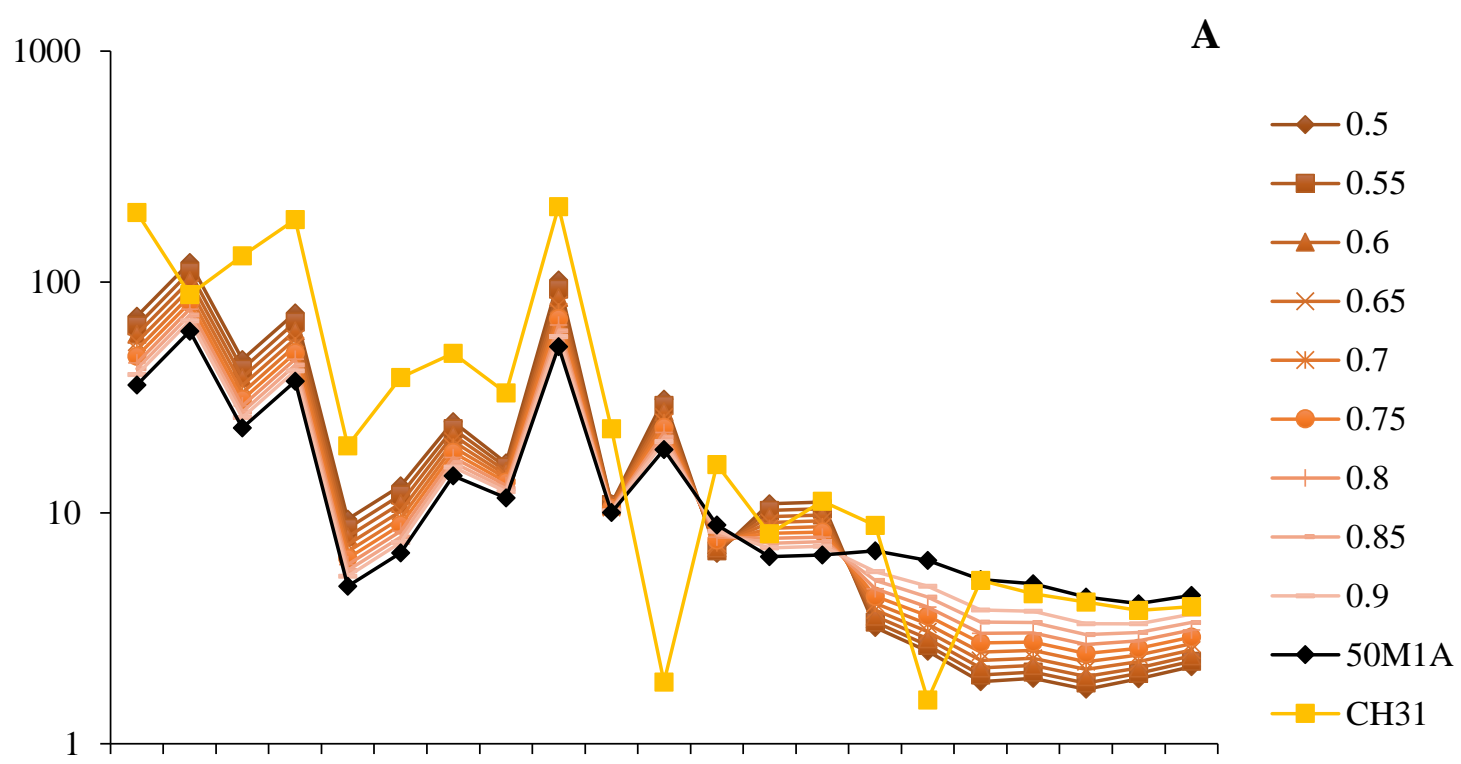

$\mathrm{Rb} \mathrm{Ba}$ Th $\mathrm{U}$ Nb Ta La Ce Pb Pr Sr Nd Zr Hf Sm Eu Tb Dy Y Yb Lu

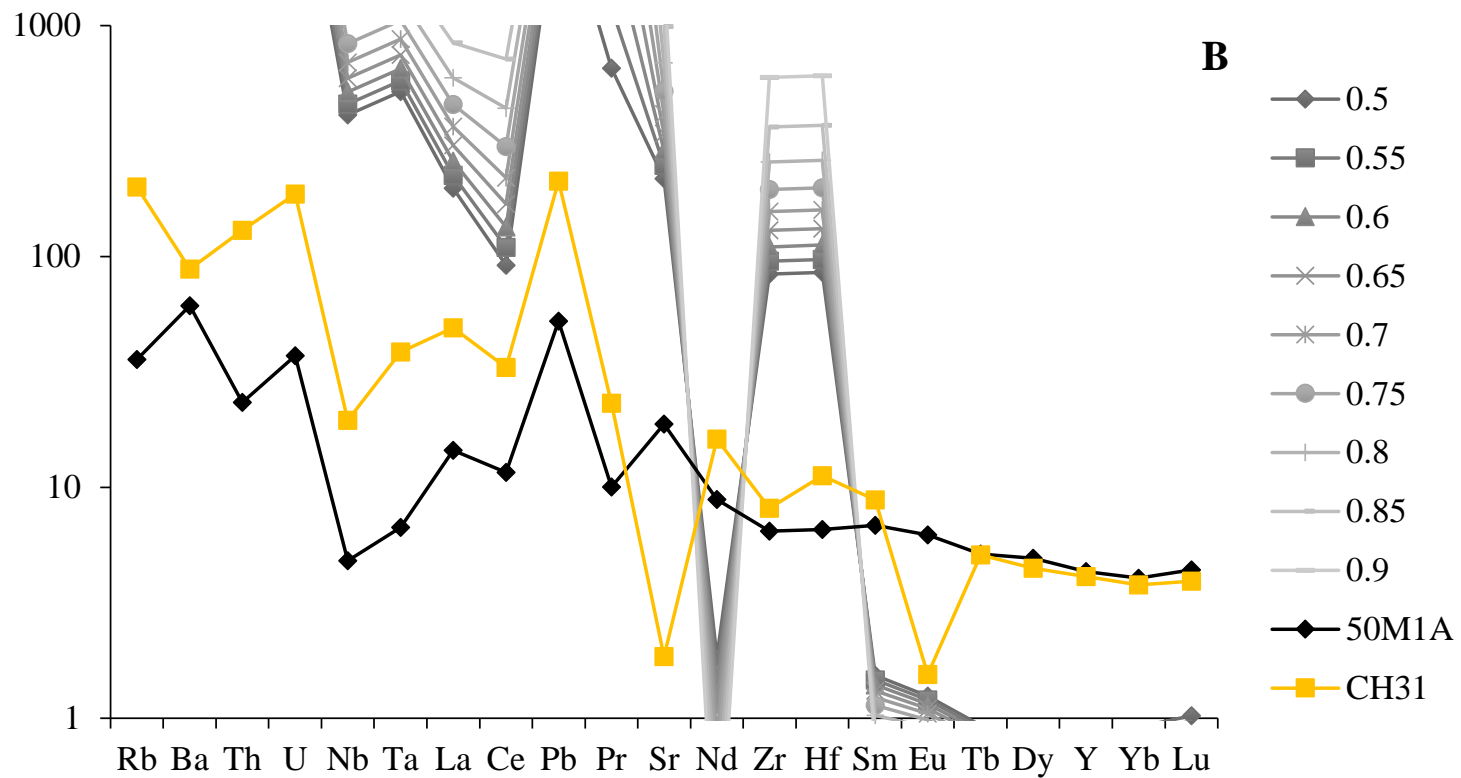

Fig. D39 Primitive mantle normalized (Sun and McDonough, 1989) spider diagram for the partial melting of experimental melt MS53. A shows the results of the batch melt model; B shows the results of the fractional melt model. The melt percentages are approximately $\pm 20 \%$ that observed in the experimental melt. The trace element concentrations of the parent material, sample 50M1A (50/50 mixture of mafic glass and biotite fels), are indicated by the black diamonds. The trace element concentrations of the Dale Tuff, sample $\mathrm{CH} 31$, which is included for comparison, are indicated by the yellow squares. For experimental melt MS53, it can be seen that the batch melt model (A) approximates the data. The fit increases as melt percent increases. 


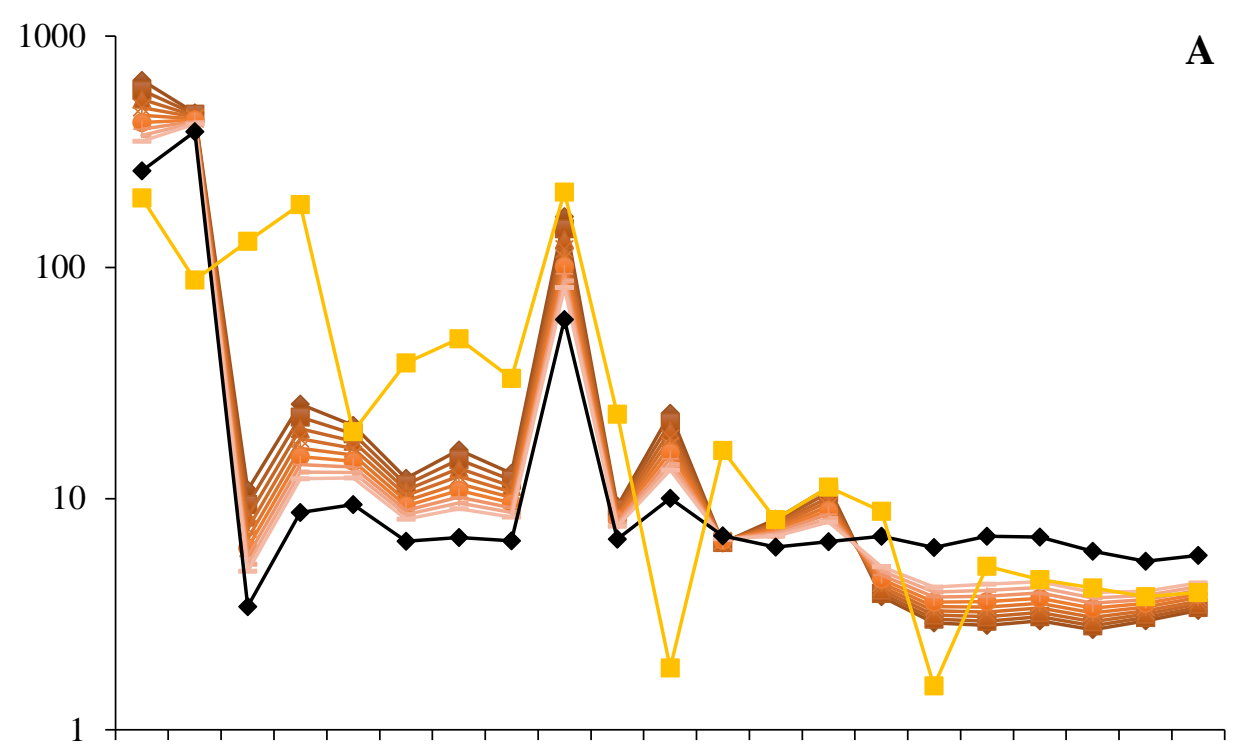

A

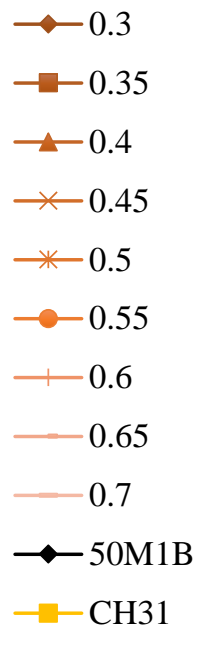

$\mathrm{Rb} \mathrm{Ba}$ Th U Nb Ta La Ce Pb Pr Sr Nd Zr Hf Sm Eu Tb Dy Y Yb Lu

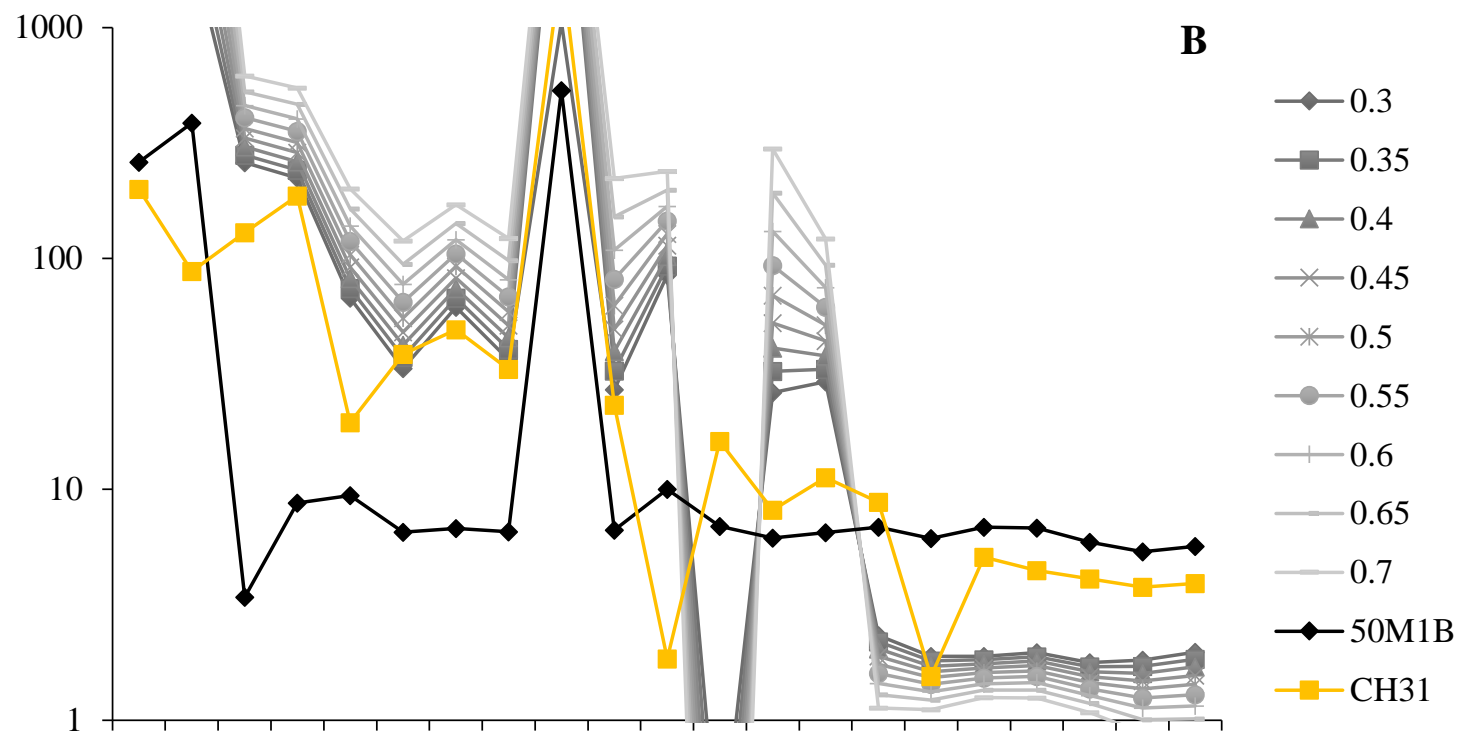

$\mathrm{Rb} \mathrm{Ba}$ Th $\mathrm{U}$ Nb Ta $\mathrm{La} \mathrm{Ce} \mathrm{Pb}$ Pr $\mathrm{Sr} \mathrm{Nd} \mathrm{Zr} \mathrm{Hf} \mathrm{Sm} \mathrm{Eu}$ Tb Dy Y Yb Lu

Fig. D40 Primitive mantle normalized (Sun and McDonough, 1989) spider diagram for the partial melting of experimental melt MS55. A shows the results of the batch melt model; B shows the results of the fractional melt model. The melt percentages are approximately $\pm 20 \%$ that observed in the experimental melt. The trace element concentrations of the parent material, sample 50M1B (50/50 mixture of mafic glass and biotite schist), are indicated by the black diamonds. The trace element concentrations of the Dale Tuff, sample $\mathrm{CH} 31$, which is included for comparison, are indicated by the yellow squares. For experimental melt MS55, it can be seen that the batch melt model (A) approximates the data. The fit increases as melt percent increases. 


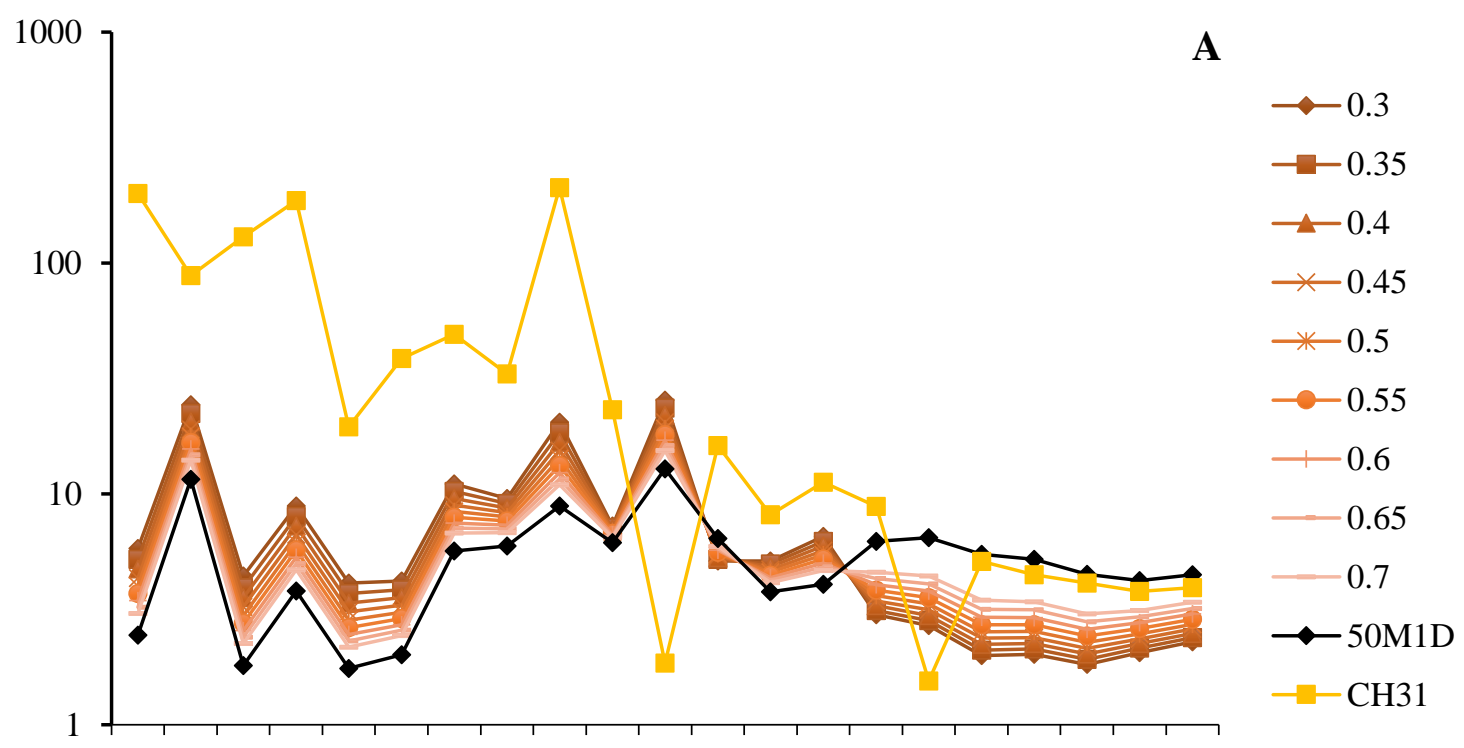

$\mathrm{Rb} \mathrm{Ba}$ Th $\mathrm{U}$ Nb Ta La Ce Pb Pr Sr Nd Zr Hf Sm Eu Tb Dy Y Yb Lu

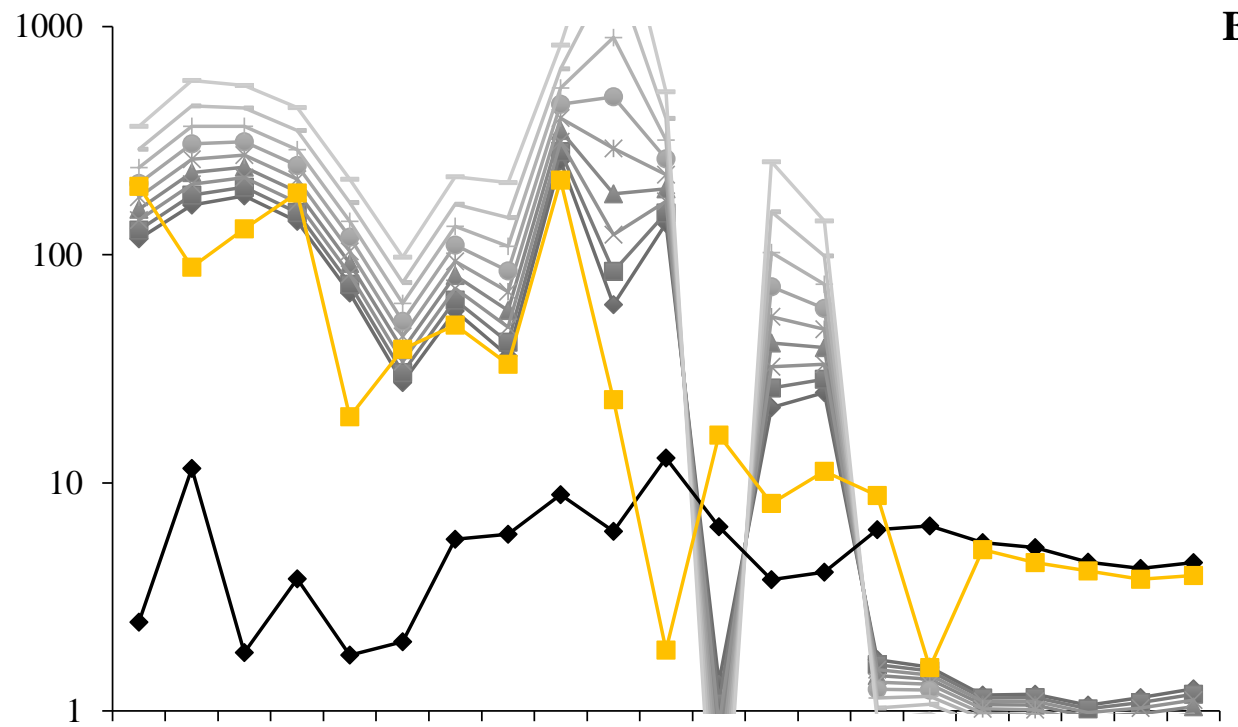

B

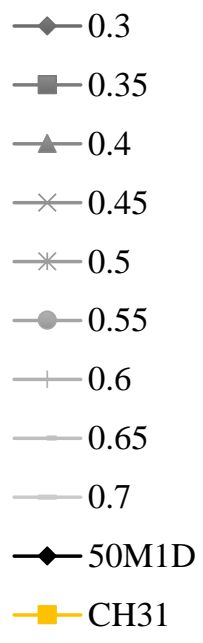

$\mathrm{Rb} \mathrm{Ba}$ Th $\mathrm{U}$ Nb Ta La Ce Pb Pr Sr Nd Zr Hf Sm Eu Tb Dy Y Yb Lu

Fig. D41 Primitive mantle normalized (Sun and McDonough, 1989) spider diagram for the partial melting of experimental melt MS57. A shows the results of the batch melt model; B shows the results of the fractional melt model. The melt percentages are approximately $\pm 20 \%$ that observed in the experimental melt. The trace element concentrations of the parent material, sample 50M1D (50/50 mixture of mafic glass and biotite fels), are indicated by the black diamonds. The trace element concentrations of the Dale Tuff, sample $\mathrm{CH} 31$, which is included for comparison, are indicated by the yellow squares. For experimental melt MS57, it can be seen that the batch melt model (A) approximates the data. The fit increases as melt percent increases. 


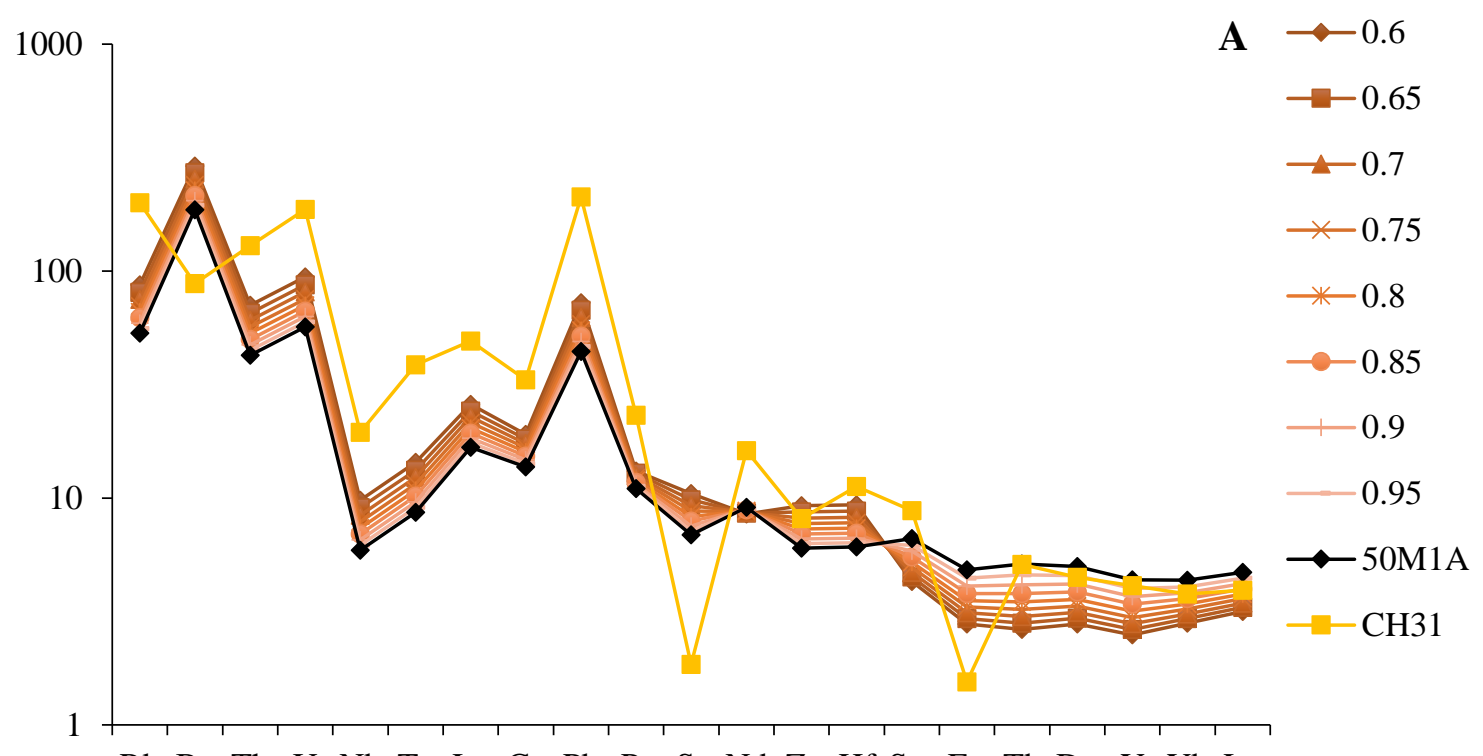

$\mathrm{Rb} \mathrm{Ba}$ Th $\mathrm{U}$ Nb Ta $\mathrm{La} \mathrm{Ce} \mathrm{Pb}$ Pr $\mathrm{Sr} \mathrm{Nd} \mathrm{Zr} \mathrm{Hf} \mathrm{Sm} \mathrm{Eu}$ Tb Dy $\mathrm{Y}$ Yb Lu

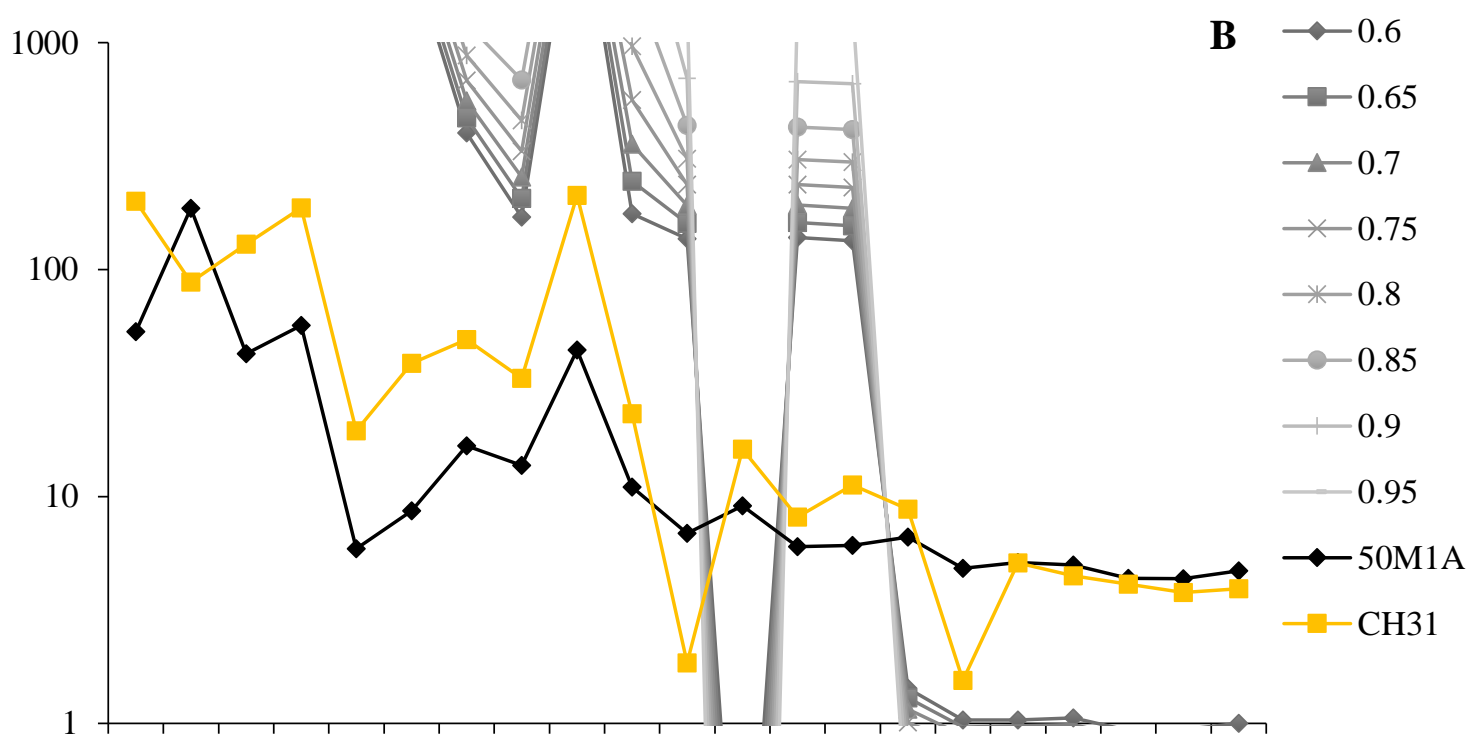

$\mathrm{Rb} \mathrm{Ba}$ Th $\mathrm{U}$ Nb Ta La Ce Pb Pr Sr Nd Zr Hf Sm Eu Tb Dy Y Yb Lu

Fig. D42 Primitive mantle normalized (Sun and McDonough, 1989) spider diagram for the partial melting of experimental melt MS58. A shows the results of the batch melt model; B shows the results of the fractional melt model. The melt percentages are approximately $\pm 20 \%$ that observed in the experimental melt. The trace element concentrations of the parent material, sample 50M1 A (50/50 mixture of mafic glass and biotite fels), are indicated by the black diamonds. The trace element concentrations of the Dale Tuff, sample $\mathrm{CH} 31$, which is included for comparison, are indicated by the yellow squares. For experimental melt MS58, it can be seen that the batch melt model (A) approximates the data. The fit increases as melt percent increases. 


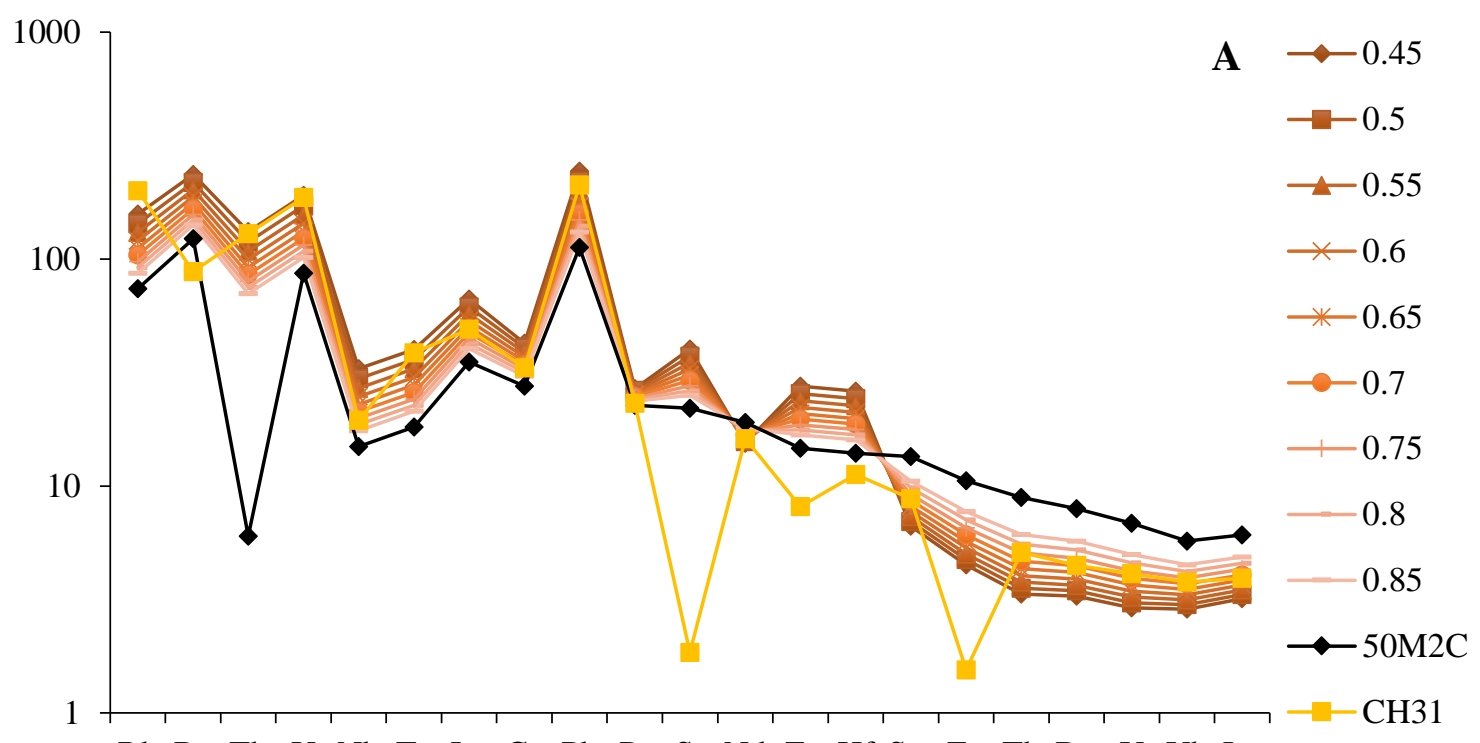

$\mathrm{Rb} \mathrm{Ba} \mathrm{Th} \mathrm{U}$ Nb Ta La Ce Pb Pr Sr Nd Zr Hf Sm Eu Tb Dy Y Yb Lu

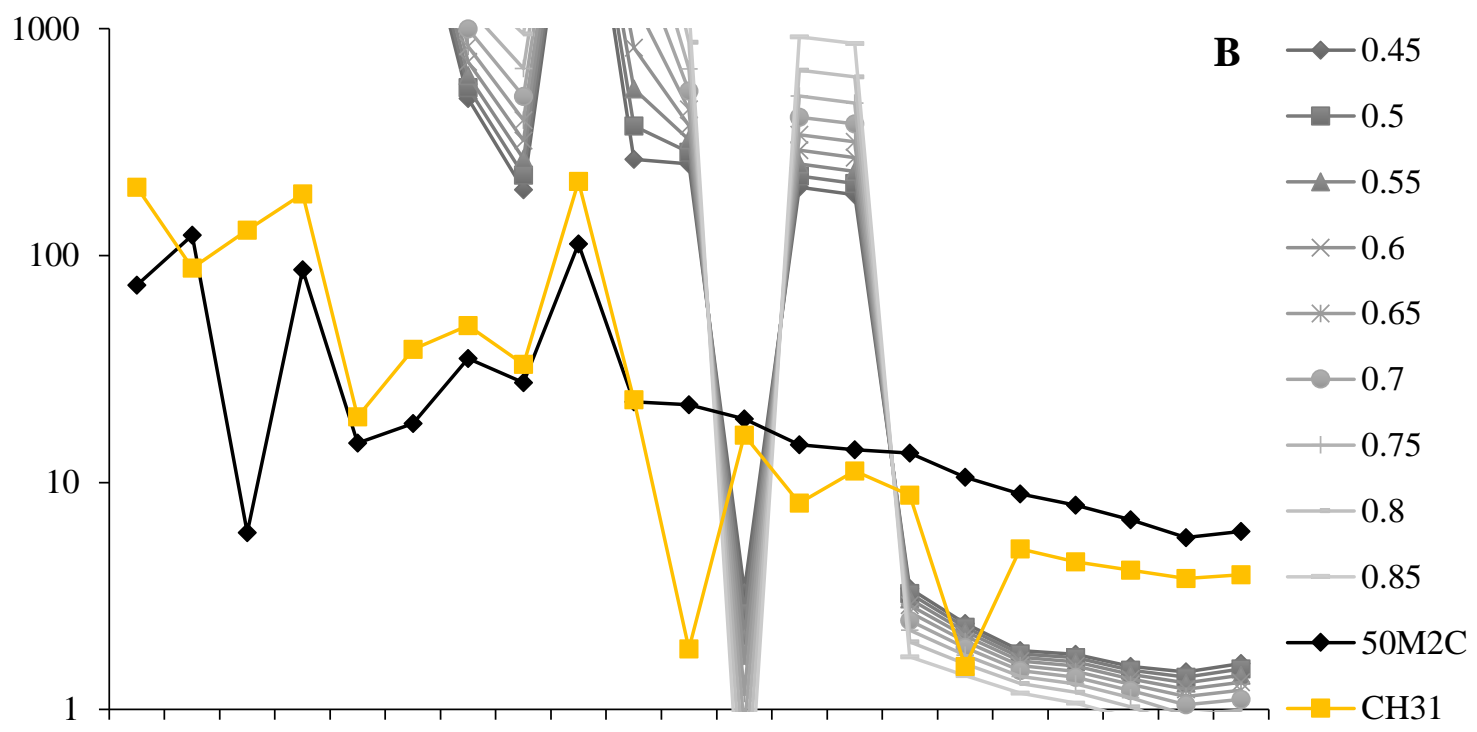

$\mathrm{Rb} \mathrm{Ba} \mathrm{Th} \mathrm{U}$ Nb Ta La Ce Pb Pr Sr Nd Zr Hf Sm Eu Tb Dy Y Yb Lu

Fig. D43 Primitive mantle normalized (Sun and McDonough, 1989) spider diagram for the partial melting of experimental melt MS59. A shows the results of the batch melt model; B shows the results of the fractional melt model. The melt percentages are approximately $\pm 20 \%$ that observed in the experimental melt. The trace element concentrations of the parent material, sample 50M2C (50/50 mixture of mafic glass and diorite), are indicated by the black diamonds. The trace element concentrations of the Dale Tuff, sample $\mathrm{CH} 31$, which is included for comparison, are indicated by the yellow squares. For experimental melt MS59, it can be seen that the batch melt model (A) approximates the data with a notable exception at Th. In general, the fit increases as melt percent increases, particularly for LREE. 


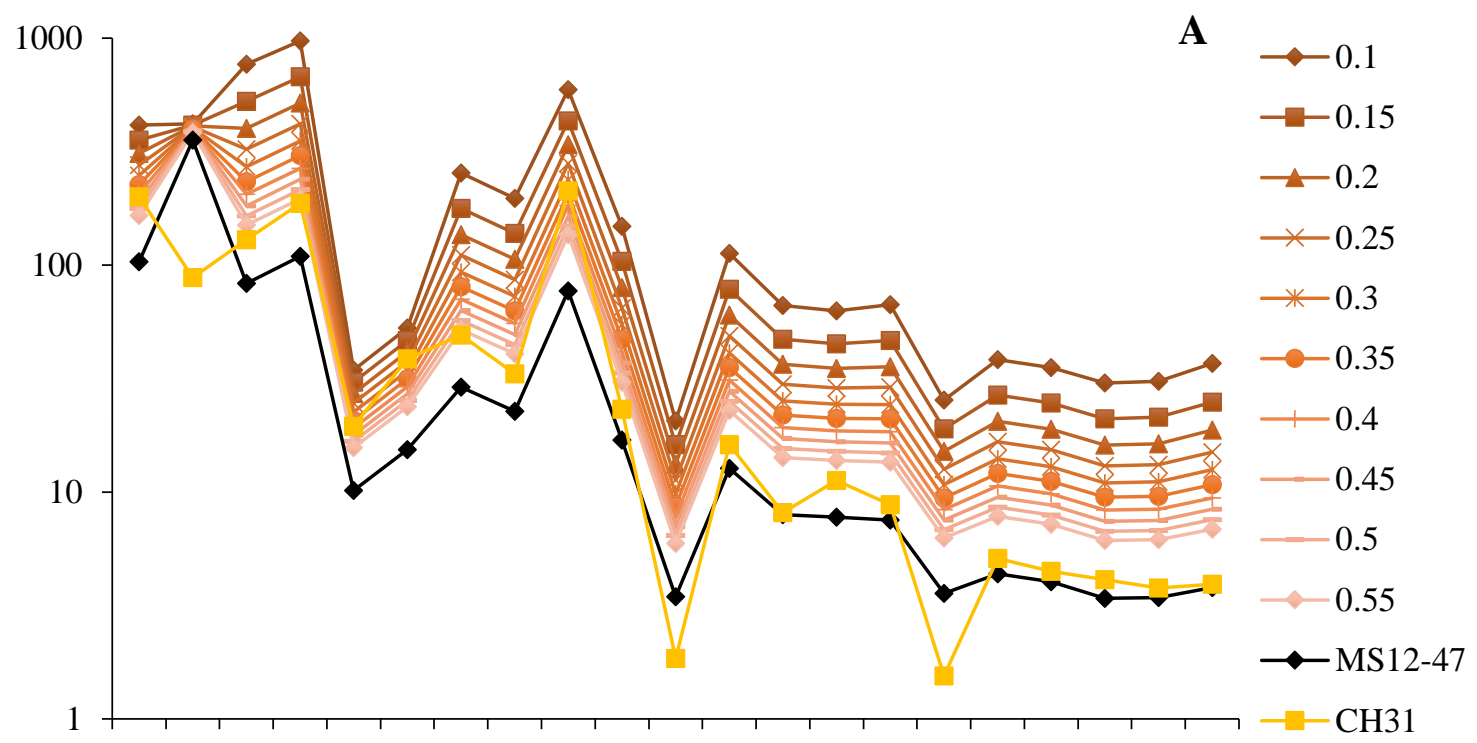

$\mathrm{Rb} \mathrm{Ba}$ Th $\mathrm{U}$ Nb Ta $\mathrm{La} \mathrm{Ce} \mathrm{Pb}$ Pr $\mathrm{Sr} \mathrm{Nd} \mathrm{Zr}$ Hf Sm Eu Tb Dy Y Yb Lu

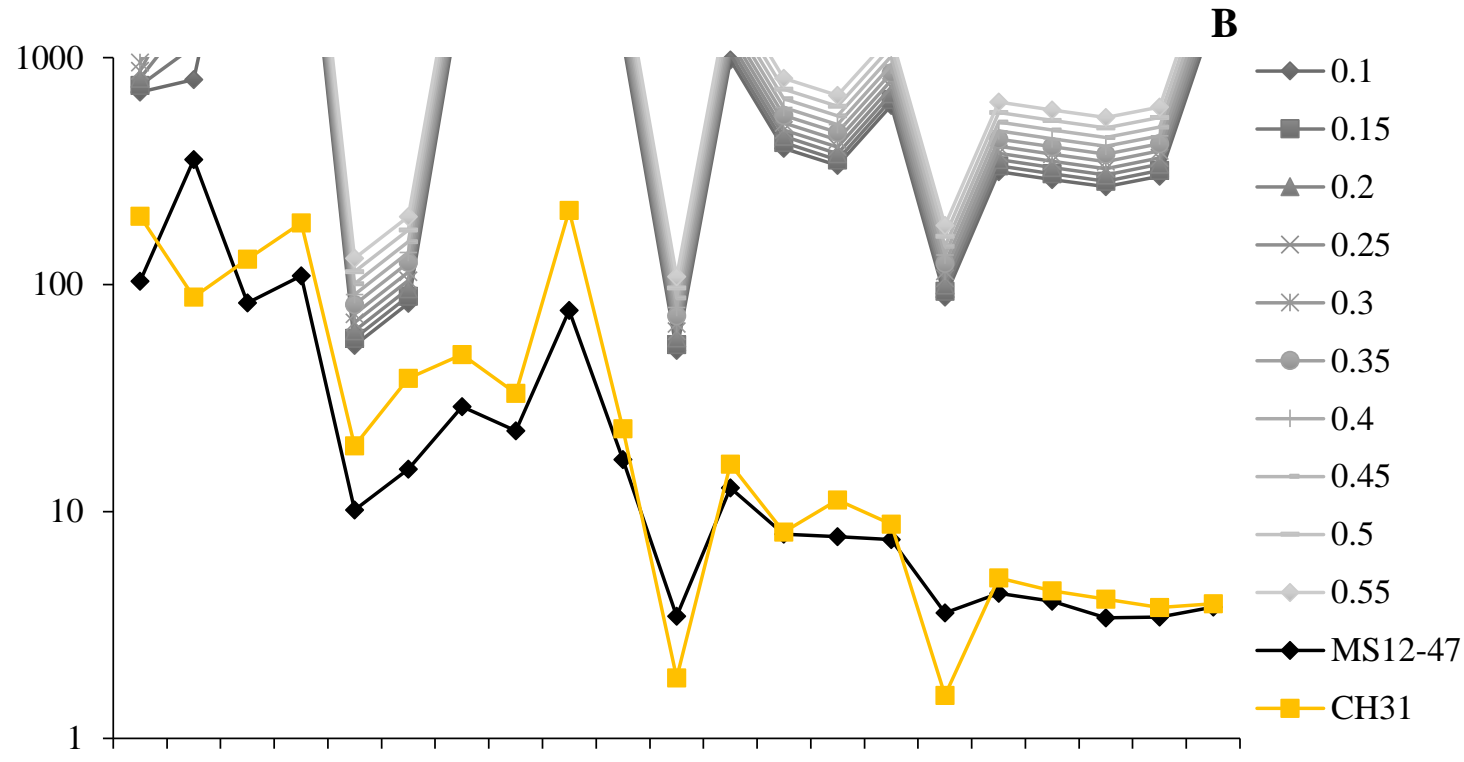

$\mathrm{Rb} \mathrm{Ba}$ Th $\mathrm{U}$ Nb Ta La Ce Pb Pr Sr Nd Zr Hf Sm Eu Tb Dy Y Yb Lu

Fig. D44 Primitive mantle normalized (Sun and McDonough, 1989) spider diagram for the partial melting of experimental melt MS60. A shows the results of the batch melt model; B shows the results of the fractional melt model. The melt percentages are approximately $\pm 20 \%$ that observed in the experimental melt. The trace element concentrations of the parent material, sample MS12-47 (biotite fels), are indicated by the black diamonds. The trace element concentrations of the Dale Tuff, sample CH31, which is included for comparison, are indicated by the yellow squares. For experimental melt MS60, it can be seen that the batch melt model (A) approximates the data. The fit increases as melt percent increases. 


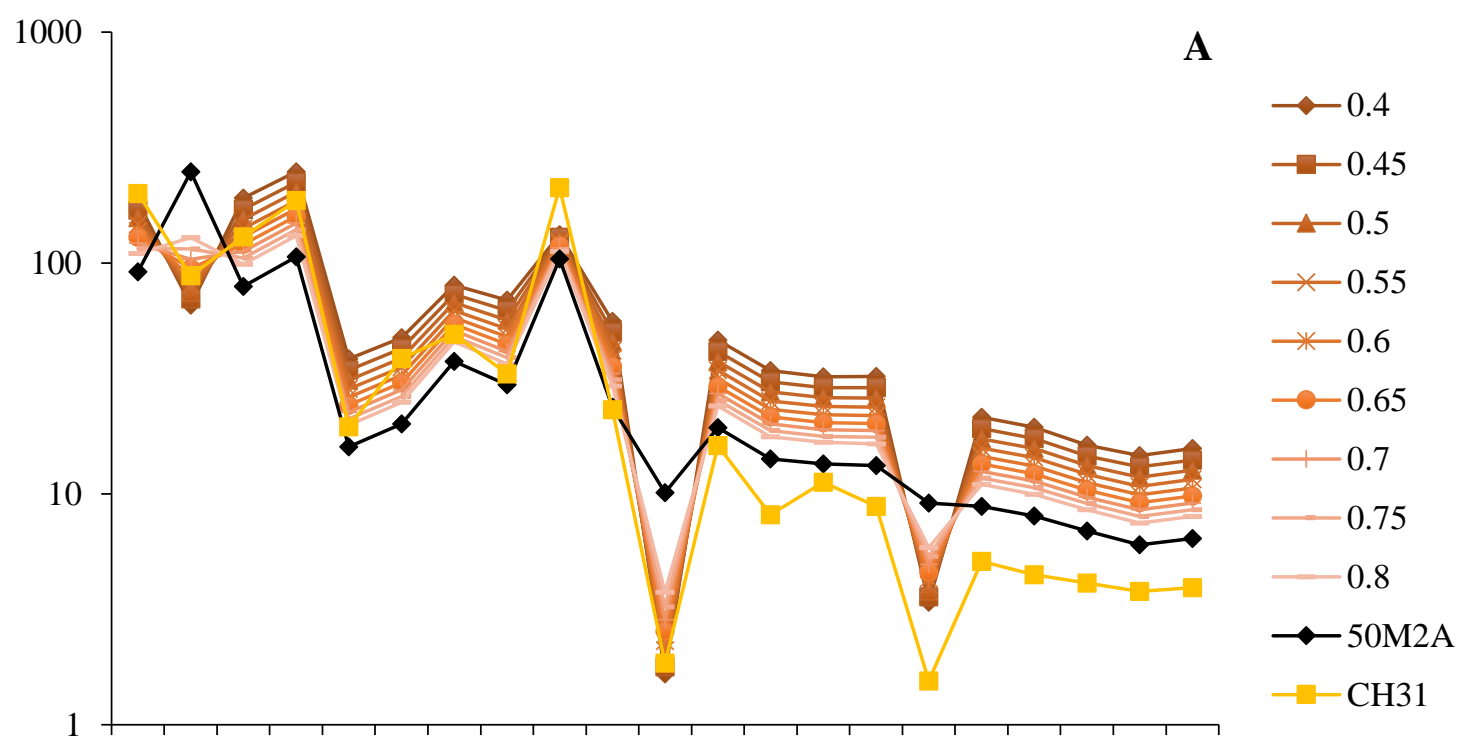

$\mathrm{Rb} \mathrm{Ba}$ Th U Nb Ta La Ce Pb Pr Sr Nd Zr Hf Sm Eu Tb Dy Y Yb Lu

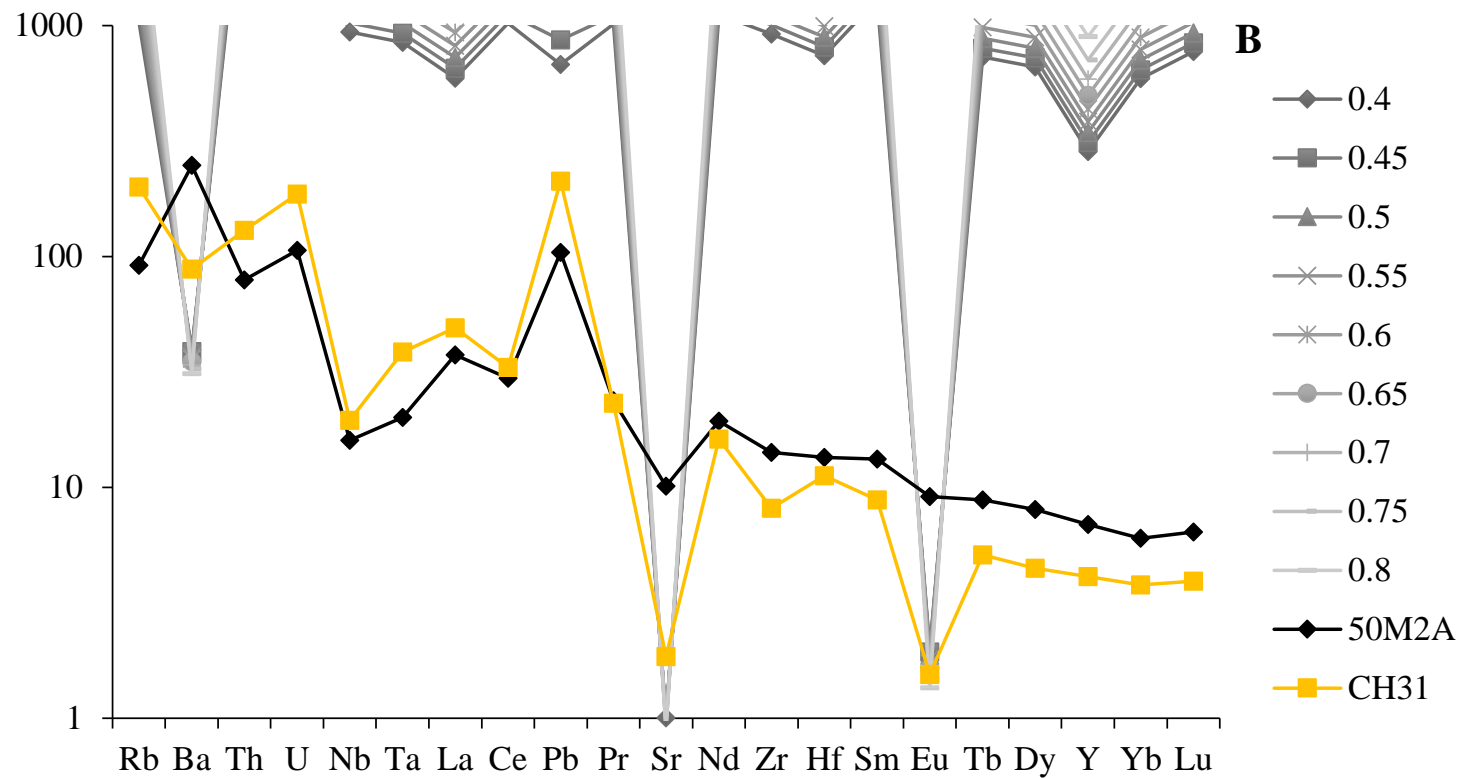

Fig. D45 Primitive mantle normalized (Sun and McDonough, 1989) spider diagram for the partial melting of experimental melt MS62. A shows the results of the batch melt model; B shows the results of the fractional melt model. The melt percentages are approximately $\pm 20 \%$ that observed in the experimental melt. The trace element concentrations of the parent material, sample 50M2A (50/50 mixture of mafic glass and biotite fels), are indicated by the black diamonds. The trace element concentrations of the Dale Tuff, sample $\mathrm{CH} 31$, which is included for comparison, are indicated by the yellow squares. For experimental melt MS62, it can be seen that the batch melt model (A) approximates the data with a notable exception at $\mathrm{Ba}$. The fit increases as melt percent increases. 


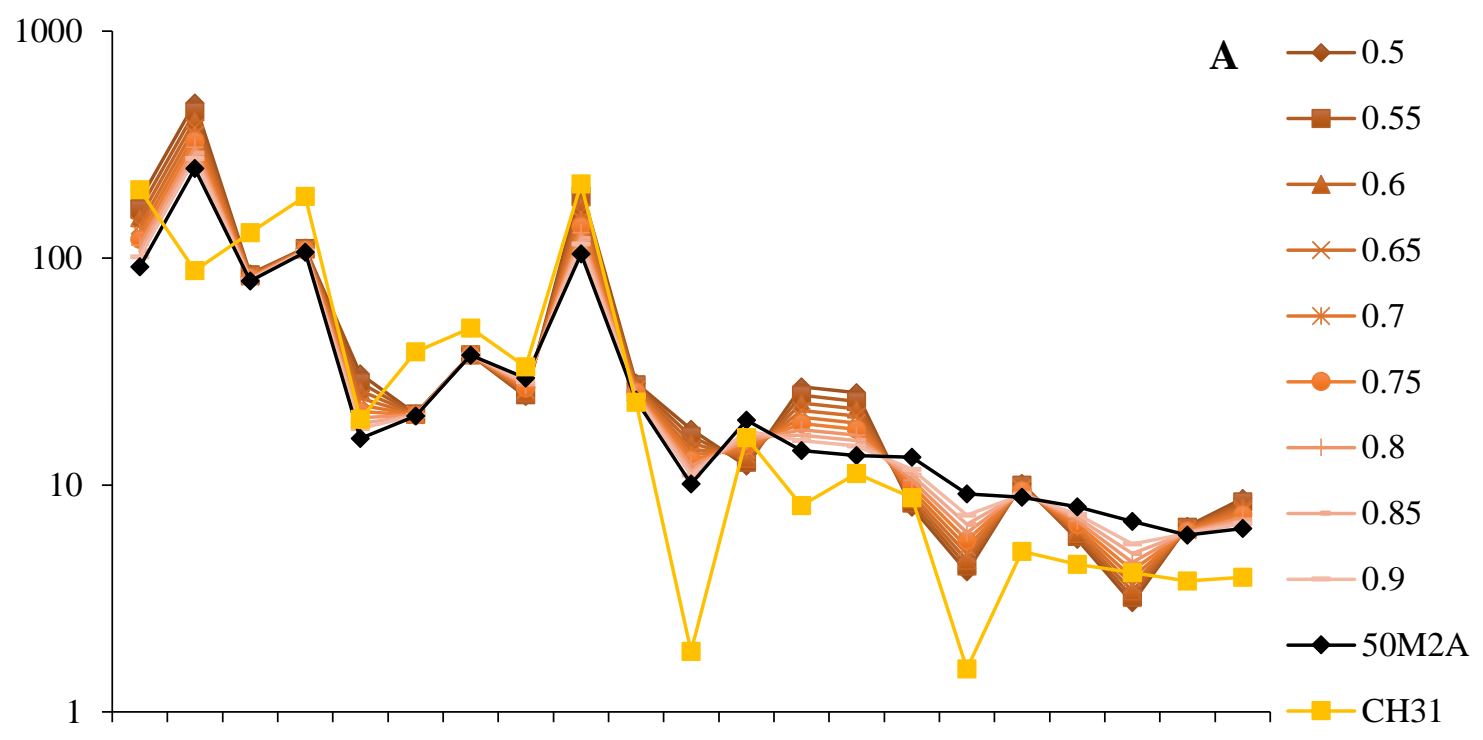

$\mathrm{Rb} \mathrm{Ba}$ Th $\mathrm{U}$ Nb Ta $\mathrm{La} \mathrm{Ce} \mathrm{Pb}$ Pr $\mathrm{Sr} \mathrm{Nd} \mathrm{Zr} \mathrm{Hf} \mathrm{Sm} \mathrm{Eu}$ Tb Dy Y Yb Lu

B

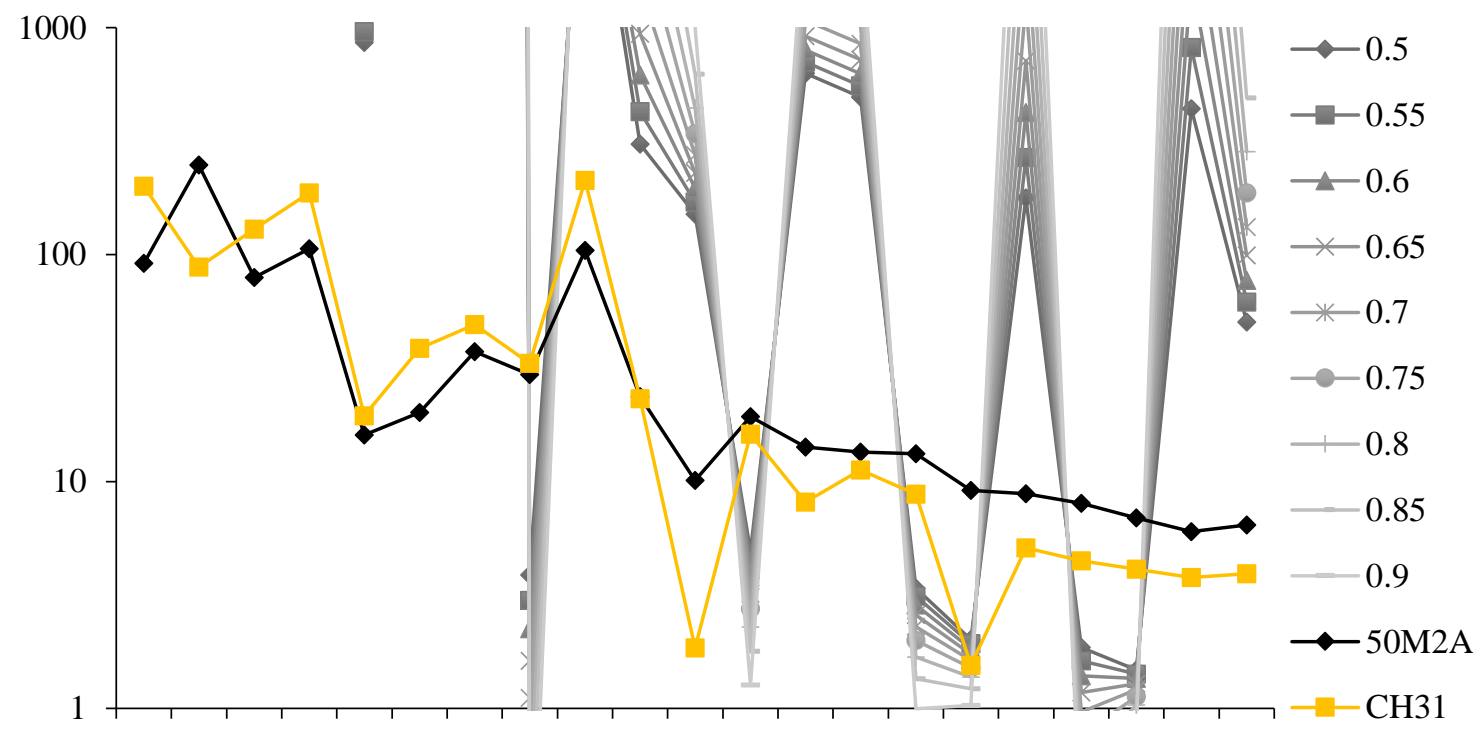

$\mathrm{Rb} \mathrm{Ba}$ Th $\mathrm{U}$ Nb Ta La Ce Pb Pr Sr Nd Zr Hf Sm Eu Tb Dy Y Yb Lu

Fig. D46 Primitive mantle normalized (Sun and McDonough, 1989) spider diagram for the partial melting of experimental melt MS63. A shows the results of the batch melt model; B shows the results of the fractional melt model. The melt percentages are approximately $\pm 20 \%$ that observed in the experimental melt. The trace element concentrations of the parent material, sample 50M2A (50/50 mixture of mafic glass and biotite fels), are indicated by the black diamonds. The trace element concentrations of the Dale Tuff, sample $\mathrm{CH} 31$, which is included for comparison, are indicated by the yellow squares. For experimental melt MS63, it can be seen that the batch melt model (A) approximates the data. The fit increases as melt percent increases. 


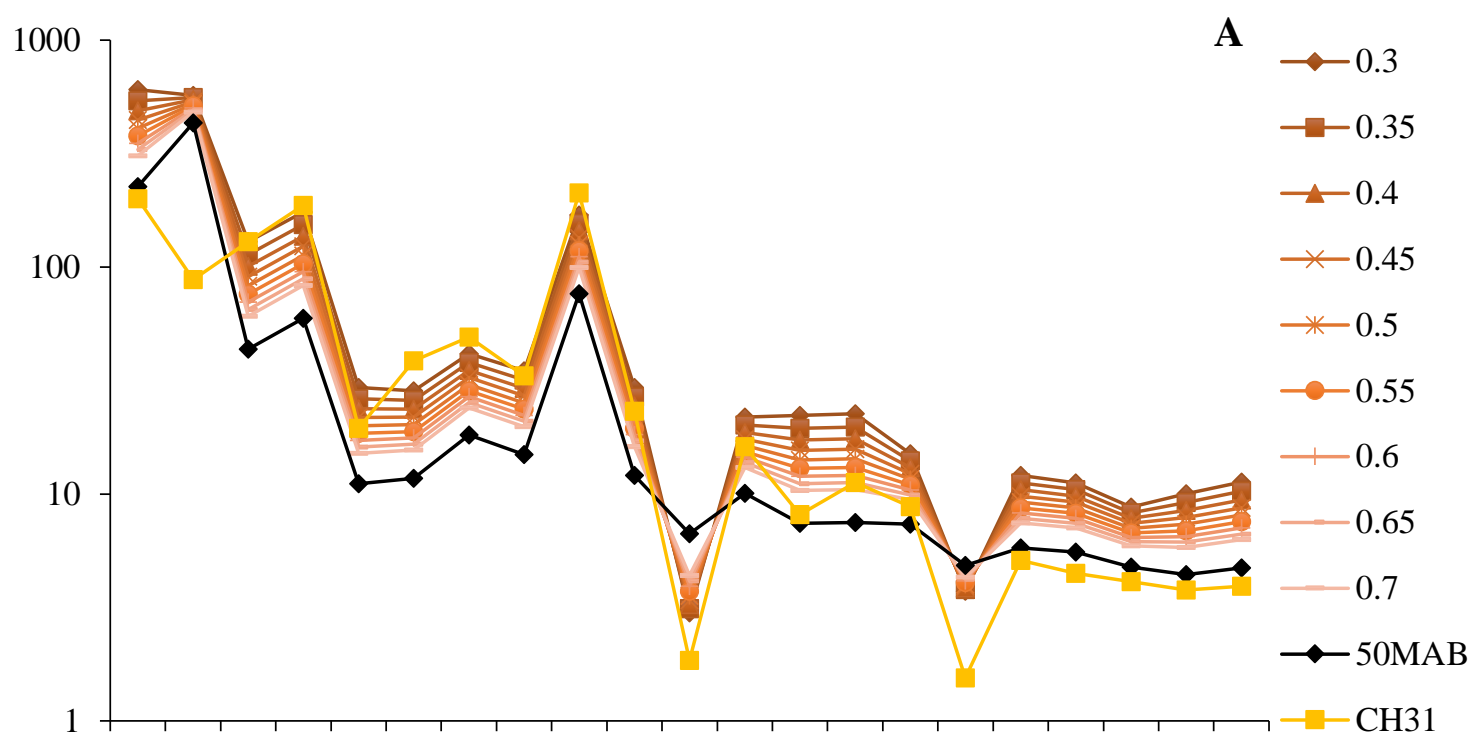

$\mathrm{Rb} \mathrm{Ba}$ Th $\mathrm{U}$ Nb Ta $\mathrm{La} \mathrm{Ce} \mathrm{Pb} \operatorname{Pr} \mathrm{Sr} \mathrm{Nd} \mathrm{Zr} \mathrm{Hf} \mathrm{Sm} \mathrm{Eu} \mathrm{Tb}$ Dy Y Yb Lu

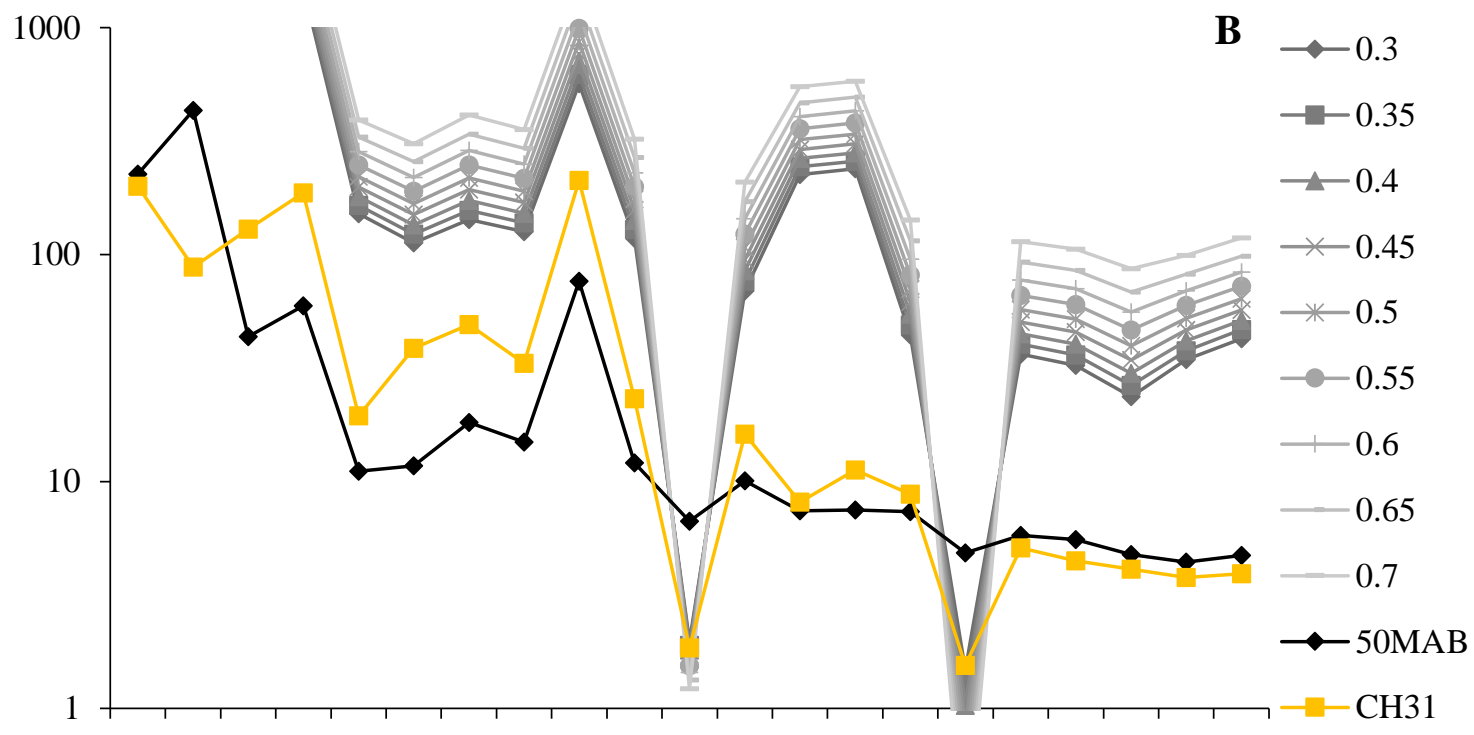

$\mathrm{Rb} \mathrm{Ba}$ Th $\mathrm{U}$ Nb Ta La Ce Pb Pr Sr Nd Zr Hf Sm Eu Tb Dy Y Yb Lu

Fig. D47 Primitive mantle normalized (Sun and McDonough, 1989) spider diagram for the partial melting of experimental melt MS65. A shows the results of the batch melt model; B shows the results of the fractional melt model. The melt percentages are approximately $\pm 20 \%$ that observed in the experimental melt. The trace element concentrations of the parent material, sample 50MAB (50/50 mixture of biotite fels and biotite schist), are indicated by the black diamonds. The trace element concentrations of the Dale Tuff, sample $\mathrm{CH} 31$, which is included for comparison, are indicated by the yellow squares. For experimental melt MS65, it can be seen that the batch melt model (A) approximates the data. The fit increases as melt percent increases. 
Table D1: Results of batch partial melting calculations

\begin{tabular}{|c|c|c|c|c|c|c|c|c|c|c|}
\hline MS1 & Element & & & & & elt Fraction & & & & \\
\hline & & 0.55 & 0.60 & 0.65 & 0.70 & 0.75 & 0.80 & 0.85 & 0.90 & 0.95 \\
\hline & $\mathrm{Rb}$ & 119.042 & 109.204 & 100.868 & 93.715 & 87.509 & 82.074 & 77.274 & 73.005 & 69.183 \\
\hline & $\mathrm{Ba}$ & 4496.786 & 4125.166 & 3810.280 & 3540.057 & 3305.624 & 3100.312 & 2919.012 & 2757.746 & 2613.365 \\
\hline & Th & 12.788 & 11.731 & 10.836 & 10.067 & 9.400 & 8.817 & 8.301 & 7.842 & 7.432 \\
\hline & $\mathrm{U}$ & 4.148 & 3.805 & 3.514 & 3.265 & 3.049 & 2.860 & 2.692 & 2.544 & 2.410 \\
\hline & $\mathrm{Nb}$ & 13.098 & 12.015 & 11.098 & 10.311 & 9.628 & 9.030 & 8.502 & 8.032 & 7.612 \\
\hline & $\mathrm{Ta}$ & 1.141 & 1.047 & 0.967 & 0.898 & 0.839 & 0.787 & 0.741 & 0.700 & 0.663 \\
\hline & $\mathrm{La}$ & 35.950 & 32.984 & 30.471 & 28.313 & 26.441 & 24.801 & 23.352 & 22.063 & 20.910 \\
\hline & $\mathrm{Ce}$ & 72.835 & 66.827 & 61.734 & 57.362 & 53.569 & 50.246 & 47.311 & 44.701 & 42.363 \\
\hline & $\mathrm{Pb}$ & 9.877 & 9.062 & 8.371 & 7.778 & 7.264 & 6.813 & 6.415 & 6.061 & 5.744 \\
\hline & $\operatorname{Pr}$ & 8.464 & 7.767 & 7.176 & 6.669 & 6.228 & 5.842 & 5.502 & 5.198 & 4.927 \\
\hline & $\mathrm{Sr}$ & 131.268 & 120.431 & 111.247 & 103.365 & 96.525 & 90.535 & 85.245 & 80.538 & 76.325 \\
\hline & $\mathrm{Nd}$ & 31.118 & 28.559 & 26.390 & 24.527 & 22.909 & 21.492 & 20.240 & 19.126 & 18.128 \\
\hline & $\mathrm{Zr}$ & 160.053 & 146.941 & 135.814 & 126.254 & 117.952 & 110.674 & 104.242 & 98.516 & 93.387 \\
\hline & $\mathrm{Hf}$ & 4.307 & 3.954 & 3.654 & 3.397 & 3.174 & 2.978 & 2.805 & 2.651 & 2.513 \\
\hline & $\mathrm{Sm}$ & 6.005 & 5.514 & 5.098 & 4.740 & 4.430 & 4.157 & 3.916 & 3.701 & 3.509 \\
\hline 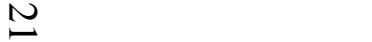 & $\mathrm{Eu}$ & 1.081 & 0.993 & 0.919 & 0.855 & 0.799 & 0.750 & 0.707 & 0.668 & 0.634 \\
\hline$\vec{A}$ & $\mathrm{~Tb}$ & 0.847 & 0.779 & 0.721 & 0.671 & 0.628 & 0.590 & 0.556 & 0.526 & 0.499 \\
\hline & Dy & 5.213 & 4.808 & 4.461 & 4.161 & 3.899 & 3.668 & 3.463 & 3.279 & 3.114 \\
\hline & Y & 26.249 & 24.363 & 22.730 & 21.302 & 20.043 & 18.924 & 17.924 & 17.024 & 16.211 \\
\hline & $\mathrm{Yb}$ & 2.738 & 2.562 & 2.407 & 2.270 & 2.147 & 2.037 & 1.938 & 1.848 & 1.766 \\
\hline & $\mathrm{Lu}$ & 0.451 & 0.422 & 0.396 & 0.373 & 0.352 & 0.334 & 0.318 & 0.303 & 0.289 \\
\hline MS3 & Element & & & & & elt Fraction & & & & \\
\hline & & 0.01 & 0.05 & 0.10 & 0.15 & 0.20 & 0.25 & 0.30 & 0.35 & 0.40 \\
\hline & $\mathrm{Rb}$ & 60.956 & 21.461 & 11.857 & 8.192 & 6.257 & 5.062 & 4.250 & 3.663 & 3.218 \\
\hline & $\mathrm{Ba}$ & 404.318 & 294.225 & 219.511 & 175.057 & 145.576 & 124.594 & 108.898 & 96.714 & 86.983 \\
\hline & Th & 6.375 & 2.132 & 1.164 & 0.800 & 0.610 & 0.493 & 0.413 & 0.356 & 0.312 \\
\hline & $\mathrm{U}$ & 3.489 & 1.167 & 0.637 & 0.438 & 0.334 & 0.270 & 0.226 & 0.195 & 0.171 \\
\hline & $\mathrm{Nb}$ & 69.870 & 22.696 & 12.308 & 8.444 & 6.426 & 5.187 & 4.348 & 3.743 & 3.286 \\
\hline & $\mathrm{Ta}$ & 4.441 & 1.443 & 0.782 & 0.537 & 0.408 & 0.330 & 0.276 & 0.238 & 0.209 \\
\hline & $\mathrm{La}$ & 20.868 & 18.303 & 15.866 & 14.002 & 12.529 & 11.337 & 10.352 & 9.525 & 8.820 \\
\hline & $\mathrm{Ce}$ & 27.920 & 26.644 & 25.204 & 23.911 & 22.745 & 21.687 & 20.723 & 19.841 & 19.031 \\
\hline & $\mathrm{Pb}$ & 4.588 & 3.347 & 2.501 & 1.997 & 1.662 & 1.423 & 1.244 & 1.105 & 0.994 \\
\hline & $\operatorname{Pr}$ & 2.283 & 2.269 & 2.252 & 2.236 & 2.219 & 2.203 & 2.187 & 2.172 & 2.156 \\
\hline & $\mathrm{Sr}$ & 277.433 & 279.052 & 281.101 & 283.182 & 285.293 & 287.436 & 289.611 & 291.819 & 294.062 \\
\hline & $\mathrm{Nd}$ & 5.882 & 5.981 & 6.110 & 6.244 & 6.385 & 6.532 & 6.686 & 6.848 & 7.017 \\
\hline & $\mathrm{Zr}$ & 214.710 & 180.933 & 151.201 & 129.862 & 113.801 & 101.275 & 91.233 & 83.003 & 76.135 \\
\hline & $\mathrm{Hf}$ & 6.407 & 5.399 & 4.512 & 3.875 & 3.396 & 3.022 & 2.722 & 2.477 & 2.272 \\
\hline
\end{tabular}




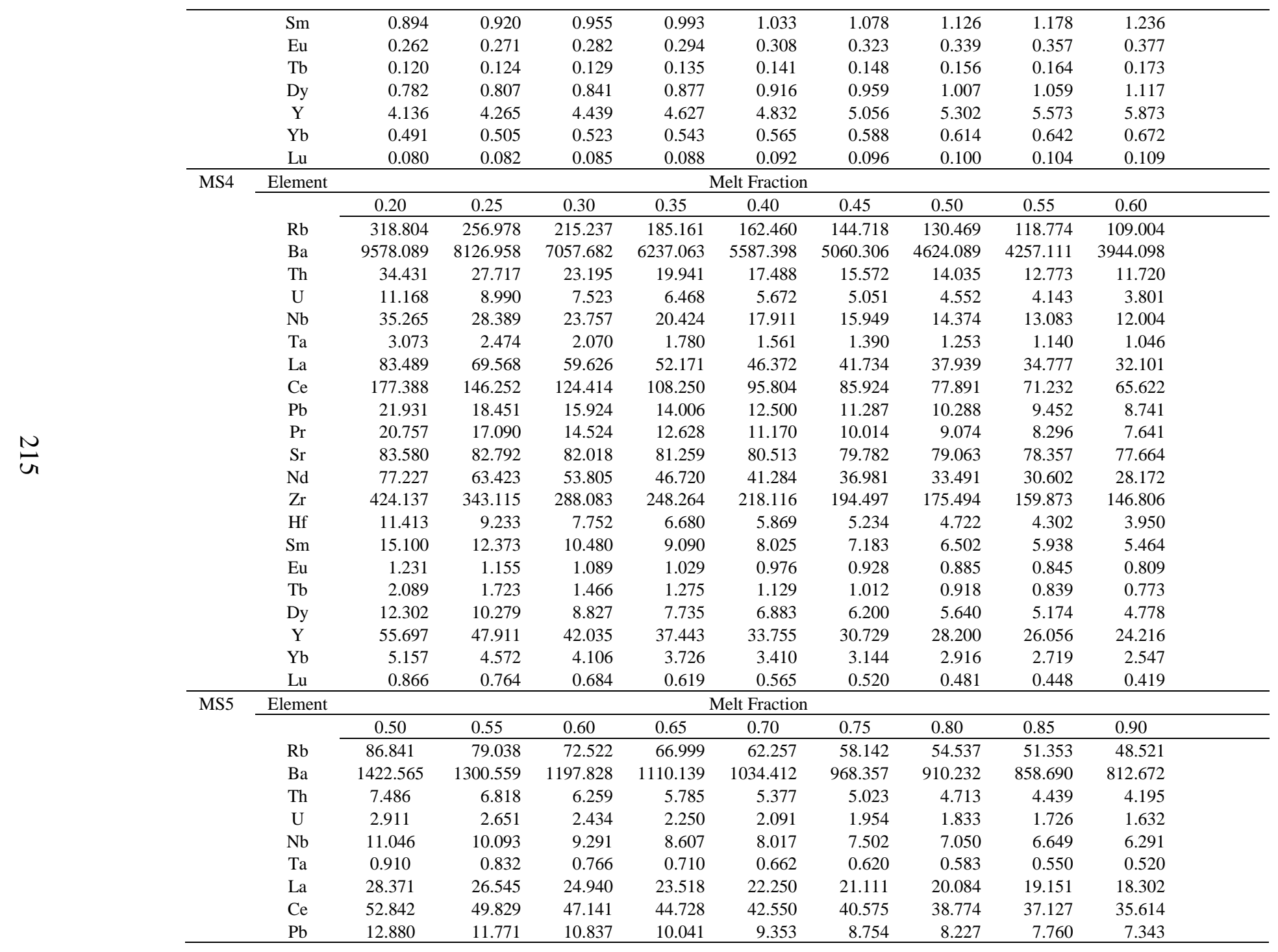




\begin{tabular}{|c|c|c|c|c|c|c|c|c|c|c|c|c|}
\hline & & $\operatorname{Pr}$ & 6.551 & 6.189 & 5.864 & 5.571 & 5.307 & 5.066 & 4.847 & 4.645 & 4.460 & \\
\hline & & $\mathrm{Sr}$ & 860.398 & 819.509 & 782.329 & 748.377 & 717.249 & 688.607 & 662.165 & 637.679 & 614.939 & \\
\hline & & $\mathrm{Nd}$ & 24.275 & 23.204 & 22.224 & 21.323 & 20.492 & 19.724 & 19.011 & 18.348 & 17.730 & \\
\hline & & $\mathrm{Zr}$ & 192.395 & 175.809 & 161.856 & 149.955 & 139.684 & 130.730 & 122.855 & 115.875 & 109.645 & \\
\hline & & $\mathrm{Hf}$ & 5.231 & 4.779 & 4.400 & 4.076 & 3.796 & 3.553 & 3.339 & 3.149 & 2.979 & \\
\hline & & $\mathrm{Sm}$ & 4.822 & 4.653 & 4.496 & 4.349 & 4.212 & 4.083 & 3.961 & 3.847 & 3.739 & \\
\hline & & $\mathrm{Eu}$ & 1.267 & 1.244 & 1.222 & 1.201 & 1.180 & 1.160 & 1.141 & 1.122 & 1.104 & \\
\hline & & $\mathrm{Tb}$ & 0.680 & 0.653 & 0.628 & 0.605 & 0.583 & 0.563 & 0.544 & 0.527 & 0.510 & \\
\hline & & Dy & 4.341 & 4.127 & 3.933 & 3.757 & 3.596 & 3.448 & 3.312 & 3.186 & 3.069 & \\
\hline & & Y & 17.947 & 17.600 & 17.266 & 16.944 & 16.634 & 16.336 & 16.048 & 15.769 & 15.501 & \\
\hline & & $\mathrm{Yb}$ & 2.107 & 2.006 & 1.914 & 1.830 & 1.753 & 1.682 & 1.617 & 1.557 & 1.501 & \\
\hline & & $\mathrm{Lu}$ & 0.369 & 0.348 & 0.329 & 0.312 & 0.297 & 0.283 & 0.271 & 0.259 & 0.249 & \\
\hline & MS6 & Element & & & & & elt Fraction & & & & & \\
\hline & & & 0.45 & 0.50 & 0.55 & 0.60 & 0.65 & 0.70 & 0.75 & 0.80 & 0.85 & \\
\hline & & $\mathrm{Rb}$ & 2.824 & 2.552 & 2.328 & 2.140 & 1.980 & 1.842 & 1.722 & 1.617 & 1.524 & \\
\hline & & $\mathrm{Ba}$ & 55.419 & 53.376 & 51.479 & 49.711 & 48.061 & 46.517 & 45.069 & 43.709 & 42.428 & \\
\hline & & Th & 0.284 & 0.256 & 0.234 & 0.215 & 0.198 & 0.185 & 0.172 & 0.162 & 0.153 & \\
\hline & & $\mathrm{U}$ & 0.153 & 0.138 & 0.126 & 0.116 & 0.107 & 0.099 & 0.093 & 0.087 & 0.082 & \\
\hline & & $\mathrm{Nb}$ & 2.921 & 2.635 & 2.399 & 2.203 & 2.036 & 1.892 & 1.768 & 1.659 & 1.562 & \\
\hline 근 & & $\mathrm{Ta}$ & 0.176 & 0.158 & 0.144 & 0.132 & 0.122 & 0.114 & 0.106 & 0.100 & 0.094 & \\
\hline$a$ & & $\mathrm{La}$ & 6.370 & 6.166 & 5.975 & 5.795 & 5.626 & 5.466 & 5.315 & 5.172 & 5.037 & \\
\hline & & $\mathrm{Ce}$ & 17.310 & 16.768 & 16.259 & 15.780 & 15.328 & 14.902 & 14.498 & 14.116 & 13.754 & \\
\hline & & $\mathrm{Pb}$ & 0.689 & 0.657 & 0.628 & 0.602 & 0.577 & 0.555 & 0.534 & 0.515 & 0.497 & \\
\hline & & $\operatorname{Pr}$ & 2.689 & 2.606 & 2.528 & 2.454 & 2.385 & 2.319 & 2.257 & 2.198 & 2.142 & \\
\hline & & $\mathrm{Sr}$ & 92.798 & 99.236 & 106.633 & 115.222 & 125.316 & 137.348 & 151.936 & 169.991 & 192.916 & \\
\hline & & $\mathrm{Nd}$ & 12.256 & 12.007 & 11.768 & 11.538 & 11.318 & 11.105 & 10.900 & 10.703 & 10.512 & \\
\hline & & $\mathrm{Zr}$ & 82.970 & 74.911 & 68.279 & 62.725 & 58.007 & 53.950 & 50.422 & 47.328 & 44.591 & \\
\hline & & $\mathrm{Hf}$ & 2.488 & 2.246 & 2.048 & 1.881 & 1.740 & 1.618 & 1.512 & 1.420 & 1.338 & \\
\hline & & $\mathrm{Sm}$ & 3.446 & 3.400 & 3.355 & 3.312 & 3.269 & 3.228 & 3.188 & 3.148 & 3.110 & \\
\hline & & $\mathrm{Eu}$ & 0.533 & 0.561 & 0.591 & 0.625 & 0.662 & 0.705 & 0.754 & 0.810 & 0.874 & \\
\hline & & $\mathrm{Tb}$ & 0.683 & 0.668 & 0.654 & 0.640 & 0.628 & 0.615 & 0.603 & 0.592 & 0.581 & \\
\hline & & Dy & 4.614 & 4.448 & 4.293 & 4.149 & 4.014 & 3.888 & 3.769 & 3.657 & 3.552 & \\
\hline & & $\mathrm{Y}$ & 17.275 & 17.212 & 17.149 & 17.086 & 17.024 & 16.962 & 16.900 & 16.839 & 16.779 & \\
\hline & & $\mathrm{Yb}$ & 2.486 & 2.358 & 2.244 & 2.139 & 2.044 & 1.958 & 1.878 & 1.804 & 1.736 & \\
\hline & & $\mathrm{Lu}$ & 0.422 & 0.395 & 0.371 & 0.350 & 0.331 & 0.314 & 0.298 & 0.285 & 0.272 & \\
\hline & MS8 & Element & & & & & Melt Fi & action & & & & \\
\hline & & & 0.25 & 0.30 & 0.35 & 0.40 & 0.45 & 0.50 & 0.55 & 0.60 & 0.65 & 0.70 \\
\hline & & $\mathrm{Rb}$ & 167.695 & 141.014 & 121.657 & 106.973 & 95.452 & 86.171 & 78.535 & 72.142 & 66.712 & 62.042 \\
\hline & & $\mathrm{Ba}$ & 2828.221 & 2375.935 & 2048.363 & 1800.172 & 1605.625 & 1449.027 & 1320.261 & 1212.512 & 1121.024 & 1042.373 \\
\hline & & Th & 3.275 & 3.304 & 3.334 & 3.365 & 3.396 & 3.427 & 3.459 & 3.492 & 3.526 & 3.560 \\
\hline & & $\mathrm{U}$ & 0.341 & 0.359 & 0.380 & 0.403 & 0.429 & 0.458 & 0.492 & 0.532 & 0.578 & 0.633 \\
\hline
\end{tabular}




\begin{tabular}{|c|c|c|c|c|c|c|c|c|c|c|c|c|}
\hline \multirow{40}{*}{$\stackrel{N}{=}$} & & $\mathrm{Nb}$ & 19.948 & 17.089 & 14.947 & 13.283 & 11.952 & 10.863 & 9.956 & 9.189 & 8.532 & 7.962 \\
\hline & & $\mathrm{Ta}$ & 1.550 & 1.345 & 1.188 & 1.064 & 0.963 & 0.880 & 0.810 & 0.750 & 0.699 & 0.654 \\
\hline & & $\mathrm{La}$ & 16.648 & 16.659 & 16.670 & 16.681 & 16.692 & 16.703 & 16.714 & 16.725 & 16.736 & 16.747 \\
\hline & & $\mathrm{Ce}$ & 24.429 & 24.857 & 25.300 & 25.759 & 26.235 & 26.729 & 27.241 & 27.774 & 28.328 & 28.905 \\
\hline & & $\mathrm{Pb}$ & 25.294 & 21.296 & 18.389 & 16.180 & 14.445 & 13.046 & 11.895 & 10.930 & 10.109 & 9.404 \\
\hline & & $\operatorname{Pr}$ & 2.834 & 2.895 & 2.958 & 3.024 & 3.093 & 3.165 & 3.240 & 3.319 & 3.403 & 3.490 \\
\hline & & $\mathrm{Sr}$ & 1793.244 & 1570.557 & 1397.068 & 1258.095 & 1144.269 & 1049.330 & 968.939 & 899.989 & 840.200 & 787.860 \\
\hline & & $\mathrm{Nd}$ & 8.170 & 8.456 & 8.764 & 9.094 & 9.451 & 9.836 & 10.254 & 10.710 & 11.208 & 11.754 \\
\hline & & $\mathrm{Zr}$ & 1.445 & 1.547 & 1.664 & 1.800 & 1.960 & 2.152 & 2.386 & 2.676 & 3.046 & 3.536 \\
\hline & & $\mathrm{Hf}$ & 0.079 & 0.085 & 0.091 & 0.098 & 0.107 & 0.117 & 0.130 & 0.145 & 0.164 & 0.190 \\
\hline & & Sm & 1.311 & 1.368 & 1.431 & 1.500 & 1.575 & 1.659 & 1.752 & 1.857 & 1.974 & 2.107 \\
\hline & & $\mathrm{Eu}$ & 0.340 & 0.356 & 0.374 & 0.394 & 0.416 & 0.440 & 0.468 & 0.499 & 0.535 & 0.576 \\
\hline & & $\mathrm{Tb}$ & 0.152 & 0.159 & 0.167 & 0.176 & 0.186 & 0.197 & 0.209 & 0.223 & 0.239 & 0.257 \\
\hline & & Dy & 0.864 & 0.906 & 0.953 & 1.004 & 1.062 & 1.126 & 1.199 & 1.282 & 1.377 & 1.487 \\
\hline & & Y & 2.642 & 2.796 & 2.968 & 3.163 & 3.386 & 3.642 & 3.941 & 4.292 & 4.712 & 5.224 \\
\hline & & $\mathrm{Yb}$ & 0.144 & 0.153 & 0.163 & 0.175 & 0.189 & 0.205 & 0.224 & 0.247 & 0.276 & 0.312 \\
\hline & & $\mathrm{Lu}$ & 0.016 & 0.017 & 0.019 & 0.020 & 0.022 & 0.024 & 0.026 & 0.029 & 0.032 & 0.037 \\
\hline & MS9 & Element & \multicolumn{10}{|c|}{ Melt Fraction } \\
\hline & & & 0.25 & 0.30 & 0.35 & 0.40 & 0.45 & 0.50 & 0.55 & 0.60 & 0.65 & \\
\hline & & $\mathrm{Rb}$ & 5.058 & 4.241 & 3.651 & 3.205 & 2.856 & 2.576 & 2.346 & 2.153 & 1.990 & \\
\hline & & $\mathrm{Ba}$ & 151.771 & 127.242 & 109.539 & 96.160 & 85.694 & 77.282 & 70.374 & 64.600 & 59.701 & \\
\hline & & Th & 0.505 & 0.424 & 0.365 & 0.320 & 0.286 & 0.258 & 0.235 & 0.215 & 0.199 & \\
\hline & & $\mathrm{U}$ & 0.272 & 0.228 & 0.196 & 0.173 & 0.154 & 0.139 & 0.126 & 0.116 & 0.107 & \\
\hline & & $\mathrm{Nb}$ & 5.189 & 4.348 & 3.742 & 3.284 & 2.925 & 2.638 & 2.402 & 2.204 & 2.037 & \\
\hline & & $\mathrm{Ta}$ & 0.312 & 0.262 & 0.225 & 0.198 & 0.176 & 0.159 & 0.144 & 0.133 & 0.123 & \\
\hline & & $\mathrm{La}$ & 11.791 & 10.703 & 9.799 & 9.035 & 8.382 & 7.817 & 7.324 & 6.889 & 6.503 & \\
\hline & & $\mathrm{Ce}$ & 21.325 & 20.414 & 19.577 & 18.806 & 18.093 & 17.432 & 16.818 & 16.246 & 15.712 & \\
\hline & & $\mathrm{Pb}$ & 1.664 & 1.411 & 1.224 & 1.081 & 0.968 & 0.876 & 0.800 & 0.737 & 0.682 & \\
\hline & & $\operatorname{Pr}$ & 2.144 & 2.133 & 2.122 & 2.111 & 2.100 & 2.090 & 2.079 & 2.069 & 2.059 & \\
\hline & & $\mathrm{Sr}$ & 780.503 & 713.485 & 657.066 & 608.915 & 567.340 & 531.080 & 499.176 & 470.888 & 445.634 & \\
\hline & & $\mathrm{Nd}$ & 6.260 & 6.420 & 6.588 & 6.764 & 6.951 & 7.148 & 7.357 & 7.578 & 7.813 & \\
\hline & & $\mathrm{Zr}$ & 99.108 & 89.512 & 81.610 & 74.990 & 69.363 & 64.522 & 60.312 & 56.619 & 53.351 & \\
\hline & & $\mathrm{Hf}$ & 2.973 & 2.685 & 2.448 & 2.250 & 2.081 & 1.936 & 1.809 & 1.699 & 1.601 & \\
\hline & & $\mathrm{Sm}$ & 1.029 & 1.076 & 1.128 & 1.184 & 1.247 & 1.317 & 1.396 & 1.484 & 1.584 & \\
\hline & & $\mathrm{Eu}$ & 0.333 & 0.350 & 0.368 & 0.389 & 0.411 & 0.437 & 0.466 & 0.499 & 0.537 & \\
\hline & & $\mathrm{Tb}$ & 0.141 & 0.148 & 0.157 & 0.166 & 0.176 & 0.188 & 0.201 & 0.216 & 0.234 & \\
\hline & & Dy & 0.917 & 0.963 & 1.015 & 1.071 & 1.135 & 1.207 & 1.288 & 1.381 & 1.488 & \\
\hline & & $\mathrm{Y}$ & 4.776 & 5.015 & 5.278 & 5.570 & 5.896 & 6.264 & 6.679 & 7.155 & 7.702 & \\
\hline & & $\mathrm{Yb}$ & 0.557 & 0.582 & 0.609 & 0.639 & 0.672 & 0.708 & 0.749 & 0.795 & 0.847 & \\
\hline & & $\mathrm{Lu}$ & 0.090 & 0.094 & 0.098 & 0.103 & 0.108 & 0.114 & 0.120 & 0.127 & 0.135 & \\
\hline & MS13 & Element & \multicolumn{10}{|c|}{ Melt Fraction } \\
\hline
\end{tabular}




\begin{tabular}{|c|c|c|c|c|c|c|c|c|c|c|c|}
\hline \multirow{41}{*}{$\frac{N}{\infty}$} & & & 0.50 & 0.55 & 0.60 & 0.65 & 0.70 & 0.75 & 0.80 & 0.85 & 0.90 \\
\hline & & $\mathrm{Rb}$ & 86.211 & 78.568 & 72.170 & 66.735 & 62.062 & 58.000 & 54.437 & 51.287 & 48.481 \\
\hline & & $\mathrm{Ba}$ & 1133.557 & 1075.036 & 1022.262 & 974.426 & 930.868 & 891.036 & 854.474 & 820.794 & 789.669 \\
\hline & & Th & 7.501 & 6.828 & 6.267 & 5.791 & 5.382 & 5.027 & 4.716 & 4.441 & 4.196 \\
\hline & & $\mathrm{U}$ & 2.917 & 2.655 & 2.437 & 2.252 & 2.093 & 1.955 & 1.834 & 1.727 & 1.632 \\
\hline & & $\mathrm{Nb}$ & 11.271 & 10.261 & 9.417 & 8.701 & 8.087 & 7.553 & 7.086 & 6.673 & 6.306 \\
\hline & & $\mathrm{Ta}$ & 0.933 & 0.849 & 0.779 & 0.720 & 0.669 & 0.625 & 0.586 & 0.552 & 0.522 \\
\hline & & $\mathrm{La}$ & 28.493 & 26.642 & 25.016 & 23.577 & 22.295 & 21.145 & 20.108 & 19.168 & 18.312 \\
\hline & & $\mathrm{Ce}$ & 58.734 & 54.466 & 50.776 & 47.555 & 44.718 & 42.200 & 39.951 & 37.929 & 36.102 \\
\hline & & $\mathrm{Pb}$ & 10.710 & 10.089 & 9.536 & 9.041 & 8.594 & 8.190 & 7.822 & 7.485 & 7.177 \\
\hline & & $\operatorname{Pr}$ & 7.430 & 6.880 & 6.406 & 5.993 & 5.631 & 5.309 & 5.022 & 4.765 & 4.533 \\
\hline & & $\mathrm{Sr}$ & 254.042 & 269.039 & 285.917 & 305.055 & 326.939 & 352.205 & 381.703 & 416.594 & 458.505 \\
\hline & & $\mathrm{Nd}$ & 30.305 & 27.997 & 26.015 & 24.296 & 22.789 & 21.459 & 20.275 & 19.215 & 18.260 \\
\hline & & $\mathrm{Zr}$ & 194.445 & 177.347 & 163.013 & 150.823 & 140.329 & 131.200 & 123.187 & 116.096 & 109.777 \\
\hline & & $\mathrm{Hf}$ & 5.283 & 4.819 & 4.429 & 4.098 & 3.813 & 3.565 & 3.347 & 3.155 & 2.983 \\
\hline & & $\mathrm{Sm}$ & 6.585 & 6.064 & 5.618 & 5.234 & 4.899 & 4.605 & 4.343 & 4.110 & 3.901 \\
\hline & & $\mathrm{Eu}$ & 0.860 & 0.877 & 0.895 & 0.913 & 0.933 & 0.953 & 0.975 & 0.997 & 1.020 \\
\hline & & $\mathrm{Tb}$ & 0.885 & 0.817 & 0.758 & 0.706 & 0.662 & 0.623 & 0.588 & 0.556 & 0.528 \\
\hline & & Dy & 5.128 & 4.751 & 4.426 & 4.142 & 3.893 & 3.672 & 3.475 & 3.297 & 3.137 \\
\hline & & $\mathrm{Y}$ & 24.846 & 23.313 & 21.958 & 20.752 & 19.672 & 18.699 & 17.817 & 17.015 & 16.282 \\
\hline & & $\mathrm{Yb}$ & 2.104 & 2.003 & 1.912 & 1.828 & 1.752 & 1.681 & 1.616 & 1.556 & 1.500 \\
\hline & & $\mathrm{Lu}$ & 0.351 & 0.303 & 0.318 & 0.303 & 0.290 & 0.278 & 0.267 & 0.257 & 0.247 \\
\hline & MS15 & Element & \multicolumn{9}{|c|}{ Melt Fraction } \\
\hline & & & 0.20 & 0.25 & 0.30 & 0.35 & 0.40 & 0.45 & 0.50 & 0.55 & 0.60 \\
\hline & & $\mathrm{Rb}$ & 321.454 & 258.549 & 216.235 & 185.823 & 162.911 & 145.029 & 130.684 & 118.921 & 109.101 \\
\hline & & $\mathrm{Ba}$ & 11594.376 & 9431.352 & 7948.497 & 6868.577 & 6047.002 & 5400.973 & 4879.657 & 4450.120 & 4090.085 \\
\hline & & Th & 34.586 & 27.810 & 23.253 & 19.980 & 17.514 & 15.591 & 14.048 & 12.782 & 11.726 \\
\hline & & $\mathrm{U}$ & 11.219 & 9.020 & 7.543 & 6.481 & 5.681 & 5.057 & 4.556 & 4.146 & 3.804 \\
\hline & & $\mathrm{Nb}$ & 31.492 & 26.032 & 22.186 & 19.330 & 17.125 & 15.372 & 13.944 & 12.759 & 11.760 \\
\hline & & Ta & 2.744 & 2.268 & 1.933 & 1.684 & 1.492 & 1.339 & 1.215 & 1.112 & 1.025 \\
\hline & & $\mathrm{La}$ & 91.495 & 74.672 & 63.074 & 54.595 & 48.126 & 43.027 & 38.905 & 35.504 & 32.649 \\
\hline & & $\mathrm{Ce}$ & 177.000 & 146.007 & 124.250 & 108.136 & 95.722 & 85.865 & 77.848 & 71.201 & 65.599 \\
\hline & & $\mathrm{Pb}$ & 26.469 & 21.337 & 17.873 & 15.376 & 13.491 & 12.018 & 10.835 & 9.864 & 9.053 \\
\hline & & $\operatorname{Pr}$ & 19.233 & 16.103 & 13.850 & 12.149 & 10.821 & 9.754 & 8.879 & 8.148 & 7.528 \\
\hline & & $\mathrm{Sr}$ & 285.642 & 241.648 & 209.397 & 184.742 & 165.280 & 149.529 & 136.518 & 125.590 & 116.282 \\
\hline & & $\mathrm{Nd}$ & 67.139 & 56.847 & 49.292 & 43.509 & 38.940 & 35.240 & 32.182 & 29.612 & 27.423 \\
\hline & & $\mathrm{Zr}$ & 399.450 & 327.952 & 278.164 & 241.500 & 213.376 & 191.119 & 173.066 & 158.130 & 145.567 \\
\hline & & $\mathrm{Hf}$ & 10.739 & 8.815 & 7.475 & 6.489 & 5.733 & 5.134 & 4.649 & 4.248 & 3.910 \\
\hline & & $\mathrm{Sm}$ & 10.780 & 9.462 & 8.432 & 7.604 & 6.924 & 6.356 & 5.874 & 5.459 & 5.100 \\
\hline & & $\mathrm{Eu}$ & 1.806 & 1.605 & 1.443 & 1.312 & 1.202 & 1.109 & 1.030 & 0.961 & 0.901 \\
\hline & & $\mathrm{Tb}$ & 1.382 & 1.233 & 1.112 & 1.013 & 0.931 & 0.860 & 0.800 & 0.748 & 0.701 \\
\hline
\end{tabular}




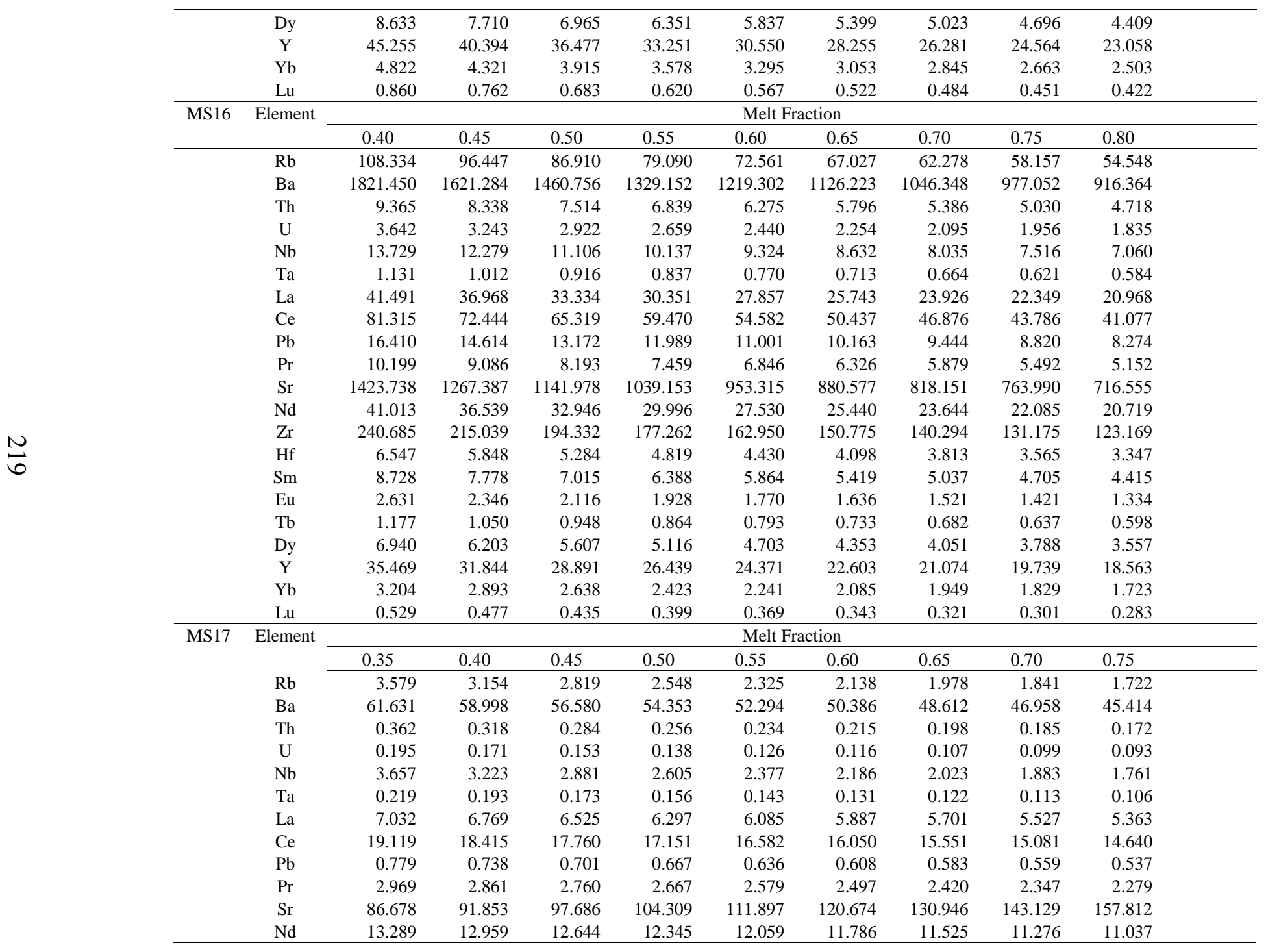




\begin{tabular}{|c|c|c|c|c|c|c|c|c|c|c|}
\hline & $\mathrm{Zr}$ & 104.244 & 91.918 & 82.199 & 74.338 & 67.85 & 62.403 & 57.766 & 53.771 & 50.292 \\
\hline & $\mathrm{Hf}$ & 3.127 & 2.757 & 2.466 & 2.23 & 2.035 & 1.872 & 1.733 & 1.613 & 1.509 \\
\hline & $\mathrm{Sm}$ & 3.693 & 3.629 & 3.566 & 3.506 & 3.448 & 3.392 & 3.337 & 3.285 & 3.233 \\
\hline & $\mathrm{Eu}$ & 0.513 & 0.535 & 0.56 & 0.588 & 0.618 & 0.651 & 0.689 & 0.731 & 0.778 \\
\hline & $\mathrm{Tb}$ & 0.74 & 0.721 & 0.703 & 0.686 & 0.669 & 0.653 & 0.638 & 0.624 & 0.61 \\
\hline & Dy & 5.112 & 4.9 & 4.704 & 4.524 & 4.357 & 4.202 & 4.057 & 3.922 & 3.796 \\
\hline & $\mathrm{Y}$ & 18.001 & 17.885 & 17.77 & 17.657 & 17.545 & 17.435 & 17.326 & 17.218 & 17.112 \\
\hline & $\mathrm{Yb}$ & 2.747 & 2.595 & 2.459 & 2.336 & 2.226 & 2.125 & 2.033 & 1.949 & 1.871 \\
\hline & $\mathrm{Lu}$ & 0.478 & 0.444 & 0.415 & 0.389 & 0.366 & 0.346 & 0.328 & 0.312 & 0.297 \\
\hline MS18 & Element & \multicolumn{9}{|c|}{ Melt Fraction } \\
\hline & & 0.05 & 0.10 & 0.15 & 0.20 & 0.25 & 0.30 & 0.35 & 0.40 & 0.45 \\
\hline & $\mathrm{Rb}$ & 955.471 & 557.846 & 393.916 & 304.449 & 248.100 & 209.353 & 181.073 & 159.524 & 142.559 \\
\hline & $\mathrm{Ba}$ & 5694.414 & 5331.491 & 5012.056 & 4728.736 & 4475.733 & 4248.427 & 4043.094 & 3856.694 & 3686.724 \\
\hline & Th & 109.069 & 61.955 & 43.265 & 33.239 & 26.985 & 22.712 & 19.607 & 17.249 & 15.397 \\
\hline & $\mathrm{U}$ & 35.378 & 20.096 & 14.034 & 10.781 & 8.753 & 7.367 & 6.360 & 5.595 & 4.994 \\
\hline & $\mathrm{Nb}$ & 89.225 & 55.874 & 40.672 & 31.973 & 26.339 & 22.393 & 19.476 & 17.231 & 15.450 \\
\hline & $\mathrm{Ta}$ & 7.775 & 4.869 & 3.544 & 2.786 & 2.295 & 1.951 & 1.697 & 1.501 & 1.346 \\
\hline & $\mathrm{La}$ & 34.773 & 33.453 & 32.229 & 31.091 & 30.031 & 29.041 & 28.114 & 27.245 & 26.427 \\
\hline & $\mathrm{Ce}$ & 64.434 & 62.460 & 60.604 & 58.854 & 57.203 & 55.642 & 54.164 & 52.762 & 51.431 \\
\hline & $\mathrm{Pb}$ & 15.402 & 14.055 & 12.924 & 11.962 & 11.134 & 10.412 & 9.779 & 9.218 & 8.718 \\
\hline & $\operatorname{Pr}$ & 7.204 & 7.005 & 6.817 & 6.639 & 6.470 & 6.309 & 6.156 & 6.010 & 5.871 \\
\hline & $\mathrm{Sr}$ & 15.832 & 16.513 & 17.254 & 18.066 & 18.958 & 19.942 & 21.034 & 22.252 & 23.621 \\
\hline & $\mathrm{Nd}$ & 20.708 & 20.491 & 20.278 & 20.069 & 19.864 & 19.664 & 19.467 & 19.275 & 19.086 \\
\hline & $\mathrm{Zr}$ & 1133.555 & 700.714 & 507.086 & 397.300 & 326.592 & 277.250 & 240.860 & 212.914 & 190.779 \\
\hline & $\mathrm{Hf}$ & 30.286 & 18.761 & 13.590 & 10.653 & 8.760 & 7.438 & 6.463 & 5.714 & 5.121 \\
\hline & $\mathrm{Sm}$ & 3.292 & 3.294 & 3.297 & 3.299 & 3.302 & 3.304 & 3.307 & 3.309 & 3.312 \\
\hline & $\mathrm{Eu}$ & 0.201 & 0.208 & 0.216 & 0.224 & 0.233 & 0.243 & 0.254 & 0.266 & 0.279 \\
\hline & $\mathrm{Tb}$ & 0.520 & 0.517 & 0.515 & 0.512 & 0.509 & 0.506 & 0.503 & 0.501 & 0.498 \\
\hline & Dy & 4.274 & 4.176 & 4.083 & 3.994 & 3.909 & 3.827 & 3.748 & 3.673 & 3.601 \\
\hline & $\mathrm{Y}$ & 12.626 & 12.749 & 12.875 & 13.003 & 13.134 & 13.268 & 13.404 & 13.543 & 13.685 \\
\hline & $\mathrm{Yb}$ & 3.447 & 3.268 & 3.107 & 2.961 & 2.828 & 2.706 & 2.595 & 2.492 & 2.397 \\
\hline & $\mathrm{Lu}$ & 0.811 & 0.738 & 0.676 & 0.624 & 0.580 & 0.541 & 0.507 & 0.477 & 0.451 \\
\hline MS19 & Element & \multicolumn{9}{|c|}{ Melt Fraction } \\
\hline & & 0.10 & 0.15 & 0.20 & 0.25 & 0.30 & 0.35 & 0.40 & 0.45 & 0.50 \\
\hline & $\mathrm{Rb}$ & 365.583 & 259.425 & 201.045 & 164.113 & 138.645 & 120.019 & 105.805 & 94.601 & 85.543 \\
\hline & $\mathrm{Ba}$ & 1339.001 & 1280.371 & 1226.659 & 1177.273 & 1131.709 & 1089.541 & 1050.402 & 1013.978 & 979.995 \\
\hline & $\mathrm{Th}$ & 33.455 & 23.295 & 17.869 & 14.493 & 12.190 & 10.518 & 9.250 & 8.254 & 7.452 \\
\hline & $\mathrm{U}$ & 13.010 & 9.059 & 6.949 & 5.636 & 4.740 & 4.090 & 3.597 & 3.210 & 2.898 \\
\hline & $\mathrm{Nb}$ & 51.029 & 35.350 & 27.041 & 21.895 & 18.394 & 15.858 & 13.937 & 12.431 & 11.219 \\
\hline & $\mathrm{Ta}$ & 4.213 & 2.921 & 2.235 & 1.810 & 1.521 & 1.312 & 1.153 & 1.028 & 0.928 \\
\hline & $\mathrm{La}$ & 30.533 & 29.208 & 27.994 & 26.876 & 25.844 & 24.889 & 24.002 & 23.175 & 22.404 \\
\hline
\end{tabular}




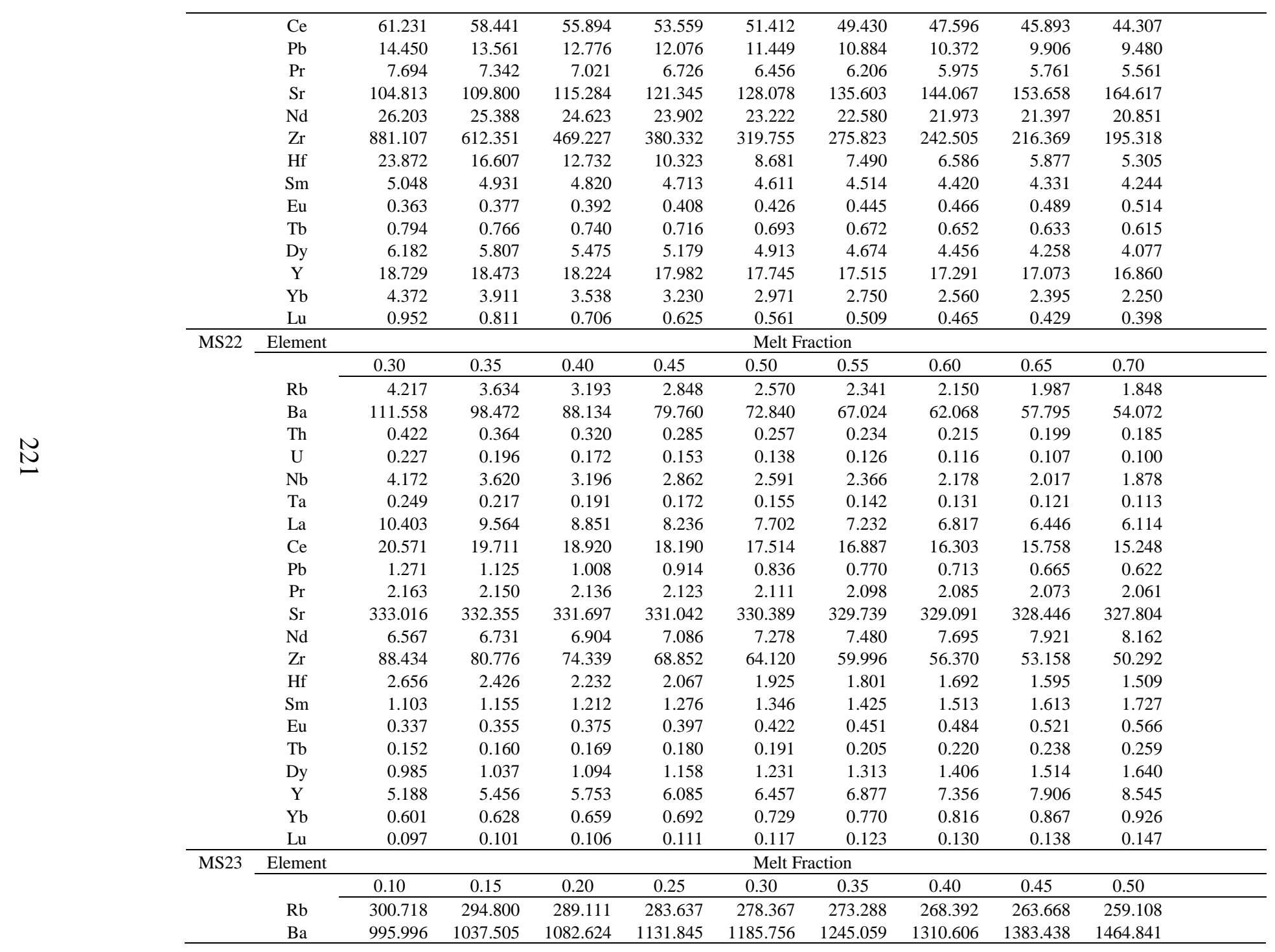




\begin{tabular}{|c|c|c|c|c|c|c|c|c|c|c|}
\hline \multirow{41}{*}{$N$} & Th & 2.845 & 1.999 & 1.541 & 1.253 & 1.056 & 0.913 & 0.804 & 0.718 & 0.648 \\
\hline & $\mathrm{U}$ & 1.810 & 1.272 & 0.980 & 0.797 & 0.672 & 0.581 & 0.511 & 0.457 & 0.413 \\
\hline & $\mathrm{Nb}$ & 9.916 & 9.829 & 9.744 & 9.661 & 9.578 & 9.498 & 9.418 & 9.340 & 9.264 \\
\hline & $\mathrm{Ta}$ & 0.378 & 0.375 & 0.372 & 0.369 & 0.366 & 0.364 & 0.361 & 0.358 & 0.355 \\
\hline & $\mathrm{La}$ & 10.011 & 9.518 & 9.071 & 8.664 & 8.292 & 7.951 & 7.637 & 7.346 & 7.077 \\
\hline & $\mathrm{Ce}$ & 20.570 & 19.891 & 19.255 & 18.659 & 18.098 & 17.571 & 17.073 & 16.602 & 16.157 \\
\hline & $\mathrm{Pb}$ & 18.078 & 15.968 & 14.299 & 12.945 & 11.826 & 10.885 & 10.082 & 9.390 & 8.787 \\
\hline & $\operatorname{Pr}$ & 3.100 & 3.007 & 2.919 & 2.836 & 2.758 & 2.684 & 2.614 & 2.547 & 2.484 \\
\hline & $\mathrm{Sr}$ & 97.668 & 100.646 & 103.812 & 107.183 & 110.781 & 114.629 & 118.754 & 123.187 & 127.963 \\
\hline & $\mathrm{Nd}$ & 11.822 & 11.704 & 11.589 & 11.476 & 11.365 & 11.256 & 11.149 & 11.044 & 10.942 \\
\hline & $\mathrm{Zr}$ & 461.891 & 361.502 & 296.960 & 251.973 & 218.823 & 193.382 & 173.240 & 156.898 & 143.373 \\
\hline & $\mathrm{Hf}$ & 13.498 & 10.539 & 8.645 & 7.327 & 6.358 & 5.616 & 5.028 & 4.552 & 4.159 \\
\hline & Sm & 3.127 & 3.132 & 3.136 & 3.141 & 3.145 & 3.150 & 3.154 & 3.159 & 3.164 \\
\hline & $\mathrm{Eu}$ & 0.523 & 0.538 & 0.554 & 0.570 & 0.588 & 0.606 & 0.626 & 0.647 & 0.670 \\
\hline & $\mathrm{Tb}$ & 0.874 & 0.868 & 0.862 & 0.857 & 0.851 & 0.846 & 0.840 & 0.835 & 0.830 \\
\hline & Dy & 7.748 & 7.546 & 7.354 & 7.172 & 6.999 & 6.834 & 6.676 & 6.526 & 6.382 \\
\hline & $\mathrm{Y}$ & 21.532 & 21.806 & 22.087 & 22.375 & 22.671 & 22.975 & 23.287 & 23.607 & 23.937 \\
\hline & $\mathrm{Yb}$ & 5.219 & 4.954 & 4.715 & 4.498 & 4.300 & 4.119 & 3.952 & 3.798 & 3.656 \\
\hline & $\mathrm{Lu}$ & 1.130 & 1.033 & 0.952 & 0.882 & 0.822 & 0.769 & 0.723 & 0.682 & 0.645 \\
\hline & Element & \multicolumn{9}{|c|}{ Melt Fraction } \\
\hline & & 0.50 & 0.55 & 0.60 & 0.65 & 0.70 & 0.75 & 0.80 & 0.85 & 0.90 \\
\hline & $\mathrm{Rb}$ & 5.192 & 4.729 & 4.343 & 4.014 & 3.732 & 3.487 & 3.272 & 3.082 & 2.913 \\
\hline & $\mathrm{Ba}$ & 92.331 & 84.225 & 77.428 & 71.646 & 66.667 & 62.335 & 58.532 & 55.166 & 52.167 \\
\hline & Th & 1700.208 & 1548.397 & 1421.474 & 1313.782 & 1221.259 & 1140.911 & 1070.482 & 1008.243 & 952.843 \\
\hline & $\mathrm{U}$ & 9.929 & 9.070 & 8.348 & 7.732 & 7.201 & 6.739 & 6.332 & 5.971 & 5.650 \\
\hline & $\mathrm{Nb}$ & 3.543 & 3.237 & 2.979 & 2.759 & 2.570 & 2.405 & 2.260 & 2.131 & 2.016 \\
\hline & $\mathrm{Ta}$ & 17.254 & 16.243 & 15.344 & 14.539 & 13.815 & 13.159 & 12.563 & 12.018 & 11.519 \\
\hline & $\mathrm{La}$ & 1.173 & 1.109 & 1.052 & 1.001 & 0.954 & 0.911 & 0.872 & 0.837 & 0.804 \\
\hline & $\mathrm{Ce}$ & 34.511 & 33.094 & 31.789 & 30.583 & 29.465 & 28.426 & 27.458 & 26.553 & 25.706 \\
\hline & $\mathrm{Pb}$ & 56.832 & 55.932 & 55.059 & 54.213 & 53.393 & 52.597 & 51.825 & 51.075 & 50.347 \\
\hline & $\operatorname{Pr}$ & 15.290 & 14.007 & 12.922 & 11.994 & 11.190 & 10.487 & 9.867 & 9.316 & 8.824 \\
\hline & $\mathrm{Sr}$ & 5.851 & 5.890 & 5.929 & 5.968 & 6.008 & 6.049 & 6.090 & 6.132 & 6.174 \\
\hline & $\mathrm{Nd}$ & 765.323 & 718.653 & 677.348 & 640.533 & 607.514 & 577.732 & 550.734 & 526.146 & 503.660 \\
\hline & $\mathrm{Zr}$ & 16.790 & 17.400 & 18.056 & 18.763 & 19.528 & 20.358 & 21.261 & 22.249 & 23.332 \\
\hline & $\mathrm{Hf}$ & 249.617 & 237.232 & 226.018 & 215.816 & 206.496 & 197.947 & 190.078 & 182.811 & 176.079 \\
\hline & Sm & 6.605 & 6.271 & 5.969 & 5.695 & 5.445 & 5.216 & 5.006 & 4.812 & 4.632 \\
\hline & $\mathrm{Eu}$ & 2.486 & 2.640 & 2.815 & 3.015 & 3.244 & 3.512 & 3.828 & 4.207 & 4.668 \\
\hline & $\mathrm{Tb}$ & 0.636 & 0.679 & 0.729 & 0.787 & 0.855 & 0.936 & 1.033 & 1.153 & 1.305 \\
\hline & Dy & 0.324 & 0.347 & 0.373 & 0.404 & 0.440 & 0.484 & 0.537 & 0.604 & 0.689 \\
\hline & Y & 2.176 & 2.322 & 2.489 & 2.682 & 2.907 & 3.174 & 3.494 & 3.886 & 4.378 \\
\hline & $\mathrm{Yb}$ & 11.146 & 11.911 & 12.788 & 13.806 & 14.999 & 16.418 & 18.133 & 20.249 & 22.924 \\
\hline
\end{tabular}




\begin{tabular}{|c|c|c|c|c|c|c|c|c|c|c|}
\hline & $\mathrm{Lu}$ & 1.324 & 1.398 & 1.481 & 1.574 & 1.680 & 1.802 & 1.942 & 2.106 & 2.300 \\
\hline \multirow[t]{23}{*}{ MS27 } & Element & \multicolumn{9}{|c|}{ Melt Fraction } \\
\hline & & 0.35 & 0.40 & 0.45 & 0.50 & 0.55 & 0.60 & 0.65 & 0.70 & 0.75 \\
\hline & $\mathrm{Rb}$ & 206.472 & 181.013 & 161.144 & 145.205 & 132.135 & 121.224 & 111.977 & 104.042 & 97.156 \\
\hline & $\mathrm{Ba}$ & 5056.958 & 4432.979 & 3946.072 & 3555.541 & 3235.348 & 2968.061 & 2741.567 & 2547.190 & 2378.551 \\
\hline & Th & 21.767 & 19.087 & 16.995 & 15.316 & 13.939 & 12.789 & 11.815 & 10.978 & 10.252 \\
\hline & $\mathrm{U}$ & 6.724 & 5.896 & 5.250 & 4.731 & 4.306 & 3.951 & 3.650 & 3.391 & 3.167 \\
\hline & $\mathrm{Nb}$ & 38.518 & 33.793 & 30.100 & 27.135 & 24.702 & 22.669 & 20.946 & 19.466 & 18.181 \\
\hline & $\mathrm{Ta}$ & 2.675 & 2.347 & 2.091 & 1.885 & 1.716 & 1.575 & 1.455 & 1.353 & 1.263 \\
\hline & $\mathrm{La}$ & 81.041 & 72.145 & 65.009 & 59.158 & 54.273 & 50.134 & 46.581 & 43.498 & 40.798 \\
\hline & $\mathrm{Ce}$ & 167.290 & 149.764 & 135.562 & 123.820 & 113.950 & 105.537 & 98.282 & 91.959 & 86.401 \\
\hline & $\mathrm{Pb}$ & 25.054 & 21.975 & 19.570 & 17.640 & 16.056 & 14.733 & 13.612 & 12.649 & 11.813 \\
\hline & $\operatorname{Pr}$ & 20.812 & 18.663 & 16.916 & 15.468 & 14.249 & 13.208 & 12.308 & 11.524 & 10.833 \\
\hline & $\mathrm{Sr}$ & 548.937 & 481.684 & 429.112 & 386.886 & 352.226 & 323.265 & 298.705 & 277.614 & 259.304 \\
\hline & $\mathrm{Nd}$ & 79.846 & 72.228 & 65.937 & 60.655 & 56.156 & 52.278 & 48.901 & 45.934 & 43.307 \\
\hline & $\mathrm{Zr}$ & 525.665 & 462.547 & 412.961 & 372.978 & 340.053 & 312.470 & 289.026 & 268.854 & 251.314 \\
\hline & $\mathrm{Hf}$ & 13.939 & 12.265 & 10.951 & 9.890 & 9.017 & 8.286 & 7.664 & 7.129 & 6.664 \\
\hline & $\mathrm{Sm}$ & 16.814 & 15.340 & 14.103 & 13.051 & 12.145 & 11.357 & 10.664 & 10.052 & 9.506 \\
\hline & $\mathrm{Eu}$ & 4.396 & 4.038 & 3.735 & 3.474 & 3.247 & 3.048 & 2.872 & 2.715 & 2.575 \\
\hline & $\mathrm{Tb}$ & 2.638 & 2.406 & 2.211 & 2.046 & 1.903 & 1.779 & 1.671 & 1.574 & 1.489 \\
\hline & Dy & 16.322 & 14.882 & 13.676 & 12.651 & 11.768 & 11.001 & 10.328 & 9.732 & 9.201 \\
\hline & $\mathrm{Y}$ & 68.259 & 64.206 & 60.607 & 57.390 & 54.497 & 51.882 & 49.506 & 47.339 & 45.353 \\
\hline & $\mathrm{Yb}$ & 6.805 & 6.389 & 6.021 & 5.693 & 5.398 & 5.133 & 4.893 & 4.674 & 4.474 \\
\hline & $\mathrm{Lu}$ & 1.141 & 1.065 & 0.998 & 0.939 & 0.886 & 0.839 & 0.797 & 0.759 & 0.725 \\
\hline \multirow[t]{16}{*}{ MS28 } & Element & \multicolumn{9}{|c|}{ Melt Fraction } \\
\hline & & 0.40 & 0.45 & 0.50 & 0.55 & 0.60 & 0.65 & 0.70 & 0.75 & 0.80 \\
\hline & $\mathrm{Rb}$ & 366.923 & 327.697 & 296.048 & 269.974 & 248.122 & 229.542 & 213.550 & 199.642 & 187.435 \\
\hline & $\mathrm{Ba}$ & 4330.965 & 4040.462 & 3786.481 & 3562.542 & 3363.612 & 3185.723 & 3025.705 & 2880.993 & 2749.492 \\
\hline & Th & 10.563 & 9.436 & 8.526 & 7.776 & 7.147 & 6.612 & 6.152 & 5.752 & 5.401 \\
\hline & $\mathrm{U}$ & 3.261 & 2.913 & 2.632 & 2.401 & 2.207 & 2.042 & 1.900 & 1.776 & 1.667 \\
\hline & $\mathrm{Nb}$ & 31.135 & 28.365 & 26.047 & 24.080 & 22.389 & 20.920 & 19.631 & 18.493 & 17.479 \\
\hline & $\mathrm{Ta}$ & 1.703 & 1.557 & 1.434 & 1.328 & 1.238 & 1.158 & 1.089 & 1.027 & 0.972 \\
\hline & $\mathrm{La}$ & 37.534 & 35.810 & 34.237 & 32.797 & 31.473 & 30.252 & 29.122 & 28.073 & 27.097 \\
\hline & $\mathrm{Ce}$ & 78.108 & 75.072 & 72.264 & 69.658 & 67.233 & 64.972 & 62.858 & 60.877 & 59.017 \\
\hline & $\mathrm{Pb}$ & 17.100 & 15.861 & 14.789 & 13.853 & 13.028 & 12.296 & 11.642 & 11.054 & 10.522 \\
\hline & $\operatorname{Pr}$ & 10.005 & 9.658 & 9.334 & 9.031 & 8.747 & 8.481 & 8.230 & 7.994 & 7.771 \\
\hline & $\mathrm{Sr}$ & 129.519 & 135.239 & 141.487 & 148.341 & 155.892 & 164.254 & 173.564 & 183.992 & 195.753 \\
\hline & $\mathrm{Nd}$ & 36.724 & 36.059 & 35.417 & 34.797 & 34.199 & 33.621 & 33.063 & 32.522 & 31.999 \\
\hline & $\mathrm{Zr}$ & 402.212 & 365.872 & 335.554 & 309.877 & 287.850 & 268.747 & 252.021 & 237.255 & 224.124 \\
\hline & $\mathrm{Hf}$ & 10.887 & 9.896 & 9.071 & 8.372 & 7.774 & 7.255 & 6.801 & 6.401 & 6.045 \\
\hline
\end{tabular}




\begin{tabular}{|c|c|c|c|c|c|c|c|c|c|c|}
\hline & Sm & 7.683 & 7.660 & 7.636 & 7.613 & 7.590 & 7.567 & 7.544 & 7.521 & 7.499 \\
\hline & $\mathrm{Eu}$ & 1.316 & 1.364 & 1.415 & 1.470 & 1.529 & 1.593 & 1.664 & 1.740 & 1.824 \\
\hline & $\mathrm{Tb}$ & 1.369 & 1.365 & 1.362 & 1.358 & 1.354 & 1.350 & 1.347 & 1.343 & 1.339 \\
\hline & Dy & 9.596 & 9.480 & 9.366 & 9.256 & 9.147 & 9.042 & 8.938 & 8.837 & 8.739 \\
\hline & $\mathrm{Y}$ & 37.467 & 37.916 & 38.375 & 38.846 & 39.328 & 39.822 & 40.329 & 40.849 & 41.383 \\
\hline & $\mathrm{Yb}$ & 4.904 & 4.833 & 4.764 & 4.697 & 4.632 & 4.569 & 4.507 & 4.447 & 4.389 \\
\hline & $\mathrm{Lu}$ & 0.853 & 0.833 & 0.814 & 0.795 & 0.777 & 0.760 & 0.744 & 0.729 & 0.714 \\
\hline \multirow[t]{23}{*}{ MS29 } & Element & \multicolumn{9}{|c|}{ Melt Fraction } \\
\hline & & 0.40 & 0.45 & 0.50 & 0.55 & 0.60 & 0.65 & 0.70 & 0.75 & 0.80 \\
\hline & $\mathrm{Rb}$ & 144.264 & 129.901 & 118.139 & 108.330 & 100.025 & 92.903 & 86.727 & 81.322 & 76.551 \\
\hline & $\mathrm{Ba}$ & 1346.574 & 1295.229 & 1247.656 & 1203.454 & 1162.276 & 1123.824 & 1087.834 & 1054.078 & 1022.353 \\
\hline & Th & 14.779 & 13.199 & 11.924 & 10.873 & 9.993 & 9.245 & 8.601 & 8.040 & 7.549 \\
\hline & $\mathrm{U}$ & 4.800 & 4.287 & 3.873 & 3.532 & 3.246 & 3.003 & 2.794 & 2.612 & 2.452 \\
\hline & $\mathrm{Nb}$ & 28.539 & 25.919 & 23.739 & 21.898 & 20.321 & 18.957 & 17.764 & 16.712 & 15.778 \\
\hline & $\mathrm{Ta}$ & 1.908 & 1.735 & 1.591 & 1.470 & 1.365 & 1.274 & 1.195 & 1.125 & 1.063 \\
\hline & $\mathrm{La}$ & 42.793 & 41.261 & 39.835 & 38.505 & 37.260 & 36.094 & 34.998 & 33.967 & 32.994 \\
\hline & $\mathrm{Ce}$ & 80.613 & 78.737 & 76.945 & 75.234 & 73.597 & 72.029 & 70.528 & 69.087 & 67.704 \\
\hline & $\mathrm{Pb}$ & 17.126 & 16.044 & 15.090 & 14.244 & 13.487 & 12.807 & 12.192 & 11.633 & 11.124 \\
\hline & $\operatorname{Pr}$ & 9.079 & 8.984 & 8.892 & 8.801 & 8.712 & 8.625 & 8.539 & 8.455 & 8.373 \\
\hline & $\mathrm{Sr}$ & 171.995 & 181.269 & 191.600 & 203.180 & 216.249 & 231.115 & 248.176 & 267.957 & 291.165 \\
\hline & $\mathrm{Nd}$ & 28.243 & 28.609 & 28.985 & 29.370 & 29.766 & 30.173 & 30.591 & 31.021 & 31.463 \\
\hline & $\mathrm{Zr}$ & 418.628 & 381.950 & 351.182 & 325.001 & 302.453 & 282.831 & 265.599 & 250.347 & 236.751 \\
\hline & $\mathrm{Hf}$ & 11.156 & 10.176 & 9.354 & 8.655 & 8.053 & 7.530 & 7.070 & 6.664 & 6.301 \\
\hline & $\mathrm{Sm}$ & 4.541 & 4.697 & 4.865 & 5.046 & 5.240 & 5.450 & 5.678 & 5.925 & 6.195 \\
\hline & $\mathrm{Eu}$ & 0.825 & 0.871 & 0.923 & 0.982 & 1.048 & 1.124 & 1.212 & 1.314 & 1.436 \\
\hline & $\mathrm{Tb}$ & 0.613 & 0.639 & 0.667 & 0.697 & 0.730 & 0.766 & 0.806 & 0.851 & 0.900 \\
\hline & Dy & 4.210 & 4.360 & 4.522 & 4.696 & 4.883 & 5.087 & 5.308 & 5.549 & 5.813 \\
\hline & $\mathrm{Y}$ & 19.385 & 20.192 & 21.069 & 22.025 & 23.073 & 24.226 & 25.499 & 26.914 & 28.496 \\
\hline & $\mathrm{Yb}$ & 2.632 & 2.689 & 2.749 & 2.812 & 2.878 & 2.947 & 3.019 & 3.095 & 3.175 \\
\hline & $\mathrm{Lu}$ & 0.454 & 0.462 & 0.470 & 0.478 & 0.486 & 0.495 & 0.504 & 0.513 & 0.523 \\
\hline \multirow[t]{11}{*}{ MS30 } & Element & \multicolumn{9}{|c|}{ Melt Fraction } \\
\hline & & 0.45 & 0.50 & 0.55 & 0.60 & 0.65 & 0.70 & 0.75 & 0.80 & 0.85 \\
\hline & $\mathrm{Rb}$ & 74.517 & 67.144 & 61.098 & 56.052 & 51.775 & 48.105 & 44.921 & 42.132 & 39.669 \\
\hline & $\mathrm{Ba}$ & 2875.793 & 2591.249 & 2357.944 & 2163.181 & 1998.137 & 1856.492 & 1733.600 & 1625.968 & 1530.919 \\
\hline & Th & 7.993 & 7.202 & 6.553 & 6.012 & 5.553 & 5.160 & 4.818 & 4.519 & 4.255 \\
\hline & $\mathrm{U}$ & 2.627 & 2.367 & 2.154 & 1.976 & 1.826 & 1.696 & 1.584 & 1.486 & 1.399 \\
\hline & $\mathrm{Nb}$ & 9.274 & 8.356 & 7.604 & 6.976 & 6.443 & 5.987 & 5.590 & 5.243 & 4.937 \\
\hline & $\mathrm{Ta}$ & 0.784 & 0.706 & 0.643 & 0.590 & 0.545 & 0.506 & 0.473 & 0.443 & 0.417 \\
\hline & $\mathrm{La}$ & 24.902 & 22.512 & 20.540 & 18.885 & 17.478 & 16.265 & 15.210 & 14.284 & 13.463 \\
\hline & $\mathrm{Ce}$ & 51.558 & 46.787 & 42.824 & 39.480 & 36.620 & 34.147 & 31.987 & 30.084 & 28.394 \\
\hline & $\mathrm{Pb}$ & 6.889 & 6.212 & 5.657 & 5.193 & 4.799 & 4.461 & 4.167 & 3.910 & 3.682 \\
\hline
\end{tabular}




\begin{tabular}{|c|c|c|c|c|c|c|c|c|c|c|}
\hline & $\mathrm{Pr}$ & 6.203 & 5.667 & 5.216 & 4.832 & 4.500 & 4.211 & 3.957 & 3.732 & 3.531 \\
\hline & $\mathrm{Sr}$ & 314.843 & 284.718 & 259.854 & 238.985 & 221.218 & 205.910 & 192.584 & 180.877 & 170.513 \\
\hline & $\mathrm{Nd}$ & 23.419 & 21.643 & 20.118 & 18.794 & 17.633 & 16.607 & 15.694 & 14.876 & 14.139 \\
\hline & $\mathrm{Zr}$ & 144.015 & 130.560 & 119.405 & 110.006 & 101.979 & 95.043 & 88.991 & 83.664 & 78.938 \\
\hline & $\mathrm{Hf}$ & 4.011 & 3.636 & 3.326 & 3.064 & 2.840 & 2.647 & 2.479 & 2.330 & 2.199 \\
\hline & $\mathrm{Sm}$ & 4.895 & 4.616 & 4.367 & 4.144 & 3.942 & 3.759 & 3.592 & 3.440 & 3.299 \\
\hline & $\mathrm{Eu}$ & 1.275 & 1.212 & 1.155 & 1.103 & 1.055 & 1.011 & 0.971 & 0.934 & 0.900 \\
\hline & $\mathrm{Tb}$ & 0.821 & 0.786 & 0.754 & 0.724 & 0.696 & 0.671 & 0.647 & 0.625 & 0.604 \\
\hline & Dy & 5.358 & 5.145 & 4.948 & 4.765 & 4.596 & 4.438 & 4.291 & 4.153 & 4.024 \\
\hline & $\mathrm{Y}$ & 26.036 & 25.320 & 24.642 & 23.999 & 23.389 & 22.809 & 22.257 & 21.731 & 21.230 \\
\hline & $\mathrm{Yb}$ & 2.543 & 2.501 & 2.459 & 2.419 & 2.380 & 2.343 & 2.306 & 2.271 & 2.237 \\
\hline & $\mathrm{Lu}$ & 0.429 & 0.420 & 0.412 & 0.404 & 0.396 & 0.389 & 0.382 & 0.375 & 0.369 \\
\hline MS33 & Element & \multicolumn{9}{|c|}{ Melt Fraction } \\
\hline & & 0.35 & 0.40 & 0.45 & 0.50 & 0.55 & 0.60 & 0.65 & 0.70 & 0.75 \\
\hline & $\mathrm{Rb}$ & 4.268 & 3.763 & 3.365 & 3.043 & 2.777 & 2.554 & 2.364 & 2.200 & 2.058 \\
\hline & $\mathrm{Ba}$ & 148.056 & 139.124 & 131.209 & 124.146 & 117.804 & 112.079 & 106.884 & 102.150 & 97.817 \\
\hline & Th & 0.427 & 0.375 & 0.335 & 0.302 & 0.276 & 0.253 & 0.234 & 0.218 & 0.204 \\
\hline & $\mathrm{U}$ & 0.222 & 0.195 & 0.174 & 0.157 & 0.143 & 0.132 & 0.122 & 0.113 & 0.106 \\
\hline & $\mathrm{Nb}$ & 1.507 & 1.484 & 1.461 & 1.439 & 1.418 & 1.397 & 1.377 & 1.358 & 1.339 \\
\hline & $\mathrm{Ta}$ & 0.099 & 0.098 & 0.096 & 0.095 & 0.093 & 0.092 & 0.091 & 0.089 & 0.088 \\
\hline & $\mathrm{La}$ & 5.688 & 5.492 & 5.309 & 5.138 & 4.978 & 4.827 & 4.685 & 4.551 & 4.425 \\
\hline & $\mathrm{Ce}$ & 10.390 & 10.402 & 10.415 & 10.428 & 10.441 & 10.453 & 10.466 & 10.479 & 10.492 \\
\hline & $\mathrm{Pb}$ & 1.567 & 1.406 & 1.275 & 1.167 & 1.075 & 0.997 & 0.930 & 0.871 & 0.819 \\
\hline & $\operatorname{Pr}$ & 1.100 & 1.130 & 1.163 & 1.197 & 1.233 & 1.271 & 1.312 & 1.356 & 1.402 \\
\hline & $\mathrm{Sr}$ & 190.750 & 195.195 & 199.852 & 204.736 & 209.866 & 215.259 & 220.937 & 226.922 & 233.241 \\
\hline & $\mathrm{Nd}$ & 3.855 & 4.027 & 4.216 & 4.423 & 4.651 & 4.904 & 5.186 & 5.503 & 5.861 \\
\hline & $\mathrm{Zr}$ & 55.806 & 54.443 & 53.145 & 51.907 & 50.725 & 49.597 & 48.517 & 47.483 & 46.493 \\
\hline & $\mathrm{Hf}$ & 1.674 & 1.632 & 1.592 & 1.554 & 1.518 & 1.484 & 1.450 & 1.419 & 1.389 \\
\hline & $\mathrm{Sm}$ & 0.666 & 0.708 & 0.754 & 0.808 & 0.869 & 0.941 & 1.026 & 1.127 & 1.251 \\
\hline & $\mathrm{Eu}$ & 0.221 & 0.235 & 0.252 & 0.271 & 0.293 & 0.319 & 0.350 & 0.387 & 0.434 \\
\hline & $\mathrm{Tb}$ & 0.110 & 0.118 & 0.126 & 0.136 & 0.147 & 0.161 & 0.177 & 0.196 & 0.221 \\
\hline & Dy & 0.765 & 0.815 & 0.873 & 0.938 & 1.015 & 1.105 & 1.213 & 1.345 & 1.508 \\
\hline & $\mathrm{Y}$ & 4.501 & 4.788 & 5.114 & 5.488 & 5.921 & 6.428 & 7.030 & 7.757 & 8.651 \\
\hline & $\mathrm{Yb}$ & 0.557 & 0.590 & 0.627 & 0.670 & 0.718 & 0.775 & 0.841 & 0.919 & 1.013 \\
\hline & $\mathrm{Lu}$ & 0.102 & 0.107 & 0.114 & 0.121 & 0.129 & 0.139 & 0.149 & 0.162 & 0.177 \\
\hline \multirow[t]{6}{*}{ MS34 } & Element & \multicolumn{9}{|c|}{ Melt Fraction } \\
\hline & & 0.40 & 0.45 & 0.50 & 0.55 & 0.60 & 0.65 & 0.70 & 0.75 & 0.80 \\
\hline & $\mathrm{Rb}$ & 63.703 & 56.777 & 51.209 & 46.636 & 42.812 & 39.568 & 36.781 & 34.361 & 32.240 \\
\hline & $\mathrm{Ba}$ & 1261.137 & 1123.757 & 1013.368 & 922.726 & 846.968 & 782.706 & 727.508 & 679.583 & 637.581 \\
\hline & Th & 8.059 & 7.183 & 6.480 & 5.902 & 5.418 & 5.008 & 4.656 & 4.350 & 4.081 \\
\hline & $\mathrm{U}$ & 2.757 & 2.457 & 2.217 & 2.019 & 1.853 & 1.713 & 1.593 & 1.488 & 1.396 \\
\hline
\end{tabular}




\begin{tabular}{|c|c|c|c|c|c|c|c|c|c|c|}
\hline & $\mathrm{Nb}$ & 20.217 & 18.119 & 16.416 & 15.005 & 13.818 & 12.804 & 11.93 & 11.167 & 10.495 \\
\hline & $\mathrm{Ta}$ & 1.313 & 1.178 & 1.068 & 0.977 & 0.9 & 0.834 & 0.778 & 0.728 & 0.684 \\
\hline & $\mathrm{La}$ & 36.359 & 33.538 & 31.123 & 29.033 & 27.206 & 25.595 & 24.164 & 22.885 & 21.735 \\
\hline & $\mathrm{Ce}$ & 60.885 & 58.141 & 55.634 & 53.334 & 51.217 & 49.262 & 47.45 & 45.767 & 44.199 \\
\hline & $\mathrm{Pb}$ & 11.747 & 10.518 & 9.522 & 8.698 & 8.006 & 7.415 & 6.906 & 6.462 & 6.072 \\
\hline & $\operatorname{Pr}$ & 5.938 & 5.868 & 5.799 & 5.731 & 5.665 & 5.601 & 5.538 & 5.477 & 5.416 \\
\hline & $\mathrm{Sr}$ & 653.676 & 606.733 & 566.081 & 530.534 & 499.187 & 471.338 & 446.433 & 424.027 & 403.763 \\
\hline & $\mathrm{Nd}$ & 16.768 & 17.133 & 17.515 & 17.914 & 18.332 & 18.77 & 19.229 & 19.711 & 20.218 \\
\hline & $\mathrm{Zr}$ & 263.682 & 243.893 & 226.867 & 212.063 & 199.073 & 187.582 & 177.346 & 168.169 & 159.895 \\
\hline & $\mathrm{Hf}$ & 6.985 & 6.459 & 6.008 & 5.615 & 5.27 & 4.966 & 4.694 & 4.451 & 4.232 \\
\hline & $\mathrm{Sm}$ & 2.498 & 2.62 & 2.756 & 2.906 & 3.074 & 3.262 & 3.475 & 3.717 & 3.996 \\
\hline & $\mathrm{Eu}$ & 0.68 & 0.717 & 0.759 & 0.806 & 0.859 & 0.919 & 0.989 & 1.07 & 1.165 \\
\hline & $\mathrm{Tb}$ & 0.332 & 0.352 & 0.374 & 0.399 & 0.427 & 0.46 & 0.498 & 0.543 & 0.598 \\
\hline & Dy & 2.188 & 2.311 & 2.449 & 2.604 & 2.78 & 2.982 & 3.216 & 3.489 & 3.813 \\
\hline & $\mathrm{Y}$ & 11.818 & 12.473 & 13.205 & 14.028 & 14.961 & 16.026 & 17.255 & 18.688 & 20.381 \\
\hline & $\mathrm{Yb}$ & 1.278 & 1.34 & 1.409 & 1.486 & 1.571 & 1.666 & 1.774 & 1.897 & 2.038 \\
\hline & $\mathrm{Lu}$ & 0.21 & 0.22 & 0.231 & 0.243 & 0.256 & 0.271 & 0.288 & 0.306 & 0.328 \\
\hline MS35 & Element & \multicolumn{9}{|c|}{ Melt Fraction } \\
\hline & & 0.45 & 0.50 & 0.55 & 0.60 & 0.65 & 0.70 & 0.75 & 0.80 & 0.85 \\
\hline & $\mathrm{Rb}$ & 89.228 & 80.539 & 73.393 & 67.411 & 62.331 & 57.962 & 54.166 & 50.837 & 47.893 \\
\hline & $\mathrm{Ba}$ & 1016.409 & 947.636 & 887.579 & 834.681 & 787.734 & 745.786 & 708.081 & 674.004 & 643.057 \\
\hline & Th & 9.310 & 8.397 & 7.647 & 7.019 & 6.487 & 6.030 & 5.633 & 5.285 & 4.978 \\
\hline & $\mathrm{U}$ & 2.789 & 2.515 & 2.291 & 2.103 & 1.943 & 1.806 & 1.688 & 1.583 & 1.491 \\
\hline & $\mathrm{Nb}$ & 23.565 & 21.251 & 19.350 & 17.762 & 16.414 & 15.257 & 14.252 & 13.371 & 12.593 \\
\hline & $\mathrm{Ta}$ & 1.489 & 1.343 & 1.223 & 1.122 & 1.037 & 0.964 & 0.900 & 0.845 & 0.796 \\
\hline & $\mathrm{La}$ & 41.212 & 38.578 & 36.261 & 34.206 & 32.371 & 30.723 & 29.235 & 27.885 & 26.653 \\
\hline & $\mathrm{Ce}$ & 82.357 & 78.330 & 74.678 & 71.352 & 68.310 & 65.516 & 62.942 & 60.562 & 58.356 \\
\hline & $\mathrm{Pb}$ & 11.772 & 10.931 & 10.202 & 9.564 & 9.001 & 8.501 & 8.054 & 7.651 & 7.286 \\
\hline & $\operatorname{Pr}$ & 8.936 & 8.715 & 8.505 & 8.305 & 8.114 & 7.931 & 7.757 & 7.590 & 7.430 \\
\hline & $\mathrm{Sr}$ & 182.231 & 189.668 & 197.739 & 206.527 & 216.133 & 226.675 & 238.299 & 251.179 & 265.532 \\
\hline & $\mathrm{Nd}$ & 28.077 & 28.245 & 28.416 & 28.589 & 28.764 & 28.941 & 29.120 & 29.302 & 29.486 \\
\hline & $\mathrm{Zr}$ & 320.258 & 294.759 & 273.021 & 254.269 & 237.927 & 223.560 & 210.828 & 199.469 & 189.271 \\
\hline & $\mathrm{Hf}$ & 8.587 & 7.903 & 7.320 & 6.818 & 6.379 & 5.994 & 5.653 & 5.348 & 5.075 \\
\hline & $\mathrm{Sm}$ & 4.428 & 4.592 & 4.770 & 4.961 & 5.168 & 5.393 & 5.639 & 5.909 & 6.205 \\
\hline & $\mathrm{Eu}$ & 0.976 & 1.030 & 1.091 & 1.159 & 1.236 & 1.325 & 1.427 & 1.546 & 1.687 \\
\hline & $\mathrm{Tb}$ & 0.579 & 0.608 & 0.640 & 0.675 & 0.715 & 0.759 & 0.810 & 0.867 & 0.933 \\
\hline & Dy & 3.798 & 3.973 & 4.166 & 4.378 & 4.613 & 4.874 & 5.167 & 5.497 & 5.872 \\
\hline & $\mathrm{Y}$ & 20.063 & 20.964 & 21.950 & 23.033 & 24.228 & 25.555 & 27.035 & 28.697 & 30.576 \\
\hline & $\mathrm{Yb}$ & 2.238 & 2.319 & 2.405 & 2.498 & 2.599 & 2.708 & 2.827 & 2.957 & 3.099 \\
\hline & $\mathrm{Lu}$ & 0.367 & 0.379 & 0.392 & 0.406 & 0.422 & 0.438 & 0.456 & 0.475 & 0.496 \\
\hline MS37 & Element & \multicolumn{9}{|c|}{ Melt Fraction } \\
\hline
\end{tabular}




\begin{tabular}{|c|c|c|c|c|c|c|c|c|c|c|}
\hline & & 0.30 & 0.35 & 0.40 & 0.45 & 0.50 & 0.55 & 0.60 & 0.65 & 0.70 \\
\hline & $\mathrm{Rb}$ & 209.370 & 210.152 & 210.939 & 211.732 & 212.531 & 213.336 & 214.147 & 214.964 & 215.788 \\
\hline & $\mathrm{Ba}$ & 820.489 & 868.208 & 921.822 & 982.492 & 1051.712 & 1131.424 & 1224.210 & 1333.574 & 1464.395 \\
\hline & Th & 1.018 & 0.884 & 0.782 & 0.701 & 0.635 & 0.580 & 0.534 & 0.495 & 0.461 \\
\hline & $\mathrm{U}$ & 0.667 & 0.579 & 0.512 & 0.459 & 0.416 & 0.380 & 0.350 & 0.324 & 0.302 \\
\hline & $\mathrm{Nb}$ & 7.028 & 7.119 & 7.212 & 7.308 & 7.406 & 7.507 & 7.611 & 7.718 & 7.827 \\
\hline & $\mathrm{Ta}$ & 0.268 & 0.272 & 0.276 & 0.280 & 0.284 & 0.288 & 0.292 & 0.296 & 0.300 \\
\hline & $\mathrm{La}$ & 5.452 & 5.432 & 5.412 & 5.392 & 5.372 & 5.352 & 5.333 & 5.313 & 5.294 \\
\hline & $\mathrm{Ce}$ & 10.744 & 10.866 & 10.990 & 11.118 & 11.248 & 11.382 & 11.519 & 11.659 & 11.802 \\
\hline & $\mathrm{Pb}$ & 12.010 & 11.031 & 10.199 & 9.484 & 8.862 & 8.317 & 7.836 & 7.407 & 7.022 \\
\hline & $\operatorname{Pr}$ & 1.606 & 1.628 & 1.651 & 1.675 & 1.699 & 1.724 & 1.750 & 1.776 & 1.804 \\
\hline & $\mathrm{Sr}$ & 147.409 & 150.587 & 153.906 & 157.374 & 161.002 & 164.801 & 168.784 & 172.964 & 177.357 \\
\hline & $\mathrm{Nd}$ & 6.117 & 6.291 & 6.476 & 6.672 & 6.881 & 7.103 & 7.339 & 7.592 & 7.863 \\
\hline & $\mathrm{Zr}$ & 201.34 & 180.372 & 163.36 & 149.28 & 137.434 & 127.33 & 118.611 & 111.008 & 104.322 \\
\hline & $\mathrm{Hf}$ & 5.874 & 5.258 & 4.760 & 4.347 & 4.001 & 3.705 & 3.450 & 3.228 & 3.003 \\
\hline & $\mathrm{Sm}$ & 1.612 & 1.672 & 1.736 & 1.805 & 1.880 & 1.961 & 2.050 & 2.147 & 2.254 \\
\hline & $\mathrm{Eu}$ & 0.383 & 0.401 & 0.421 & 0.442 & 0.467 & 0.494 & 0.524 & 0.559 & 0.598 \\
\hline & $\mathrm{Tb}$ & 0.408 & 0.422 & 0.437 & 0.454 & 0.472 & 0.491 & 0.512 & 0.535 & 0.560 \\
\hline & Dy & 4.015 & 4.082 & 4.152 & 4.225 & 4.300 & 4.377 & 4.458 & 4.542 & 4.628 \\
\hline & $\mathrm{Y}$ & 11.553 & 12.056 & 12.606 & 13.208 & 13.870 & 14.602 & 15.416 & 16.326 & 17.350 \\
\hline & $\mathrm{Yb}$ & 3.143 & 3.103 & 3.064 & 3.026 & 2.989 & 2.953 & 2.918 & 2.883 & 2.850 \\
\hline & $\mathrm{Lu}$ & 0.704 & 0.672 & 0.642 & 0.615 & 0.590 & 0.567 & 0.546 & 0.526 & 0.508 \\
\hline \multirow[t]{19}{*}{ MS38 } & Element & \multicolumn{9}{|c|}{ Melt Fraction } \\
\hline & & 0.30 & 0.35 & 0.40 & 0.45 & 0.50 & 0.55 & 0.60 & 0.65 & 0.70 \\
\hline & $\mathrm{Rb}$ & 139.176 & 120.388 & 106.070 & 94.795 & 85.687 & 78.176 & 71.876 & 66.515 & 61.898 \\
\hline & $\mathrm{Ba}$ & 1322.771 & 1251.089 & 1186.777 & 1128.753 & 1076.139 & 1028.211 & 984.370 & 944.115 & 907.023 \\
\hline & Th & 12.096 & 10.453 & 9.203 & 8.220 & 7.427 & 6.774 & 6.226 & 5.760 & 5.359 \\
\hline & $\mathrm{U}$ & 4.704 & 4.065 & 3.579 & 3.197 & 2.888 & 2.634 & 2.421 & 2.240 & 2.084 \\
\hline & $\mathrm{Nb}$ & 17.736 & 15.401 & 13.610 & 12.191 & 11.041 & 10.089 & 9.288 & 8.604 & 8.015 \\
\hline & $\mathrm{Ta}$ & 1.458 & 1.267 & 1.121 & 1.005 & 0.911 & 0.833 & 0.767 & 0.711 & 0.662 \\
\hline & $\mathrm{La}$ & 23.454 & 22.810 & 22.200 & 21.622 & 21.074 & 20.553 & 20.056 & 19.584 & 19.133 \\
\hline & $\mathrm{Ce}$ & 41.744 & 40.961 & 40.206 & 39.479 & 38.778 & 38.102 & 37.448 & 36.817 & 36.206 \\
\hline & $\mathrm{Pb}$ & 13.076 & 12.227 & 11.481 & 10.821 & 10.233 & 9.706 & 9.230 & 8.799 & 8.406 \\
\hline & $\operatorname{Pr}$ & 4.969 & 4.898 & 4.829 & 4.762 & 4.696 & 4.633 & 4.571 & 4.511 & 4.452 \\
\hline & $\mathrm{Sr}$ & 170.973 & 180.000 & 190.034 & 201.253 & 213.879 & 228.196 & 244.567 & 263.468 & 285.536 \\
\hline & $\mathrm{Nd}$ & 15.900 & 15.949 & 15.998 & 16.047 & 16.097 & 16.147 & 16.197 & 16.247 & 16.298 \\
\hline & $\mathrm{Zr}$ & 298.075 & 260.639 & 231.557 & 208.313 & 189.310 & 173.485 & 160.101 & 148.634 & 138.700 \\
\hline & $\mathrm{Hf}$ & 8.099 & 7.082 & 6.292 & 5.660 & 5.144 & 4.714 & 4.350 & 4.039 & 3.769 \\
\hline & $\mathrm{Sm}$ & 2.742 & 2.787 & 2.833 & 2.881 & 2.931 & 2.982 & 3.035 & 3.090 & 3.147 \\
\hline & $\mathrm{Eu}$ & 0.398 & 0.417 & 0.437 & 0.460 & 0.485 & 0.513 & 0.545 & 0.580 & 0.621 \\
\hline & $\mathrm{Tb}$ & 0.379 & 0.385 & 0.391 & 0.397 & 0.404 & 0.410 & 0.417 & 0.424 & 0.431 \\
\hline
\end{tabular}




\begin{tabular}{|c|c|c|c|c|c|c|c|c|c|c|}
\hline & Dy & 2.700 & 2.711 & 2.722 & 2.733 & 2.744 & 2.755 & 2.766 & 2.778 & 2.789 \\
\hline & $\mathrm{Y}$ & 9.743 & 9.992 & 10.255 & 10.533 & 10.825 & 11.135 & 11.462 & 11.810 & 12.179 \\
\hline & $\mathrm{Yb}$ & 1.634 & 1.615 & 1.596 & 1.577 & 1.559 & 1.542 & 1.525 & 1.508 & 1.492 \\
\hline & $\mathrm{Lu}$ & 0.315 & 0.307 & 0.299 & 0.292 & 0.285 & 0.278 & 0.272 & 0.266 & 0.260 \\
\hline \multirow[t]{23}{*}{ MS39 } & Element & \multicolumn{9}{|c|}{ Melt Fraction } \\
\hline & & 0.70 & 0.75 & 0.80 & 0.85 & 0.90 & 0.95 & & & \\
\hline & $\mathrm{Rb}$ & 62.125 & 58.046 & 54.47 & 51.309 & 48.494 & 45.972 & & & \\
\hline & $\mathrm{Ba}$ & 1044.175 & 975.472 & 915.252 & 862.035 & 814.666 & 772.232 & & & \\
\hline & Th & 5.371 & 5.019 & 4.71 & 4.437 & 4.194 & 3.976 & & & \\
\hline & $\mathrm{U}$ & 2.089 & 1.952 & 1.832 & 1.726 & 1.631 & 1.546 & & & \\
\hline & $\mathrm{Nb}$ & 7.941 & 7.447 & 7.011 & 6.623 & 6.276 & 5.963 & & & \\
\hline & $\mathrm{Ta}$ & 0.655 & 0.615 & 0.579 & 0.547 & 0.519 & 0.493 & & & \\
\hline & $\mathrm{La}$ & 22.112 & 21.008 & 20.009 & 19.1 & 18.27 & 17.51 & & & \\
\hline & $\mathrm{Ce}$ & 38.886 & 37.748 & 36.675 & 35.661 & 34.702 & 33.793 & & & \\
\hline & $\mathrm{Pb}$ & 9.341 & 8.745 & 8.221 & 7.756 & 7.34 & 6.967 & & & \\
\hline & $\operatorname{Pr}$ & 4.166 & 4.16 & 4.154 & 4.148 & 4.142 & 4.136 & & & \\
\hline & $\mathrm{Sr}$ & 743.067 & 708.297 & 676.635 & 647.682 & 621.106 & 596.625 & & & \\
\hline & $\mathrm{Nd}$ & 12.971 & 13.463 & 13.993 & 14.567 & 15.19 & 15.868 & & & \\
\hline & $\mathrm{Zr}$ & 129.19 & 122.941 & 117.269 & 112.098 & 107.363 & 103.012 & & & \\
\hline & Hf & 3.513 & 3.342 & 3.188 & 3.047 & 2.918 & 2.799 & & & \\
\hline & $\mathrm{Sm}$ & 1.904 & 2.063 & 2.251 & 2.476 & 2.752 & 3.097 & & & \\
\hline & $\mathrm{Eu}$ & 0.512 & 0.56 & 0.619 & 0.692 & 0.785 & 0.905 & & & \\
\hline & $\mathrm{Tb}$ & 0.209 & 0.231 & 0.258 & 0.292 & 0.335 & 0.395 & & & \\
\hline & Dy & 1.331 & 1.461 & 1.62 & 1.817 & 2.068 & 2.401 & & & \\
\hline & $\mathrm{Y}$ & 7.171 & 7.854 & 8.68 & 9.701 & 10.994 & 12.685 & & & \\
\hline & $\mathrm{Yb}$ & 0.777 & 0.84 & 0.913 & 1 & 1.105 & 1.235 & & & \\
\hline & $\mathrm{Lu}$ & 0.132 & 0.142 & 0.154 & 0.168 & 0.184 & 0.205 & & & \\
\hline \multirow[t]{14}{*}{ MS40 } & Element & \multicolumn{9}{|c|}{ Melt Fraction } \\
\hline & & 0.05 & 0.10 & 0.15 & 0.20 & 0.25 & 0.30 & 0.35 & 0.40 & 0.45 \\
\hline & $\mathrm{Rb}$ & 20.674 & 11.586 & 8.048 & 6.166 & 4.997 & 4.200 & 3.623 & 3.185 & 2.842 \\
\hline & $\mathrm{Ba}$ & 226.253 & 180.612 & 150.294 & 128.691 & 112.518 & 99.956 & 89.918 & 81.712 & 74.878 \\
\hline & Th & 2.102 & 1.169 & 0.809 & 0.619 & 0.501 & 0.421 & 0.363 & 0.319 & 0.285 \\
\hline & $\mathrm{U}$ & 1.132 & 0.629 & 0.436 & 0.333 & 0.270 & 0.227 & 0.195 & 0.172 & 0.153 \\
\hline & $\mathrm{Nb}$ & 18.076 & 10.872 & 7.773 & 6.049 & 4.951 & 4.191 & 3.633 & 3.206 & 2.869 \\
\hline & $\mathrm{Ta}$ & 1.052 & 0.642 & 0.462 & 0.361 & 0.296 & 0.251 & 0.218 & 0.192 & 0.172 \\
\hline & $\mathrm{La}$ & 14.013 & 12.678 & 11.575 & 10.649 & 9.860 & 9.180 & 8.588 & 8.067 & 7.606 \\
\hline & $\mathrm{Ce}$ & 23.149 & 22.199 & 21.325 & 20.516 & 19.767 & 19.070 & 18.421 & 17.815 & 17.247 \\
\hline & $\mathrm{Pb}$ & 2.733 & 2.157 & 1.781 & 1.517 & 1.321 & 1.170 & 1.050 & 0.953 & 0.871 \\
\hline & $\operatorname{Pr}$ & 2.195 & 2.184 & 2.172 & 2.160 & 2.149 & 2.137 & 2.126 & 2.115 & 2.104 \\
\hline & $\mathrm{Sr}$ & 201.355 & 205.448 & 209.711 & 214.155 & 218.791 & 223.632 & 228.692 & 233.987 & 239.533 \\
\hline & $\mathrm{Nd}$ & 6.035 & 6.163 & 6.297 & 6.437 & 6.583 & 6.736 & 6.896 & 7.064 & 7.240 \\
\hline
\end{tabular}




\begin{tabular}{|c|c|c|c|c|c|c|c|c|c|c|}
\hline & $\mathrm{Zr}$ & 186.057 & 154.396 & 131.943 & 115.192 & 102.215 & 91.865 & 83.419 & 76.395 & 70.462 \\
\hline & $\mathrm{Hf}$ & 5.594 & 4.640 & 3.964 & 3.460 & 3.069 & 2.758 & 2.504 & 2.293 & 2.115 \\
\hline & $\mathrm{Sm}$ & 0.968 & 1.004 & 1.043 & 1.084 & 1.129 & 1.178 & 1.232 & 1.290 & 1.355 \\
\hline & $\mathrm{Eu}$ & 0.270 & 0.282 & 0.294 & 0.308 & 0.322 & 0.339 & 0.357 & 0.376 & 0.399 \\
\hline & $\mathrm{Tb}$ & 0.133 & 0.139 & 0.145 & 0.152 & 0.159 & 0.167 & 0.175 & 0.185 & 0.196 \\
\hline & Dy & 0.880 & 0.915 & 0.954 & 0.995 & 1.040 & 1.090 & 1.144 & 1.204 & 1.271 \\
\hline & $\mathrm{Y}$ & 4.481 & 4.660 & 4.854 & 5.065 & 5.295 & 5.547 & 5.824 & 6.130 & 6.470 \\
\hline & $\mathrm{Yb}$ & 0.555 & 0.574 & 0.595 & 0.618 & 0.642 & 0.668 & 0.696 & 0.727 & 0.761 \\
\hline & $\mathrm{Lu}$ & 0.091 & 0.094 & 0.097 & 0.101 & 0.105 & 0.109 & 0.113 & 0.118 & 0.123 \\
\hline \multirow[t]{23}{*}{ MS41 } & Element & \multicolumn{9}{|c|}{ Melt Fraction } \\
\hline & & 0.05 & 0.10 & 0.15 & 0.20 & 0.25 & 0.30 & 0.35 & 0.40 & \\
\hline & $\mathrm{Rb}$ & 20.871 & 11.645 & 8.075 & 6.18 & 5.006 & 4.206 & 3.627 & 3.188 & \\
\hline & $\mathrm{Ba}$ & 234.079 & 185.297 & 153.341 & 130.785 & 114.015 & 101.056 & 90.743 & 82.339 & \\
\hline & Th & 2.019 & 1.144 & 0.798 & 0.613 & 0.497 & 0.419 & 0.361 & 0.318 & \\
\hline & $\mathrm{U}$ & 1.087 & 0.616 & 0.43 & 0.33 & 0.268 & 0.225 & 0.195 & 0.171 & \\
\hline & $\mathrm{Nb}$ & 17.520 & 10.678 & 7.680 & 5.996 & 4.918 & 4.168 & 3.617 & 3.194 & \\
\hline & $\mathrm{Ta}$ & 1.006 & 0.625 & 0.453 & 0.356 & 0.293 & 0.249 & 0.216 & 0.191 & \\
\hline & $\mathrm{La}$ & 10.552 & 9.896 & 9.316 & 8.801 & 8.340 & 7.925 & 7.549 & 7.207 & \\
\hline & $\mathrm{Ce}$ & 17.949 & 17.574 & 17.214 & 16.869 & 16.537 & 16.218 & 15.911 & 15.616 & \\
\hline & $\mathrm{Pb}$ & 2.803 & 2.198 & 1.808 & 1.535 & 1.334 & 1.180 & 1.057 & 0.958 & \\
\hline & $\operatorname{Pr}$ & 1.841 & 1.848 & 1.856 & 1.863 & 1.871 & 1.878 & 1.886 & 1.893 & \\
\hline & $\mathrm{Sr}$ & 210.401 & 214.357 & 218.464 & 222.731 & 227.169 & 231.787 & 236.597 & 241.611 & \\
\hline & $\mathrm{Nd}$ & 5.219 & 5.354 & 5.495 & 5.644 & 5.802 & 5.969 & 6.145 & 6.332 & \\
\hline & $\mathrm{Zr}$ & 181.875 & 151.654 & 130.046 & 113.827 & 101.205 & 91.103 & 82.835 & 75.942 & \\
\hline & $\mathrm{Hf}$ & 5.465 & 4.555 & 3.905 & 3.418 & 3.038 & 2.735 & 2.486 & 2.279 & \\
\hline & $\mathrm{Sm}$ & 0.872 & 0.906 & 0.942 & 0.982 & 1.025 & 1.072 & 1.123 & 1.180 & \\
\hline & $\mathrm{Eu}$ & 0.248 & 0.259 & 0.271 & 0.283 & 0.297 & 0.313 & 0.330 & 0.349 & \\
\hline & $\mathrm{Tb}$ & 0.124 & 0.129 & 0.135 & 0.141 & 0.148 & 0.156 & 0.164 & 0.174 & \\
\hline & Dy & 0.830 & 0.864 & 0.901 & 0.941 & 0.985 & 1.033 & 1.086 & 1.145 & \\
\hline & $\mathrm{Y}$ & 4.018 & 4.184 & 4.366 & 4.564 & 4.780 & 5.019 & 5.282 & 5.574 & \\
\hline & $\mathrm{Yb}$ & 0.531 & 0.550 & 0.571 & 0.593 & 0.617 & 0.642 & 0.671 & 0.701 & \\
\hline & $\mathrm{Lu}$ & 0.088 & 0.091 & 0.095 & 0.098 & 0.102 & 0.106 & 0.110 & 0.115 & \\
\hline \multirow[t]{9}{*}{ MS43 } & Element & \multicolumn{9}{|c|}{ Melt Fraction } \\
\hline & & 0.35 & 0.40 & 0.45 & 0.50 & 0.55 & 0.60 & 0.65 & 0.70 & 0.75 \\
\hline & $\mathrm{Rb}$ & 381.918 & 335.118 & 298.536 & 269.155 & 245.038 & 224.888 & 207.800 & 193.126 & 180.387 \\
\hline & $\mathrm{Ba}$ & 5611.690 & 5041.245 & 4576.074 & 4189.496 & 3863.145 & 3583.964 & 3342.414 & 3131.369 & 2945.392 \\
\hline & Th & 9.489 & 8.329 & 7.421 & 6.692 & 6.093 & 5.592 & 5.168 & 4.803 & 4.487 \\
\hline & $\mathrm{U}$ & 3.346 & 2.937 & 2.617 & 2.359 & 2.148 & 1.972 & 1.822 & 1.694 & 1.582 \\
\hline & $\mathrm{Nb}$ & 24.964 & 23.070 & 21.443 & 20.030 & 18.792 & 17.698 & 16.725 & 15.853 & 15.067 \\
\hline & $\mathrm{Ta}$ & 1.396 & 1.290 & 1.199 & 1.120 & 1.050 & 0.989 & 0.935 & 0.886 & 0.842 \\
\hline & $\mathrm{La}$ & 31.645 & 29.974 & 28.471 & 27.112 & 25.876 & 24.748 & 23.715 & 22.764 & 21.887 \\
\hline
\end{tabular}




\begin{tabular}{|c|c|c|c|c|c|c|c|c|c|c|}
\hline & $\mathrm{Ce}$ & 53.344 & 51.856 & 50.449 & 49.117 & 47.853 & 46.653 & 45.511 & 44.424 & 43.387 \\
\hline & $\mathrm{Pb}$ & 20.107 & 17.734 & 15.862 & 14.348 & 13.097 & 12.047 & 11.153 & 10.383 & 9.712 \\
\hline & $\operatorname{Pr}$ & 5.882 & 5.822 & 5.764 & 5.706 & 5.650 & 5.595 & 5.541 & 5.488 & 5.436 \\
\hline & $\mathrm{Sr}$ & 432.513 & 415.372 & 399.538 & 384.867 & 371.235 & 358.536 & 346.677 & 335.577 & 325.166 \\
\hline & $\mathrm{Nd}$ & 19.325 & 19.540 & 19.760 & 19.985 & 20.215 & 20.450 & 20.691 & 20.937 & 21.190 \\
\hline & $\mathrm{Zr}$ & 330.539 & 303.453 & 280.469 & 260.723 & 243.573 & 228.541 & 215.256 & 203.431 & 192.837 \\
\hline & $\mathrm{Hf}$ & 8.834 & 8.107 & 7.491 & 6.962 & 6.503 & 6.100 & 5.745 & 5.428 & 5.145 \\
\hline & $\mathrm{Sm}$ & 3.296 & 3.410 & 3.532 & 3.662 & 3.803 & 3.955 & 4.120 & 4.299 & 4.494 \\
\hline & $\mathrm{Eu}$ & 0.856 & 0.891 & 0.929 & 0.970 & 1.016 & 1.065 & 1.120 & 1.181 & 1.249 \\
\hline & $\mathrm{Tb}$ & 0.549 & 0.571 & 0.595 & 0.621 & 0.650 & 0.681 & 0.716 & 0.754 & 0.797 \\
\hline & Dy & 3.841 & 3.980 & 4.130 & 4.291 & 4.466 & 4.655 & 4.861 & 5.086 & 5.333 \\
\hline & $\mathrm{Y}$ & 19.102 & 19.853 & 20.665 & 21.546 & 22.506 & 23.555 & 24.707 & 25.978 & 27.386 \\
\hline & $\mathrm{Yb}$ & 2.482 & 2.537 & 2.594 & 2.654 & 2.717 & 2.782 & 2.851 & 2.924 & 3.000 \\
\hline & $\mathrm{Lu}$ & 0.455 & 0.461 & 0.467 & 0.473 & 0.479 & 0.486 & 0.492 & 0.499 & 0.506 \\
\hline MS44 & Element & \multicolumn{9}{|c|}{ Melt Fraction } \\
\hline & & 0.55 & 0.60 & 0.65 & 0.70 & 0.75 & 0.80 & 0.85 & 0.90 & 0.95 \\
\hline & $\mathrm{Rb}$ & 85.258 & 78.202 & 72.224 & 67.096 & 62.647 & 58.752 & 55.313 & 52.254 & 49.516 \\
\hline & $\mathrm{Ba}$ & 1547.948 & 1421.137 & 1313.531 & 1221.073 & 1140.775 & 1070.386 & 1008.179 & 952.805 & 903.198 \\
\hline & Th & 9.222 & 8.462 & 7.818 & 7.265 & 6.785 & 6.364 & 5.993 & 5.662 & 5.367 \\
\hline & $\mathrm{U}$ & 3.291 & 3.020 & 2.790 & 2.593 & 2.421 & 2.271 & 2.139 & 2.021 & 1.915 \\
\hline & $\mathrm{Nb}$ & 19.007 & 17.479 & 16.177 & 15.056 & 14.081 & 13.224 & 12.465 & 11.789 & 11.182 \\
\hline & $\mathrm{Ta}$ & 1.332 & 1.224 & 1.133 & 1.055 & 0.986 & 0.926 & 0.873 & 0.826 & 0.783 \\
\hline & $\mathrm{La}$ & 36.201 & 34.303 & 32.594 & 31.047 & 29.640 & 28.355 & 27.177 & 26.093 & 25.092 \\
\hline & $\mathrm{Ce}$ & 66.404 & 63.874 & 61.529 & 59.350 & 57.320 & 55.425 & 53.651 & 51.987 & 50.423 \\
\hline & $\mathrm{Pb}$ & 14.413 & 13.228 & 12.224 & 11.360 & 10.611 & 9.955 & 9.375 & 8.859 & 8.396 \\
\hline & $\operatorname{Pr}$ & 7.885 & 7.664 & 7.455 & 7.257 & 7.069 & 6.891 & 6.722 & 6.560 & 6.407 \\
\hline & $\mathrm{Sr}$ & 783.473 & 727.795 & 679.506 & 637.226 & 599.899 & 566.703 & 536.988 & 510.235 & 486.020 \\
\hline & $\mathrm{Nd}$ & 27.230 & 27.069 & 26.911 & 26.754 & 26.599 & 26.446 & 26.294 & 26.144 & 25.996 \\
\hline & $\mathrm{Zr}$ & 285.819 & 264.028 & 245.324 & 229.095 & 214.880 & 202.326 & 191.158 & 181.158 & 172.153 \\
\hline & $\mathrm{Hf}$ & 7.510 & 6.938 & 6.446 & 6.020 & 5.647 & 5.317 & 5.023 & 4.761 & 4.524 \\
\hline & $\mathrm{Sm}$ & 4.998 & 5.090 & 5.187 & 5.287 & 5.391 & 5.499 & 5.612 & 5.730 & 5.852 \\
\hline & $\mathrm{Eu}$ & 1.337 & 1.374 & 1.414 & 1.456 & 1.500 & 1.547 & 1.598 & 1.651 & 1.709 \\
\hline & $\mathrm{Tb}$ & 0.743 & 0.762 & 0.782 & 0.804 & 0.826 & 0.850 & 0.875 & 0.902 & 0.930 \\
\hline & Dy & 4.979 & 5.064 & 5.151 & 5.242 & 5.336 & 5.433 & 5.534 & 5.638 & 5.747 \\
\hline & $\mathrm{Y}$ & 23.394 & 24.060 & 24.765 & 25.513 & 26.307 & 27.152 & 28.053 & 29.016 & 30.047 \\
\hline & $\mathrm{Yb}$ & 2.860 & 2.855 & 2.851 & 2.846 & 2.842 & 2.837 & 2.833 & 2.829 & 2.824 \\
\hline & $\mathrm{Lu}$ & 0.489 & 0.484 & 0.480 & 0.475 & 0.471 & 0.466 & 0.462 & 0.458 & 0.454 \\
\hline MS45 & Element & \multicolumn{9}{|c|}{ Melt Fraction } \\
\hline & & 0.35 & 0.40 & 0.45 & 0.50 & 0.55 & 0.60 & 0.65 & 0.70 & 0.75 \\
\hline & $\mathrm{Rb}$ & 190.693 & 169.653 & 152.794 & 138.983 & 127.462 & 117.705 & 109.335 & 102.077 & 95.722 \\
\hline & $\mathrm{Ba}$ & 3361.918 & 3148.471 & 2960.509 & 2793.726 & 2644.732 & 2510.826 & 2389.826 & 2279.952 & 2179.737 \\
\hline
\end{tabular}




\begin{tabular}{|c|c|c|c|c|c|c|c|c|c|c|c|}
\hline \multirow{40}{*}{ w } & & Th & 21.781 & 19.097 & 17.002 & 15.321 & 13.943 & 12.792 & 11.817 & 10.980 & 10.254 \\
\hline & & $\mathrm{U}$ & 6.728 & 5.899 & 5.252 & 4.733 & 4.307 & 3.951 & 3.650 & 3.392 & 3.167 \\
\hline & & $\mathrm{Nb}$ & 35.050 & 31.286 & 28.252 & 25.755 & 23.663 & 21.885 & 20.356 & 19.027 & 17.860 \\
\hline & & Тa & 2.432 & 2.171 & 1.961 & 1.788 & 1.643 & 1.520 & 1.414 & 1.322 & 1.241 \\
\hline & & $\mathrm{La}$ & 84.149 & 74.404 & 66.682 & 60.411 & 55.219 & 50.849 & 47.119 & 43.900 & 41.092 \\
\hline & & $\mathrm{Ce}$ & 181.846 & 160.372 & 143.434 & 129.732 & 118.420 & 108.922 & 100.835 & 93.865 & 87.797 \\
\hline & & $\mathrm{Pb}$ & 23.522 & 20.874 & 18.762 & 17.039 & 15.605 & 14.394 & 13.357 & 12.460 & 11.675 \\
\hline & & $\operatorname{Pr}$ & 22.851 & 20.151 & 18.022 & 16.300 & 14.878 & 13.685 & 12.668 & 11.792 & 11.030 \\
\hline & & $\mathrm{Sr}$ & 291.626 & 280.918 & 270.969 & 261.700 & 253.045 & 244.944 & 237.345 & 230.204 & 223.479 \\
\hline & & $\mathrm{Nd}$ & 92.470 & 81.522 & 72.891 & 65.913 & 60.154 & 55.321 & 51.207 & 47.662 & 44.576 \\
\hline & & $\mathrm{Zr}$ & 523.428 & 460.946 & 411.791 & 372.109 & 339.403 & 311.982 & 288.660 & 268.583 & 251.117 \\
\hline & & $\mathrm{Hf}$ & 13.886 & 12.228 & 10.923 & 9.870 & 9.002 & 8.274 & 7.656 & 7.123 & 6.659 \\
\hline & & $\mathrm{Sm}$ & 20.451 & 18.042 & 16.141 & 14.602 & 13.331 & 12.263 & 11.354 & 10.571 & 9.888 \\
\hline & & $\mathrm{Eu}$ & 4.142 & 3.839 & 3.578 & 3.349 & 3.149 & 2.970 & 2.811 & 2.669 & 2.540 \\
\hline & & $\mathrm{Tb}$ & 3.124 & 2.769 & 2.486 & 2.255 & 2.064 & 1.902 & 1.764 & 1.645 & 1.541 \\
\hline & & Dy & 18.439 & 16.475 & 14.888 & 13.580 & 12.484 & 11.551 & 10.748 & 10.050 & 9.436 \\
\hline & & Y & 85.933 & 78.164 & 71.683 & 66.195 & 61.487 & 57.404 & 53.830 & 50.675 & 47.869 \\
\hline & & $\mathrm{Yb}$ & 7.423 & 6.886 & 6.421 & 6.015 & 5.657 & 5.340 & 5.056 & 4.801 & 4.570 \\
\hline & & $\mathrm{Lu}$ & 1.212 & 1.121 & 1.043 & 0.975 & 0.915 & 0.862 & 0.815 & 0.773 & 0.735 \\
\hline & MS46 & Element & \multicolumn{9}{|c|}{ Melt Fraction } \\
\hline & & & 0.40 & 0.45 & 0.50 & 0.55 & 0.60 & 0.65 & 0.70 & 0.75 & 0.80 \\
\hline & & $\mathrm{Rb}$ & 161.199 & 160.259 & 159.330 & 158.411 & 157.504 & 156.606 & 155.719 & 154.842 & 153.975 \\
\hline & & $\mathrm{Ba}$ & 712.392 & 756.094 & 805.508 & 861.833 & 926.627 & 1001.955 & 1090.615 & 1196.488 & 1325.126 \\
\hline & & Th & 10.460 & 9.360 & 8.470 & 7.734 & 7.116 & 6.589 & 6.135 & 5.739 & 5.392 \\
\hline & & $\mathrm{U}$ & 3.230 & 2.890 & 2.615 & 2.388 & 2.197 & 2.034 & 1.894 & 1.772 & 1.665 \\
\hline & & $\mathrm{Nb}$ & 12.828 & 12.942 & 13.057 & 13.175 & 13.294 & 13.416 & 13.540 & 13.666 & 13.795 \\
\hline & & $\mathrm{Ta}$ & 0.703 & 0.710 & 0.718 & 0.725 & 0.733 & 0.741 & 0.749 & 0.757 & 0.765 \\
\hline & & $\mathrm{La}$ & 37.312 & 35.625 & 34.084 & 32.670 & 31.369 & 30.168 & 29.055 & 28.021 & 27.059 \\
\hline & & $\mathrm{Ce}$ & 75.163 & 72.567 & 70.145 & 67.879 & 65.755 & 63.760 & 61.882 & 60.112 & 58.440 \\
\hline & & $\mathrm{Pb}$ & 16.970 & 15.758 & 14.708 & 13.788 & 12.977 & 12.257 & 11.612 & 11.031 & 10.506 \\
\hline & & $\operatorname{Pr}$ & 9.759 & 9.447 & 9.154 & 8.879 & 8.621 & 8.376 & 8.146 & 7.927 & 7.720 \\
\hline & & $\mathrm{Sr}$ & 193.209 & 197.578 & 202.149 & 206.937 & 211.958 & 217.227 & 222.766 & 228.594 & 234.736 \\
\hline & & $\mathrm{Nd}$ & 35.782 & 35.224 & 34.683 & 34.158 & 33.649 & 33.155 & 32.675 & 32.209 & 31.756 \\
\hline & & $\mathrm{Zr}$ & 364.228 & 336.601 & 312.871 & 292.265 & 274.207 & 258.249 & 244.048 & 231.326 & 219.865 \\
\hline & & $\mathrm{Hf}$ & 9.906 & 9.142 & 8.487 & 7.920 & 7.424 & 6.986 & 6.597 & 6.249 & 5.936 \\
\hline & & $\mathrm{Sm}$ & 7.714 & 7.688 & 7.662 & 7.636 & 7.610 & 7.584 & 7.559 & 7.534 & 7.509 \\
\hline & & $\mathrm{Eu}$ & 1.602 & 1.642 & 1.684 & 1.728 & 1.774 & 1.823 & 1.875 & 1.930 & 1.988 \\
\hline & & $\mathrm{Tb}$ & 1.471 & 1.458 & 1.445 & 1.432 & 1.419 & 1.407 & 1.394 & 1.382 & 1.370 \\
\hline & & Dy & 10.852 & 10.589 & 10.340 & 10.101 & 9.873 & 9.656 & 9.448 & 9.248 & 9.057 \\
\hline & & $\mathrm{Y}$ & 39.882 & 40.172 & 40.466 & 40.765 & 41.068 & 41.376 & 41.688 & 42.005 & 42.327 \\
\hline
\end{tabular}




\begin{tabular}{|c|c|c|c|c|c|c|c|c|c|c|}
\hline & $\mathrm{Yb}$ & 6.578 & 6.276 & 6.000 & 5.748 & 5.516 & 5.302 & 5.104 & 4.920 & 4.750 \\
\hline & $\mathrm{Lu}$ & 1.177 & 1.105 & 1.041 & 0.984 & 0.933 & 0.887 & 0.846 & 0.808 & 0.773 \\
\hline \multirow[t]{23}{*}{ MS47 } & Element & \multicolumn{9}{|c|}{ Melt Fraction } \\
\hline & & 0.50 & 0.55 & 0.60 & 0.65 & 0.70 & 0.75 & 0.80 & 0.85 & 0.90 \\
\hline & $\mathrm{Rb}$ & 121.257 & 110.679 & 101.798 & 94.237 & 87.721 & 82.048 & 77.064 & 72.651 & 68.716 \\
\hline & $\mathrm{Ba}$ & 1720.089 & 1580.234 & 1461.411 & 1359.208 & 1270.365 & 1192.424 & 1123.494 & 1062.098 & 1007.064 \\
\hline & Th & 11.664 & 10.678 & 9.846 & 9.134 & 8.518 & 7.980 & 7.506 & 7.085 & 6.709 \\
\hline & $\mathrm{U}$ & 3.789 & 3.468 & 3.198 & 2.967 & 2.767 & 2.592 & 2.438 & 2.301 & 2.179 \\
\hline & $\mathrm{Nb}$ & 22.811 & 21.182 & 19.770 & 18.535 & 17.445 & 16.476 & 15.609 & 14.829 & 14.123 \\
\hline & $\mathrm{Ta}$ & 1.539 & 1.429 & 1.334 & 1.251 & 1.177 & 1.112 & 1.053 & 1.000 & 0.953 \\
\hline & $\mathrm{La}$ & 22.493 & 23.046 & 23.628 & 24.240 & 24.884 & 25.563 & 26.281 & 27.040 & 27.844 \\
\hline & $\mathrm{Ce}$ & 35.804 & 37.408 & 39.163 & 41.090 & 43.217 & 45.576 & 48.207 & 51.162 & 54.501 \\
\hline & $\mathrm{Pb}$ & 18.234 & 16.688 & 15.384 & 14.268 & 13.304 & 12.461 & 11.719 & 11.061 & 10.472 \\
\hline & Pr & 3.959 & 4.171 & 4.407 & 4.672 & 4.971 & 5.310 & 5.699 & 6.149 & 6.677 \\
\hline & $\mathrm{Sr}$ & 601.900 & 581.486 & 562.412 & 544.548 & 527.785 & 512.023 & 497.175 & 483.164 & 469.921 \\
\hline & $\mathrm{Nd}$ & 11.432 & 12.237 & 13.163 & 14.240 & 15.511 & 17.029 & 18.878 & 21.177 & 24.113 \\
\hline & $\mathrm{Zr}$ & 313.170 & 295.161 & 279.111 & 264.717 & 251.734 & 239.965 & 229.248 & 219.447 & 210.449 \\
\hline & $\mathrm{Hf}$ & 8.32 & 7.843 & 7.418 & 7.037 & 6.693 & 6.381 & 6.097 & 5.837 & 5.598 \\
\hline & $\mathrm{Sm}$ & 1.799 & 1.948 & 2.123 & 2.333 & 2.589 & 2.908 & 3.317 & 3.859 & 4.613 \\
\hline & $\mathrm{Eu}$ & 0.462 & 0.502 & 0.55 & 0.607 & 0.678 & 0.768 & 0.886 & 1.045 & 1.276 \\
\hline & $\mathrm{Tb}$ & 0.261 & 0.282 & 0.309 & 0.34 & 0.378 & 0.426 & 0.489 & 0.572 & 0.69 \\
\hline & Dy & 1.893 & 2.043 & 2.22 & 2.43 & 2.683 & 2.996 & 3.391 & 3.906 & 4.607 \\
\hline & $\mathrm{Y}$ & 7.454 & 8.102 & 8.873 & 9.807 & 10.961 & 12.422 & 14.332 & 16.938 & 20.7 \\
\hline & $\mathrm{Yb}$ & 1.308 & 1.396 & 1.497 & 1.613 & 1.749 & 1.91 & 2.104 & 2.342 & 2.639 \\
\hline & $\mathrm{Lu}$ & 0.248 & 0.263 & 0.279 & 0.298 & 0.32 & 0.345 & 0.374 & 0.408 & 0.45 \\
\hline \multirow[t]{16}{*}{ MS48 } & Element & \multicolumn{9}{|c|}{ Melt Fraction } \\
\hline & & 0.50 & 0.55 & 0.60 & 0.65 & 0.70 & 0.75 & 0.80 & 0.85 & 0.90 \\
\hline & $\mathrm{Rb}$ & 140.093 & 128.301 & 118.340 & 109.814 & 102.435 & 95.984 & 90.298 & 85.248 & 80.733 \\
\hline & $\mathrm{Ba}$ & 2973.230 & 2788.149 & 2624.761 & 2479.462 & 2349.405 & 2232.313 & 2126.338 & 2029.969 & 1941.957 \\
\hline & Th & 15.325 & 13.946 & 12.795 & 11.819 & 10.981 & 10.254 & 9.618 & 9.056 & 8.556 \\
\hline & $\mathrm{U}$ & 4.734 & 4.308 & 3.952 & 3.651 & 3.392 & 3.167 & 2.971 & 2.797 & 2.643 \\
\hline & $\mathrm{Nb}$ & 25.910 & 23.781 & 21.975 & 20.424 & 19.077 & 17.897 & 16.855 & 15.927 & 15.096 \\
\hline & $\mathrm{Ta}$ & 1.798 & 1.651 & 1.526 & 1.418 & 1.325 & 1.243 & 1.171 & 1.107 & 1.049 \\
\hline & $\mathrm{La}$ & 61.742 & 56.216 & 51.597 & 47.680 & 44.316 & 41.395 & 38.836 & 36.574 & 34.562 \\
\hline & $\mathrm{Ce}$ & 131.593 & 119.811 & 109.966 & 101.616 & 94.445 & 88.219 & 82.763 & 77.943 & 73.653 \\
\hline & $\mathrm{Pb}$ & 17.562 & 15.998 & 14.690 & 13.579 & 12.625 & 11.796 & 11.069 & 10.426 & 9.854 \\
\hline & $\operatorname{Pr}$ & 16.503 & 15.030 & 13.799 & 12.754 & 11.856 & 11.076 & 10.392 & 9.788 & 9.251 \\
\hline & $\mathrm{Sr}$ & 382.034 & 348.598 & 320.544 & 296.669 & 276.104 & 258.205 & 242.486 & 228.571 & 216.166 \\
\hline & $\mathrm{Nd}$ & 66.558 & 60.637 & 55.683 & 51.478 & 47.863 & 44.723 & 41.969 & 39.535 & 37.367 \\
\hline & $\mathrm{Zr}$ & 371.095 & 338.643 & 311.411 & 288.232 & 268.265 & 250.885 & 235.620 & 222.106 & 210.058 \\
\hline & $\mathrm{Hf}$ & 9.844 & 8.982 & 8.260 & 7.645 & 7.115 & 6.654 & 6.249 & 5.890 & 5.570 \\
\hline
\end{tabular}




\begin{tabular}{|c|c|c|c|c|c|c|c|c|c|c|}
\hline & $\mathrm{Sm}$ & 14.667 & 13.380 & 12.300 & 11.382 & 10.591 & 9.903 & 9.299 & 8.764 & 8.288 \\
\hline & $\mathrm{Eu}$ & 3.969 & 3.627 & 3.340 & 3.095 & 2.884 & 2.699 & 2.537 & 2.393 & 2.265 \\
\hline & $\mathrm{Tb}$ & 2.253 & 2.062 & 1.901 & 1.764 & 1.644 & 1.540 & 1.449 & 1.367 & 1.294 \\
\hline & Dy & 13.475 & 12.403 & 11.490 & 10.702 & 10.015 & 9.411 & 8.875 & 8.398 & 7.969 \\
\hline & $\mathrm{Y}$ & 64.760 & 60.369 & 56.535 & 53.160 & 50.164 & 47.489 & 45.084 & 42.911 & 40.938 \\
\hline & $\mathrm{Yb}$ & 5.789 & 5.476 & 5.195 & 4.942 & 4.713 & 4.503 & 4.312 & 4.136 & 3.974 \\
\hline & $\mathrm{Lu}$ & 0.941 & 0.888 & 0.841 & 0.798 & 0.760 & 0.725 & 0.693 & 0.664 & 0.638 \\
\hline \multirow[t]{23}{*}{ MS49 } & Element & \multicolumn{9}{|c|}{ Melt Fraction } \\
\hline & & 0.75 & 0.80 & 0.85 & 0.90 & 0.95 & & & & \\
\hline & $\mathrm{Rb}$ & 96.911 & 90.953 & 85.685 & 80.994 & 76.789 & & & & \\
\hline & $\mathrm{Ba}$ & 2378.792 & 2231.025 & 2100.542 & 1984.478 & 1880.569 & & & & \\
\hline & Th & 10.210 & 9.587 & 9.035 & 8.544 & 8.103 & & & & \\
\hline & $\mathrm{U}$ & 3.154 & 2.961 & 2.791 & 2.639 & 2.503 & & & & \\
\hline & $\mathrm{Nb}$ & 16.970 & 16.188 & 15.476 & 14.823 & 14.223 & & & & \\
\hline & $\mathrm{Ta}$ & 1.166 & 1.115 & 1.069 & 1.026 & 0.987 & & & & \\
\hline & $\mathrm{La}$ & 40.860 & 38.458 & 36.322 & 34.411 & 32.691 & & & & \\
\hline & $\mathrm{Ce}$ & 87.179 & 82.029 & 77.453 & 73.361 & 69.680 & & & & \\
\hline & $\mathrm{Pb}$ & 11.741 & 11.030 & 10.401 & 9.839 & 9.335 & & & & \\
\hline & $\operatorname{Pr}$ & 10.957 & 10.308 & 9.732 & 9.217 & 8.754 & & & & \\
\hline & $\mathrm{Sr}$ & 259.293 & 243.252 & 229.081 & 216.470 & 205.175 & & & & \\
\hline & $\mathrm{Nd}$ & 44.263 & 41.644 & 39.318 & 37.238 & 35.367 & & & & \\
\hline & $\mathrm{Zr}$ & 239.168 & 227.255 & 216.473 & 206.667 & 197.712 & & & & \\
\hline & $\mathrm{Hf}$ & 6.360 & 6.039 & 5.749 & 5.486 & 5.245 & & & & \\
\hline & $\mathrm{Sm}$ & 9.797 & 9.224 & 8.714 & 8.258 & 7.847 & & & & \\
\hline & $\mathrm{Eu}$ & 2.663 & 2.511 & 2.376 & 2.254 & 2.145 & & & & \\
\hline & $\mathrm{Tb}$ & 1.516 & 1.432 & 1.356 & 1.288 & 1.226 & & & & \\
\hline & Dy & 9.153 & 8.691 & 8.273 & 7.893 & 7.547 & & & & \\
\hline & $\mathrm{Y}$ & 44.972 & 43.246 & 41.647 & 40.163 & 38.780 & & & & \\
\hline & $\mathrm{Yb}$ & 4.125 & 4.029 & 3.937 & 3.849 & 3.765 & & & & \\
\hline & $\mathrm{Lu}$ & 0.668 & 0.650 & 0.634 & 0.619 & 0.604 & & & & \\
\hline \multirow[t]{11}{*}{ MS50 } & Element & \multicolumn{9}{|c|}{ Melt Fraction } \\
\hline & & 0.45 & 0.50 & 0.55 & 0.60 & 0.65 & 0.70 & 0.75 & 0.80 & 0.85 \\
\hline & $\mathrm{Rb}$ & 128.014 & 115.373 & 105.004 & 96.345 & 89.006 & 82.705 & 77.238 & 72.448 & 68.218 \\
\hline & $\mathrm{Ba}$ & 3674.545 & 3334.890 & 3052.714 & 2814.564 & 2610.883 & 2434.692 & 2280.777 & 2145.166 & 2024.776 \\
\hline & Th & 14.879 & 13.407 & 12.200 & 11.193 & 10.339 & 9.606 & 8.970 & 8.414 & 7.922 \\
\hline & $\mathrm{U}$ & 4.923 & 4.436 & 4.037 & 3.703 & 3.421 & 3.178 & 2.968 & 2.784 & 2.621 \\
\hline & $\mathrm{Nb}$ & 22.807 & 20.908 & 19.301 & 17.924 & 16.730 & 15.685 & 14.763 & 13.944 & 13.210 \\
\hline & $\mathrm{Ta}$ & 1.649 & 1.512 & 1.396 & 1.296 & 1.210 & 1.134 & 1.067 & 1.008 & 0.955 \\
\hline & $\mathrm{La}$ & 53.797 & 48.932 & 44.874 & 41.437 & 38.490 & 35.934 & 33.696 & 31.720 & 29.964 \\
\hline & $\mathrm{Ce}$ & 105.920 & 96.988 & 89.444 & 82.990 & 77.404 & 72.523 & 68.221 & 64.400 & 60.985 \\
\hline & $\mathrm{Pb}$ & 16.170 & 14.596 & 13.301 & 12.217 & 11.296 & 10.504 & 9.816 & 9.213 & 8.680 \\
\hline
\end{tabular}




\begin{tabular}{|c|c|c|c|c|c|c|c|c|c|c|}
\hline & $\operatorname{Pr}$ & 12.409 & 11.472 & 10.666 & 9.966 & 9.352 & 8.810 & 8.327 & 7.894 & 7.504 \\
\hline & $\mathrm{Sr}$ & 389.025 & 361.972 & 338.436 & 317.774 & 299.490 & 283.196 & 268.583 & 255.404 & 243.458 \\
\hline & $\mathrm{Nd}$ & 47.412 & 44.152 & 41.310 & 38.813 & 36.600 & 34.626 & 32.854 & 31.254 & 29.803 \\
\hline & $\mathrm{Zr}$ & 324.683 & 296.587 & 272.967 & 252.831 & 235.462 & 220.326 & 207.018 & 195.226 & 184.706 \\
\hline & $\mathrm{Hf}$ & 8.503 & 7.766 & 7.147 & 6.619 & 6.163 & 5.767 & 5.418 & 5.109 & 4.833 \\
\hline & $\mathrm{Sm}$ & 9.015 & 8.598 & 8.219 & 7.871 & 7.551 & 7.257 & 6.984 & 6.731 & 6.496 \\
\hline & $\mathrm{Eu}$ & 2.207 & 2.123 & 2.045 & 1.972 & 1.904 & 1.841 & 1.782 & 1.726 & 1.674 \\
\hline & $\mathrm{Tb}$ & 1.341 & 1.293 & 1.249 & 1.208 & 1.169 & 1.133 & 1.099 & 1.067 & 1.036 \\
\hline & Dy & 8.154 & 7.882 & 7.627 & 7.389 & 7.165 & 6.954 & 6.755 & 6.567 & 6.389 \\
\hline & $\mathrm{Y}$ & 42.388 & 41.081 & 39.851 & 38.693 & 37.601 & 36.568 & 35.591 & 34.664 & 33.785 \\
\hline & $\mathrm{Yb}$ & 3.797 & 3.702 & 3.613 & 3.527 & 3.445 & 3.367 & 3.293 & 3.222 & 3.153 \\
\hline & $\mathrm{Lu}$ & 0.650 & 0.629 & 0.609 & 0.591 & 0.573 & 0.557 & 0.541 & 0.527 & 0.513 \\
\hline MS51 & Element & \multicolumn{9}{|c|}{ Melt Fraction } \\
\hline & & 0.75 & 0.80 & 0.85 & 0.90 & 0.95 & & & & \\
\hline & $\mathrm{Rb}$ & 77.190 & 72.415 & 68.196 & 64.441 & 61.079 & & & & \\
\hline & $\mathrm{Ba}$ & 2244.413 & 2119.329 & 2007.451 & 1906.793 & 1815.747 & & & & \\
\hline & Th & 8.969 & 8.412 & 7.921 & 7.484 & 7.092 & & & & \\
\hline & $\mathrm{U}$ & 2.967 & 2.783 & 2.621 & 2.476 & 2.347 & & & & \\
\hline & $\mathrm{Nb}$ & 14.194 & 13.533 & 12.932 & 12.381 & 11.876 & & & & \\
\hline & $\mathrm{Ta}$ & 1.026 & 0.979 & 0.935 & 0.895 & 0.859 & & & & \\
\hline & $\mathrm{La}$ & 33.009 & 31.231 & 29.635 & 28.194 & 26.887 & & & & \\
\hline & $\mathrm{Ce}$ & 65.804 & 62.663 & 59.807 & 57.201 & 54.812 & & & & \\
\hline & $\mathrm{Pb}$ & 9.782 & 9.189 & 8.663 & 8.195 & 7.774 & & & & \\
\hline & $\operatorname{Pr}$ & 7.852 & 7.548 & 7.266 & 7.005 & 6.762 & & & & \\
\hline & $\mathrm{Sr}$ & 249.024 & 241.004 & 233.484 & 226.420 & 219.770 & & & & \\
\hline & $\mathrm{Nd}$ & 30.429 & 29.467 & 28.564 & 27.715 & 26.915 & & & & \\
\hline & $\mathrm{Zr}$ & 201.192 & 191.053 & 181.886 & 173.559 & 165.961 & & & & \\
\hline & $\mathrm{Hf}$ & 5.267 & 5.001 & 4.761 & 4.542 & 4.343 & & & & \\
\hline & $\mathrm{Sm}$ & 6.065 & 6.027 & 5.990 & 5.953 & 5.916 & & & & \\
\hline & $\mathrm{Eu}$ & 1.511 & 1.516 & 1.521 & 1.525 & 1.530 & & & & \\
\hline & $\mathrm{Tb}$ & 0.925 & 0.931 & 0.937 & 0.943 & 0.949 & & & & \\
\hline & Dy & 5.686 & 5.730 & 5.774 & 5.818 & 5.864 & & & & \\
\hline & Y & 30.176 & 30.412 & 30.652 & 30.896 & 31.143 & & & & \\
\hline & $\mathrm{Yb}$ & 2.791 & 2.824 & 2.858 & 2.893 & 2.928 & & & & \\
\hline & $\mathrm{Lu}$ & 0.470 & 0.471 & 0.472 & 0.473 & 0.474 & & & & \\
\hline MS52 & Element & \multicolumn{9}{|c|}{ Melt Fraction } \\
\hline & & 0.45 & 0.50 & 0.55 & 0.60 & 0.65 & 0.70 & 0.75 & 0.80 & 0.85 \\
\hline & $\mathrm{Rb}$ & 73.964 & 66.735 & 60.794 & 55.824 & 51.605 & 47.979 & 44.829 & 42.067 & 39.626 \\
\hline & $\mathrm{Ba}$ & 2379.345 & 2213.016 & 2068.422 & 1941.565 & 1829.369 & 1729.431 & 1639.847 & 1559.086 & 1485.907 \\
\hline & $\mathrm{Th}$ & 7.967 & 7.183 & 6.539 & 6.001 & 5.545 & 5.154 & 4.814 & 4.516 & 4.253 \\
\hline & $\mathrm{U}$ & 2.619 & 2.361 & 2.150 & 1.973 & 1.823 & 1.694 & 1.582 & 1.485 & 1.398 \\
\hline
\end{tabular}




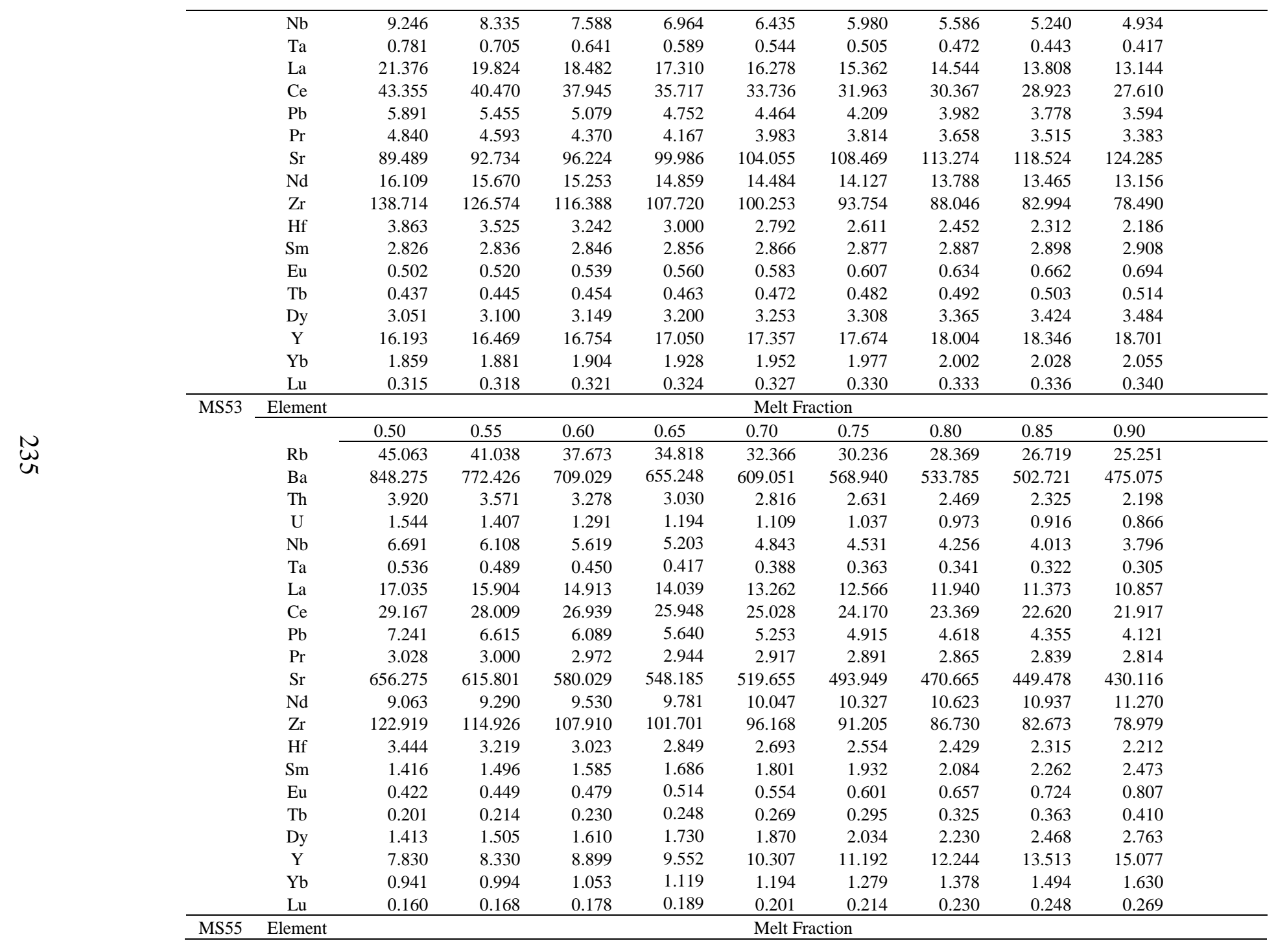




\begin{tabular}{|c|c|c|c|c|c|c|c|c|c|c|}
\hline & & 0.30 & 0.35 & 0.40 & 0.45 & 0.50 & 0.55 & 0.60 & 0.65 & 0.70 \\
\hline & $\mathrm{Rb}$ & 422.038 & 380.193 & 345.897 & 317.277 & 293.031 & 272.227 & 254.182 & 238.380 & 224.428 \\
\hline & $\mathrm{Ba}$ & 3519.827 & 3445.117 & 3373.513 & 3304.824 & 3238.877 & 3175.510 & 3114.575 & 3055.934 & 2999.461 \\
\hline & Th & 0.941 & 0.811 & 0.713 & 0.636 & 0.574 & 0.523 & 0.480 & 0.444 & 0.413 \\
\hline & $\mathrm{U}$ & 0.594 & 0.512 & 0.450 & 0.401 & 0.362 & 0.330 & 0.303 & 0.280 & 0.260 \\
\hline & $\mathrm{Nb}$ & 14.827 & 13.648 & 12.642 & 11.775 & 11.019 & 10.354 & 9.765 & 9.239 & 8.767 \\
\hline & $\mathrm{Ta}$ & 0.580 & 0.536 & 0.497 & 0.464 & 0.435 & 0.409 & 0.387 & 0.366 & 0.348 \\
\hline & $\mathrm{La}$ & 12.077 & 10.845 & 9.840 & 9.007 & 8.303 & 7.701 & 7.181 & 6.726 & 6.326 \\
\hline & $\mathrm{Ce}$ & 23.473 & 21.884 & 20.497 & 19.276 & 18.192 & 17.223 & 16.352 & 15.565 & 14.851 \\
\hline & $\mathrm{Pb}$ & 12.894 & 11.242 & 9.966 & 8.949 & 8.121 & 7.433 & 6.853 & 6.357 & 5.927 \\
\hline & $\operatorname{Pr}$ & 2.703 & 2.615 & 2.533 & 2.456 & 2.384 & 2.315 & 2.251 & 2.190 & 2.132 \\
\hline & $\mathrm{Sr}$ & 492.466 & 449.764 & 413.876 & 383.292 & 356.917 & 333.938 & 313.739 & 295.844 & 279.881 \\
\hline & $\mathrm{Nd}$ & 8.901 & 8.932 & 8.963 & 8.994 & 9.026 & 9.057 & 9.089 & 9.121 & 9.153 \\
\hline & $\mathrm{Zr}$ & 165.394 & 150.378 & 137.861 & 127.268 & 118.187 & 110.315 & 103.427 & 97.348 & 91.944 \\
\hline & $\mathrm{Hf}$ & 4.849 & 4.405 & 4.036 & 3.724 & 3.456 & 3.225 & 3.022 & 2.844 & 2.685 \\
\hline & $\mathrm{Sm}$ & 1.698 & 1.754 & 1.813 & 1.876 & 1.944 & 2.017 & 2.095 & 2.180 & 2.273 \\
\hline & $\mathrm{Eu}$ & 0.490 & 0.509 & 0.530 & 0.552 & 0.577 & 0.603 & 0.632 & 0.664 & 0.700 \\
\hline & $\mathrm{Tb}$ & 0.315 & 0.328 & 0.343 & 0.359 & 0.377 & 0.396 & 0.418 & 0.442 & 0.469 \\
\hline & Dy & 2.288 & 2.380 & 2.481 & 2.590 & 2.709 & 2.840 & 2.984 & 3.143 & 3.321 \\
\hline & $\mathrm{Y}$ & 12.395 & 12.892 & 13.431 & 14.016 & 14.655 & 15.355 & 16.124 & 16.976 & 17.922 \\
\hline & $\mathrm{Yb}$ & 1.413 & 1.461 & 1.514 & 1.570 & 1.630 & 1.695 & 1.765 & 1.842 & 1.925 \\
\hline & $\mathrm{Lu}$ & 0.235 & 0.243 & 0.251 & 0.259 & 0.269 & 0.279 & 0.290 & 0.301 & 0.314 \\
\hline MS57 & Element & & & & & Melt $\mathrm{Fl}_{1}$ & action & & & \\
\hline & & 0.40 & 0.45 & 0.50 & 0.55 & 0.60 & 0.65 & 0.70 & 0.75 & 0.80 \\
\hline & $\mathrm{Rb}$ & 3.827 & 3.411 & 3.077 & 2.803 & 2.573 & 2.378 & 2.211 & 2.066 & 1.938 \\
\hline & $\mathrm{Ba}$ & 199.230 & 177.504 & 160.051 & 145.723 & 133.750 & 123.594 & 114.872 & 107.300 & 100.665 \\
\hline & Th & 0.377 & 0.336 & 0.303 & 0.276 & 0.254 & 0.235 & 0.218 & 0.204 & 0.191 \\
\hline & $\mathrm{U}$ & 0.196 & 0.175 & 0.158 & 0.144 & 0.132 & 0.122 & 0.113 & 0.106 & 0.099 \\
\hline & $\mathrm{Nb}$ & 2.859 & 2.582 & 2.355 & 2.164 & 2.002 & 1.862 & 1.741 & 1.634 & 1.540 \\
\hline & Ta & 0.186 & 0.168 & 0.154 & 0.141 & 0.131 & 0.122 & 0.114 & 0.107 & 0.101 \\
\hline & $\mathrm{La}$ & 7.923 & 7.291 & 6.753 & 6.289 & 5.885 & 5.529 & 5.214 & 4.933 & 4.680 \\
\hline & $\mathrm{Ce}$ & 17.194 & 16.338 & 15.563 & 14.859 & 14.215 & 13.625 & 13.082 & 12.580 & 12.116 \\
\hline & $\mathrm{Pb}$ & 1.513 & 1.355 & 1.227 & 1.121 & 1.032 & 0.956 & 0.890 & 0.833 & 0.783 \\
\hline & $\operatorname{Pr}$ & 2.063 & 2.026 & 1.990 & 1.956 & 1.923 & 1.891 & 1.860 & 1.830 & 1.801 \\
\hline & $\mathrm{Sr}$ & 536.574 & 496.053 & 461.223 & 430.963 & 404.430 & 380.974 & 360.089 & 341.376 & 324.511 \\
\hline & $\mathrm{Nd}$ & 7.034 & 7.147 & 7.264 & 7.385 & 7.510 & 7.639 & 7.773 & 7.912 & 8.055 \\
\hline & $\mathrm{Zr}$ & 82.619 & 76.485 & 71.199 & 66.596 & 62.552 & 58.972 & 55.779 & 52.914 & 50.329 \\
\hline & $\mathrm{Hf}$ & 2.469 & 2.285 & 2.126 & 1.988 & 1.867 & 1.760 & 1.664 & 1.578 & 1.501 \\
\hline & $\mathrm{Sm}$ & 1.341 & 1.401 & 1.467 & 1.540 & 1.620 & 1.708 & 1.807 & 1.918 & 2.044 \\
\hline & $\mathrm{Eu}$ & 0.453 & 0.476 & 0.502 & 0.530 & 0.562 & 0.598 & 0.639 & 0.687 & 0.741 \\
\hline & $\mathrm{Tb}$ & 0.219 & 0.231 & 0.245 & 0.260 & 0.277 & 0.297 & 0.320 & 0.346 & 0.378 \\
\hline
\end{tabular}




\begin{tabular}{|c|c|c|c|c|c|c|c|c|c|c|}
\hline & Dy & 1.534 & 1.615 & 1.705 & 1.805 & 1.918 & 2.045 & 2.192 & 2.360 & 2.557 \\
\hline & $\mathrm{Y}$ & 8.327 & 8.759 & 9.239 & 9.774 & 10.375 & 11.055 & 11.830 & 12.722 & 13.759 \\
\hline & $\mathrm{Yb}$ & 0.995 & 1.040 & 1.090 & 1.144 & 1.204 & 1.271 & 1.345 & 1.429 & 1.524 \\
\hline & $\mathrm{Lu}$ & 0.166 & 0.173 & 0.181 & 0.189 & 0.199 & 0.209 & 0.221 & 0.234 & 0.248 \\
\hline \multirow[t]{23}{*}{ MS58 } & Element & \multicolumn{9}{|c|}{ Melt Fraction } \\
\hline & & 0.60 & 0.65 & 0.70 & 0.75 & 0.80 & 0.85 & 0.90 & 0.95 & \\
\hline & $\mathrm{Rb}$ & 55.988 & 51.734 & 48.080 & 44.909 & 42.130 & 39.674 & 37.490 & 35.533 & \\
\hline & $\mathrm{Ba}$ & 2159.714 & 1995.599 & 1854.665 & 1732.324 & 1625.124 & 1530.418 & 1446.143 & 1370.665 & \\
\hline & Th & 6.003 & 5.547 & 5.155 & 4.815 & 4.517 & 4.254 & 4.020 & 3.810 & \\
\hline & $\mathrm{U}$ & 1.973 & 1.823 & 1.695 & 1.583 & 1.485 & 1.398 & 1.321 & 1.252 & \\
\hline & $\mathrm{Nb}$ & 6.968 & 6.438 & 5.983 & 5.588 & 5.242 & 4.937 & 4.665 & 4.421 & \\
\hline & $\mathrm{Ta}$ & 0.589 & 0.544 & 0.506 & 0.472 & 0.443 & 0.417 & 0.394 & 0.374 & \\
\hline & $\mathrm{La}$ & 17.711 & 16.587 & 15.598 & 14.719 & 13.934 & 13.229 & 12.592 & 12.013 & \\
\hline & $\mathrm{Ce}$ & 33.811 & 32.234 & 30.797 & 29.483 & 28.276 & 27.165 & 26.137 & 25.184 & \\
\hline & $\mathrm{Pb}$ & 5.151 & 4.767 & 4.437 & 4.150 & 3.897 & 3.674 & 3.474 & 3.296 & \\
\hline & $\operatorname{Pr}$ & 3.635 & 3.549 & 3.466 & 3.387 & 3.312 & 3.240 & 3.171 & 3.105 & \\
\hline & $\mathrm{Sr}$ & 220.155 & 206.826 & 195.020 & 184.488 & 175.036 & 166.505 & 158.767 & 151.716 & \\
\hline & $\mathrm{Nd}$ & 11.490 & 11.586 & 11.684 & 11.784 & 11.885 & 11.989 & 12.094 & 12.200 & \\
\hline & $\mathrm{Zr}$ & 103.382 & 96.910 & 91.201 & 86.127 & 81.588 & 77.503 & 73.808 & 70.449 & \\
\hline & Hf & 2.883 & 2.703 & 2.544 & 2.402 & 2.275 & 2.162 & 2.058 & 1.965 & \\
\hline & $\mathrm{Sm}$ & 1.904 & 1.992 & 2.088 & 2.194 & 2.311 & 2.441 & 2.587 & 2.751 & \\
\hline & $\mathrm{Eu}$ & 0.468 & 0.494 & 0.523 & 0.556 & 0.594 & 0.636 & 0.686 & 0.743 & \\
\hline & $\mathrm{Tb}$ & 0.290 & 0.309 & 0.329 & 0.353 & 0.381 & 0.413 & 0.451 & 0.496 & \\
\hline & Dy & 2.046 & 2.166 & 2.302 & 2.455 & 2.630 & 2.833 & 3.069 & 3.348 & \\
\hline & $\mathrm{Y}$ & 11.095 & 11.742 & 12.470 & 13.294 & 14.235 & 15.319 & 16.582 & 18.072 & \\
\hline & $\mathrm{Yb}$ & 1.333 & 1.399 & 1.472 & 1.553 & 1.643 & 1.745 & 1.859 & 1.990 & \\
\hline & $\mathrm{Lu}$ & 0.225 & 0.235 & 0.247 & 0.259 & 0.273 & 0.289 & 0.306 & 0.326 & \\
\hline \multirow[t]{14}{*}{ MS59 } & Element & \multicolumn{9}{|c|}{ Melt Fraction } \\
\hline & & 0.45 & 0.50 & 0.55 & 0.60 & 0.65 & 0.70 & 0.75 & 0.80 & 0.85 \\
\hline & $\mathrm{Rb}$ & 103.472 & 93.300 & 84.950 & 77.971 & 72.052 & 66.968 & 62.555 & 58.687 & 55.269 \\
\hline & $\mathrm{Ba}$ & 1887.999 & 1702.408 & 1550.039 & 1422.704 & 1314.701 & 1221.940 & 1141.406 & 1070.830 & 1008.475 \\
\hline & Th & 11.216 & 10.113 & 9.208 & 8.452 & 7.810 & 7.259 & 6.781 & 6.361 & 5.991 \\
\hline & $\mathrm{U}$ & 4.003 & 3.609 & 3.286 & 3.016 & 2.787 & 2.590 & 2.420 & 2.270 & 2.138 \\
\hline & $\mathrm{Nb}$ & 23.409 & 21.104 & 19.213 & 17.633 & 16.293 & 15.142 & 14.143 & 13.268 & 12.494 \\
\hline & $\mathrm{Ta}$ & 1.640 & 1.478 & 1.346 & 1.235 & 1.141 & 1.061 & 0.991 & 0.929 & 0.875 \\
\hline & $\mathrm{La}$ & 45.582 & 42.183 & 39.256 & 36.709 & 34.472 & 32.492 & 30.728 & 29.145 & 27.717 \\
\hline & $\mathrm{Ce}$ & 75.540 & 71.985 & 68.750 & 65.793 & 63.080 & 60.582 & 58.274 & 56.135 & 54.148 \\
\hline & $\mathrm{Pb}$ & 17.228 & 15.586 & 14.230 & 13.091 & 12.120 & 11.284 & 10.556 & 9.916 & 9.349 \\
\hline & $\operatorname{Pr}$ & 7.406 & 7.285 & 7.168 & 7.054 & 6.944 & 6.837 & 6.734 & 6.633 & 6.536 \\
\hline & $\mathrm{Sr}$ & 844.448 & 785.870 & 734.892 & 690.124 & 650.498 & 615.175 & 583.491 & 554.910 & 528.999 \\
\hline & $\mathrm{Nd}$ & 20.866 & 21.238 & 21.624 & 22.024 & 22.439 & 22.870 & 23.318 & 23.784 & 24.269 \\
\hline
\end{tabular}




\begin{tabular}{|c|c|c|c|c|c|c|c|c|c|c|c|}
\hline & $\mathrm{Zr}$ & 307.527 & 284.864 & 265.311 & 248.270 & 233.286 & 220.008 & 208.160 & 197.522 & 187.919 & \\
\hline & $\mathrm{Hf}$ & 8.082 & 7.486 & 6.972 & 6.525 & 6.131 & 5.782 & 5.471 & 5.191 & 4.939 & \\
\hline & Sm & 2.949 & 3.092 & 3.248 & 3.422 & 3.615 & 3.832 & 4.076 & 4.353 & 4.671 & \\
\hline & $\mathrm{Eu}$ & 0.755 & 0.796 & 0.843 & 0.895 & 0.954 & 1.021 & 1.098 & 1.189 & 1.295 & \\
\hline & $\mathrm{Tb}$ & 0.366 & 0.388 & 0.412 & 0.440 & 0.472 & 0.509 & 0.552 & 0.603 & 0.665 & \\
\hline & Dy & 2.399 & 2.535 & 2.688 & 2.860 & 3.055 & 3.280 & 3.539 & 3.844 & 4.205 & \\
\hline & $\mathrm{Y}$ & 12.989 & 13.716 & 14.529 & 15.445 & 16.484 & 17.673 & 19.047 & 20.652 & 22.553 & \\
\hline & $\mathrm{Yb}$ & 1.374 & 1.441 & 1.515 & 1.597 & 1.689 & 1.791 & 1.907 & 2.039 & 2.191 & \\
\hline & $\mathrm{Lu}$ & 0.229 & 0.240 & 0.251 & 0.264 & 0.279 & 0.295 & 0.313 & 0.333 & 0.356 & \\
\hline MS60 & Element & \multicolumn{10}{|c|}{ Melt Fraction } \\
\hline & & 0.10 & 0.15 & 0.20 & 0.25 & 0.30 & 0.35 & 0.40 & 0.45 & 0.50 & 0.55 \\
\hline & $\mathrm{Rb}$ & 270.623 & 230.655 & 200.973 & 178.060 & 159.836 & 144.997 & 132.679 & 122.289 & 113.409 & 105.731 \\
\hline & $\mathrm{Ba}$ & 2942.088 & 2912.175 & 2882.863 & 2854.136 & 2825.976 & 2798.367 & 2771.291 & 2744.734 & 2718.682 & 2693.119 \\
\hline & Th & 67.560 & 45.770 & 34.608 & 27.823 & 23.262 & 19.986 & 17.519 & 15.594 & 14.050 & 12.784 \\
\hline & $\mathrm{U}$ & 21.914 & 14.846 & 11.225 & 9.025 & 7.545 & 6.483 & 5.682 & 5.058 & 4.557 & 4.147 \\
\hline & $\mathrm{Nb}$ & 27.672 & 23.916 & 21.057 & 18.809 & 16.994 & 15.499 & 14.246 & 13.180 & 12.263 & 11.465 \\
\hline & $\mathrm{Ta}$ & 2.411 & 2.084 & 1.835 & 1.639 & 1.481 & 1.351 & 1.241 & 1.148 & 1.069 & 0.999 \\
\hline & $\mathrm{La}$ & 189.306 & 128.453 & 97.206 & 78.187 & 65.392 & 56.196 & 49.268 & 43.860 & 39.522 & 35.965 \\
\hline & $\mathrm{Ce}$ & 383.566 & 260.268 & 196.956 & 158.420 & 132.495 & 113.863 & 99.824 & 88.868 & 80.078 & 72.871 \\
\hline & $\mathrm{Pb}$ & 45.591 & 32.372 & 25.096 & 20.490 & 17.313 & 14.989 & 13.215 & 11.816 & 10.685 & 9.752 \\
\hline & $\operatorname{Pr}$ & 44.587 & 30.255 & 22.895 & 18.415 & 15.402 & 13.236 & 11.604 & 10.330 & 9.309 & 8.471 \\
\hline & $\mathrm{Sr}$ & 457.590 & 353.983 & 288.632 & 243.650 & 210.798 & 185.753 & 166.027 & 150.088 & 136.942 & 125.913 \\
\hline & $\mathrm{Nd}$ & 164.154 & 111.386 & 84.291 & 67.799 & 56.704 & 48.730 & 42.722 & 38.033 & 34.271 & 31.187 \\
\hline & $\mathrm{Zr}$ & 819.053 & 562.647 & 428.503 & 346.009 & 290.151 & 249.820 & 219.334 & 195.478 & 176.303 & 160.554 \\
\hline & $\mathrm{Hf}$ & 21.995 & 15.109 & 11.507 & 9.292 & 7.792 & 6.709 & 5.890 & 5.249 & 4.734 & 4.312 \\
\hline & $\mathrm{Sm}$ & 31.821 & 21.592 & 16.340 & 13.143 & 10.992 & 9.446 & 8.282 & 7.373 & 6.643 & 6.045 \\
\hline & $\mathrm{Eu}$ & 5.089 & 3.595 & 2.779 & 2.265 & 1.911 & 1.653 & 1.457 & 1.302 & 1.177 & 1.073 \\
\hline & $\mathrm{Tb}$ & 4.458 & 3.030 & 2.295 & 1.847 & 1.545 & 1.328 & 1.164 & 1.037 & 0.934 & 0.850 \\
\hline & Dy & 27.954 & 19.028 & 14.423 & 11.612 & 9.718 & 8.356 & 7.328 & 6.526 & 5.882 & 5.353 \\
\hline & Y & 144.834 & 98.892 & 75.077 & 60.506 & 50.672 & 43.587 & 38.241 & 34.063 & 30.708 & 27.954 \\
\hline & $\mathrm{Yb}$ & 15.686 & 10.743 & 8.169 & 6.590 & 5.523 & 4.753 & 4.171 & 3.717 & 3.351 & 3.051 \\
\hline & $\mathrm{Lu}$ & 2.577 & 1.770 & 1.348 & 1.089 & 0.913 & 0.786 & 0.690 & 0.615 & 0.555 & 0.505 \\
\hline MS62 & Element & \multicolumn{10}{|c|}{ Melt Fraction } \\
\hline & & 0.40 & 0.45 & 0.50 & 0.55 & 0.60 & 0.65 & 0.70 & 0.75 & 0.80 & \\
\hline & $\mathrm{Rb}$ & 116.782 & 107.702 & 99.931 & 93.207 & 87.330 & 82.151 & 77.551 & 73.439 & 69.742 & \\
\hline & $\mathrm{Ba}$ & 458.886 & 488.836 & 522.967 & 562.224 & 607.851 & 661.539 & 725.630 & 803.471 & 900.020 & \\
\hline & $\mathrm{Th}$ & 16.237 & 14.530 & 13.149 & 12.007 & 11.048 & 10.230 & 9.526 & 8.912 & 8.372 & \\
\hline & $\mathrm{U}$ & 5.220 & 4.696 & 4.267 & 3.910 & 3.608 & 3.349 & 3.125 & 2.929 & 2.756 & \\
\hline & $\mathrm{Nb}$ & 27.343 & 24.493 & 22.181 & 20.268 & 18.658 & 17.286 & 16.101 & 15.069 & 14.160 & \\
\hline & $\mathrm{Ta}$ & 1.944 & 1.747 & 1.586 & 1.452 & 1.339 & 1.242 & 1.158 & 1.085 & 1.021 & \\
\hline & $\mathrm{La}$ & 54.985 & 50.215 & 46.207 & 42.791 & 39.845 & 37.279 & 35.023 & 33.025 & 31.243 & \\
\hline
\end{tabular}




\begin{tabular}{|c|c|c|c|c|c|c|c|c|c|c|}
\hline & $\mathrm{Ce}$ & 122.453 & 110.258 & 100.271 & 91.943 & 84.893 & 78.846 & 73.604 & 69.016 & 64.966 \\
\hline & $\mathrm{Pb}$ & 9.403 & 9.195 & 8.996 & 8.805 & 8.622 & 8.447 & 8.279 & 8.117 & 7.962 \\
\hline & $\operatorname{Pr}$ & 15.418 & 13.849 & 12.570 & 11.507 & 10.610 & 9.843 & 9.179 & 8.599 & 8.088 \\
\hline & $\mathrm{Sr}$ & 35.049 & 37.673 & 40.722 & 44.307 & 48.585 & 53.778 & 60.213 & 68.398 & 79.157 \\
\hline & $\mathrm{Nd}$ & 62.647 & 56.124 & 50.831 & 46.450 & 42.765 & 39.621 & 36.908 & 34.543 & 32.462 \\
\hline & $\mathrm{Zr}$ & 382.523 & 342.410 & 309.911 & 283.046 & 260.468 & 241.225 & 224.630 & 210.171 & 197.462 \\
\hline & $\mathrm{Hf}$ & 9.940 & 8.908 & 8.071 & 7.377 & 6.794 & 6.295 & 5.865 & 5.490 & 5.160 \\
\hline & $\mathrm{Sm}$ & 14.330 & 12.798 & 11.561 & 10.543 & 9.689 & 8.963 & 8.339 & 7.795 & 7.319 \\
\hline & $\mathrm{Eu}$ & 0.570 & 0.602 & 0.637 & 0.677 & 0.721 & 0.773 & 0.832 & 0.900 & 0.982 \\
\hline & $\mathrm{Tb}$ & 2.317 & 2.071 & 1.872 & 1.708 & 1.570 & 1.453 & 1.352 & 1.265 & 1.188 \\
\hline & Dy & 14.337 & 12.814 & 11.584 & 10.569 & 9.718 & 8.994 & 8.370 & 7.827 & 7.350 \\
\hline & $\mathrm{Y}$ & 73.911 & 66.416 & 60.301 & 55.217 & 50.924 & 47.250 & 44.070 & 41.292 & 38.843 \\
\hline & $\mathrm{Yb}$ & 7.226 & 6.454 & 5.830 & 5.316 & 4.886 & 4.520 & 4.205 & 3.931 & 3.690 \\
\hline & $\mathrm{Lu}$ & 1.163 & 1.038 & 0.937 & 0.854 & 0.784 & 0.725 & 0.675 & 0.630 & 0.592 \\
\hline MS63 & Element & \multicolumn{9}{|c|}{ Melt Fraction } \\
\hline & & 0.50 & 0.55 & 0.60 & 0.65 & 0.70 & 0.75 & 0.80 & 0.85 & 0.90 \\
\hline & $\mathrm{Rb}$ & 114.538 & 104.381 & 95.878 & 88.657 & 82.447 & 77.050 & 72.316 & 68.130 & 64.402 \\
\hline & $\mathrm{Ba}$ & 3401.388 & 3102.687 & 2852.213 & 2639.159 & 2455.722 & 2296.128 & 2156.011 & 2032.012 & 1921.500 \\
\hline & Th & 13.139 & 12.000 & 11.042 & 10.226 & 9.523 & 8.910 & 8.371 & 7.893 & 7.467 \\
\hline & $\mathrm{U}$ & 4.347 & 3.970 & 3.653 & 3.384 & 3.151 & 2.948 & 2.770 & 2.612 & 2.471 \\
\hline & $\mathrm{Nb}$ & 22.068 & 20.183 & 18.594 & 17.238 & 16.065 & 15.043 & 14.142 & 13.343 & 12.630 \\
\hline & $\mathrm{Ta}$ & 1.596 & 1.459 & 1.344 & 1.246 & 1.162 & 1.088 & 1.023 & 0.965 & 0.913 \\
\hline & $\mathrm{La}$ & 25.879 & 25.861 & 25.842 & 25.824 & 25.805 & 25.787 & 25.768 & 25.750 & 25.732 \\
\hline & $\mathrm{Ce}$ & 43.778 & 44.525 & 45.299 & 46.101 & 46.931 & 47.791 & 48.684 & 49.611 & 50.573 \\
\hline & $\mathrm{Pb}$ & 14.608 & 13.309 & 12.223 & 11.301 & 10.508 & 9.819 & 9.215 & 8.681 & 8.205 \\
\hline & $\operatorname{Pr}$ & 5.206 & 5.314 & 5.427 & 5.544 & 5.667 & 5.795 & 5.929 & 6.070 & 6.217 \\
\hline & $\mathrm{Sr}$ & 388.902 & 359.377 & 334.019 & 312.004 & 292.711 & 275.665 & 260.495 & 246.908 & 234.668 \\
\hline & $\mathrm{Nd}$ & 16.432 & 17.066 & 17.752 & 18.495 & 19.303 & 20.185 & 21.151 & 22.214 & 23.390 \\
\hline & $\mathrm{Zr}$ & 304.843 & 279.231 & 257.588 & 239.060 & 223.018 & 208.993 & 196.628 & 185.645 & 175.824 \\
\hline & $\mathrm{Hf}$ & 7.962 & 7.295 & 6.731 & 6.249 & 5.830 & 5.465 & 5.142 & 4.856 & 4.599 \\
\hline & $\mathrm{Sm}$ & 3.064 & 3.218 & 3.389 & 3.578 & 3.790 & 4.029 & 4.299 & 4.609 & 4.967 \\
\hline & $\mathrm{Eu}$ & 0.699 & 0.740 & 0.785 & 0.836 & 0.894 & 0.961 & 1.039 & 1.130 & 1.239 \\
\hline & $\mathrm{Tb}$ & 0.551 & 0.576 & 0.602 & 0.631 & 0.664 & 0.699 & 0.739 & 0.783 & 0.833 \\
\hline & Dy & 4.243 & 4.366 & 4.496 & 4.635 & 4.782 & 4.939 & 5.107 & 5.287 & 5.479 \\
\hline & $\mathrm{Y}$ & 13.519 & 14.335 & 15.256 & 16.304 & 17.506 & 18.899 & 20.534 & 22.478 & 24.829 \\
\hline & $\mathrm{Yb}$ & 2.709 & 2.733 & 2.757 & 2.781 & 2.806 & 2.831 & 2.857 & 2.883 & 2.910 \\
\hline & $\mathrm{Lu}$ & 0.549 & 0.540 & 0.532 & 0.524 & 0.517 & 0.509 & 0.502 & 0.495 & 0.488 \\
\hline MS65 & Element & \multicolumn{9}{|c|}{ Melt Fraction } \\
\hline & & 0.30 & 0.35 & 0.40 & 0.45 & 0.50 & 0.55 & 0.60 & 0.65 & 0.70 \\
\hline & $\mathrm{Rb}$ & 411.182 & 362.745 & 324.518 & 293.579 & 268.027 & 246.566 & 228.287 & 212.531 & 198.810 \\
\hline & $\mathrm{Ba}$ & 4020.363 & 3927.559 & 3838.942 & 3754.237 & 3673.188 & 3595.566 & 3521.156 & 3449.763 & 3381.208 \\
\hline
\end{tabular}




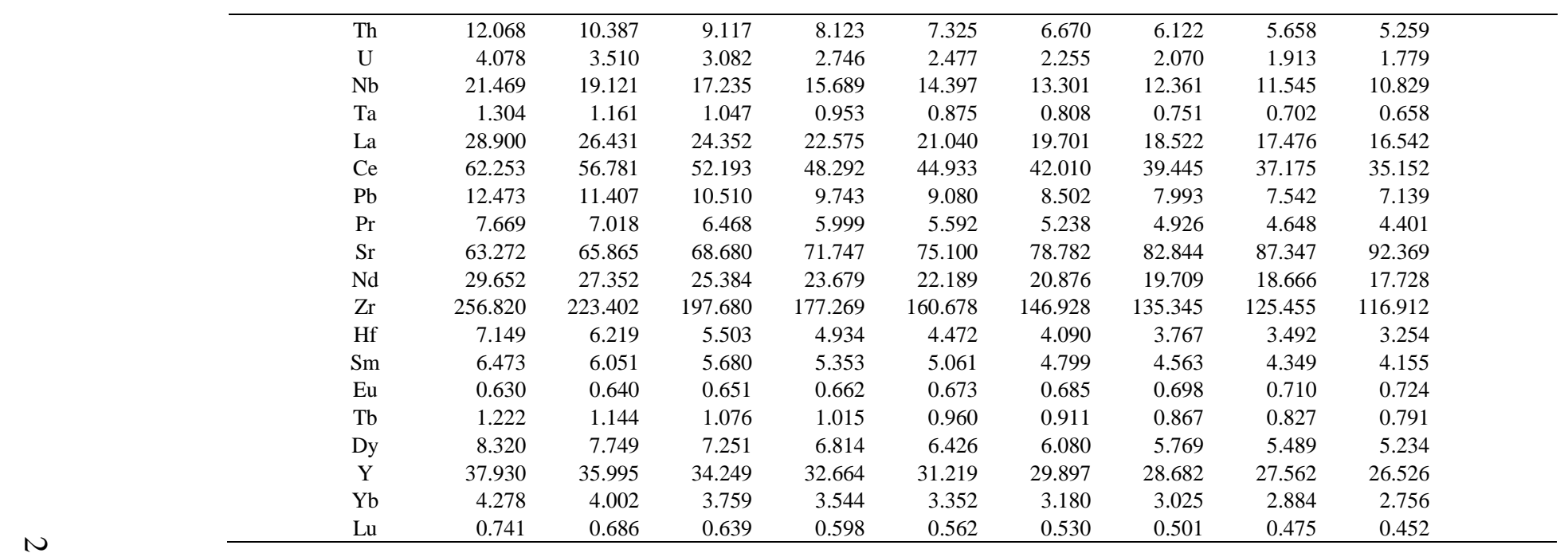

Table D2. Results of fractional partial melting calculations

\begin{tabular}{|c|c|c|c|c|c|c|c|c|c|c|}
\hline \multirow[t]{15}{*}{ MS1 } & \multirow{3}{*}{$\begin{array}{c}\text { Element } \\
\mathrm{Rb}\end{array}$} & \multicolumn{9}{|c|}{ Melt Fraction } \\
\hline & & 0.55 & 0.60 & 0.65 & 0.70 & 0.75 & 0.80 & 0.85 & 0.90 & 0.95 \\
\hline & & 0.000 & 0.000 & 0.000 & 0.000 & 0.000 & 0.000 & 0.000 & 0.000 & 0.000 \\
\hline & $\mathrm{Ba}$ & 0.000 & 0.000 & 0.000 & 0.000 & 0.000 & 0.000 & 0.000 & 0.000 & 0.000 \\
\hline & Th & 0.000 & 0.000 & 0.000 & 0.000 & 0.000 & 0.000 & 0.000 & 0.000 & 0.000 \\
\hline & $\mathrm{U}$ & 0.000 & 0.000 & 0.000 & 0.000 & 0.000 & 0.000 & 0.000 & 0.000 & 0.000 \\
\hline & $\mathrm{Nb}$ & 0.000 & 0.000 & 0.000 & 0.000 & 0.000 & 0.000 & 0.000 & 0.000 & 0.000 \\
\hline & $\mathrm{Ta}$ & 0.000 & 0.000 & 0.000 & 0.000 & 0.000 & 0.000 & 0.000 & 0.000 & 0.000 \\
\hline & $\mathrm{La}$ & 0.000 & 0.000 & 0.000 & 0.000 & 0.000 & 0.000 & 0.000 & 0.000 & 0.000 \\
\hline & $\mathrm{Ce}$ & 0.000 & 0.000 & 0.000 & 0.000 & 0.000 & 0.000 & 0.000 & 0.000 & 0.000 \\
\hline & $\mathrm{Pb}$ & 0.000 & 0.000 & 0.000 & 0.000 & 0.000 & 0.000 & 0.000 & 0.000 & 0.000 \\
\hline & $\operatorname{Pr}$ & 0.000 & 0.000 & 0.000 & 0.000 & 0.000 & 0.000 & 0.000 & 0.000 & 0.000 \\
\hline & $\mathrm{Sr}$ & 0.000 & 0.000 & 0.000 & 0.000 & 0.000 & 0.000 & 0.000 & 0.000 & 0.000 \\
\hline & $\mathrm{Nd}$ & 0.000 & 0.000 & 0.000 & 0.000 & 0.000 & 0.000 & 0.000 & 0.000 & 0.000 \\
\hline & $\mathrm{Zr}$ & 0.000 & 0.000 & 0.000 & 0.000 & 0.000 & 0.000 & 0.000 & 0.000 & 0.000 \\
\hline
\end{tabular}




\begin{tabular}{|c|c|c|c|c|c|c|c|c|c|c|}
\hline & $\mathrm{Hf}$ & 0.000 & 0.000 & 0.000 & 0.000 & 0.000 & 0.000 & 0.000 & 0.000 & 0.000 \\
\hline & $\mathrm{Sm}$ & 0.000 & 0.000 & 0.000 & 0.000 & 0.000 & 0.000 & 0.000 & 0.000 & 0.000 \\
\hline & $\mathrm{Eu}$ & 0.000 & 0.000 & 0.000 & 0.000 & 0.000 & 0.000 & 0.000 & 0.000 & 0.000 \\
\hline & $\mathrm{Tb}$ & 0.000 & 0.000 & 0.000 & 0.000 & 0.000 & 0.000 & 0.000 & 0.000 & 0.000 \\
\hline & Dy & 0.000 & 0.000 & 0.000 & 0.000 & 0.000 & 0.000 & 0.000 & 0.000 & 0.000 \\
\hline & $\mathrm{Y}$ & 0.043 & 0.013 & 0.003 & 0.001 & 0.000 & 0.000 & 0.000 & 0.000 & 0.000 \\
\hline & $\mathrm{Yb}$ & 0.123 & 0.063 & 0.030 & 0.012 & 0.004 & 0.001 & 0.000 & 0.000 & 0.000 \\
\hline & $\mathrm{Lu}$ & 0.015 & 0.007 & 0.003 & 0.001 & 0.000 & 0.000 & 0.000 & 0.000 & 0.000 \\
\hline \multirow[t]{22}{*}{ MS3 } & Element & \multicolumn{9}{|c|}{ Melt Fraction } \\
\hline & & 0.01 & 0.05 & 0.10 & 0.15 & 0.20 & 0.25 & 0.30 & 0.35 & 0.40 \\
\hline & $\mathrm{Ba}$ & 402.099 & 262.716 & 150.371 & 83.366 & 44.594 & 22.910 & 11.241 & 5.232 & 2.290 \\
\hline & Th & 4.691 & 0.079 & 0.000 & 0.000 & 0.000 & 0.000 & 0.000 & 0.000 & 0.000 \\
\hline & $\mathrm{U}$ & 2.567 & 0.043 & 0.000 & 0.000 & 0.000 & 0.000 & 0.000 & 0.000 & 0.000 \\
\hline & $\mathrm{Nb}$ & 49.042 & 0.566 & 0.002 & 0.000 & 0.000 & 0.000 & 0.000 & 0.000 & 0.000 \\
\hline & $\mathrm{Ta}$ & 3.117 & 0.036 & 0.000 & 0.000 & 0.000 & 0.000 & 0.000 & 0.000 & 0.000 \\
\hline & $\mathrm{La}$ & 20.851 & 17.952 & 14.753 & 11.988 & 9.620 & 7.611 & 5.925 & 4.528 & 3.386 \\
\hline & $\mathrm{Ce}$ & 27.917 & 26.555 & 24.871 & 23.206 & 21.562 & 19.939 & 18.339 & 16.764 & 15.213 \\
\hline & $\mathrm{Pb}$ & 4.563 & 2.994 & 1.723 & 0.961 & 0.517 & 0.268 & 0.132 & 0.062 & 0.027 \\
\hline & $\operatorname{Pr}$ & 2.283 & 2.269 & 2.250 & 2.231 & 2.211 & 2.189 & 2.167 & 2.143 & 2.117 \\
\hline & $\mathrm{Sr}$ & 277.435 & 279.097 & 281.290 & 283.627 & 286.128 & 288.814 & 291.714 & 294.860 & 298.297 \\
\hline & $\mathrm{Nd}$ & 5.882 & 5.983 & 6.118 & 6.264 & 6.423 & 6.597 & 6.787 & 6.999 & 7.234 \\
\hline & $\mathrm{Zr}$ & 214.408 & 175.209 & 134.464 & 101.645 & 75.543 & 55.078 & 39.291 & 27.336 & 18.474 \\
\hline & $\mathrm{Hf}$ & 6.398 & 5.228 & 4.012 & 3.033 & 2.254 & 1.643 & 1.172 & 0.816 & 0.551 \\
\hline & $\mathrm{Sm}$ & 0.894 & 0.921 & 0.956 & 0.996 & 1.039 & 1.087 & 1.141 & 1.202 & 1.272 \\
\hline & $\mathrm{Eu}$ & 0.262 & 0.271 & 0.282 & 0.295 & 0.309 & 0.325 & 0.343 & 0.363 & 0.387 \\
\hline & $\mathrm{Tb}$ & 0.120 & 0.124 & 0.129 & 0.135 & 0.142 & 0.149 & 0.157 & 0.167 & 0.178 \\
\hline & Dy & 0.782 & 0.807 & 0.841 & 0.879 & 0.921 & 0.967 & 1.019 & 1.078 & 1.146 \\
\hline & Y & 4.136 & 4.266 & 4.443 & 4.639 & 4.855 & 5.097 & 5.369 & 5.677 & 6.029 \\
\hline & $\mathrm{Yb}$ & 0.491 & 0.505 & 0.524 & 0.545 & 0.568 & 0.594 & 0.623 & 0.655 & 0.692 \\
\hline & $\mathrm{Lu}$ & 0.080 & 0.082 & 0.085 & 0.089 & 0.092 & 0.096 & 0.101 & 0.106 & 0.112 \\
\hline \multirow[t]{10}{*}{ MS4 } & Element & \multicolumn{9}{|c|}{ Melt Fraction } \\
\hline & & 0.20 & 0.25 & 0.30 & 0.35 & 0.40 & 0.45 & 0.50 & 0.55 & 0.60 \\
\hline & $\mathrm{Rb}$ & 0.000 & 0.000 & 0.000 & 0.000 & 0.000 & 0.000 & 0.000 & 0.000 & 0.000 \\
\hline & $\mathrm{Ba}$ & 2061.647 & 920.343 & 388.607 & 153.925 & 56.611 & 19.084 & 5.800 & 1.554 & 0.357 \\
\hline & Th & 0.000 & 0.000 & 0.000 & 0.000 & 0.000 & 0.000 & 0.000 & 0.000 & 0.000 \\
\hline & $\mathrm{U}$ & 0.000 & 0.000 & 0.000 & 0.000 & 0.000 & 0.000 & 0.000 & 0.000 & 0.000 \\
\hline & $\mathrm{Nb}$ & 0.000 & 0.000 & 0.000 & 0.000 & 0.000 & 0.000 & 0.000 & 0.000 & 0.000 \\
\hline & $\mathrm{Ta}$ & 0.000 & 0.000 & 0.000 & 0.000 & 0.000 & 0.000 & 0.000 & 0.000 & 0.000 \\
\hline & $\mathrm{La}$ & 4.767 & 1.307 & 0.328 & 0.074 & 0.015 & 0.003 & 0.000 & 0.000 & 0.000 \\
\hline & $\mathrm{Ce}$ & 1.982 & 0.311 & 0.043 & 0.005 & 0.001 & 0.000 & 0.000 & 0.000 & 0.000 \\
\hline
\end{tabular}




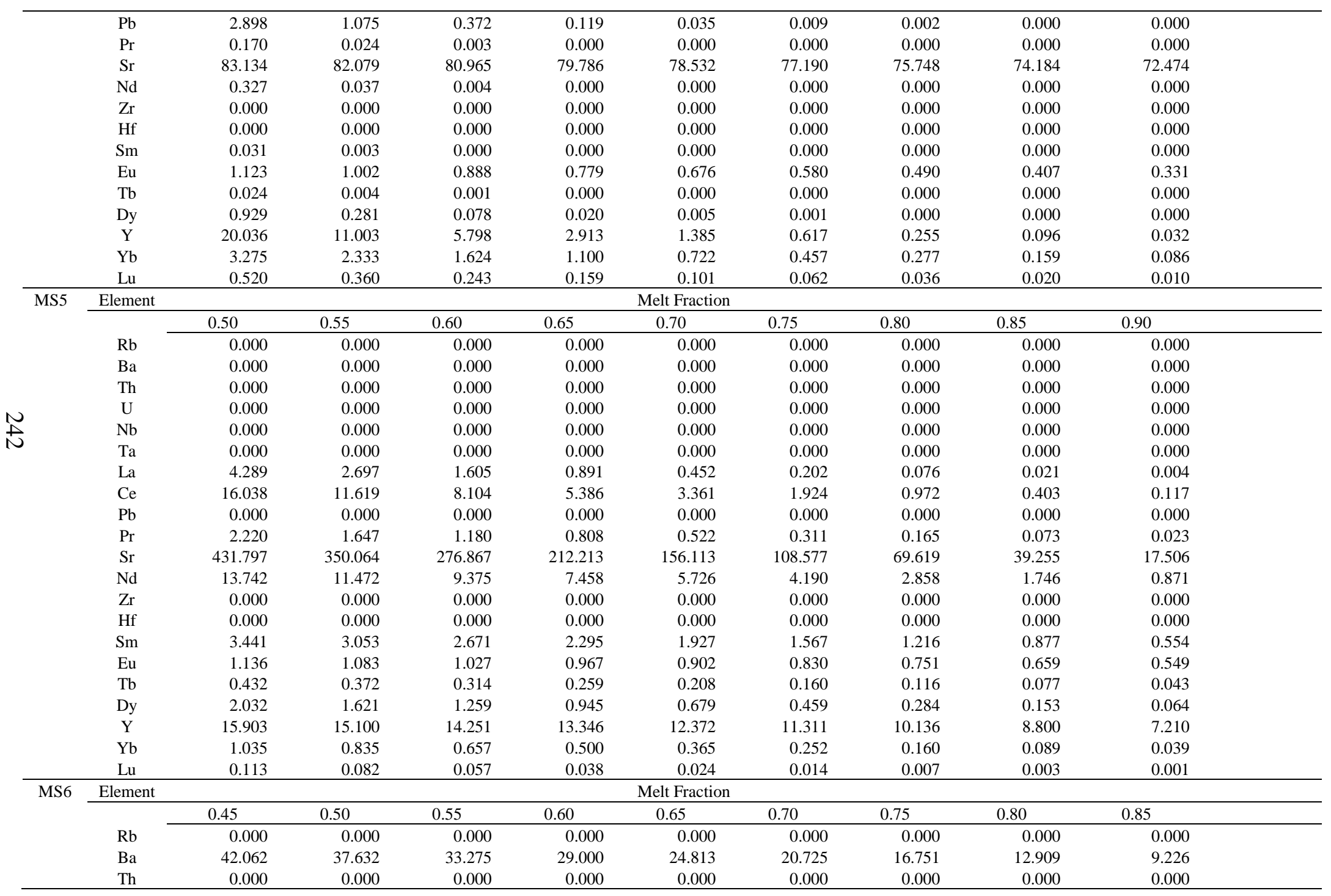




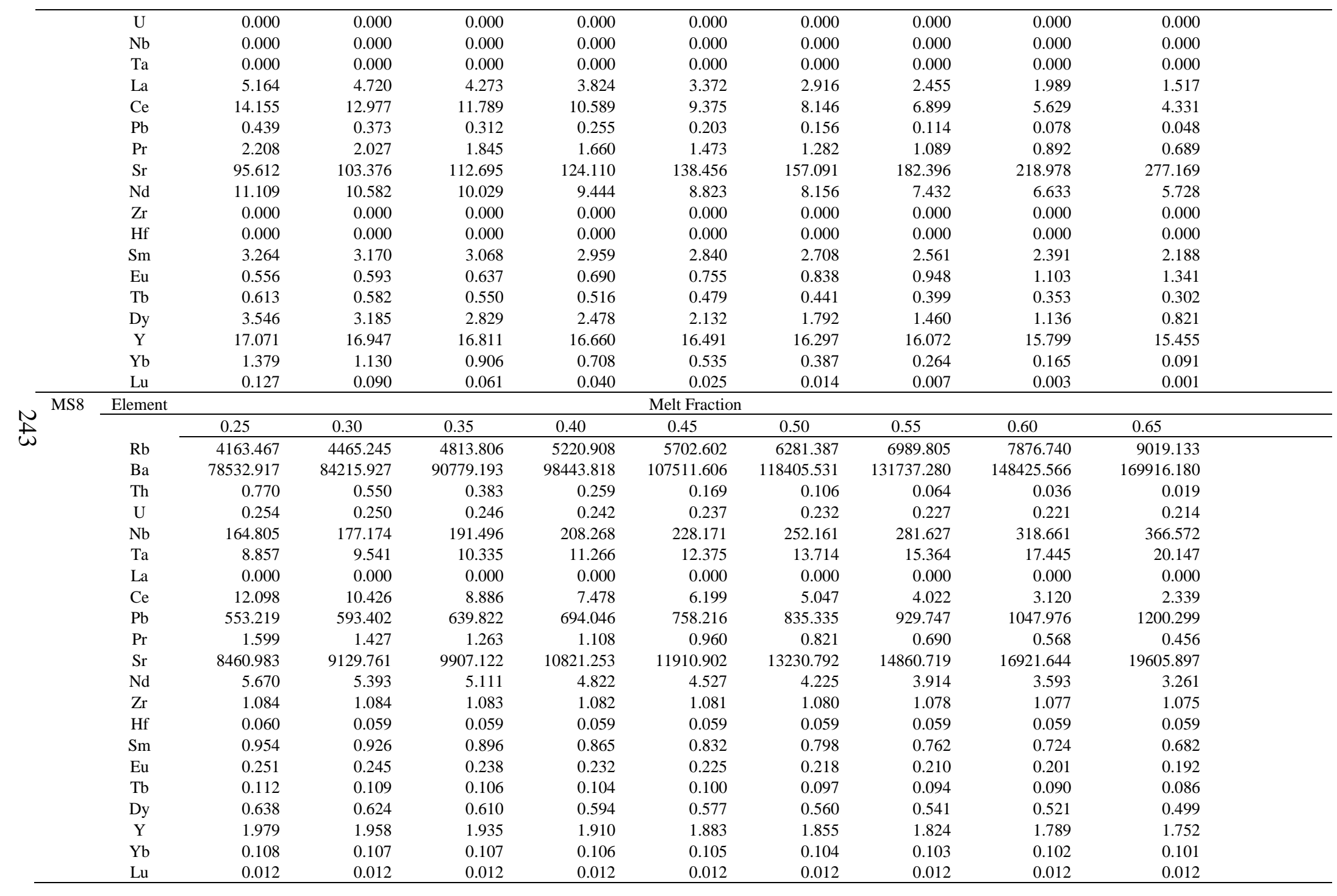




\begin{tabular}{|c|c|c|c|c|c|c|c|c|c|c|}
\hline \multirow[t]{2}{*}{ MS9 } & Element & \multicolumn{9}{|c|}{ Melt Fraction } \\
\hline & & 0.25 & 0.30 & 0.35 & 0.40 & 0.45 & 0.50 & 0.55 & 0.60 & 0.65 \\
\hline & $\mathrm{Rb}$ & 185.494 & 198.874 & 214.322 & 232.358 & 253.690 & 279.310 & 310.654 & 349.876 & 400.363 \\
\hline & $\mathrm{Ba}$ & 5613.883 & 6018.766 & 6486.253 & 7032.048 & 7677.586 & 8452.894 & 9401.386 & 10588.244 & 12116.008 \\
\hline & Th & 18.076 & 19.380 & 20.885 & 22.644 & 24.723 & 27.220 & 30.276 & 34.099 & 39.021 \\
\hline & $\mathrm{U}$ & 9.733 & 10.435 & 11.246 & 12.193 & 13.312 & 14.657 & 16.302 & 18.361 & 21.011 \\
\hline & $\mathrm{Nb}$ & 211.290 & 226.515 & 244.093 & 264.614 & 288.883 & 318.028 & 353.680 & 398.288 & 455.703 \\
\hline & $\mathrm{Ta}$ & 12.709 & 13.625 & 14.682 & 15.917 & 17.376 & 19.129 & 21.274 & 23.957 & 27.411 \\
\hline & $\mathrm{La}$ & 34.274 & 37.340 & 40.939 & 45.218 & 50.378 & 56.708 & 64.635 & 74.816 & 88.311 \\
\hline & $\mathrm{Ce}$ & 47.014 & 53.486 & 61.434 & 71.350 & 83.954 & 100.328 & 122.170 & 152.262 & 195.436 \\
\hline & $\mathrm{Pb}$ & 22.282 & 23.919 & 25.813 & 28.026 & 30.648 & 33.803 & 37.669 & 42.517 & 48.773 \\
\hline & $\operatorname{Pr}$ & 44.748 & 92.160 & 200.251 & 463.015 & 1151.620 & 3124.386 & 9417.323 & 32328.525 & 130877.607 \\
\hline & $\mathrm{Sr}$ & 2128.259 & 2325.126 & 2556.920 & 2833.302 & 3167.739 & 3579.543 & 4097.347 & 4765.364 & 5655.351 \\
\hline & $\mathrm{Nd}$ & 3.873 & 3.550 & 3.233 & 2.922 & 2.618 & 2.322 & 2.032 & 1.752 & 1.480 \\
\hline & $\mathrm{Zr}$ & 303.107 & 329.642 & 360.738 & 397.627 & 442.020 & 496.354 & 564.222 & 651.136 & 765.971 \\
\hline & $\mathrm{Hf}$ & 9.091 & 9.887 & 10.820 & 11.926 & 13.258 & 14.888 & 16.924 & 19.531 & 22.975 \\
\hline & $\mathrm{Sm}$ & 0.754 & 0.734 & 0.713 & 0.691 & 0.668 & 0.643 & 0.617 & 0.590 & 0.560 \\
\hline & $\mathrm{Eu}$ & 0.247 & 0.242 & 0.236 & 0.231 & 0.224 & 0.218 & 0.211 & 0.204 & 0.195 \\
\hline & $\mathrm{Tb}$ & 0.105 & 0.103 & 0.101 & 0.099 & 0.097 & 0.095 & 0.092 & 0.089 & 0.086 \\
\hline & Dy & 0.680 & 0.667 & 0.652 & 0.637 & 0.621 & 0.604 & 0.586 & 0.566 & 0.544 \\
\hline & $\mathrm{Y}$ & 3.538 & 3.465 & 3.388 & 3.307 & 3.221 & 3.129 & 3.031 & 2.924 & 2.808 \\
\hline & $\mathrm{Yb}$ & 0.407 & 0.395 & 0.383 & 0.371 & 0.357 & 0.344 & 0.329 & 0.313 & 0.296 \\
\hline & $\mathrm{Lu}$ & 0.066 & 0.064 & 0.061 & 0.059 & 0.057 & 0.055 & 0.052 & 0.049 & 0.046 \\
\hline \multirow[t]{18}{*}{ MS13 } & Element & \multicolumn{9}{|c|}{ Melt Fraction } \\
\hline & & 0.50 & 0.55 & 0.60 & 0.65 & 0.70 & 0.75 & 0.80 & 0.85 & 0.90 \\
\hline & $\mathrm{Rb}$ & 6400.084 & 7121.688 & 8025.104 & 9188.686 & 10743.264 & 12924.823 & 16206.514 & 21695.777 & 32728.685 \\
\hline & $\mathrm{Ba}$ & 6650.175 & 7722.198 & 9126.447 & 11029.717 & 13725.546 & 17776.639 & 24395.981 & 36689.870 & 65213.101 \\
\hline & Th & 958.676 & 1066.093 & 1200.484 & 1373.447 & 1604.331 & 1928.004 & 2414.308 & 3226.488 & 4855.442 \\
\hline & $\mathrm{U}$ & 372.818 & 414.592 & 466.855 & 534.118 & 623.906 & 749.779 & 938.898 & 1254.745 & 1888.228 \\
\hline & $\mathrm{Nb}$ & 1440.550 & 1601.960 & 1803.902 & 2063.804 & 2410.740 & 2897.107 & 3627.849 & 4848.267 & 7296.009 \\
\hline & $\mathrm{Ta}$ & 119.200 & 132.557 & 149.267 & 170.773 & 199.480 & 239.725 & 300.192 & 401.177 & 603.719 \\
\hline & $\mathrm{La}$ & 217.550 & 247.375 & 285.582 & 336.082 & 405.583 & 506.563 & 664.974 & 944.397 & 1548.388 \\
\hline & $\mathrm{Ce}$ & 597.347 & 673.446 & 770.049 & 896.433 & 1068.338 & 1314.690 & 1694.786 & 2351.285 & 3730.023 \\
\hline & $\mathrm{Pb}$ & 69.160 & 79.417 & 92.694 & 110.450 & 135.218 & 171.776 & 230.227 & 335.846 & 571.824 \\
\hline & $\operatorname{Pr}$ & 80.696 & 90.858 & 103.740 & 120.567 & 143.414 & 176.087 & 226.371 & 312.944 & 493.965 \\
\hline & $\mathrm{Sr}$ & 123.878 & 118.803 & 113.376 & 107.522 & 101.140 & 94.078 & 86.102 & 76.809 & 65.389 \\
\hline & $\mathrm{Nd}$ & 371.863 & 417.839 & 475.997 & 551.781 & 654.391 & 800.652 & 1024.862 & 1408.959 & 2206.615 \\
\hline & $\mathrm{Zr}$ & 10971.474 & 12214.468 & 13771.449 & 15777.983 & 18460.563 & 22228.014 & 27900.713 & 37400.776 & 56526.355 \\
\hline & $\mathrm{Hf}$ & 298.114 & 331.888 & 374.194 & 428.715 & 501.605 & 603.973 & 758.110 & 1016.243 & 1535.918 \\
\hline & $\mathrm{Sm}$ & 99.662 & 111.688 & 126.858 & 146.562 & 173.144 & 210.874 & 268.416 & 366.354 & 567.939 \\
\hline & $\mathrm{Eu}$ & 0.174 & 0.141 & 0.111 & 0.084 & 0.061 & 0.042 & 0.027 & 0.015 & 0.007 \\
\hline
\end{tabular}




\begin{tabular}{|c|c|c|c|c|c|c|c|c|c|c|}
\hline & $\mathrm{Tb}$ & 12.160 & 13.642 & 15.514 & 17.950 & 21.240 & 25.918 & 33.069 & 45.273 & 70.487 \\
\hline & Dy & 54.196 & 61.052 & 69.749 & 81.115 & 96.560 & 118.665 & 152.719 & 211.424 & 334.387 \\
\hline & $\mathrm{Y}$ & 173.789 & 198.472 & 230.237 & 272.440 & 330.868 & 416.352 & 551.584 & 792.670 & 1321.444 \\
\hline & $\mathrm{Yb}$ & 11.922 & 13.955 & 16.641 & 20.317 & 25.580 & 33.592 & 46.888 & 72.074 & 132.111 \\
\hline & $\mathrm{Lu}$ & 2.025 & 2.360 & 2.799 & 3.397 & 4.247 & 5.532 & 7.645 & 11.601 & 20.881 \\
\hline \multirow[t]{21}{*}{ MS15 } & Element & \multicolumn{9}{|c|}{ Melt Fraction } \\
\hline & & 0.20 & 0.25 & 0.30 & 0.35 & 0.40 & 0.45 & 0.50 & 0.55 & 0.60 \\
\hline & $\mathrm{Rb}$ & 15004.318 & 16010.298 & 17160.414 & 18487.994 & 20037.496 & 21869.570 & 24069.164 & 26759.047 & 30123.472 \\
\hline & $\mathrm{Ba}$ & 176114.640 & 188074.133 & 201758.586 & 217568.720 & 236039.529 & 257901.573 & 284179.202 & 316354.502 & 356654.708 \\
\hline & Th & 1712.673 & 1827.463 & 1958.696 & 2110.176 & 2286.973 & 2496.006 & 2746.963 & 3053.850 & 3437.680 \\
\hline & $\mathrm{Nb}$ & 246.491 & 263.576 & 283.152 & 305.802 & 332.305 & 363.728 & 401.568 & 447.996 & 506.280 \\
\hline & $\mathrm{Ta}$ & 21.478 & 22.967 & 24.673 & 26.647 & 28.956 & 31.694 & 34.991 & 39.037 & 44.116 \\
\hline & $\mathrm{La}$ & 1162.833 & 1242.112 & 1332.850 & 1437.712 & 1560.258 & 1705.352 & 1879.813 & 2093.513 & 2361.295 \\
\hline & $\mathrm{Ce}$ & 1477.960 & 1580.111 & 1697.133 & 1832.502 & 1990.866 & 2178.580 & 2404.570 & 2681.768 & 3029.645 \\
\hline & $\mathrm{Pb}$ & 870.612 & 929.127 & 996.037 & 1073.285 & 1163.462 & 1270.106 & 1398.171 & 1554.819 & 1750.803 \\
\hline & $\operatorname{Pr}$ & 109.397 & 117.122 & 125.985 & 136.253 & 148.285 & 162.574 & 179.810 & 200.998 & 227.653 \\
\hline & $\mathrm{Sr}$ & 1335.840 & 1431.776 & 1541.966 & 1669.790 & 1819.779 & 1998.148 & 2213.655 & 2479.036 & 2813.542 \\
\hline & $\mathrm{Nd}$ & 309.463 & 331.720 & 357.288 & 386.950 & 421.760 & 463.162 & 513.190 & 574.807 & 652.486 \\
\hline & $\mathrm{Zr}$ & 3928.059 & 4197.872 & 4506.835 & 4864.081 & 5281.813 & 5776.709 & 6372.176 & 7102.120 & 8017.548 \\
\hline & $\mathrm{Sm}$ & 31.508 & 33.955 & 36.782 & 40.082 & 43.978 & 48.645 & 54.328 & 61.385 & 70.365 \\
\hline & $\mathrm{Eu}$ & 4.744 & 5.125 & 5.567 & 6.084 & 6.696 & 7.432 & 8.330 & 9.451 & 10.883 \\
\hline & $\mathrm{Tb}$ & 3.520 & 3.806 & 4.138 & 4.527 & 4.989 & 5.543 & 6.222 & 7.070 & 8.155 \\
\hline & Dy & 21.750 & 23.528 & 25.590 & 28.006 & 30.872 & 34.322 & 38.544 & 43.819 & 50.575 \\
\hline & $\mathrm{Y}$ & 114.441 & 123.780 & 134.608 & 147.296 & 162.348 & 180.459 & 202.624 & 230.308 & 265.758 \\
\hline & $\mathrm{Yb}$ & 11.825 & 12.803 & 13.939 & 15.271 & 16.853 & 18.760 & 21.097 & 24.020 & 27.770 \\
\hline & $\mathrm{Lu}$ & 2.326 & 2.511 & 2.725 & 2.975 & 3.272 & 3.627 & 4.061 & 4.602 & 5.292 \\
\hline \multirow[t]{13}{*}{ MS16 } & Element & \multicolumn{9}{|c|}{ Melt Fraction } \\
\hline & & 0.40 & 0.45 & 0.50 & 0.55 & 0.60 & 0.65 & 0.70 & 0.75 & 0.80 \\
\hline & $\mathrm{Rb}$ & 12964.303 & 14149.851 & 15573.244 & 17313.938 & 19491.184 & 22292.499 & 26030.642 & 31269.057 & 39135.772 \\
\hline & $\mathrm{Ba}$ & 247304.665 & 269903.930 & 297035.389 & 330212.675 & 371707.399 & 425091.268 & 496320.983 & 596126.627 & 745987.469 \\
\hline & Th & 1038.699 & 1133.730 & 1247.831 & 1387.373 & 1561.921 & 1786.512 & 2086.232 & 2506.274 & 3137.124 \\
\hline & $\mathrm{U}$ & 403.939 & 440.895 & 485.268 & 539.534 & 607.414 & 694.755 & 811.312 & 974.662 & 1219.993 \\
\hline & $\mathrm{Nb}$ & 418.654 & 457.646 & 504.535 & 561.980 & 633.973 & 726.810 & 851.012 & 1025.585 & 1288.698 \\
\hline & $\mathrm{Ta}$ & 30.676 & 33.543 & 36.991 & 41.216 & 46.513 & 53.347 & 62.493 & 75.356 & 94.756 \\
\hline & $\mathrm{La}$ & 3278.860 & 3579.634 & 3940.847 & 4382.715 & 4935.584 & 5647.191 & 6597.188 & 7929.130 & 9930.577 \\
\hline & $\mathrm{Ce}$ & 6654.747 & 7265.002 & 7997.866 & 8894.341 & 10015.981 & 11459.608 & 13386.766 & 16088.599 & 20148.262 \\
\hline & $\mathrm{Pb}$ & 1654.243 & 1805.688 & 1987.535 & 2209.943 & 2488.163 & 2846.178 & 3323.997 & 3993.707 & 4999.656 \\
\hline & $\operatorname{Pr}$ & 839.849 & 916.861 & 1009.345 & 1122.476 & 1264.020 & 1446.196 & 1689.389 & 2030.336 & 2542.624 \\
\hline & $\mathrm{Sr}$ & 181912.013 & 198541.027 & 218505.503 & 242919.533 & 273455.163 & 312741.489 & 365163.352 & 438619.752 & 548923.639 \\
\hline
\end{tabular}




\begin{tabular}{|c|c|c|c|c|c|c|c|c|c|c|}
\hline & $\mathrm{Nd}$ & 3338.033 & 3644.153 & 4011.778 & 4461.477 & 5024.130 & 5748.306 & 6715.045 & 8070.403 & 10106.928 \\
\hline & $\mathrm{Zr}$ & 8827.856 & 9646.523 & 10630.650 & 11835.800 & 13345.481 & 15291.211 & 17892.731 & 21546.722 & 27049.275 \\
\hline & $\mathrm{Hf}$ & 249.694 & 272.831 & 300.643 & 334.698 & 377.355 & 432.327 & 505.819 & 609.029 & 764.429 \\
\hline & $\mathrm{Sm}$ & 635.969 & 694.352 & 764.473 & 850.257 & 957.600 & 1095.775 & 1280.260 & 1538.950 & 1927.731 \\
\hline & $\mathrm{Eu}$ & 159.940 & 174.652 & 192.325 & 213.950 & 241.016 & 275.864 & 322.405 & 387.686 & 485.836 \\
\hline & $\mathrm{Tb}$ & 62.652 & 68.425 & 75.361 & 83.849 & 94.475 & 108.160 & 126.440 & 152.089 & 190.664 \\
\hline & Dy & 238.795 & 260.971 & 287.632 & 320.285 & 361.196 & 413.932 & 484.455 & 583.532 & 732.774 \\
\hline & $\mathrm{Y}$ & 676.181 & 740.170 & 817.233 & 911.792 & 1030.509 & 1183.901 & 1389.583 & 1679.452 & 2117.744 \\
\hline & $\mathrm{Yb}$ & 39.178 & 42.984 & 47.579 & 53.233 & 60.351 & 69.580 & 82.002 & 99.587 & 126.320 \\
\hline & $\mathrm{Lu}$ & 6.848 & 7.511 & 8.310 & 9.293 & 10.531 & 12.134 & 14.291 & 17.342 & 21.976 \\
\hline MS17 & Element & \multicolumn{9}{|c|}{ Melt Fraction } \\
\hline & & 0.35 & 0.40 & 0.45 & 0.50 & 0.55 & 0.60 & 0.65 & 0.70 & 0.75 \\
\hline & $\mathrm{Rb}$ & 99.442 & 107.908 & 117.930 & 129.980 & 144.737 & 163.227 & 187.062 & 218.937 & 263.718 \\
\hline & $\mathrm{Ba}$ & 192.165 & 221.415 & 258.284 & 305.751 & 368.438 & 453.849 & 574.864 & 755.213 & 1042.871 \\
\hline & Th & 13.646 & 14.801 & 16.168 & 17.810 & 19.820 & 22.337 & 25.579 & 29.912 & 35.992 \\
\hline & $\mathrm{U}$ & 7.348 & 7.970 & 8.706 & 9.590 & 10.672 & 12.028 & 13.773 & 16.106 & 19.380 \\
\hline & $\mathrm{Nb}$ & 97.893 & 106.233 & 116.108 & 127.982 & 142.525 & 160.749 & 184.242 & 215.664 & 259.816 \\
\hline & $\mathrm{Ta}$ & 5.427 & 5.890 & 6.439 & 7.098 & 7.906 & 8.919 & 10.225 & 11.973 & 14.429 \\
\hline & $\mathrm{La}$ & 22.244 & 25.970 & 30.731 & 36.955 & 45.311 & 56.909 & 73.687 & 99.294 & 141.296 \\
\hline & $\mathrm{Ce}$ & 60.671 & 70.962 & 84.138 & 101.394 & 124.617 & 156.928 & 203.804 & 275.583 & 393.767 \\
\hline & $\mathrm{Pb}$ & 2.496 & 2.823 & 3.227 & 3.736 & 4.393 & 5.266 & 6.466 & 8.195 & 10.848 \\
\hline & $\operatorname{Pr}$ & 9.442 & 11.056 & 13.124 & 15.835 & 19.490 & 24.581 & 31.981 & 43.333 & 62.068 \\
\hline & $\mathrm{Sr}$ & 56.118 & 55.062 & 53.936 & 52.729 & 51.427 & 50.009 & 48.448 & 46.707 & 44.729 \\
\hline & $\mathrm{Nd}$ & 49.804 & 61.377 & 77.027 & 98.785 & 130.057 & 176.871 & 250.629 & 374.785 & 603.211 \\
\hline & $\mathrm{Zr}$ & 2641.038 & 2866.366 & 3133.172 & 3454.009 & 3847.045 & 4339.597 & 4974.706 & 5824.317 & 7018.379 \\
\hline & $\mathrm{Hf}$ & 79.152 & 85.905 & 93.901 & 103.517 & 115.297 & 130.059 & 149.094 & 174.558 & 210.345 \\
\hline & $\mathrm{Sm}$ & 18.749 & 24.738 & 33.437 & 46.512 & 66.991 & 100.729 & 159.948 & 272.780 & 512.877 \\
\hline & $\mathrm{Eu}$ & 0.315 & 0.302 & 0.289 & 0.275 & 0.260 & 0.245 & 0.228 & 0.210 & 0.191 \\
\hline & $\mathrm{Tb}$ & 2.704 & 3.310 & 4.124 & 5.248 & 6.849 & 9.224 & 12.927 & 19.087 & 30.261 \\
\hline & Dy & 15.960 & 18.439 & 21.573 & 25.620 & 30.983 & 38.318 & 48.755 & 64.385 & 89.459 \\
\hline & $\mathrm{Y}$ & 688.747 & 1343.992 & 2779.782 & 6162.072 & 14855.873 & 39730.997 & 121194.902 & 439168.353 & 2013544.341 \\
\hline & $\mathrm{Yb}$ & 8.894 & 10.033 & 11.436 & 13.199 & 15.466 & 18.465 & 22.574 & 28.466 & 37.450 \\
\hline & $\mathrm{Lu}$ & 1.798 & 1.996 & 2.236 & 2.533 & 2.906 & 3.390 & 4.036 & 4.936 & 6.262 \\
\hline \multirow[t]{8}{*}{ MS18 } & \multicolumn{10}{|c|}{ Melt Fraction } \\
\hline & & 0.05 & 0.10 & 0.15 & 0.20 & 0.25 & 0.30 & 0.35 & 0.40 & 0.45 \\
\hline & $\mathrm{Rb}$ & 3505.393 & 3704.170 & 3926.581 & 4177.091 & 4461.361 & 4786.679 & 5162.587 & 5601.830 & 6121.809 \\
\hline & $\mathrm{Ba}$ & 6661.799 & 7297.026 & 8034.554 & 8898.430 & 9920.381 & 11142.997 & 12624.605 & 14446.921 & 16727.495 \\
\hline & Th & 479.693 & 506.773 & 537.067 & 571.179 & 609.877 & 654.150 & 705.292 & 765.030 & 835.722 \\
\hline & $\mathrm{U}$ & 155.594 & 164.378 & 174.204 & 185.269 & 197.821 & 212.182 & 228.770 & 248.147 & 271.077 \\
\hline & $\mathrm{Nb}$ & 233.397 & 246.814 & 261.837 & 278.772 & 298.006 & 320.037 & 345.518 & 375.325 & 410.650 \\
\hline & $\mathrm{Ta}$ & 20.338 & 21.507 & 22.816 & 24.291 & 25.967 & 27.887 & 30.107 & 32.705 & 35.783 \\
\hline
\end{tabular}




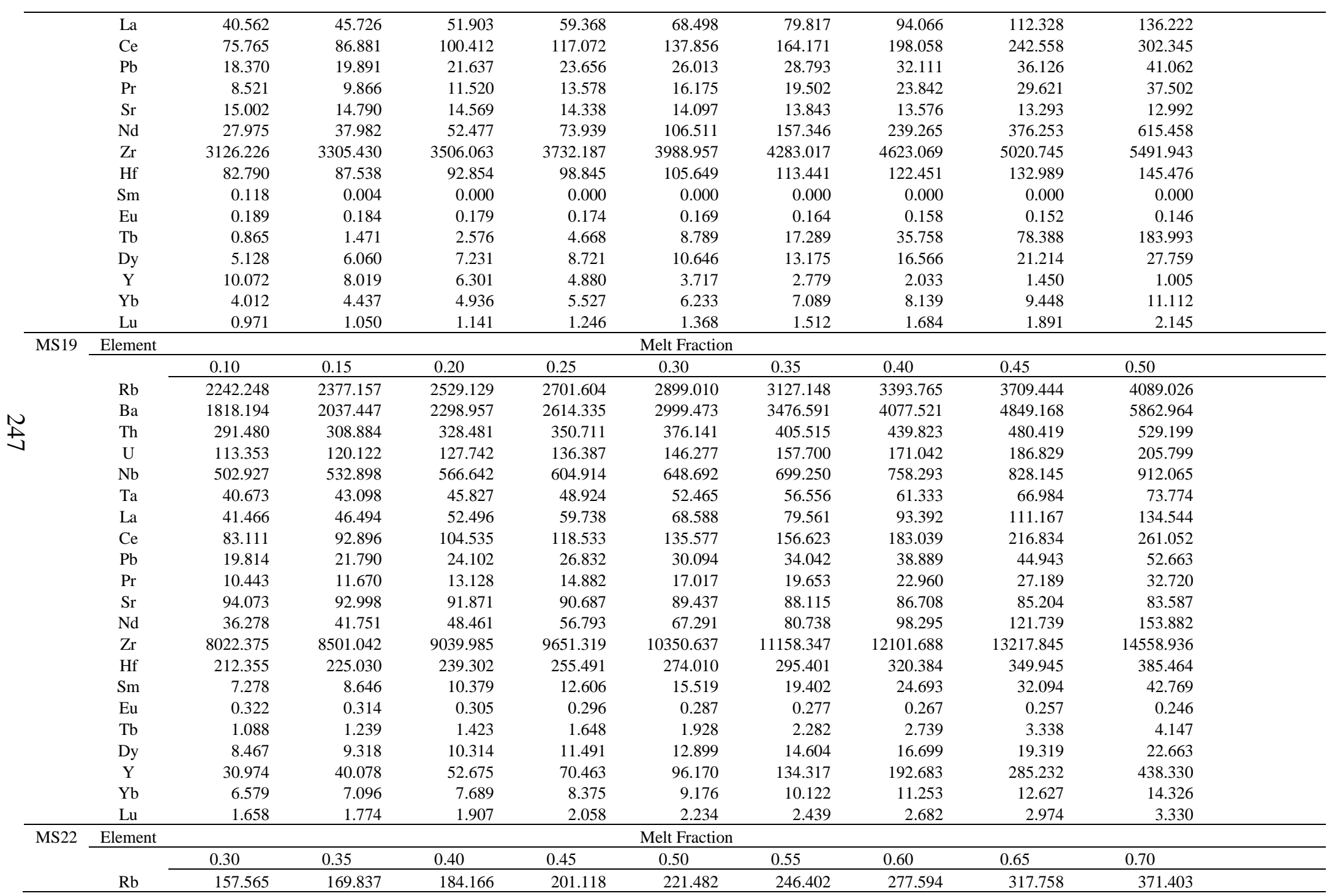




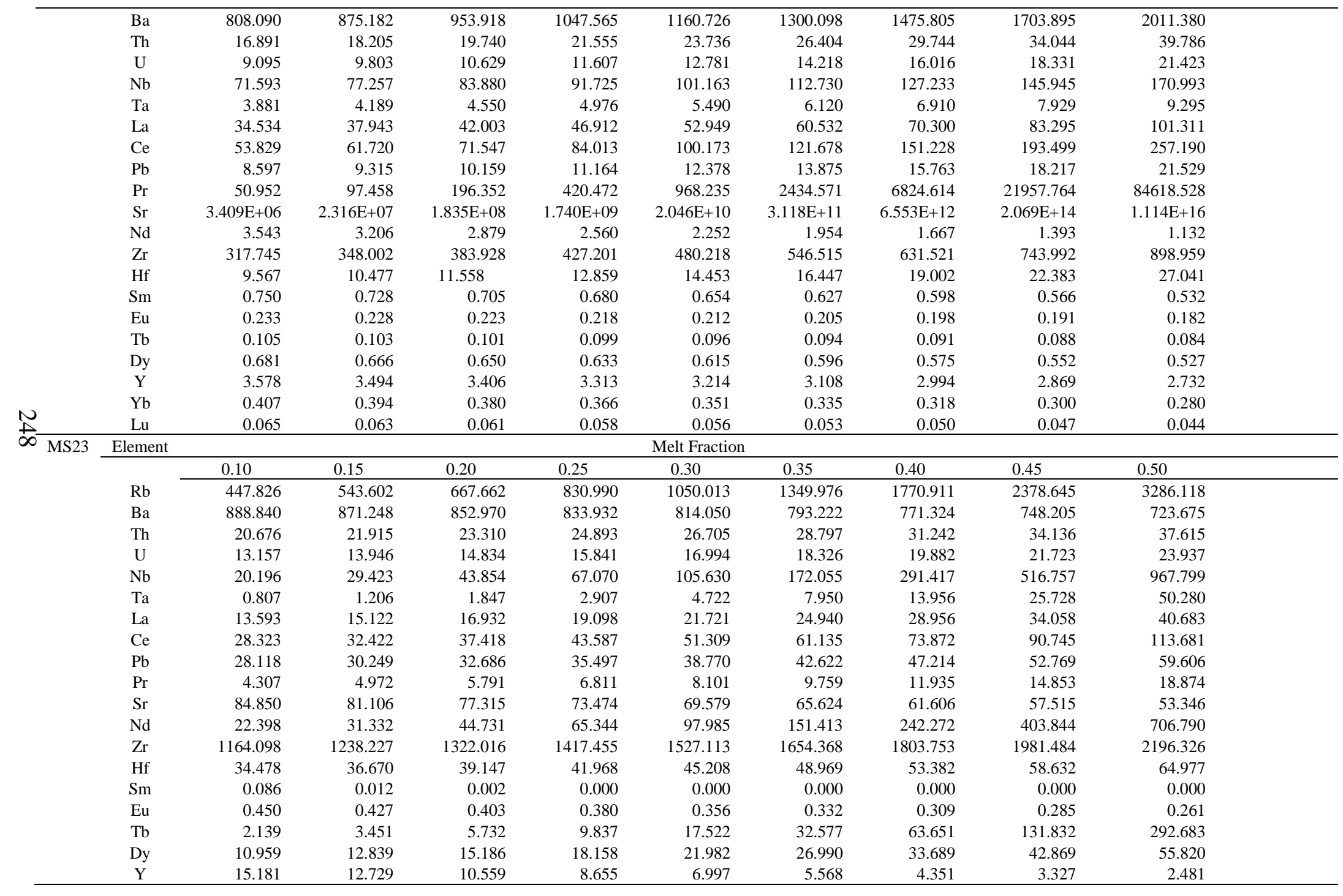




\begin{tabular}{|c|c|c|c|c|c|c|c|c|c|c|}
\hline & $\mathrm{Yb}$ & 7.091 & 7.875 & 8.802 & 9.909 & 11.247 & 12.885 & 14.924 & 17.509 & 20.856 \\
\hline & $\mathrm{Lu}$ & 1.619 & 1.757 & 1.916 & 2.102 & 2.320 & 2.580 & 2.893 & 3.277 & 3.756 \\
\hline \multirow[t]{23}{*}{ MS26 } & Element & \multicolumn{9}{|c|}{ Melt Fraction } \\
\hline & & 0.50 & 0.55 & 0.60 & 0.65 & 0.70 & 0.75 & 0.80 & 0.85 & 0.90 \\
\hline & $\mathrm{Rb}$ & 4979.662 & 5544.354 & 6251.761 & 7163.525 & 8382.643 & 10095.050 & 12673.921 & 16993.765 & 25693.286 \\
\hline & $\mathrm{Ba}$ & 175053.453 & 194708.362 & 219304.413 & 250967.676 & 293246.186 & 352535.983 & 441651.940 & 590561.516 & 889432.333 \\
\hline & Th & 380.489 & 424.019 & 478.603 & 549.032 & 643.318 & 775.947 & 976.036 & 1311.945 & 1990.470 \\
\hline & $\mathrm{U}$ & 135.782 & 151.317 & 170.796 & 195.929 & 229.576 & 276.907 & 348.311 & 468.184 & 710.324 \\
\hline & $\mathrm{Nb}$ & 112.774 & 129.374 & 150.840 & 179.515 & 219.460 & 278.327 & 372.273 & 541.625 & 918.767 \\
\hline & $\mathrm{Ta}$ & 7.128 & 8.235 & 9.678 & 11.621 & 14.353 & 18.427 & 25.017 & 37.104 & 64.670 \\
\hline & $\mathrm{La}$ & 191.752 & 228.589 & 278.208 & 347.608 & 449.516 & 609.265 & 883.966 & 1428.275 & 2808.678 \\
\hline & $\mathrm{Ce}$ & 824.292 & 1205.147 & 1842.673 & 2982.039 & 5198.298 & 10030.326 & 22422.415 & 63255.048 & 272845.246 \\
\hline & $\mathrm{Pb}$ & 375.898 & 419.687 & 474.703 & 545.849 & 641.340 & 776.066 & 980.056 & 1324.087 & 2023.390 \\
\hline & $\operatorname{Pr}$ & 0.038 & 0.018 & 0.008 & 0.003 & 0.001 & 0.000 & 0.000 & 0.000 & 0.000 \\
\hline & $\mathrm{Sr}$ & 5264.110 & 6017.751 & 6988.674 & 8280.204 & 10070.693 & 12694.474 & 16853.283 & 24285.613 & 40641.920 \\
\hline & $\mathrm{Nd}$ & 6.541 & 5.932 & 5.319 & 4.700 & 4.074 & 3.441 & 2.798 & 2.143 & 1.472 \\
\hline & $\mathrm{Zr}$ & 1434.574 & 1672.731 & 1986.068 & 2412.860 & 3020.816 & 3940.499 & 5455.347 & 8297.602 & 14984.792 \\
\hline & $\mathrm{Hf}$ & 38.304 & 44.575 & 52.808 & 63.996 & 79.890 & 103.857 & 143.183 & 216.611 & 388.222 \\
\hline & $\mathrm{Sm}$ & 1.226 & 1.181 & 1.133 & 1.080 & 1.023 & 0.958 & 0.885 & 0.799 & 0.692 \\
\hline & $\mathrm{Eu}$ & 0.319 & 0.310 & 0.300 & 0.289 & 0.277 & 0.263 & 0.247 & 0.228 & 0.203 \\
\hline & $\mathrm{Tb}$ & 0.163 & 0.159 & 0.154 & 0.149 & 0.143 & 0.137 & 0.129 & 0.120 & 0.108 \\
\hline & Dy & 1.089 & 1.055 & 1.019 & 0.980 & 0.936 & 0.887 & 0.831 & 0.763 & 0.677 \\
\hline & $\mathrm{Y}$ & 5.595 & 5.434 & 5.258 & 5.066 & 4.853 & 4.613 & 4.335 & 4.001 & 3.574 \\
\hline & $\mathrm{Yb}$ & 0.637 & 0.608 & 0.577 & 0.544 & 0.508 & 0.468 & 0.424 & 0.374 & 0.312 \\
\hline & $\mathrm{Lu}$ & 0.106 & 0.100 & 0.095 & 0.089 & 0.082 & 0.075 & 0.067 & 0.058 & 0.048 \\
\hline \multirow[t]{16}{*}{ MS27 } & Element & \multicolumn{9}{|c|}{ Melt Fraction } \\
\hline & & 0.35 & 0.40 & 0.45 & 0.50 & 0.55 & 0.60 & 0.65 & 0.70 & 0.75 \\
\hline & $\mathrm{Rb}$ & 20556.190 & 22279.023 & 24316.037 & 26761.682 & 29752.459 & 33493.232 & 38306.136 & 44728.444 & 53728.046 \\
\hline & $\mathrm{Ba}$ & 530750.389 & 575219.815 & 627797.532 & 690920.857 & 768112.063 & 864657.385 & 988868.725 & 1154608.639 & 1386849.287 \\
\hline & Th & 1940.544 & 2103.293 & 2295.733 & 2526.789 & 2809.367 & 3162.833 & 3617.642 & 4224.594 & 5075.212 \\
\hline & $\mathrm{U}$ & 599.415 & 649.687 & 709.130 & 780.501 & 867.786 & 976.969 & 1117.455 & 1304.936 & 1567.684 \\
\hline & $\mathrm{Nb}$ & 2798.632 & 3033.695 & 3311.674 & 3645.480 & 4053.776 & 4564.581 & 5221.958 & 6099.423 & 7329.452 \\
\hline & $\mathrm{Ta}$ & 184.889 & 200.425 & 218.797 & 240.861 & 267.848 & 301.613 & 345.069 & 403.077 & 484.398 \\
\hline & $\mathrm{La}$ & 932.677 & 1014.901 & 1112.526 & 1230.272 & 1374.988 & 1557.010 & 1792.682 & 2109.439 & 2557.081 \\
\hline & $\mathrm{Ce}$ & 1471.505 & 1604.019 & 1761.641 & 1952.127 & 2186.757 & 2482.590 & 2866.667 & 3384.513 & 4119.028 \\
\hline & $\mathrm{Pb}$ & 2009.600 & 2178.264 & 2377.708 & 2617.190 & 2910.093 & 3276.503 & 3748.009 & 4377.307 & 5259.348 \\
\hline & $\operatorname{Pr}$ & 171.171 & 186.690 & 205.160 & 227.494 & 255.025 & 289.763 & 334.903 & 395.826 & 482.342 \\
\hline & $\mathrm{Sr}$ & 37399.411 & 40542.354 & 44259.263 & 48722.859 & 54182.816 & 61013.957 & 69805.837 & 81542.083 & 97995.379 \\
\hline & $\mathrm{Nd}$ & 495.104 & 541.716 & 597.378 & 664.933 & 748.537 & 854.501 & 992.888 & 1180.740 & 1449.307 \\
\hline & $\mathrm{Zr}$ & 18183.535 & 19724.724 & 21548.630 & 23740.600 & 26424.096 & 29784.627 & 34114.253 & 39900.780 & 48024.421 \\
\hline & $\mathrm{Hf}$ & 482.176 & 523.043 & 571.408 & 629.533 & 700.692 & 789.803 & 904.613 & 1058.055 & 1273.471 \\
\hline
\end{tabular}




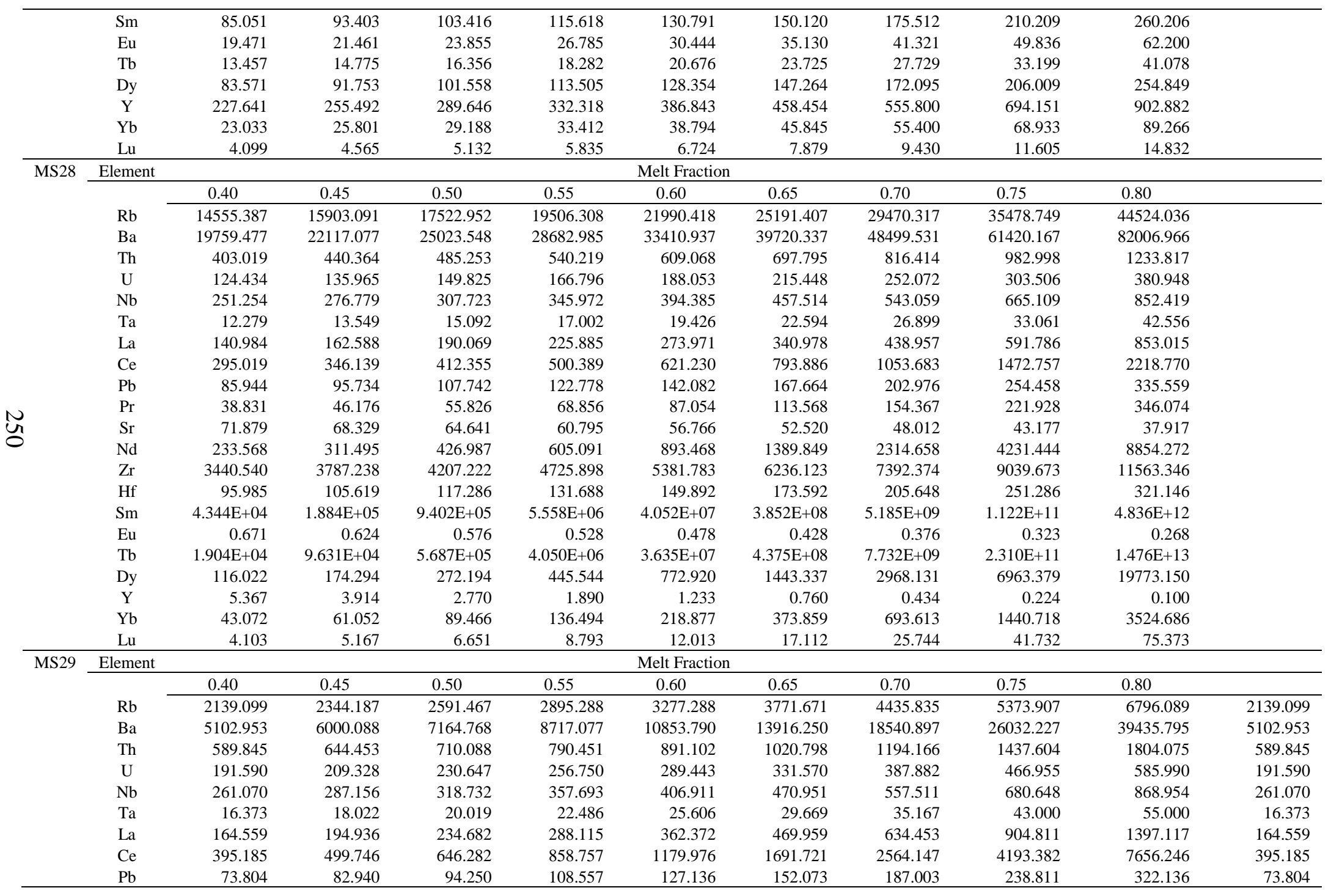




\begin{tabular}{|c|c|c|c|c|c|c|c|c|c|c|c|}
\hline & $\operatorname{Pr}$ & 152.171 & 242.303 & 403.326 & 708.414 & 1329.727 & 2715.206 & 6190.391 & 16407.487 & 54093.958 & 152.171 \\
\hline & $\mathrm{Sr}$ & 100.649 & 97.399 & 93.958 & 90.296 & 86.371 & 82.127 & 77.487 & 72.336 & 66.495 & 100.649 \\
\hline & $\mathrm{Nd}$ & 4.727 & 3.545 & 2.586 & 1.825 & 1.236 & 0.795 & 0.477 & 0.261 & 0.125 & 4.727 \\
\hline & $\mathrm{Zr}$ & 3201.658 & 3529.588 & 3927.463 & 4419.690 & 5043.333 & 5857.446 & 6962.007 & 8540.271 & 10966.730 & 3201.658 \\
\hline & $\mathrm{Hf}$ & 86.107 & 94.913 & 105.597 & 118.812 & 135.552 & 157.401 & 187.038 & 229.374 & 294.441 & 86.107 \\
\hline & $\mathrm{Sm}$ & 2.265 & 2.095 & 1.923 & 1.750 & 1.574 & 1.396 & 1.216 & 1.032 & 0.845 & 2.265 \\
\hline & $\mathrm{Eu}$ & 0.487 & 0.472 & 0.457 & 0.441 & 0.424 & 0.405 & 0.384 & 0.361 & 0.335 & 0.487 \\
\hline & $\mathrm{Tb}$ & 0.333 & 0.314 & 0.295 & 0.276 & 0.255 & 0.234 & 0.211 & 0.188 & 0.162 & 0.333 \\
\hline & Dy & 2.137 & 1.985 & 1.830 & 1.673 & 1.514 & 1.351 & 1.185 & 1.015 & 0.840 & 2.137 \\
\hline & $\mathrm{Y}$ & 10.533 & 9.953 & 9.354 & 8.734 & 8.089 & 7.416 & 6.708 & 5.957 & 5.151 & 10.533 \\
\hline & $\mathrm{Lu}$ & 0.114 & 0.092 & 0.073 & 0.056 & 0.042 & 0.030 & 0.021 & 0.013 & 0.008 & 0.114 \\
\hline \multirow[t]{23}{*}{ MS30 } & Element & \multicolumn{9}{|c|}{ Melt Fraction } & \\
\hline & & 0.45 & 0.50 & 0.55 & 0.60 & 0.65 & 0.70 & 0.75 & 0.80 & 0.85 & \\
\hline & $\mathrm{Rb}$ & 11600.773 & 12767.341 & 14193.910 & 15978.186 & 18273.796 & 21336.969 & 25629.279 & 32074.759 & 42832.030 & \\
\hline & $\mathrm{Ba}$ & 447703.918 & 492724.779 & 547779.786 & 616639.604 & 705233.181 & 823448.943 & 989100.316 & 1237848.091 & 1652999.088 & \\
\hline & Th & 1244.290 & 1369.416 & 1522.428 & 1713.808 & 1960.034 & 2288.587 & 2748.977 & 3440.315 & 4594.132 & \\
\hline & $\mathrm{U}$ & 409.035 & 450.167 & 500.467 & 563.379 & 644.321 & 752.326 & 903.669 & 1130.932 & 1510.226 & \\
\hline & $\mathrm{Nb}$ & 1460.470 & 1607.325 & 1786.909 & 2011.521 & 2300.501 & 2686.100 & 3226.420 & 4037.773 & 5391.870 & \\
\hline & $\mathrm{Ta}$ & 123.445 & 135.857 & 151.036 & 170.021 & 194.447 & 227.039 & 272.709 & 341.288 & 455.741 & \\
\hline & $\mathrm{La}$ & 1038.252 & 1144.342 & 1274.279 & 1437.078 & 1646.940 & 1927.597 & 2321.900 & 2915.869 & 3911.144 & \\
\hline & $\mathrm{Ce}$ & 1167.847 & 1289.578 & 1438.964 & 1626.540 & 1868.938 & 2194.021 & 2652.249 & 3345.272 & 4512.398 & \\
\hline & $\mathrm{Pb}$ & 618.964 & 681.468 & 757.932 & 853.613 & 976.776 & 1141.215 & 1371.794 & 1718.323 & 2297.266 & \\
\hline & $\operatorname{Pr}$ & 79.556 & 88.169 & 98.780 & 112.160 & 129.534 & 152.964 & 186.204 & 236.870 & 323.043 & \\
\hline & $\mathrm{Sr}$ & 12152.117 & 13396.105 & 14919.988 & 16829.652 & 19291.925 & 22585.665 & 27214.520 & 34189.857 & 45883.338 & \\
\hline & $\mathrm{Nd}$ & 179.021 & 199.939 & 225.918 & 258.976 & 302.342 & 361.511 & 446.610 & 578.484 & 807.519 & \\
\hline & $\mathrm{Zr}$ & 3685.977 & 4068.201 & 4537.019 & 5125.352 & 5885.143 & 6903.358 & 8337.367 & 10503.891 & 14147.734 & \\
\hline & $\mathrm{Hf}$ & 102.661 & 113.307 & 126.364 & 142.751 & 163.912 & 192.271 & 232.211 & 292.553 & 394.041 & \\
\hline & $\mathrm{Sm}$ & 24.461 & 27.891 & 32.246 & 37.924 & 45.579 & 56.357 & 72.440 & 98.497 & 146.374 & \\
\hline & $\mathrm{Eu}$ & 5.927 & 6.843 & 8.020 & 9.578 & 11.713 & 14.776 & 19.449 & 27.224 & 41.999 & \\
\hline & $\mathrm{Tb}$ & 3.725 & 4.366 & 5.204 & 6.332 & 7.910 & 10.226 & 13.856 & 20.096 & 32.455 & \\
\hline & Dy & 24.421 & 28.871 & 34.739 & 42.721 & 54.011 & 70.802 & 97.520 & 144.304 & 239.157 & \\
\hline & Y & 139.554 & 174.034 & 222.144 & 291.834 & 397.631 & 568.289 & 866.963 & 1453.792 & 2830.973 & \\
\hline & $\mathrm{Yb}$ & 23.907 & 33.270 & 47.944 & 72.130 & 114.609 & 195.601 & 368.089 & 798.016 & 2164.053 & \\
\hline & $\mathrm{Lu}$ & 3.139 & 4.172 & 5.716 & 8.127 & 12.110 & 19.193 & 33.091 & 64.450 & 152.220 & \\
\hline \multirow[t]{6}{*}{ MS33 } & Element & \multicolumn{9}{|c|}{ Melt Fraction } & \\
\hline & & 0.35 & 0.40 & 0.45 & 0.50 & 0.55 & 0.60 & 0.65 & 0.70 & 0.75 & \\
\hline & $\mathrm{Rb}$ & 109.982 & 119.362 & 130.468 & 143.822 & 160.181 & 180.680 & 207.112 & 242.468 & 292.155 & \\
\hline & $\mathrm{Ba}$ & 497.629 & 557.922 & 631.781 & 723.950 & 841.564 & 995.805 & 1205.129 & 1502.061 & 1949.046 & \\
\hline & Th & 16.228 & 17.601 & 19.226 & 21.178 & 23.569 & 26.561 & 30.416 & 35.566 & 42.795 & \\
\hline & $\mathrm{U}$ & 8.431 & 9.144 & 9.988 & 11.003 & 12.244 & 13.799 & 15.801 & 18.477 & 22.233 & \\
\hline
\end{tabular}




\begin{tabular}{|c|c|c|c|c|c|c|c|c|c|c|}
\hline \multirow{40}{*}{ MS34 } & $\mathrm{Nb}$ & 8.802 & 11.955 & 16.676 & 24.011 & 35.929 & 56.379 & 93.961 & 169.448 & 340.345 \\
\hline & $\mathrm{Ta}$ & 0.579 & 0.787 & 1.097 & 1.580 & 2.365 & 3.711 & 6.184 & 11.152 & 22.400 \\
\hline & $\mathrm{La}$ & 18.345 & 21.618 & 25.841 & 31.419 & 38.997 & 49.652 & 65.294 & 89.571 & 130.183 \\
\hline & $\mathrm{Ce}$ & 0.000 & 0.000 & 0.000 & 0.000 & 0.000 & 0.000 & 0.000 & 0.000 & 0.000 \\
\hline & $\mathrm{Pb}$ & 12.508 & 13.646 & 15.001 & 16.639 & 18.659 & 21.209 & 24.524 & 29.000 & 35.360 \\
\hline & $\operatorname{Pr}$ & 0.551 & 0.500 & 0.450 & 0.401 & 0.354 & 0.307 & 0.261 & 0.217 & 0.174 \\
\hline & $\mathrm{Sr}$ & 84.539 & 74.701 & 65.301 & 56.356 & 47.887 & 39.916 & 32.472 & 25.588 & 19.304 \\
\hline & $\mathrm{Nd}$ & 2.373 & 2.276 & 2.176 & 2.071 & 1.961 & 1.844 & 1.721 & 1.589 & 1.445 \\
\hline & $\mathrm{Zr}$ & 211.622 & 261.559 & 329.298 & 423.789 & 560.095 & 764.987 & 1089.304 & 1638.122 & 2654.152 \\
\hline & $\mathrm{Hf}$ & 6.246 & 7.689 & 9.638 & 12.345 & 16.229 & 22.035 & 31.167 & 46.507 & 74.665 \\
\hline & $\mathrm{Sm}$ & 0.433 & 0.426 & 0.418 & 0.410 & 0.401 & 0.392 & 0.381 & 0.369 & 0.355 \\
\hline & $\mathrm{Eu}$ & 0.144 & 0.142 & 0.140 & 0.138 & 0.135 & 0.133 & 0.130 & 0.127 & 0.123 \\
\hline & $\mathrm{Tb}$ & 0.072 & 0.071 & 0.070 & 0.069 & 0.068 & 0.067 & 0.066 & 0.064 & 0.062 \\
\hline & Dy & 0.499 & 0.492 & 0.485 & 0.478 & 0.470 & 0.461 & 0.451 & 0.440 & 0.427 \\
\hline & Y & 2.929 & 2.886 & 2.840 & 2.791 & 2.737 & 2.679 & 2.614 & 2.541 & 2.457 \\
\hline & $\mathrm{Yb}$ & 0.360 & 0.354 & 0.346 & 0.339 & 0.330 & 0.321 & 0.311 & 0.300 & 0.287 \\
\hline & $\mathrm{Lu}$ & 0.065 & 0.064 & 0.062 & 0.061 & 0.059 & 0.057 & 0.055 & 0.052 & 0.050 \\
\hline & Element & & & & & Melt Fraction & & & & \\
\hline & & 0.40 & 0.45 & 0.50 & 0.55 & 0.60 & 0.65 & 0.70 & 0.75 & 0.80 \\
\hline & $\mathrm{Rb}$ & 4432.013 & 4839.077 & 5327.993 & 5926.151 & 6674.673 & 7638.257 & 8924.866 & 10729.125 & 13440.972 \\
\hline & $\mathrm{Ba}$ & 95995.274 & 104804.126 & 115383.433 & 128325.394 & 144519.126 & 165363.251 & 193191.531 & 232210.412 & 290846.311 \\
\hline & Th & 530.344 & 579.084 & 637.627 & 709.255 & 798.894 & 914.297 & 1068.399 & 1284.524 & 1609.404 \\
\hline & $\mathrm{U}$ & 181.410 & 198.082 & 218.107 & 242.608 & 273.270 & 312.745 & 365.457 & 439.385 & 550.513 \\
\hline & $\mathrm{Nb}$ & 464.581 & 508.221 & 560.742 & 625.140 & 705.924 & 810.208 & 949.892 & 1146.505 & 1443.348 \\
\hline & $\mathrm{Ta}$ & 27.056 & 29.608 & 32.681 & 36.450 & 41.180 & 47.288 & 55.476 & 67.008 & 84.434 \\
\hline & $\mathrm{La}$ & 204.584 & 226.991 & 254.361 & 288.474 & 332.053 & 389.473 & 468.214 & 582.136 & 759.943 \\
\hline & $\mathrm{Ce}$ & 228.326 & 263.795 & 308.999 & 368.038 & 447.488 & 558.496 & 721.305 & 976.161 & 1413.661 \\
\hline & $\mathrm{Pb}$ & 304.204 & 332.666 & 366.908 & 408.878 & 461.504 & 529.404 & 620.304 & 748.166 & 941.056 \\
\hline & $\operatorname{Pr}$ & 74.378 & 112.444 & 176.826 & 291.667 & 510.335 & 962.297 & 2001.230 & 4757.703 & 13731.145 \\
\hline & $\mathrm{Sr}$ & 3242.429 & 3613.801 & 4069.580 & 4640.606 & 5374.316 & 6347.393 & 7691.791 & 9653.986 & 12749.121 \\
\hline & $\mathrm{Nd}$ & 5.876 & 5.049 & 4.276 & 3.558 & 2.897 & 2.295 & 1.754 & 1.276 & 0.865 \\
\hline & $\mathrm{Zr}$ & 1398.665 & 1554.797 & 1745.891 & 1984.583 & 2290.251 & 2694.110 & 3249.666 & 4056.413 & 5321.183 \\
\hline & $\mathrm{Hf}$ & 37.180 & 41.325 & 46.398 & 52.733 & 60.845 & 71.561 & 86.299 & 107.696 & 141.231 \\
\hline & $\mathrm{Sm}$ & 1.431 & 1.374 & 1.314 & 1.251 & 1.184 & 1.113 & 1.036 & 0.951 & 0.857 \\
\hline & $\mathrm{Eu}$ & 0.399 & 0.387 & 0.374 & 0.360 & 0.345 & 0.329 & 0.311 & 0.291 & 0.269 \\
\hline & $\mathrm{Tb}$ & 0.197 & 0.192 & 0.187 & 0.181 & 0.175 & 0.168 & 0.160 & 0.152 & 0.142 \\
\hline & Dy & 1.290 & 1.253 & 1.213 & 1.171 & 1.125 & 1.075 & 1.021 & 0.959 & 0.890 \\
\hline & $\mathrm{Y}$ & 6.952 & 6.742 & 6.520 & 6.282 & 6.027 & 5.750 & 5.447 & 5.108 & 4.722 \\
\hline & $\mathrm{Yb}$ & 0.731 & 0.702 & 0.671 & 0.638 & 0.604 & 0.567 & 0.527 & 0.483 & 0.435 \\
\hline & $\mathrm{Lu}$ & 0.119 & 0.114 & 0.108 & 0.103 & 0.097 & 0.090 & 0.083 & 0.076 & 0.068 \\
\hline MS35 & Element & & & & & Melt Fraction & & & & \\
\hline
\end{tabular}




\begin{tabular}{|c|c|c|c|c|c|c|c|c|c|c|}
\hline & & 0.45 & 0.50 & 0.55 & 0.60 & 0.65 & 0.70 & 0.75 & 0.80 & 0.85 \\
\hline & $\mathrm{Rb}$ & 5628.941 & 6199.787 & 6898.432 & 7773.054 & 8899.478 & 10404.299 & 12515.848 & 15691.890 & 21003.728 \\
\hline & $\mathrm{Ba}$ & 6146.442 & 6916.835 & 7881.317 & 9119.571 & 10760.283 & 13024.698 & 16325.614 & 21524.671 & 30741.835 \\
\hline & Th & 811.986 & 894.005 & 994.349 & 1119.913 & 1281.548 & 1497.363 & 1799.995 & 2254.838 & 3014.797 \\
\hline & $\mathrm{U}$ & 243.247 & 267.818 & 297.878 & 335.493 & 383.915 & 448.566 & 539.226 & 675.484 & 903.146 \\
\hline & $\mathrm{Nb}$ & 2175.160 & 2394.750 & 2663.383 & 2999.517 & 3432.184 & 4009.831 & 4819.780 & 6036.962 & 8070.364 \\
\hline & $\mathrm{Ta}$ & 137.423 & 151.296 & 168.268 & 189.504 & 216.839 & 253.334 & 304.505 & 381.405 & 509.872 \\
\hline & $\mathrm{La}$ & 230.118 & 260.033 & 297.647 & 346.174 & 410.824 & 500.611 & 632.460 & 841.975 & 1217.603 \\
\hline & $\mathrm{Ce}$ & 380.883 & 440.368 & 516.993 & 618.540 & 757.992 & 958.509 & 1265.189 & 1777.083 & 2753.823 \\
\hline & $\mathrm{Pb}$ & 78.444 & 87.947 & 99.797 & 114.945 & 134.918 & 162.327 & 202.019 & 264.036 & 372.874 \\
\hline & $\operatorname{Pr}$ & 52.321 & 66.546 & 86.811 & 116.853 & 163.667 & 241.479 & 382.530 & 671.715 & 1388.115 \\
\hline & $\mathrm{Sr}$ & 87.317 & 81.487 & 75.494 & 69.314 & 62.918 & 56.265 & 49.298 & 41.934 & 34.039 \\
\hline & $\mathrm{Nd}$ & 0.248 & 0.118 & 0.052 & 0.021 & 0.007 & 0.002 & 0.001 & 0.000 & 0.000 \\
\hline & $\mathrm{Zr}$ & 2838.767 & 3160.968 & 3559.866 & 4065.677 & 4726.577 & 5624.211 & 6908.387 & 8885.656 & 12291.877 \\
\hline & $\mathrm{Hf}$ & 76.115 & 84.754 & 95.449 & 109.011 & 126.731 & 150.799 & 185.231 & 238.247 & 329.576 \\
\hline & $\mathrm{Sm}$ & 2.019 & 1.862 & 1.703 & 1.542 & 1.377 & 1.208 & 1.035 & 0.857 & 0.672 \\
\hline & $\mathrm{Eu}$ & 0.521 & 0.502 & 0.481 & 0.459 & 0.435 & 0.410 & 0.381 & 0.348 & 0.311 \\
\hline & $\mathrm{Tb}$ & 0.300 & 0.286 & 0.271 & 0.256 & 0.239 & 0.221 & 0.202 & 0.180 & 0.156 \\
\hline & Dy & 1.919 & 1.815 & 1.707 & 1.594 & 1.475 & 1.348 & 1.212 & 1.065 & 0.900 \\
\hline & Y & 10.026 & 9.457 & 8.865 & 8.247 & 7.599 & 6.913 & 6.182 & 5.391 & 4.519 \\
\hline & $\mathrm{Yb}$ & 1.001 & 0.920 & 0.838 & 0.754 & 0.670 & 0.584 & 0.496 & 0.407 & 0.315 \\
\hline & $\mathrm{Lu}$ & 0.157 & 0.143 & 0.129 & 0.115 & 0.100 & 0.086 & 0.072 & 0.058 & 0.043 \\
\hline \multirow[t]{19}{*}{ MS37 } & Element & \multicolumn{9}{|c|}{ Melt Fraction } \\
\hline & & 0.30 & 0.35 & 0.40 & 0.45 & 0.50 & 0.55 & 0.60 & 0.65 & 0.70 \\
\hline & $\mathrm{Rb}$ & 2.170 & 0.843 & 0.304 & 0.100 & 0.030 & 0.008 & 0.002 & 0.000 & 0.000 \\
\hline & $\mathrm{Ba}$ & 572.475 & 563.648 & 554.266 & 544.245 & 533.476 & 521.819 & 509.088 & 495.031 & 479.285 \\
\hline & Th & 15.657 & 16.901 & 18.356 & 20.079 & 22.153 & 24.696 & 27.886 & 32.004 & 37.519 \\
\hline & $\mathrm{U}$ & 9.964 & 10.755 & 11.681 & 12.778 & 14.098 & 15.716 & 17.746 & 20.366 & 23.876 \\
\hline & $\mathrm{Nb}$ & 2.072 & 1.632 & 1.261 & 0.953 & 0.701 & 0.500 & 0.342 & 0.222 & 0.135 \\
\hline & $\mathrm{Ta}$ & 0.084 & 0.067 & 0.052 & 0.040 & 0.030 & 0.022 & 0.015 & 0.010 & 0.006 \\
\hline & $\mathrm{La}$ & 854.669 & 2431.633 & 7522.520 & 25676.127 & 98525.127 & 435660.692 & 2295457.793 & 15103897.279 & 132939597.581 \\
\hline & $\mathrm{Ce}$ & 2.632 & 1.992 & 1.474 & 1.063 & 0.743 & 0.500 & 0.321 & 0.194 & 0.109 \\
\hline & $\mathrm{Pb}$ & 40.307 & 44.262 & 48.971 & 54.660 & 61.652 & 70.427 & 81.724 & 96.738 & 117.531 \\
\hline & $\operatorname{Pr}$ & 0.518 & 0.416 & 0.329 & 0.254 & 0.192 & 0.141 & 0.099 & 0.067 & 0.042 \\
\hline & $\mathrm{Sr}$ & 72.066 & 63.682 & 55.719 & 48.188 & 41.102 & 34.474 & 28.322 & 22.665 & 17.524 \\
\hline & $\mathrm{Nd}$ & 3.542 & 3.264 & 2.989 & 2.716 & 2.446 & 2.178 & 1.913 & 1.652 & 1.394 \\
\hline & $\mathrm{Zr}$ & 1000.806 & 1088.234 & 1191.262 & 1314.355 & 1463.831 & 1648.930 & 1883.693 & 2190.522 & 2607.384 \\
\hline & $\mathrm{Hf}$ & 29.555 & 32.130 & 35.163 & 38.786 & 43.185 & 48.630 & 55.535 & 64.555 & 76.804 \\
\hline & $\mathrm{Sm}$ & 1.032 & 0.980 & 0.926 & 0.871 & 0.814 & 0.756 & 0.696 & 0.633 & 0.568 \\
\hline & $\mathrm{Eu}$ & 0.259 & 0.251 & 0.243 & 0.235 & 0.226 & 0.216 & 0.206 & 0.195 & 0.183 \\
\hline & $\mathrm{Tb}$ & 0.258 & 0.243 & 0.229 & 0.214 & 0.199 & 0.184 & 0.168 & 0.151 & 0.134 \\
\hline
\end{tabular}




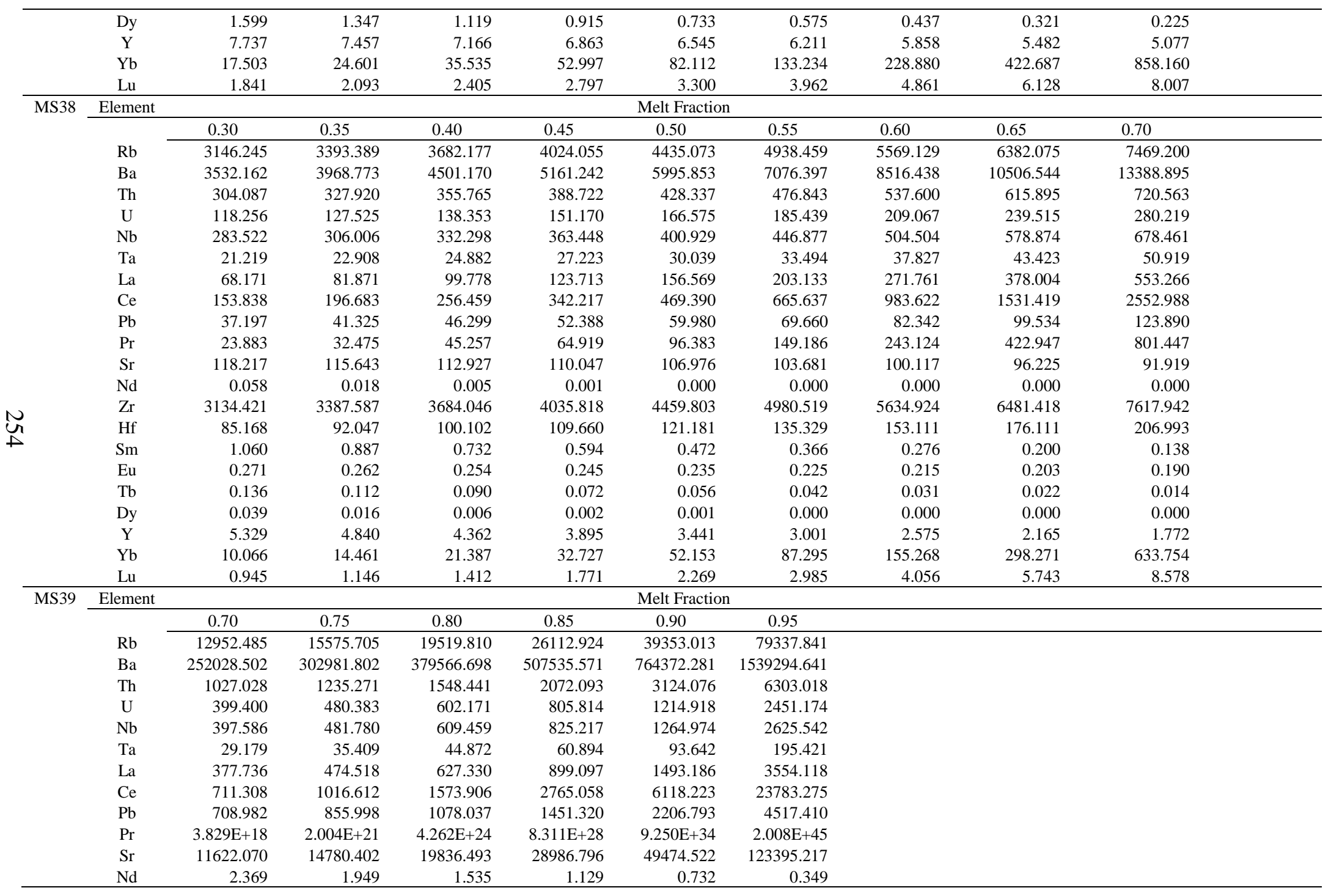




\begin{tabular}{|c|c|c|c|c|c|c|c|c|c|c|}
\hline & $\mathrm{Zr}$ & 2100.887 & 2654.938 & 3535.622 & 5115.171 & 8608.417 & 20959.490 & & & \\
\hline & $\mathrm{Hf}$ & 57.342 & 72.426 & 96.389 & 139.335 & 234.216 & 569.121 & & & \\
\hline & $\mathrm{Sm}$ & 0.602 & 0.565 & 0.522 & 0.472 & 0.410 & 0.322 & & & \\
\hline & $\mathrm{Eu}$ & 0.166 & 0.158 & 0.148 & 0.137 & 0.123 & 0.101 & & & \\
\hline & $\mathrm{Tb}$ & 0.068 & 0.066 & 0.062 & 0.058 & 0.053 & 0.045 & & & \\
\hline & Dy & 0.433 & 0.412 & 0.389 & 0.361 & 0.325 & 0.271 & & & \\
\hline & $\mathrm{Y}$ & 2.322 & 2.209 & 2.077 & 1.919 & 1.717 & 1.419 & & & \\
\hline & $\mathrm{Yb}$ & 0.243 & 0.227 & 0.209 & 0.187 & 0.161 & 0.124 & & & \\
\hline & $\mathrm{Lu}$ & 0.041 & 0.038 & 0.035 & 0.031 & 0.026 & 0.020 & & & \\
\hline \multirow[t]{22}{*}{ MS40 } & Element & \multicolumn{9}{|c|}{ Melt Fraction } \\
\hline & & 0.05 & 0.10 & 0.15 & 0.20 & 0.25 & 0.30 & 0.35 & 0.40 & 0.45 \\
\hline & $\mathrm{Rb}$ & 105.751 & 111.705 & 118.365 & 125.863 & 134.368 & 144.096 & 155.331 & 168.452 & 183.975 \\
\hline & $\mathrm{Ba}$ & 319.704 & 340.188 & 363.272 & 389.469 & 419.438 & 454.030 & 494.370 & 541.977 & 598.943 \\
\hline & Th & 11.933 & 12.604 & 13.354 & 14.199 & 15.157 & 16.252 & 17.518 & 18.995 & 20.743 \\
\hline & $\mathrm{U}$ & 6.425 & 6.787 & 7.191 & 7.645 & 8.161 & 8.751 & 9.433 & 10.228 & 11.169 \\
\hline & $\mathrm{Nb}$ & 126.939 & 134.072 & 142.049 & 151.029 & 161.214 & 172.862 & 186.313 & 202.020 & 220.599 \\
\hline & $\mathrm{Ta}$ & 7.635 & 8.064 & 8.544 & 9.084 & 9.697 & 10.398 & 11.207 & 12.152 & 13.269 \\
\hline & $\mathrm{La}$ & 16.961 & 18.315 & 19.865 & 21.651 & 23.731 & 26.175 & 29.081 & 32.583 & 36.871 \\
\hline & $\mathrm{Ce}$ & 27.010 & 30.281 & 34.170 & 38.841 & 44.519 & 51.508 & 60.244 & 71.350 & 85.757 \\
\hline & $\mathrm{Pb}$ & 3.990 & 4.243 & 4.527 & 4.850 & 5.219 & 5.644 & 6.140 & 6.724 & 7.423 \\
\hline & $\mathrm{Sr}$ & 181.972 & 167.323 & 153.117 & 139.365 & 126.080 & 113.276 & 100.967 & 89.170 & 77.905 \\
\hline & $\mathrm{Nd}$ & 5.484 & 5.070 & 4.667 & 4.274 & 3.892 & 3.521 & 3.162 & 2.816 & 2.482 \\
\hline & $\mathrm{Zr}$ & 263.631 & 281.011 & 300.633 & 322.944 & 348.517 & 378.099 & 412.677 & 453.585 & 502.667 \\
\hline & $\mathrm{Hf}$ & 7.902 & 8.423 & 9.011 & 9.680 & 10.446 & 11.333 & 12.370 & 13.596 & 15.068 \\
\hline & $\mathrm{Sm}$ & 0.912 & 0.890 & 0.868 & 0.844 & 0.820 & 0.795 & 0.768 & 0.741 & 0.713 \\
\hline & $\mathrm{Eu}$ & 0.256 & 0.252 & 0.248 & 0.243 & 0.239 & 0.234 & 0.229 & 0.224 & 0.218 \\
\hline & $\mathrm{Tb}$ & 0.126 & 0.124 & 0.122 & 0.120 & 0.117 & 0.115 & 0.112 & 0.110 & 0.107 \\
\hline & Dy & 0.831 & 0.816 & 0.799 & 0.783 & 0.765 & 0.747 & 0.728 & 0.708 & 0.687 \\
\hline & $\mathrm{Y}$ & 4.232 & 4.153 & 4.071 & 3.985 & 3.896 & 3.803 & 3.705 & 3.603 & 3.494 \\
\hline & $\mathrm{Yb}$ & 0.522 & 0.507 & 0.492 & 0.477 & 0.461 & 0.445 & 0.428 & 0.410 & 0.392 \\
\hline & $\mathrm{Lu}$ & 0.085 & 0.083 & 0.080 & 0.077 & 0.074 & 0.072 & 0.069 & 0.065 & 0.062 \\
\hline \multirow[t]{8}{*}{ MS41 } & Element & \multicolumn{9}{|c|}{ Melt Fraction } \\
\hline & & 0.05 & 0.10 & 0.15 & 0.20 & 0.25 & 0.30 & 0.35 & 0.40 & \\
\hline & $\mathrm{Rb}$ & 105.872 & 111.833 & 118.501 & 126.007 & 134.521 & 144.260 & 155.508 & 168.644 & \\
\hline & $\mathrm{Ba}$ & 336.856 & 358.270 & 382.391 & 409.751 & 441.033 & 477.119 & 519.176 & 568.776 & \\
\hline & Th & 9.039 & 9.549 & 10.119 & 10.762 & 11.491 & 12.325 & 13.288 & 14.413 & \\
\hline & U & 4.867 & 5.142 & 5.449 & 5.795 & 6.187 & 6.636 & 7.155 & 7.761 & \\
\hline & $\mathrm{Nb}$ & 51.397 & 54.335 & 57.623 & 61.329 & 65.536 & 70.353 & 75.922 & 82.434 & \\
\hline & $\mathrm{Ta}$ & 2.713 & 2.868 & 3.043 & 3.239 & 3.462 & 3.718 & 4.013 & 4.359 & \\
\hline
\end{tabular}




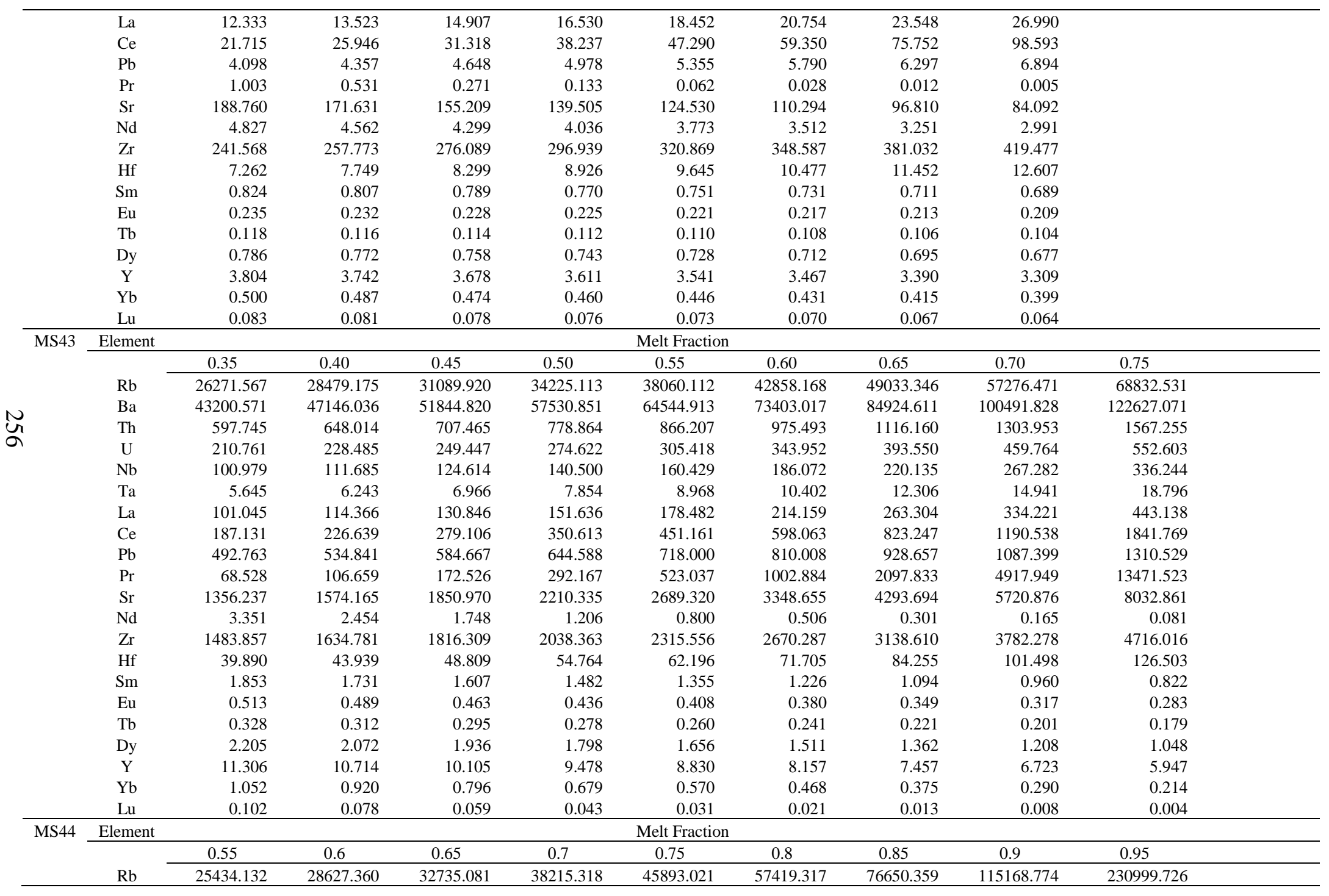




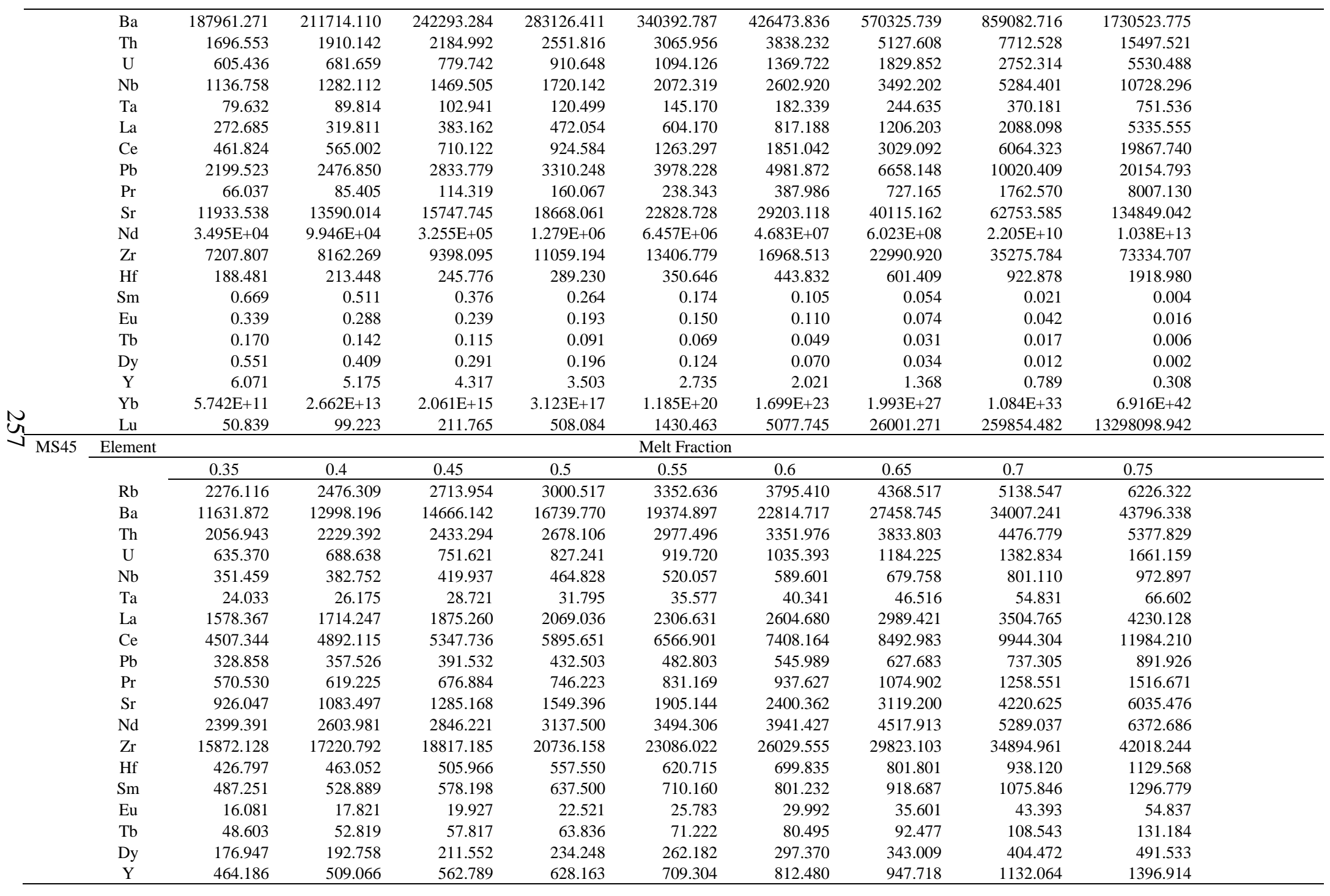




\begin{tabular}{|c|c|c|c|c|c|c|c|c|c|c|}
\hline & $\mathrm{Yb}$ & 28.523 & 31.628 & 35.387 & 40.020 & 45.850 & 53.379 & 63.420 & 77.382 & 97.914 \\
\hline & $\mathrm{Lu}$ & 4.842 & 5.358 & 5.982 & 6.750 & 7.713 & 8.954 & 10.603 & 12.888 & 16.235 \\
\hline MS46 & Element & \multicolumn{9}{|c|}{ Melt Fraction } \\
\hline & & 0.4 & 0.45 & 0.5 & 0.55 & 0.6 & 0.65 & 0.7 & 0.75 & 0.8 \\
\hline & $\mathrm{Rb}$ & 17896.087 & 39591.832 & 94480.747 & 247122.900 & 723962.672 & 2448690.938 & 9997032.136 & 52778412.928 & 404416796.363 \\
\hline & $\mathrm{Ba}$ & 425.453 & 415.753 & 405.381 & 394.217 & 382.100 & 368.812 & 354.047 & 337.344 & 317.970 \\
\hline & Th & 295.012 & 322.545 & 355.662 & 396.242 & 447.112 & 512.726 & 600.534 & 723.995 & 910.153 \\
\hline & $\mathrm{U}$ & 91.087 & 99.588 & 109.813 & 122.342 & 138.048 & 158.307 & 185.418 & 223.538 & 281.015 \\
\hline & $\mathrm{Nb}$ & 0.882 & 0.565 & 0.347 & 0.203 & 0.111 & 0.056 & 0.026 & 0.010 & 0.003 \\
\hline & $\mathrm{Ta}$ & 0.070 & 0.048 & 0.032 & 0.020 & 0.012 & 0.007 & 0.003 & 0.002 & 0.001 \\
\hline & $\mathrm{La}$ & 139.961 & 161.647 & 189.277 & 225.348 & 273.867 & 341.626 & 440.947 & 596.318 & 862.820 \\
\hline & $\mathrm{Ce}$ & 292.160 & 347.630 & 420.547 & 519.076 & 656.790 & 857.602 & 1166.906 & 1679.688 & 2623.257 \\
\hline & $\mathrm{Pb}$ & 83.537 & 93.136 & 104.921 & 119.691 & 138.679 & 163.872 & 198.699 & 249.563 & 329.858 \\
\hline & $\operatorname{Pr}$ & 39.058 & 46.949 & 57.434 & 71.769 & 92.069 & 122.110 & 169.173 & 248.761 & 398.775 \\
\hline & $\mathrm{Sr}$ & 70.273 & 60.816 & 51.912 & 43.577 & 35.834 & 28.706 & 22.221 & 16.415 & 11.331 \\
\hline & $\mathrm{Nd}$ & 279.025 & 386.857 & 553.340 & 821.910 & 1279.137 & 2111.998 & 3767.887 & 7472.163 & 17273.143 \\
\hline & $\mathrm{Zr}$ & 1967.103 & 2185.351 & 2452.299 & 2785.502 & 3211.865 & 3774.690 & 4548.138 & 5669.958 & 7426.150 \\
\hline & $\mathrm{Hf}$ & 55.171 & 61.232 & 68.639 & 77.873 & 89.674 & 105.231 & 126.575 & 157.473 & 205.734 \\
\hline & $\mathrm{Sm}$ & $1.885 \mathrm{E}+04$ & $7.086 \mathrm{E}+04$ & $3.022 \mathrm{E}+05$ & $1.502 \mathrm{E}+06$ & $9.017 \mathrm{E}+06$ & $6.880 \mathrm{E}+07$ & $7.184 \mathrm{E}+08$ & $1.152 \mathrm{E}+10$ & $3.436 \mathrm{E}+11$ \\
\hline & $\mathrm{Eu}$ & 0.636 & 0.560 & 0.487 & 0.418 & 0.352 & 0.289 & 0.231 & 0.177 & 0.128 \\
\hline & $\mathrm{Tb}$ & 34.635 & 58.550 & 104.061 & 196.510 & 399.975 & 895.260 & 2269.332 & 6818.176 & 26206.663 \\
\hline & Dy & 51.556 & 64.747 & 83.099 & 109.497 & 149.049 & 211.429 & 316.553 & 510.223 & 915.148 \\
\hline & $\mathrm{Y}$ & 1.489 & 0.859 & 0.470 & 0.241 & 0.115 & 0.049 & 0.019 & 0.006 & 0.001 \\
\hline & $\mathrm{Yb}$ & 24.706 & 28.493 & 33.311 & 39.590 & 48.021 & 59.771 & 76.953 & 103.756 & 149.576 \\
\hline & $\mathrm{Lu}$ & 4.948 & 5.573 & 6.348 & 7.331 & 8.610 & 10.333 & 12.756 & 16.364 & 22.196 \\
\hline \multirow[t]{16}{*}{ MS47 } & Element & \multicolumn{9}{|c|}{ Melt Fraction } \\
\hline & & 0.5 & 0.55 & 0.6 & 0.65 & 0.7 & 0.75 & 0.8 & 0.85 & 0.9 \\
\hline & $\mathrm{Rb}$ & 5569.486 & 6203.429 & 6997.911 & 8022.372 & 9392.890 & 11319.139 & 14222.196 & 19089.629 & 28904.463 \\
\hline & $\mathrm{Ba}$ & 31300.085 & 35016.691 & 39696.311 & 45762.407 & 53926.727 & 65482.972 & 83048.734 & 112820.216 & 173746.427 \\
\hline & Th & 312.314 & 348.542 & 394.037 & 452.839 & 531.715 & 642.920 & 811.152 & 1094.571 & 1669.812 \\
\hline & $\mathrm{U}$ & 101.444 & 113.211 & 127.989 & 147.089 & 172.709 & 208.830 & 263.474 & 355.532 & 542.379 \\
\hline & $\mathrm{Nb}$ & 219.162 & 247.398 & 283.291 & 330.320 & 394.400 & 486.420 & 628.749 & 875.351 & 1395.476 \\
\hline & $\mathrm{Ta}$ & 14.786 & 16.691 & 19.113 & 22.286 & 26.609 & 32.818 & 42.420 & 59.058 & 94.150 \\
\hline & $\mathrm{La}$ & 6.061 & 5.131 & 4.259 & 3.448 & 2.702 & 2.025 & 1.423 & 0.903 & 0.476 \\
\hline & $\mathrm{Ce}$ & 15.793 & 14.723 & 13.612 & 12.454 & 11.239 & 9.954 & 8.579 & 7.084 & 5.407 \\
\hline & $\mathrm{Pb}$ & 509.824 & 568.844 & 642.948 & 738.701 & 867.106 & 1048.082 & 1321.754 & 1782.572 & 2717.225 \\
\hline & $\operatorname{Pr}$ & 1.878 & 1.785 & 1.686 & 1.581 & 1.467 & 1.344 & 1.207 & 1.050 & 0.864 \\
\hline & $\mathrm{Sr}$ & 3520.266 & 4311.451 & 5408.198 & 6992.659 & 9407.264 & 13360.607 & 20525.950 & 35703.764 & 77902.955 \\
\hline & $\mathrm{Nd}$ & 5.758 & 5.602 & 5.433 & 5.247 & 5.040 & 4.806 & 4.535 & 4.207 & 3.785 \\
\hline & $\mathrm{Zr}$ & 2004.780 & 2303.794 & 2691.160 & 3209.666 & 3933.655 & 5003.524 & 6716.589 & 9817.605 & 16763.246 \\
\hline & $\mathrm{Hf}$ & 53.045 & 60.978 & 71.259 & 85.026 & 104.259 & 132.696 & 178.260 & 260.813 & 445.934 \\
\hline
\end{tabular}




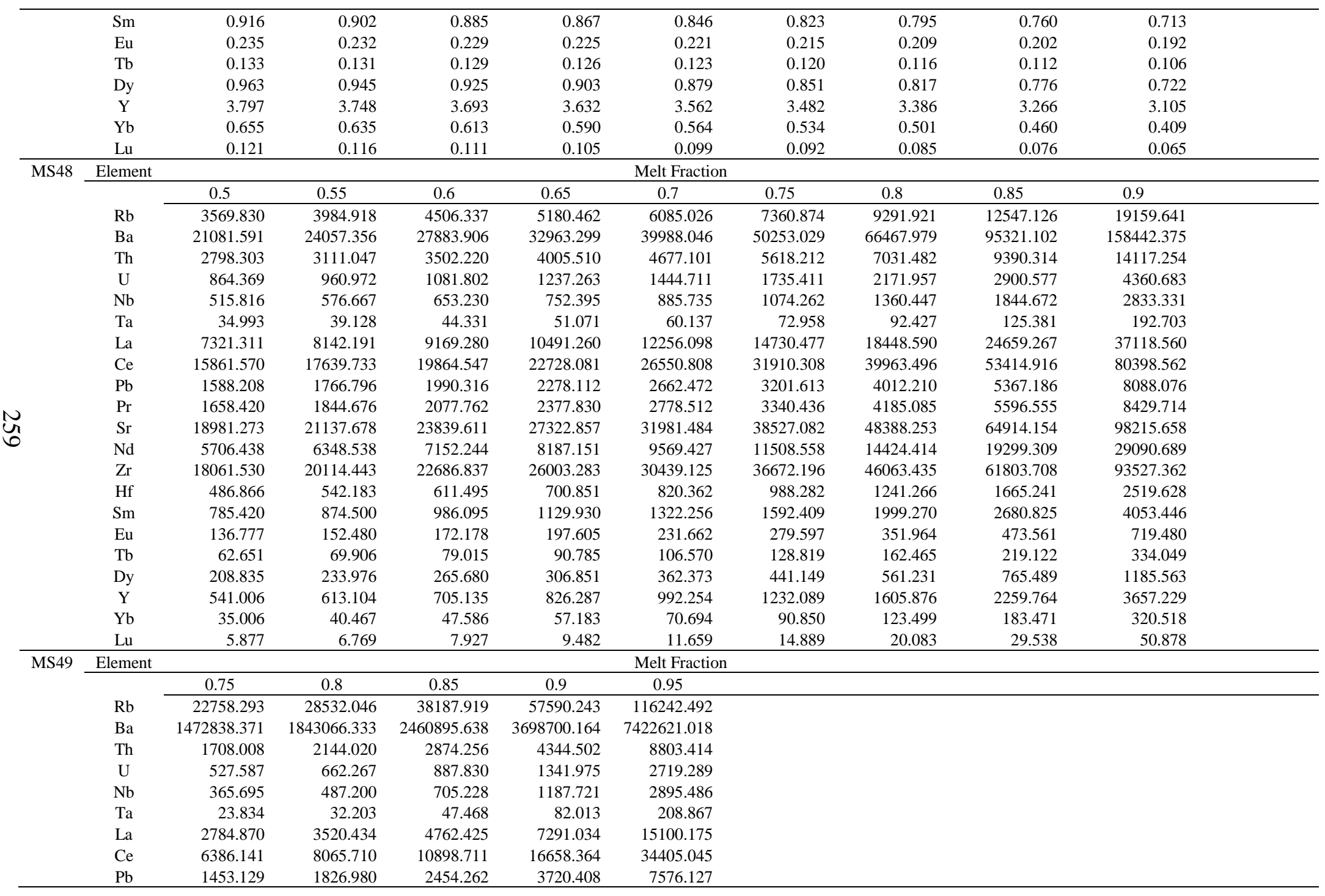




\begin{tabular}{|c|c|c|c|c|c|c|c|c|c|c|}
\hline & $\operatorname{Pr}$ & 828.045 & 1045.446 & 1411.993 & 2156.777 & 4449.477 & & & & \\
\hline & $\mathrm{Sr}$ & 96429.821 & 120759.363 & 161395.049 & 242903.683 & 488593.163 & & & & \\
\hline & $\mathrm{Nd}$ & 3320.764 & 4192.969 & 5663.683 & 8652.415 & 17854.718 & & & & \\
\hline & $\mathrm{Zr}$ & 5938.595 & 7768.675 & 10983.784 & 17895.177 & 41221.618 & & & & \\
\hline & $\mathrm{Hf}$ & 163.430 & 213.173 & 300.268 & 486.629 & 1110.872 & & & & \\
\hline & $\mathrm{Sm}$ & 622.432 & 787.586 & 1066.754 & 1635.984 & 3398.290 & & & & \\
\hline & $\mathrm{Eu}$ & 127.510 & 162.144 & 221.025 & 342.030 & 721.485 & & & & \\
\hline & $\mathrm{Tb}$ & 61.917 & 79.041 & 108.284 & 168.754 & 360.295 & & & & \\
\hline & Dy & 235.833 & 307.547 & 433.079 & 701.595 & 1600.521 & & & & \\
\hline & $\mathrm{Y}$ & 899.902 & 1258.418 & 1938.985 & 3566.092 & 10105.552 & & & & \\
\hline & $\mathrm{Yb}$ & 165.471 & 279.109 & 547.641 & 1415.999 & 7183.758 & & & & \\
\hline & $\mathrm{Lu}$ & 21.762 & 35.173 & 65.317 & 156.280 & 694.378 & & & & \\
\hline \multirow[t]{23}{*}{ MS50 } & Element & & & & & Melt Fraction & & & & \\
\hline & & 0.45 & 0.5 & 0.55 & 0.6 & 0.65 & 0.7 & 0.75 & 0.8 & 0.85 \\
\hline & $\mathrm{Rb}$ & 16808.657 & 18500.704 & 20570.080 & 23158.636 & 26489.439 & 30934.578 & 37164.449 & 46521.362 & 62141.774 \\
\hline & $\mathrm{Ba}$ & 82130.885 & 90697.042 & 101209.942 & 114411.332 & 131472.290 & 154355.005 & 186613.192 & 235406.396 & 317592.278 \\
\hline & Th & 2262.389 & 2489.924 & 2768.175 & 3116.202 & 3563.973 & 4161.473 & 4998.744 & 6256.054 & 8354.527 \\
\hline & $\mathrm{U}$ & 748.535 & 823.818 & 915.880 & 1031.028 & 1179.178 & 1376.867 & 1653.887 & 2069.881 & 2764.183 \\
\hline & $\mathrm{Nb}$ & 240.949 & 267.599 & 300.501 & 342.095 & 396.255 & 469.526 & 573.865 & 733.623 & 1006.898 \\
\hline & $\mathrm{Ta}$ & 17.422 & 19.349 & 21.728 & 24.735 & 28.651 & 33.949 & 41.493 & 53.045 & 72.804 \\
\hline & $\mathrm{La}$ & 959.872 & 1061.195 & 1185.695 & 1342.243 & 1544.864 & 1817.097 & 2201.645 & 2784.730 & 3769.902 \\
\hline & $\mathrm{Ce}$ & 1189.938 & 1320.571 & 1481.729 & 1685.281 & 1950.071 & 2307.889 & 2816.763 & 3594.687 & 4922.704 \\
\hline & $\mathrm{Pb}$ & 1018.979 & 1122.318 & 1248.792 & 1407.124 & 1611.039 & 1883.456 & 2265.709 & 2840.669 & 3802.276 \\
\hline & $\operatorname{Pr}$ & 93.938 & 104.939 & 118.604 & 135.999 & 158.824 & 189.977 & 234.802 & 304.300 & 425.077 \\
\hline & $\mathrm{Sr}$ & 2463.005 & 2766.449 & 3145.580 & 3631.242 & 4273.139 & 5156.496 & 6439.854 & 8452.956 & 12003.504 \\
\hline & $\mathrm{Nd}$ & 294.456 & 330.986 & 376.664 & 435.230 & 512.715 & 619.470 & 774.776 & 1018.792 & 1450.061 \\
\hline & $\mathrm{Zr}$ & 4194.844 & 4648.678 & 5207.720 & 5912.629 & 6827.870 & 8061.977 & 9812.605 & 12480.600 & 17017.524 \\
\hline & $\mathrm{Hf}$ & 111.022 & 123.021 & 137.801 & 156.435 & 180.626 & 213.240 & 259.496 & 329.977 & 449.800 \\
\hline & $\mathrm{Sm}$ & 41.164 & 47.860 & 56.538 & 68.114 & 84.130 & 107.357 & 143.237 & 203.853 & 321.297 \\
\hline & $\mathrm{Eu}$ & 10.126 & 12.027 & 14.547 & 17.993 & 22.899 & 30.247 & 42.038 & 62.893 & 105.724 \\
\hline & $\mathrm{Tb}$ & 6.279 & 7.534 & 9.215 & 11.543 & 14.899 & 20.005 & 28.347 & 43.427 & 75.267 \\
\hline & Dy & 39.070 & 47.269 & 58.350 & 73.839 & 96.428 & 131.225 & 188.924 & 295.117 & 524.475 \\
\hline & $\mathrm{Y}$ & 211.088 & 258.372 & 323.060 & 414.728 & 550.487 & 763.346 & 1123.676 & 1803.678 & 3319.840 \\
\hline & $\mathrm{Yb}$ & 22.102 & 28.077 & 36.577 & 49.159 & 68.735 & 101.211 & 159.951 & 280.060 & 576.596 \\
\hline & $\mathrm{Lu}$ & 3.155 & 3.832 & 4.750 & 6.041 & 7.932 & 10.863 & 15.757 & 24.841 & 44.673 \\
\hline \multirow[t]{6}{*}{ MS51 } & Element & & & & & Melt Fraction & & & & \\
\hline & & 0.75 & 0.8 & 0.85 & 0.9 & 0.95 & & & & \\
\hline & $\mathrm{Rb}$ & 28736.764 & 35987.067 & 48096.640 & 72386.414 & 145602.112 & & & & \\
\hline & $\mathrm{Ba}$ & 89563.221 & 114407.818 & 156867.782 & 244756.605 & 523616.274 & & & & \\
\hline & Th & 4488.301 & 5618.012 & 7503.827 & 11283.590 & 22662.716 & & & & \\
\hline & $\mathrm{U}$ & 1485.002 & 1858.778 & 2482.720 & 3733.295 & 7498.198 & & & & \\
\hline
\end{tabular}




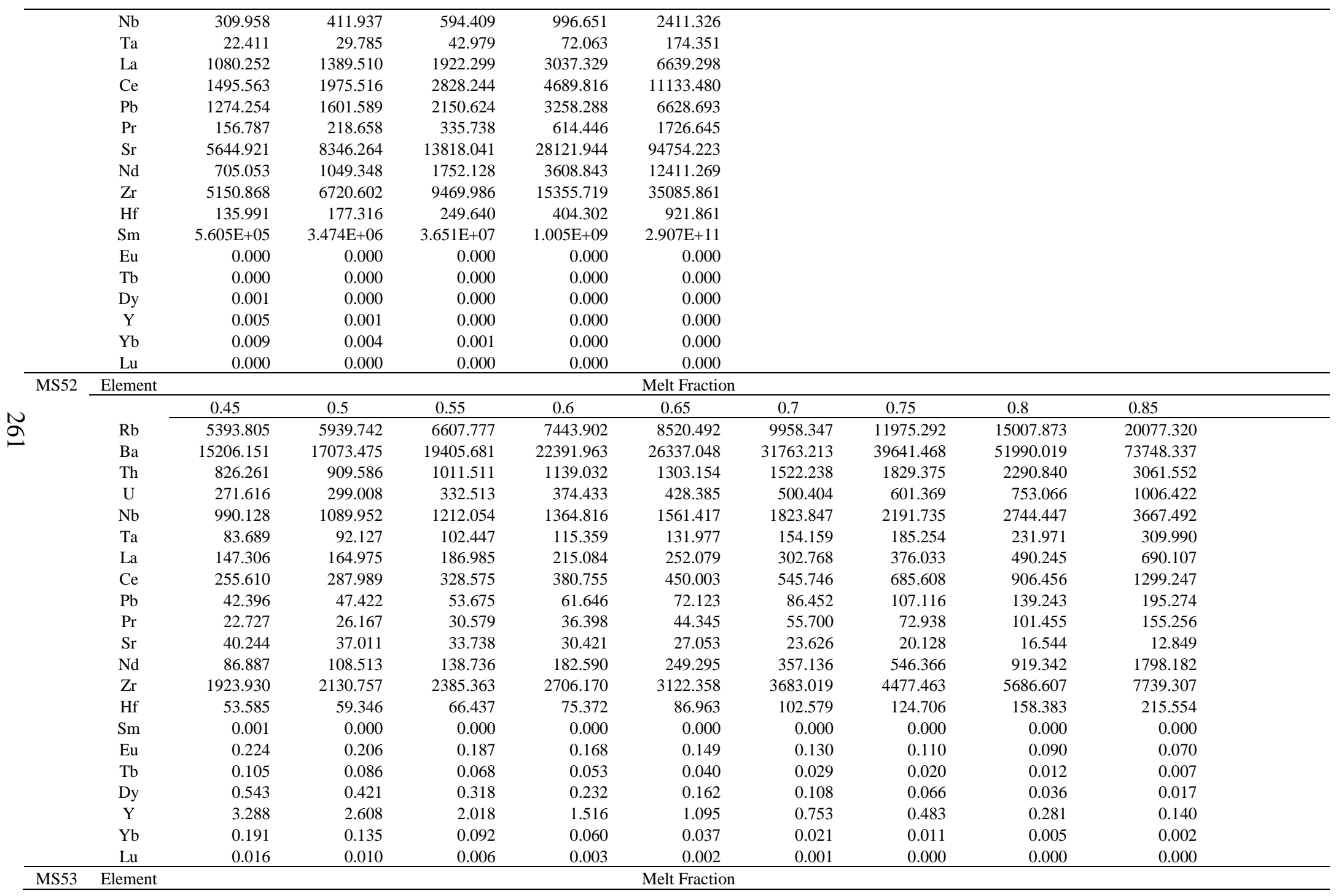




\begin{tabular}{|c|c|c|c|c|c|c|c|c|c|c|}
\hline \multirow{41}{*}{$\hat{N}$} & & 0.5 & 0.55 & 0.6 & 0.65 & 0.7 & 0.75 & 0.8 & 0.85 & 0.9 \\
\hline & $\mathrm{Rb}$ & 4726.762 & 5257.376 & 5921.370 & 6776.129 & 7917.419 & 9517.870 & 11923.347 & 15942.618 & 24009.006 \\
\hline & $\mathrm{Ba}$ & 94592.855 & 105204.999 & 118483.816 & 135576.362 & 158396.676 & 190394.786 & 238482.079 & 318817.960 & 480012.410 \\
\hline & Th & 395.594 & 440.021 & 495.618 & 567.191 & 662.763 & 796.793 & 998.257 & 1334.915 & 2010.658 \\
\hline & $\mathrm{U}$ & 155.840 & 173.342 & 195.243 & 223.439 & 261.088 & 313.888 & 393.253 & 525.876 & 792.077 \\
\hline & $\mathrm{Nb}$ & 292.658 & 326.012 & 367.818 & 421.734 & 493.876 & 595.293 & 748.176 & 1004.591 & 1521.859 \\
\hline & $\mathrm{Ta}$ & 21.227 & 23.653 & 26.694 & 30.618 & 35.871 & 43.259 & 54.402 & 73.103 & 110.868 \\
\hline & $\mathrm{La}$ & 135.780 & 154.129 & 177.593 & 208.542 & 251.036 & 312.606 & 408.872 & 577.961 & 941.348 \\
\hline & $\mathrm{Ce}$ & 162.608 & 194.696 & 238.118 & 299.170 & 389.359 & 531.735 & 778.654 & 1273.219 & 2546.227 \\
\hline & $\mathrm{Pb}$ & 276.573 & 308.217 & 347.898 & 399.099 & 467.644 & 564.066 & 709.534 & 953.753 & 1447.085 \\
\hline & $\operatorname{Pr}$ & 180.262 & 330.415 & 650.494 & 1402.041 & 3402.345 & 9708.616 & 35034.525 & 183244.531 & 1886900.216 \\
\hline & $\mathrm{Sr}$ & 4588.165 & 5239.950 & 6078.786 & 7193.319 & 8736.379 & 10994.058 & 14565.850 & 20933.913 & 34902.358 \\
\hline & $\mathrm{Nd}$ & 2.499 & 2.124 & 1.771 & 1.441 & 1.136 & 0.858 & 0.608 & 0.390 & 0.209 \\
\hline & $\mathrm{Zr}$ & 939.459 & 1068.207 & 1233.132 & 1451.111 & 1751.086 & 2186.895 & 2870.512 & 4076.218 & 6682.044 \\
\hline & $\mathrm{Hf}$ & 26.383 & 29.995 & 34.622 & 40.737 & 49.151 & 61.373 & 80.541 & 114.339 & 187.360 \\
\hline & $\mathrm{Sm}$ & 0.682 & 0.652 & 0.619 & 0.584 & 0.546 & 0.504 & 0.458 & 0.404 & 0.338 \\
\hline & $\mathrm{Eu}$ & 0.209 & 0.202 & 0.194 & 0.185 & 0.176 & 0.165 & 0.153 & 0.139 & 0.121 \\
\hline & $\mathrm{Tb}$ & 0.101 & 0.098 & 0.094 & 0.091 & 0.087 & 0.083 & 0.078 & 0.072 & 0.064 \\
\hline & Dy & 0.703 & 0.680 & 0.655 & 0.628 & 0.598 & 0.564 & 0.525 & 0.479 & 0.421 \\
\hline & $\mathrm{Y}$ & 3.885 & 3.752 & 3.608 & 3.451 & 3.279 & 3.086 & 2.866 & 2.604 & 2.276 \\
\hline & $\mathrm{Yb}$ & 0.452 & 0.431 & 0.409 & 0.385 & 0.360 & 0.332 & 0.300 & 0.264 & 0.220 \\
\hline & $\mathrm{Lu}$ & 0.076 & 0.072 & 0.068 & 0.064 & 0.059 & 0.054 & 0.049 & 0.042 & 0.035 \\
\hline & Element & & & & & Melt Fractio & & & & \\
\hline & & 0.3 & 0.35 & 0.4 & 0.45 & 0.5 & 0.55 & 0.6 & 0.65 & 0.7 \\
\hline & $\mathrm{Rb}$ & 1875.668 & 2043.178 & 2240.947 & 2477.710 & 2765.855 & 3123.537 & 3578.419 & 4174.750 & 4987.768 \\
\hline & $\mathrm{Ba}$ & 11820.646 & 14769.889 & 18787.115 & 24402.788 & 32497.619 & 44604.918 & 63552.046 & 94936.512 & 150887.121 \\
\hline & Th & 34.436 & 37.119 & 40.251 & 43.957 & 48.410 & 53.858 & 60.678 & 69.459 & 81.189 \\
\hline & $\mathrm{U}$ & 21.725 & 23.418 & 25.394 & 27.732 & 30.541 & 33.978 & 38.281 & 43.821 & 51.221 \\
\hline & $\mathrm{Nb}$ & 48.578 & 53.406 & 59.162 & 66.124 & 74.695 & 85.467 & 99.359 & 117.859 & 143.538 \\
\hline & $\mathrm{Ta}$ & 1.849 & 2.036 & 2.259 & 2.529 & 2.863 & 3.282 & 3.825 & 4.549 & 5.557 \\
\hline & $\mathrm{La}$ & 57.000 & 62.025 & 67.951 & 75.036 & 83.649 & 94.324 & 107.879 & 125.617 & 149.750 \\
\hline & $\mathrm{Ce}$ & 68.235 & 75.633 & 84.526 & 95.385 & 108.886 & 126.045 & 148.448 & 178.699 & 221.365 \\
\hline & $\mathrm{Pb}$ & 157.642 & 170.277 & 185.063 & 202.597 & 223.715 & 249.631 & 282.173 & 324.225 & 380.622 \\
\hline & $\operatorname{Pr}$ & 7.395 & 8.700 & 10.369 & 12.548 & 15.465 & 19.483 & 25.225 & 33.805 & 47.399 \\
\hline & $\mathrm{Sr}$ & 1772.476 & 1941.148 & 2141.404 & 2382.599 & 2678.083 & 3047.552 & 3521.242 & 4147.917 & 5011.277 \\
\hline & $\mathrm{Nd}$ & 0.063 & 0.023 & 0.008 & 0.002 & 0.001 & 0.000 & 0.000 & 0.000 & 0.000 \\
\hline & $\mathrm{Zr}$ & 633.178 & 692.103 & 761.921 & 845.830 & 948.383 & 1076.280 & 1239.781 & 1455.381 & 1751.300 \\
\hline & $\mathrm{Hf}$ & 18.776 & 20.516 & 22.578 & 25.056 & 28.082 & 31.855 & 36.676 & 43.029 & 51.744 \\
\hline & $\mathrm{Sm}$ & 1.042 & 0.976 & 0.909 & 0.842 & 0.774 & 0.705 & 0.636 & 0.565 & 0.493 \\
\hline & $\mathrm{Eu}$ & 0.319 & 0.304 & 0.289 & 0.274 & 0.258 & 0.241 & 0.224 & 0.206 & 0.186 \\
\hline & $\mathrm{Tb}$ & 0.210 & 0.202 & 0.194 & 0.185 & 0.177 & 0.167 & 0.157 & 0.147 & 0.136 \\
\hline
\end{tabular}




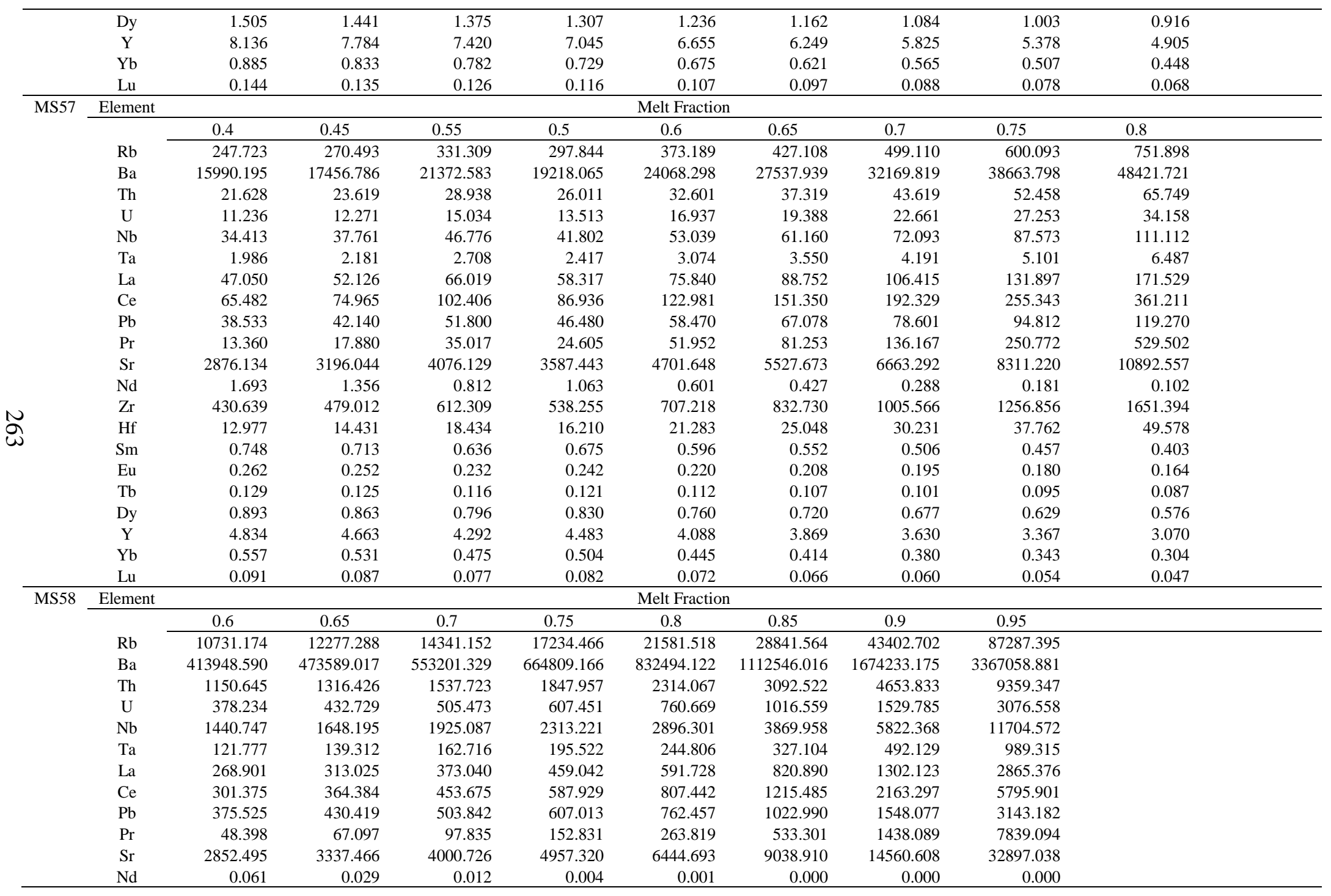




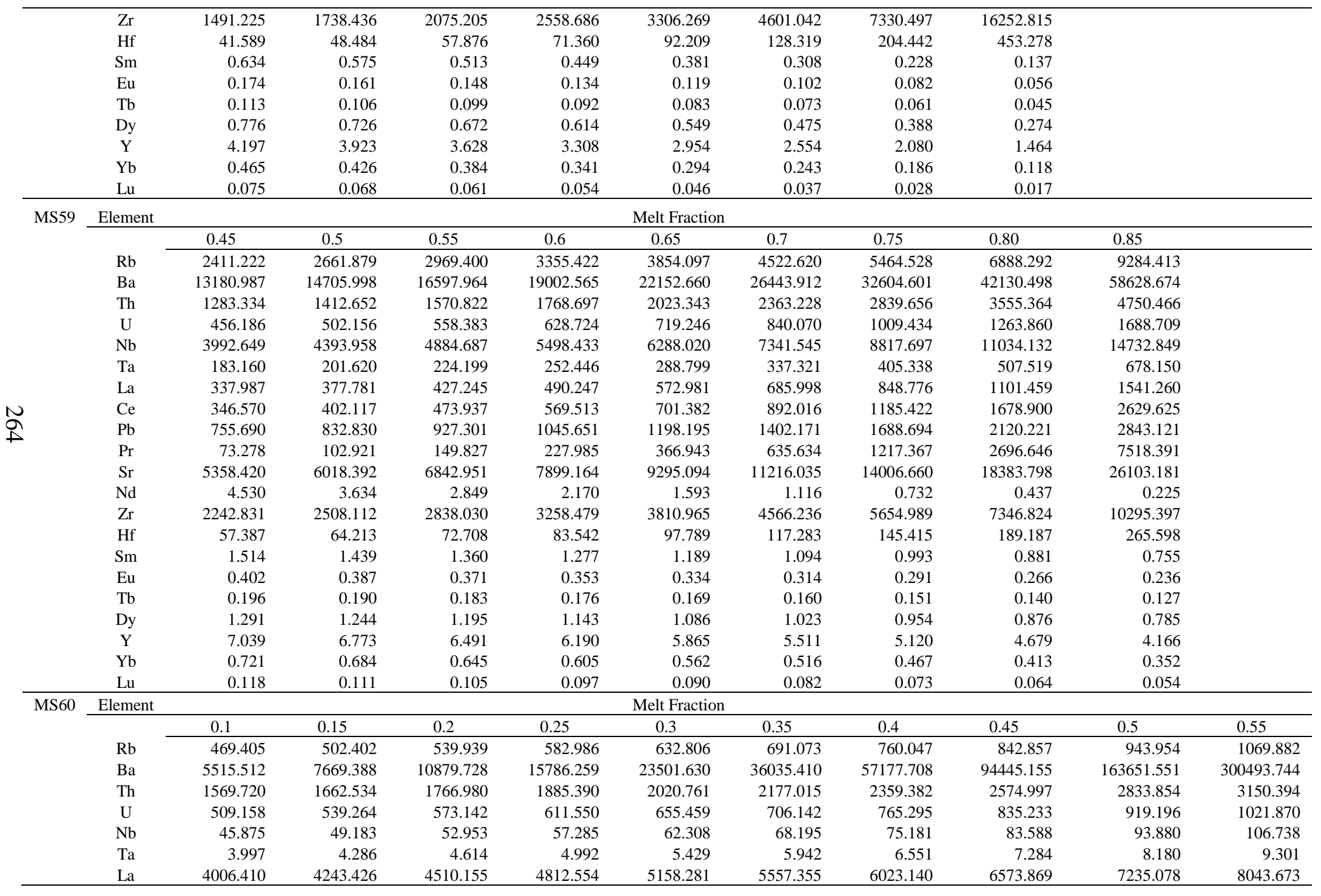




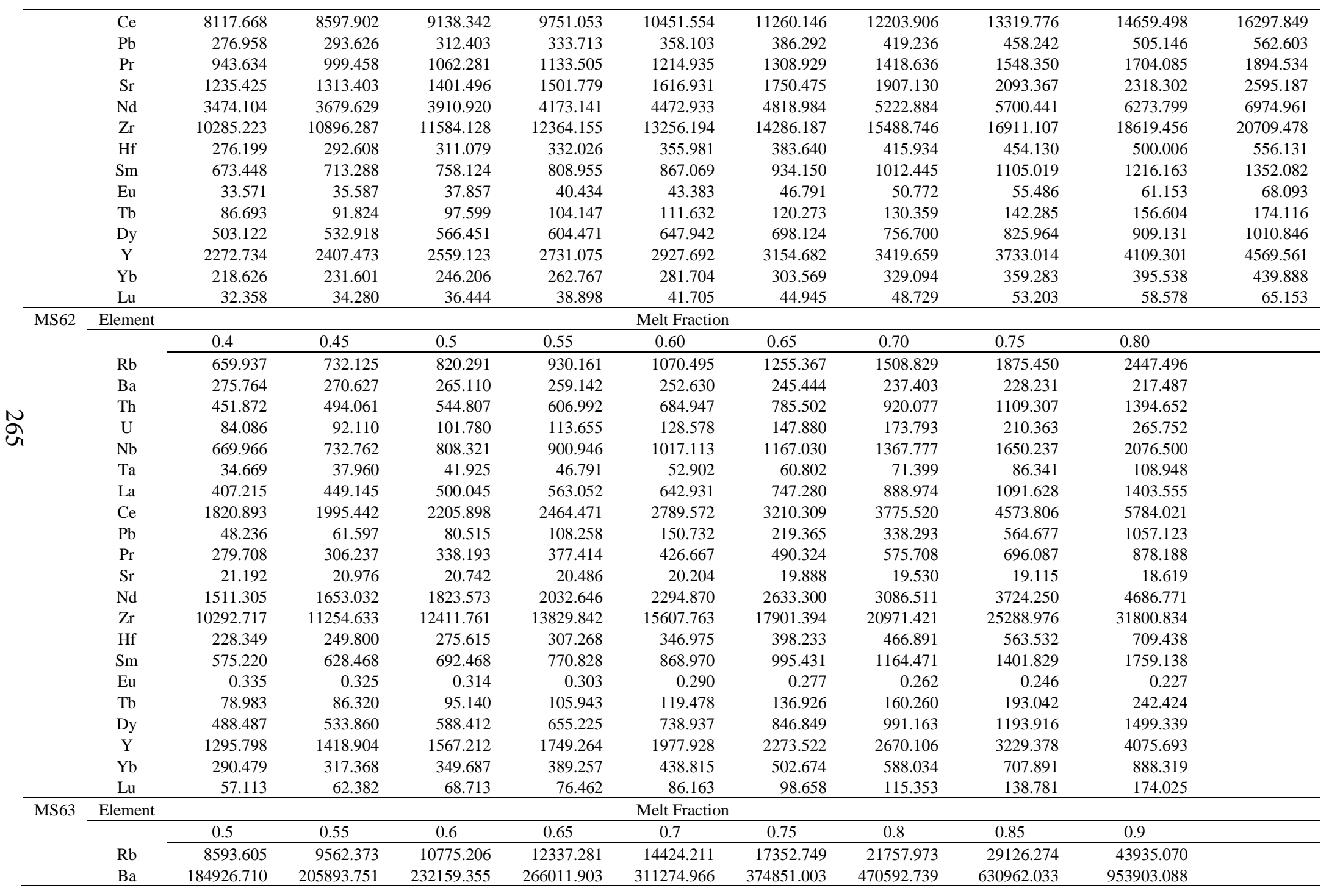




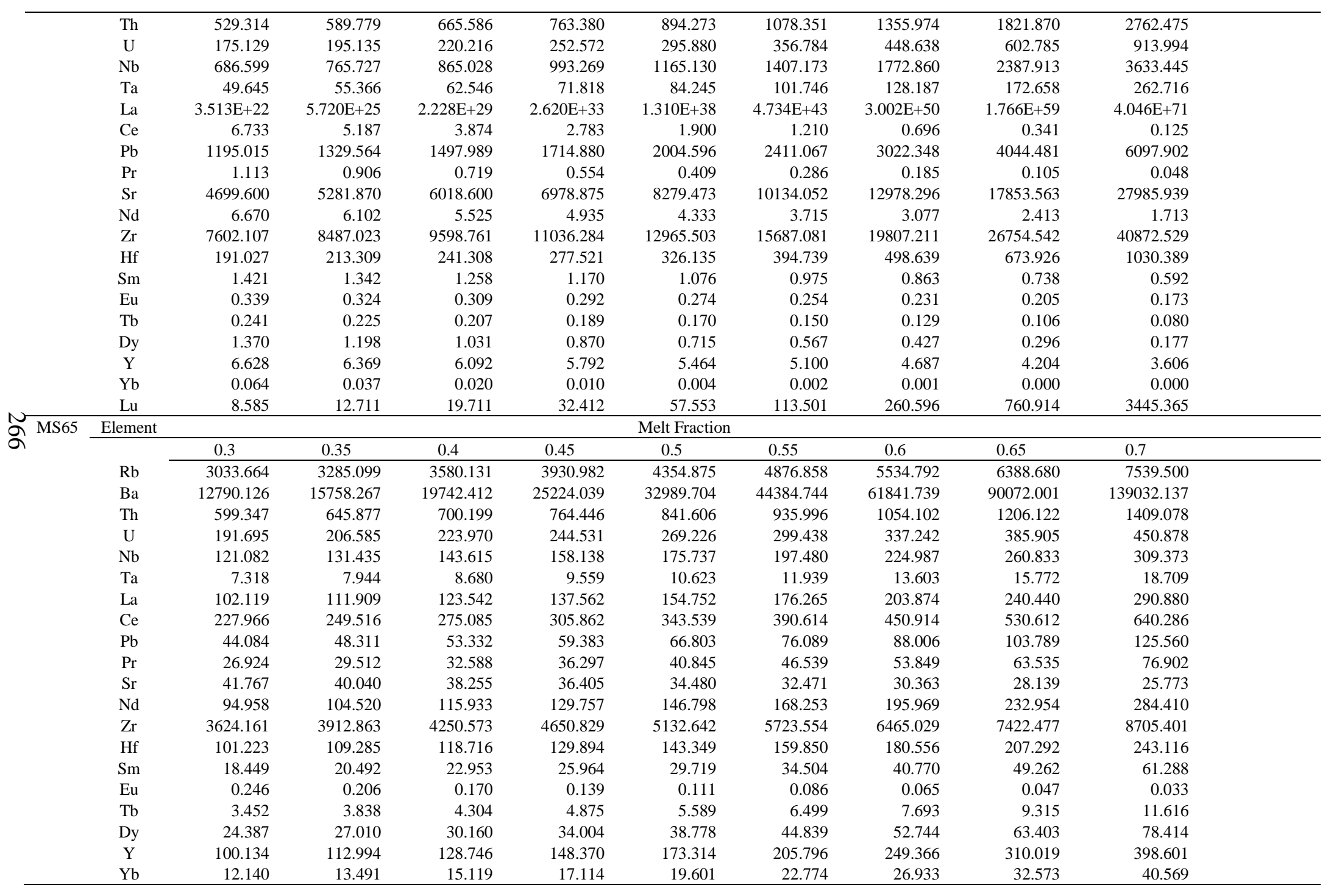




\begin{tabular}{lllllllll}
\hline $\mathrm{Lu}$ & 2.284 & 2.520 & 2.802 & 3.144 & 3.568 & 4.103 & 4.797 & 5.726 \\
\hline
\end{tabular}




\section{APPENDIX E: FRACTIONAL CRYSTALIZATION OF BASALT}

Table E1. Unnormalized trace element concentrations

\begin{tabular}{crrrrrrrrr}
\hline \hline & \multicolumn{8}{c}{ melt fraction remaining } \\
\hline $\mathrm{ppm}$ & \multicolumn{1}{c}{0.05} & \multicolumn{1}{c}{0.045} & \multicolumn{1}{c}{0.04} & \multicolumn{1}{c}{0.035} & \multicolumn{1}{c}{0.03} & \multicolumn{1}{c}{0.025} & \multicolumn{1}{c}{0.02} & 0.015 & \multicolumn{1}{c}{0.01} \\
\hline $\mathrm{Rb}$ & 158.74 & 176.32 & 198.30 & 226.55 & 264.20 & 316.89 & 395.88 & 527.43 & 790.30 \\
$\mathrm{Ba}$ & 2515.17 & 2786.23 & 3123.97 & 3556.66 & 4131.19 & 4931.66 & 6125.39 & 8100.31 & 12010.48 \\
$\mathrm{Th}$ & 10.34 & 11.49 & 12.92 & 14.76 & 17.22 & 20.65 & 25.81 & 34.39 & 51.54 \\
$\mathrm{U}$ & 3.18 & 3.53 & 3.97 & 4.54 & 5.29 & 6.35 & 7.93 & 10.57 & 15.84 \\
$\mathrm{Nb}$ & 160.48 & 178.24 & 200.43 & 228.94 & 266.93 & 320.09 & 399.76 & 532.41 & 797.33 \\
$\mathrm{Ta}$ & 10.44 & 11.60 & 13.04 & 14.89 & 17.36 & 20.82 & 25.99 & 34.61 & 51.81 \\
$\mathrm{La}$ & 117.62 & 130.33 & 146.18 & 166.50 & 193.48 & 231.09 & 287.22 & 380.15 & 564.33 \\
$\mathrm{Ce}$ & 287.33 & 318.20 & 356.65 & 405.88 & 471.23 & 562.24 & 697.87 & 922.09 & 1365.56 \\
$\mathrm{~Pb}$ & 12.12 & 13.44 & 15.07 & 17.17 & 19.96 & 23.85 & 29.66 & 39.28 & 58.37 \\
$\mathrm{Pr}$ & 42.11 & 46.54 & 52.05 & 59.08 & 68.40 & 81.32 & 100.51 & 132.08 & 194.10 \\
$\mathrm{Sr}$ & 2149.55 & 2304.51 & 2491.01 & 2720.77 & 3012.47 & 3398.11 & 3937.92 & 4762.26 & 6225.25 \\
$\mathrm{Nd}$ & 184.93 & 203.55 & 226.59 & 255.89 & 294.45 & 347.62 & 425.93 & 553.47 & 800.61 \\
$\mathrm{Zr}$ & 1601.29 & 1776.95 & 1996.25 & 2277.76 & 2652.47 & 3176.00 & 3959.36 & 5260.93 & 7853.02 \\
$\mathrm{Hf}$ & 41.36 & 45.91 & 51.57 & 58.85 & 68.54 & 82.08 & 102.34 & 136.00 & 203.07 \\
$\mathrm{Sm}$ & 44.55 & 48.64 & 53.65 & 59.96 & 68.16 & 79.34 & 95.53 & 121.38 & 170.11 \\
$\mathrm{Eu}$ & 10.14 & 10.88 & 11.77 & 12.87 & 14.27 & 16.11 & 18.71 & 22.67 & 29.73 \\
$\mathrm{~Tb}$ & 7.09 & 7.69 & 8.42 & 9.33 & 10.52 & 12.11 & 14.38 & 17.96 & 24.57 \\
$\mathrm{Dy}$ & 47.01 & 51.11 & 56.11 & 62.39 & 70.51 & 81.49 & 97.28 & 122.23 & 168.63 \\
$\mathrm{Y}$ & 243.96 & 265.48 & 291.80 & 324.80 & 367.56 & 425.46 & 508.88 & 641.01 & 887.48 \\
$\mathrm{Yb}$ & 26.14 & 28.57 & 31.55 & 35.30 & 40.19 & 46.86 & 56.54 & 72.03 & 101.33 \\
$\mathrm{Lu}$ & 4.21 & 4.60 & 5.09 & 5.70 & 6.50 & 7.59 & 9.17 & 11.71 & 16.53 \\
\hline & & & & & & & & &
\end{tabular}


Table E2. Primitive mantle normalized trace element concentrations

\begin{tabular}{cccccccccc}
\hline \hline & \multicolumn{7}{c}{ melt fraction remaining } \\
\cline { 2 - 11 } & 0.05 & 0.045 & 0.04 & 0.035 & 0.03 & 0.025 & 0.02 & 0.015 & 0.01 \\
\cline { 2 - 11 } $\mathrm{Rb}$ & 249.98 & 277.67 & 312.29 & 356.77 & 416.07 & 499.04 & 623.43 & 830.60 & 1244.57 \\
$\mathrm{Ba}$ & 359.88 & 398.66 & 446.98 & 508.89 & 591.10 & 705.63 & 876.43 & 1159.01 & 1718.48 \\
$\mathrm{Th}$ & 121.65 & 135.14 & 152.00 & 173.67 & 202.56 & 242.99 & 303.60 & 404.58 & 606.40 \\
$\mathrm{U}$ & 151.39 & 168.18 & 189.15 & 216.11 & 252.04 & 302.33 & 377.73 & 503.33 & 754.33 \\
$\mathrm{Nb}$ & 225.08 & 249.99 & 281.11 & 321.09 & 374.38 & 448.93 & 560.67 & 746.71 & 1118.27 \\
$\mathrm{Ta}$ & 254.74 & 282.89 & 318.07 & 363.27 & 423.49 & 507.73 & 633.97 & 844.09 & 1263.60 \\
$\mathrm{La}$ & 171.20 & 189.71 & 212.79 & 242.35 & 281.63 & 336.38 & 418.08 & 553.35 & 821.44 \\
$\mathrm{Ce}$ & 161.88 & 179.27 & 200.93 & 228.67 & 265.48 & 316.75 & 393.17 & 519.49 & 769.33 \\
$\mathrm{~Pb}$ & 170.73 & 189.23 & 212.30 & 241.87 & 281.17 & 335.97 & 417.78 & 553.30 & 822.13 \\
$\mathrm{Pr}$ & 152.58 & 168.63 & 188.58 & 214.07 & 247.81 & 294.65 & 364.17 & 478.55 & 703.26 \\
$\mathrm{Sr}$ & 101.87 & 109.22 & 118.06 & 128.95 & 142.77 & 161.05 & 186.63 & 225.70 & 295.04 \\
$\mathrm{Nd}$ & 136.58 & 150.33 & 167.35 & 188.99 & 217.46 & 256.73 & 314.57 & 408.77 & 591.30 \\
$\mathrm{Zr}$ & 142.97 & 158.66 & 178.24 & 203.37 & 236.83 & 283.57 & 353.51 & 469.73 & 701.16 \\
$\mathrm{Hf}$ & 133.87 & 148.56 & 166.91 & 190.46 & 221.82 & 265.63 & 331.19 & 440.14 & 657.17 \\
$\mathrm{Sm}$ & 100.35 & 109.54 & 120.83 & 135.04 & 153.52 & 178.69 & 215.16 & 273.38 & 383.14 \\
$\mathrm{Eu}$ & 60.36 & 64.76 & 70.07 & 76.61 & 84.92 & 95.92 & 111.34 & 134.94 & 176.94 \\
$\mathrm{~Tb}$ & 65.61 & 71.18 & 77.96 & 86.43 & 97.36 & 112.09 & 133.19 & 166.34 & 227.54 \\
$\mathrm{Dy}$ & 63.78 & 69.34 & 76.14 & 84.65 & 95.67 & 110.56 & 131.99 & 165.85 & 228.81 \\
$\mathrm{Y}$ & 53.62 & 58.35 & 64.13 & 71.38 & 80.78 & 93.51 & 111.84 & 140.88 & 195.05 \\
$\mathrm{Yb}$ & 53.03 & 57.95 & 63.99 & 71.60 & 81.52 & 95.04 & 114.68 & 146.11 & 205.54 \\
$\mathrm{Lu}$ & 56.88 & 62.20 & 68.75 & 77.01 & 87.79 & 102.51 & 123.91 & 158.23 & 223.32 \\
\hline & & & & & & & & &
\end{tabular}




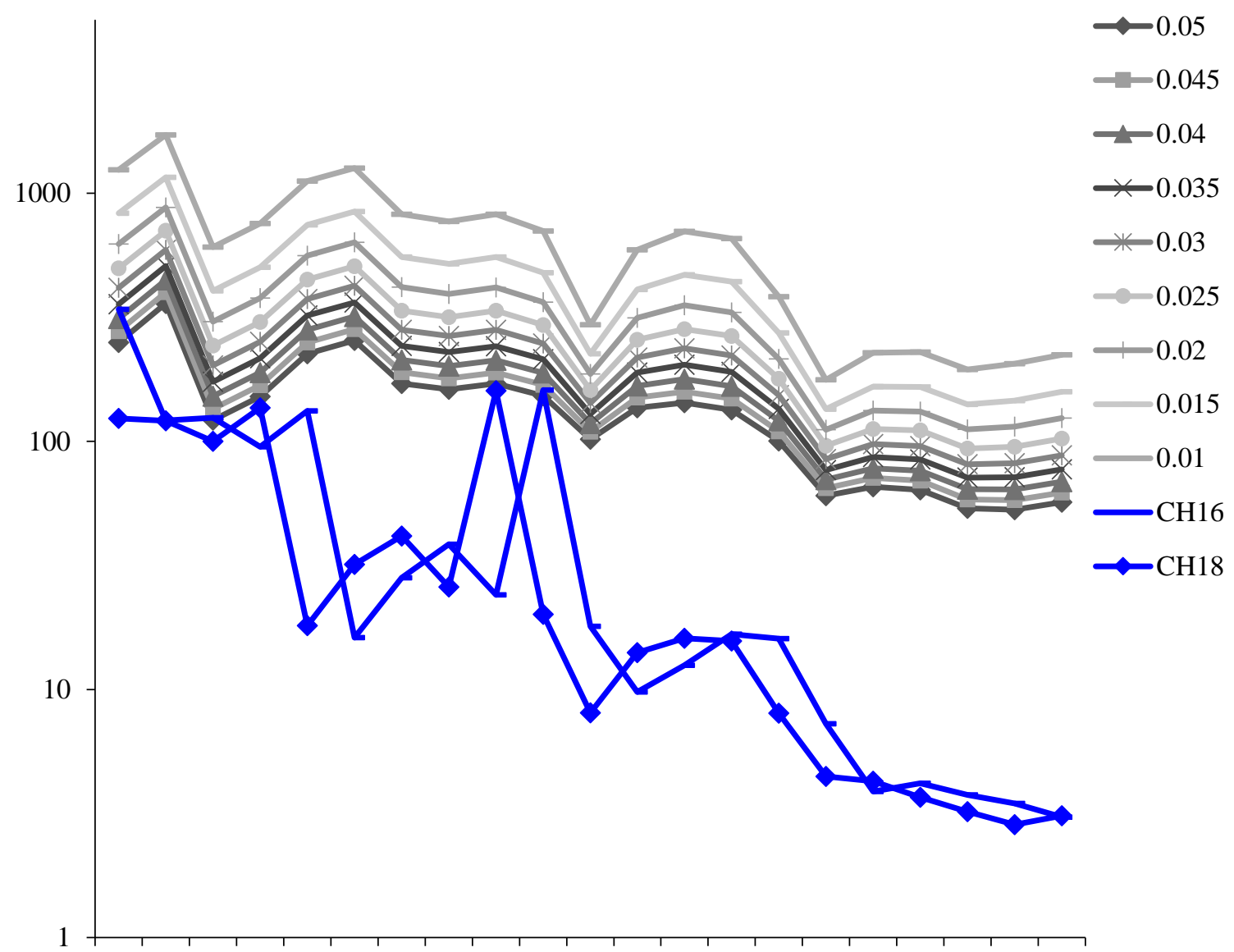

$\mathrm{Rb} \mathrm{Ba}$ Th $\mathrm{U}$ Nb Ta $\mathrm{La} \mathrm{Ce} \mathrm{Pb}$ Pr $\mathrm{Sr} \mathrm{Nd} \mathrm{Zr}$ Hf Sm Eu Tb Dy Y Yb Lu

Fig. E1 Primitive mantle normalized (Sun and McDonough, 1989) spider diagram of the results of fractional crystallization modeling of basalt sample G314-09-2. The trace element concentrations at differing melt fractions (5-1\%) are represented by the greyscale lines with the remaining melt fractions listed in the key. The normalized trace element concentrations of low-silica rhyolite samples are also plotted (color lines). With few exceptions, the trace element concentrations are higher for the model as compared to the rhyolites. The modeled concentrations do not share the lead peak shown by the low-silica rhyolites. The model shows troughs at thorium and europium, which the rhyolites do not share. $\mathrm{CH} 18$ has a trough at niobium, while $\mathrm{CH} 16$ has a trough at tantalum; the model shows troughs at neither. The fit between the low-silica rhyolites and the model is unsatisfactory. 


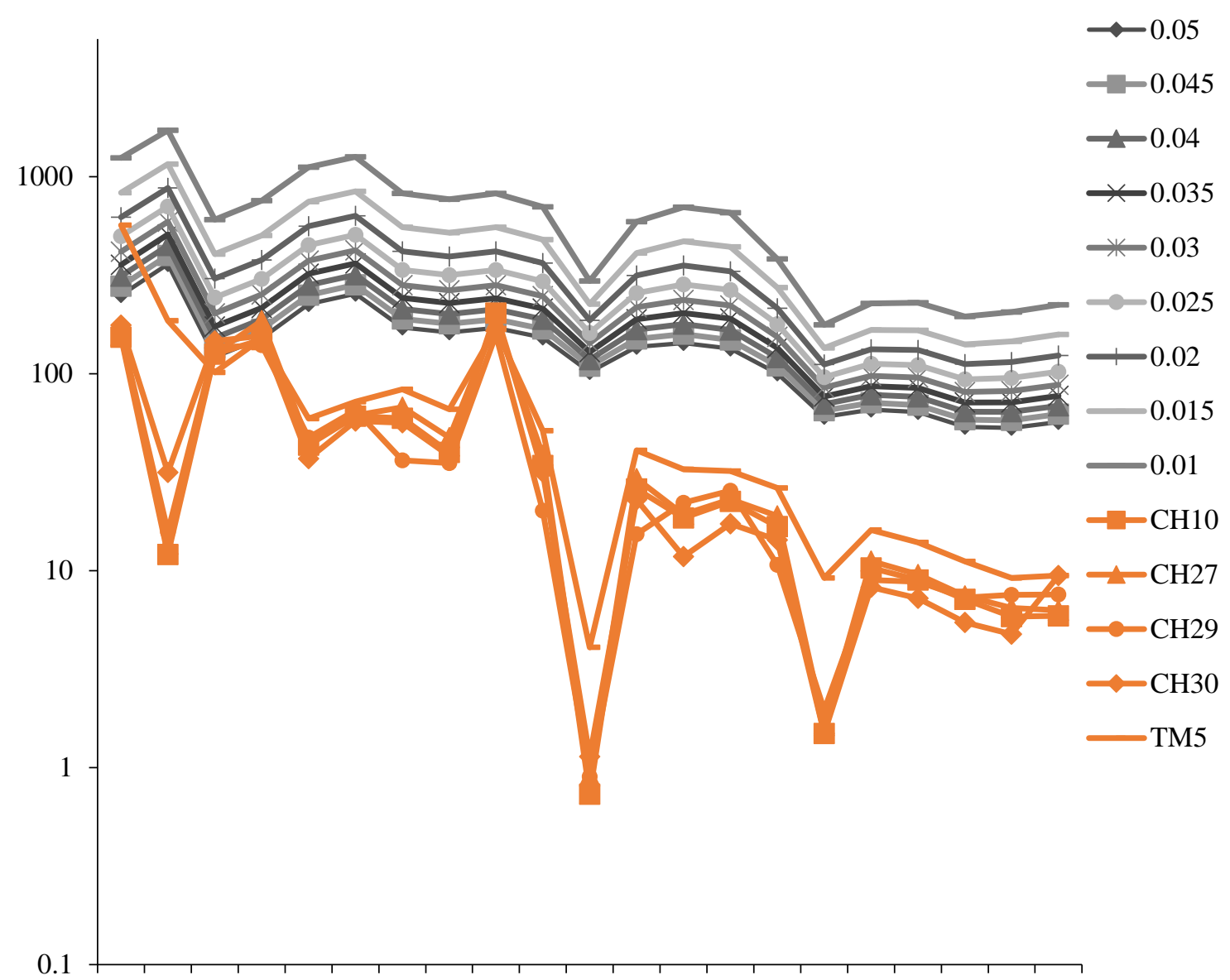

$\mathrm{Rb} \mathrm{Ba}$ Th $\mathrm{U}$ Nb Ta $\mathrm{La} \mathrm{Ce} \mathrm{Pb}$ Pr $\mathrm{Sr} \mathrm{Nd} \mathrm{Zr} \mathrm{Hf} \mathrm{Sm} \mathrm{Eu}$ Tb Dy Y Yb Lu

Fig. E2 Primitive mantle normalized (Sun and McDonough, 1989) spider diagram of the results of fractional crystallization modeling of basalt sample G314-09-2. The trace element concentrations at differing melt fractions (5-1\%) are represented by the greyscale lines with the remaining melt fractions listed in the key. The normalized trace element concentrations of A-like rhyolite samples are also plotted (color lines). With few exceptions, the trace element concentrations are higher for the model as compared to the rhyolites. The modeled concentrations do not share the lead peak shown by the A-like rhyolites. The model shows a trough at thorium, which the rhyolites do not share. The A-like rhyolites show a trough at barium not shared by the model. The fit between the A-like rhyolites and the model is unsatisfactory. 


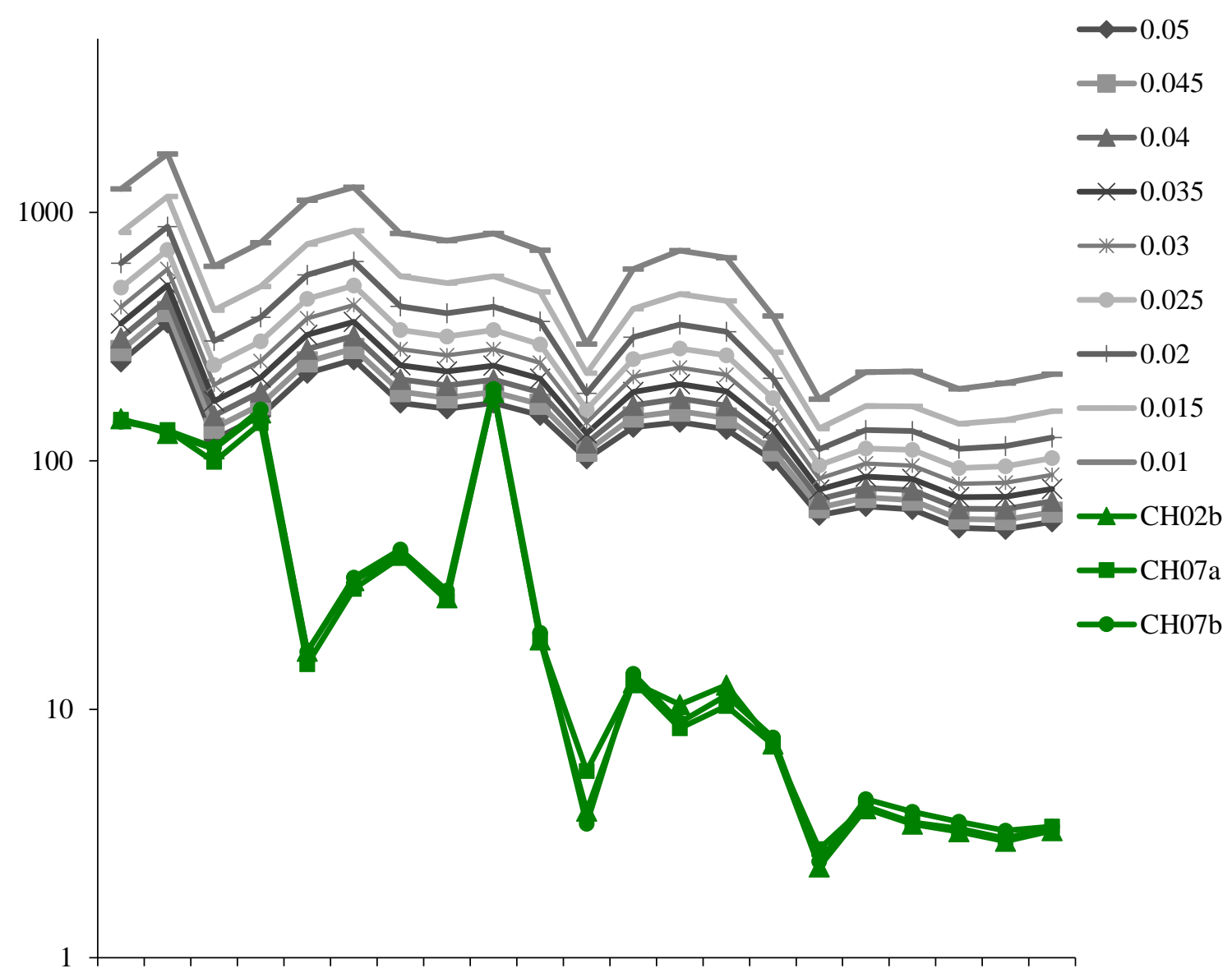

$\mathrm{Rb} \mathrm{Ba}$ Th $\mathrm{U}$ Nb Ta La Ce Pb Pr $\mathrm{Sr} \mathrm{Nd} \mathrm{Zr} \mathrm{Hf} \mathrm{Sm} \mathrm{Eu} \mathrm{Tb} \mathrm{Dy} \mathrm{Y} \mathrm{Yb} \mathrm{Lu}$

Fig E3. Primitive mantle normalized (Sun and McDonough, 1989) spider diagram of the results of fractional crystallization modeling of basalt sample G314-09-2. The trace element concentrations at differing melt fractions $(5-1 \%)$ are represented by the greyscale lines with the remaining melt fractions listed in the key. The normalized trace element concentrations of Dale Tuff-like rhyolite samples are also plotted (color lines). With few exceptions, the trace element concentrations are higher for the model as compared to the rhyolites. The modeled concentrations do not share the lead peak shown by the Dale Tufflike rhyolites. The fit between the Dale Tuff-like rhyolites and the model is unsatisfactory. 


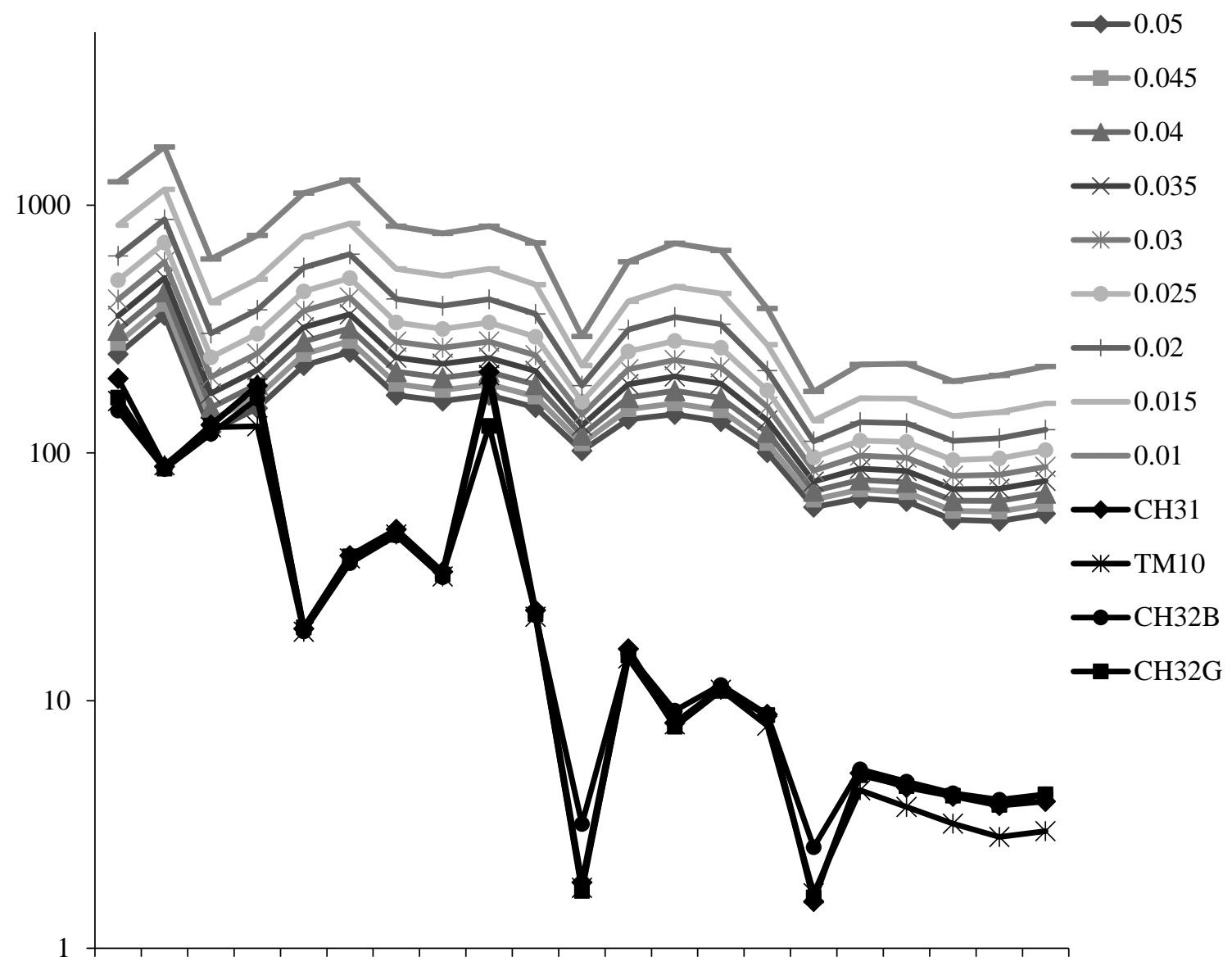

$\mathrm{Rb} \mathrm{Ba}$ Th $\mathrm{U}$ Nb Ta $\mathrm{La} \mathrm{Ce} \mathrm{Pb}$ Pr $\mathrm{Sr} \mathrm{Nd} \mathrm{Zr} \mathrm{Hf} \mathrm{Sm} \mathrm{Eu}$ Tb Dy Y Yb Lu

Fig. E4 Primitive mantle normalized (Sun and McDonough, 1989) spider diagram of the results of fractional crystallization modeling of basalt sample G314-09-2. The trace element concentrations at differing melt fractions (5-1\%) are represented by the greyscale lines with the remaining melt fractions listed in the key. The normalized trace element concentrations of Dale Tuff rhyolite samples are also plotted (color lines). With few exceptions, the trace element concentrations are higher for the model as compared to the rhyolites. The modeled concentrations do not share the lead peak shown by the Dale Tuff rhyolites and the inverse concentration pattern is shown at barium. The fit between the Dale Tuff rhyolites and the model is unsatisfactory. 


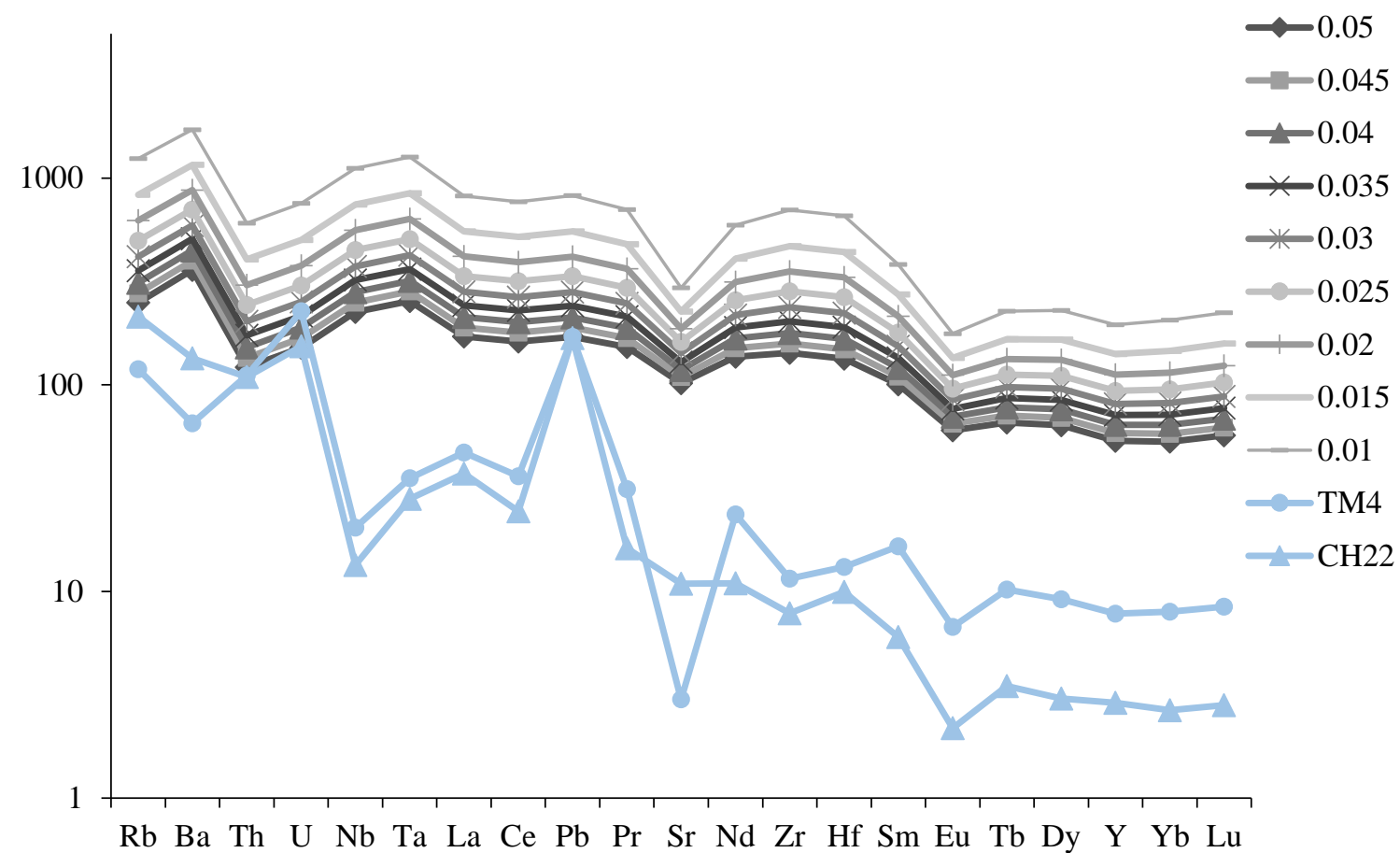

Fig. E5 Primitive mantle normalized (Sun and McDonough, 1989) spider diagram of the results of fractional crystallization modeling of basalt sample G314-09-2. The trace element concentrations at differing melt fractions (5-1\%) are represented by the greyscale lines with the remaining melt fractions listed in the key. The normalized trace element concentrations of high-silica rhyolite samples are also plotted (color lines). With few exceptions, the trace element concentrations are higher for the model as compared to the rhyolites. The modeled concentrations do not share the lead peak shown by the high-silica rhyolites and the inverse concentration pattern is shown at barium. The fit between the high-silica rhyolites and the model is unsatisfactory. 
APPENDIX F: ZIRCONS AND ISOTOPIC DATING

A

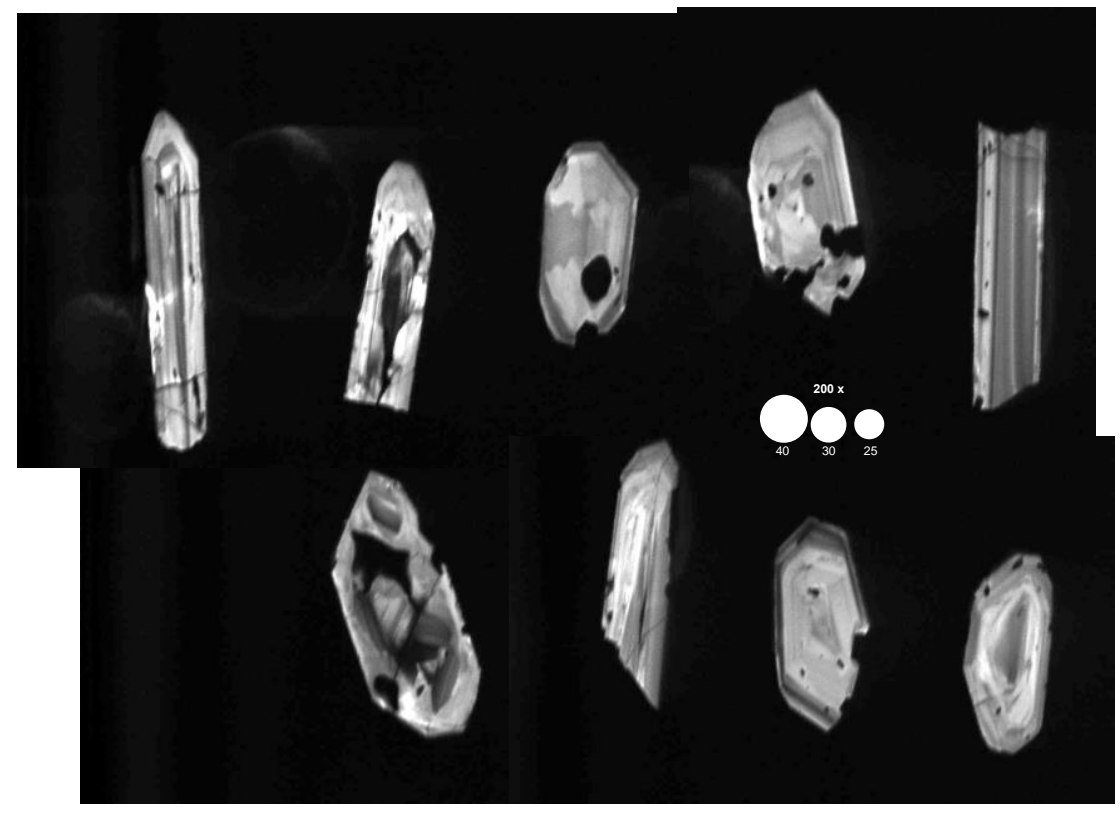

B

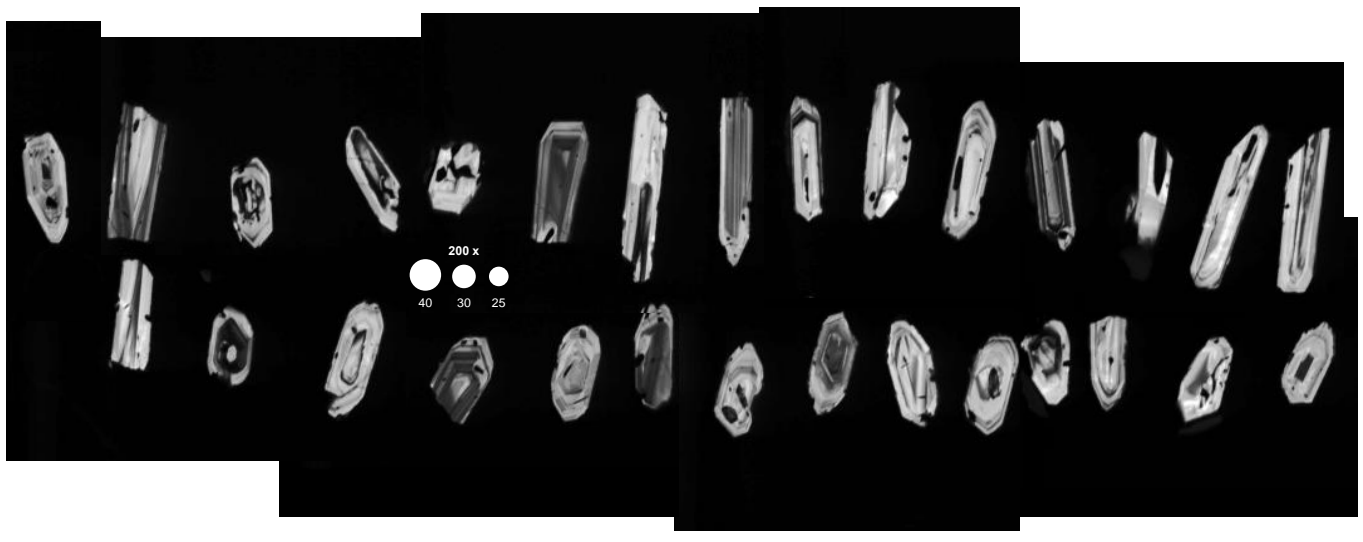



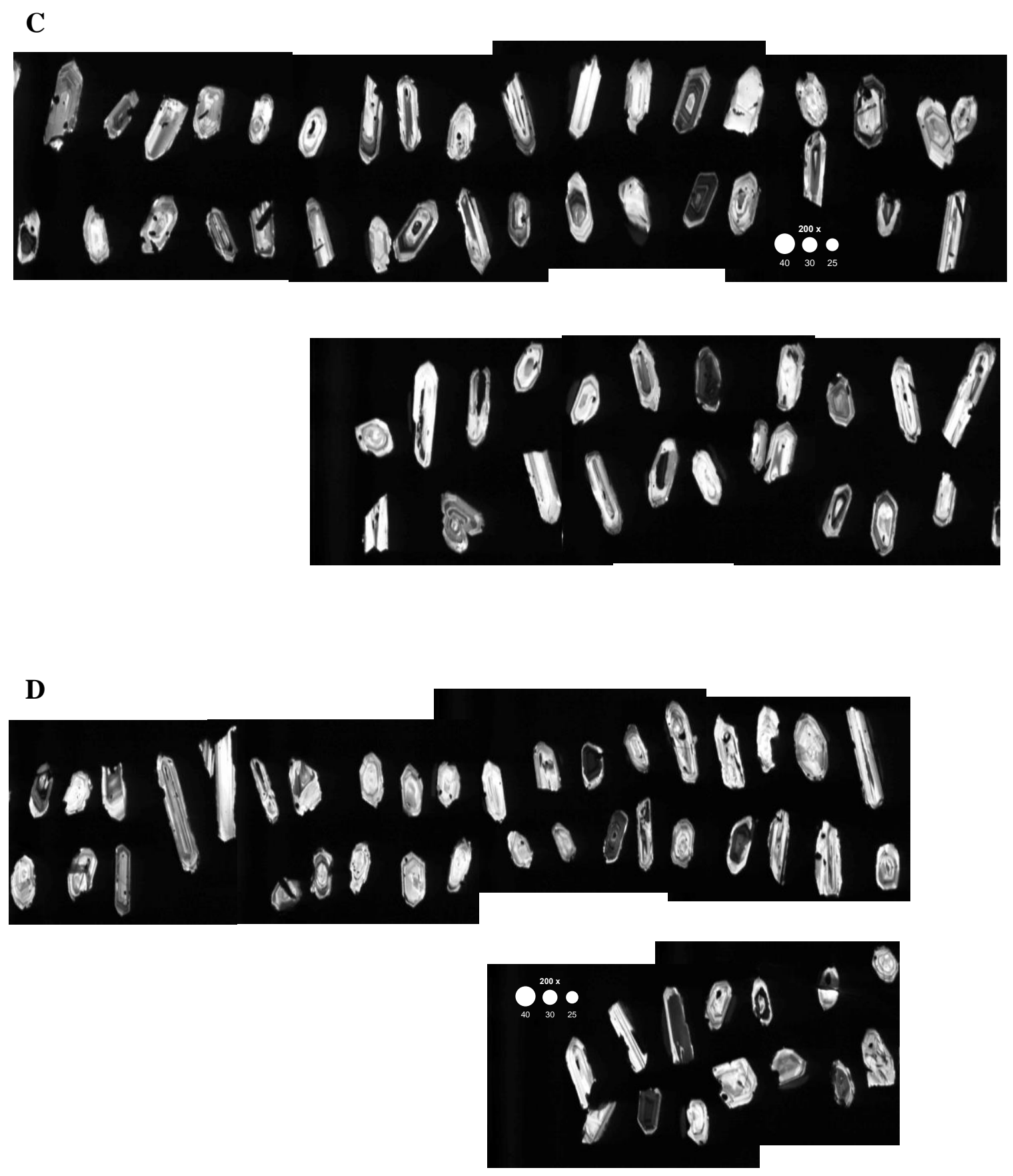

Fig. F1 A-D. Cathodoluminescence (CL) montage images taken via scanning electron microscope, which reveal oscillatory/sector zoning typical of magmatic zircon. The bright ("active") areas are relatively high in $\mathrm{REE}^{3+}$ and/or low in U, while dark ("inactive") areas indicate the reverse. The white circles labeled 40, 35 , and 25 indicate diameter in millimeters. All are shown at a magnification of 200x. All zircons were picked by hand from sample CH07a (Dale Tuff-like rhyolite). 

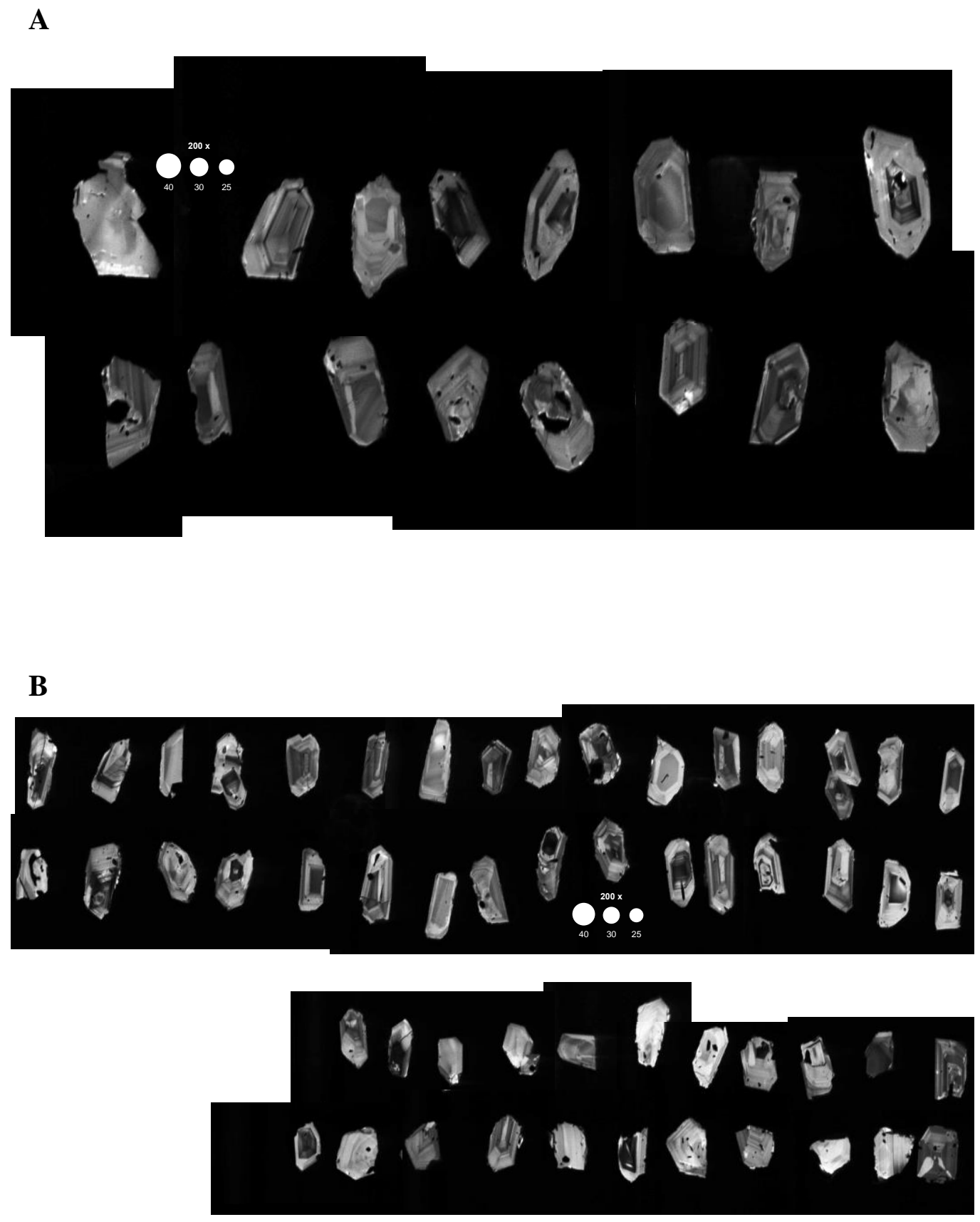
C
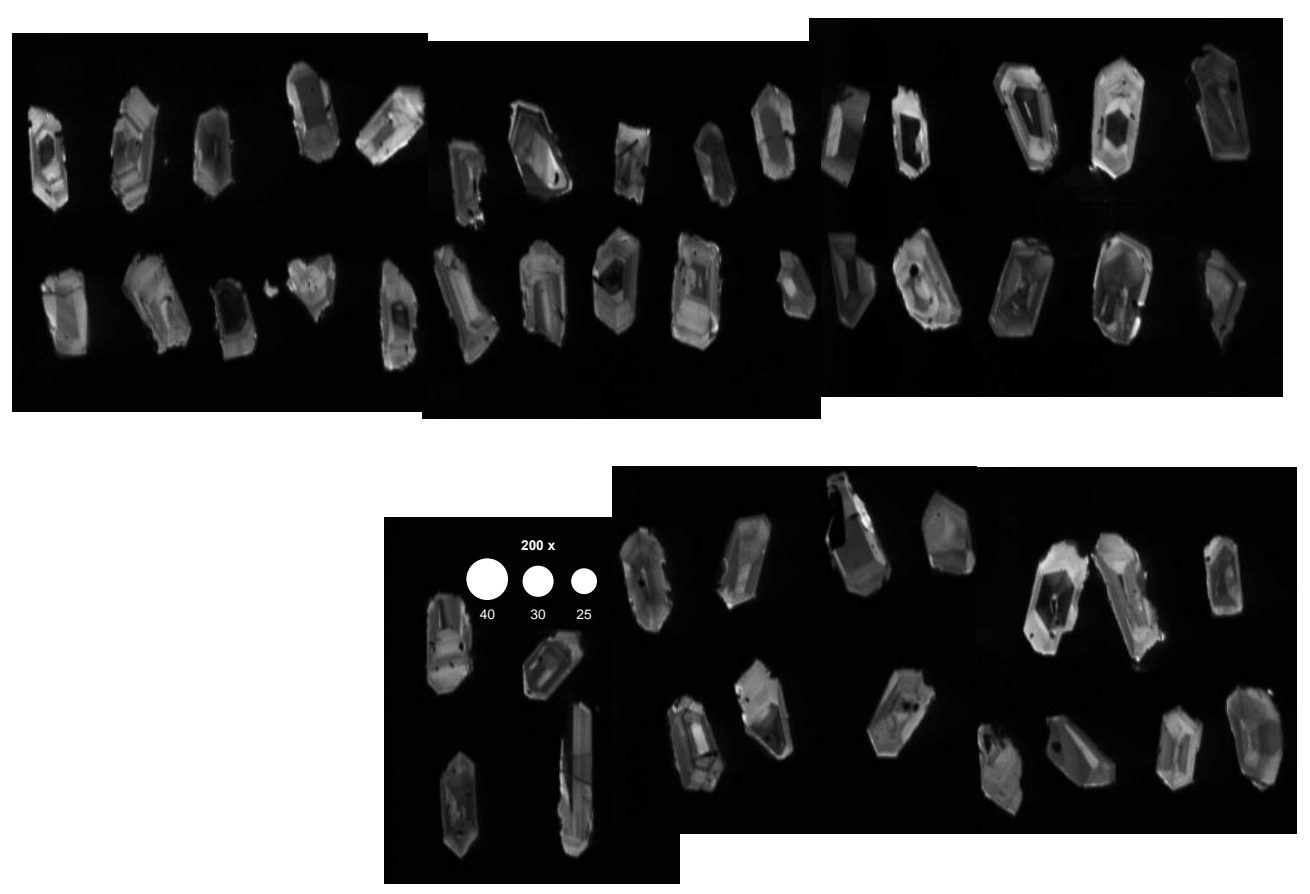

\section{D}
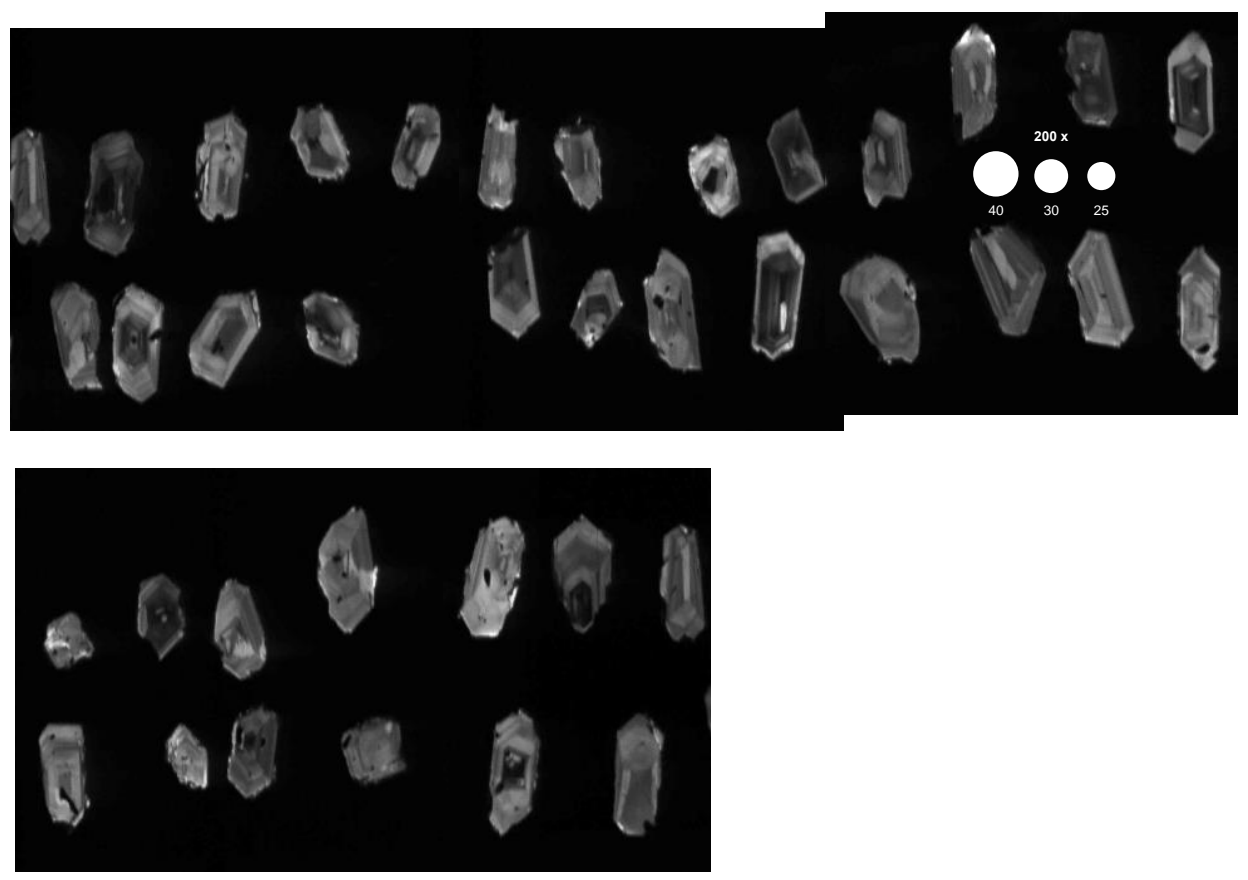

Fig. F2 A-D. Cathodoluminescence (CL) montage images taken via scanning electron microscope, which reveal oscillatory/sector zoning typical of magmatic zircon. The bright ("active") areas are relatively high in $\mathrm{REE}^{3+}$ and/or low in $\mathrm{U}$. The white circles labeled 40, 35, and 25 indicate diameter in millimeters. All are shown at a magnification of 200x. All zircons were picked by hand from sample CH08a (A-like rhyolite). 
A

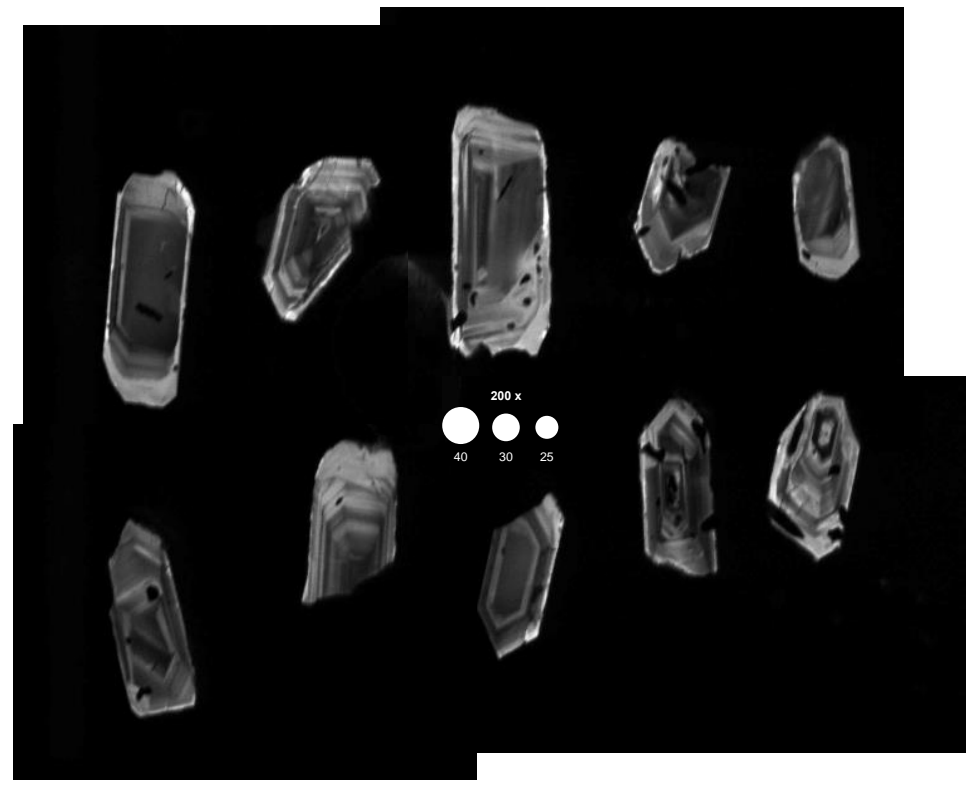

B

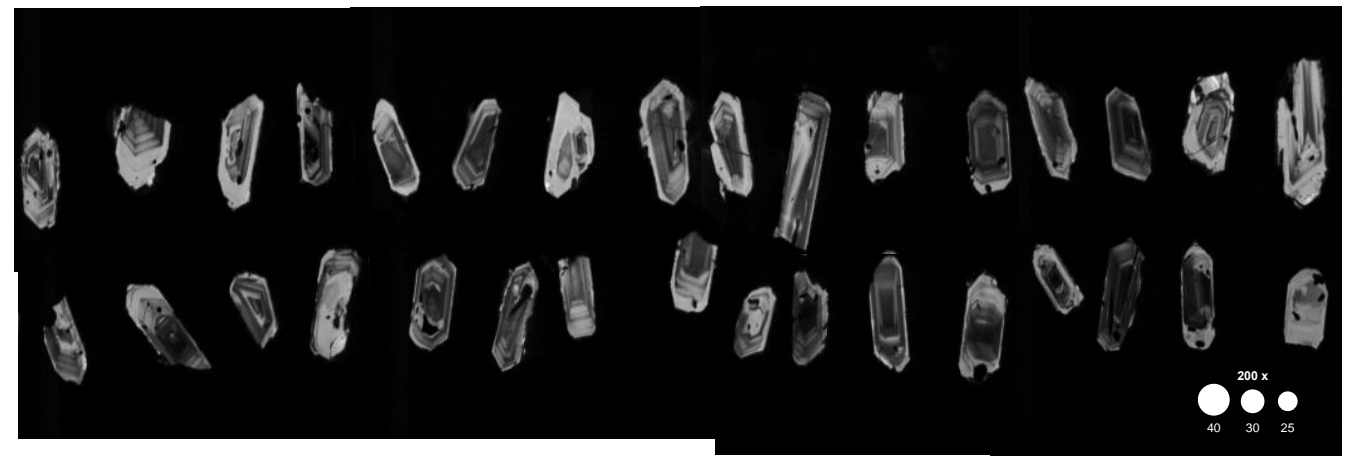



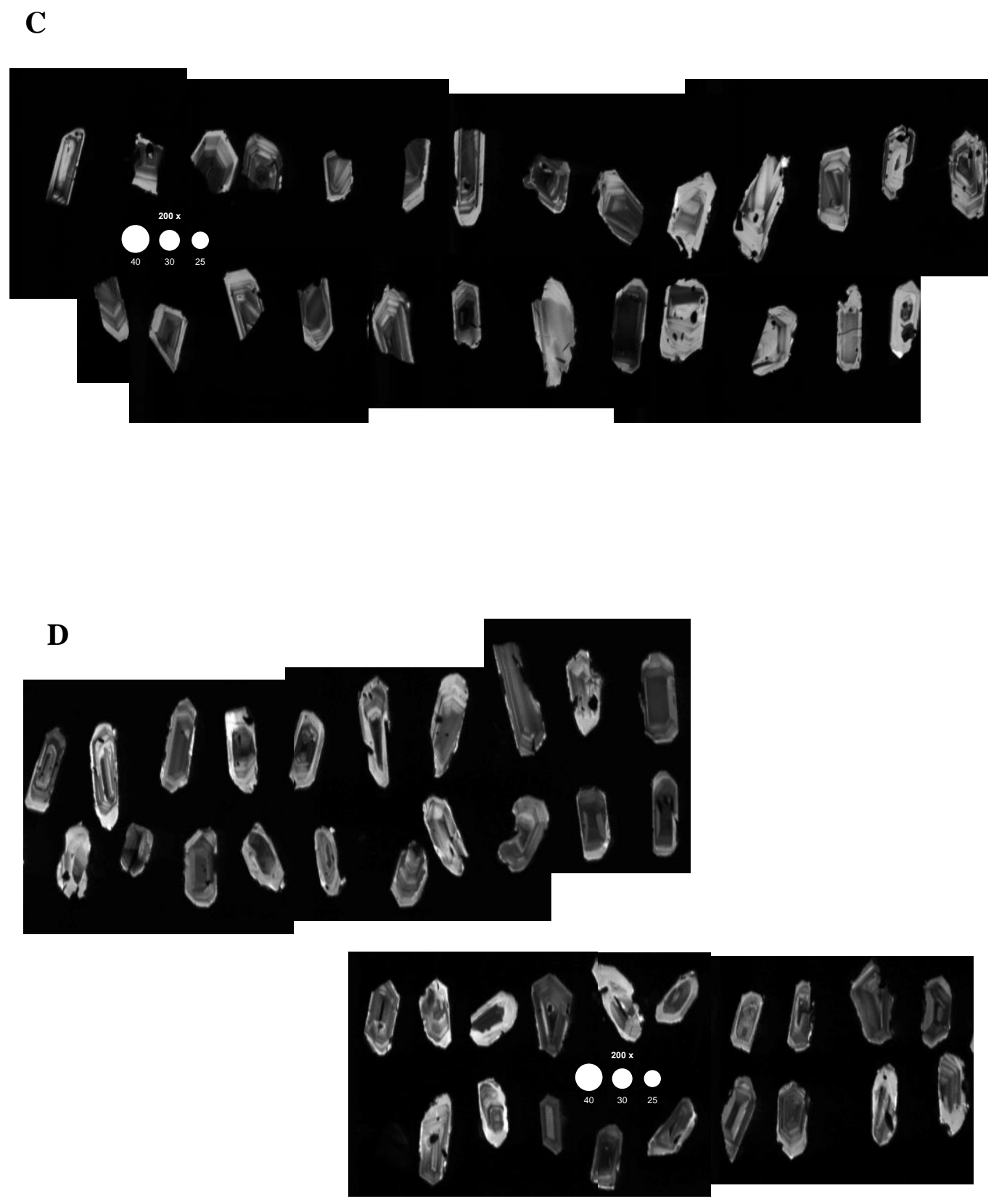


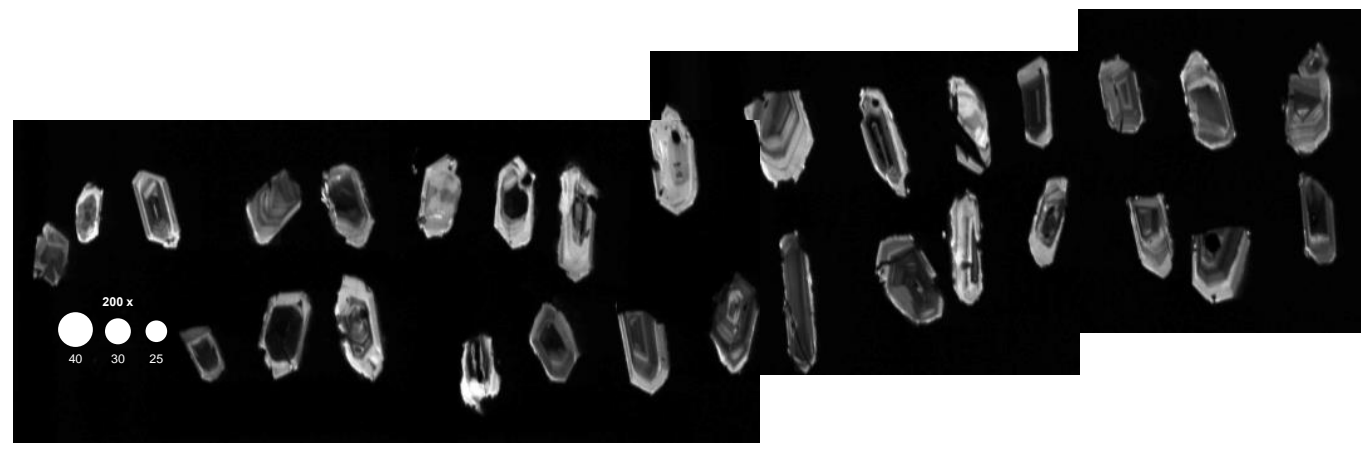

Fig. F3 A-E. Cathodoluminescence (CL) montage images taken via scanning electron microscope, which reveal oscillatory/sector zoning typical of magmatic zircon. The bright ("active") areas are relatively high in $\mathrm{REE}^{3+}$ and/or low in $\mathrm{U}$. The white circles labeled 40, 35, and 25 indicate diameter in millimeters. All are shown at a magnification of 200x. All zircons were picked by hand from sample TM5 (A-like rhyolite). 
Table F1. Zircon U-Pb isotopic compositions

\begin{tabular}{|c|c|c|c|c|c|c|}
\hline $\begin{array}{c}\text { Sample } \\
\text { (a) }\end{array}$ & $\begin{array}{l}\frac{\mathrm{Th}}{\mathrm{U}} \\
\text { (b) }\end{array}$ & $\begin{array}{c}{ }^{206} \mathrm{~Pb}^{*} \\
\mathrm{x} 10^{-13} \text { mole } \\
\text { (c) }\end{array}$ & $\begin{array}{l}\text { mole fraction } \\
{ }^{206} \mathrm{~Pb}^{*} \\
\text { (c) }\end{array}$ & $\begin{array}{l}\frac{\mathrm{Pb}^{*}}{\mathrm{~Pb}_{\mathrm{c}}} \\
\text { (c) }\end{array}$ & $\begin{array}{l}\mathrm{Pb}_{\mathrm{c}} \\
(\mathrm{pg}) \\
(\mathrm{c})\end{array}$ & $\begin{array}{c}\frac{{ }^{206} \mathrm{~Pb}}{{ }^{204} \mathrm{~Pb}} \\
\text { (d) }\end{array}$ \\
\hline \multicolumn{7}{|l|}{ CH08a } \\
\hline z8 (180) & 0.425 & 0.1216 & 0.9670 & 8.6 & 0.34 & 547 \\
\hline Z7 (171) & 0.666 & 0.0858 & 0.9354 & 4.5 & 0.49 & 279 \\
\hline z1 (39/40) & 0.694 & 0.2455 & 0.9800 & 15.5 & 0.42 & 904 \\
\hline z6 (168) & 0.514 & 0.1855 & 0.9743 & 11.4 & 0.41 & 703 \\
\hline z5 (101) & 0.479 & 0.0960 & 0.9548 & 6.3 & 0.38 & 399 \\
\hline z2 (43/44) & 0.437 & 0.2524 & 0.9686 & 9.1 & 0.68 & 574 \\
\hline z4 (104) & 0.419 & 0.1670 & 0.9803 & 14.6 & 0.28 & 915 \\
\hline \multicolumn{7}{|l|}{ TM5 } \\
\hline z6 (188) & 0.482 & 0.0829 & 0.9619 & 7.5 & 0.27 & 474 \\
\hline z5 (117) & 0.461 & 0.2146 & 0.9778 & 13.1 & 0.40 & 815 \\
\hline z3 (108) & 0.424 & 0.1980 & 0.9669 & 8.6 & 0.56 & 544 \\
\hline z7 (189) & 0.451 & 0.1407 & 0.9775 & 12.9 & 0.27 & 803 \\
\hline z1 (54/55) & 0.429 & 0.2917 & 0.9907 & 31.2 & 0.23 & 1933 \\
\hline z2 (58/59) & 0.475 & 0.3565 & 0.9939 & 48.9 & 0.18 & 2977 \\
\hline z4 (128) & 0.563 & 0.5419 & 0.9932 & 44.3 & 0.31 & 2640 \\
\hline \multicolumn{7}{|l|}{ CH07a } \\
\hline z3 (6) & 0.325 & 0.1702 & 0.9627 & 7.4 & 0.55 & 484 \\
\hline z8 (136) & 0.430 & 0.0458 & 0.9298 & 3.9 & 0.29 & 257 \\
\hline z1 (1/2) & 0.406 & 0.1160 & 0.9482 & 5.4 & 0.53 & 348 \\
\hline z7 (132/133) & 0.431 & 0.1924 & 0.9825 & 16.5 & 0.28 & 1030 \\
\hline z5 (86/87) & 0.535 & 0.0953 & 0.9474 & 5.5 & 0.44 & 343 \\
\hline z2 (3/4/5) & 0.364 & 0.7549 & 0.9940 & 47.8 & 0.38 & 3001 \\
\hline z4 (88) & 0.397 & 0.2148 & 0.9856 & 20.0 & 0.26 & 1255 \\
\hline \multicolumn{7}{|l|}{ MS12-49 } \\
\hline $\mathrm{z} 9(25)$ & 0.406 & 0.2218 & 0.9850 & 19.2 & 0.28 & 1199 \\
\hline z6(14/15) & 0.389 & 0.3585 & 0.9787 & 13.5 & 0.64 & 857 \\
\hline z10(31) & 0.346 & 0.4640 & 0.9730 & 10.5 & 1.06 & 684 \\
\hline
\end{tabular}




\begin{tabular}{llllrrr}
\hline z15(86) & 0.351 & 0.0908 & 0.9560 & 6.3 & 0.35 & 410 \\
z8(23) & 0.361 & 0.5710 & 0.9877 & 23.3 & 0.59 & 1478 \\
z2(7) & 0.392 & 0.4625 & 0.9828 & 16.7 & 0.67 & 1059 \\
z1(11) & 0.347 & 0.8206 & 0.9874 & 22.7 & 0.86 & 1454 \\
z4(17/18) & 0.299 & 0.2843 & 0.9789 & 13.2 & 0.51 & 856 \\
z14(47) & 0.394 & 0.6815 & 0.9900 & 28.8 & 0.57 & 1807 \\
z12(39) & 0.480 & 1.0336 & 0.9953 & 63.9 & 0.40 & 3879 \\
z7(12) & 0.350 & 0.9319 & 0.9874 & 22.8 & 0.98 & 1466 \\
z1(32) & 0.426 & 1.0777 & 0.9957 & 68.5 & 0.38 & 4216 \\
z20(37) & 0.354 & 0.7854 & 0.9958 & 69.2 & 0.27 & 4346 \\
z16(22) & 0.352 & 1.0303 & 0.9953 & 60.9 & 0.40 & 3828 \\
z21(50) & 0.340 & 0.3881 & 0.9907 & 30.7 & 0.30 & 1949 \\
z19(29) & 0.395 & 1.1988 & 0.9962 & 76.6 & 0.38 & 4757 \\
z18(27) & 0.377 & 0.4491 & 0.9927 & 39.6 & 0.27 & 2482 \\
\hline
\end{tabular}

(a) Labels for partial zircon crystals annealed and chemically abraded after Mattinson (2005); parentheses contain laser spot numbers on the same crystal, ex. Sample CH08a, zircon number 8 , spot number 180 .

(b) Model $\mathrm{Th} / \mathrm{U}$ ratio iteratively calculated from the radiogenic ${ }^{208} \mathrm{~Pb} /{ }^{206} \mathrm{~Pb}$ ratio and ${ }^{206} \mathrm{~Pb} /{ }^{238} \mathrm{U}$ age.

(c) $\mathrm{Pb}^{*}$ and $\mathrm{Pb}_{\mathrm{c}}$ represent radiogenic and common $\mathrm{Pb}$, respectively; mole fraction ${ }^{206} \mathrm{~Pb}^{*}$ with respect to radiogenic, blank, and initial common $\mathrm{Pb}$.

(d) Measured ratio corrected for spike and fractionation only. Fractionation estimated at $(0.16 \pm 0.03 \%)$ / atomic mass unit for Daly analyses, based on analysis of the NBS-981 and NBS-982 $\mathrm{Pb}$ isotope standards.

Table F2. Zircon radiogenic isotope ratios

\begin{tabular}{|c|c|c|c|c|c|c|c|c|}
\hline \multirow[b]{2}{*}{$\begin{array}{c}\text { Sample } \\
\text { (a) }\end{array}$} & \multirow{2}{*}{$\begin{array}{c}\frac{{ }^{208} \mathrm{~Pb}}{{ }^{206} \mathrm{~Pb}} \\
\text { (b) }\end{array}$} & \multicolumn{2}{|l|}{${ }^{207} \mathrm{~Pb}$} & \multicolumn{2}{|l|}{${ }^{207} \mathrm{~Pb}$} & \multicolumn{2}{|l|}{${ }^{206} \mathrm{~Pb}$} & \multirow{2}{*}{$\begin{array}{l}\text { correlation } \\
\text { coefficien }\end{array}$} \\
\hline & & $\begin{array}{c}{ }^{206} \mathrm{~Pb} \\
\text { (b) }\end{array}$ & $\begin{array}{l}\% \\
\text { error } \\
\text { (c) }\end{array}$ & $\begin{array}{l}{ }^{235} \mathrm{U} \\
\text { (b) }\end{array}$ & $\begin{array}{l}\% \\
\text { error } \\
\text { (c) }\end{array}$ & $\begin{array}{l}{ }^{238} \mathrm{U} \\
\text { (b) }\end{array}$ & $\begin{array}{l}\% \\
\text { error } \\
\text { (c) }\end{array}$ & \\
\hline \multicolumn{9}{|l|}{ CH08a } \\
\hline z8 (180) & 0.137 & 0.04697 & 0.9 & 0.0320 & 1.0 & 0.004946 & 0.121 & 0.709 \\
\hline z7 (171) & 0.214 & 0.04637 & 2 & 0.0313 & 2.2 & 0.004899 & 0.217 & 0.663 \\
\hline z1 (39/40) & 0.223 & 0.04675 & 0.7 & 0.0315 & 0.7 & 0.004889 & 0.100 & 0.688 \\
\hline z6 (168) & 0.165 & 0.04644 & 0.9 & 0.0313 & 1.0 & 0.004889 & 0.115 & 0.679 \\
\hline z5 (101) & 0.154 & 0.04634 & 1.4 & 0.0312 & 1.5 & 0.004888 & 0.163 & 0.678 \\
\hline z2 (43/44) & 0.141 & 0.04670 & 0.8 & 0.0315 & 0.9 & 0.004887 & 0.119 & 0.662 \\
\hline z4 (104) & 0.135 & 0.04608 & 0.7 & 0.0310 & 0.7 & 0.004883 & 0.103 & 0.666 \\
\hline \multicolumn{9}{|l|}{ TM5 } \\
\hline z6 (188) & 0.155 & 0.04640 & 1.8 & 0.0317 & 1.9 & 0.004950 & 0.158 & 0.692 \\
\hline z5 (117) & 0.148 & 0.04682 & 0.6 & 0.0319 & 0.7 & 0.004949 & 0.104 & 0.676 \\
\hline
\end{tabular}




\begin{tabular}{lllllllll}
\hline z3 (108) & 0.136 & 0.04685 & 0.9 & 0.0319 & 1.0 & 0.004945 & 0.128 & 0.705 \\
z7 (189) & 0.145 & 0.04613 & 0.9 & 0.0314 & 1.0 & 0.004945 & 0.110 & 0.718 \\
z1 (54/55) & 0.138 & 0.04646 & 0.4 & 0.0317 & 0.4 & 0.004944 & 0.079 & 0.745 \\
z2 (58/59) & 0.153 & 0.04656 & 0.3 & 0.0317 & 0.3 & 0.004944 & 0.078 & 0.698 \\
z4 (128) & 0.181 & 0.04656 & 0.3 & 0.0317 & 0.4 & 0.004943 & 0.083 & 0.615
\end{tabular}

\section{CH07a}

$\begin{array}{lllllllll}\text { z3 (6) } & 0.104 & 0.04673 & 1 & 0.0325 & 1.1 & 0.005037 & 0.130 & 0.697 \\ \text { z8 (136) } & 0.138 & 0.04649 & 3.1 & 0.0322 & 3.2 & 0.005020 & 0.236 & 0.519 \\ \text { z1 (1/2) } & 0.131 & 0.04636 & 1.5 & 0.0321 & 1.6 & 0.005016 & 0.173 & 0.677 \\ \text { z7 (132/133) } & 0.139 & 0.04678 & 0.5 & 0.0324 & 0.6 & 0.005016 & 0.093 & 0.697 \\ \text { z5 (86/87) } & 0.172 & 0.04659 & 1.6 & 0.0322 & 1.7 & 0.005008 & 0.172 & 0.676 \\ \text { z2 (3/4/5) } & 0.117 & 0.04670 & 0.2 & 0.0322 & 0.3 & 0.005002 & 0.073 & 0.764 \\ \text { z4 (88) } & 0.128 & 0.04562 & 0.9 & 0.0315 & 0.9 & 0.005001 & 0.154 & 0.552\end{array}$

MS12-49

$\begin{array}{lllllllll}\text { z9(25) } & 0.130 & 0.04662 & 0.7 & 0.0334 & 0.7 & 0.005200 & 0.097 & 0.672 \\ \text { z6(14/15) } & 0.125 & 0.04637 & 0.5 & 0.0331 & 0.6 & 0.005178 & 0.109 & 0.626 \\ \text { z10(31) } & 0.111 & 0.04678 & 0.4 & 0.0333 & 0.5 & 0.005163 & 0.087 & 0.701 \\ \text { z15(86) } & 0.113 & 0.04667 & 1.3 & 0.0332 & 1.4 & 0.005157 & 0.153 & 0.696 \\ \text { z8(23) } & 0.116 & 0.04646 & 0.4 & 0.0330 & 0.4 & 0.005153 & 0.112 & 0.610 \\ \text { z2(7) } & 0.126 & 0.04675 & 0.4 & 0.0332 & 0.4 & 0.005152 & 0.083 & 0.710 \\ \text { z1(11) } & 0.112 & 0.04672 & 0.2 & 0.0332 & 0.3 & 0.005150 & 0.076 & 0.746 \\ \text { z4(17/18) } & 0.096 & 0.04668 & 0.6 & 0.0330 & 0.7 & 0.005130 & 0.106 & 0.639 \\ \text { z14(47) } & 0.127 & 0.04673 & 0.3 & 0.0325 & 0.3 & 0.005040 & 0.078 & 0.727 \\ \text { z12(39) } & 0.154 & 0.04684 & 0.2 & 0.0325 & 0.2 & 0.005038 & 0.076 & 0.742 \\ \text { z7(12) } & 0.113 & 0.04653 & 0.3 & 0.0323 & 0.3 & 0.005033 & 0.084 & 0.637 \\ \text { z1(32) } & 0.137 & 0.04677 & 0.2 & 0.0324 & 0.2 & 0.005032 & 0.076 & 0.806 \\ \text { z20(37) } & 0.114 & 0.04672 & 0.2 & 0.0324 & 0.2 & 0.005031 & 0.076 & 0.753 \\ \text { z16(22) } & 0.113 & 0.04680 & 0.2 & 0.0325 & 0.2 & 0.005030 & 0.076 & 0.784 \\ \text { z21(50) } & 0.109 & 0.04665 & 0.3 & 0.0324 & 0.3 & 0.005029 & 0.082 & 0.676 \\ \text { z19(29) } & 0.127 & 0.04673 & 0.2 & 0.0324 & 0.2 & 0.005029 & 0.092 & 0.712 \\ \text { z18(27) } & 0.121 & 0.04687 & 0.3 & 0.0325 & 0.4 & 0.005027 & 0.082 & 0.657\end{array}$

(a) Labels for partial zircon crystals annealed and chemically abraded after Mattinson (2005); parentheses contain laser spot numbers on the same crystal, ex. Sample CH08a, zircon number 8, spot number 180.

(b) Calculations are based on the decay constants of Jaffey et al. (1971). ${ }^{206} \mathrm{~Pb} /{ }^{238} \mathrm{U}$ and ${ }^{207} \mathrm{~Pb} /{ }^{206} \mathrm{~Pb}$ ages corrected for initial disequilibrium in ${ }^{230} \mathrm{Th} /{ }^{238} \mathrm{U}$ using $\mathrm{Th} / \mathrm{U}$ [magma] $=3$.

(c) Errors are $2 \sigma$, propagated using the algorithms of Schmitz and Schoene (2007). 
Table F3. Zircon isotopic ages

\begin{tabular}{|c|c|c|c|c|c|c|c|c|}
\hline & ${ }^{207} \mathrm{~Pb}$ & & ${ }^{207} \mathrm{~Pb}$ & & ${ }^{206} \mathrm{~Pb}$ & & & age, millions of \\
\hline $\begin{array}{l}\text { Sample } \\
\text { (a) }\end{array}$ & $\begin{array}{l}{ }^{206} \mathrm{~Pb} \\
\text { (b) }\end{array}$ & $\begin{array}{l} \pm \\
\text { (c) }\end{array}$ & $\begin{array}{l}{ }^{235} \mathrm{U} \\
\text { (b) }\end{array}$ & $\begin{array}{l} \pm \\
\text { (c) }\end{array}$ & $\begin{array}{l}{ }^{238} \mathrm{U} \\
\text { (b) }\end{array}$ & $\begin{array}{l} \pm \\
\text { (c) }\end{array}$ & $\begin{array}{l}\text { MSWD } \\
\text { (d) }\end{array}$ & $\begin{array}{c}\text { years } \\
\text { (e) }\end{array}$ \\
\hline CH08a & & & & & & & 0.78 & $31.426 \pm 0.016$ \\
\hline z8 (180) & 48 & 21 & 32.02 & 0.31 & 31.807 & 0.038 & & \\
\hline z7 (171) & 17 & 49 & 31.31 & 0.67 & 31.501 & 0.068 & & \\
\hline z1 (39/40) & 36 & 16 & 31.50 & 0.22 & 31.439 & 0.031 & & \\
\hline z6 (168) & 20 & 21 & 31.29 & 0.30 & 31.438 & 0.036 & & \\
\hline z5 (101) & 16 & 34 & 31.23 & 0.47 & 31.431 & 0.051 & & \\
\hline z2 (43/44) & 34 & 19 & 31.46 & 0.27 & 31.424 & 0.037 & & \\
\hline z4 (104) & 2 & 16 & 31.03 & 0.22 & 31.403 & 0.032 & & \\
\hline TM5 & & & & & & & 0.88 & $31.798 \pm 0.012$ \\
\hline z6 (188) & 18 & 42 & 31.65 & 0.58 & 31.829 & 0.05 & & \\
\hline z5 (117) & 40 & 15 & 31.93 & 0.22 & 31.824 & 0.033 & & \\
\hline z3 (108) & 41 & 22 & 31.93 & 0.31 & 31.802 & 0.04 & & \\
\hline z7 (189) & 4 & 21 & 31.44 & 0.30 & 31.799 & 0.035 & & \\
\hline z1 (54/55) & 22 & 9 & 31.66 & 0.14 & 31.792 & 0.025 & & \\
\hline z2 (58/59) & 27 & 7 & 31.72 & 0.10 & 31.79 & 0.025 & & \\
\hline z4 (128) & 27 & 8 & 31.72 & 0.12 & 31.787 & 0.026 & & \\
\hline CH07a & & & & & & & 0.25 & $32.169 \pm 0.024$ \\
\hline z3 (6) & 36 & 23 & 32.43 & 0.34 & 32.389 & 0.042 & & \\
\hline z8 (136) & 23 & 74 & 32.16 & 1.01 & 32.277 & 0.076 & & \\
\hline z1 (1/2) & 16 & 36 & 32.05 & 0.50 & 32.256 & 0.056 & & \\
\hline z7 (132/133) & 38 & 13 & 32.33 & 0.19 & 32.254 & 0.03 & & \\
\hline z5 (86/87) & 28 & 39 & 32.15 & 0.55 & 32.201 & 0.055 & & \\
\hline $\mathrm{z} 2(3 / 4 / 5)$ & 34 & 5 & 32.18 & 0.08 & 32.162 & 0.023 & & \\
\hline z4 (88) & -22 & 21 & 31.45 & 0.29 & 32.159 & 0.05 & & \\
\hline \multicolumn{9}{|l|}{ MS12-49 } \\
\hline $\mathrm{z} 9(25)$ & 30 & 16 & 33.38 & 0.24 & 33.432 & 0.032 & 0.99 & $32.345 \pm 0.010$ \\
\hline$z 6(14 / 15)$ & 17 & 13 & 33.07 & 0.20 & 33.291 & 0.036 & & \\
\hline z10(31) & 38 & 10 & 33.26 & 0.15 & 33.197 & 0.029 & & \\
\hline z15(86) & 32 & 31 & 33.14 & 0.46 & 33.159 & 0.051 & & \\
\hline z8(23) & 22 & 9 & 32.98 & 0.14 & 33.133 & 0.037 & & \\
\hline $\mathrm{z} 2(7)$ & 37 & 9 & 33.17 & 0.14 & 33.124 & 0.028 & & \\
\hline z1(11) & 35 & 5 & 33.14 & 0.09 & 33.115 & 0.025 & & \\
\hline $\mathrm{z} 4(17 / 18)$ & 33 & 15 & 32.98 & 0.23 & 32.983 & 0.035 & & \\
\hline $\mathrm{z} 14(47)$ & 35 & 6 & 32.44 & 0.10 & 32.407 & 0.025 & & \\
\hline
\end{tabular}




\begin{tabular}{lllllll}
\hline z12(39) & 41 & 4 & 32.51 & 0.07 & 32.392 & 0.025 \\
z7(12) & 25 & 7 & 32.27 & 0.11 & 32.363 & 0.027 \\
z1(32) & 38 & 4 & 32.43 & 0.07 & 32.354 & 0.025 \\
z20(37) & 35 & 4 & 32.39 & 0.07 & 32.351 & 0.024 \\
z16(22) & 39 & 4 & 32.43 & 0.07 & 32.344 & 0.025 \\
z21(50) & 31 & 7 & 32.33 & 0.10 & 32.34 & 0.027 \\
z19(29) & 35 & 4 & 32.38 & 0.07 & 32.338 & 0.03 \\
z18(27) & 42 & 7 & 32.46 & 0.11 & 32.322 & 0.026 \\
\hline
\end{tabular}

(a) Labels for partial zircon crystals annealed and chemically abraded after Mattinson (2005); parentheses contain laser spot numbers on the same crystal, ex. Sample CH08a, zircon number 8 , spot number 180 .

(b) Calculations are based on the decay constants of Jaffey et al. (1971). ${ }^{206} \mathrm{~Pb} /{ }^{238} \mathrm{U}$ and

${ }^{207} \mathrm{~Pb} / 206 \mathrm{~Pb}$ ages corrected for initial disequilibrium in ${ }^{230} \mathrm{Th} /{ }^{238} \mathrm{U}$ using $\mathrm{Th} / \mathrm{U}$ [magma] $=3$.

(c) Errors are $2 \sigma$, propagated using the algorithms of Schmitz and Schoene (2007).

(d) Mean square weighted deviation.

(e) $95 \%$ confidence interval $=2 \sigma *$ Student's T-test $*(M S W D)^{0.5}$ 
Table F4. $\mathrm{C} 1$ chondrite normalized ${ }^{1}$ REE concentrations

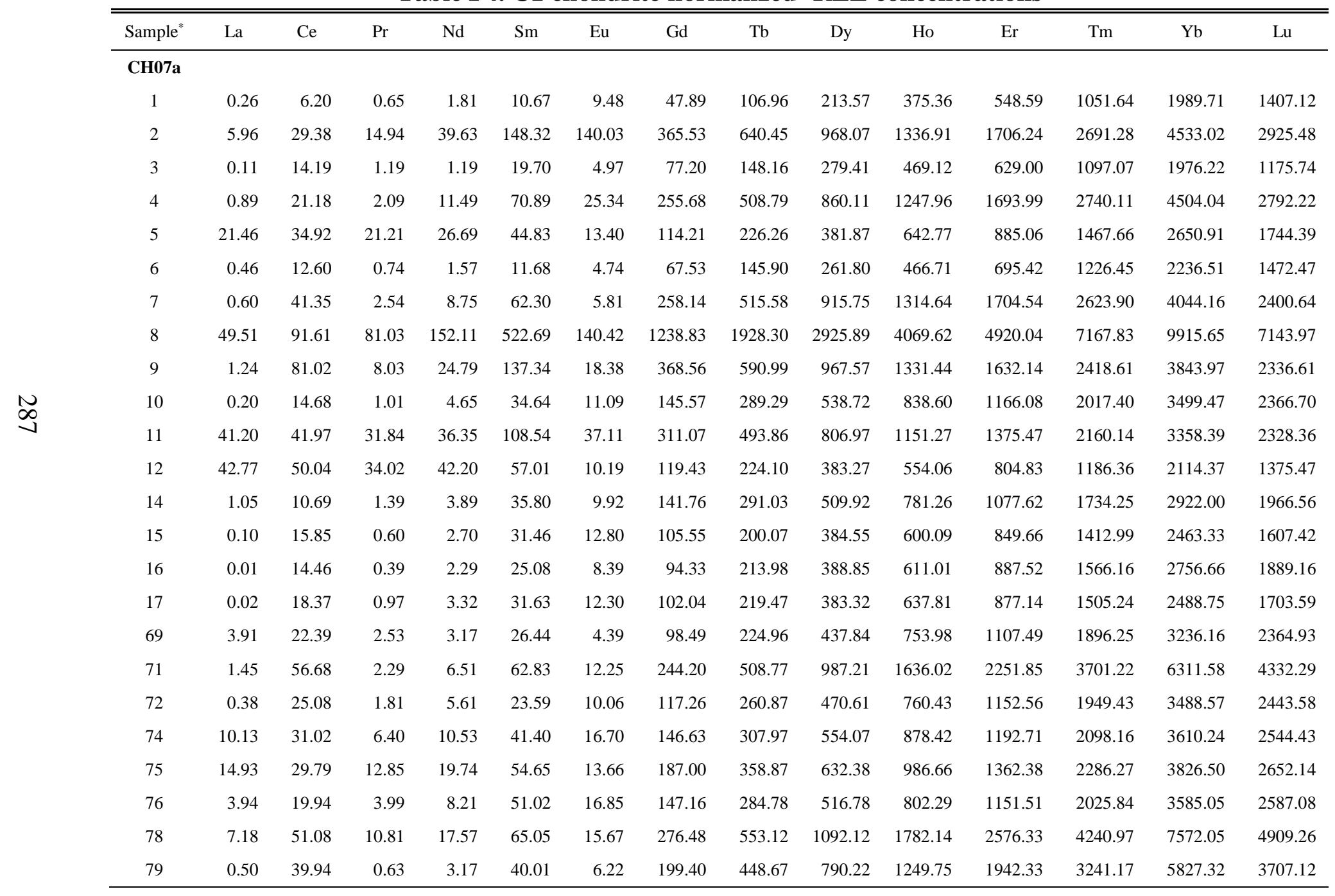




\begin{tabular}{|c|c|c|c|c|c|c|c|c|c|c|c|c|c|c|}
\hline 80 & 19.21 & 34.20 & 15.23 & 15.77 & 26.96 & 13.47 & 89.66 & 157.45 & 279.45 & 465.90 & 726.77 & 1232.36 & 2172.38 & 1541.50 \\
\hline 81 & 0.02 & 16.88 & 0.96 & 3.60 & 33.92 & 12.57 & 124.17 & 269.30 & 494.75 & 799.63 & 1125.36 & 1945.89 & 3363.29 & 2345.73 \\
\hline 83 & 3.54 & 20.63 & 6.21 & 10.21 & 35.81 & 23.53 & 130.94 & 258.69 & 449.98 & 799.44 & 1154.22 & 2000.24 & 3617.01 & 2486.66 \\
\hline 84 & 0.38 & 22.08 & 2.24 & 7.57 & 48.49 & 15.55 & 203.12 & 417.35 & 733.45 & 1186.83 & 1646.57 & 2689.83 & 4661.44 & 3132.64 \\
\hline 85 & 0.47 & 25.74 & 0.81 & 3.32 & 29.99 & 6.04 & 163.92 & 341.80 & 699.24 & 1176.35 & 1826.18 & 3186.20 & 5670.96 & 3892.07 \\
\hline 86 & 2.72 & 120.02 & 8.84 & 26.91 & 204.02 & 8.04 & 592.76 & 1226.36 & 2044.77 & 2950.70 & 3763.47 & 5745.16 & 8765.70 & 4716.91 \\
\hline 87 & 0.85 & 144.70 & 12.83 & 45.38 & 267.29 & 13.65 & 856.02 & 1641.03 & 2685.88 & 3889.12 & 4873.97 & 7399.41 & 10766.15 & 6548.97 \\
\hline 88 & 0.27 & 8.99 & 0.46 & 1.81 & 10.29 & 16.15 & 53.38 & 113.43 & 210.15 & 376.78 & 597.74 & 1185.80 & 2294.67 & 1841.41 \\
\hline 89 & 0.76 & 43.52 & 9.87 & 31.16 & 208.52 & 114.08 & 587.25 & 1031.74 & 1637.56 & 2283.10 & 2944.53 & 4570.13 & 7787.74 & 4586.34 \\
\hline 90 & 0.58 & 16.42 & 4.52 & 20.39 & 139.18 & 94.28 & 406.23 & 629.15 & 1011.05 & 1441.04 & 1902.22 & 2948.47 & 4974.50 & 3160.93 \\
\hline 129 & 3.94 & 24.69 & 3.69 & 5.62 & 23.18 & 20.50 & 105.89 & 231.13 & 433.38 & 741.91 & 1132.31 & 1858.78 & 3068.79 & 2534.92 \\
\hline 130 & 0.19 & 11.11 & 1.14 & 4.30 & 38.17 & 13.39 & 126.40 & 268.42 & 448.31 & 737.23 & 1095.02 & 1836.92 & 2899.02 & 2365.68 \\
\hline 131 & 5.09 & 16.00 & 5.41 & 18.72 & 104.68 & 57.56 & 381.30 & 604.18 & 959.55 & 1474.45 & 1990.53 & 3104.72 & 4413.24 & 3676.92 \\
\hline 132 & 17.15 & 24.01 & 17.67 & 18.20 & 57.93 & 19.93 & 172.10 & 307.52 & 527.92 & 827.04 & 1196.05 & 1794.83 & 2892.38 & 2588.94 \\
\hline 133 & 0.02 & 26.20 & 0.92 & 6.51 & 54.88 & 13.45 & 226.04 & 406.24 & 761.45 & 1281.57 & 1835.71 & 2888.28 & 4323.56 & 3848.83 \\
\hline 134 & 0.86 & 10.12 & 4.74 & 13.45 & 83.21 & 44.58 & 286.47 & 535.90 & 900.64 & 1340.19 & 1875.08 & 2851.79 & 4051.90 & 3544.45 \\
\hline 135 & 7.18 & 22.94 & 3.65 & 11.29 & 55.76 & 19.72 & 200.43 & 437.23 & 735.85 & 1137.76 & 1614.19 & 2496.34 & 3820.12 & 3320.52 \\
\hline 136 & 0.99 & 14.47 & 1.78 & 7.28 & 41.24 & 20.72 & 149.46 & 320.63 & 571.18 & 885.72 & 1226.93 & 1886.08 & 2959.31 & 2523.21 \\
\hline 137 & 0.05 & 14.64 & 0.77 & 3.01 & 32.95 & 11.70 & 139.56 & 290.61 & 561.92 & 919.26 & 1397.89 & 2295.34 & 3633.71 & 3081.97 \\
\hline 138 & 4.75 & 27.88 & 3.56 & 5.56 & 37.47 & 9.88 & 139.08 & 289.60 & 577.38 & 939.57 & 1476.37 & 2558.32 & 4172.02 & 3252.82 \\
\hline 139 & 5.84 & 90.43 & 10.07 & 29.28 & 179.91 & 15.53 & 646.87 & 1185.14 & 1984.31 & 2866.27 & 3657.13 & 5411.35 & 8065.29 & 5348.98 \\
\hline 140 & 1.63 & 15.32 & 1.80 & 4.65 & 25.33 & 8.47 & 104.63 & 211.89 & 422.31 & 713.16 & 1075.27 & 1825.90 & 3151.88 & 2436.47 \\
\hline 141 & 44.59 & 269.28 & 108.81 & 209.76 & 806.06 & 347.38 & 2159.60 & 3794.41 & 5885.00 & 8313.13 & 10641.91 & 15238.72 & 21960.72 & 16187.70 \\
\hline 143 & 42.98 & 101.52 & 34.50 & 61.09 & 151.54 & 23.68 & 395.97 & 879.53 & 1549.24 & 2506.79 & 3561.40 & 5497.66 & 8440.66 & 7061.11 \\
\hline 145 & 0.01 & 16.09 & 0.37 & 2.25 & 12.48 & 5.34 & 81.32 & 184.90 & 334.29 & 601.43 & 933.14 & 1530.66 & 2466.49 & 2191.06 \\
\hline 146 & 37.88 & 78.02 & 29.14 & 41.34 & 93.33 & 29.09 & 333.95 & 644.05 & 1133.12 & 1815.40 & 2585.80 & 4128.86 & 6261.65 & 5370.17 \\
\hline 148 & 10.99 & 14.24 & 9.93 & 15.85 & 59.04 & 52.32 & 219.34 & 379.20 & 605.75 & 960.39 & 1358.20 & 2053.03 & 3030.77 & 2854.06 \\
\hline
\end{tabular}




\begin{tabular}{|c|c|c|c|c|c|c|c|c|c|c|c|c|c|c|}
\hline 150 & 24.76 & 53.15 & 42.74 & 65.29 & 243.32 & 82.89 & 689.78 & 1239.66 & 1999.37 & 2897.39 & 3782.69 & 5117.06 & 6772.80 & 5751.62 \\
\hline 151 & 6.89 & 55.74 & 8.02 & 21.29 & 96.38 & 18.08 & 382.61 & 645.97 & 1068.78 & 1493.17 & 2024.25 & 2684.46 & 3848.13 & 3022.16 \\
\hline 153 & 2.85 & 20.19 & 1.82 & 6.18 & 28.99 & 10.69 & 155.63 & 354.57 & 658.38 & 1123.63 & 1785.63 & 2876.79 & 4416.69 & 4368.46 \\
\hline 154 & 0.43 & 61.02 & 7.40 & 35.14 & 237.45 & 19.51 & 796.39 & 1390.09 & 2125.02 & 3058.33 & 4001.46 & 5343.63 & 7108.08 & 5786.24 \\
\hline 156 & 1.08 & 12.43 & 1.38 & 5.89 & 45.36 & 19.56 & 172.76 & 356.25 & 620.97 & 1027.22 & 1523.15 & 2439.35 & 3688.92 & 3468.53 \\
\hline 157 & 1.32 & 138.47 & 3.08 & 13.94 & 158.28 & 22.13 & 808.47 & 1636.26 & 2901.64 & 4703.95 & 6734.93 & 10475.79 & 16056.14 & 12947.91 \\
\hline 158 & 25.67 & 47.39 & 47.79 & 75.09 & 165.91 & 74.24 & 459.67 & 693.71 & 1155.25 & 1678.78 & 2287.25 & 3529.03 & 5521.68 & 4337.70 \\
\hline 159 & 0.07 & 22.44 & 2.91 & 9.69 & 79.90 & 33.37 & 271.22 & 515.74 & 909.55 & 1373.20 & 1986.25 & 3121.88 & 5018.64 & 3676.92 \\
\hline 160 & 11.28 & 21.58 & 10.76 & 16.37 & 37.80 & 11.31 & 131.34 & 240.79 & 421.69 & 697.22 & 993.18 & 1618.09 & 2770.68 & 2165.59 \\
\hline 161 & 0.36 & 64.38 & 1.38 & 6.88 & 65.26 & 10.57 & 374.61 & 767.67 & 1437.35 & 2351.43 & 3392.86 & 5280.84 & 8168.01 & 6541.63 \\
\hline 162 & 0.97 & 10.84 & 1.04 & 3.87 & 34.46 & 10.66 & 139.21 & 263.13 & 511.56 & 850.28 & 1245.61 & 2002.46 & 3006.35 & 2918.33 \\
\hline 163 & 0.03 & 24.37 & 1.06 & 5.66 & 43.63 & 15.53 & 211.50 & 452.66 & 787.90 & 1293.64 & 1829.17 & 2817.81 & 4307.76 & 3522.61 \\
\hline \multicolumn{15}{|c|}{ CH08a } \\
\hline 19 & 10.94 & 29.52 & 8.06 & 12.49 & 44.76 & 9.87 & 150.24 & 293.09 & 507.88 & 739.97 & 985.53 & 1530.35 & 2488.82 & 1596.50 \\
\hline 21 & 0.03 & 37.01 & 1.30 & 6.45 & 55.04 & 3.53 & 201.71 & 357.69 & 642.08 & 967.70 & 1331.59 & 2047.04 & 3244.13 & 2006.07 \\
\hline 23 & 0.08 & 42.69 & 2.99 & 8.87 & 64.92 & 12.93 & 277.44 & 533.30 & 886.87 & 1295.77 & 1647.31 & 2550.06 & 4029.67 & 2481.97 \\
\hline 24 & 31.63 & 37.72 & 23.36 & 32.59 & 104.07 & 12.70 & 312.44 & 538.22 & 834.36 & 1266.04 & 1619.33 & 2581.67 & 4123.23 & 2408.46 \\
\hline 25 & 0.02 & 19.00 & 0.93 & 3.73 & 38.19 & 5.01 & 135.72 & 262.98 & 473.61 & 720.59 & 967.97 & 1577.62 & 2447.24 & 1621.14 \\
\hline 27 & 19.28 & 47.66 & 17.64 & 20.76 & 45.62 & 6.24 & 192.66 & 365.20 & 617.68 & 952.76 & 1208.69 & 1951.90 & 3114.64 & 1846.08 \\
\hline 28 & 22.34 & 39.08 & 22.23 & 30.91 & 98.54 & 10.57 & 332.52 & 562.60 & 977.15 & 1479.59 & 1904.58 & 2880.14 & 4421.04 & 2817.19 \\
\hline 29 & 444.26 & 369.10 & 330.02 & 364.10 & 301.64 & 13.19 & 322.94 & 412.47 & 625.65 & 961.20 & 1265.30 & 1885.42 & 2965.32 & 1874.11 \\
\hline 30 & 69.98 & 112.17 & 53.97 & 60.32 & 96.85 & 5.04 & 305.04 & 605.45 & 1023.11 & 1541.61 & 1988.35 & 3033.03 & 4706.94 & 2974.70 \\
\hline 31 & 1.50 & 53.78 & 3.57 & 9.45 & 65.32 & 6.17 & 279.30 & 492.68 & 891.62 & 1333.04 & 1782.83 & 2600.09 & 4157.48 & 2582.56 \\
\hline 33 & 0.86 & 31.56 & 5.17 & 18.51 & 108.93 & 9.75 & 425.71 & 808.47 & 1365.13 & 1980.59 & 2569.98 & 3877.41 & 5970.38 & 3650.32 \\
\hline 36 & 49.55 & 76.23 & 34.54 & 67.76 & 182.45 & 11.27 & 545.81 & 1002.43 & 1655.95 & 2473.10 & 3127.12 & 4656.99 & 7311.32 & 4486.15 \\
\hline 37 & 2.24 & 21.10 & 2.30 & 4.71 & 27.38 & 2.67 & 105.60 & 232.71 & 371.89 & 604.85 & 861.56 & 1287.85 & 2095.01 & 1378.35 \\
\hline
\end{tabular}




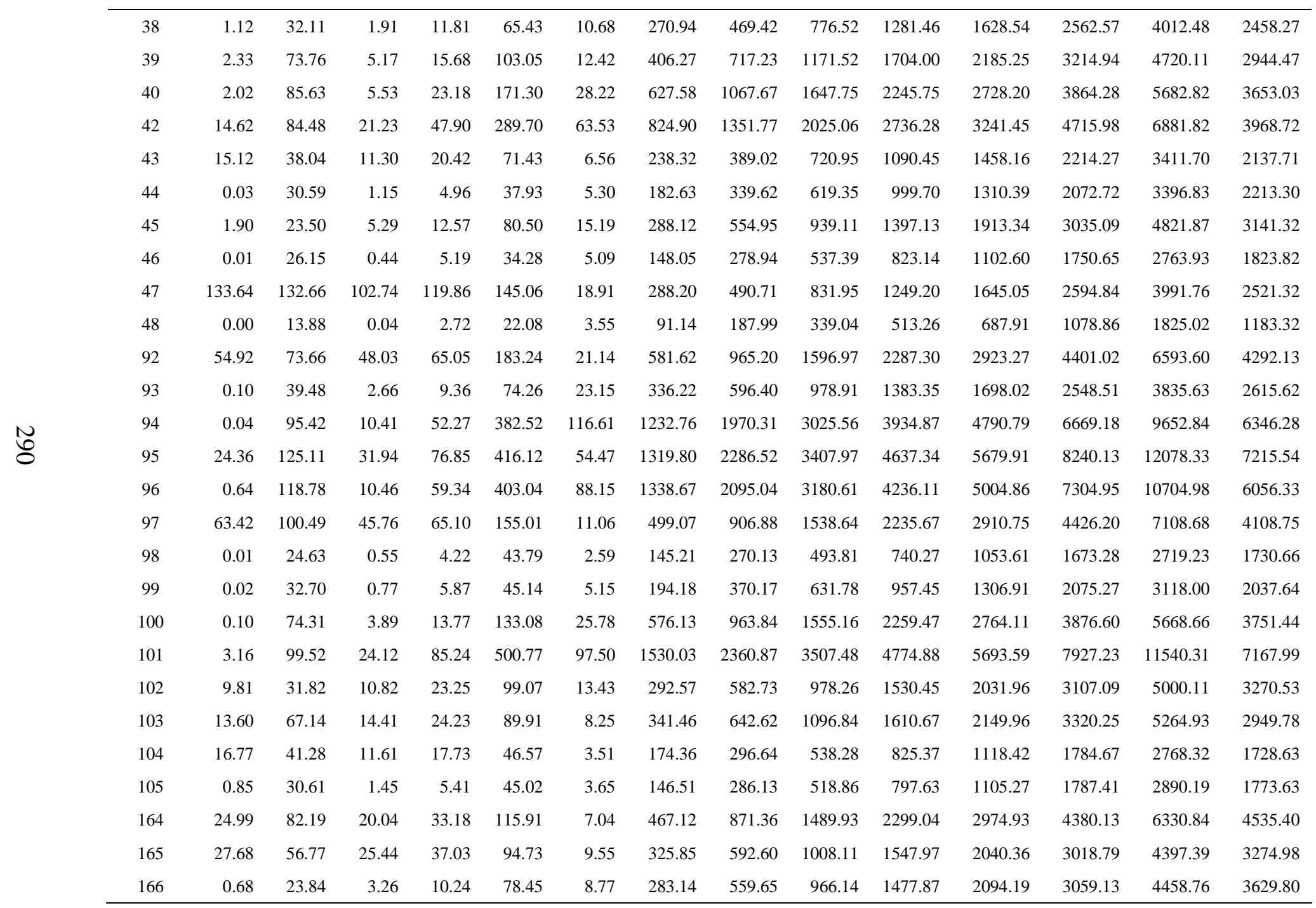




\begin{tabular}{|c|c|c|c|c|c|c|c|c|c|c|c|c|c|c|}
\hline 167 & 1.19 & 55.01 & 8.16 & 36.29 & 288.76 & 48.21 & 1042.04 & 1660.61 & 2757.77 & 3833.54 & 4916.01 & 6732.26 & 9490.70 & 7357.46 \\
\hline 168 & 38.60 & 93.67 & 36.50 & 61.51 & 181.89 & 15.13 & 648.03 & 1133.76 & 1862.31 & 2742.44 & 3479.45 & 5034.19 & 7353.05 & 4910.35 \\
\hline 169 & 4.03 & 72.84 & 9.47 & 41.94 & 245.15 & 27.92 & 910.59 & 1571.76 & 2518.04 & 3604.36 & 4628.67 & 6403.95 & 9150.71 & 6623.75 \\
\hline 170 & 52.68 & 88.11 & 48.77 & 56.96 & 107.66 & 9.23 & 321.45 & 608.43 & 991.38 & 1502.96 & 2116.50 & 2974.38 & 4115.85 & 3314.40 \\
\hline 171 & 0.12 & 43.04 & 3.54 & 17.70 & 158.64 & 35.52 & 638.02 & 988.08 & 1582.41 & 2225.19 & 2802.54 & 3894.18 & 5529.90 & 4086.62 \\
\hline 172 & 29.37 & 184.07 & 35.53 & 73.82 & 388.87 & 57.02 & 1480.68 & 2495.42 & 3797.66 & 5282.36 & 6655.86 & 8465.46 & 11156.55 & 8765.56 \\
\hline 174 & 17.71 & 46.60 & 17.25 & 20.45 & 71.21 & 5.77 & 236.96 & 428.35 & 741.60 & 1165.15 & 1603.21 & 2358.46 & 3491.55 & 2744.55 \\
\hline 175 & 13.31 & 95.27 & 15.09 & 26.20 & 97.72 & 5.35 & 466.30 & 848.07 & 1486.76 & 2159.75 & 2870.58 & 4057.21 & 6061.33 & 4357.35 \\
\hline 176 & 21.47 & 40.12 & 16.95 & 20.70 & 57.43 & 5.74 & 211.59 & 411.27 & 687.07 & 1067.90 & 1450.98 & 2175.86 & 3129.34 & 2598.05 \\
\hline 177 & 15.98 & 221.00 & 28.14 & 102.16 & 634.36 & 140.25 & 2078.24 & 3279.41 & 4932.37 & 6674.44 & 8099.56 & 10609.97 & 13941.12 & 9913.94 \\
\hline 178 & 0.06 & 64.52 & 2.33 & 9.08 & 68.18 & 3.01 & 362.57 & 713.29 & 1273.96 & 1937.10 & 2690.59 & 3924.36 & 5571.35 & 4077.85 \\
\hline 179 & 19.48 & 97.71 & 19.09 & 37.43 & 137.16 & 6.45 & 564.80 & 1062.38 & 1809.13 & 2637.31 & 3455.69 & 5080.23 & 7512.99 & 5170.00 \\
\hline 180 & 40.15 & 195.03 & 36.37 & 74.23 & 337.22 & 53.94 & 1226.41 & 1944.91 & 3105.70 & 4156.23 & 5104.81 & 7056.96 & 9774.54 & 6340.29 \\
\hline 181 & 0.37 & 36.97 & 4.44 & 25.13 & 168.06 & 25.70 & 735.11 & 1269.77 & 2077.02 & 3105.63 & 4124.79 & 5656.29 & 7695.30 & 6406.86 \\
\hline 182 & 16.43 & 129.94 & 26.89 & 77.49 & 450.95 & 188.83 & 1487.80 & 2188.01 & 3381.16 & 4529.56 & 5282.36 & 6934.38 & 9457.03 & 6995.89 \\
\hline \multicolumn{15}{|l|}{ TM5 } \\
\hline 50 & 102.64 & 135.74 & 92.30 & 136.13 & 329.77 & 50.80 & 888.33 & 1492.24 & 2368.46 & 3382.65 & 4185.60 & 6124.02 & 9253.46 & 5476.17 \\
\hline 52 & 505.64 & 440.25 & 420.64 & 497.32 & 540.87 & 72.20 & 849.19 & 1301.17 & 1918.11 & 2648.33 & 3378.91 & 4787.66 & 7012.18 & 4236.27 \\
\hline 53 & 2.57 & 40.27 & 3.49 & 11.68 & 67.47 & 11.22 & 271.79 & 497.81 & 859.55 & 1305.66 & 1688.28 & 2477.24 & 3893.15 & 2351.43 \\
\hline 54 & 0.04 & 54.85 & 1.59 & 9.86 & 86.74 & 11.18 & 359.09 & 730.62 & 1258.41 & 1855.32 & 2372.98 & 3622.62 & 5191.75 & 3218.69 \\
\hline 55 & 648.97 & 569.95 & 617.37 & 705.54 & 730.19 & 64.43 & 1049.43 & 1568.04 & 2413.16 & 3325.29 & 4156.88 & 5923.08 & 8711.41 & 5292.08 \\
\hline 56 & 40.19 & 67.72 & 38.73 & 59.31 & 188.45 & 34.62 & 631.32 & 1079.05 & 1808.05 & 2572.30 & 3237.58 & 4855.68 & 7240.52 & 4439.79 \\
\hline 58 & 61.92 & 97.36 & 58.98 & 89.19 & 212.97 & 26.58 & 676.09 & 1178.16 & 1922.37 & 2917.24 & 3742.93 & 5381.20 & 8509.28 & 4975.23 \\
\hline 59 & 0.69 & 28.24 & 1.33 & 4.88 & 53.92 & 6.72 & 247.52 & 431.74 & 772.88 & 1115.98 & 1539.38 & 2269.10 & 3591.05 & 2119.34 \\
\hline 60 & 19.41 & 35.39 & 14.21 & 24.43 & 66.11 & 11.92 & 184.66 & 339.24 & 574.61 & 851.50 & 1127.93 & 1719.07 & 2562.86 & 1560.61 \\
\hline 61 & 61.70 & 74.72 & 52.51 & 79.65 & 223.02 & 34.21 & 646.81 & 1084.05 & 1757.30 & 2471.78 & 3127.75 & 4565.81 & 6908.93 & 4131.26 \\
\hline
\end{tabular}




\begin{tabular}{|c|c|c|c|c|c|c|c|c|c|c|c|c|c|c|}
\hline 62 & 27.68 & 49.42 & 22.82 & 33.93 & 70.00 & 13.35 & 226.16 & 419.21 & 707.25 & 1000.57 & 1325.31 & 1932.60 & 2994.71 & 1926.90 \\
\hline 63 & 5.82 & 39.51 & 9.87 & 32.52 & 195.74 & 37.90 & 758.39 & 1352.57 & 2156.76 & 3070.01 & 3831.17 & 5634.01 & 8199.78 & 5248.04 \\
\hline 64 & 0.02 & 14.14 & 0.71 & 1.90 & 32.82 & 5.64 & 117.13 & 241.83 & 402.71 & 605.48 & 845.93 & 1246.00 & 1999.66 & 1319.69 \\
\hline 66 & 4.16 & 32.71 & 3.76 & 11.50 & 58.08 & 9.75 & 242.99 & 435.30 & 782.64 & 1120.90 & 1498.33 & 2300.07 & 3692.79 & 2043.98 \\
\hline 67 & 0.23 & 23.24 & 2.03 & 6.51 & 58.03 & 7.45 & 206.45 & 365.81 & 634.69 & 950.20 & 1273.65 & 1861.27 & 2897.79 & 1802.75 \\
\hline 68 & 0.08 & 51.22 & 4.10 & 16.48 & 139.57 & 19.36 & 535.79 & 968.42 & 1597.45 & 2336.56 & 2971.00 & 4413.57 & 6615.59 & 4000.63 \\
\hline 107 & 156.20 & 192.93 & 135.86 & 175.47 & 225.69 & 22.60 & 497.15 & 847.90 & 1360.08 & 1951.31 & 2454.79 & 3632.13 & 5616.24 & 3327.26 \\
\hline 108 & 56.30 & 108.75 & 50.50 & 83.06 & 248.45 & 39.19 & 690.05 & 1247.95 & 1948.30 & 2777.21 & 3607.78 & 5327.07 & 8218.89 & 4596.12 \\
\hline 109 & 0.05 & 22.24 & 2.10 & 8.85 & 77.35 & 37.35 & 267.57 & 456.24 & 719.55 & 1024.17 & 1253.53 & 1916.84 & 2973.34 & 1722.16 \\
\hline 110 & 82.96 & 96.12 & 69.30 & 93.27 & 164.45 & 24.76 & 450.08 & 798.43 & 1367.90 & 1981.89 & 2449.42 & 3663.50 & 5436.72 & 3235.97 \\
\hline 111 & 5.25 & 9.01 & 8.52 & 20.86 & 144.33 & 103.30 & 362.90 & 620.58 & 979.21 & 1346.67 & 1667.64 & 2488.93 & 3753.17 & 2555.55 \\
\hline 114 & 17.40 & 19.41 & 17.93 & 19.18 & 35.85 & 11.95 & 87.42 & 157.25 & 280.13 & 426.70 & 561.66 & 886.20 & 1469.79 & 885.14 \\
\hline 115 & 1.94 & 35.99 & 3.88 & 11.04 & 80.95 & 18.59 & 283.56 & 539.42 & 902.90 & 1327.20 & 1664.87 & 2518.27 & 3957.67 & 2438.96 \\
\hline 117 & 987.83 & 869.94 & 771.65 & 968.40 & 900.04 & 107.84 & 1271.61 & 1684.78 & 2493.18 & 3344.14 & 4058.49 & 5830.06 & 8588.27 & 4993.82 \\
\hline 118 & 27.92 & 36.54 & 23.35 & 35.32 & 77.13 & 35.21 & 217.33 & 355.84 & 593.75 & 857.66 & 1043.78 & 1634.02 & 2479.98 & 1524.06 \\
\hline 121 & 0.02 & 25.95 & 0.89 & 7.07 & 53.98 & 8.91 & 201.61 & 394.09 & 673.61 & 1007.22 & 1326.34 & 2054.23 & 3291.70 & 1860.23 \\
\hline 122 & 13.50 & 98.50 & 12.31 & 31.60 & 163.69 & 15.95 & 669.35 & 1273.70 & 2186.36 & 3085.64 & 3939.02 & 5746.85 & 8479.37 & 5031.63 \\
\hline 123 & 27.79 & 71.59 & 22.33 & 36.26 & 140.75 & 23.48 & 490.98 & 917.65 & 1429.28 & 2140.84 & 2597.67 & 4035.92 & 6245.75 & 3584.71 \\
\hline 124 & 288.18 & 307.36 & 252.29 & 286.71 & 340.79 & 33.40 & 591.87 & 961.59 & 1523.43 & 2112.76 & 2687.51 & 4035.57 & 6071.67 & 3594.70 \\
\hline 125 & 1.31 & 95.30 & 3.92 & 14.13 & 164.84 & 13.78 & 741.81 & 1385.43 & 2425.97 & 3548.60 & 4476.13 & 6714.03 & 10499.01 & 6210.00 \\
\hline 126 & 0.01 & 14.21 & 0.50 & 2.68 & 29.47 & 7.86 & 118.54 & 230.15 & 426.69 & 664.58 & 958.30 & 1609.69 & 2630.91 & 1625.92 \\
\hline 127 & - & 11.57 & 0.79 & 3.52 & 23.61 & 5.95 & 97.56 & 192.32 & 334.39 & 519.61 & 714.30 & 1211.68 & 2207.59 & 1420.87 \\
\hline 128 & 5.19 & 107.74 & 12.29 & 46.63 & 329.39 & 23.53 & 1247.00 & 2239.77 & 3766.94 & 5381.28 & 6720.27 & 10031.06 & 14787.57 & 8463.58 \\
\hline 183 & 0.36 & 42.30 & 2.81 & 13.59 & 106.42 & 25.91 & 440.57 & 845.02 & 1377.20 & 2066.06 & 2557.59 & 3802.39 & 5304.49 & 3788.03 \\
\hline 184 & 277.85 & 222.81 & 176.17 & 221.25 & 333.56 & 104.94 & 855.03 & 1381.09 & 2297.85 & 3275.08 & 4138.56 & 5881.39 & 8079.76 & 6100.92 \\
\hline 185 & 0.02 & 8.60 & 0.74 & 3.67 & 33.71 & 12.83 & 128.57 & 247.07 & 433.33 & 678.29 & 910.09 & 1380.89 & 2046.32 & 1599.16 \\
\hline 187 & 0.09 & 63.29 & 3.38 & 20.02 & 174.51 & 15.79 & 794.52 & 1477.05 & 2502.82 & 3809.91 & 5187.70 & 6996.85 & 9291.31 & 7494.30 \\
\hline
\end{tabular}




\begin{tabular}{|c|c|c|c|c|c|c|c|c|c|c|c|c|c|c|}
\hline 188 & 0.05 & 31.83 & 1.99 & 7.86 & 58.95 & 17.62 & 316.81 & 594.22 & 967.20 & 1437.96 & 1932.96 & 2716.48 & 3638.13 & 2938.25 \\
\hline 189 & 46.37 & 65.37 & 37.53 & 45.42 & 122.21 & 29.22 & 378.42 & 643.16 & 1104.20 & 1661.13 & 2081.48 & 3174.86 & 4510.07 & 3213.22 \\
\hline 191 & 11.30 & 58.52 & 16.53 & 39.75 & 242.14 & 26.03 & 1020.09 & 1902.38 & 3071.62 & 4460.76 & 5886.05 & 8114.74 & 10834.04 & 8260.41 \\
\hline 192 & 10.55 & 52.59 & 14.93 & 25.81 & 123.92 & 18.58 & 472.46 & 864.61 & 1489.44 & 2189.02 & 2878.65 & 3846.77 & 5255.74 & 4357.99 \\
\hline 193 & 2.54 & 36.84 & 9.80 & 34.73 & 209.37 & 40.25 & 827.63 & 1530.86 & 2495.57 & 3667.32 & 4806.42 & 6540.61 & 8897.91 & 6807.73 \\
\hline 194 & 2.88 & 41.93 & 5.14 & 23.61 & 161.26 & 27.61 & 611.40 & 1077.44 & 1839.55 & 2598.70 & 3419.60 & 4826.59 & 6791.55 & 4730.10 \\
\hline 195 & 46.63 & 77.37 & 45.80 & 71.47 & 197.40 & 21.14 & 587.72 & 1109.09 & 1804.06 & 2671.82 & 3504.24 & 4753.72 & 6436.37 & 5031.98 \\
\hline 196 & 29.36 & 197.94 & 40.53 & 115.50 & 742.92 & 108.65 & 2482.55 & 4035.44 & 6321.18 & 8844.66 & 10983.20 & 14286.96 & 18662.14 & 13712.24 \\
\hline 197 & 0.09 & 42.21 & 2.17 & 10.16 & 82.43 & 14.37 & 358.42 & 680.53 & 1150.75 & 1785.08 & 2353.75 & 3194.96 & 4424.12 & 3511.05 \\
\hline 198 & 3.35 & 12.50 & 2.72 & 6.55 & 32.26 & 13.92 & 116.31 & 224.88 & 401.07 & 586.25 & 817.84 & 1194.26 & 1701.32 & 1334.88 \\
\hline 199 & 0.40 & 89.26 & 3.77 & 17.04 & 158.48 & 14.99 & 718.91 & 1381.15 & 2315.59 & 3407.16 & 4371.35 & 6010.76 & 8083.52 & 6176.21 \\
\hline 200 & 2.99 & 63.61 & 7.83 & 28.32 & 234.51 & 31.50 & 988.82 & 1704.63 & 2949.06 & 4362.93 & 5555.32 & 7663.45 & 10133.48 & 7982.60 \\
\hline 201 & 160.76 & 156.56 & 134.61 & 189.67 & 325.01 & 46.62 & 888.19 & 1451.77 & 2325.53 & 3357.80 & 4431.15 & 5940.08 & 8151.75 & 6340.78 \\
\hline 202 & 0.05 & 31.25 & 1.41 & 6.84 & 60.95 & 6.57 & 296.45 & 550.93 & 1015.06 & 1471.41 & 1961.86 & 2817.74 & 3743.08 & 2958.35 \\
\hline 203 & 140.69 & 252.83 & 122.04 & 171.11 & 377.16 & 42.24 & 1120.72 & 2015.53 & 3266.53 & 4691.34 & 5800.07 & 8125.42 & 11656.86 & 7980.55 \\
\hline & \multicolumn{14}{|c|}{$\begin{array}{c}\text { MS12- } \\
47\end{array}$} \\
\hline 1 & 9.05 & 21.19 & 5.59 & 6.32 & 14.62 & 10.97 & 59.64 & 145.37 & 247.65 & 517.23 & 797.29 & 1488.01 & 2565.44 & 2882.82 \\
\hline 2 & 1.19 & 10.67 & 1.37 & 1.89 & 12.75 & 11.03 & 52.50 & 106.34 & 209.52 & 364.40 & 652.20 & 1224.02 & 2063.89 & 2361.98 \\
\hline 3 & 1.22 & 10.07 & & 1.75 & 17.13 & 16.12 & 51.80 & 140.35 & 250.16 & 475.39 & 880.07 & 1438.82 & 2577.97 & 2677.24 \\
\hline 4 & & 6.54 & & 0.35 & 3.37 & 7.04 & 38.06 & 103.76 & 192.24 & 362.12 & 625.73 & 1095.99 & 1981.18 & 2247.04 \\
\hline 5 & & 22.11 & 0.26 & 1.18 & 12.77 & 14.51 & 85.89 & 196.27 & 368.41 & 722.57 & 1279.91 & 2130.97 & 3909.76 & 4487.77 \\
\hline 6 & 0.00 & 9.90 & 0.06 & 1.00 & 6.56 & 5.97 & 56.12 & 101.86 & 223.14 & 381.39 & 745.84 & 1232.41 & 2244.16 & 2619.34 \\
\hline 7 & & 24.53 & 0.55 & 2.46 & 13.10 & 11.40 & 98.93 & 187.10 & 413.93 & 833.76 & 1473.04 & 2477.96 & 3919.43 & 4705.14 \\
\hline 8 & 4.07 & 14.82 & 2.93 & 1.97 & 11.62 & 8.25 & 64.19 & 119.00 & 220.96 & 423.37 & 711.49 & 1241.45 & 2062.19 & 2345.84 \\
\hline 9 & & 15.32 & 0.36 & 1.61 & 9.68 & 9.73 & 79.93 & 154.34 & 341.91 & 639.79 & 1062.98 & 1844.83 & 3049.36 & 3703.81 \\
\hline 10 & & 9.70 & & 0.58 & 5.68 & 6.06 & 48.12 & 111.31 & 217.66 & 406.06 & 769.70 & 1236.60 & 2099.17 & 2725.81 \\
\hline
\end{tabular}




\begin{tabular}{|c|c|c|c|c|c|c|c|c|c|c|c|c|c|c|}
\hline 11 & 0.13 & 18.30 & 0.76 & 0.76 & 10.99 & 8.18 & 54.39 & 159.87 & 304.65 & 623.52 & 985.49 & 1702.82 & 2867.67 & 3647.55 \\
\hline 12 & 0.04 & 51.34 & 1.72 & 6.58 & 66.69 & 7.91 & 373.91 & 724.36 & 1346.22 & 2334.33 & 3464.80 & 5230.86 & 7531.16 & 8068.55 \\
\hline 13 & 0.01 & 20.48 & 0.33 & 1.01 & 9.80 & 12.15 & 104.03 & 209.21 & 405.09 & 755.78 & 1365.26 & 2302.02 & 3665.62 & 4291.52 \\
\hline 14 & 0.02 & 17.86 & 0.88 & 6.22 & 41.35 & 24.21 & 179.76 & 301.64 & 525.55 & 872.54 & 1350.35 & 2086.03 & 3139.37 & 3932.08 \\
\hline 15 & 3.17 & 21.29 & 1.36 & 6.58 & 41.87 & 27.91 & 166.78 & 332.99 & 532.54 & 911.49 & 1388.18 & 2162.52 & 3508.36 & 4044.57 \\
\hline 16 & 3.96 & 18.70 & 3.42 & 11.37 & 42.25 & 27.03 & 153.90 & 259.12 & 475.78 & 852.07 & 1375.62 & 2151.18 & 3197.09 & 3893.97 \\
\hline 17 & 0.00 & 13.66 & 0.15 & 1.71 & 18.40 & 6.33 & 70.69 & 141.07 & 280.09 & 535.91 & 930.97 & 1636.75 & 2867.86 & 3478.21 \\
\hline 18 & 8 & 14.26 & 0.18 & 0.79 & 11.72 & 6.64 & 70.62 & 134.42 & 295.89 & 549.78 & 971.82 & 1690.13 & 2784.30 & 3442.49 \\
\hline 19 & 9 & 17.97 & 0.41 & 1.82 & 10.90 & 10.12 & 90.48 & 198.68 & 363.05 & 738.69 & 1232.63 & 2081.67 & 3418.93 & 3746.65 \\
\hline 20 & 0 & 24.65 & 0.76 & 3.41 & 26.05 & 1.78 & 187.49 & 392.03 & 726.24 & 1253.09 & 2059.95 & 3079.09 & 4895.27 & 4788.01 \\
\hline 21 & 1 & 13.68 & 0.12 & 0.53 & 11.27 & 5.04 & 57.36 & 120.02 & 236.36 & 473.52 & 797.44 & 1399.50 & 2380.56 & 2538.86 \\
\hline 22 & 0.03 & 33.39 & 1.30 & 3.92 & 26.70 & 4.74 & 166.13 & 396.72 & 725.61 & 1250.83 & 1852.22 & 3119.32 & 5079.27 & 4194.55 \\
\hline 23 & 1.65 & 14.35 & 1.19 & 2.55 & 10.83 & 7.58 & 59.55 & 127.73 & 247.81 & 472.01 & 753.02 & 1348.06 & 2549.66 & 2427.87 \\
\hline 24 & 4 & 10.09 & 0.06 & 0.28 & 9.29 & 8.38 & 49.37 & 94.98 & 218.81 & 391.83 & 610.26 & 1193.84 & 2360.19 & 2182.63 \\
\hline 25 & 0.02 & 13.94 & 0.90 & 6.50 & 32.10 & 25.68 & 105.59 & 195.46 & 360.93 & 605.66 & 907.79 & 1559.88 & 2628.26 & 2292.45 \\
\hline 26 & 0.47 & 61.22 & 1.81 & 6.46 & 59.61 & 5.76 & 330.11 & 622.06 & 1161.71 & 1912.47 & 2774.22 & 4360.60 & 7096.19 & 5636.61 \\
\hline 27 & 0.02 & 18.44 & 0.68 & 3.80 & 28.80 & 5.84 & 149.43 & 288.04 & 540.51 & 930.46 & 1407.70 & 2319.63 & 3842.69 & 3226.21 \\
\hline 28 & 0.95 & 26.78 & 1.23 & 2.90 & 28.67 & 18.60 & 122.45 & 238.48 & 450.22 & 768.01 & 1259.96 & 2306.52 & 3852.34 & 3400.02 \\
\hline 29 & 0.03 & 52.82 & 1.00 & 4.89 & 50.18 & 5.77 & 297.66 & 624.70 & 1075.15 & 1791.73 & 2613.81 & 4112.54 & 6613.50 & 5282.57 \\
\hline 30 & 0 & 20.11 & 0.34 & 1.50 & 7.24 & 11.93 & 79.44 & 186.68 & 348.32 & 636.19 & 1060.01 & 1955.89 & 3506.79 & 3219.92 \\
\hline 31 & 0.01 & 16.60 & 0.57 & 1.58 & 15.45 & 15.47 & 57.87 & 135.97 & 270.99 & 516.08 & 866.63 & 1585.11 & 2884.56 & 2748.18 \\
\hline 32 & 0.01 & 28.80 & 0.41 & 3.27 & 28.89 & 5.24 & 149.17 & 343.68 & 651.40 & 1116.59 & 1738.65 & 3119.64 & 4954.07 & 4116.26 \\
\hline 33 & 0.35 & 20.24 & 1.26 & 2.91 & 16.90 & 4.54 & 65.97 & 183.12 & 304.18 & 604.47 & 1022.69 & 1893.31 & 3681.18 & 3174.53 \\
\hline 34 & 0.01 & 14.80 & 0.45 & 1.08 & 19.75 & 1.39 & 126.61 & 260.54 & 540.76 & 1027.29 & 1518.68 & 2543.17 & 4241.86 & 3583.29 \\
\hline 35 & 5 & 12.27 & 0.18 & 0.82 & 10.61 & 11.55 & 71.81 & 140.99 & 264.95 & 496.57 & 795.48 & 1425.96 & 2428.98 & 2192.30 \\
\hline 36 & 0.02 & 15.30 & 0.90 & 4.21 & 25.35 & 19.62 & 109.79 & 205.12 & 403.12 & 646.06 & 1022.66 & 1680.23 & 3007.43 & 2781.71 \\
\hline 37 & 0.02 & 37.82 & 0.89 & 3.23 & 34.44 & 5.37 & 199.07 & 390.16 & 738.62 & 1274.06 & 1809.70 & 2909.91 & 4609.01 & 3862.07 \\
\hline
\end{tabular}




\begin{tabular}{|c|c|c|c|c|c|c|c|c|c|c|c|c|c|c|}
\hline 38 & 0.02 & 15.22 & 0.95 & 4.10 & 24.87 & 16.68 & 91.72 & 157.41 & 326.50 & 576.83 & 942.49 & 1673.36 & 3039.39 & 2583.62 \\
\hline 39 & 9 & 14.45 & 0.42 & 1.86 & 31.39 & 3.39 & 160.86 & 335.80 & 638.29 & 1082.72 & 1718.86 & 2851.37 & 5049.75 & 4111.23 \\
\hline 40 & 0.21 & 14.76 & 0.69 & 3.45 & 24.09 & 17.12 & 129.70 & 220.33 & 405.42 & 643.90 & 1000.76 & 1837.30 & 3058.80 & 2713.63 \\
\hline 41 & 0.01 & 22.17 & 0.21 & 2.00 & 14.55 & 12.80 & 91.71 & 180.89 & 358.47 & 667.69 & 1092.50 & 2059.01 & 3741.82 & 3256.15 \\
\hline 42 & 2 & 25.52 & 0.80 & 3.58 & 26.59 & 2.91 & 157.06 & 367.36 & 743.55 & 1226.49 & 1947.67 & 3247.63 & 5367.05 & 4272.83 \\
\hline 43 & 0.05 & 23.87 & 1.96 & 6.90 & 60.20 & 8.36 & 258.26 & 471.15 & 873.20 & 1439.57 & 2249.04 & 3693.75 & 5914.74 & 4740.67 \\
\hline 44 & 0.01 & 23.71 & 0.60 & 2.98 & 27.00 & 3.98 & 118.99 & 234.14 & 458.13 & 813.73 & 1204.71 & 1984.42 & 3303.04 & 2724.34 \\
\hline 45 & 5 & 11.11 & 0.23 & 1.02 & 12.37 & 9.15 & 62.53 & 134.12 & 246.84 & 477.14 & 734.83 & 1436.61 & 2622.27 & 2632.08 \\
\hline 46 & 0.01 & 42.73 & 0.25 & 4.59 & 43.08 & 3.96 & 248.58 & 507.20 & 960.05 & 1548.93 & 2355.59 & 3805.10 & 6076.02 & 5100.18 \\
\hline 47 & 0.02 & 36.17 & 0.93 & 2.58 & 48.60 & 2.93 & 230.32 & 501.19 & 942.89 & 1619.88 & 2517.69 & 4072.98 & 6527.53 & 5340.94 \\
\hline 48 & 0.48 & 11.71 & 0.35 & 2.02 & 18.60 & 2.26 & 125.04 & 263.64 & 533.83 & 895.19 & 1423.83 & 2570.06 & 4261.65 & 3443.47 \\
\hline 50 & 0.01 & 22.41 & 0.47 & 1.64 & 22.36 & 2.75 & 124.88 & 271.28 & 501.22 & 791.70 & 1230.78 & 2172.10 & 3621.28 & 2878.33 \\
\hline 51 & 1 & 15.90 & 0.23 & 1.02 & 15.15 & 12.82 & 62.71 & 121.12 & 234.52 & 463.21 & 790.54 & 1489.90 & 2793.04 & 2390.76 \\
\hline 52 & 0.01 & 7.31 & 0.37 & 1.26 & 6.42 & 14.47 & 57.62 & 132.31 & 220.71 & 400.35 & 635.45 & 1158.08 & 2261.15 & 1861.60 \\
\hline 53 & 3 & 10.29 & 0.47 & 2.11 & 16.60 & 15.38 & 89.26 & 160.49 & 348.44 & 649.22 & 1003.32 & 1863.65 & 2889.33 & 2837.91 \\
\hline 54 & 0.02 & 34.10 & 0.83 & 4.11 & 28.68 & 6.32 & 171.57 & 367.57 & 689.53 & 1177.62 & 1732.30 & 2619.20 & 4290.93 & 3665.42 \\
\hline 55 & 26.56 & 81.76 & 18.09 & 24.43 & 91.80 & 10.25 & 386.84 & 752.96 & 1456.27 & 2295.29 & 3262.25 & 5113.97 & 7988.61 & 6684.00 \\
\hline 56 & 6 & 21.31 & 0.86 & 3.87 & 36.28 & 4.65 & 202.78 & 468.25 & 888.45 & 1582.28 & 2326.37 & 3808.89 & 6414.82 & 5365.36 \\
\hline 57 & 0.02 & 39.03 & 0.90 & 6.65 & 57.74 & 5.15 & 278.80 & 500.99 & 944.41 & 1535.43 & 2399.06 & 3688.24 & 6064.47 & 4978.98 \\
\hline 58 & 0.01 & 42.80 & 0.56 & 4.63 & 56.68 & 2.78 & 249.71 & 479.14 & 861.92 & 1461.56 & 2118.49 & 3367.40 & 5409.12 & 4529.00 \\
\hline 59 & 0.02 & 33.03 & 0.88 & 4.30 & 52.78 & 3.05 & 285.40 & 590.26 & 1143.47 & 1921.14 & 2868.84 & 4713.43 & 7910.33 & 6522.57 \\
\hline 60 & 0.03 & 35.08 & 1.17 & 9.90 & 57.86 & 12.62 & 228.74 & 471.57 & 815.97 & 1332.77 & 1934.61 & 3156.51 & 4926.02 & 4053.65 \\
\hline 61 & 0.00 & 24.41 & 0.17 & 3.95 & 31.14 & 2.55 & 133.05 & 268.28 & 547.05 & 882.24 & 1341.89 & 2160.49 & 3554.14 & 2950.16 \\
\hline 62 & 0.02 & 24.76 & 0.79 & 1.32 & 17.78 & 16.95 & 97.20 & 173.95 & 383.52 & 685.86 & 1043.40 & 1782.53 & 3065.58 & 2934.70 \\
\hline 63 & 0.02 & 41.65 & 0.93 & 4.04 & 49.19 & 4.85 & 215.22 & 465.04 & 847.70 & 1363.47 & 2052.76 & 3161.75 & 4819.17 & 4229.26 \\
\hline 66 & 0.84 & 42.73 & 2.73 & 8.67 & 51.74 & 22.69 & 229.52 & 498.89 & 894.33 & 1529.90 & 2210.98 & 3529.47 & 5771.81 & 4954.35 \\
\hline 67 & 3.97 & 23.24 & 3.87 & 13.28 & 50.98 & 12.18 & 218.17 & 428.79 & 791.90 & 1342.68 & 2056.43 & 3343.12 & 5487.37 & 4859.06 \\
\hline
\end{tabular}




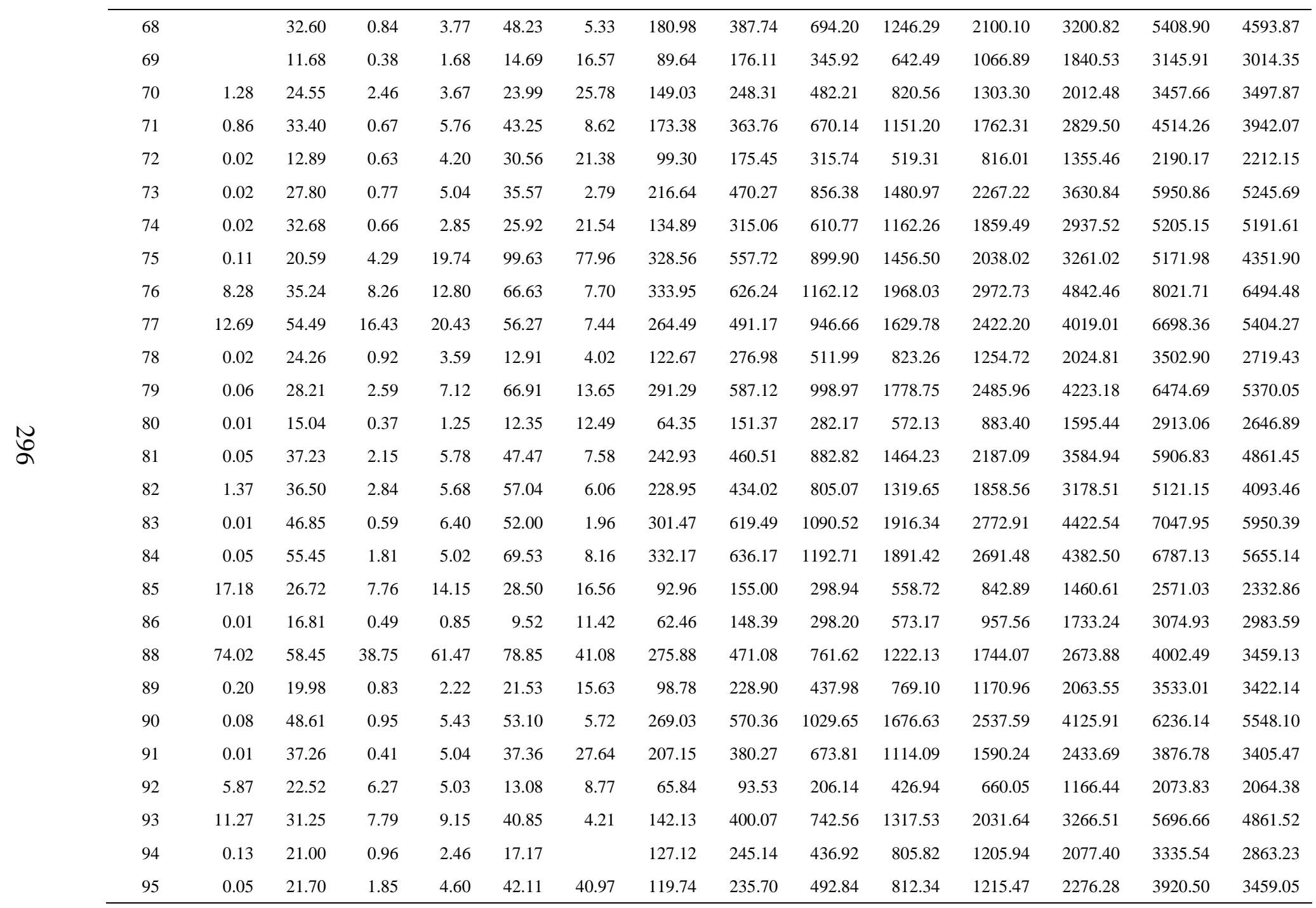




\begin{tabular}{|c|c|c|c|c|c|c|c|c|c|c|c|c|c|c|}
\hline 96 & 0.04 & 23.63 & 1.77 & 4.90 & 36.98 & 28.90 & 121.55 & 265.58 & 437.85 & 710.66 & 1147.48 & 1939.17 & 3527.64 & 3033.19 \\
\hline 97 & 0.28 & 44.08 & 5.05 & 16.77 & 69.88 & 67.08 & 294.43 & 481.74 & 858.20 & 1355.40 & 2007.66 & 3295.50 & 6086.75 & 5036.76 \\
\hline 98 & 0.05 & 67.48 & 1.87 & 5.46 & 75.59 & 7.08 & 323.11 & 695.04 & 1277.52 & 2222.21 & 3226.85 & 5140.46 & 8431.41 & 6700.46 \\
\hline 99 & 0.01 & 34.75 & 0.56 & 3.65 & 26.92 & 7.27 & 178.98 & 383.50 & 733.98 & 1180.14 & 1777.41 & 2842.55 & 4577.00 & 3687.02 \\
\hline 100 & 0.07 & 17.08 & 2.83 & 16.28 & 69.86 & 95.53 & 278.87 & 471.09 & 729.21 & 1206.21 & 1746.00 & 2804.50 & 4681.85 & 3780.97 \\
\hline 101 & 3.35 & 30.05 & 1.48 & 4.02 & 29.42 & 27.15 & 123.52 & 306.13 & 521.95 & 911.31 & 1512.12 & 2646.06 & 4483.20 & 4100.83 \\
\hline 102 & 0.02 & 13.19 & 0.79 & 0.76 & 12.16 & 11.81 & 61.25 & 134.67 & 220.95 & 423.53 & 711.16 & 1262.38 & 2314.02 & 2315.46 \\
\hline 103 & 0.01 & 39.84 & 0.32 & 6.33 & 38.33 & 4.03 & 241.70 & 505.79 & 952.64 & 1518.23 & 2240.30 & 3568.23 & 5633.91 & 4703.39 \\
\hline 104 & 0.02 & 44.24 & 0.97 & 3.12 & 57.85 & 4.35 & 280.93 & 536.80 & 975.42 & 1637.52 & 2390.12 & 3614.21 & 5805.08 & 5087.37 \\
\hline 106 & 0.03 & 7.63 & 1.19 & 5.85 & 43.65 & 33.41 & 137.15 & 241.81 & 408.90 & 673.45 & 964.88 & 1632.97 & 2585.30 & 2493.86 \\
\hline 107 & 07 & 22.34 & 0.61 & 2.71 & 21.66 & 2.33 & 134.65 & 265.43 & 525.93 & 880.61 & 1402.78 & 2163.74 & 3428.56 & 3092.95 \\
\hline 108 & 0.03 & 43.84 & 1.05 & 6.05 & 60.76 & 6.04 & 288.09 & 567.85 & 1053.32 & 1749.80 & 2528.41 & 4177.65 & 6902.57 & 5620.39 \\
\hline 109 & 0.01 & 25.54 & 0.55 & 2.17 & 19.59 & 18.96 & 94.92 & 204.70 & 435.38 & 806.68 & 1273.99 & 2249.02 & 4112.36 & 3538.58 \\
\hline 110 & 0.72 & 30.79 & 1.78 & 4.21 & 37.60 & 4.40 & 164.16 & 339.94 & 600.09 & 1054.14 & 1578.09 & 2571.64 & 4199.18 & 3259.70 \\
\hline 111 & 11 & 17.37 & 0.32 & 1.42 & 14.23 & 14.97 & 71.41 & 157.47 & 318.78 & 612.60 & 995.16 & 1839.32 & 3426.46 & 3041.04 \\
\hline 112 & 12 & 9.11 & 0.35 & 1.56 & 8.53 & 13.40 & 63.35 & 102.84 & 205.97 & 381.38 & 685.53 & 1227.67 & 2386.61 & 2004.18 \\
\hline 113 & 0.04 & 25.10 & 1.64 & 6.79 & 53.28 & 42.07 & 200.75 & 327.68 & 601.13 & 928.82 & 1442.35 & 2328.07 & 4011.83 & 3524.91 \\
\hline 114 & 0.01 & 42.98 & 0.52 & 8.23 & 52.72 & 4.21 & 288.16 & 594.32 & 1106.57 & 1784.87 & 2661.76 & 4439.02 & 7626.49 & 6183.89 \\
\hline 115 & 4.15 & 17.07 & 2.60 & 4.24 & 22.70 & & 149.35 & 306.92 & 590.21 & 1014.66 & 1522.37 & 2614.91 & 4413.88 & 3886.35 \\
\hline 116 & 0.30 & 13.44 & 0.65 & 3.03 & 24.98 & 8.26 & 118.65 & 239.58 & 453.40 & 815.54 & 1271.47 & 2171.87 & 3477.73 & 3151.31 \\
\hline 117 & 0.38 & 10.60 & 1.46 & 3.91 & 34.32 & 27.74 & 156.66 & 290.76 & 496.87 & 784.11 & 1176.53 & 1843.04 & 3081.86 & 2709.32 \\
\hline
\end{tabular}




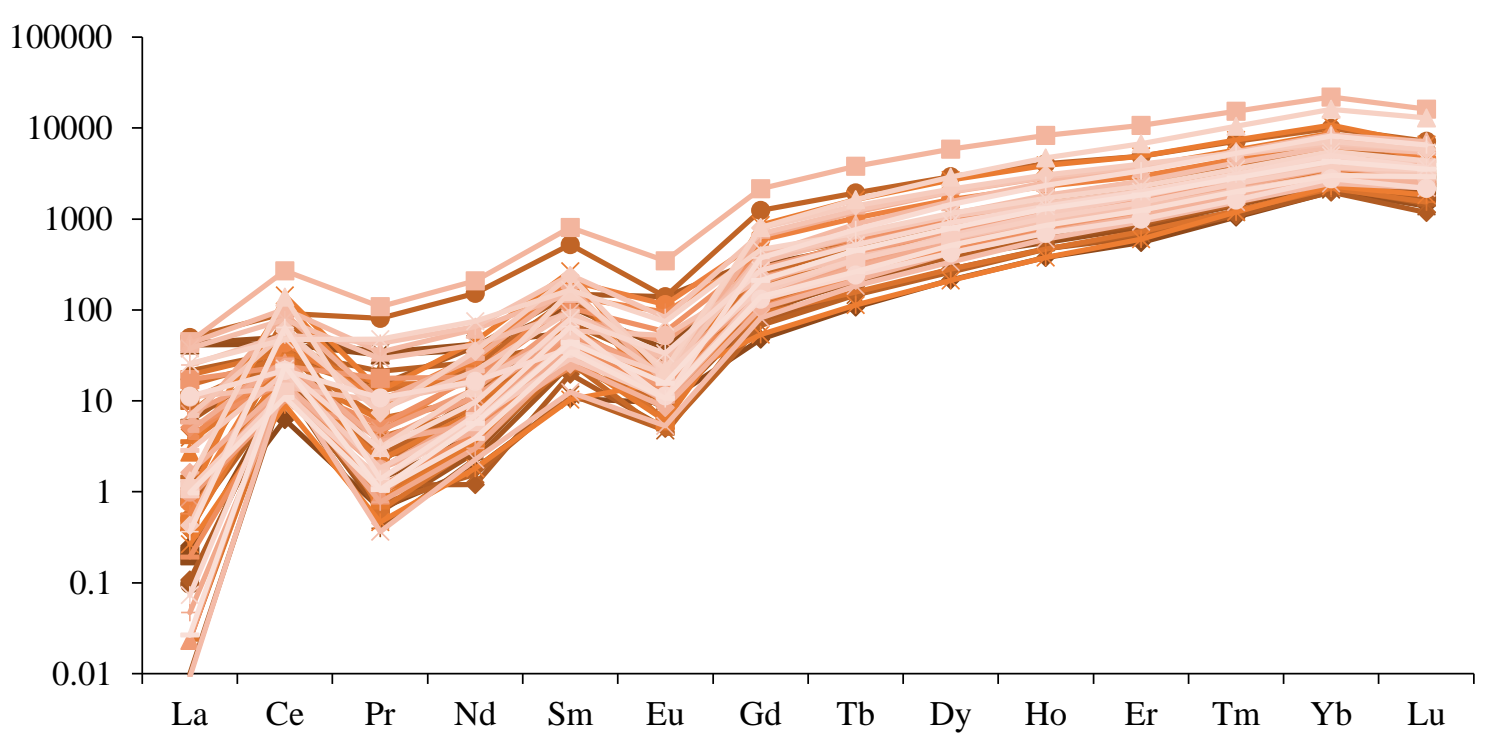

Fig. F4 C1 chondrite normalized (Sunn and McDonough, 1989) REE spider diagram for zircons from sample $\mathrm{CH} 07 \mathrm{a}$. Values plotted here are those listed in Table 4E. Compositional data was obtained via laser ablation inductively coupled plasma mass spectrometry (LA-ICPMS). Overall, the values increase from La to Lu. The values show the greatest spread at La. There are notable peaks at $\mathrm{Ce}$ and $\mathrm{Sm}$ and troughs at Pr and Eu.

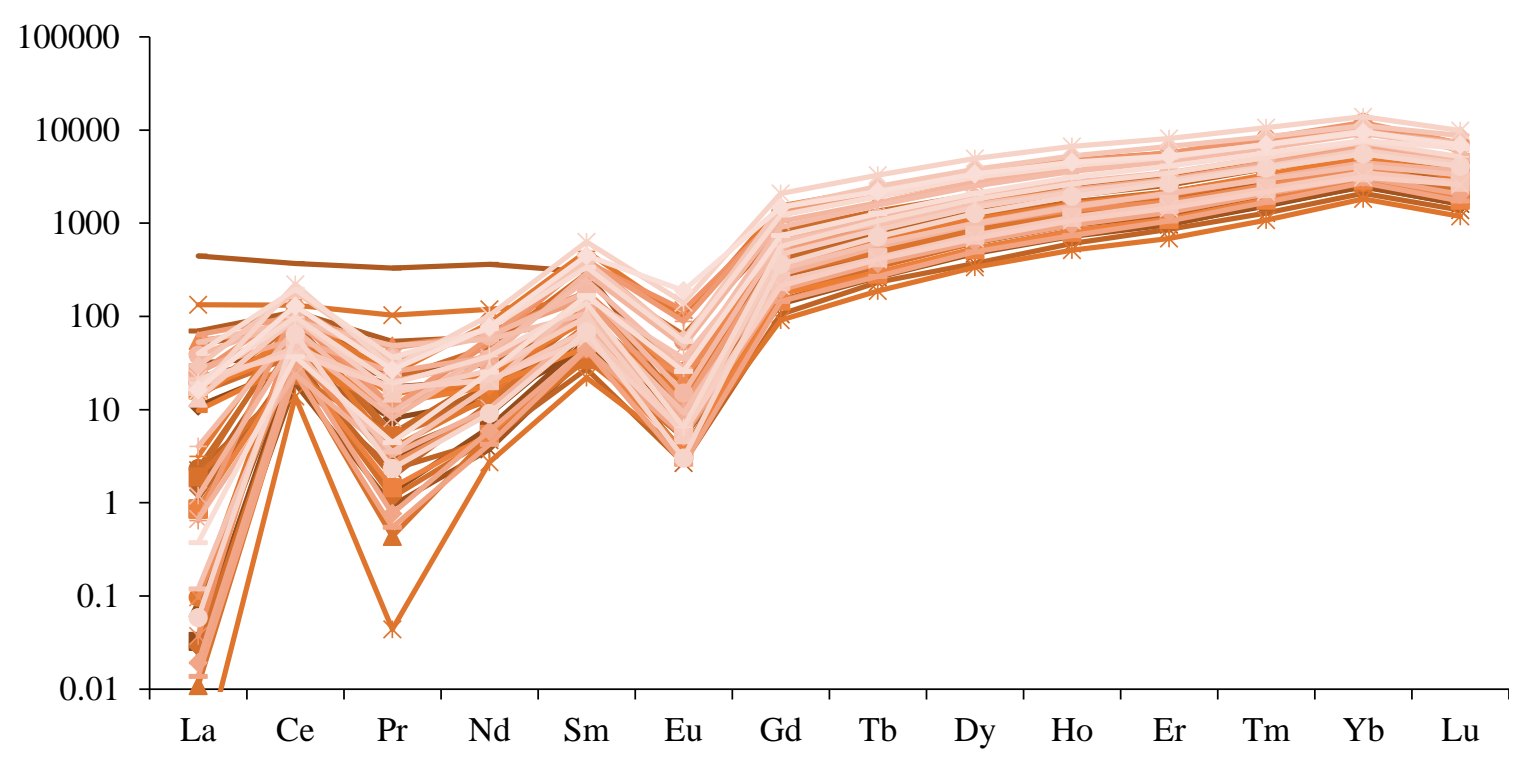

Fig. F5 C1 chondrite normalized (Sunn and McDonough, 1989) REE spider diagram for zircons from sample CH08a. Values plotted here are those listed in Table 4E. Compositional data was obtained via laser ablation inductively coupled plasma mass spectrometry (LA-ICPMS). Overall, the values increase from La to Lu. The values show the greatest spread at La. There are notable peaks at $\mathrm{Ce}$ and $\mathrm{Sm}$ and troughs at Pr and Eu. 


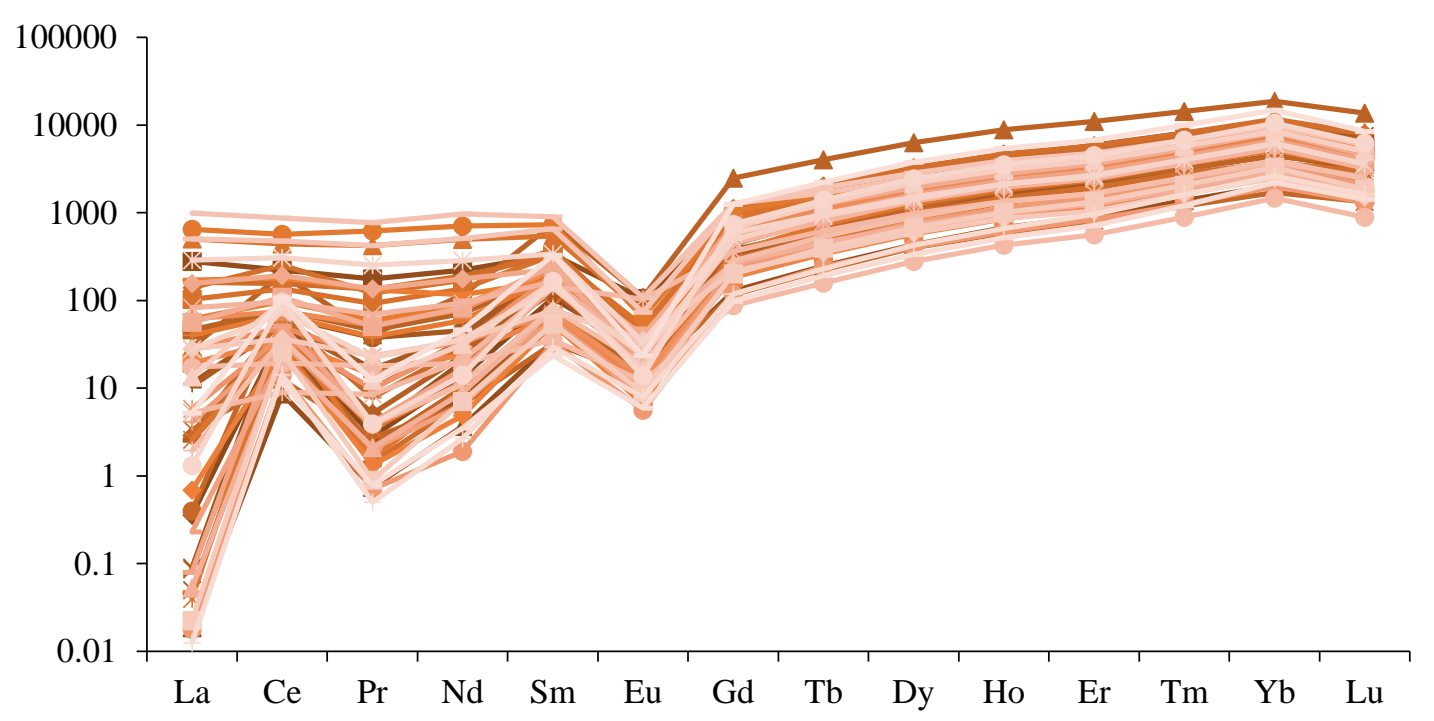

Fig. F6 C1 chondrite normalized (Sunn and McDonough, 1989) REE spider diagram for zircons from sample TM5. Values plotted here are those listed in Table 4E. Compositional data was obtained via laser ablation inductively coupled plasma mass spectrometry (LA-ICPMS).

Overall, the values increase from $\mathrm{La}$ to $\mathrm{Lu}$. The values show the greatest spread at $\mathrm{La}$. There are notable peaks at $\mathrm{Ce}$ and $\mathrm{Sm}$ and troughs at $\mathrm{Pr}$ and $\mathrm{Eu}$. 


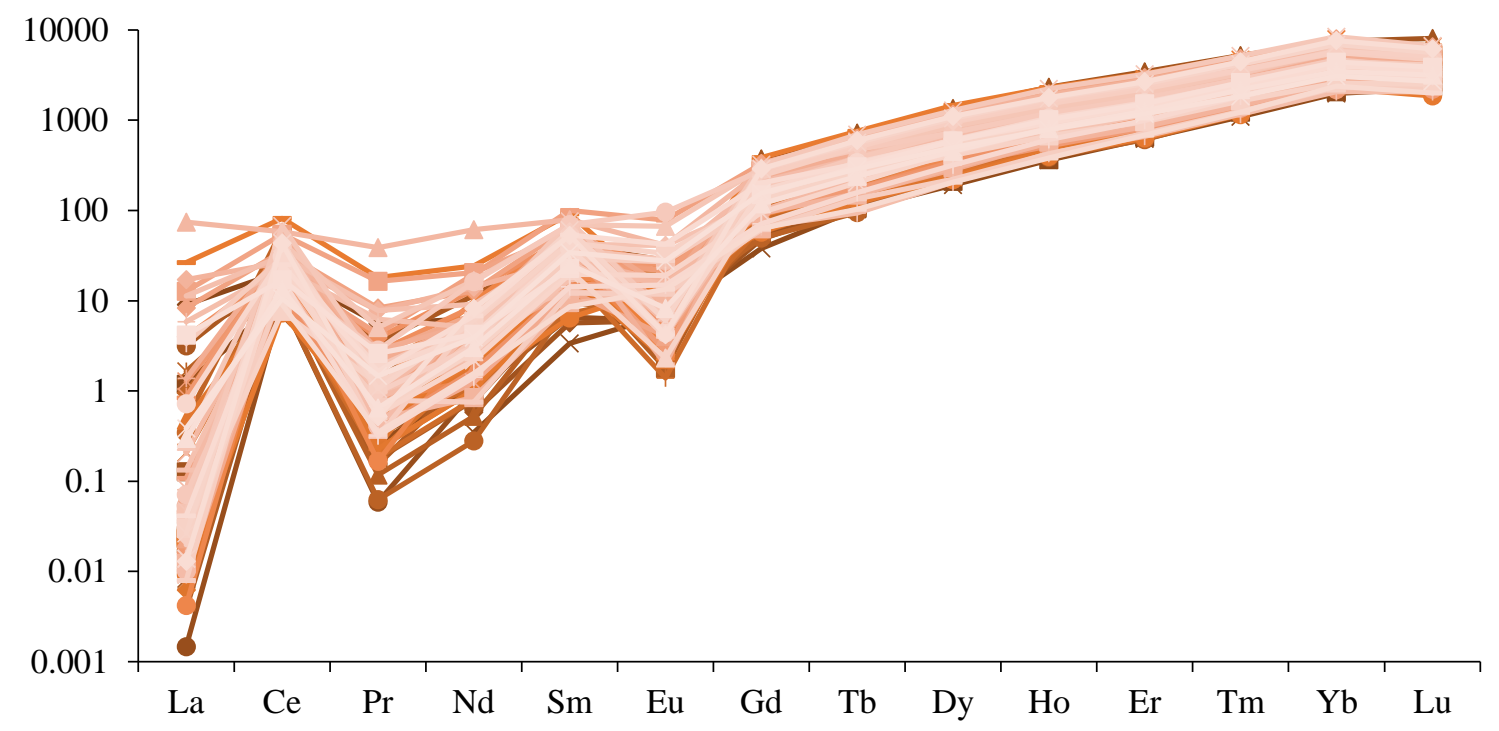

Fig. F7 C1 chondrite normalized (Sunn and McDonough, 1989) REE spider diagram for zircons from sample MS12-47. Values plotted here are those listed in Table 4E. Compositional data was obtained via laser ablation inductively coupled plasma mass spectrometry (LA-ICPMS). Overall, the values increase from $\mathrm{La}$ to $\mathrm{Lu}$. The values show the greatest spread at $\mathrm{La}$. There are notable peaks at $\mathrm{Ce}$ and $\mathrm{Sm}$ and troughs at $\mathrm{Pr}$ and Eu. 
Table F5. Ti-in-zircon formation temperatures

\begin{tabular}{|c|c|c|c|c|c|c|c|}
\hline Sample* & ${ }^{\circ} \mathrm{C}$ & Sample $^{*}$ & ${ }^{\circ} \mathrm{C}$ & Sample $^{*}$ & ${ }^{\circ} \mathrm{C}$ & Sample* & ${ }^{\circ} \mathrm{C}$ \\
\hline CH07a & & CH08a & & TM5 & & MS12-47 & \\
\hline 1 & 711 & 19 & 681 & 50 & 693 & 1 & 659 \\
\hline 2 & 661 & 21 & 655 & 52 & 763 & 2 & 708 \\
\hline 3 & 1104 & 23 & 697 & 53 & 715 & 3 & 692 \\
\hline 4 & 823 & 24 & 757 & 54 & 672 & 4 & 653 \\
\hline 5 & 779 & 25 & 656 & 55 & 697 & 5 & 687 \\
\hline 6 & 720 & 27 & 670 & 56 & 701 & 6 & 664 \\
\hline 7 & 703 & 28 & 679 & 58 & 653 & 7 & 687 \\
\hline 8 & 704 & 29 & 662 & 59 & 666 & 8 & 720 \\
\hline 9 & 791 & 30 & 676 & 60 & 1140 & 9 & 747 \\
\hline 10 & 687 & 31 & 657 & 61 & 719 & 10 & 682 \\
\hline 11 & 731 & 33 & 628 & 62 & 672 & 11 & 671 \\
\hline 12 & 710 & 36 & 653 & 63 & 747 & 12 & 649 \\
\hline 14 & 673 & 37 & 661 & 64 & 681 & 13 & 711 \\
\hline 15 & 693 & 38 & 677 & 66 & 742 & 14 & 700 \\
\hline 16 & 1203 & 39 & 688 & 67 & 697 & 15 & 676 \\
\hline 17 & 759 & 40 & 788 & 68 & 716 & 16 & 741 \\
\hline 69 & 838 & 42 & 723 & 107 & 729 & 17 & 698 \\
\hline 71 & 658 & 43 & 675 & 108 & 672 & 18 & 691 \\
\hline 72 & 673 & 44 & 659 & 109 & 847 & 19 & 719 \\
\hline 74 & 668 & 45 & 756 & 110 & 721 & 20 & 631 \\
\hline 75 & 643 & 46 & 668 & 111 & 1885 & 21 & 695 \\
\hline 76 & 761 & 47 & 693 & 114 & 772 & 22 & 660 \\
\hline 78 & 696 & 48 & 664 & 115 & 708 & 23 & 686 \\
\hline 79 & 670 & 92 & 737 & 117 & 722 & 24 & 667 \\
\hline 80 & 708 & 93 & 698 & 118 & 735 & 25 & 732 \\
\hline 81 & 697 & 94 & 666 & 121 & 849 & 26 & 656 \\
\hline 83 & 687 & 95 & 664 & 122 & 665 & 27 & 661 \\
\hline 84 & 612 & 96 & 738 & 123 & 782 & 28 & 727 \\
\hline 85 & 696 & 97 & 644 & 124 & 669 & 29 & 659 \\
\hline 86 & 829 & 98 & 671 & 125 & 663 & 30 & 721 \\
\hline 87 & 701 & 99 & 727 & 126 & 683 & 31 & 707 \\
\hline 88 & 667 & 100 & 753 & 127 & 704 & 32 & 656 \\
\hline 89 & 714 & 101 & 701 & 128 & 718 & 33 & 731 \\
\hline 90 & 791 & 102 & 776 & 183 & 681 & 34 & 616 \\
\hline 129 & 743 & 103 & 626 & 184 & 668 & 35 & 729 \\
\hline 130 & 672 & 104 & 645 & 185 & 676 & 36 & 683 \\
\hline 131 & 765 & 105 & 669 & 187 & 657 & 37 & 638 \\
\hline 132 & 654 & 164 & 661 & 188 & 714 & 38 & 785 \\
\hline
\end{tabular}




\begin{tabular}{|c|c|c|c|c|c|c|c|}
\hline 133 & 676 & 165 & 680 & 189 & 634 & 39 & 576 \\
\hline 134 & 771 & 166 & 659 & 191 & 779 & 40 & 791 \\
\hline 135 & 843 & 167 & 709 & 192 & 697 & 41 & 748 \\
\hline 136 & 705 & 168 & 671 & 193 & 703 & 42 & 648 \\
\hline 137 & 652 & 169 & 694 & 194 & 697 & 43 & 645 \\
\hline 138 & 700 & 170 & 659 & 195 & 722 & 44 & 682 \\
\hline 139 & 677 & 171 & 738 & 196 & 654 & 45 & 679 \\
\hline 140 & 657 & 172 & 736 & 197 & 684 & 46 & 659 \\
\hline 141 & 799 & 174 & 667 & 198 & 681 & 47 & 611 \\
\hline 143 & 711 & 175 & 670 & 199 & 696 & 48 & 658 \\
\hline 145 & 643 & 176 & 696 & 200 & 724 & 50 & 644 \\
\hline 146 & 727 & 177 & 781 & 201 & 672 & 51 & 726 \\
\hline 148 & 836 & 178 & 657 & 202 & 609 & 52 & 687 \\
\hline 150 & 1655 & 179 & 654 & 203 & 649 & 53 & 758 \\
\hline 151 & 732 & 180 & 991 & & & 54 & 671 \\
\hline 153 & 691 & 181 & 799 & & & 55 & 672 \\
\hline 154 & 694 & 182 & 773 & & & 56 & 621 \\
\hline 156 & 710 & & & & & 57 & 667 \\
\hline 157 & 752 & & & & & 58 & 667 \\
\hline 158 & 881 & & & & & 59 & 641 \\
\hline 159 & 668 & & & & & 60 & 677 \\
\hline 160 & 663 & & & & & 61 & 640 \\
\hline 161 & 656 & & & & & 62 & 714 \\
\hline 162 & 694 & & & & & 63 & 661 \\
\hline \multirow[t]{17}{*}{163} & 685 & & & & & 66 & 756 \\
\hline & & & & & & 67 & 723 \\
\hline & & & & & & 68 & 676 \\
\hline & & & & & & 69 & 739 \\
\hline & & & & & & 70 & 679 \\
\hline & & & & & & 71 & 658 \\
\hline & & & & & & 72 & 750 \\
\hline & & & & & & 73 & 631 \\
\hline & & & & & & 74 & 769 \\
\hline & & & & & & 75 & 759 \\
\hline & & & & & & 76 & 654 \\
\hline & & & & & & 77 & 672 \\
\hline & & & & & & 78 & 636 \\
\hline & & & & & & 79 & 637 \\
\hline & & & & & & 80 & 768 \\
\hline & & & & & & 81 & 667 \\
\hline & & & & & & 82 & 721 \\
\hline
\end{tabular}




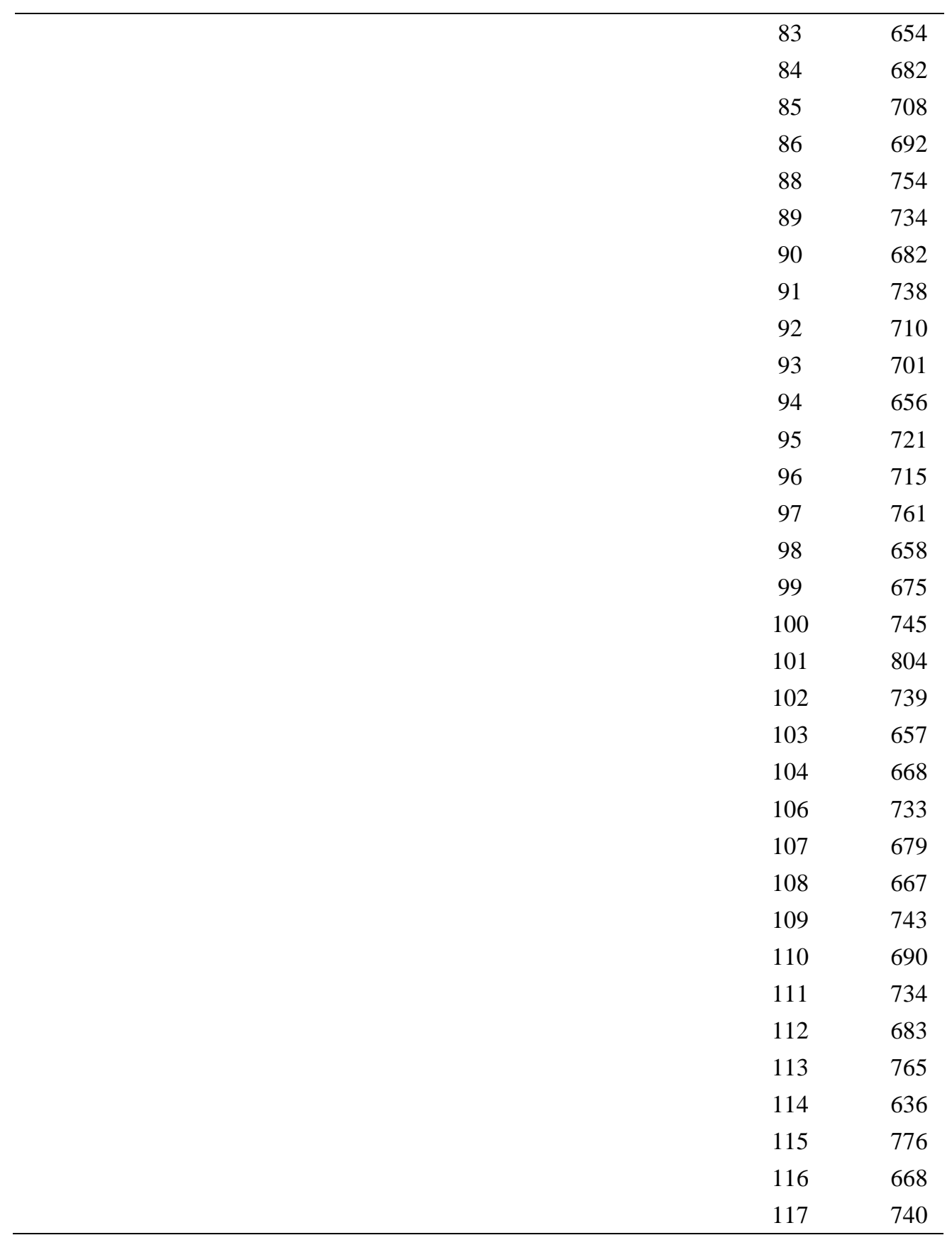

* Laser spot numbers. Spots with data that was incomplete or judged to be unusable due to sampling error (i.e. laser burning through zircon, hitting an inclusion) were removed. 
Table F6. ${ }^{40} \mathrm{Ar} /{ }^{39} \mathrm{Ar}$ age dating

\begin{tabular}{|c|c|c|c|c|c|c|c|c|c|c|}
\hline & $40(a) / 36(a)$ & $\pm 2 \sigma$ & $40(\mathrm{r}) / 39(\mathrm{k})$ & $\pm 2 \sigma$ & $\begin{array}{l}\text { Age } \\
(\mathrm{Ma})\end{array}$ & $\pm 2 \sigma$ & MSWD & $\begin{array}{c}39 \operatorname{Ar}(\mathrm{k}) \\
(\%, \mathrm{n})\end{array}$ & $\mathrm{K} / \mathrm{Ca}$ & $\begin{array}{l} \pm \\
2 \sigma\end{array}$ \\
\hline $\begin{array}{l}\text { Age Plateau } \\
\text { Error Mean }\end{array}$ & & & 10.41868 & $\begin{array}{l} \pm 0.08699 \\
\pm 0.83 \%\end{array}$ & 32.66 & $\begin{array}{l} \pm 0.36 \\
\pm 1.09 \%\end{array}$ & $\begin{array}{r}91.75 \\
0 \%\end{array}$ & $\begin{array}{c}86.96 \\
5\end{array}$ & 10.7 & $\begin{array}{l} \pm \\
3.8\end{array}$ \\
\hline & & & \multicolumn{3}{|c|}{ Minimal External Error } & \pm 0.63 & 2.41 & \multicolumn{3}{|c|}{$2 \sigma$ Confidence Limit } \\
\hline & & & \multicolumn{3}{|c|}{ Analytical Error } & \pm 0.27 & 9.5787 & \multicolumn{3}{|c|}{ Error Magnification } \\
\hline \multirow[t]{3}{*}{ Total Fusion Age } & & & 10.19654 & $\begin{array}{l} \pm 0.00949 \\
\pm 0.09 \%\end{array}$ & 31.97 & $\begin{array}{l} \pm 0.23 \\
\pm 0.71 \%\end{array}$ & & 8 & 9.7 & $\begin{array}{l} \pm \\
0.2\end{array}$ \\
\hline & & & \multicolumn{3}{|c|}{ Minimal External Error } & \pm 0.56 & & & & \\
\hline & & & & \multicolumn{2}{|c|}{ Analytical Error } & \pm 0.03 & & & & \\
\hline \multirow[t]{3}{*}{$\begin{array}{l}\text { Normal Isochron } \\
\text { No Convergence }\end{array}$} & \multirow[t]{3}{*}{218.54} & $\begin{array}{l} \pm 73.06 \\
\pm 33.43 \%\end{array}$ & 10.53987 & $\begin{array}{l} \pm 0.13330 \\
\pm 1.26 \%\end{array}$ & 33.04 & $\begin{array}{l} \pm 0.48 \\
\pm 1.44 \%\end{array}$ & $\begin{array}{r}69.67 \\
0 \%\end{array}$ & \multicolumn{3}{|l|}{$\begin{array}{c}86.96 \\
5\end{array}$} \\
\hline & & & \multicolumn{3}{|c|}{ Minimal External Error } & \pm 0.71 & 2.63 & \multicolumn{3}{|c|}{$2 \sigma$ Confidence Limit } \\
\hline & & & \multicolumn{2}{|r|}{ Analytic } & Error & \pm 0.41 & $\begin{array}{r}8.3471 \\
500 \\
885 \mathrm{E}-05\end{array}$ & $\begin{array}{l}\text { Error Ma } \\
\text { Number } \\
\text { Converge }\end{array}$ & $\begin{array}{l}\text { nificati } \\
\text { f Iterati } \\
\text { nce }\end{array}$ & \\
\hline \multirow{4}{*}{$\begin{array}{l}\text { Inverse Isochron } \\
\text { Error Chron }\end{array}$} & \multirow{3}{*}{234.13} & \pm 67.83 & \multirow{2}{*}{10.51273} & \pm 0.12564 & \multirow{2}{*}{32.95} & \pm 0.45 & 65.31 & \multicolumn{3}{|l|}{86.96} \\
\hline & & $\pm 28.97 \%$ & & $\pm 1.20 \%$ & & $\pm 1.38 \%$ & $0 \%$ & \multicolumn{2}{|l|}{5} & \\
\hline & & & \multicolumn{3}{|c|}{ Minimal External Error } & \pm 0.69 & 2.63 & \multicolumn{3}{|c|}{$2 \sigma$ Confidence Limit } \\
\hline & & & & \multicolumn{2}{|c|}{ Analytical Error } & \pm 0.39 & $\begin{array}{r}8.0817 \\
7\end{array}$ & \multicolumn{3}{|c|}{$\begin{array}{l}\text { Error Magnification } \\
\text { Number of Iterations }\end{array}$} \\
\hline
\end{tabular}


5.24E-08 Convergence

8\% Spreading Factor 